Hers 


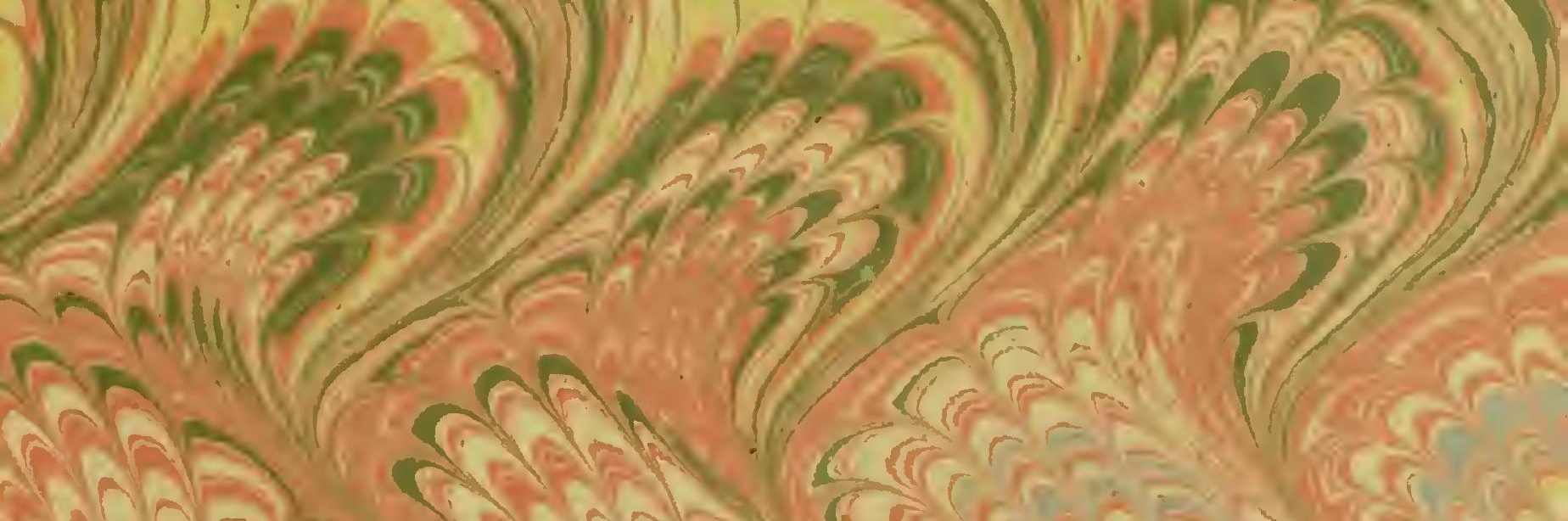
Man

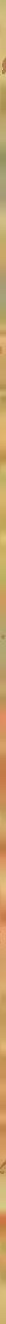

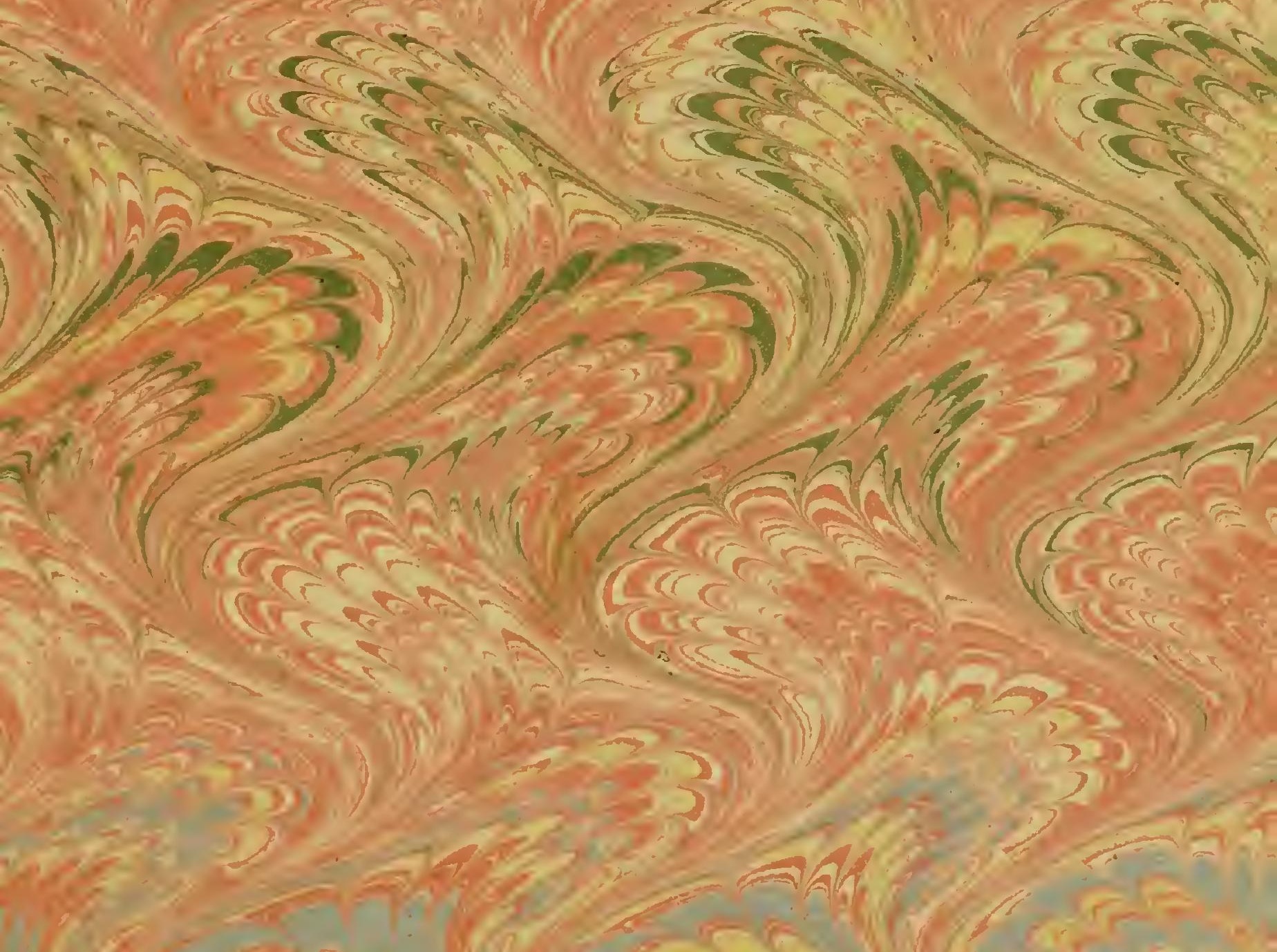







\title{
LES PLANTES
}

\author{
DANS LANTIQUITÉ ET AU MOYEN AGE
}




\section{PUBLIGATIONS PRINGIPALES DU MËME AUTEUR}

La loi des finales en espagnol. Nogent-le-Rotrou, 1872, in-8.

Du c dans les langues romanes. Paris, 1874, in-8.

De rhotacismo in indocuropacis ac praesertim in germanicis linguis. Parisiis, 1875 , in-8.

Herder et la Renaissance littéraire en Allemagne au $\mathrm{xr}^{\mathrm{r}}{ }^{\mathrm{e}}$ siècle. Paris, 1875 , in- 8 .

Épuisé.

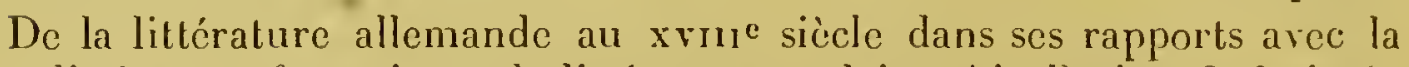
littérature française et la littérature anglaise. Aix-Paris, 1876, in-8.

Essai sur le patois du Bessin, suivi d'un dictionnaire étymologique. Paris, 1881, in-8.

Épuisé.

Du caractère et de l'extension du patois normand. Étude de phonétique et d'ethnographic, suivic d'une carte. Paris, 1883, in-8.

Mélanges de phonétique normande. Paris, $18 \&_{4}$, in- 8 .

Des rapports intellectuels et littéraires de la France arec l'Allemagne avant 1789. Paris, 188́, in-8.

Épuisć.

J.-B. Tavernier, ćcuyer, baron d'Aubonne, chambelian du GrandÉlecteur. Paris, 1886, in-8.

La Flore populaire de la Normandie. Caen-Paris, 1887, in-8.

Les incantations botaniques du manuscrit F. 277 de la Bibliothèque de l'École de Médecine de Montpellicr, etc. Paris, 1888, in-8.

Le voyageur Tavernier d'après des documents inédits. (1670-1685). Paris, 1890, in-8.

Le P. Guevarre et les bureaux de charité au $x_{\text {vil }}$ e siècle. Paris, 1889-1890, in-8.

Pierre et Nicolas Formont. Un banquier et un correspondant du Grand-Électeur. Paris, 1890 , in-8.

La légende de la Rose au moyen àge clıez les nations romanes et germaniques. Màcon, 1891, in-8.

La Rose dans. l'antiquité el au moyen àge. Histoire, légendes et symbolisme. Paris, 1892, in-8.

J. de Séranon, orateur, voyageur, archéologue, historien. Aix, 1893. in-8.

Fabri de Peiresc, humaniste, archéologue, natủaliste. Aix, 1894, in-8,

Les Jardins dans l'Égypte ancienne. Le Puy, 1894, in-8.

De la représentation du papyrus sur les monuments de l'Égyple ancienne. Mícon, 1895, ill-8.

Caen et Rouen. Étude étymologique. Caen, 1895, in-8.

Le comte du Manoir et la cour de Weimar. Paris, 1896, in-8.

CHARTRES. - IMPHMERIE DURAND, RUE FULBLIT. - 1. VI. 97. 


\section{LES PLANTES}

\section{DANS L'ANTIQUITE ET AU MOYEN AGE}

HISTOIRE, USAGES ET SYMBOLISME

PREMIERE PARTIE

LES PIANTES DANS J'ORIENT GLASSTQUE:

I

Égypte, Chaldée, Assyrie, Judée, Phénicie

PAB

Citartes JORET

Profosseur a l'Université d' $\Lambda \mathrm{ix}$

Correspondant de l'Institut

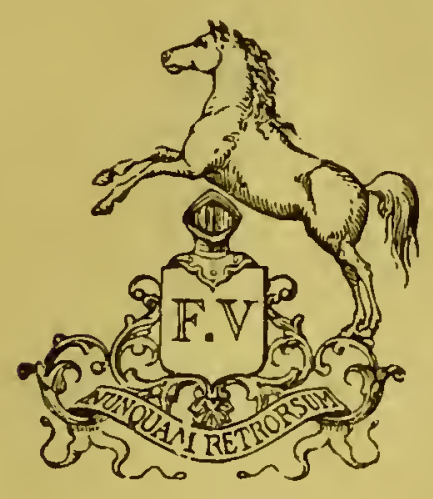

PARIS

LIBRAIRIE ÉMILE BOUILLON, ÉDITEUR

67, RUE DE RICHELIEU, AU PREMIER

1897

rOUS DHOITS MĹSERVÉS 
Galears

(2) $A Q, 2$ 


\section{PRÉFAGE}

"Les arbres, dit Pline au douzième livre de son Histoire naturelle ${ }^{1}$, par leurs sucs plus agréables que les céréales, ont adouci la nature de l'homme. Ce sont eux qui fournissent la liqueur de l'olive, qui assouplit les membres, le vin, qui ranime les forces; chaque année ils produisent spontanément et en abondance les fruits savoureux, qui composent le second service de nos tables... Les arbres sont employés en outre à mille usages indispensables à la vie. Avec eux nous sillonnons les mers et nous rapprochons ainsi les pays éloignés; arec eux nous construisons nos édifices; avec le bois des arbres on fait aussi ou on a fait longtemps les statues des Dieux."

Ce que Pline dit ici des arbres, il aurait, avec plus de raison encore, pu le dire du règne végétal pris dans son ensemble; sans le monde des plantes, la vie humaine ne serait pas possible; elles en sont les auxiliaires les plus utiles et la condition première; sans la culture des plantes alimentaires, comme sans l'élevage des animaux domestiques, l'homme serait fatalement resté à l'état sauvage; l'invention de l'agriculture

1. Chapitre II (1). 
a marqué ses débuts dans la civilisation, et chaque nouvelle plante qu'il a acclimatée ou appropriée à son usage a contribué à accroitre son bien-être, et lui a fait faire un pas nouveau dans la voie du progrès.

Ce ne sont pas seulement des aliments fortifiants, des breuvages délicieux ou des matériaux de construction, que les végétaux, comme le remarquait Pline, nous procurent; ils nous fournissent encore les tissus dont nous nous revêtons, les condiments qui assaisomnent nos mets; leurs fleurs parent nos demeures et les temples de la divinité; leurs parfums les embaument, et les matières colorantes qu'ils recèlent servent à teindre nos vêtements; enfin nous leur devons les remèdes les plus efficaces, qui guérissent les maladies dont nous sommes atteints. Comment s'étonner après cela de l'estime dans laquelle les hommes des premiers âges ont tenu les plantes et de la place considérable qu'ils leur ont faite dans leurs croyances, comme dans leur existence réelle!

Dans leur penchant au merveilleux et leur reconnaissance, ils ont vu en elles " un présent de la puissance invisible, qui fait naître et croitre " toutes choses ${ }^{1}$; elles leur sont apparues comme une manifestation de la sagesse divine; ils leur attribuèrent une vie analogue ḋ la leur propre et les regardèrent comme douées, ainsi qu'eux-mêmes, de pensée et de sentiment ${ }^{2}$. Tantòt ils rirent dans les plantes des êtres semblables à eux, transformés en végétaux après leur

l. Schweinfurth, De la flore pharaonique. (Bulletin de l'Institut igyptien, 2e série, $n^{\circ} 3$ (an. 1882). Le Caire, 1884. in-8, p. 53.)

2. "Ce sont des êtres, des personnes actives, conscientes, vivantes. "James Darmesteter, IIaurvalâl el Amerelât. Essai sur. la mylhologic de l'Avesta. Paris, 1875, in-8, p. 56. 
mort ou par une intervention divine, mais ayant conservé quelque chose de leurs attributions premières; tantòt ils crurent qu'un génie ou un démon particulier résidait en elles, qu'il en faisait du moins son séjour habituel, qu'elles en étaient le corps, comme il en était à son tour l'âme ou en personnifiait la vie végétative et sensible'. Il n'y arait qu'un pas, et on ne tarda point i le faire, pour roir dans ces symboles de vie des ètres d'une nature supérieure et pour les invoquer comme de véritables divinités.

C'est ainsi que l'histoire des plantes se trouve, dès les premiers temps, mêlée à celle même du genre humain; on les rencontre dans les mythes les plus anciens; elles figurent dans les traditions religieuses et profanes des nations les plus diverses. Les arts leur ont emprunté les motifs de décoration les plus gracieux, la poésie, les fictions les plus ingénieuses et les plus belles comparaisons. En un mot, elles ont leur place marquée dans l'histoire de la civilisation des différents peuples. Mais cette place, on le comprend, est dans un rapport étroit avec le génie et la manière de vivre de chacun d'eux; elle dépend aussi et surtout de la flore particulière à chaque pays; elle varie avec sa richesse ou sa pauvreté en plantes alimentaires ou industrielles, richesse et pauvreté qui varient ellesmêmes avec le climat, la nature du sol, l'altitude, le degré plus ou moins grand de chaleur, de sécheresse ou d'humidité, circonstances dont l'ensemble constitue le caractère propre de la flore d'une contrée et la distingue de celles des contrées voisines,

Il existe ainsi à la surface du globe un certain

1. Mannhardt, Der Buumcullus der Germanen und ihrer Nachbarstümme. Berlin, 1875, in-8, p. 5. 
nombre de régious naturelles, caractérisées par une flore et par un climat particuliers. A. Grisebach ${ }^{1}$ en a distingué vingt-quatre, dont cinq dans le monde connu des Anciens: la région des moussons, qui lui appartient à peine et qui comprend, outre les îles de la Sonde et l'Indo-Chine, la grande presqu'ile hindoustanique. La région des steppes, qui s'étend de la Chine centrale presque jusqu'aux bords de la Méditerranée, en comprenant le Turkestan dans son entier, le plateau de l'Iran, l'Arménie, la Mésopotamie et la plus grande partie de l'Asie Mineure et de la Syrie ${ }^{2}$. La région saharienne, qui embrasse la contrée brûlante voisine des bouches de l'Indus, la presqu'ile arabique, moins la côte du sud-ouest, et tout le nord de l'Afrique, à l'exception de l'étroite vallée du Nil, de-la Cyrénaïque et de la Mauritanie. Au sud de cette région s'étend celle du Soudan, restée à peu près inconnue des royageur's et des géographes de l'antiquité, raison pour laquelle je ne la compte pas ici. La région méditerranéenne qui embrasse les rivages méridionaux du Pont-Enxin et tous ceux de la Méditerranée, y compris dans leur plus grande partie les presqu'iles balkanique, italique et ibérique, région à laquelle Oscar Drude rattache les rivages océaniens de l'Europe méridionale et de l'Afrique septentrionale, ainsi que la région des steppes de l'Asie antérieure, des còtes de la Syrie et de la mer Égée à la chaîne des monts Sou-

1. Die Vegetation der Erde nach ihrer klimalischen Anordmung. Ein.Abriss der vergleichenden Geographie der Pflansen. Leipzig, $2^{\mathrm{e}}$ éd., 1884, t. I, p. 1.

2. C'est la "Région orientale propremont dite » d'Edmond Boissier, Flora orientalis. Basileae-Genevae, 1867, in-8, t. I, préf., p. vir. 
leiman et à l'Hindokousch '. La région forestière, qui s'étend des rivages septentrionaux de l'Océan atlantique à ceux du Grand Océan, en comprenant presque toute la Gaule, la Bretagne, la Germanie, la Scandinavie, le centre et le nord de la Russie avec la Sibérie, moins les côtes de l'Océan glacial, qui forment la région arctique, restée inconnue des Anciens, ainsi que la plus grande partie de la région des forèts.

Ces diverses régions sont caractérisées chacune par une flore particulière, différente dans son ensemble de celles des contrées voisines par des espèces qui lui sont propres $^{2}$; et dont les conditions climatériques, avec les obstacles, - déserts, montagnes ou mers, - qui l'empêchent de se répandre au delà de son centre de formation, assureraient la persistance, si l'action de l'homme n'intervenait pour en modifier le caractère et en détruire l'unité. A l'exception de la région des tropiques et des moussons, aucune contrée ne renferme assez de plantes alimentaires ou industrielles pour suffire aux besoins d'un peuple arrivé à un certain degré de civilisation; les nations qui ont peuplé les steppes de l'Asie, les forêts de l'Europe ou même les rivages bénis de la Méditerranée, ont dû emprunter pour leur alimentation, leur industrie ou leur agrément, un certain nombre de végétaux à la flore des autres contrées. Mais l'homme ne s'est pas borné à faire " vivre et reproduire sous sa protection" des espèces transportées " loin de leur lieu natal ${ }^{3}$ ", il a anobli par la culture

1. Handbuch der Pflanzengeographie. Stuttgart, 1890, in-8, p. 386.

2. "Divisae arboribus patriae. " Virgile, Georg., lib. II, v. 116.

3. Alphonse de Candolle, Géographie botanique raisonnée. Paris, 1855, in-8, préface, p. xI.

$x$. 
certaines espèces indigènes; il en a abandonné d'autres comme inférieures à des espèces exotiques analogues qui présentent plus d'avantages ${ }^{1}$; enfin il en a détruit d'autres encore par une exploitation excessive ou inintelligente. C'est ainsi que, à part la flore des déserts proprement dits et des steppes stériles, qu'il lui était dès lors impossible de modifier, le défrichement, la culture de plus en plus étendue du sol, enfin l'acclimatation de plantes étrangères, ont changé peu à peu la végétation de la plupart des pays habités.

On l'a bien vu en Europe depuis la découverte de l'Amérique ${ }^{2}$; le peuplier de Virginie (Populus Virginiana Desf.), importé des bords du Missipi et du Missouri, le platane d'Occident et le robinier ou faux-acacia (Robinia pseudo-acacia L.), ce bel arbre aux fleurs si suaves, apportés de la même région que le peuplier de Virginie, ont, avec le peuplier pyramidal - le soidisant peuplier d'Italie - (Populus fastigiata Poir.) et le platane d'Orient (Platanus occidentalis L.), originaires l'un et l'autre de l'Asie antérieure, changé l'aspect de nos campagnes. Le pin de lord Weymouth (Pinus Strobus L.), le catalpa (Bignonia catalpa L.), le tulipier (Liriodendron tulipiferum L.), le magnolier à grandes fleurs (Magnolia grandiflora L.), importés aussi de l'Amérique du Nord sont venus successivement transformer nos jardins et nos parcs; la vigne vierge (Ampelopsis hederacea Michx., Cissus quinquefolia. Pers.), apportée de la même contrée et la capucine (Tro-

1. Alphonse de Candolle, Origine des plantes cultivées. Paris, 1886, in-8, p. 2.

2. Victor Hehn, Kulturpflanzen und Hausthiere in ihrem Uebergang aus Asien nach Griechenland"und Italien sowie in das übrige Europa. Berlin, 1894, in-8, p. 502. 
paeolum majus L.), cette jolie plante du Chili, les ont embellis. L'acacia de Farnèse (Acacia Farnesiana Willd.), le poivrier (Schinus molle L.), l'arbre à corail (Erythrina corallodendron L.), autres végétaux américains de la région des tropiques, ornent de leur feuillage élégant et de leurs fleurs parfumées ou de leurs fruits éclatants les promenades du Midi. L'oponce (Opuntia ficus indica Webb.), vulgairement figuier de Barbarie, et le maguey ou aloès (Agave americana L.), tous deux originaires du Mexique ${ }^{1}$, ont animé d'une vie inconnue les rochers stériles ou les lieux sablonneux et déserts de la région méditerranéenne.

La culture des champs de l'Ancien Monde n'a pas été moins profondément modifiée ou enrichie que celle des jardins et des parcs par les plantes alimentaires importées de l'Amérique, à laquelle il a donné en retour le froment et l'orge. Les habitants de l'Europe néridionale et ceux du bassin de la Méditerranée tout entier ont reçu avec le maïs, ceux de l'Europe centrale, avec la pomme de terre, solanée originaire du Chili, un aliment substantiel et précieux. Une autre solanée, la tomate (Solanumi lycopersicum L.), originaire peut-être du Pérou, leur a procuré encore un condiment agréable et recherché ${ }^{2}$; et l'on sait quel narcotique aussi funeste qu'aimé - le tabac - l'Europe et l'Ancien Monde tout entier ont emprunté aux sauvages dégénérés du Nouveau ${ }^{3}$.

1. A. de Candolle, Orjgine des plantes, p. 218 et 122 . - Id., Géographie botanique, t. II, p. 725 et 739.

2. A. de Candolle, Origine des Plantes, p. 231. Il faudrait y joindre les piments ou poivrons (Capsicum annuum L.).

3. Il n'est question dans ce qui précède que des espèces américaines cultivées, mais bien d'autres plantes du Nouveau 
Ce qui s'est ainsi fait à l'origine des temps modernes, s'était produit déjà au moyen âge, comme dans l'antiquité et dès les premiers siècles de l'histoire. Les peuples de l'Ancien Monde: touraniens, sémites, ariens, pour ne parler que de ceux-là, ont porté avec eux dans leurs migrations quelques-unes des plantes de leurs pays d'origine ou des contrées qu'ils avaient traversées ou successivement habitées; ils ont emprunté à leurs voisins ou aux peuples qu'ils avaient subjugués les plantes alimentaires ou industrielles qu'ils ne possédaient pas. C'est ce que firent, nous le verrons, les Assyriens, quand ils étendirent leurs conquêtes de l'Élam et de la Médie, aux bords de l'Halys et du Nil, les Pharaons d'Égypte dans leurs expéditions au pays de Pount et en Syrie, enfin les Médo-Perses, lorsqu'ils eurent soumis à leur domination le bassin de l'Indus et toute l'Asie occidentale. Les expéditions et les établissements des Phéniciens dans l'Archipel et sur presque toutes les côtes de la Méditerranée portèrent dans ces contrées nombre de plantes utiles, originaires de l'Asie antérieure, dont les colons grecs à leur tour contribuèrent à répandre. encore la culture ou l'emploi dans l'Europe méridionale.

Les" conquêtes d'Alexandre, en mettant en rapport le monde occidental et le monde oriental, achevèrent ce qu'avaient commencé les établissements des Phéniciens et les expéditions des Perses; elles firent connaître en Grèce plusieurs plantes du plateau de l'Iran ou même de l'Inde. De Grèce, où elles furent d'abord

Monde se sont acclimatées dans l'Ancien; A. de Candolle, Géographie bolanique, t. II, p. 723-742, n'en compte pas moins de 49 . 
recues, ces plantes pénétrèrent en Italie, de lì elles se répandirent en Espagne et en Gaule, puis, après notre ère, en Germanie et dans la Grande-Bretagne. Ainsi peu à peu la flore indigène des différents pays du monde connu des Anciens a été transformée par les migrations des peuples qui s'y sont établis, par leurs expéditions guerrières ou les conquètes pacifiques de leurs marchands et de leurs royageurs.

Mais rien n'a plus contribué à enrichir la flore agricole de l'occident que la domination des Arabes '. Déjà dans l'antiquité, les trafiquants de cette nation avaient importé en Égypte et dans l'Asie antérieure quelquesuns des régétaux les plus précieux de la péninsule hindoustanique; devenus maitres d'un empire qui s'étendait des bords de l'Indus aux rivages de l'Atlantique, ils furent les promoteurs d'un échange international sans égal jusque-là, que devaient continuer les croisades et les entreprises maritimes des Vénitiens et des Génois.

Ce sont les Arabes qui ont introduit le coton et la canne i sucre sur les còtes de la Méditerranée; ils y ont apporté le bigaradier et contribué à y répandre le citronnier, le caroubier et le palmier ${ }^{2}$; ils y ont fait connaitre le jasmin sambac, cet arbuste parfumé de l'Inde, qu'ils avaient peut-être déjà révélé à l'Égypte ancienne, et le mar'gousier (Melia azedarach L.), originaire de l'Iran. Les Turcs, qui ont remplacé les Arabes dans la domination de l'Asie antérieure, n'ont pas moins servi que ce peuple à la diffusion des espèces régétales les plus bclles ou les plus utiles ${ }^{3}$. C'est à

1. Victor Hehn, op. laud., p. 497.

2. A. de Candolle, Géographic bolanique, t. II, p. 626.

3. Victor Hehn, op. laud., p. 499. 
eux que nous devons la connaissance du lilas, de l'Hibiscus de Syrie, cette malvacée aux fleurs brillantes, de la rose jaune, de l'hyacinthe d'Orient, qui a donné de si nombreuses rariétés, de la couronne impériale (Fritillaria imperialis L.), belle liliacée de la Perse, apportée, comme la tulipe cultivée et la renoncule d'Asie, des jardins de Constantinople dans l'Europe occidentale. Les Turcs encore ont planté les premiers dans leurs jardins le marronnier d'Inde, quils avaient trouvé dans les montagnes de la Péninsule balkanique et de l'Asie-Mineure, et si ce n'est pas à eux, c'est à un peuple de leur race, les Tartares de Russie, que l'Europe centrale est redevable de la culture du blé noir ou sarrasin ${ }^{1}$ (Polygonum fagopyrum L.), cette polygonacée, originaire de la Sibérie, ainsi que l'espèce qui porte le nom de ce pays.

Tandis que les Turcs propageaient ainsi en Europe les plantes de l'Asie antérieure, les Portugais y importaient celles de la région des moussons et de l'Extrême-Orient; ce sont eux, en particulier, qui y ont fait connaitre l'orange douce ${ }^{2}$. Leur exemple fut suivi par les navigateurs des autres nations, qui pénétrèrent à leur tour dans l'Océan indien et la mer de Chine; c'est ainsi que le mandarinier, originaire de la Cochinchine, le néflier du Japon (Mespilus L. ou Eriobotrya japonica Lindl.), le camélia, venu de la même contrée, les rosiers de Bengale et de Banks³ ${ }^{3}$ ont pénétré

1. A. de Candolle, Giographie bolanique, p. 953-955. Id., Origine des planles cullivées, p. 280.

2. Victor Hehn, op. laud., p. 437. Il faut dire toutefois que De Candolle, après Gallesio, attribue cet honneur aux Génois.

3. Ces deux espèces, ainsi que la rose thé, n'ont été introduites de Chine en Europe que depuis le commencement du siècle. 
successivement dans les jardins de l'Europe tempérée. L'Australie aussi devait en enrichir les promenades de régétaux inconnus ì l'Ancien Monde; il suffit de citer l'eucalyptus, qui s'est si rapidement acclimaté dans la région méditerranéenne.

On le roit, àtoutes les époques, les plantes ont été entre les divers pays l'objet d'échanges continuels; par là, leur histoire est liée étroitement à celle du commerce international. Par le bien-être qu'elles procurent à l'homme, les ressources alimentaires ou industrielles qu'elles lui offrent, les emplois si divers qu'il en fait, elles premment place également dans l'histoire générale de la civilisation, dont elles ont été, surtout dans le passé, les auxiliaires les plus puissants. Dans tous les ordres de l'activité humaine on rencontre leur action salutaire et bienfaisante; leur culture a été l'occupation la plus noble des peuples primitifs, comme elle l'est encore des nations les plus civilisées; leurs légendes comptent parmi les fictions les plus gracieuses, et dans leurs formes élégantes les artistes ont trouré les plus beaux motifs de décoration. Aussi à quelque point de vue qu'on les considère, les plantes méritent de fixer l'attention du penseur et de l'historien comme du naturaliste.

Je ne me propose pas d'en exposer la nomenclature ou d'en étudier les caractères; - c'est là l'œuvre d'un botaniste et qu'un botaniste seul, comme l'a fait M. H. Baillon dans un ouvrage justement estimé ${ }^{1}$, peut entreprendre. - Mon dessein est autre; je voudrais essayer de retracer l'histoire agricole, industrielle, poétique, artistique et pharmacologique des

1. II istoive des plantes. Paris, in-8, 1866-1896, t. I-Xill. 
espèces végétales connues des différentes nations de l'antiquité classique et du moyen àge. Je ne me dissimule pas les difficultés de cette entreprise; elles sont d'autant plus grandes que personne jusqu'ici n'a abordé ce vaste sujet. Victor Hehn a consacré á une soixantaine de plantes des monographies, qui sont des chefs-d'ourre d'analyse el d'érudition; Alphonse de Candolle a étudié, avec un soin et une science qu'on ne saurait trop admirer, l'origine de 447 espèces cultivées; mais ni l'un ni l'autre n'en ont fait connaître les emplois si divers, encore muins le symbolisme. Les historiens de la botanique n'ont point darantage étudié ce còté si plein d'intérêt de la vie des plantes; ils se sont bornés, comme Kurt Sprengel ${ }^{1}$ et Ernest Meyer ${ }^{2}$, pour ne parler que des plus célèbres ${ }^{3}$, à nous apprendre quelles étaient les connaissances botaniques des naturalistes, médecins et agronomes de l'antiquité, comme des temps modernes, et quels progrès ils araient fait faire à la science; ils n'ont point songé à exposer en détail ce qu'a été, aux différentes époques, la culture des plantes alimentaires et d'agrément, ni à rechercher quels emprunts ont faits oü ce que doivent au règne végétal les diverses industries et l'art de

1. Ilistoria rei herbariae. Amstedolami, 1807-1808, 2 vol. in-8. - Geschichte der Bolanik. Leipzig, 1817-18, 2 vol. in-8.

2. Geschichte der Botanili, Studien von Ernst Meyer. Kœenigsberg; 1854-58, 4 vol. in-8. Nalheureusement cette œuvre inagistrale s'arrête à la fin du $\mathrm{XVI}^{\mathrm{c}}$ siècle.

3. Il faut encore citer: Karl F. B. Jessen, Bolanik der Gegenwart und Vorseil in culturhislorischer Entwickelung. Ein Beitrag sur Geschichle der abendlündischen Völker. Leipzig, 186', in-8, résumé excellent dont le titre fait connaitre l'esprit et le but. Quant à l'Histoire de la Bolanique de Ferd. Hoefer, Paris, 1872, in-12, je ne la mentionne que pour ne pas être trop incomplet. 
guérir ${ }^{\prime}$, encore moins les arts du dessin et la poésie; ils ne se sont pas demandé non plus, et ils n'avaient pas, il est vrai, a l'essayer, - c'est affaire au folkloriste, - quelle place le monde si varié des plantes occupe dans les croyances et les légendes des différents peuples. C'est cette lacune que j'ai voulu combler.

Inutile de dire comment, il y a dix ans, au lendemain de la publication de ma Flore populaire de la Normandie, j'ai été amené à entreprendre cetouvrage, devant lequel j'aurais reculé, si j'en avais prévu les développements et l'étendue; depuis lors j'y ai consacré la plus grande partie des loisirs que me laissent mes devoirs professionnels. J'ai beaucoup herborisé autrefois, et il y a plus de quarante ans, je me suis livré à des recherches d'histoire, que l'état de ma santé et d'autres études m'ont forcé pendant longtemps d'interrompre. Arec celte Histoire des Plantes, je suis revenu à ces occupations farorites de ma première jeunesse; je ne forme qu'un vœu, c'est qu'il me soit donné de la conduire à bonne fin. Si je puis achever cet ourrage, ainsi que l'Histoire des rapports intellectuels et littéraires de la France et de l'Allemagne, que j'ai projetée, il y a près de trente ans, et dont j'ai, à plusieurs reprises, publié des fragments ou fait connaitre des épisodes détachés, j'y verrai le couronnement le plus cher d'une vie consacrée, tout entière, à l'étude et à l'enseignement.

1. Inutile de rappeler ici tous les traités spéciaux de matière médicale, dans lesquels on trouve l'énumération des plantes employées dans la pharmacopée; j’ai eu à citer déjà et j'aurai plus tard à parler longuement de celui de Dioscoride; parmi les modernes, je me bornerai à mentionner F. A. Flückiger et D. Hanbury, Ifisloire des drogues d'origine végétale, trad. par le Dr Lanessan. Paris, 1878, 2 vol. in-8. 
Il me reste en terminant à adresser mes remerciements aux personnes qui m'ont aidé de leurs conseils ou soutenu de leurs encouragements; j'en dois de tout particuliers à M. Tictor Loret, l'égyptologue connu de Lyon, qui a bien voulu se charger de reroir les épreuves de la partie de ce volume qui traite des plantes chez les Égyptiens. Je ne puis oublier dans l'expression de ma gratitude mon éditeur, qui n'a pas hésité à entreprendre la publication d'un ouvrage, dont il était encore impossible de prévoir l'étendue, et dont le plan n'était pas même arrêté dans toutes ses parties: puisse l'accueil fait à mon livre récompenser sa généreuse initiative!

Un mot encore au sujet de l'orthographe que j'ai adoptée dans la transcription des noms propres et des mots d'origine étrangère; j'ai suivi en geénéral celle de M. Maspero dans la quatrième édition de son excellente Histoire ancienne des peuples de l'Orient; je ne pouvais prendre un meilleur guide. Comme lui, je représente par ou l'u des textes chaldéo-assyriens et hébraïques et par $k h$ l'h sémitique fortement aspiré, le cheth de l'alphabet hébreu. L'étendue de la matière m'a forcé d'ajourner l'index général, par lequel devait se terminer ce volume; on le trouvera à la fin du second, qui sera consacré à l'histoire des plantes chez les Perses et chez les Hindous et ne tardera pas, je l'espère, à paraître.

Aix, Ler mai 1897. 


\section{ADDITIONS ET CORRECTIONS.}

Page 30, ligne 15, Kussemel, lire : Koussemeth.

Page 3', ligne 7. Le dokhan, dont il est question dans la Bible (Ezechiel, IV, 9), est, non une espèce de sorgho, mais le millet, comme je l'ai dit p. 387 et 389.

Page 50, note '́, Tité,

lire : 'éti.

- 93, ligne 26, Chanan,

- Canaan.

- 106, - 21, en les dëfendant, — en défendant.

- 115, - 20, tainat, - teenah.

- 118, - 13, rimmoun, - rimmon.

- 124, - 3, Delie, - Delile.

- 134, - 24, lappoukh, - tappouakh.

- 143, - 6, $11^{\mathrm{c}}$ degré, $\quad-\quad 21^{\mathrm{c}}$ degré.

Page 155. Je ne crois pas, toute réflexion faite, que le nom asmi doive faire croire à une culture ancienne du Jasminum sambac en Égypte.

Page 163. La plante désignée par l'hẻbr. shoshan parait bien avoir été en général une liliacée ou une iridacée; mais ce mot ne servit sans doute qu'assez tard à désigner le lis blanc et il a été parfois employé comme nom du lotus.

Page 180. Il est à peine besoin de remarquer que la supposition de Wilkinson est absolument arbitraire.

Page 184, ligne 19, siliques, lire : gousses.

- 186, - 3, une épaisse, lire : une espèce.

Page 195. Si l'huile d'olive fut apprèciée, elle ne fut néanmoins guère employée ou l'on n'en fabriqua point en Égypte; les comptes administratifs de Ptolémée Philadelphe n'en font du moins pas mention, tandis qu'ils parlent d'huile de sésame, de ricin, de lin, de cnicus et de coloquinte. Grenfell, Revenue Laws of Plolemy Philadelphus... ediled from a greal Papyrus, etc. Oxford, 1896, in-8, pp. 40, 41, 42, 45, 47, 48, etc.

Pagre 196, liłrne 11, manière, lire : matière.

- 196, note 1, oxyocanthoüles, lire : oxyacanthoïdes.

Page 235. Il va sans dire que si je la mentionne, je n'accepte point l'explication donnée par M. Goodyear de l'origine de la
rosette. 
Page 241, ligne 16, gatlon, lire : gallon.

Page 303, note 2, El Berseh, lire : El Bersheh.

Page 312, note 2. Il faut ajouter, à propos des identifications arbitraires de WIM. Joachim et lüring, que la Pistic stralioles, plante de l'Hindoustan, n'a pu figurer dans la pharmacopée de l'Égypte ancienne et que le Chamacrops humilis, palmier de la région occidentale du bassin de la léditerranée, n'a pas dù y prendre place davantage.

Page 329, lignes 3 et 10, Chypre, lire : Cypre.

- 355, - 11, des arbustes, - les arbustes.

- 362, - 6, Khasdim, - Kasdim.

Pages 378, - 26 et 379, ligne 3, Khonsour, lire : Khousour.

Page 380, - 19, carthamme, lire : carthame.

Pages 319 et 500 . Outre les deux identifications dont j'ai parlé pour l'aspalathe, on en a proposé une autre toute différente; on a voulu y voir une espèce de genêt, le Genisla aspalathoüdes P., - G. accinthoclada DC. d'après Fraas, Synopsis plantarum forae classicae, p. 49; - mais cette papillonacée ne se rapporte point à la description que Pline, XII, 52, a donnée de l'aspalathe. Le mieux est donc d'avouer, avec Ed. Lefèvre dans la Grande Encyclopédie, "qu'on ne connait pas la plante qui fournit le bois d'aspalathe. ”

Page 422, ligne 19, latiforme, lire: lotiforme.

- $472, \quad$ - 25 et 173 , ligne 4 , Ningirsou, lire: Nin-Ghirsour.

On ne trouvera point de figures dans ce volume; pour remédier à cet inconvénient, j'ai renvoyé, toutes les fois que j'ai parlé des mêmes monuments, à l'IIistoire de l'ar'l de II. Perrot, ouvrage magistral, auquel je clois tant et qui se trouve dans toutes les bibliothèques. J'ai eu le regret de ne pouvoir citer la thèse de M. Georges Foucart, Hisloire de lordre lotiforme; la partie de mon livre, qui traite du mème sujet, était imprimée quand a paru ce travail remarquable. 


\section{LIVRE PREMIER}

\section{LES PLANTES CHEZ LES ÉGYPTIENS.}

\section{CHAPITRE PREMIER}

\section{LA FLORE PHARAONIQUE.}

Au nord-est du vaste désert qui va des rivages de l'Atlantique à ceux de la mer Rouge et comprend presque toutel'Afrique septentrionale, s'étend, semblable à une oasis immense, entre les dunes du désert de Libye à l'occident et la chaine des monts arabiques à l'orient, l'étroite vallée, qui, peut-être d'un des noms sacrés HâKou-Phtah - demeure ou château de Phtah - de la ville de Memphis - Mannofri - fut appelée Égypte par les Grecs ${ }^{1}$. Formée par les alluvions du fleuve, dont les débordements périodiques la fertilisent, cette " terre des merveilles » est, suivant l'expression d'Hérodote $^{2}$, un " présent du Nil."

Sorti de la région des lacs intérieurs de l'Afrique, ce fleuve, l'un des plus grands cours d'eau qui existent,

1 G. Maspero, Histoire ancienne des peuples de l'Orient. Paris, $4^{\mathrm{c}}$ éd. 1886, in-12, p. 23. - Id., IIisloire ancienne des peuples de l'Orient. Paris, 1895, in-8, t. I, p. 43.

2. Historiae, lib. II, cap. 7. 
après avoir reçu les nombreuses rivières qui arrosent la région tropicale comprise entre le $4^{c}$ et le $9^{c}$ degré de latitude, coule au nord, sous le nom de Fleuve-Blanc - Bahr-el-Abyâd - jusqu’à Khartoum, où il reçoit le Fleuve-Bleu - Bahr-el-Azrak, l'Astapos de Ptolémée, - qui lui apporte, avec l'Atbara - l'ancien Astaboras - le dernier de ses affluents, les eaux et les alluvions des montagnes de l'Abyssinie actuelle ${ }^{1}$. Se frayant avec peine un passage à travers les rochers qui barrent son cours tortueux, le Nil, arrivéà AbouHamed, décrit autour du désert de Nubie un vaste demi-cercle jusquà Korosko; à partir de cette ville il reprend la direction du nord, qu'il ne quitte plus, et, après avoir franchi une dernière cataracte, celle d'Assouan - l'ancienne Syène, - il entre, au $24^{\mathrm{e}}$ degré de latitude, dans l'Égypte proprement dite? Désormais rien n'arrête son cours et ses eaux coulent sans obstacle jusqu'au point où, divisé en deux branches principales, la Canopique et la Pélusiaque, il se jette dans la Méditerranée.

La région qu'enserre la branche pélusiaque à l'est et la branche canopique à l'ouest était, à une époque antéhistorique, recouverte par les eaux; la mer renait alor's baigner de ses flots le pied du plateau sablonneux que domine la grande Pyramide et le Nil se terminait un peu au nord de l'emplacement où s'éleva plus tard la ville de Memphis ${ }^{3}$. Les allurions du theuve,

1. Ptoléméc, Geographia, lib. IV, cap. 8, tab. 4. - Dümichen, Geschichte des allen Aegyplens. Berlin, 1879, in-8, p. 6. - Cav. Antonio Figari-Bey, Studii scientifici sull' Egillo e sue adiacenze. Lucca, 1864, in-8, vol. I. Introduzione, p. Xxir.

2. Élisée Reclus, Nonvelle géographie universelle. Paris, 1885, t. X, p. 73 et suivantes.

3. G. Maspero, Mistoire ancinne, in-12, p. 6. 
en comblant peü à peu le golfe dans lequel il se dérersait, ont donné naissance au Delta, vaste plaine marécageuse sans cesse grandissant, qui compte aujourd'hui 23,000 kilomètres carrés et n'a pas moins de 2to kilomètres et demi à sa base'.

Ces dimensions considérables, la vallée du Nil ne les atteint qu'aux bords de la Méditerranée; à partir de Memphis, sa plus grande largeur ne dépasse pas, y compris la vallée secondaire de Bahr-Yousef, 20 à 25 kilomètres; au sud de Thèbes même, elle n'en atteint guère que 15; elle se rétrécit encore au deli d'Edfou, et jusqu'à Assouan elle ne compte plus que 3 ou 4 kilomètres en moyenne ${ }^{2}$. Au-dessous d'Ombos même la chaine des hauteurs qui borne la vallée sur la droite, le Gebel Silsileh — le Silsilis des Grecs - s'arance jusqu'aux bords du Nil, et le thalweg se confond prosque arec le lit du fleuve. Il est encore moins large à Assouan, où les rochers granitiques de la rive orientale, en se prolongeant à travers le Nil, forment la première cataracte. Là finit l'Égypte historique, et les premiers habitants de la contrée y placèrent, avec la limite méridionale de leur pays, les sources dı Heure "deux fois pur et mystérieux ", qui le féconde ${ }^{3}$.

Depuis son confluent avec l'Atbara, où il quitte la région des steppes, jusqu'à son embouchure dans la Méditerranée, le Nil coule au milieu d'une contrée aride et déserte; à l'exception du fond de la vallée,

1. Élisée Reclus, op. laud., t. X, p. 93. - Dümichen, op. lauel., p. 12, dit 40 milles allemands; Strabon, Géographie, liv. XVII, ch. 6 , lui attribuait 1.300 stades.

2. Élisée Reclus, op. laud., t. X, p. 472.

3. Dümichen, Geographische Inschriften, pl. 79. 
que ses eaux recouvrent chaque année de leur limon fertilisant, toute la région présente, pendant neuf mois de l'année, l'aspect de la stérilitè. La rive droite du fleuve, d'Assouan au Caire, est dominée par les monts arabiques; prolongement des hauteurs qui forment la frontière orientale de la Nubie, ils envoient vers l'est de nombreux rameaux, qui les rattachent à la chaine bordière de la mer Rouge et s'élèvent par place à près de 2,000 metres. Ces monts sont divisés en plusieurs massifs d'une composition géologique différente; l'un d'eux, qui appartient encore à la Nubie, court de l'est à l'ouest jusqu'au delà du Nil et donne ainsi naissance aux cataractes ${ }^{1}$. Plus bas est le massif du Silsileh, entaillé de carrières aujourd'hui abandonnées, d'où sont sortis les matériaux de milliers de temples et de palais.

Les montagnes de la Nubie, ainsi que les hauteurs du désert arabique, sont composées d'un grès quartzeux, que traversent d'immenses filons de roches primitives ${ }^{2}$, granit, gneiss, micaschiste, porphyre et diorite $^{3}$. Au nord d'Edfou, les grès font place à des calcaires de divers àges, les uns de la période crétacée, les autres de la période éocène; ce sont des roches crétacées qui se dressent en falaises au-dessus du Nil, présentant avec leurs assises d'aspect monumental, séparées par de sombres ravins, les formes les plus pittoresques. Les dernières roches, qui se terminent au Caire même par le Gebel Moqattam, sont presque en entier composées de nummulites et d'au-

1. Élisée Reclus, op. laul., t. X, p. 472-74.

2. Wiedemann, Aegyptische Geschichte. Leipzig, 18sí, vol. I, p. 12. - Figari, op. leud., p. 20, 159, 163, 164 et 167.

3. Elisée Reclus, op. lauld, vol. X, p. 477 . 
tres coquillagos unis par un ciment calcaire ${ }^{1}$. Au deli s'étend le désert de sable mouvant de l'isthme.

A l'onest du Nil, les terrains primitifs s'enfoncent sous le plateau nummulitique du désert de Libye, plaine sans fin, parsemée de dunes et dont la stérile uniformité n'est interrompue que par quelques oasis lointaines. Atteignant une hauteur de 250 mètres aux bords du fleuve, ce plateau incline vers l'ouest sa plaine calcaire, que recourre entièrement un sable quartzeux, et s'abaisse dans la région des oasis au-dessous du niveau de la Méditerranée?

Telle est l'Égypte dans son ensemble, une vallée d'une merreilleuse fertilité entre un désert de pierre, qui l'empèche de s'étendre à l'est, et un désert de sable, qui la menace du côté de l'Occident. Le Nil, qui l'a formée, la conserve aussi, en arrêtant l'envahissement du désert libyque et en lui fournissant l'humidité doublement nécessaire à la végétation dans une contrée où la pluie ne tombe presque jamais. C'est le Nil - le dieu Hapi - "dont les flots, en se répandant sur les vergers, donnent la vie à l'Égypte»; " maître de tous les germes, stabiliteur des vrais biens, il crée, comme dit un ancien hymne ${ }^{3}$, toutes les bonnes choses ». Le génie des habitants devait compléter l'œuvre du fleuve, ce "dieu caché», père du pays qu'ils ont colonisé.

D'origine incertaine, mais appartenant, suivant toute

1. J. Williamson Dawson, Egypt and Syria. Their physical features in relation with Bible history. London, 1885, in-12, p. 25.

2. Élisée Reclus, op. laud., t. X, p. 478. - A. Figari Bey, op. laud., t. I, p. 70.

3. Papyrus Sallier II, ap. Maspero, Histoire ancienne, in-8, t. I, p. $40-42$. 
vraisemblance, aux races blanches, qui occupent, de toute antiquité, la région méditérranéenne du continent libyen ${ }^{1}$, ils se répandirent peu à peu, en se mêlant peut-être à des colons asiatiques venus à travers l'isthme de Suez, dans la vallée inférieure du Nil ${ }^{?}$. Cette contrée était loin alors d'offrir l'aspect qu'elle a pris depuis; le Delta, pent-être encore en formation, était sillonné par les nombreux bras du fleuve et couvert de marécages; plus loin les débordements non réglés du Nil laissaient, en se retirant, de vastes éten- dues de terrain submergées ${ }^{3}$, tandis que les parties hautes de la vallée qu'il n'atteignait pas restaient presque stériles. Les eaux du fleuve et des lacs, il est vrai, étaient remplies de poissons, d'innombrables oiseaux en couvraient la surface; les fauves en fréquentaient les rives, offrant une proie facile et abondante aux nouveaux habitants; mais quelles ressources agricoles ou industrielles trouvaient-ils dans la végétation indigène?

I.

La flore de l'ancienne Égypte n’était pas aussi pauvre en végétaux qu'on l'á sourent répété: sans doute elle était loin de renfermer les treize cents espèces que

1. Ed. Meyer, Geschichte des alten Aegyptens. Berlin, 1889, in-8, p. 18-23. - G. Maspero, IIstoire ancienne, t. I, p. 45.

2. Gardner Wilkinson, The manners and customs of the ancient Egyptians. A new ed. revised by Sam. Birch. London, 1878, in-8, vol. I, p. 2; Max Duncker, Geschichte des Alterthums. Berlin, 1887, in-8, vol. I, p. 11 ; Brugsch, Geschichte Aegyptens. Leipzig, 1877, in-8, p. 8 ; Wiedemann, op. laud., p. 21 , les font venir de l'Asie antérieure.

3. G. Maspero, op. laud., in-12, p. 17. 
Schweinfurth lui attribue de nos jours ${ }^{1}$; beaucoup d'entre elles y ont pénétré des contrées étrangères; la plupart toutefois y sont indigènes. Mais ces plantes n'étaient pas réparties également sur le sol de l'Égrpte. C'était dans la vallée dı Nil proprement dite, en particulier dans le Delta, qu'on en trouvait le plus grand nombre: mais la nature de la flore changeait, quand on s'avançait des bords de la Méditerranée aux confins méridionaux de la terre de Qimit, où elle prenait le caractère tropical; elle rariait encore plus, si l'on quittait les terres inondées de la vallée du Nil pour pénétrer dans le double désert qui la borde. Mème dans le Delta elle différait beaucoup, lorsque de la région calcaire de la Marmaritique, avec sa végétation presque entièrement méditerranéenne, on passaitdans la région sablonneuse de l'isthme, couverte d'espèces déjà plus asiatiques ${ }^{2}$.

Dans le Delta on trourait en abondance des renonculacées, des crucifères -- Matthiola, Malcomia, Lepidium, etc., - des caryophyllées, tamariscinées, frankéniacées, malvacées - Alcaea, Abutilon, Hibiscus, - des géraniacées, zygophyllées, de nombreuses papilionacées, - Ononis, Trigonella, Medicago, Melilotus, Trifolium, Lotus, Astragalus, Vicia, Lathyrus, - des lythrariées, plusieurs Mesembrian-

1. Sur la flore des anciens jardins arabes de l'Égyple. (Bulletin de l'Instiut égygtien, no 8 (an. 1887), p. 294.)

2. A. Figari, Studii scientifici sull'Egillo, t. I, p. $203 .-$ P. Ascherson, Florula Rhinocolurea. (Kémoires de l'Institut épyptien, t. II (an 1889). 2e partie, p. 786). - G. Schweinfurth, Planzengoographische Slizze des gesammen Nil-Gebiets und der Uferlüncier des Rothen Meeres. (Milheilungen aus Juslus Perlhes' geographischer Anstall, etc. von $\Lambda$. Petermann, 1868, p. 116). 
themum, des ombellifères, des composées variées telle que le Sphaeranthes suaveolens, corymbifère aquatique au délicieux parfum, des Inula, Gnaphalium, Anthemis, Chrysanthemum, en particulier le coronarium, plusieurs Senecio, des Echinopus, Carthamus, Cichorium, Picris, Sonchus, la Ceruana pratensis, astérö̈dée particulière à la vallée du Nil ${ }^{1}$, etc., - des convolvulacées, comme la Ciressa cretica, répandue dans toute l'Égypte, et de nombreuses espèces du genre type, des Borraginées - Anchusa, Heliotropium, Echium, - des Solanées et des Scrofulariées, des Labiées, - salvia, Lavendula, etc., - de beaux Statice, d'abondantes plantaginées et chénopodées - Atriplex, Suaeda, Salsola, — des polygonées et des euphorbiacées, le saule africain safsaf, des liliacées - Allium, Asphodelus, Muscari - l'Alisma ${ }^{2}$, des joncs, comme le Juncus acutus L. - l'asir des textes hiéroglyphiques ${ }^{3}$; - enfin des cypéracées et de nombreuses graminées. Parmi celles-ci il faut citer le Panicum obtusifolium, l'A rundo donax, le Phragmiles Isiaca, le Saccharum aegyptiacum, l'Eragrostis aegyptiaca, des Bromus, etc.; parmi les premières, les Cyperus papyrus, esculentus, auricomus, alopecuroüdes, rotundus, etc., le Scirpus maritimus", plantes aquati-

1. G. Schweinfurth, Die letsten botanischen Entdeckungen in den Gräbern Aegyptens. (Botanische Jahrbücher, t. VIII (an. 1884), p. 3.)

2. Victor Loret, La Flore pharaonique, d'après les documents hiéroglyphiques, 2c édition. Paris, 1892, in-8, p. 32.

3. Victor Loret, Fecherches sur plusieurs planles, XII, p. 11. (Recueil de travaux relatifs à la Philologie et à l'Archiologie égyptiennes el assyriennes, t. XVI).

4. P. Ascherson et G. Schweinfurth, Illustration de la flore d'Égypte, passim. (Mémoires de l'Institut égyptien, t. II, $1^{\text {re }}$ partie (an. 1889), p. 25-180). 
ques qui remplissaient les bas-fonds des marécalges et des lagunes que le Nil formait en se retirant, tandis que des lotus blancs et bleus en courraient la surface.

Une partie de ces plantes, surtout celles qui sont particulières à la flore égyptienne', se retrouvaient dans la vallée moyenne ou supérieure du Nil, ainsi que dans le Fayoum actuel ou mème dans les oasis de l'ouest; mais on rencontrait aussi dans ces régions des espèces qui leur appartenaient en propre, tel que les Polygala erioptera, Hibiscus vermucosus, Corchorus tridens, Astragalus falcinellus, Vigna nilotica, Cassia obovata et acutifolia, Potentilla supina, Vahlia viscosa, l'ashour (Calotropis procera), asclépiadée vénéneuse qu'on trouve aussi dans les déserts libyque et arabique, les Heliotropium pallens, Solanum coagulans, Polygonum limbatum, Panicum Petiveri, Andropogon annulatus, Eragrostis nutans, aegyp. tiaca et cynosuroüdes ${ }^{2}$, Bromus macrostachys, etc ${ }^{3}$. Sans doute on n'y royail pas ces forêts, dont, on l'a prétendu*, l'Égypte aurait été couverte à l'origine;

1. Outre celles qui sont déjá nommées, on pourrait encore citer les Senebiera nilotica, Silene villosa, Mesembryanthemum coplicum, Ammania aegypliaca, Psoralea plicata, Trigonella hamosa el laciniala, Crepis senecioïdes, Senecio arabicus, Erigeron aegyptiacum, Conyza Dioscoridis, Echium Ranwolfi, etc. - G. Schweinfurth. Pflanzengeographische Shizze des Nil-Gebiels. (Op. laud., p. 119-120.)

2. Cette dernière espèce " caractéristique du pays de Qimit », appelée aussi Leptochloa bipinnala, est souvent désignée sous le nom de halfa. Berichle der bolanischen Gesellschaft, t. II, p. 371.

3. Illustration de la Flore d'Égyple. (Hémoires, t. II, p. 25180.)

4. Ad. Erman, Aegyplen und aegyplisches Leben im Allerthum. Tübingen, s. d., in-8, vol. I, p. 27. 
néanmoins des espèces arborescentes variées, le saule safsaf, diverses espèces de tamaris - Tamarix nilotica, tetragona, arborea, articulata -- des acacias, Acacia nilotica, seyal, tortilis, Ehrenbergiana, - le figuier faux-sycomore, sinon le figuier sycomore, qui n'est plus que cultivé aujourd hui ' ${ }^{1}$, et que Schweinfurth croil originaire d'Arabie ${ }^{2}$, presque partout aussi peutètre le dattier, mais à l'état encore sauvage ${ }^{3}$, et au sud le palmier doum y croissaient en abondance.

Quelques-uns des végétaux, arbres et plantes herbacées, de la vallée du Nil, se rencontraient aussi dans les parties non complètement arides du double désert qui la borde. Mais la les choses changeaient; a la fertilité du Delta et dos terres inondées par le Nil, faisait place une rare et maigre végétation. Ce n'est pas que la stérilité régnàt partout; les déserts libyque, isthmique et arabique, eux aussi, ont leur flore, celle du Sahara", caractérisée par des espèces particulières, mais qui varient du nord an sud, et sont plus nombreuses à l'orient qu'à l'occident de la vallée du Nil. Tout aride qu'il est, le désert libyque est loin cependant d'être dépourvu de toute végétation, dans

1. Illustration de la Flore d'Égypte, p. 141.

2. Verhandhungon der Berliner Gesellschaf't für Anthropologie, Ethnologie und Urgeschichte, an. 1891. (Zeitschrift (iir Ethnologie, an. 1891, p. 657.)

3. Il y a au Musée du Louvre douze dattes qui paraissent appartenir à la forme non cultivée du Phencix dactylifera L., sinon à une des espèces non comestibles $P$. reclinala. $P$. sylvestris ou P. canariensis. Victor Loret et Jules Poisson, Les Végétaux antiques du Musie ég!lptien du Louvre. (Recueil de Iravaux, t. XIII (an. 1895), p. 183). - Lillustration de la Flore d'Égyple signale, p. 1'́7, l'existence du P. canariensis

* L. dans la vallée du Nil, mais seulement comme cultivé.

4. H. Schirmer, Le Sahara. Paris, 1893, in-8, p. 187. 
les parties où la dune ne porte pas la stérilité et la mort, on rencontre des crucifères, telle que la prétendue rose de Jéricho (Anastatica hierochuntina L."), quion retrouve d'ailleur's à l'orient de la vallée du Nil, commeà l'occident; plusieurs tamaris, diverses espèces de Fagonia, plantes de la famille des zygophyllées, lesquelles, arec le genre qui en est le type, affectionnent la région saharienne, des papilionacées aux tiges épineuses et presque sans feuilles, comme l'agol (Alhagi manniferum Desv.), répandu, ainsi que le curieux retem (Retama raetam IVebb.), dans les deux déserts égyptiens, plusieurs espèces d'acacias, la coloquinte, quelques composées aux feuilles velues, des plantaginées frutescentes, de nombreuses chénopodées, en particulier du genre Salsola, le Cormulaca monacantha - le had - plante farorite des chameaux, des Ephedra, mais surtout des graminées rigides et relues telles que les Aristida, dont une espèce, l'Aristida Zittelii Asch., est particulière au désert libyque ${ }^{2}$.

Ces plantes se retrouvent presque toutes dans le désert arabique, mais il en renferme bien d'autres, comme le Cocculus leaeba, ménispermée rampante aux fleurs d'un beau jaune, des crucifères, comme la Farsetia longisiliqua et la Moricandia sinaica, les Reseda Boisserii et muricata, le Corchorus antichorus, les Fagonia latifolia et glutinosa, de nombreuses légumineuses,

1. D'après G. Schweinfurth, la vraie rose de Jéricho est l'Aslericus pygmaeus Coss., plante du désert arabique. Bulletin de l'Institul égyplien, n' 4, (ann. 1883), p. 92.

2. Illustralion de la Flore d'Egypte, p. 168. On y rencontre aussi entre autres les Aristida ciliala, pungens, plumosa, scoparia, etc. Cf. Gerhard Rohlfs, Drei monate in der libyschen Wüsle mil Beitrïgen von P. Ascherson, W. Jordan und K. Zittel. Cassel, 1875, in-8, p. 53 et 71. 
entre autres des astragales, des acacias - comme les Acacia spirocarpa et albida -, végétation arborescente, qui était représentée aussi par des tamaris Tamarix passerinoüdes et macrocarpa.-, le Pistacia atlantica, le Balanites aegyptiaca, le Moringa aptera et diverses capparidées, comme le Capparis galeata ot surtout le Maema uniflora, qu'on rencontre en particulier dans le pays des Bisharis, sur les côtes de la mer Rouge, où il atteint une hauteur de 10 à 12 mètres. Ses rameaux qui retombent en berceau offrent aux pàtres un asile recherché contre les ardeurs du soleil'. A ces représentants de la flore arborescente du désert, il faut ajouter le figuier plumeux ${ }^{2}$, qu'on voit escalader les roches porphyriques les plus arides, et le pseudo-sycomore.

Les composées comptaient également de nombreuses espèces particulières à la même région, tels que les A stericus pygmaens et graveolens, I'Achillaea fragrantissima, les Artemisia herba-alba et judaïca, les Echinopus spinosus et glaberrimus, la Centaurea eryngioüdes, les Zollikoferia fallax et massavensis, etc. On y rencontrait aussi des borraginées, scrofulariées et labiées étrangères au désert libyque et à la vallée du Nil, par exemple les Heliotropium arbainense, Scrofularia deserti, Salvia aegyptiaca, les Lavendula multifida, pubescens et coronopifolia, le Stachys aegyptiaca, la Kallota damascena, les Teucrium sinäcum et leucocladum, des plantaginées, comme le

1. G. Schweinfurth, Die letsten botanischen Entdectungen. (Engler's Botanische Jahrbïcher für Pflanzengeschichte und. Pflanzengeographie, an. 1886. Berlin, in-8, p. 4.)

2. A. Figari, op laud., vol. I, p. 211. L'Illustration de la Flore d'Égyple ne mentionne pas cette espèce. 
Planlayo stricta, des chénopodées - Atriplex halimus et leucocladum, Suacda vermiculata, Salsola longifolia, - l'Euphorbia dracunculoüdes et l'Andrachne aspera, l'Allium desertorum, l'Urginea undulata; enfin d'assez nombreuses graminées, comme les Panicum Teneriffae et dichotomum, le Pennisetum oricntale, le Tragus Berteroanus, les Andropogon foveolatus, hirtus et laniger, les Aristida hirlighuma, caloptila, funiculatu, Schweinfurthii, l'Eragrostis ciliaris et le Poa sinaïca ${ }^{1}$. La flore de cette région avait dì être, à l'origine, bien plus riche, surtout en végétaux arborescents que la sécheresse plus grande du climat et la main d'homme ont contribué à faire disparaitre depuis de longs siècles ${ }^{2}$.

II.

Quelque variée que soit la flore indigène de l'Égypte, elle ne pouvait néanmoins, dépourvue qu'elle ètait des espèces les plus nécessaires à la vie, suffire aux besoins d'un peuple arrivè à un certain degré de culture. Les céréales lui faisaient défaut, depuis le riz, originaire de la région des moussons et resté si longtemps inconnu de l'Asie antérieure, jusqu'au froment et à l'épeautre, dont la patrie incertaine pourrait bien

1. Illuslration de la Flore d'Égyple, p. 25-180.

2. Quant aux restes fossiles de la Nicolia aegypliaca Unger, dont on retrouve les débris silicifiés dans le désert situé au sud.est du Caire, ils n'appartiennent pas à la flore égyptienne et semblent avoir été apportés par les eaux de régions plus tropicales. F. Unger, Der versteinerte Wald bei Cairo. (Silsungsherichle der Kaiserlichen Aliademie der Wissenschaften, t. XXXIII (an. 1858), 1. 209-229.) 
être la Mésopotamie; elle ne possédait pas davantage l'orge, natif peut-être aussi de l'Asie antérieure, ni le millet, venu, il semble. d'une région plus orientale, ni mème le sorgho, encore qu'il paraisse originaire de l'Afrique tropicale ${ }^{1}$. La plupart des légumes et nos arbres à fruits étaient également inconnus de l'Égypte préhistorique. Aussi pauvre en plantes alimentaires, la flore égyptienne pouvait encore satisfaire aux rares besoins des populations primitives que les ancêtres des Égyptiens paraissent avoir rencontrées à leur arrivée dans la vallée du Nil et qu'ils refoulèrent dans l'intérieur du continent ${ }^{2}$. De race négroïde probablement, restés sans doute encore à l'àge de pierre $^{3}$, et, comme toutes les tribus sauvages, vivant surtout de chasse et de pêche, ils ne demandaient au règne végétal, pour compléter leur alimentation, que quelques fruits sauvages et quelques racines. La rallée du Nil les leur offrait. Mais si c'était assez pour ces peuplades inférieureś, il n'en était pas de mème pour les colons de race supérieure qui leur succédèrent.

Sans doute les Rotou, "les hommes " - c'est ainsi que s'appelaient les nouveaux habitants — n'étaient pas

1. A. de Candolle, Origine des Plantes cultivées. Paris, 1853, in-8, p. $284-310$.

2. Lepsius, Ueber die Annahme eines sogenamnten prehistorischen Steinalters in Acgypten. (Zeitschrift für aegyptische Sprache (an. 1870), p. 113.)

3. “Es hat eine Steinzeit gegeben, welche weit über jede historische Nachricht, weit selbst über die Anfänge ägyptischer Geschichte zurückreicht. " W. Reiss, Frunde ans der. Steinzeit Aegyptens. Berlin, 1890, in-8. (Abhandlungen der. Berliner anthropologischen Gesellschaft. Sitzung vom 16 nov. 1889, p. 712.) 
le peuple heureux qu'ont rèvé lcur's descendants; divisés en un grand nombre de tribus, dont les nomes de l'Égypte pharaonique ont conservé le souvenir, ils durent mettre de longues années à s'élever au haut degré de civilisation où nous les montre l'histoire la plus reculée; mais s'ils étaient encore étrangers à l'agriculture, à l'époque de leur établissement dans la terre de Qimit, "la terre noire ",-c'est sous ce nom que les anciennes inscriptions désignent l'Egypte', - ils ne durent pas tarder a s'y livrer. Leurs relations avec les populations sémites de l'Asie antérieure les mirent bien rite en possession des céréales et des légumes les plus utiles, originaires de cette contrée ou de la région du Caucase.

Ces plantes alimentaires, quelqu'en ait été le nombre, suffirent sans doute à ces "serviteurs d'Horus» Shosou Hor - pendant la première période de leur établissement, celle où, partagés en tribus indépendantes, ils colonisèrent lentement la vallée du Nil et la mirent en état de receroir les cultures nouvelles qu'ils lui apportaient; mais quand toute la terre de Qimit ent été réunie sous un mème sceptre, que la BasseEgypte - To-miri ou pays du Nord - et la HauteÉgypte - To-risi ou pays du Sud ${ }^{2}$ - formèrent sous Menès un seul empire, celui des Pharaons, les espèces végétales apportées par leurs ancêtres ne purent, pas plus que les plantes indigènes de l'Égypte, contenter les habitants du jeune et puissant État; leurs besoins et leur luxe croissant, ils demandèrent de nouveaux régétaux aux pays avec lesquels ils entrèrent tour à

1. Brugsch, Geschichle Aegyplens, p. 14 et 20.

2. Maspero, IIistoire ancienne, in-12, p. 18. 
tour en relation. Leur agriculture, dès longtemps prospère - elle avait été la condition première de la grandeur de leur empire - fit de nouveaux progrès et s'enrichit d'espèces exotiques rapportées par les Pharaons de leurs expéditions dans les contrées étrangères, en Nubie et en Éthiopie, dans le désert arabique et la presqu'île du Sinaï, ainsi que dans la Syrie méridionale.

Amenemhat I, fondateur de la douzième dynastie, se vante, dans un papyrus, d'être le "créateur", c'està-dire sans doute l'introducteur de "trois espèces de grains ${ }^{1} »$. A cette époque, comme nous l'ont révélé les fouilles de M. Flinders Petrie ${ }^{2}$, les Égyptiens connaissaient et probablement aussi cultivaient déjà, outre le dattier, le palmier doum (Hyphaene thebaica Mart.), l'Hyphaene argun Mart., palmier de la vallée du désert de Nubie, ainsi que l'hegelig (Balanites aegyptiaca Del.), arbre de l'Afrique tropicale, le $M i$ musops Schimperi Hochst., - le perséa des Anciens d'après Schweinfurth ${ }^{3}$ - indigène dans l'Abyssinie et dans l'Arabie heureuse, où vient aussi le caroubier, que le même botaniste a supposé originaire de ce pays ${ }^{4}$, mais qui croît spontanément, dans toute la région orientale de la Méditerranée ${ }^{3}$, enfin le lin, importé sans doute depuis longtemps de la région du Caucase, le

1. Papyrus Sallier, II, pl. 1, 7-9, ap. Maspero. Hisloire ancienne, in-12, p. 95.

2. Kahun, Gurob and IIavara. London, 1890, in-4, p. 49-50.

3. Bulletin de l'Institul égyptien, $n^{\circ} 3$ (an. 1882), p. 67.

4. Bulletin de l'Institut égyptien, no 8 (an. 1887), p. 306.

5. A. de Candolle, Origine des plantes cultivées, p. 270. Engler, ap. V. Hehn, Kullurpflanzen, p. 443, le croit encore indigène dans la Cyrénaïque, l'Algérie et la Sicile. 
concombre, les radis, les petits pois et la fève, dont la patrie est incommue, mais presque certainement asiatique.

Ce n'est là probablement qu'une faible partie des végétaux exotiques importés jusqu'au $\mathrm{xxx}^{\mathrm{e}}$ siècle avant notre ère dans la vallée du Nil; il est vraisemblable que les Égyptiens en avaient déjà recu d'autres, dès le temps de l'ancien Empire. Faut-il ranger parmi ces végétaux, le figuier commun et l'arbre baq, cultivés, au temps de la douzième dynastie, dans le pays d'Edom, comme nous le savons par le récit du séjour que l'exilé Sinouhit fit alors chez un des chefs de cette contrée ${ }^{1}$. Si l'Égypte ne les possédait pas encore, on peut croire que l'invasion des Hyksos dut y introduire ces arbres qu'ils connaissaient et cultivaient peut-être eux-mêmes dans leur pays.

Plus tard, les conquêtes des Ahmessides et des Ramessides ne purent manquer aussi de contribuer à emrichir la flore horticole et agricole de l'Égypte; poussées au sud jusqu'aux derniers confins de l'Éthiopie, à l'est jusqu'aux bords de la mer Rouge, au nord au delà de l'Euphrate, elles avaient révélé à ces princes l'existence de nombreuses plantes utiles, étrangères à l'Égypte, et, comme leurs prédécesseurs, ils durent songer à en introduire quelques-unes dans leur propre pays. Un document célèbre permet de nous faire une idée du soin avec lequel les Pharaons cherchaient à acclimater en Égypte les végétaux utiles des contrées étrangères, même de celles qu'ils n'avaient pas visitées.

1. G. Maspero, Histoire ancienne, in-12, p. 97. - Mélanges d'archéologie égyptienne el assyrienne, t. III, p. 133. 
Parmi celles-ci, il y en avait une, longtemps à demifabuleuse ${ }^{1}$, dont ils avaient souvent entendu parler ${ }^{2}$, la "terre divine», où l'on recueillait les aromates, que le commerce des caravanes ou la navigation de la mer Rouge $^{3}$ leur apportait de temps immémorial : le pays de Pount, - la côte occidentale de la mer Rouge de Souakim à Massouah, plus tard le pays des Somalis et même l'Arabie heureuse ${ }^{4}$. - La fille de Thoutmès I, second roi de la dix-huitième dynastie, la reine Hatshopsitou ou Hatasou, résolut d'y envoyer chercher quelques-uns de ces végétaux précieux dont elle ne connaissait que les produits. Cinq vaisseaux furent équipés dans un des ports de la mer Rouge et, comme nous l'apprennent les inscriptions du temple de Deirel-Bahari, élevé par la souveraine égyptienne en souvenir de cette expédition ${ }^{5}$, ils abordèrent au pays de

1. Le conte du papyrus de Saint-Pétersbourg, qui date du moyen empire, en fait une île inaccessible gouvernée par un serpent à tête humaine; mais il mentionne déji la mỵrhe, l'encens, les huiles et les arbres précieux qu'on y trouvait. Erman, Aegypten, t. II, p. 671.

2. "La grande déesse de Poun est citée dans le papyrus de l'ancien empire. » E. Chabas, Éludes sur l'antiquili historique. Châlon-Paris, 1872, in-8, p. 149.

3. "Une inscription du règne de Sonkh-Kara de la $\mathrm{xi}^{\mathrm{e}} \mathrm{dy}$ nastie nous relate l'envoi de vaisseaux à Poun pour recueillir de l'ana. » E. Chabas, op. laud., p. 150.

4. Krall, Das Land Punt. Wien,-1890, in-8, p. 75. - J. Lieblein, Handel und Schiffarl auf dem rolhen Meere in allen Zeilen. Leipzig, 1886, in-8, p. 75.

5. Joh. Dümichen, Dic Flolte einer aegyplischen Koenigin aus dem xvir. Jahrhunderl vor unserer Zeitrechmung. Leipzig. 1868, in-fol., pl. I-XV et p. 17-18. - Aug. Nariette-Bey, Deirel-Bahari. Documents topographiques, historiques el ellnographiques recucillis dans ce temple. Paris-Leipzig, 1877, in-4 et atlas, in-fol. Pl. IV-VII et p. 13-18. 
Pount; les indigènes n'hésitèrent pas à entrer en relations avec eux et bientòt les échanges commencèrent.

Les Ėyptiens recurent en retour de leurs présents des cynocéphales et des cercopithèques, des lévrier's, des peaux de léopards, de l'ivoire et de l'or, des aromates et de l'encens, toutes sortes de bois précieux du Tonoutri, - bois d'ébène, bois taas ou toshp, bois lihisit, - enfin trente et un "sycomores à anti », qui, arrachés arec leurs racines et mis dans des corbeilles, furent transportés en Égypte . "Jamais on n'avait ramené chose pareille à aucun roi, qui eût été depuis que la terre existe ». Ces riches présents furent portés à Thèbes, et dans une grande fête, célébrée en l'honneur d'Amon, offerts au dieu, "maitre de Karnak ». Les trente et un sycomores à encens furent ensuite plantés dans le jardin sacré, et comme s'ils y eussent repris et grandi, "le monument nous montre, remarque M. Maspero, quelques-uns d'entre eux arrivés à leur taille normale. "

Cet essai d'acclimatation, s'il est le plus ancien que les monuments nous font connaitre, ne fut ni le premier, ni le dernier qui fut tenté en Égypte ${ }^{2}$. Il avait été précédé de l'introduction des céréales, puis de légumes et d'arbres à fruits, inconnus dans la vallée du

1. G. Maspero, De quelques navigations des Égypliens sur les côles de la mer Érylhréc. (Revue hislorique, t. IX (an. 1879), p. 24). - Lieblein, op. laud., p. 2'-37.

2. Une peinture du tombeau de Rekhmara nous montre, parmi les présents apportés à Thoutmès III, un sycomore ou arbre à cunli, et Ramsès III rappelle à Phtah, dans le Papyrus Harris, les sycomores d'aromates qu'il avait plantés dans sa ville de Memphis, après les avoir rapportés lui-même des "terres divines " de Pount. Wilkinson, The manners, t. I, pl. IIa. G. Maspero, De quelques navigations, p. 30. 
Nil; il fut suivi par celle de bien d'autres végétaux rares ou utiles. Commencées dès les premiers temps de son établissement, ces importations se continuèrent jusqu'à la destruction de l'empire des Pharaons et bien au delà : c'est seulement à l'époque de la domination perse et sous les Ptolémées que la flore égyptienne s'enrichit de plusieurs des espèces végétales les plus belles qu'elle ait possédées au commencement de notre ère. 


\section{CHAPITRE II.}

\section{L'AGRICULTURE DANS L'ÉGYPTE ANCIENNE}

Aucun document n'est venu nous renseigner sur l'ètat de civilisation auquel les ancêtres des Ėgyptiens étaient arrivés, quand ils s'établirent dans la vallée du Nil ; mais tout fait supposer que, s'ils menaient encore la vie pastorale et vinrent dans leur nouvelle patrie avec leurs troupeaux de chèvres et de bœufs, les seuls animaux domestiques qu'ils ont, avec l'àne, -- les monuments en font foi - dû posséder tout d'abord, ils ne restèrent pas longtemps étrangers aux occupations agricoles; la nature du pays qu'ils venaient coloniser décida de leur manière de vivre ${ }^{1}$. Dans une contrée soumise à des inondations périodiques et presque sans pâturages naturels, une existence nomade était impossible, la nécessité de demander à la culture du sol leur nourriture, et celle de leur bétail, contraignit les nouveaux habitants à une vie sédendaire et en fit un peuple de laboureurs ${ }^{2}$ et la nation la plus industrieuse que l'ancien monde ait connue.

1. F. Unger, Bolanische Streifzüge. (Silzungsberichte der kais. Akademie der Wissenschaflen, t. XXXVIII, p. 70 et suiv.), adrnet l'existence de pâturages, à l'arrivée des ancêtres des Egyptiens dans la vallée du Nil, mais il reconnait en même temps la nécessité où furent les nouveaux venus de se livrer à l'agriculture.

2. Ed. Neyer, Geschichte des alten Aegyplens, p. 25. 
Le Nil, non contenu par des digues, dévastait alor's la vallée qu'il fertilise aujourd'hui; de vastes marécages, laissés par l'inondation, en couvraient les parties basses, tandis que les parties hautes, où elle n'arrivait pas, restaient arides et impropres à la culture. Il fallait contenir et régler les débordements du fleuve, en amener les eaux bienfaisantes sur les terres sablonneuses qu'il ne pouvait atteindre et le renfermer dans son lit; c'est ce qu'entreprirent de faire les nouveaux arrivants ${ }^{1}$, et, grâce à un système de canaux bien entendu et à une habile distribution des eaux sur tout le territoire, l'Égypte devint une des contrées les mieux appropriées à une agriculture perfectionnée, source première de son étonnante civilisation.

C'est aux générations sans histoire des premiers temps de l'Égypte, aux "serviteurs d'Horus ", que revient l'honneur d'avoir commencé les travaux, qui, maîtrisant le Nil, en firent le génie tutélaire et bienfaisant de la vallée qu'il avait ravagée jusque-là; ces travaux, inaugurés à l'époque des origines de la nation, furent, pendant de longues générations, continués par les Pharaons, qui se succédèrent sur le tròne. Ménès, le fondateur de l'ancien empire, fit creuser, près de Memphis, une digue qui existe encore et sert de clef aux réservoirs d'inondation de la Haute-Égypte ${ }^{2}$.

Plusieurs des plus grands princes de la XII ${ }^{\mathrm{e}}$ dynastie rivalisèrent avec lui et le surpassèrent. Ousirtesen I, fils d'Amenemhat I, le premier d'entre eux, fit construire des digues Je long de la rive occidentale du

1. G. Maspero, IIisloire ancicnne, in-12, p. 17. - Ed. Meyer, op. laud., p. 24.

2. G. Maspero, Histoire ancienne, in-12, p. 43. 
Nil. Amenemhat III alla plus loin; frappé du peu de résultats que donnaient les réservoirs de faible dimension échelonnés le long du fleuve, il forma le projet de les remplacer ou d'en compléter l'effet par la création d'un réservoir immense, où l'excédant des eaux, accumulé pendant les années d'abondance, resterait emmagasiné, pour subrenir aux crues trop faibles, menace de stérilité et de disette pour le pays ${ }^{1}$. Dans une dépression naturelle du sol, située à l'ouest et ì quelque distance du Nil, - le Fayoum actuel, - il établit, au moyen de digues, le lac artificiel de Miri - le Mœris des Grecs, -- et le mit en communication avec le fleuve, à l'aide d'un double canal, pourvu d'écluses, qui permettaient d'y faire entrer les eaux du Nil débordé ou de faire écouler celles qui y étaient accumulées.

Ces entreprises colossales, destinées à faciliter l'irrigation régulière des terres, peurent nous donner une idée de l'importance que les Pharaons attachaient à tout ce qui pouvait favoriser l'agriculture. "J'ai fait labourer le pays jusqu'à Abou - Abydos, - dit l'un deux, Amenembat I, dans une inscription ${ }^{2}, \ldots$ je suis l'ami de Nopri ${ }^{3}$... Le Nil a accordé à mes prières l'inondation sur les champs. "Cette inondation était, sous un climat brûlant, la condition première de la fertilité de l'Égypte; sans elle, le sol était condamné à la stérilité; arrosé, au contraire, par les eaux du fleuve et enrichi par leur limon, il devenait d'une surprenante fécondité.

1. G. Maspero, Histoire ancienne, in-12, p. 108.

2. Papyrus Sallier II, pl. I, 1. 7-9, ap. Maspero, op. laud., p. 95.

3. La divinité des grains. 
Sa mise en culture était d'ailleurs de la plus grande simplicité. Quand les eaux du fleuve s'étaient retirées et que les vents du nord-ouest avec les chauds rayons du soleil d'automne les avaient suffisamment asséchés, les Égyptiens donnaient avec un hoyau en bois un simple labour à leurs champs, puis ils y répandaient la semence que des chèvres ${ }^{1}$ - Hérodote dit des porcs ${ }^{2}$, ce qui ne dut ètre vrai qu'exceptionnellement et assez $\operatorname{tard~}^{3}$ - venaient ensuite, remplaçant nos herses, fouler aux pieds pour la faire pénétrer dans le sol. D'autres fois ils se servaient pour labourer leurs terres d'une charrue légère, traìnée par deux boufs", mais en se bornant à y tracer un sillon peu profond; tout au plus quelques ouvriers venaient ensuite avec un hoyau écraser les mottes, pour achever de préparer le sol à recevoir la semence qu'on voulait lui confier.

Quoi qu'il en soit, cinq ou six mois après ce labour primitif, les moissons arrivaient à maturité, l'orge d'abord, puis les diverses espèces de froment. On procédait alors à la récolte; les chaumes, coupés avec une faucille à une certaine hauteur du sol ${ }^{5}$, étaient

1. Rosellịni, Monumenti, t. II, pl. XXXII, 1. - Lepsius, Denkmïler; t. III, pl. XVI. Peinture de Giseh (IV dynastie), tombe 15.

2. II istoriae, lib. II, cap. 14. Hérodote dit que les Égyptiens ne retournaient pas le sol; mais les peintures des hypogées nous les montrent toujours lui donnant un premier labour.

3. Wilkinson, The manners, t. II, p. 100.

4. Rosellini, Monumenti, t. II, pl. XXXII, 2 et 7. - Lepsius, Denkmäler, t. V, pl. 106. Peinture de Zaouiet-el-Mlaiétin (ve dynastie), tombe 2. - Bas-relief du tombeau de Ti. G. Maspero, IIistoire ancienne, t. I, p. 67. - Plus tard on se servit aussi de chevaux, comme on le voit d'après une peinture de Thèbes. G. Ebers, L'Égypte, trad. par Naspero, t. II, p. 211.

5. Rosellini, Monumenti, t. II, pl.XXXV, 1 et 2, et pl. XXXVI, 
liés en gerbe, puis portés sur l'aire et là foulés aux pieds des bœufs ${ }^{1}$ ou, dans la Basse-Égypte ${ }^{2}$, par des ines. Le grain était ensuile déposé dans de vastes greniers ${ }^{3}$, en altendant qu'on le livràt au commerce ou à la consommation.

Tels étaient les procédés employés par les anciens Egyptiens pour la culture et la récolte des céréales dans les parties de la vallée où les débordements du Nil fournissaient une humidité suffisante à la végétation. Dans les parties insuffisamment inondées ou pour les cultures qui réclamaient de fréquents arrosages, on les semait dans des carrés entourés de rigoles qu'on remplissait d'eau à l'aide d'une machine analogue à la shadouf actuelle des fellahs ${ }^{4}$, simple levier posé sur un support et à l'une des extrémités duquel était attaché une espèce d'auge, qu'on élevait ou abaissait par un mouvement de bascule. Souvent aussi, travail. plus pénible, on puisait directement l'eau dans les bassins ou réservoirs avec des cruches qu'on transportait, fixées aux deux extrémités d'une espèce de joug, au lieu qu'il fallait arroser. C'est ainsi qu'on voit sur une peinture de Béni-Hassan ${ }^{\check{2}}$ deux jardiniers, dont le premier a l'un de ces jougs posé sur les épaules, tandis que le second est représenté incliné, remplis-

2. - Lepsius, Dentimäler, t. IV, 106. Peintures de Zaouïet-elMaiétin, tombe 2.

1. Rosellini, Monumenti, t. II, pl. XXXIII, 2 et 3.

2. Wilkinson, The manners, t. Il, p. 101.

3. Rosellini, Monumenti, t. II, pl. XXXIV, 2.

4. Wilkinson, The manners of the ancient Aegyptians, t. I, 281. - Mémoires publies par les membres de la mission archéologique française au Caire, t. V, fasc. IV, p. 60\%, pl. 1.

5. Champollion, Les Monuments de l'Égyple el de la Nubie, t. II, pl. 185. 
sant avec l'eau de ses cruches les rigoles de son jardin.

I.

\section{Les Céréales.}

Des céréales, cultivées dans l'Égypte pharaonique, le froment était de beaucoup la plus répandue; il avait probablement été importé dans la vallée du Nil dès les premiers temps de la colonisation de cette région par ses nouveaux habitants; en tout cas il n'y était pas indigène; mais il s'était bien vite acclimaté dans cette contrée, où il trouvait les mêmes conditions de végétation que dans son pays supposé d'origine, la région de l'Asie antérieure, dont la Mésopotamie est le centre ${ }^{1}$, et sa culture devint une des sources principales de la richesse et de la prospérité de l'Égypte. De temps immémorial elle avait été, comme nous le montre l'histoire de Joseph ${ }^{2}$, le grenier où venaient s'approvisionner les peuples voisins menacés par la disette. Pendant deux mille ans sa fertilité ne se démentit pas; après son asservissement à Rome, elle expédiait encore chaque année vingt millions de boisseaux de froment en Italie ${ }^{3}$.

1. Unger, Sitzungsberichte, etc., t. XXXVIII, (1859), p. 79. - A. de Candolle, Urigine des plantes cultivées, pl. 288. - "Je considère comme la patrie vraisemblable du froment la région qui s'étend du Caucase, de la mer Caspienne et de la Perse à la mer Méditerranée et à la mer Rouge. "Fr. Körnicke, Die Arlen und Varietäten des Getreides. Bonn, 1885, in-8, p. 34.

2. Genèse, cap. XLI, v. 57. Sous Rhamsès I, on voit également les Khiti venir s'approvisionner en Égypte pendant une disette.

3. Dr. A. Thaer, Die alt-ägyptische Landwirthschaft. Ein 
Le froment, qu'on a trouvé dans les tombes les plus anciennes de l'Égyte et dont tous les musées possèdent des échantillons, ne diffère point de l'espèce de blé ou froment cultivée de nos jour's ' c'est le Trilicum vulgare Vill.; il portait dans la langue hiéroglyphique le nom de sou, copte sour ${ }^{2}$. La race connue des anciens Egyptiens était le blé dur (Triticum durum Desf.), l'emraï des Coptes ${ }^{3}$. Il faut ajouter que les artistes pharaoniques ont représenté les épis de froment, parfois sans barbes, le plus souvent avec des barbes ${ }^{*}$ : quelles variétés avaient-ils en vue dans cette double représentation? C'est ce que je ne saurais dire.

Le Triticum vulgare n'était pas la seule espèce de froment cultivée dans l'Égypte ancienne; Alph. de Candolle" a cru reconnaitre le gros blé ou blé poulard (Trilicum turgidum L.) parmi des graines tirées de cercueils de momies. Unger ${ }^{0}$ a aussi prétendu en avoir découvert des fragments dans une brique d'El-Kab. On vient d'en trouver quelques grains parmi les échantillons de blé conservés au Musée du Louvre; ils se distinguent par leur aspect corné et leur teinte rougeàtre. Comme les anciens Égyptiens divisaient le froment en sou blanc et en sou rouge, M. Victor Loret incline à voir

Beitrag zur Geschichte der Agricultur. Berlin, 1881, in-8, p. 18.

1. Schweinfurth, Ueber Pflanzenreste, elc. (Berichte der deutschen botanischen Gesellschaft, t. II (an. 1884), p. 370.)

2. Victor Loret, La flore pharaonique, p. 23, no 13. Schweinfurth, Zeilschrift /ür Ethnologie, an. 1891, p. 655.

3. Victor Loret, op. laud., p. 23, no 14 .

4. Rosellini, Monumenti, pl. XXXIII, 1 et 2, représente avec des barbes les épis qui sont répandus sur l'aire, sans barbes ceux qui sont encore sur leurs tiges.

5. Origine des plantes cultivées, p. 288.

6. Silzungsberichte, etc., t. XLV (an. 1862), p. 79. 
dans ce dernier le gros blé, tandis que le sou blanc serait le blé tendre ${ }^{1}$. Il est probable que le blé poulard aura pénétré en Égypte à l'époque de la domination des Hiksos ; très répandue de nos jours dans la vallée dı Nil, cette espèce semble y avoir été beaucoup moins cultivée autrefois, encore que Unger incline ${ }^{2}$, il est vrai, sans en donner de preuves, à l'y supposer indigène.

L'épeautre (Triticum spelta L.) était-il aussi cultivé en Égypte? Unger l'a admis, en s'appuyant sur le témoignage des auteurs classiques. Malheureusement ce témoignage n'est rien moins que clair. Hérodote ${ }^{3}$,

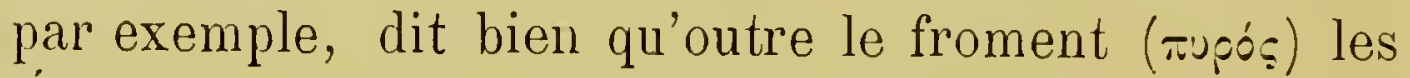
Égyptiens cultivaient encore l'olyra ("zrupa), que " quelques-uns appellent zeia (५sía) ); mais quelle céréale l'historien grec désigne-t-il par les mots olyra ou zeia? Tout ce qu'il nous en apprend, c'est que les Égyptiens s'en servaient pour fabriquer leur pain, et, fait bien propre pour surprendre, il ajoute qu'ils regardaient comme une honte de se nourrir de froment, ainsi que d'orge. Il semble d'après cela que l'olyra ou zeia était assez différent du froment; mais c'est tout ce que nous en pouvons conclure. Théophraste connait aussi le nom zeia, de même que celui d'olyra, mais il mentionne de plus une autre céréale qu'il appelle tiphe (tor.i. $)^{4}$ et qu'il regardait, au rapport de Galien, avec la zeia, comme ressemblant le plus au fro-

1. Victor Loret et Jules Poisson, Les végétaux antiques du Musée die Louvre. (Recueil de travaux, t. XVII, p. 180.)

2. Silzungsberichle, etc., t. XXXVIII (an. 1859), p. 79.

3. IIistoricae, lib. II, cap. 36.

4. Historia plantarum, lib. II, cap. 4, 1 et lib. VIII, cap. 4,1 . 
ment. Ce dernier nous apprend' que Dioclès plaçait, au point de vue des qualités nutritives, l'olyra et la tipha immédiatement après le froment, et qu'il en faisait deux espècés différentes, tandis que Mnésithée les regardait comme une seule et même espèce, mais en distinguait la seia, qui pour lui était une céréale des pays froids. Dioscoride ${ }^{2}$, qui ne parait pas connaitre la tipha, dit que l'olyra domne une farine plus grossière que la zeia; quant à cette dernière il y en aurait deux variétés, qu'on pourrait peut-être identifier avec les T. monococcum L. - notre blé locular ou engrain - et $T$. dicoccum Schrank - l'amidonnier - simples races peut-être de l'épeautre. Pline ${ }^{3}$, qui mentionne les noms de la zeia, de l'olyra et de la tipha, ne donne aucun renseignement sur les plantes qu'ils désignent.

On voit combien la question est obscure. Dans une étude sur " l'histoire des céréales ", Fink ${ }^{4}$ arrive à cette conclusion que les Anciens ne savaient pas ce que c'était que la zeia et qu'on ne voit pas quelle différence existait entre l'olyra et la tipha, mais qu'on peut, arec Sprengel, regarder la dernière comme étant l'engrain $(T \text {. monococcum L. })^{5}$. On n'a point rencontré

1 De alimentorum facullatibus, lib. I, cap. 13. Ed. Kühn. (Medicorum graecorum opera. Lipsiae, 1823, t. VI, p. 512.)

2. De maleria medica, lib. II, cap. 111 et 113.

3. Hisloria naluralis, lib. XVIII, cap. 19 (6) et 20 (10).

4. Ueber dieälteren Geschichten der Getreidearten. (Abhandlungen der kön. Akademie der Wissenschaften zu Berlin, an. 1826, p. 76.$)$

5. Fr. Körnicke, op. laud., p. 77, croit que les mots olyra et zeia servaient également à désigner l'épeautre, mais que le second de ces noms se perdit et que le mot olyra persista seul, et p. 109, il regarde tipha comme le nom grec de l'engrain. 
cette dernière céréale dans les hypogées égyptiens, mais Schweinfurth a reconnu des épis et des grains de l'amidonnier ( $T$. dicoccum Schrank), au milieu d'offrandes trouvées par M. Maspero dans une tombe de Gébéleïn ${ }^{1}$; ils appartenaient à la variété iricoccum. Il est donc probable que cette espèce était cultivée dans l'Égypte pharaonique. L'épeautre proprement dit y fut-il aussi connu et cultivé? Unger a prétendu en avoir trouvé des grains dans des tombes antiques ${ }^{2}$, mais Schweinfurth a affirmé ne l'avoir jamais rencontré parmi les restes végétaux des hypogées égyptiens; de Candolle a nié également que cette céréale ait été connue des contemporains des Pharaons ${ }^{3}$.

Il existe un mot copte bôti ou bôte, donné comme équiralent du grec ó̀upx et de l'hébreu kussemet ${ }^{4}$, qu'on traduit d'ordinaire par épeautre; Schweinfurth considère ce nom comme désignant plutôt l'amidonnier, l'emmer des Allemands ( $T$. dicoccum Schr.), espèce qu'il regarde d'ailleurs comme une simple variété de l'épeautre; elle aurait, ajoute-t-il, été trouvée par Th. Kotschy à l'état réellement sauvage sur les flancs de l'Hermon". Il y aurait eu aussi, dit-il, d'après une communication de M. Brugsch, deux espèces de bóti, le bóti blanc et le bôti rouge; mais il ne nous fait point connaître en quoi elles pouvaient différer. Le

1. Bulletin de l'Institut égyptien, no 7 (an. 1886), p. 420 et 424 .

2. Schreiben von Dr. Steudner : Ueber die Flore in und um Alexandrien. (Petermanns Nitheilungen, an. 1861, p. 310.)

3. Origine des plantes cultivies, p. 291.

4. Une Scala traduisant boti par al-hommos: pois chiche, ou al-dour $\hat{a}:$ sorgho, on voit que la signification de boti est loin d'être certaine.

5. Zeilschrift fïr Elhnologie, an. 1891, p. 655. 
bóti serrait à désigner le mois de lybi, - le lubi du calendrier copte, - qui va du 17 novembre au 16 décembre '; ce mois était personnifié sous la figure d'un homme, tenant comme emblême un épi de la main droite, ce qui permet de conclure que cette céréale était déjà arrivée à sa maturité et montre avec quelle rapidité elle croissait.

Après le froment, l'orge - ati-occupait la première place parmi les céréales de la vallée du Nil; comme lui, il y avait, à une époque antéhistorique, été importé de l'Asie antérieure, on on l'a, sur les points les plus divers, rencontré à l'état spontané une de ses variétés, lorge à deux rangs (Hordeum distichon L.) ${ }^{2}$. Les Égyptiens en connaissaient plusieurs races. Unger a découvert des fragments de l'orge ordinaire (Hordeumvulgare L.) dans une brique d'El-Kab (Eileithyia)', ainsi que de l'orge à six rangs ( $\boldsymbol{H}$. hexastichon L.) dans une brique de la pyramide de Dahshour", contemporaine de la XII dynastie. Parmi les mets funèbres d'une tombe de Saqqarah, qui date de la cinquième dynastie, Mariette a trouvé une coupe remplie d'épis d'orge à moitié brisés et décomposés, que Schweinfurth regarde comme appartenant à l'espèce vulgaire $^{5}$. Les épis et les grains d'orge trouvés parmi

1. Schweinfurth, Zeilschrift für Elhnologie, an.1891, p. 654.

2. A. de Candolle, op. laud., p. 295, lui attribue pour patrie la région qui va de la mer Rouge au Caucase et à la mer Caspienne. L'espèce sauvage (IIordeum sponlaneum C. Koch), dit $\mathrm{Fr}$. Körnicke, op. laud., p. 141, a été trouvée dans la région qui s'étend du Caucase à la Perse.

3. Silaungsberichle, t. XLV (an. 1862), p. 79.

4. Silzungsberichle, t. LIV, 1 (an. 1866), p. 40.

5. Berichte der deutschen botanischen Gesellschaft, t. II (an. 1884), p. 370. - Bullelin de l'Inslilul égyplien, no 5 (an. 1884), p. 4. 
les offrandes découvertes par M. Maspero dans les anciens tombeaux de Gébéleïn appartiennent, au contraire, à l'espèce à six rang's $(\boldsymbol{H} \text {. hexastichon L. })^{1}$. Le Musée de Boulaq renferme aussi plusieurs morceaux de pâte faite de grains d'orge - Schweinfurth ne dit pas de quelle variété - grossièrement moulus ${ }^{2}$. On a même découvert, dans les fouilles d'Abd-el-Gournah, autour du cou de la momie d'un égyptien du nom de Kent, une espèce de guirlande composée de grains d'orge à moitié germés ${ }^{3}$; mais Schweinfurth, qui en a fait mention, n'a pas déterminé l'espèce à laquelle ils appartiennent. M. Flinders Petrie a découvert à Kahoun ${ }^{4}$ des grains d'orge plus petits que ceux des variétés cultivées de nos jours. Les Égyptiens distinguaient deux espèces d'orge, comme de blé, l'ati blanc et l'ati rouge ; mais nous ignorons ce qui en faisait la différence.

Wilkinson ${ }^{6}$ et Unger ${ }^{7}$, à l'opinion desquels se sont rangés Lepsius ${ }^{8}$, Erman ${ }^{9}$ et Franz Wœnig ${ }^{10}$, ont cru reconnaître dans les représentations de scènes champêtres de Thèbes et d'El-Kab, ainsi que parmi des

1. G. Schweinfurth, Sur les dernières trouvailles, etc. Bulletin, no 7 (an. 1886), p. 420. Le Musée du Louvre renferme aussi plusieurs centaines de grains de cette espèce. Recueil de travaux, t. XVII (an. 1895), p. 180.

2. Bulletin de l'Institut égyptien, no 5 (an. 1884), p. 4.

3. Botanische Jahrbücher, VIII (an. 1886), p. 12. Kent parait avoir vécu au temps de la $x^{\mathrm{e}}$ dynastie.

4. Kahun, Gurob and Hawara, p. 30, 2.

5. Victor Loret, op. laud., p. 23, n० 18.

6. The manners and customs, t. II, p. 427 et 428 .

7. Sitzungsberichte, t. XXXVIII (an. 1859), p. 99.

8. Denkmüler, t. III, pl. 78.

9. Aegypten, t. II, p. 578.

10. Die Pflansen im allen Aegypten, p. 172. 
offrandes de Saqqarah et de Gizel, une autre céréale dont Hérodote et Théophraste ne parlent pas, ct que Pline a mentionnée le premier', le sorgho, ar. dourah

- Holcus ou Androgopogon sorghum Brotero, Sorghum vulgare Persoon, - cultivée aujourd'hui sur une grande échelle dans la Haute-Égyte, la Nubie, l'Abyssinie et le Soudan. Malheureusement les peintures que l'on invoque ne donnent aucune idée du dourah; la plante qu'elles représentent ne lui ressemblent ni par la hauteur des chaumes, ni par la forme des épis, courts et arrondis dans la céréale de ces peintures, tandis que ceux du sorgho s'étalent en panicule.

Ce qui a pu faire supposer qu'il s'agit de cette céréale, c'est la manière dont la récolte en est représentée; d'après une peinture d'EI-Kab on ne coupe point la plante comme on le faisait pour l'orge ou le froment, à l'aide d'une faucille, mais on l'arrache avec ses racines, et on en sépare les grains au moyen d'une espèce de séran. Comme l'a dit Schweinfurth, il s'agit tout simplement de la récolte du lin ${ }^{2}$. Rosellini avait rapporté d'une tombe de Thèbes des semences parmi lesquelles Hannerd crut découvrir des grains de sorgho ${ }^{3}$; mais depuis on a mis ce fait en doute ${ }^{4}$. Pickering prétendait aussi avoir vu dans une chambre funéraire de Saqqarah des tiges de dourah mêlées à des fragments de papyrus, mais elles n'auraient remonté

1. "Milium intra hos decem annos ex India in Italiam invectum est, nigrum colore, amplum grano, harundinaceum culmo. Adolescit ad pedes altitudine septem, praegrandibus comis : jubas vocant. " Lib. XVIII, cap. 55.

2. Zeitschrift für Ethnologie, an. 1891, p. 654.

3. Silsungsberichte, t. XXXVIII (an. 1859), p. 100.

4. S. Birch, ap. Wilkinson, op. laud., t. II, p. 427, note. 
qu'au temps de la domination romaine ${ }^{1}$. Schweinfurth n'indique point cette céréale parmi les plantes indigènes de l'Égypte ${ }^{2}$. On connait deux espèces de sorgho, cultivées de nos jours dans la vallée du Nil, le sorgho vulgaire, le dourah des Arabes, et le sorgho sucré (Sorghum succharatum P.), mentionné, supposet-on, dans la Bible sous le nom de dokhan, le dokhn des Arabes; de Candolle admet que c'est lá l'espèce décrite par Pline; il la croit originaire de l'Afrique tropicale $^{3}$; le dourah n'avait peut-être pas néanmoins été importé de cette région en Égypte, et Pline l'y fait venir de l'Inde.

Outre ces céréales, Unger a mentionné encore, comme appartenant à la flore de l'ancienne Égypte ${ }^{4}$, le Pennisetum typhoideum DC., le Panicum miliaceum L., dont l'origine est obscure, et le Panicum italicum L., qui parait indigène de l'Asie orientale". Mais le botaniste viennois avoue lui-mème qu'on ne sait rien de certain sur l'ancienne culture du Pennisetum dans la vallée du Nil, et le passage d'Hérodote sur lequel il s'appuie pour prouver celle du millet ne parait guère s'appliquer à l'Égypte. Quant au panicaut d'Italie, Charles Pickering, qui, à la vérité, ne se piquait guère d'exactitude, croit l'avoir reconnu dans les peintures de la tombe de Ramsès et à El-Kíab ${ }^{6}$;

1. "I have seen dourra stems intermingled with those of the Papyrus in a parcel exhumed at Saccara, possibly as ancient as the time of the Romans. " The races of man, p. 399.

2. Illustration de la Flore d'Égypte, p. 163.

3. Origine des planles cullivées,, $\mathrm{p} .307$.

4. Silzungsberichte, t. XXXVIII (an. 1859), p. 100.

5. A. de Candolle, op. laud., p. 303 et 305.

6. The races of man, p. 376 . 
mais son témoignage est resté isolé. Une brique de cette dernière localité, il est vrai, examinée par Unger', renfermait, aflirme-t-il, des caryopses d'un panicaut; mais comme il n'y avait pas de graine, il n'a pu en déterminer l'espèce et rien ne dit que ces débris appartiennent à l'une des deux précédentes.

Au cour's de recherches ultérieures ${ }^{2}$, Unger a cru encore découvrir des graines d'une autre céréale, l'Eragrostis ou Poa abyssinica Link, dans deux briques de Dahshour et de Tell-el-Maskhouta; cette plante cultivée fréquemment aujourd'hui en Abyssinie, donne un excellent pain; mais Schweinfurth préfère rapporter à l'Eragrostis aegyptiaca Link, espèce non alimentaire, les grains étudiés par le botaniste viennois ${ }^{3}$; on peut donc mettre en doute que l'E. abyssinica Link ait été réellement cultivée dans l'ancienne Égypte.

Il faut rapprocher des céréales utilisées dans la rallée du Nil pour la nourriture de l'homme l'avoine rigide (Avena strigosa Schreb.); M. Flinders Petrie en a trouvé quatre grains, mêlés aux offrandes d'orge, dans la nécropole de Kahoun (XII ${ }^{\mathrm{e}}$ dynastie) ${ }^{4}$; il en a découvert aussi des restes dans le cimetière grécoromain de Hawara". Cette espèce n'a pas été trouvée a l'état spontané et $\mathrm{A}$. de Candolle la regarde comme une simple variété de l'avoine cultivée ordinaire ${ }^{6}$; on la rencontre parfois encore aujourd'hui en Égypte

1. Sitsungsberichle, t. XLV (an. 1862), p. 81.

2. Sitzungsberichle, t. LIV (an. 1866), p. 42 et LV (an. 1867), p. 202 .

3. Victor Loret, La Flore pharaonique, p. 21, no 11.

4. Kahun, Gurob and IIawara, p. 50.

5. Hawara, Biahmu and Arsinoë, p. 49.

6. Oigine des planles cullivies, p. 301. 
dans les champs abandonnés, suivant Percy Newberry ${ }^{1}$; mais ce n'est pas une raison pour qu'elle y ait été cultivée autrefois. L'était-elle en Asie-Mineure, ainsi que parait le supposer le botaniste anglais ${ }^{2}$, en l'identifiant avec le Bpópros - l'avoine - dont parle Galien ${ }^{3}$, comme d'une plante abondante dans cette contrée et en particulier dans la Mysie, au-dessus de Pergane? " Il sert, dit le médecin grec, d'aliment aux chevaux, non aux hommes, excepté toutefois en temps de disette, où l'on en fait alors du pain ». Évidemment rien n'indique que le Bromos de Galien fût l'Avena strigosa; mais que cette espèce ou une autre ait été cultivée en Mysie, c'est probablement d'Asie que l'aroine rugueuse a été importée dans la vallée du Nil.

II.

\section{Plantes fourragères.}

Possesseurs de troupeaux et de bêtes de somme d'espèces diverses, les Égyptiens furent naturellement obligés de cultiver des plantes fourragères propres à nourrir ces nombreux animaux pendant la durée de l'inondation, commeà la fin de la saison sèche; il y avait là pour eux une source nouvelle d'occupations agricoles, auxquelles on a jusqu'ici fait trop peu attention.

1. Il faut dire toutefois que Schweinfurth ne la mentionne pas comme indigène dans l'Illustration de la Flore d'Égyple.

2. Howara, Biahmu and Arsinoë, p. 49.

3. De facullalibus alimentorum, lib. I, cap. 14́, éd. Kühn, t. VI, p. 522 . 
Ce lurent des légumineuses surtout qui, dès les temps les plus reculés, furent employées à cet usage, comme elles servent encore chez nous. Parmi celles que nous ont fait connaitre les fouilles archéologiques, il faut mentionner le trèfle d'Alexandrie, la vesce et la gesse cultivées, ainsi pent-être que la gesse velue, enfin le pois des champs.

M. Flinders Petrie a découvert seize graines de trèfle d'Alexandrie (Trifolium Alexandrinum L.), à Kahoun '; cette plante, originaire de l'Asie-Mineure ${ }^{2}$, arait donc été importée en Égypte dès le temps de l'ancien empire ${ }^{3}$; elle y existait sous la XII ${ }^{e}$ dynastie et elle n'a pas cessé d'y ètre cultivée depuis; comme à Kahoun, des graines de ce trèfle ont été trouvées aussi par le mème égyptologue dans la nécropole grècoromaine de Hawara ${ }^{4}$.

M. Flinders Petrie a également découvert des graines - buit - de pois gris (Pisum arvense L.) à Kahoun ${ }^{5}$; il en a aussi rencontré, ce qui est moins surprenant, dans la nécropole de Hawara ${ }^{6}$. Avant lui, Unger avait reconnu des fragments de cette légumineuse dans une brique de Dahshour ${ }^{7}$, qui remonte à l'époque de la $\mathrm{XII}{ }^{e}$ dynastie; on ne peut guère douter dès lors que le pois gris n'ait été, de même que le petit pois, nous le

1. Kahun, Gurob and Hawara, p. 50.

2. A. de Candolle, op. laud., p. 85.

3. Schweinfurth, Zeilschrift für Ellhnologie, an. 1891, p. 666, qui croit le trèfle d'Alexandrie originaire de la presqu'ile des Balkans, n'admet pas qu'il ait pu être importé en Egypte avant l'époque gréco-romaine.

4. Iawara, Biahmu and Arsinoë, p. 52.

5. Kahun, Gurob and Hanvara, p. 50.

6. Llawara, Biahmu and Arsinoë, p. 52.

7. Silsungsberichte, t. LIV, 1 (an. 1866), p. 43. 
verrons, cultivé très anciennement en Égypte. Il semble d'après cela qu'il soit bien plutòt originaire du Caucase, que de la Péninsule italique, ainsi que l'a cru A. de Candolle 1 .

Une autre légumineuse, originaire de la région située au midi du Caucase et de la Caspienne ${ }^{2}$, la gesse cultivée (Lathyrus sativus L.) parait bien aussi avoir été connue dans l'ancienne Egypte. Son nom copte pihouf semble à M. Victor Loret ${ }^{3}$ d'origine hiéroglyphique. Schiaparelli a trouvé des graines de cette gesse dans la nécropole de Drah-Abou'l-Neggah; mais Schweinfurth a mis en doute leur ancienneté ${ }^{4}$. M. Maspero en a découvert aussi dans les tombes de Gébéleïn", relativement récentes, il est vrai. Des restes de cette plante ont été rencontrés également par M. Flinders Petrie, dans la nécropole gréco-romaine de Hawara ${ }^{6}$.

- Toutefois comme la gesse se rencontre souvent à l'état subspontané dans les moissons, on peut se demander si les graines, trouvées dans ces tombes, provenaient de pieds saurages ou cultivés. On pourrait faire aussi la même remarque au sujet des gousses et des graines de gesse velue (Lathyrus hirsutus L.) recueillies aussi par Schiaparelli dans les tombes de DrahAbou'l-Neggah ${ }^{\top}$; mais Schweinfurth n'admet pas leur

1. Origine des plantes cultivées, p. 262.

2. A. de Candolle, op. laud., p. 88.

3. La Flore pharaonique, p. 94, no 159.

4. Bulletin de l'Instilul égyplien, $\mathrm{n}^{\circ} 6$ (an. 1885), p. 265. Bolanische Jahrbücher, t. VIII (an. 1886), p. 6.

5. Bulletin de l'Institul égyptien, no 6. p. 260. - Bolanische Jahrbücher, t. VIII, p. 3.

6. Kahun, Gurob and IIawara, p. 47, 2.

7. Bulletin de l'Institul égyptien, n 6, (an. 1885), p. 265.Bolanische Jahrbücher, t. VIII, p. 6. 
authenticité et croit qu'elles proviennent de battages récents. On ne peut dès lors qu'hésiter à considérer cette espèce comme ayant été anciennement cultivée en Égyte.

La vesce commune (Vicia sativa L.), au contraire, l'y était-clle réellement? Schweinfurth a trouvé, dans une armoire du musée de Boulaq, un fragment de gousse de cette papilionacée provenant des fouilles de DrahAbou'l-Neggah '; Unger en a découvert aussi des débris dans une brique de la pyramide de Dahshour ${ }^{2}$. Cette plante existait donc en Égypte à une époque reculée; mais y était-elle cultivée ou y croissait-elle spontanément? C'est là une question qu'il est difficile de résoudre dans l'état actuel de nos connaissances en botanique égyptologique, surtout à cause de la facilité avec laquelle la vesce devient spontanée. On la rencontre dans presque toute l'Asie antérieure et jusque dans l'Inde ; on comprend qu'elle ait pu facilement pénétrer en Égypte.

Ces papilionacées ne furent pas les seules plantes employées comme fourrages par les anciens Égyptiens; sans parler des céréales, comme l'orge, dont la paille, sinon le grain, dut leur servir pour cet usage; ils eurent recours, dans le même but, à bien d'autres représentants de la flore indigène. Théophraste dit même, sans doute en exagérant, que toutes les plantes du pays servaient également de fourrages aux bœufs et aux brebis. Mais il en cite deux qui, parait-il, étaient surtout employées comme tels:

1. Bulletin de l'Institut égyplien, no 5 (an. 1884), p. 10.

2. Silzungsberichte, t. LIV, 1 (an. 1866), p. 54.

3. A. de Candolle, Origine des plantes cullivées, p. 86. 
" Il y a, dit-il", une plante qui croit spontanément et en abondance au milien des moissons, on en recueille lasemence, quand on sarcle celles-ci, et en hiver on la dépose dans la terre humide. Elle lève et pousse rapidement; on coupe alors la plante, on la fait sécher et on la donne aux boufs, aux chevaux et aux bêtes de somme. La graine est de la grosseur du sésame, mais ronde, de couleur verte et exceptionnellement bonne. "

Sprengel ${ }^{2}$ a cru reconnaître dans cette plante le Corchorus aestuans L.; mais cette tiliacée n'est mentionnée dans la flore d'Égypte, ni par Schweinfurth, ni par Delile; peut-être est-ce le Corchorus trilocularis L. ou antichorus Raeusch ${ }^{3}$. Il est difficile d'ailleurs de se prononcer d'après une description aussi vague que celle de Théophraste. Quant à la seconde plante mentionnée par le naturaliste grec, elle semble bien avoir été une graminée:

"Il croît, remarque-t-il", dans les lagunes et les marécages une plante fort précieuse pour la nourriture du bétail; elle se mange verte et en hiver on la donne sèche aux bœufs qui travaillent; ils s'en trouvent très bien, même quand ils ne mangent rien autre chose. ")

Sprengel a supposé qu'il s'agissait du Panicum appressum Lam. ou grossarium L., espèces qui ne sont mentionnées, ni par Delile, ni par Schweinfurth, ni

1. IIistoria plantarum, lib. IV, cap. 8, 14 .

2. Theophrasts Nahurgeschichte der Gewüchse überselzt und crlëulert, ap. Fr. Woenig, op. laud., p. 134.

3. Illustration de la Flore d'Égyple, p. 53. - Boissier, Flora orienlalis, t. I, p. 816 .

4. IIistoria plantarum, lib. IV, cap. 8, 13. 
par Boissier; mais il peut bien se faire qu'il s'agisse d'un panicum, peut-ètre le $P$. paspaliodes Pers., ( $P$. geminatum Forsk.), ou du Saccharum aegyptiacum Willd.', plantes communes dans les mar'écages de l'Egypte.

III.

Plantes industrielles.

A còté des céréales et des plantes fourragères, les plantes industrielles occupaient une place considérable dans l'agriculture égyptienne; la plus grande était prise par le lin. D'une origine incertaine, il avait sans doute été importé de l'Asie antérieure dans la vallée du Nil ${ }^{2}$. Il y apparaît à l'époque la plus reculée. Les peintures tombales des Pharaons de la XII e dynastie à Kom-el-Ahmar et à Béni-Hassan ${ }^{3}$ nous font assister aux direrses scènes de sa récolte et au rouissage du lin, ainsi qu'au filage et au tissage de ses produits; il était à cette époque et probablement depuis longtemps déjà employé à la fabrication des étoffes. L'examen microscopique a montré que les tissus dont sont enveloppées les momies étaient en pur lin ${ }^{4}$, et Hérodote nous apprend que les prètres ne devaient porter que

1. Illustration de la Flore d'igyple, p. 159 et 163. Boissier, Flora orientalis, t. V, p. 436 et 454.

2. A. de Candolle, op. laud., p. 97 et 101 . - Victor Hehn, Die Kullurpflanzen, p. 161.

3. Rosellini, Monumenti dell' Egillo, t. II, pl. 35, 41, 42.

4. H. Brugsch, Ueber die äyyptischen Benennungen fï̈. Sindon und Byssus. (Allgemeine Monalschrift. Braunschweig, 1854, in-8, p. 633.) 
des vètements faits avec ce textile ${ }^{1}$. On a trouvé en quantité dans les anciennes tombes des capsules de cette plante si utile. Unger a attribué celles qu'il a observées à l'espèce commune (Linum usitatissimum L. $)^{2}$; Schweinfurth, qui a étudié un grand nombre de graines de lin trouvées par Mariette à Drah-Abou']Neggah, les considère, lui, comme appartenant à l'espèce à tige basse ( $L$. humile Miller) ${ }^{3}$, qui riest d'ailleur's peut-être qu'une variété plus petite du $L$. usitatissimum. C'est une intermédiaire entre l'espèce. el cette variété qui aujourd'hui est, dit-on, exclusivement cultivée en Égypte et en Abyssinie *. Sur trois graines de lin, conservées au musée de Berlin, Braun en a reconnu deux comme appartenant à l'espèce à basse tige, mais la troisième lui a paru se rapporter au lin à feuilles étroites (L. angustifolium DC. $)^{5}$. C'est, paraitil, à cette espèce aussi qu'appartenaient la plupart des graines de lin trourées par M. Flinders Petrie à Kahoun ${ }^{6}$ - 143 sur 163 -, tandis que les 20 autres, ainsi que les quatre capsules découvertes à Hawara ${ }^{\top}$, se rapportent au Linum humiles.

1. Historiae, lib. II, cap. 37.

2. Sitzungsberichte, t. XXXVIII (an. 1859), p. 128, et LIV (an. 1866), p. 46.

3. Berichte der botanischen Gesellschaft, t. III (1884), p. 360.

4. Supplément à l'Illustration de la Flore d'Égypte. (Mémoires de l'Institut égyptien, t. II, $2^{\mathrm{e}}$ partie, p. 751).

5. Die Pflanzenreste des ägyptischen Museums in Berlin. (Zeitschrift für Ethnologie. Berlin, in-8, t. IX (an. 1877), p. 290.) Le Linum angustifolium croît spontanément du Caucase à l'Atlantique.

6. Kahun, Gurob and Hawara, p. 50.

7. Hawara, Biahmu and Arsinö̈, p. 50.

8. Pline parle de quatre espèces de lin d'Égypte, mais il s'agit seulement de formes locales sans importance : "Quatuor" 
Une plante aussi anciemmement connue derait porter un nom indigène; suivant M. Victor Loret ${ }^{1}$, clle s'appelait mihi dans la langue hiéroglyphique, dénomination qui s'est conservée en copte. Le lin représcntait arec l'orge et le froment les principales cultures de l'Égypte pharaonique. "Son commencement, dit une inscription en parlant du champ d'Osiris², est semé d'orge, son extrémité est semée d'épeautre et son milieu est semé de lin ».

Les anciens Égyptiens connaissaient-ils d'autres plantes textiles que le lin? Le chanvre, son rival dans les pays tempérés, parait leur ètre toujours resté inconnu comme textile et même, quoi qu'on en ait dit ${ }^{3}$, comme stupéfiant; mais on trouve dans la vallée du Nil des malvacées dont ils ont pu utiliser les fibres, tel que l'Hibiscus, dont une espèce, le cannabinus, est cultivée de nos jours en Égypte". Cependant rien ne nous dit qu'on en ait autrefois tiré parti. Il n'en a pas été ainsi d'une autre plante que Pline appelle gossipion ou $x y$ lon; le polygraphe latin en a domné une description",

ibi genera: Tanaticum ac Pelusiacum, Buticum, Tentyricum, cum regionum nominibus, in quibus nascuntur. " Lib. XIX, cap. 2.

1. La Flore pharaonique, p. 107, no 177.

2. Victor Loret, Les fêtes d'Osiris an mois de Khoiak. (Recucil de liavaux, t. IV, p. 2'x.)

2. Unger a voulu y voir le népenthès donné par Polydamna a Ilélène pour lui faire oublier ses maux passés. Silaungsberichte, t. XXXVIII (an. 1859), p. 110.

f. P. Ascherson et G. Schweinfurth, Illustralion de la Flore d'Éyypte, s. v. Dans une communication faite, en 1891, à la Société d anthropologie et d'ethnogénie de Berlin, M. Schweinfurth a paru regarder comme très ancienne la culture de cette plante. Zeilschrift für Elhnologie, an. 1891, p. 656.

5. Lib. XIX, cap. 2 : "Superior pars Aegypti... gignit fruticem, quem aliqui gossipion vocant, plures xylon, et ideo 
dans laquelle il est impossible de ne pas reconnaitre le cotonnier arborescent (Gossypium arboreum L.), plante de la même famille que l'Hibiscus. Cet arbuste, auquel Virgile fait allusion dans un vers bien connu des Géorgiques ${ }^{1}$, est indigène dans l'Afrique intertropicale; il n'y a donc rien de surprenant qu'on le rencontràt dans la Haute-Égypte.

Les contemporains des Pharaons ont-ils su utiliser cette plante textile? Pline dit que les prêtres égyptiens se servaient de préférence de vêtements en coton; H. Brugsch, de son côté, incline à croire que le shent ou tablier égyptien était fait d'une étoffe de même

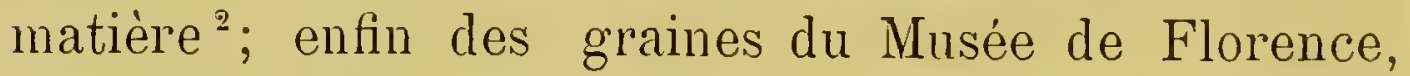
trouvées par Rosellini dans un vase de Thèbes, ont été reconnues par Parlatore, qui les a examinées avec soin, comme appartenant au Gossypium arboreum ${ }^{3}$. Il semble donc hors de doute que les anciens Égyptiens ont connu et probablement cultivé le cotonnier arborescent. Pollux, qui était originaire de la région, le dit expressément ${ }^{4}$, et, comme Pline, il affirme qu'ils tissaient des étoffes avec l'espèce de laine que renferment ses fruits, mais peut-être ne l'ont-ils fait qu'assez tard, ce qui expliquerait l'absence de tissus en coton dans les tombes pharaoniques ${ }^{5}$ ?

lina inde facta xylina. Parvus est, similemque barbatae nucis defert fructum, cujus ex interiore bombyce lanugo netur; nec ulla sunt eis candore mollitiave praeferenda. »

1. Nemora Aethiopum molli canentia lana. Lib. II, v. 120.

2. Allgemeine Monatschrift, an. 1854, p. 652.

3. Specie di coloni, p. 16, ap. A. de Candolle, op. laud., p. 326.

4. Onomasticon, I, 75, éd. Bekker. Berlin, 1846, in-8, p. 293.

5. M. Victor Loret, op. laud., p. 105, dit, mais sans donner aucune référence ni date, qu'on a reconnu, en les examinant au microscope, que " quelques-unes» des bandelettes trouvées 
Outre les plantes textiles, les Egyptiens cultivaient aussi un certain nombre d'autres végétaux pour en retirer l'huile ou les matières colorantes nécessaires aux usages domestiques ou a l'industric. Parni les premicrs se rangent, outre l'olivier et l'arbre à noix de ben, dont il sera question plus loin, le ricin et le sésame; parmi les seconds, le carthame, le henné et l’indigotier.

Le ricin (Ricinus communis L.) n'est point indigène en Égypte; y a-t-il été ancionnement connu ou cultivé? Unger ${ }^{-1}$ a cru reconnaitre des plants de ricin dans des peintures de Thèbos et de Tell-el-Amarna ${ }^{2}$, et il affirme qu'il se trouve au musée égyptien de Vienne, ainsi que dans la collection Passalacqua, une graine de cette euphorbiacée; il en existe aussi plusieurs au musée du Lourre ${ }^{3}$. Schiaparelli a également trouvé des graines de ricin à Drah-Abou'l-Neggah, mais Schweinfurth en a mis en doutc l'ancienneté ${ }^{4}$, et on ignore l'origine de celles du Lourre. Quant aux peintures de Thèbes et de Tell-el-Amarna qui, d'après Franz Woenig " ${ }^{\circ}$, représenteraient probablement des ricins, l'une, celle de Thèbes, est trop conventionnelle ou trop inexacte pour qu'on puisse en tirer aucune conclusion, et l'autre, celle de

dans les tombes égyptiennes étaient en coton; Unger, Silzungsberichte, t. XXXVIII (an. 1859), p. 129, affirme, au contraire, que l'examen microscopique des étoffes, déconvertes dans les hypogées pharaoniques, a montré qu'elles étaient en fil de lin et non en coton.

1. Silzungsberichte, t. XXXVIII (an. 1859), p. 127.

2. Wilkinson, The manners of ancient Egyplians, t. II, p. 413, affirme aussi qu'on a trouvé le ricin dans des tombes de Thèbes.

3. Recueil de travaux, t. XVII (an. 1895), 17, p. 188.

4. Bolanische Jahrbücher, t. VIII, p. 6.

5. Die Pflanzen im allen Aegypten, p. 338. 
Tell-el-Amarna, représente certainement non un ricin, mais, une vigne ${ }^{1}$. On ne saurait trouver la une preuve que le ricin ait été cultivé en Égypte au temps de l'ancien empire; toutefois il est vraisemblable qu'il l'a été à une époque reculée.

Cette plante, qui, dans les pays chauds, devient arborescente et vivace - Dioscoride ${ }^{2}$ compare le ricin à un figuier de petite taille ${ }^{3}-$, tandis que dans nos climats elle est herbacée et annuelle, paraît être originaire de l'Afrique tropicale ${ }^{4}$; il n'y aurait donc rien de surprenant qu'elle eût pénétré de bonne heure dans la vallée du Nil. C'est aussi ce qui arriva; la mention faite des graines et de l'huile de ricin dans des recettes du papyrus Ebers prouve qu'on connaissait et qu'on cultivait sans doute déjà cette plante sous la XIX ${ }^{\mathrm{e}}$ dynastie $^{5}$. Un officier d'Apriès, pharaon de la XXVI ${ }^{\mathrm{e}}$, Nesher, gouverneur des provinces méridionales, se vante, dans une inscription, d'avoir donné de l'huile de teqem, c'est-à-dire de ricin, pour l'éclairage du sanctuaire

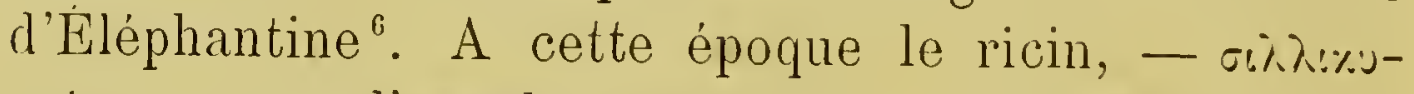

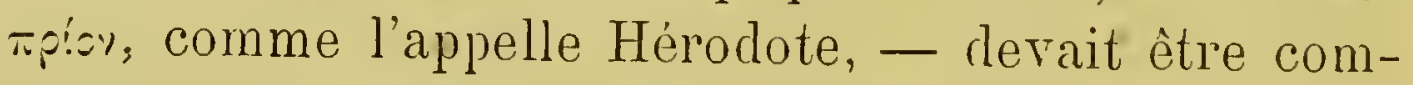
mun dans la vallée du Nil : "On le cultive, dit l'his-

1. Il en est de même probablement de celle de Thèbes, tant elle ressemble à une vigne grossièrement dessinée.

2. De materia medica, lib. IV, cap. 161 (15'́).

3. Pline dit qu'il atteint la hauteur de l'olivier, lib. XV, cap. 7. J'ai vu à Vintimiglia des ricins qui avaient trois à quatre mètres de haut.

4. A. de Candolle, op. laud., p. 340.

5. Chabás, Nolice du papyrus Ebers. (L'Égyplologie, t. I (an. 1876), p. 178).

6. Paul Pierret, Études égyptologiques, fasc. II, p. 23, lit "huile de l'arbre desher ". NI. E. Revillout, Revue égyplologique. t. II (an. 1882), p. 82, de "l'huile de teqem." 


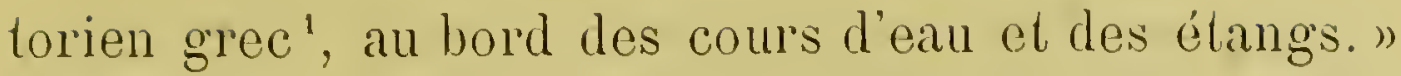
D'après lui, le fruit portait le nom de likik; c'était celui qu'il avait aussi en copte ${ }^{2}$. Strabon ${ }^{3}$, ainsi que Dioscoride ${ }^{4}$, attribuaient ce nom au ricin lui-mème, et le premier affirme qu'on le semait dans les champs. Le nom hiéroglyphique du ricin teqem, teliam on deqam, atteste la haute antiquité de sa culture en Égypte et parait bien indiquer qu'il n'y arait pas été importé par l'intermédiaire des Sémites.

Pas plus que le ricin, le sésame (Sesamum indicum DC.) n'est indigène dans la vallée du Nil. A quelle époque y a-t-il été importé? D'après A. de Candolle cette plante oléagineuse est originaire des iles de la Sonde"; ce serait de là qu'à une époque préhistorique elle aurait passé dans l'Inde, d'où elle se serait ensuite répandue successivement dans la vallée de l'Euphrate et plus tard dans celle du Nil. Cette hypothèse n'a rien d'invraisemblable, mais il faut aire toutefois que toutes les espèces de sésame sont indigènes dans l'Afrique tropicale ${ }^{6}$, et que par suite la variété cultivée pourrait bien renir de cette région. Le sésame porte en arabe le nom semsem ou simsim; faut-il y voir l'origine du mot hiéroglyphique shemshem, qui, d'après M. Yictor Loret', désigne une plante dontles

1. Historiae, lib. II, cap. 94.

2. Victor Loret, La Flore pharaonique, p. 49, no 6x. Dans la même langue, le nom de la plante est djismis.

3. Geographica, lib. XVII, cap. 2, 5.

4. De maleria medica, lib. IV, cap. 161. Il est surprenant que Dioscoride dise que le ricin portait le nom de Syslanna en égyptien.

5. Origine des plantes cullivées, p. 339.

6. Schweinfurth, Zcilschrifl für Elhnologie, an. 1891, p. 659.

7. La Flore placraonique, p. 57, no 91. 
graines se mangent, c'est-à-dire le sésame? S'il en était ainsi, le sésame serait venu en Égypte par l'intermédiaire des Sémites. 'Toutefois comme le nom copte de celte plante, olie', a une autre origine et qu'on rencontre dans les textes hiéroglyphiques une plante appelée alke, dont on retirait de l'huile, le savant égyptologue lyonnais s'est demandé si ce mot ake ne désignerait pas le sésame; dans ce cas, l'existence de cette plante oléagineuse dans la vallée du Nil remonterait nécessairement à une époque très reculée. Peutêtre y avait-elle été introduite, comme le pense A. de Candolle, à la suite des expéditions des Ramessides dans la Mésopotamie, où sa culture parait avoir été très ancienne; mais ce n'est là qu'une supposition.

Unger $^{2}$ et Franz Wonig ${ }^{3}$ ont roulu voir des semences de sésames dans les petites graines dont un apprenti boulanger, sur une peinture du tombeau de Ramsès III, saupoudre un pain qu'on va mettre au four; mais si on se sert aujourd'hui de graines de cette plante pour cet usage, on en emploie également d'autres; rien ne dit, ainsi que l'a fait remarquer $A$. de Candolle, qu'il n'en fût pas de même dans l'antiquité, et il est impossible de reconnaître à quelle espèce végétale appartiennent les graines des peintures de la $\mathrm{XX}^{\mathrm{c}}$ dynastie. Hérodote ne mentionne pas le sésame parmi les plantes de la vallée du Nil; Théophraste, qui revient à plusieurs reprises sur les caractères de cette plante, en particulier sur la forme de sa tige et

1. Cettc forme est indiquée par M. Loret; Kircher, Lingua aeg!ppliacr restilula, p. 194, donne pi-phati.

2. Silzungsberichle, t. XXXVIII (an. 1859), p. 112.

3. Die Pflanzen im allen Aegyplen, p. 178. 
de sesfruits, sur son inflorescence ${ }^{1}$, ne nous apprend lien sur les contrées où on la cultivait. Mais Pline ${ }^{2}$ lindique en Égyte; il en est de mème de Dioscoride ${ }^{3}$.

Si on est si peu renseigné sur la culture du sésame dans la vallée du Nil, de nombreux documents, au contraire, nous font connaitre celle dont le carthame ( $\mathrm{Car}$ thamus inctorius L.) y était l'objet; mais si les habitants du pays retiraient aussi de l'huile de ses graines", c'était surtout à cause de ses propriétés colorantes qu'ils cultivaient cette composée. D'où l'avaient-ils reçue, ainsi que les autres peuples de l'ancien monde? Le carthame n'a été trouré nulle part à l'état spontané, mais A. de Candolle incline à le croire originaire de l'Arabie ${ }^{5}$. Quoi qu'il en soit, cette plante fut connue de bonne heure en Égypte, M. Schiaparelli a trouvé dans une tombe de Shéikh Abd-el-Gournah, qui remonte à la XIX ${ }^{e}$ dynastie, un fragment d'une guirlande formée de feuilles de saule entremêlées arec des fleurs de carthame ${ }^{6}$. La momie d'Amenhotpou I, découverte en 1881 par M. Maspero à Deir-el-Bahari, portait sur la

1. Hislofia planlarum, lib. VIII, cap. 3.

2. Hisloria naluralis, lib. XV, cap. 7, 5 .

3. De maleria medica, lib. II, cap. 121.

4. " Naxime celebrant (Aegypti) cnicon, Italiae ignotam, ipsis autem oleo, non cibo, gratam : hoc faciunt e semine eius. » Pline, lib. XXI, cap. 53.

5. Origine des plantes cullivées, p. 131. G. Schweinfurtn, Zeilschrift für Elhnologie, an. 1891, p. 665, le regarde comme venant de la Syrie ou de l'Arménie, où croît à l'état sauvage le Carlhamus flavescens IV., le type peut-être de l'espèce cultivée.

6. Bolanische Jahrbïcher, t. VIII (an. 1886), p. 9. - Bullelin de l'Instilul égyplien, no 6 (an. 1886). p. 280. 
poitrine une couronne semblable', dont les fleurs avaient conservé presque tout leur éclat. Enfin le mot nasi ou nasti, rencontré par Dümichen dans une inscription hiéroglyphique ${ }^{2}$, désigne une plante dont une partie de la fleur servait à teindre en rouge. M. Victor Loret veut, non sans raison, y voir le carthame ${ }^{3}$. Or le mème mot, avec l'orthoglaphe nas, se retrouve dans une inscription de la pyramide du roi Téti, qui appartenait à la VI ${ }^{e}$ dynastie". Si ce vocable nas ( $i$ ) est bien le nom du carthame, on a lá une preuve de l'époque reculée à laquelle cette plante aurait été connue en Égypte. Pline, qui donne au carthame le nom de cnicos, distingue deux espèces de cette plante précieuse, la sauvage et la cultivée; la graine, dit-il, en est blanche, grosse et amère. Cette graine fournissait aux habitants de la vallée du Nil une huile estimée, tandis que de la fleur on retirait la couleur rouge dont leur industrie avait besoin.

Un arbuste, "l'arbre au henné " - Shagarat-elHenné des Arabes - (Lawsonia inermis L.), leur donnait une couleur d'un rouge orange non moins recherchée. Cette ly thrariée, qui peut atteindre trois à quatre mètres de haut, a des feuilles lancéulées, semblables a celles de l'olivier, mais plus larges, moins rigides et plus vertes, opposées et serrées contre la tige, arec des fleurs à quatre pétales blancs, réunies en corymbe

1. Bulletin de l'Institul égyptien, $\mathrm{n}^{\circ} 3$ (an. 1882), p. 70. Berichte der botanischen Gesellschaft, t. II (an. 1884), p. 365.

2. Geographische Inschriften allägyptischer Dentimäler. (H. Brugsch, Recueil de monuments égypliens. Leipzig, t. IV (an. 1886), pl. 90.)

3. La Flore pharaonique, p. $66, n^{\circ} 108$.

4. G. Maspero, La Pyramide du roi Tile. (Recueil de travalu. t. V (an. 1881), p. 50). Cf. Victor Loret, (p). laud.. p. 67. 
i l'extrémité des rameaux et d'une odeur délicieuse; elle se rencontre dans toute la région, qui s'étend de l'Inde ì la Nubie, sans qu'on puisse dire quel est au juste son pays d'origine'; Emin Pacha a cru la trouver à l'état spontané, à l'est ảe Latouka au $4^{\circ}$ degré de latitude septentrionale ${ }^{2}$; mais Schweinfurth a mis en doute cette découverte, et il inclineraiti faire venir le henné de la Perse ${ }^{3}$, ou il aurait été importé de l'Inde antérieure. En 1820, Minutoli en trouva des feuilles dans des tombes antiques" ${ }^{4}$ M. Maspero a découvert à son tour des boutons et des fleurs épanouies de Lawsonia dans les hypogées probablement peu anciens, il est vrai, de Gébéleïn", et plus récemment $M$. Flinder's Petrie en a trouvé aussi des fragments dans la nécropole gréco-romaine de Hawara ${ }^{6}$.

Théophraste ne parle pas du Lausonia; Dioscoride *, qui l'appelle \%úropṣ et l'a très bien décrit, dit qu'il réussissait surtout à Canope, et Pline $^{8}$ vante le parfum qu'on fabriquait dans cette ville avec ses fleurs. L'arbre au henné portait dans la langue hiéroglyphique le nom de pouqer, hébreu kopher par transposition de lettres, en copte koupher ou kouper; M. Victor Loret ${ }^{9} y$ voit l'origine probable du nom grec жúrpos et peut-être de l'arabe faghou ou faghiah. Le mot persan henné désigne

1. A. de Candolle, Origine des plantes cultivées, p. 110.

2. Percy Newberry, ap. Flinders Petrie, IIawara, Biahmu and Arsinoë. p. 50.

3. Zeitschrift für Ethnologie, an. 1891, p. 658.

4. Reise zum Tempel des Ammon, p. 350.

5. Berichle der bolanischen Gesellschaft, t. II (an. 1884), p. 360.

6. Hawara, Biahmu and Arsinoë, p. 50.

7. De materia medica, lib. I, cap. 124.

8. IIistoria naturalis, lib. XII, cap. 51.

9. La Flore pharaonique, p. 80, no 134. 
la poudre rouge orange obtenue avec les feuilles desséchées et broyées du Lawsonia; Prospero Alpini, qui en parle, la nomme archenda ${ }^{1}$. Avant lui Pierre Belon avait aussi remarqué le Lawsonia, " petit arbre d'Égypte tousiours verd qui teinct en rouge s et dont les habitants, qui le nomment Henne ou Alcanna, ditil ${ }^{2}$, "font de beaux petits bois taillis ".

Le Lawsonia fut peut-être cultivé dans l'ancienne Égypte autant pour le parfum de ses fleurs que pour la couleur qu'on extrayait de ses feuilles; l'indigotier ne le fut que pour cette dernière raison. Quel est le pays d'origine de cette plante? Elle n'est pas indigène en Égypte; mais une espèce à feuilles argentées, l'Indigofera argentea L., croit spontanément dans l'Abyssinie, ainsi qu'au Kordofan et à Sennaar ${ }^{3}$; peutêtre a-t-elle été importée de l'une de ces contrées dans la vallée du Nil. Un texte hiéroglyphique, relatif à la teinture, fait connaître le nom égyptien de l'indigo; il s'appelait dinkon, mot qui signifie littéralement "qui chasse les tranchées ", propriété astringente attribuée par Dioscoride à l'indigo". M. Victor Loret a voulu voir dans le vocable dinkon l'origine du nom grec ¿yò:óy, latin indicum, donné par les anciens à l'indigo"; pour qu'il en fùt ainsi, il faudrait que les Grecs

1..De plantis aegypliacis, cap. XIII, p. 46. Alpini donne au henné les noms de "troène égyptien ", elhanne, tamarhendi ou " alcanna d'Avicenne."

2. Les observalions de plusieurs singularilés el choses mémorables trouvées en Grèce, Asie, Judée, Égypte, Arabie et autres pays étranges. Paris, $2^{2}$ éd., 1554, in-fol. Livre II, chap. Lximin, p. 35.

3. A. de Candolle, Origine des planles cullivées, p. 109.

4. De maleria medica, lib. V, cap. 107.

5. La Flore pharaonique, p. 90, no 150. Dans cette hypo- 
eussent reçu d'Egypte cette plante tinctoriale, ce qui n'est rien moins que prouré.

Le dinkon, qu'on trouve mentionné plusieurs fois dans les papyrus médicaux, a été connu et peut-être cultivé, anciennement, dans le pays de Qimit; Pickering a supposé que les pains cylindriques de couleur azurée qu'on voit, sur plusieurs peintures pharaoniques, portés sulennellement dans des corbeilles, étaient de l'indigo'. D'où était-il apporté en Égypte? Probablement de l'Abyssinie, de l'Éthiopie ou de la Nubie, ou il croit spontanément; mais son nom moderne nil, dérivé du sanscrit nili, fait supposer qu'après avoir été importé de ces régions dans l'antiquité, il l'a été de l'Inde au moyen ìge et peut-ètre déjà à l'époque grécoromaine.

thèse, le mot dinkon aurait été transformé en indicon par une étymologie populaire.

1. The races of man, p. 375. 


\section{CHAPITRE III.}

\section{L'HORTICULTURE DANS L'ÉGYPTE ANCIENNE}

I.

La culture des jardins n'occupait pas dans la vie des Égyptiens une place moins considélable que celle des champs; tandis que la dernière leur procurait les céréales et les plantes industrielles, la première leur fournissait les plantes potagères, non moins nécessaires que les céréales à l'alimentation. Si les sujets des Pharaons n'avaient pas un régime aussi exclusivement végétal que l'a dit Pline', les légumes entraient cependant, avec les produits des céréales, pour une très grande part dans leur alimentation; les graines de quelques légumineuses, les racines ou les feuilles de plusieurs alliacées ou crucifères, les fruits de certaines cucurbitacées étaient recherchés par eux; aussi les cultivaient-ils avec un grand soin dans leurs potagers ou leurs vergers.

Les plantes potagères, comme nous le montrent les peintures des hypogées, étaient cultivées en platesbandes ou carrés, situés à proximité d'une pièce d'eau, où l'on pouvait puiser l'eau nécessaire pour les arro-

1. Ilistoria naturalis, lib. XXI, cap. 15 (50). 
ser. ${ }^{1}$. Il nous est impossible d'énumérer toutes les plantes qu'on trouvait dans les potagers égyptiens, mais le nombre des especes que nous ont fait connaitre les monuments ou les anciens textes est déja considérable; parlons d'abord des légumineuses, dont les graines étaient un élément important de l'alimentation des Égyptiens.

Une de celles qui furent le plus anciennement connues et pent-ètre, quoique Hérodote, par une confusion d'espèces ${ }^{2}$, ait dit le contraire, le plus généralement cultirées, est la fèré; parmi les offrandes funèbres découvertes par Mariette à Drah-Abou'l-Neggah (XII ${ }^{\circ}$ dynastie), Schweinfurth a reconnu deux fèves, absolument semblables à la petite race que de nos jours on cultive sur une si vaste échelle en Égypte ${ }^{3}$. M. Flinders Petrie a ég'alement trouvé des fères dans la nécropole de Kahoun "qui, comme les tombes de Drah-Abou'l-Neggah, remonte à l'époque de la XII dynastie. Ainsi trois mille ans avant notre ère la fève existait en Égypte; elle n'y est pas indigène; d'où était-elle venue? L'origine de cette légumineuse est obscure, comme celle de tant de plantes cultivées, mais il semble bien que son berceau se troure dans la région située au sud de la mer Caspienne . De là elle se sera, à une époque reculée, répandue dans l'Asie antérieure, d'où les Égyptiens l'ont reçue ou importée, au temps de leurs premières relations avec la Syrie.

C'est de l'Asie antérieure aussi, où elle paraît avoir

1. Rosellini, op. laud., t. II, pl. XL, 1.

2. Historiae, lib. II, cap. 37,6 .

3. Berichteder botanischen Gesellschaft, t. II (an.1884), p.362).

'. Kahun, Gurob and IIawara, p. 50.

5. A. de Candolle, op. laud., p. 256. 
été cultivée à une époque préhistorique ${ }^{1}$, et où une espèce voisine (Lens Schnittspahni Alefeld) croit à l'état sauvage ${ }^{2}$, que la lentille (Lens esculcnta Mœnch) pénétra en Égypte, à une époque reculée. Parmi les offrandes funéraires trouvées, dans des coupes en terre cuite, à Drah-Abou'l-Neggah, on aperçoit des espèces de gâteaux composés de lentilles réduites en une bouillie grossière. Ces lentilles incomplètement écrasées ont paru à Schweinfurth ne différer en rien de l'espèce cultivée encore de nos jours dans la vallée du $\mathrm{Nil}^{3}$. On vient d'en trouver une graine, mêlée à deux fragments d'un fruit de genévriera, au musée du Louvre". Les lentilles paraissent avoir été appelées arshana dans la langue hiéroglyphique; ce nom apparait d'abord sous la XIX e dynastie; c'est le copte arshin ${ }^{5}$. A l'époque des Ptolémées on semble avoir cultivé en grand ce légume dans le Delta; Phacussa, la ville des lentilles, du grec oxwós, leur devait son nom ${ }^{6}$. Pline dit qu'il y avait en Égypte deux espèces de lentilles ${ }^{\top}$ : l'une qui avait la forme de l'espèce ordinaire, l'autre plus ronde et plus noire.

Les lentilles et les fèves si répandues dans l'antiquité n'étaient pas les seules légummeuses que connussent ou cultivassent les sujets des Pharaons; M. Flinders Petrie a découvert dans la nécropole de Kahoun ${ }^{8}$ une variété de petits pois (Pisum sativum L.),

1. A. de Candolle, op. laud., p. 258.

2. Engler, ap. Victor Hehn, Die Kullurplanzen, p. 215.

3. Berichle der bolanischen Gesellschaft, t. Il, p. 362.

4. Recueil de lravaux, t. XVII (an. 1895), 25, p. 192.

5. Victor Loret, La Flore pharaonique, p. 93, no 156.

6. Victor Hehn, Die Kullurpflanzen, p. 209.

7. Hisloria naluralis, lib. XVIII, cap. 30.

8. Kahun, Gurob and Hawara, p. 50, 1. M. Flinders Petrie 
moins grossc que l'espèce cultivée aujourd'hui'. Il l'a, ce qui est moins surprenant, retrouvée également dans le cimetière de Hawara. Cette légumineuse si recherchée était donc déjà connue dans la vallée du Nil dès le temps de la XI ${ }^{\circ}$ dynastie. Elle y arait sans doute été importée de l'Asic antérieure, son berceau peut-ètre, encore qu'elle ne s'y trouve plus à l'état spontané?

Ainsi que le petit pois, les anciens Égyptiens paraissent avoir cultivé le pois chiche (Cicer arietinumL.) et une espèce de lupin - le Lupinus termis Forsk. - On ne connait pas le pois chiche à l'état spontané. A. de Candolle suppose qüil est peut-être originaire de la région située au sud du Caucase et au nord de la Perse ${ }^{3}$; c'est de là qu'il serait passé en Égypte; on l'y cultivait à l'époque gréco-romaine. M. Flinders Petrie en a trouvé des graines dans la nécropole de Hawara ${ }^{4}$, qui date du $\mathrm{I}^{\text {er }}$ siècle de notre ère. M. Victor Loret ${ }^{5}$ a cru pouroir supposer que le pois chiche portait en ancien égyptien le nom de arshá.

Indigène, il semble, dans les sables de la région méditerranéenne $^{6}$, le lupin (Lupinus termis Forsk.), a sans

a aussi trouvé à Kahoun une graine de pois, que M. Percy Newberry n'a pu déterminer; serait-ce la var. elatius, de l'espèce cultivée, que Schweinfurth (Illustration de la lilore d'Égypte, p. 69) indique dans le Delta?

1. On en a trouvé aussi une espèce plus petite dans les stations lacustres de la Suisse et de la Savoie.

2. A. de Candolle, op. laud., p. 263.

3. Origine des planles cultivées, p. 260.

4. IIawara, Bialımu and Arsinö̈, p. 53.

5. La Flore pharconique, p. 92, no 152.

6. A. de Candolle, op. laud., p. 261. - Schweinfurth (Illusuration de la Flore d'Égypte, p. 60) le regarde seulement comme subspontané en Égyte. 
doute aussi, comme le pois chiche, été cultivé dans l'Egypte ancienne, ainsi qu'il l'est aujourd'hui, où on mange ses graines cuites, après les avoir fait macérer quelque temps dans de l'eau salée. M. Flinders Petrie en a découvert de nombreuses graines dans la nécropole gréco-romaine de Hawara ${ }^{1}$; mais on n'a pas trouvé de restes authentiques de celte légumineuse dans les tombes pharaoniques.

Le cajan (Cajanus indicus L.) a été, tout au contraire, reconnu par G. Schweinfurth parmi les restes végétaux trouvés par Mariette à Drah-: bou'l-Neggah; l'existence de cette papilionacée frutescente en Égypte remonte donc au moins à l'époque de la XII dynastie ${ }^{2}$. Elle y arait sans doute été importée de l'Afrique tropicale, où elle croit spontanément ${ }^{3}$; Schweinfurth assure même qu'on la rencontre à l'état sauvage dans la Haute-Égypte. On Ja cultive encore de nos jours dans la Nubie et au Soudan, à cause de ses graines, qui ressemblent aux petits pois, mais n'en ont ni le goût, ni les qualités nutritives.

En même temps qu'ils cultivaient ces légumineuses pour leurs graines alimentaires, les anciens Égyptiens cultivaient pour leurs fruits d'autres espèces régétales, en particulier diverses espèces de cucurbitacées. Une des plus anciennement connues et plantées dans la vallée du Nil, a été la pastèque (Citrullus vulgaris Schrad.); originaire de l'Afrique intertropicale $^{4}$, recherchée par les indigènes de cette région,

1. Kahun, Gurob and Hawara, p. 47, 2.

2. Berichte der botanischen Gesellschaft, t. II, p. 363.

3. A. de Candolle, op. laud., p; 267, dit par erreur que la culture en est toute moderne en Égypte.

4. G. Schweinfurlh, Beiträge sur Flor Acthiopiens. Berlin, 
elle ne pouvait manquer de pénétrer de bonne heure en Egypte, et c'est de là peut-ètre qu'elle s'est répandue dans l'Asie antéricure et l'Europe méridionale. Unger a cru recomaitre des fruits de cette - plante parmi les offrandes funèbres représentées dans plusieur's hypogées'. On en voit bien un, il semble, dans la tombe $n^{\circ} 17$ de Saqqarah, qui date de la IV ${ }^{e} d y$ nastie? Dans le cercueil du prètre Nebseni, découvert en 1881 à Deir-el-Bahari, monument contemporain de la $\mathrm{XX}^{\circ}$ dynastie, se trouraient des fragments de feuilles d'une cucurbitacée, que Schweinfurth a identifiée avec la rariété colocynthoüdes du Citrullus vulgaris", variété cultivée de nos jours, sous le nom do gouiourma dans la Haute-Egypte. Ces feuilles ramollies dans de l'eau tiède ont pu ètre étalées sans peine sur le papier et se sont fait remarquer par leur vive couleur verte. On a découvert aussi des graines de pastèque dans une tombe pharaonique, mais d'une date postérieure. Dans la collection Passalacqua se trouvent, sous le $n^{\circ} 459$, des graines d'une cucurbitacéc que Kunth n'a rattachée à aucune espèce particulière ${ }^{4}$, mais que Braun regarde comme appartenant au $\mathrm{C} i$ trullus vulgarig $"$. La pastèque, nous le savons par le témoignage de la Bible, était cultivée en Égypte dès le temps de Moïse ${ }^{6}$. Il existe en ancien égyptien une

1877, in-8, p. 250. Engler, ap. V. Hehn, Kullurp/anzen, p. 312.

1. Silsungsberichle, etc., t. XXXVIII (an. 1859), p. 12'.

2. Lepsius, Dentimïler, t. II, pl. 68.

3. Berichte der bolanischen Gesellschaft, t. II, p. 361.

4. Calalogue raisonné el historique des antiquilés découverles en Egyple. Paris, 1826, in-8, p. 229.

5. Ucber Pflanzenreste. (Zeitschrift /ür Filhnologie). Berlin 1877, t. IX, p. 303.)

6. Numeri, cap. XI, v. 5. 
plante du nom de betlou-ka ${ }^{1}$; il est difficile de ne pas y voir l'origine du copte betuke, ar. battikh: pastèque.

Les anciens Égyptiens ont-ils possédé aussi le melon (Cucumis melo L.)? Unger a cru le reconnaitre, tout aussi bien que la pastèque, dans de nombreuses peintures de monuments antiques ${ }^{2}$; on n'a pas découvert toutefois de restes de cette plante dans les tombes égyptiennes avant l'époque gréco-romaine; mais M. Flinders Petrie en a trouvé une fleur dans la nécropole de $\mathrm{Hawara}^{3}$; le melon existait donc dans la vallée du Nil au I I $^{\text {er }}$ siècle de notre ère. D'où y avait-il été importé? Egalement indigène en Afrique et en Asie, dans toute la région des tropiques", il avait donc pu être introduit en Égypte, soit de la vallée supérieure du Nil, soit de l'Inde; mais on ignore à quelle époque.

Ainsi que le melon, Unger a cru aussi reconnaître le concombre chate (Cucumis chate L.) dans les peintures d'anciens monuments égyptiens, en particulier dans celles du temple de Deir-el-Bahari; toutefois il n'est pas, comme il le dit", fait mention dans l'Exode de cette cucurbitacée, mais du concombre ordinaire. Le melon chate ressemble par le gout et la forme au concombre, mais ses feuilles et ses fleurs le rapprochent du melon

1. Victor Loret, La Flore pharaonique, p. 73, no 125. Comme le copte betulic est traduit dans les Scalce par aubergine sauvage, M. V. Loret s'est demandé si le mot hiéroglyphique bellou-ka n'avait pas la même signification, mais comment ce mot aurait-il pu désigner une plante étrangère à l'ancienne Égypte? Il faut ajouter que Kircher donne à la citrouille le nom de mapiepon et non celui de betuke.

2. Sitzungsberichte, etc., t. XXXVIII (an. 1859), p. 124.

3. Hawara, Biahmu and Arsinoë, p. 52.

4. A. de Candolle, Origine des plantes cultivées, p. 208. Engler, ap. Victor Hehn, op. laud., p. 312.

5. Sitzungsberichte, t. XXXVIII (an. 1859), p. 124. 
commun. Le nom arabe du cucumis chate est qatta, dont chate semble itre une simple déformation; or M. Victor Loret a cru retrouver l'analogue de ce vocable dans l'ancien égyptien qadi, qui signifie une plante "poussant sur son ventre", et il se demande si ce mot ne désignerait pas le melon chate', mais comme qassa, autre forme de qatta, a été employé pour désigner le concombre, on voit qu'il est difficile de se prononcer sur l'existence du melon chate dans l'ancienne Égypte.

Celle du concombre (Cucumis salivus L.) y a été mise hors de doute, grâce à la découverte faite à Kahoun par M. Flinder's Petrie de feuilles et de tiges de cette cucurbitacée ${ }^{2}$; elle existait donc dans la vallée du Nil dès le temps de la XII ${ }^{e}$ dynastie. D'vù y était-elle renue? A. de Candolle croit que la patrie du concombre se trouve au nord-ouest de la presqu'ile hindoustanique ${ }^{3}$; de là il aurait été apporté, sans doute par les Touraniens, dans l'Asie antérieure, d'où il aurait passé en Égypte: on roit combien sa propagation vers loccident a été rapide. On a cru que le concombre portait en ancien égyptien le nom de shoupi $i^{4}$ - copte shop ; - majs le sens du mot hiéroglyphique n'est pas entièrement certain ; il est donc impossible de rien affirmer sur ce point.

Une autre plante de la famille des cucurbitacées, la calebasse (Lagenaria vulgaris Ser.), se rencontre fréquemment parmi les offrandes funéraires des hypogées

1. La Flore pharcuonique, p. 75, no 128.

2. Kahun, Gurob and Hawara, p. 50.

3. L'origine des plantes cullive'es, p. 211.

4. Victor Loret, op. laud., p. 75, no 129.

5. Kircher, op. laud., p. 197, donne la forme pi-shopi. 
égyptiens; on en a trouvé, en particulier à DrahAbou'l-Neggah', des fruits qui sont ainsi contemporains des Pharaons de la XII ${ }^{\mathrm{e}}$ dynastie. Unger ${ }^{2}$ et Franz. Woenig ${ }^{3}$ ont cru aussi reconnaitre une tige de calebasse dans une peinture de Thèbes. L'existence de cette cucurbitacée dans l'ancienne Égypte parait donc probable; si elle est, comme le suppose A. de Candolle", originaire de l'Inde, son introduction dans la vallée du Nil prouverait à quelle date reculée remontent les relations et les échanges de ce pays avec la péninsule hindoustanique ${ }^{5}$.

Tandis que les cucurbitacées ont été cultivées en Egypte pour leurs fruits, diverses espèces d'aulx et de crucifères l'étaient, comme chez nous, pour leurs racines. La plus répandue et peut-ètre la plus anciennement connue était l'oignon (Allium cepa L.). La patrie de l'oignon est incertaine, mais il n'est point indigène dans la vallée du Nil: de quel pays y avaitil été importé? On paraît l'avoir trouvè à l'état sauvage dans l'Afghanistan et le Béloutchistan et Boissieren a reçu un échantillon des montagnes du Khorassan ${ }^{6}$; Hassel quist l'a même rencon tré aux environs de Jérichō', mais il est douteux qu'il y fìt spontané. Quoi qu'il en

1. G. Schweinfurth, Berichte der botunischen Gesellschaft, t. II, p. 361 .

2. Sitzungsherichte, t. XXXVIII (an. 1859), p. 125.

3. Die Pflansen im alten Aegypten, p. 206.

4. Origine des plantes cultiveres, p. 196.

5. Schweinfurth, Zeitschrift für Ethnologie, an. 1891, p. 656, semble indiquer une autre espèce de cucurbitacée, la Luffa cylindrica Ser., comme anciennement cultivée en Égyte, mais il ne donne aucun renseignement détaillé à ce sujet.

6. A. de Candolle, op. laud., p. 54.

7. Voyage en Palestine, ap. Franz, Woenig, op. laud., p. 194. 
soit, loignon parait originaire du platean de l'Iran '; de lì il s'est répandu dans tout le sud-onest de l'Asie antérieure et en Égypte. Il y était connu dès la plus haute antiquité. Si l'on en croit Hérodote ${ }^{2}$, il aurait eté cultivé sur les bords du Nil plus de trois mille ans arant notre ère.

Les Égyptiens firent de temps immémorial une grande consommation de cette alliacée, qui était dans leur pays de qualité supérieure. L'estime cn laquelle les sujets des Pharaons tenaient l'oignon explique le ròle considérable qu'il occupait dans les offrandes divines ou funéraires. Unger parle d'un oignon trouvé dans la main d'une momie ${ }^{3}$; mais il ne dit pas à quelle époque cette dernière remontait, ce qu'il eût mieux valu nous apprendre que de rechercher si l'oignon que tenait cotte momie ent pu repousser. On n'a pas encore retrouré, dans les texte égyptiens, d'une manière certaine, le nom hiéroglyphique de l'oignon; mais comme le signe qui représente cette plante se prononce houdj, M. Victor Loret' s'est demande si ce vocable ne serait pas le nom, ou l'un des noms, de l'oignon. En hébreu il s'appelle bezel, ar. basal; on peut rapprocher de ce rocable le mot badjar que M. Maspero a lu, dans un tombeau de Thèbes, écrit à côté d'un personnage qui porte une botte d'oignons.

Les oignons sont une des plantes qu'on rencontre le plus sourent sur les monuments de l'ancienne Égypte.

1. L'Asie antérieure, dit Schweinfurth, Zeitschrift für Ethnologic, an. 1891, p. 666, a des droits particuliers pour être leur patrie.

2. Historice, lii. Il, cap. 125.

3. Silzungsberichte, etc., t. XXXVIII (an. 1859), p. 108.

1. La Flore pharaonique, p. 37, no 42. 
Une peinture de Béni-Hassan nous fait assister à leur récolte $^{1}$; un jardinier les arrache et en fait des bottes de quatre à six pieds chacune; c'était ainsi sans doute qu'on les portait au marché. Sur un bas-relief de Saqqarah, reproduit en gypse dans le Musée égypticn de Berlin², on voit une marchande, qui se rend a la ville, portant une corbeille de légumes sur la tète et trois bottes d'oignons garnis de leurs feuilles sur l'épaule.

Les artistes égyptiens paraissent avoir adopté trois types différents pour la représentation de l'oignon; tantôt ils lui donnent des feuilles courtes et étroites, qui enveloppent jusqu'à sa moitié la tige comme d'une gaine; d'autres fois ils le figurent avec de longues feuilles renflées et des bulbes arrondis, mais quelque peu anguleux, ou encore arec un bulbe obovale et une tige longue et cylindrique. M. Franz Wonig ${ }^{3}$ regarde la première forme comme étant celle de l'ail proprement dit (Allium sativum L.); la seconde, à ses yeux, représente la ciboule (Zipolle) ou oignon d'été ( $A$. cepa L.), et dans la troisième il veut voir l'échalote (A. ascalonicum L.). Unger avait cru aussi dans la dernière reconnaître cette alliacée. Mais l'échalote n'a été trouvée dans aucune tombe et n'est mentionnée dans aucun texte hiéroglyphique; elle ne se rencontre. même pas de nos jours dans la vallée du Nil; il est douteux qu'on l'y ait jamais cultivée. Elle ne parait d'ailleurs ètre qu'une forme modifiée et persistante ou une race de l'oignon, et $A$. de Candolle ${ }^{4}$ ne croit pas

1. Rosellini, Monumenti civili, t. II, pl. 40.

2. Franz Wœnig, op. laud., p. 196.

3. Die Pflanzen im allen Aegyplen, p. 197.

4. Origine des planles cullivies, p. 52. 
qu'elle remonte plus haut qu'au commencement de notre ère. Quant ì l'échaloigne (Ascalonia) de Théophraste 'et de Pline ${ }^{2}$ ce n'était qu'une variété d'oignon, qui ne parait avoir rien de commun arec notre échalote.

Reste l'ail. Hérodote en mentionne l'existence en Égypte dès le temps de l'ancien empire ${ }^{3}$; la Bible, du temps de Moïse ${ }^{4}$; le Talmud en parle souvent; Pline aussi en fait mention et aujourd'hui encore cette plante est une de celles qu'on cultive le plus dans la vallée du Nil: d'où y a-t-elle été importée? On l'ignore, mais comme elle pourrait bien n'être qu'une variété cultivée, ainsi que le suppose $\mathrm{A}$. de Candolle", de direrses espèces d'aulx mal définies qu'on rencontre depuis la Tartarie jusqu'en Espagne, cette plante serait encore renue de l'Asie an térieure dans l'ancienne Égypte, où l'on ne peut douter qu'elle ait existé. Schiaparelli a trouvé dans une tombe de l'Assassif près Thèbes, un faisceau de tiges d'un Allium, garnies encore de feuilles; l'examen microscopique a montré au Dr. Tolkens que, malgré les différences qu'elles présentaient, ces tiges appartenaient à l'ail proprement dit ${ }^{6}$. L'égyptologue italien a également découvert

1. Historia plantarum, lib. VII, cap. 4, 11.

2. Historia naturalis, lib. XIX, cap. 32.

3. Hisloricue, lib. II, cap. 125.

4. Numeri, cap. XI, v. 5.

5. Origine des plantes cultivies, p. 52.

6. Bulletin de l'Institut égyptien, no 6, p. 274. - Bolanische Jahrbiucher, t. VIII, p. 10. Dans le Bulletin, Schweinfurth dit que " le tissu de la tige de l'ancien Allium correspond entièrement avec celui du poireau, mais diffère sous des rapports essentiels de l'ail. " Dans les Jahrbücher, on lit, au contraire: "Dr. Volkens kam schliesslich zu dem Ergebniss, dass diese Pflanze... dem Knoblauch (A.Sativum L.),
angehört." 
dans un des tombeaux de Drah-Abou'l Neggah trois paquets formés de tiges et de feuilles du même Allium, pelotonnées sur elles-mêmes et attachées au moyen de fibres de feuilles du dattier ${ }^{1}$.

Schweinfurth avait pris d'abord l'Allium de l'Assassif pour lo poireau (Allium porrum L.); cette espèce fut aussi anciennement cultivée en Égypte, comme nous le savons par le témoignage de la Bible $^{2}$, et Pline dit que le poireau d'ígypte était supérieur à celui de toutes les, autres contrées ${ }^{3}$. On arait cru que le poireau n'était point mentionné dans les textes hiéroglyphiques; mais, d'après M. Victor Loret", il en est question, sous le nom aaqi - le copté eshé, igi ou idji - dans plusieurs papyrus. L'Allium promum n'est point indigène dans la vallée du Nil: de quelle région y arait-il éts introduit? Le poireau parait n'ètre qu'une varièté cultivée de l'Allium ampeloprasum L., commun dans l'Asie antérieure et la région méditerranéenne ${ }^{5}$; c'est probablement de ces contrées qüil aura été importé en Égyte.

Les différentes espèces d'aulx sont plutòt des condiments que des aliments réritables; une plante ì racine varaiment comestible, et qui, indigène dans la rallée du Nil, y fut peut-ètre aussi cultivée dès me haute antiquité, comme elle l'est de nos jours, est le souchet esculent (Cyperus esculcutus L.); il $y$ a au musée de Boulaq une coupe remplie de bulbes de celte cypéracée,

1. Bulletin, $n^{\circ}$ 6, p. 275. Bolanische Jahrbücher, t. VIII, p. 10.

2. Numeri, cap. XI, v. 5.

3. "Laudatissimus in Aegypto. " Itist. naturalis, lib. XIX, cap. 33.

4. Recueil de travaux, etc., t. XVII, p. 181.

5. 1 . de Candolle, op. Inud.. p. 81 . 
que Mariette a probablemont, trourée à Drah-Abou'lNeggah, dans un tombeau de la XII dynastie ${ }^{\mathrm{C}}$. On a également découvert des bulbes de la mème plante ì Crébéleïn ${ }^{2}$, dans une tombe qu'on a supposé être celle d'Ani, contemporain de la XX' dyuastie. Il en existe des bulbes au Musée de Berlin; mais Braun les regardait comme appartenant à une variété particulière et non cultivée, le $C$. melanom i cetic forme que $\mathrm{M}$. Poisson rapporte les tubercules de cette cypéracée, qui sont au Musée du Lourre". Théophraste parle du $C$. esculentus comme d'une plante saurage, qui croissait dans les terrains sablonneux roisins du Nil ${ }^{3}$; il lui donne le nom de malinathalle. Suirant M. Tictor Loret ${ }^{6}$, dans la langue hiéroglyphique, cette cypéracée s'appelait gaïou, comme le souchet a racine arrondie, et ses rhizomes portaient le nom de shabin; les Arabes qui en font un grand commerce leur domnent celui de habb-el-azis, c'est-àdire "grains exquis ".

Une autre plante à racine également comestible, mais qui, quoique exotique en Égypte, semble aussi néanmoins y aroir été cultivée, assez tard toutefois, est la Colocase (Arum esculentum L. ). Originaire de l'Inde ${ }^{\top}$, elle n'a été connue des écrivains anciens que

1. G. Schweinfurth, Notice sur les restes de végétanux conlemus dans une armoire du Wusée de Boulag. (Bulletin de l'Instilut égyplien. no 5 (an. 1886), p. 5.)

2. G. Schweinfurth, Die lelslen bolanischen Entdeckungen. (Bolanische Johrbücher, t. VIII, p. 15). Bulletin de l'Institul igylien, no 6 (an. 1885), p. 260.

3. Zeilscheifl für Ellhnologie, t. IX, p. 296.

4. Recueil de lravaur, t. XVII (an. 1895), 5, p. 181.

5. IIistoria planlarum, lib. IV, cap. 8, 12 .

6. La Flore pharaonique, p. 27, no 26.

7. A. de Candolie, op. laud., p. 59. 
par l'Égypte, où elle avait pénétré sans doute vers le commencement de notre ère. Diodore ${ }^{1}$ et Dioscoride ${ }^{2}$ l'ont confondue avec le lotus; mais Pline l'a bien décrite. C'est, dit-il" " " une plante appelée aron en Égypte; elle a la feuille de la patience; la tige est droite, longue de deux coudées; la racine est douce, au point qu'elle peut même se manger crue ". Impossible de méconnaître à cette description la colocase; mais on voit que cette plante, par suite de son introduction tardive dans la vallée du Nil, n'appartient point à la flore pharaonique.

Le radis, au contraire, en fait partie. Hérodote avait bien parlé de l'existence ancienne de cette crucifère en Égypte ${ }^{4}$; mais on avait mis en doute la véracité de son récit; la découverte, par M. Flinders Petrie, d'un radis à Kahoun "justifie l'historien grec; cette crucifère a véritablement existé en Égypte dès les temps de l'ancien empire. Originaire probablement du nord de la région syro-arménienne ${ }^{6}$, c'est de là qu'il avail été importé en Égypte à une époque, on le voit, très reculée.

Le radis et les diverses espèces d'aulx étaient-ils, avec le souchet comestible, les seules plantés que les anciens Égyptiens cultirassent pour leurs racines? On a admis parfois qu'ils connaissaient aussi la betterave; Charles Pickering s'est demandé si une plante qui se

1. Bibliotheca historica, l. I, cap. 34.

2. De materia medica, I. IV, cap. 414 (1. III, cap. 157)

3. IIistoria naluralis, lib. XIX, cap. 30.

4. II istoriae, lib. II, cap. 125, 5.

5. Kahun, Gurob and IIavara, p. 50.

6. G. Schweinfurth, Zeilschrifl für Ellnologie, an. 1891, p. 665 . 
troure représentée sur une peinture de Béni-Hassan était une betterave ou un radis ${ }^{1}$; Unger inclinait à $\mathrm{y}$ voir un radis ${ }^{2}$; Franz Wœnig n'a pas hésité à la regarder comme une betterave ${ }^{3}$; Rosellini l'a prise pour un chou palmiste ${ }^{4}$; le mieux est de s'abstenir. Quant au naret, il n'est pas rraisemblable qu'on ait jamais, avant notre ère, cultivé cette crucifère en Égpte; le radis était la seule plante de cette famille qui y füt semée à cause de ses racines comestibles; mais on en cultivait d'autres pour leurs feuilles alimentaires; tel était le chou (Brassica oleracea L.).

Originaire de l'Europe ${ }^{5}$, cette crucifère fut pent-être importée dans la vallée du Nil dès une haute antiquité; M. Victor Loret a cru en trouver la mention dans un passage du papyrus Sallier ${ }^{6}$; il aurait porté le nom hiéroglyphique shaïou, analogue au copte pi-shíou, qui désigne ce légume dans une Scala d'Oxford. En tout cas le chou était cultivé en Égypte à l'époque gréco-romaine; M. Flinders Petrie a trouvé des feuilles et des graines de cette crucifère dans la nécropole de Hawara ${ }^{7}$. Pline ${ }^{8}$ dit qu'on ne mangeait pas le chou en Égypte, à cause de son amertume. Athénée se borne à remarquer ${ }^{9}$ qu'au bout d'un an il devenait, sous l'influence du climat, amer à Alexandrie.

1. The races of man, p. 371 .

2. Sitzungsberichte, etc., t. XXXVIII (an. 1859), p. 117.

3. Die Pflanzen im alten Aegypten, p. 28.

4. I monumenti dell' Egillo e della Nubia. Monumenti civili. Pisa, 1834, in-8, t. I, p. 388.

5. A. de Candolle, op. laud., p. 68.

6. Recherches sur plusieurs plantes connues des anciens Égyptiens, X, Le poireau, p. 3. (Recueil de travaux, t. XVI.)

7. Kahun, Gurob and Hawara, p. 47.

8. IIistoria naturalis, lib. XX, cap. 68 (35).

9. Deipnosophistae, lib. IX, cap. 9 (369). 
Indigène en Perse', naturalisé dès longtemps dans l'Asie antérieure, l'Europe mérirlionale et le nord de l'Afrique, le cardame des Anciens - notre cresson alénois - Lepidium sativum L.) a dù aussi, et même assez tôt, ètre cultivé dans la vallée du Nil; Migliarini a cru en reconnaitre des graines ${ }^{2}$ parmi celles qui se trouvent au Musée égyptien de Florence, il en existe aussi une vingtaine au Musée du Louvre ${ }^{3}$, et M. Tictor Loret rapporte à une racine égyptienne le nom copte pi-ghleimi de cette plante". Il y a donc des raisons d'admettre la culture du cardame dans l'ancienne Égypte.

L'endive (Cichorium endiriu L.), quion a voulu identifier avec la seris de Pline", est indigène dans tout le bassin de la Méditerranée ${ }^{6}$; elle se rencontre aussi à l'ètat sauvage dans la vallée du Nil' ${ }^{\prime}$; Maillet trouvait celle qui y croit ainsi spontanément préférable à la variété qu'on cultive en Frances. Mais cette plante a-t-elle aussi été un objet de culture dans l'ancienne Égypte comme elle l'esi parfois dans l'Égy pte actuelle? Je ne saurais le dire, comme j’ignore si la laitue faisait partie des plantes potagères de ce pays.

Il existe deux espèces indigènes de laitue en

1. A. de Candolle, op. laud., p. 69.

2. Indication succincte des monuments égyptiens du Musie de Florence. Florence, 1859, in-8, p. 75, no 3624.

3. Recueil de travaux, t. XVII (an. 1895), 33, p. 199.

4. La Flore pharaonique, p. 110, n" 189.

5. Historia naturalis, lib. XX, p. 32 .

6. A. de Candolle, op. laud., p. 77.

7. D'après Schweinfurth, Zeilschrift für Ethnologie, an. 1891 , p. 662, cette espèce sauvage est le C. divaricatum Schousb., type, pour lui, du C. endivia L.

8. Descriplion de l'Égyple. La Haye, 1740, in-12, t II, p. 101. "Il croit en Égypte, dans les campagnes, une chicorée mille fois plus donce que celle de nos jardins. " 
Figrpte : mais on n'y rencontre pas la scarole (Lacluca scariola L.), qui est rogardée comme le type de la variété cultivé2. Celte espèce a-t-clle été importée dans la vallée du Nil? Le fait n'est pas invraisemblable, mais n’a dù peut-ètre se produire qu'à l'époque grecque. Des graines de la laitue cultivée ont été, il est rrai, trouvées au Musée égyptien de Berlin; mais A. Braun, qui les a déterminées, doute de leur ancienneté $^{3}$.

Parmi les plantes figurées le plus souvent dans les anciens hypogées, au milieu des offrandes funèbres, M. Tictor Loret a cru reconnaitre des laitues pommées ${ }^{4}$ - évidemment de la variété appelée scarole. La supposition peut ètre vraie de quelques-unes de ces représentations", mais elle n'est guère admissible pour les plantes aux feuilles agglomérées réunies au bout d'une longue tige. Il faut dire toutefois, en faveur de l'existence ancienne de la laitue en Égypte, que cette plante porte en copte le nom pi-ôb ${ }^{6}$, qui semble d'origine hiéroglyphique; M. Victor Loret le rapproche des plantes abou ou afa, mentionnées toutes deux dans les papyrus médicaux, mais qu'on n'a pu encore identifier.

1. Illustration de la Flore d'Égypte. p. 99.

2. A. de Candolle, op. laud., p. 75.

3. Zeitschrift für Ethnologie, t. IX (an. 1877), p. 290.

'. La Flore pharcronique, p. 68, no 113. Il est à remarquer que Maillet, op. laud., dit que les laitues ne pomment pas en Erypte.

5. Par exemple des plantes à feuilles vertes ramassées en paquet qu'on voit parmi les offrandes du tombeau de Rokhou. Mémoires publiris prir les membres de la Mission archéologique d" Caire. t. I, pl. IV.

6. Kircher, op. Iaud. p. 196. 
S'il n'est pas impossible que les anciens Égyptiens aient cultivé la laitue, est-il probable qu'ils aient aussi cultivé l'ache ou céleri (Apium graveolens L.)? Indigène dans presque toute l'Europe, ainsi que dans l'Afrique septentrionale et l'Asie antérieure ", l'ache se rencontre dans les marécages de l'Égypte, et sa présence dans la guirlande que la momie de Kent, officier de la $\mathrm{XX}^{\mathrm{e}}$ dynastie, portait sur la poitrine montre qu'on lui attribuait peut-être chez les Égyptiens, comme plus tard chez les Grecs et les Romains, une signification symbolique; mais c'est l'espèce saurage, Schweinfurth l'a reconnu ${ }^{2}$, que les contemporains des Pharaons employaient dans les cérémonies funèbres.

D'après M. Victor Loret ${ }^{3}$, qui croit que l'Apium portait en égyptien le nom de mati, copte mit, les habitants de la vallée du Nil auraient aussi connu l'ache cultivée, ainsi même que le persil - le " mati du Nord »- mentionnés l'un et l'autre, avec l'ache sauvage - le " matı des marais " - et une autre ombellifère appelée "mati de montagne " -- peutêtre le Crithmum pyrenaicum Forsk. - , dans les papyrus médicaux. De l'identité du copte mit, qui ne désigne que l'ache cultivée, tandis que l'ache saurage est appelée kram dans cette langue *, avec l'égyptien mati, M. Victor Loret conclut que ce dernier mot,

1. A. de Candolle, op. laud., p. 71.

2. Bolanische Jahrbücher, t. VIII, p. 13.

3. Recherches sur plusieurs plantes connues des anciens Égyptiens, XI, p. 8. (Recueil de travaux, etc., ı. XVI.)

4. Kircher, op. laud., p. 195, donne à l'ache cultivée le nom de pi-mil, à l'ache sauvage celui de pi-liram et le nom de pi-serinou au persil. 
employé seul, désigne, lui aussi, l'ache cultivée; celte plante aurait done pris place dans les jardins égyptiens.

Le pourpier (Portulaca oleracea L.) s'y trouvait-il également? Originaire de lia région qui s’étend de l'Himalaya à la Grèce', cette plante a été connue de l'Égrpte pharaonique. M. Náspero a trouvé dans un texte hiéroglyphique le nom makhmakhaï, qu'il faut rapprocher du copte mehmouhi, traduit en grec par

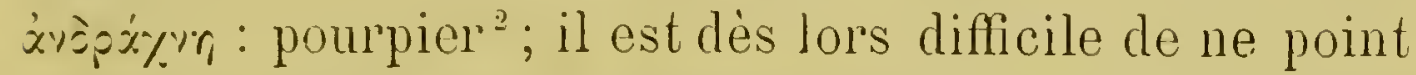
admetire que le mot makhmakhä̈ ne désigne pas cette plante. Le Pseudo-Apulée attribuait aussi au pourpier le nom égyptien mothmutim ${ }^{3}$, qui n'en est évidemment qu'une variante. Le Portulaca oleracea existait donc à une époque reculée dans la vallée du Nil; Schweinfurth l'y regarde comme indigène ${ }^{4}$, et il n'est pas impossible qu'il y ait été anciennement cultivé.

Une autre plante qui dut aussi pénétrer en Égypte, mais à une date relativement récente, est la coranète ou jute, ar. molokhiéh (Corchorus olitorius L.). Théophraste ${ }^{5}$, qui parait bien avoir connu cette plante, parle de son amertume passée en proverbe el ajoute que ses feuilles ressemblaient à celles du basilic. Pline de son côté nous apprend qu'on la mangeait à Alexandrie ${ }^{6}$; le Corchorus était dès lors cultivé de son temps en Égypte. De Candolle ne croit pas qu'il y fût connu

1. A. de Candolle, op. laud., p. 70.

2. Victor Loret, La Flore pharconique, p. 73, no 12'.

3. De herbarum virtutibus, cap. 10'.

4. Illustration de la Flore d'Égypte, p. 50.

5. II istoria plantarum, lib. VII, cap. 7, 2.

6. IIistoria naturalis, lib. XXI, cap. 52. 
anciennement '; Schweinfurth ne le considere aussi que comme subspontané dans la vallée du Nil²; peutètre y fut-il importé de la région tropicale sous les Ptolémées.

Les figures, dans lesquelles M. Victor Loret a cru reconnaitre des laitues, ont été prises par Unger pour des représentations d'articliauts ${ }^{3}$. Quelque grande qu'ait été en botanique l'autorité du naturaliste riennois, il est difficile, bien que M. Franz Tronig nait pas hésité à l'admettre, de se ranger à sa manière de voir ${ }^{4}$, et il est plus que douteux que l'articliaut ait été comnu, encore moins cultivé, en Égypte, arant liépoque gréco-romaine.

A côté des plantes plus on moins alimentaires dont il vient d'ètre question, les anciens Égyptiens en cultivaient d'autres comme condiments, à cause de leur's qualités aromatiques: tels étaient l'anis, la coriandre, le cumin et probablement l'aneth et le fenouil. Suivant Pline et Dioscoride", l'anis d'Égypte ne le cédait qu'i celui de Crète; cette ombellifère croissait donc en Egypte, nais elle n'y est pas indigène; à quelle époque y fut-elle importée? L'anis (Pimpinella anisum L.) porte en copte le nom emlii ou mlii, découvert par M. Victor Loret dans deux manuscrits de la Bibliothèque nationale ${ }^{6}$; or on trouve dans le Papyrus

1. Origine des plantes cullivies. p. 105.

2. Illustration de la Flore d'Égyple. p. 53 .

3. Silzungsheriche, t. XXXVIII (an. 1859), p. 112.

4. C'est aussi l'avis d'A. de Candolle, op. lanul. p. 75.

5. "Laudatissimum est Creticum, proximum Aegyptium ». lib. XX, cap. 33. - De malcria medica, lib. III. cap. 58.

6. Recherches sur phusieur's plantes, etc., XIII, p. 5. (Recueil. de trovaur, t. XVI.) 
Elbers le reme muqrä, nom d'une substance qui, "dessechéc. broye et triturée n, itait recommandie contre les "oppressions de l'estomac \#; l'habile egyptologue lyomnais y a cru hourer la forme antique de emlii ef par suite le nom de l'anis; celte plante a done éte introduite dans la vallée du Nil, au plus tard, au commencement de la XVII dynastic; clle y fut sans loute cultive depuis lor's, comme clle l'est encorce aujourdhui, surtout dans le Fayoun et la HanteEgypte'.

La coriandre (Coriandrum saticum L.) dut l'ètre également au. plus tard vers la mime époque; son nom hieroglyphique ounshaou, qu'on rencontre liéquemment, ainsi que le nom de sa graine, ousshiz, dans les papyrus médicaux, en est la preuve. Il existo, au Musee de Leyde, deux paquets de graines de corriandre trourées, dit-on, dans des tombes pharaoniques: mais l'origine en est douteuse ${ }^{3}$. On a découvert dans un hypogre de la XXII dynastie, situé à Deir-elBahari, des fragments de cetto plante que Schweinfurth a pu identifier". Enfin plus récemment M. Flinder's Petrie a, ce qui ne samrait surprentre, trouvé une capsule de coriandre dans la nécropole gréco-romaine de Hawara. Pline regardait la coriandre d'Egyte comme préférable à celle des autres pays ${ }^{6}$; mais sui-

1. Figari, Studii sull Egillo, t. II, p. 97. - Illustration de la Flore d'Egyple, p. 80.

2. Victor Loreh, Recherehes sur plusieur's planles, VI. (Recueil de truvaur, t. XV.) Dioscoride, liv. III, chap. 4'í (71). dit que Ies Fyptiens appclaient ochion la coriandre.

3. Communication de M. Pleyte.

4. Berichte der botanischen G̈escllscha/t, t. II, p. 359.

5. Iranerer, Birehmu and Arsinoï, p. 5\%.

6. Historia naturalis, lib. XX, cap. 82. 
vant lui, cette plante n'existait pas à l'état sauvage dans la vallée du $\mathrm{Nil}$; elle ne pouvait donc qu'y être cultivée. Une inscription parle de la graine ounshi du district troïque ${ }^{1}$, situé en face de Memphis.

Le cumin (Cuminum cyminum L.), " le meilleur des condiments ", comme l'appelle Pline ${ }^{2}$, fut aussi anciennement connu et sans doute cultivé en Égypte; Dioscoride dit que le cumin qui croissait dans ce pays prenait place pour sa bonté tout après celui d'Éthiopie ${ }^{3}$. On conserve au Musée de Florence une graine de cette ombellifère trouvée dans une to mbe pharaonique 4 . Le cumin portait dans la langue hiéroglyphique un double nom, celui de tapnen, qu'on rencontre souvent dans les papyrus médicaux, et le nom de qamnimi, qui paraît emprunté aux langues sémitiques", - hébreu kammon, ar. lammoún. - C'est de Syrie, en effet, que cette ombellifère, originaire du Turkestan, fut probablement importée en Égypte.

Le cumin est renommé pour ses vertus carminatives; il en est de même de l'aneth (Anethum graveolens L.) et pour cette raison cette plante fut, ainsi que le cumin, cultivée dans l'ancienne Égypte, comme elle l'est dans l'Égypte actuelle ${ }^{6}$. L'aneth portait, dans la langue hiéroglyphique, le nom d'ammisi, qu'on rencontre dans les Papyrus Ebers et de Berlin; il faut rapprocher de ce mot le vocable amisi, emisé de

1. Victor Loret, Recherches sur plusieurs planles, VI, p. 3.

2. Ilistoria naturalis, lib. XIX, cap. 57.

3. De materia medica, lib. III, cap. 61 (78).

4. A. Migliarini, Indicalion succincte des monuments égygliens, p. 75, no 3628 .

5. Victor Loret, La Flore pharaonique, p. 72, no 122.

6. Illustration de la Flore d'Égyple, p. 81. 
quelques Scalae coptes, rendu par le grec ävinoo\%, ar. shebel'; on voit donc que l'aneth était connu et probablement cultivé dans la patrie des Pharaons dès les premiers temps de la XVIII ${ }^{\circ}$ dynastie; plante indigène dans l'Asie antérieure et dans la région méditerranéenne ${ }^{2}$, il n'est pas surprenant qu'il ait pénétré en Egypte à une époque aussi reculée.

Le fenouil (Fœniculum officinale L.) y dut pénétrer aussi à une date ancienne. Il est fait mention dans le Papyrus gnostique de Leyde de la plante shamari hoout; or M. Victor Loret ${ }^{3}$ a trouvé dans une Scala de la Bibliothèque nationale le nom copte shamar hoout, traduit par l'arabe shamàr berri " fenouil sauvage »", ce qui parait bien prouver que la plante du Papyrus de Leyde est le fenouil. M. Loret croit aussi que le mot shamair, qui se rencontre une fois dans le grand Papyrus Harris, désigne probablement la mème ombellifère. Il incline à la retrouver dans la plante besbes des Papyrus Ebers et de Berlin, nom qui se serait conservé dans l'arabe bisbas, une des appellations du fenouil.

II.

On vient de voir combien fut considérable - encore ai-je dû en oublier — le nombre des plantes potagères cultivées dans les jardins égyptiens; mais elles étaient

1. Victor Loret, La Flore pharaonique, p. 71, no 120.

2. Boissier, Flora orienlalis. Genève, 1872, in-8, t. II, p. 1026.

3. La Flore pharaonique, p. 71, no 121.

4. Kircher, op. laud., p. 193, donne au fenouil ordinaire le nom de pi-aneoumor et celui de malatron au fenouil sauvage. 
loin d'ètre les soules qu'on y rencontràt. Si, à côté de la culture agricole proprement dite, avait pris place une culture maraichère, quil no lui cédait guère en importance, les habilants de la vallée du Nil ne s'en timrent pas là, et, ¿ ces deux cultures si prospères, ils joignirent, les monuments on font foi, d'aborrl celles des arbies fruitiers, et plus lard celle des plantes d'agrément et des fleurs. Quel a été l'état successif de ces dernières cultures dans l'Égypte ancienne?

Les peintures d'une tombe de Béni-Hassan, qui représentent la propriété de Khnoumbotpou, contemporain de la XII dynastie, peuvent nous donner une idée de ce qu'était, à cette époque, la culture maraìchère et fruitière dans la vallee du Nil' '. A ganche, on roit une vigne, dont trois rendangeurs cueillent les raisins dejai mûrs, qu'un quatrième emporte dans des corbeilles; au delà, s'élèvent deux figuiers, l'un courert de fruits, que cueillent deux ourriers, aidés dans leur besogne par trois singes cynocéphales, l'autre qui en ost dépouillé. Puis vient le potager, courert de plantes disposées en plates-bandes quadrangulaires, que demx ouvriers sont occupés à arroser, tandis qu'un troisieme personnage, le jardinier de Ḱlınoumhotpou lui-mème, - il s'appelait Noufirhotpou —, met en botles des oignons nouvellement arrachés". Plus loin, a droite, se dresse un dattier qui complète le tableau.

Le jardin pharaonique, tel que l'a peint l'artiste de

1. Champollion le Jeune, Honuments de l'Égyple et de la Nubie, t. IY, pl. CCCVII, 3; CCCLVII. 1, 2. - Rosellini, Monnmenti civili (Atlas), t. II, pl. XL. - Lepsius, Dentimëler., t. IV, pl. 127, tombe 2, face occidentale B. - Percy E. Newberry, Bmi-IIasan. London, 1893-9', in-fol., t. I, pl. XXXIX.

2. Liosellini, Monumenli civili (texte). t. I, p. 383-85. 
la XII" dynastice, renfermatil a la fois, on le roit, des légumes et des arbres a fruit. La culture des légumes remonte sans doute aux temps les plus reculés de l'histoire egyptienne; colle des arbres à fruits n'est guere moins ancicnne. Sur une peinture tombale de Gizeh', qui date de la IV dynastic, se trouve díjic représcntée la cucillette des fruits du sycomore, ainsi que la fabrication du vin. Une peinture de zaouiet-elMaiétin², un peu postérieure, - elle remonte seulement i la VI dynastie, - mais encore fort ancienne, nous fait assister aux différentes scènes de la vendange: on y roit aussi des sycomores, qu'on est en train d'abattre et dont plus loin on débite le bois. Sur une tombe de Béni-Hassan ${ }^{3}$, qui remonte à la XII dynastie, sont représentés des dattiers que des ourriers riennent abattre; sur une autre tombe, igalcment de. Béni-Hassan ${ }^{4}$, celle de Khati, est sculptée de nouveau une vigne dont on cueille le raisin.

La culture des arbres irruitiers arait donc pris de bonne heure, à coté des cultures agricoles ou maraîchères, une place qui, modeste d'abord, ira toujours grandissant; on les plantait aux bords des jardins potagers ou des réservoir's aménagés partout où le Nil ne portait pas, en débordant, ses caux bienfaisantes. C'est ainsi que sur une peinture tombale de Shéikh Abd-el-Gournah", contemporaine le la XVIII dynas-

1. Lepsius, Denlimäler, t. III, pl. 53, tombe 16.

2. Lepsius, Denlimïler, t. IV, pl. 111, tombe 14.

3. Lepsius, Denlimïler, t. IV, pl. 126, tombe 2.

4. Rosellini, Monumenti civili (atlas), t. II, no 37, 1 et 2 ; (texte), t. I, p. 358.

5. Champollion le Jeune, Monuments de l'Egyple cl de la Nubie, t. II, pl. 185, 2. - Rosellini, Mommenli civili, 1. II, no 40,2 . 
tie, on aperçoit un sycomore qui ombrage de son épais feuillage un réservoir, oì un jardinier vient puiser de l'eau; au delà se dresse un arbre, que Rosellini a pris pour un acacia ou un tamaris, plus loin un palmier doum. Désormais il n'y aura pas une pièce d'eau, nous en verrons tout à l'heure de nombreux exemples, qui ne soit entourée d'arbres ${ }^{1}$. Mais ce n'est pas là seulement qu'on les trouvait.

Les jardins, tels que celui de Khnoumhotpou, où aux légumes utiles se joignaient quelques arbres à fruits, suffisaient peut-être aux besoins des habitants de l'ancien Empire; il n'en fut plus de mème pour ceux du nouveau, il leur fallait des jardins d'un tout autre caractère. L'antique culture maraîchère ne fut plus assez pour eux; si le colon des campagnes s'en contentait toujours, si elle fournissait à l'artisan des villes une ample ressource pour son alimentation, elle ne pouvait satisfaire au luxe croissant et aux gouts nouveaux des grands; leurs riches habitations de la ville, leurs maisons champêtres surtout réclamaient autre chose que des jardins destinés aux besoins ordinaires de la vie; il fallait à ces résidences somptueuses de l'ombre et de la fraicheur; des arbres seuls pouvaient leur en donner, et ceux-ci n'occupaient qu'une place restreinte dans les jardins potagers; aussi, outre ces jardins, que les grands faisaient cultiver par leurs métayers ou par leurs serviteurs, ils en avaient de tout différents, consacrés à des cultures

1. C'est ainsi que, sur une peinture thébaine de la XVIIIo dynastie, le réservoir, où des captifs cccupés à construire le temple d'Amon puisent de l'eau, est entouré d'une rang'ée de sycomores. Prisse d'Avennes, Histoive de l'art égyptien d'après les monuments. Paris, 1878, in-fol., pl. 59. 
tout autres, et qui, tenant à la fois du parc et du verger, étaient l'accompagnement obligé et l'ornement ordinaire de leurs palais et de leurs villas ${ }^{1}$.

C'était là qu'après les fatigues de la guerre ou le soin des affaires les pharaons et leurs officiers allaicnt goûter le repos; là, au bord des pièces d'eau qu'animaient de leurs ébats des oiseaux aquatiques, à l'ombre des arbres rares on utiles qu'ils avaient réunis à grand'peine, ils allaient arec leurs femmes " faire un jour heureux " et prendre le frais ${ }^{2}$, jouissance si délicieuse dans un pays dévoré par les ardeurs du soleil, qu'on n'en concevait pas de plus grande au delì de la tombe: "Que je me promène, fait dire à un mort son inscription funéraire ${ }^{3}$, chaque jour, sans cesse, au bord de mes étangs, que mon àme repose sur les branches des arbres que j'ai plantés, que je me rafraichisse sous mes sycomores. " Ce n'était pas seulement pour les hommes occupés que les jardins étaient ainsi un lieu de délassement et de repos, les dames aussi y trouvaient une retraite charmante et recherchée; elles aimaient à se promener dans les nombreuses allées qui y étaient ménagées; elles $\mathrm{y}$ recevaient les visites de leurs amies et y passaient dans de longs entretiens les heures brûlantes du jour.

Les Égyptiens aussi, et cela se comprend, mettaient

1. "J'ai planté ta ville de Thèbes-», dit Ramsès dans le Papyrus IIarris, "d'arbres, d'arbustes, de fleurs hura, menhet, pour ton nez. " Pl. VII. Zeitschrift für ägyplische Sprache, t. XI (an. 1873), p. 54.

2. G. Maspero, Le tombeau de Nakhti. (Mémoires publiés par. les membres de la mission archéologique française au Caire, t. V, p. 412).

3. Karl Piehl, Notes de critique. (Recueil de travaux, etc. t. I, n०3, p. 196.) 
le plus grand soin à entretenir et à embellir ces lieux de prédilection; ils les ornaient d'arbres amenés parfois de lointaines contrées. Un officier de Thoutmès I ${ }^{\mathrm{er}}$ (XVIII ${ }^{\circ}$ dynastie), Anna, intendant des greniers d'Amon et directeur des travaux du roi, rappelle avec complaisance, dans son inscription funèbre ${ }^{1}$, les arbres nombreux et plusieurs d'essence rare dont il avait rempli son jardin. Dans le papyrus moral de Boulaq, un vieux scribe, Khonsouhotpou, félicite son fils Ani du domaine qu'il avait acquis et accru. "Tu as, lui ditil ${ }^{2}$, mis en état tes champs, entouré de haies ton domaine; tu as planté autour de ta demeure des sycomores en allées. Tu remplis ta main de toutes les fleurs que tu vois."

Les jardins, aux yeux des Égyptiens, étaient d'un si grand prix que, dans leur anthropomorphisme, ils ne croyaient pouvoir offrir aux dieux rien de plus grand ou de plus auguste. Parmi les dons que Ramsès III fit aux temples d'Égypte, il $y$ avait cinq cent quatorze parcs ou bois sacrés. "Je t'ai aménagé d'immenses jardins avec des arbres magnifiques et des rignes ", dit le Pharaon dans la charte de donation, en parlant des parterres ufferts aux divinités d'Héliopolis.... " J'ai fait planter pour toi des bosquets remplis d'arbres divers

1. Henri Brugsch, Recueil de monuments égypliens. Leipzig, 1862, in-4, $1^{\text {re }}$ partie, p. 48, pl. XXXVI. - Charles E. IIoldenke, Ueber die in allügyptischen Texten erwühnten Büume und deren Ververthung. Leipzig, 1886, in-8, p. 18. - H. Boussac, Le tombeau d'Anna. (Mémoires de la mission archéologique au Caire, 1896, t. XVIII, fasc. 1, pl. s. n.).

2. E. de Rougé, Étude sur le Papyrus 4 du musée de Boulaq. (Comptes rendus de l'Acadimie des Inscriptions, an. 1861, p. 345). - Amélineau, La morale égyplienne quinse siècles avant nolre ère. Paris, 1892, in-8, p. 90. 
et de dattiers, arec de vastes bassins couverts de fleurs de lotus et de joncs ${ }^{1}$. "

Ces textes nous laissent déji entrevoir ce qu'était un jardin dạns l'ancienne Égypte; les peintures des hypogées achèvent de nous le faire connaitre ${ }^{2}$. Établi, pour en faciliter l'arrosage, dans le voisinage de quelque canal, entouré, ainsi que la villa dont il dépendait, de murs qui en défendaient l'accès, le jardin pharaonique était d'ordinaire divisé en plusieurs sections, consacrées chacune à une culture particulière et séparées les unes des autres par une muraille peu élevée; des bassins y entretenaient une fraîcheur perpétuelle, en même temps qu'ils foumissaient l'eau nécessaire aux plantes qu'on y cultivait. Dans le roisinage de ces bassins des espèces de kiosques entourés d'arbres permettaient de jouir en paix du repos et de l'ombre. Une large porte donnait accès dans le jardin et une allée conduisait à la maison d'habitation située le plus sourent à l'extrémité opposée de l'enceinte. Éloignée du tumulte de la ville et protégée contre les ardeurs du soleil, une pareille retraite offrait à son possesseur un intérieur calme et paisible, où il vivait exempt du souci des affaires et loin des bruits du monde. Elle pouvait d'ailleurs offrir les dispositions les plus diverses.

Une peinture de Thèbes, par exemple ${ }^{3}$, nous montre

1. Aus dem grossen Papyrus Harris von Aug. Eisenlohr, pl. XXVII. (Zeitschrift für ägyplische Sprache, t. XI (an. 1873),
p. 98-99

2. G. Wilkinson, The manners and customs of the ancient Ey!plians, t. I, p. 377. - Perrot et Chipie\%, IIisloire de l'arl dans l'antiquité. Paris, 1882, in-8, t. I. L'Égyple, p. 482. 3. J. Rosellini, Monumenti civili, t. II, p. 382-84. Atlas,
t. II, pl. LXVIII, 2. 
une enceinte quadrangulaire, dans laquelle on pénètre par deux portes; celle de droite donne accès dans la maison, celle du côté gauche conduit dans le jardin; celui-ci parait, par le manque de perspective, divisé en trois sections; en haut on voit une treille formée de trois vignes couvertes de grappes de raisins déjà mùres; de chaque côté se dresse un arbre sans caractère bien défini, mais probablement un sycomore. Dans la seconde section se voient un grenadier et peut-ètre un perséa. Au delà de ce jardin si simple s'étend une cour, au fond de laquelle se trouve une espèce d'office garni de vaisselle et de victuailles; au bas se dresse une table d'offrandes. A droite de la cour règne une galerie pourvue d'une large fenêtre et plus loin apparait l'habitation. Le moment où l'artiste a peint cette villa est celui où la maitresse de la maison et les femmes de sa famille reçoivent la visite de dames étrangères, qui viennent prendre part à une fète. Celles-ci sont entrées par la porte du jardin et s'avancent, en s'inclinant, vers la maitresse de la maison, debout sur le seuil de la porte, et lui offrent leurs hommages et des présents.

Une autre peinture, qui se troure également dans une tombe thébaine ${ }^{1}$, représente à droite une résidence d'été précédée d'un portique, devant lequel se dressent un obélisque et une espèce de colonne ou de mât. Au delà s'étend le jardin, avec son bassin central, alimenté par les eaux d'un canal extérieur, arec lequel il communique par une large tranchée ou fossé intérieur. De chaque còté de ce fossé et du bassin s'alignent des arbres sans caractère distinctif, mais

1. G. Wilkinson, op. laud., t. I, p. 366, pl. 136. 
dans lesquels il faut voir sans doute des sycomores; on en voit également le long de la façade septentrionale de la maison; parallèlement ì la tranchée intérieure sont plantés des papyrus ${ }^{1}$ et un arbuste sans forme précise, mais qui représente peut-être un cep de vigne. L'artiste ne s'est pas borné à donner cette esquisse; il a représenté dans la partie du jardin, voisine de la villa, une visite faite à la maitresse de la maison par des dames, à chacune desquelles elle offre un bouquet.

Dans ces deux peintures l'artiste pharaonique a tenu à nous faire assister à des scènes d'intérieur qui nous initient ì la vie intime de ses contemporains; dans quatre autres peintures, également thébaines et de la mème époque, l'une qui se trouve au British Muserım, la seconde publiée par les membres de la mission archéologique française au Caire, la troisième donnée dans les Monuments de Lepsius et la quatrième repro-duite par Rosellini, l'artiste, au contraire, a laissé de côté les scènes d'intérieur et ne s'est attaché qu'à représenter, dans leurs moindres détails, les villas et surtout les jardins de son temps.

Le jardin de la peinture conservée au British Museum ${ }^{2}$ est d'une grande simplicité. Composé d'un enclos rectangulaire allongé, au centre s'étend un bassin de même forme, sur lequel on voit des oies prendre leurs ébats, tandis que des poissons se jouent dans ses eaux, au milieu desquelles poussent six touffes de lotus. Autour de ce bassin se trouve une

1. Il semble aussi qu'il y a seize touffes de papyrus dans le bassin.

2. Northern Egyptian gallery, n" 177. 
rangée de plantes aquatiques et d'arbustes; j'ai compté cinq touffes de papyrus, un arbuste à fleurs ou fruits rouges à peine distincts et un autre arbuste à fleurs blanches. Je reviendrai plus loin sur ces deux arbustes. Au delà et parallèlement à chacun des murs du jardin, règne une rangée d'arbres; en haut, d'abord un sycomore, un arbre à basse tige et à feuilles longues, avec des fruits semblables à des dattes ${ }^{1}$, un palmier doum, puis encore un sycomore, un dattier, peut-ètre un perséa, suivi d'un second palmier doum, enfin un troisième sycomore, au pied duquel une femme range des figues dans une corbeille. A gauche on voit un sycomore entre deux perséas. En bas il y a sept arbres analogues à ceux de la partie haute du jardin, mais distribués d'une manière un peu différente; d'abord un arbre méconnaissable, ensuite un dattier, un sycomore, peut-être un perséa, puis encore un dattier, un sycomore et un dattier. Le côté droit est détruit. Là se trouvait sans doute la demeure du propriétaire du jardin; elle était probablement aussi simple que celui-ci.

Le jardin de Rekhmara, officier de Thoutmès III, à Shéikh Abd-el-Gournah, - j'aurai encore occasion d'en parler dans un autre chapitre - offre un aspect bien plus opulent ${ }^{2}$. Le vaste bassin qui en forme le centre est entouré d'une première rangée de dix-neuf à vingt arbres sans caractère distinctif, mais qui pourraient bien être des jeunes sycomores; au delà règne une seconde rangée d'arbres composée de vingt-six à

1. On serait tenté d'y voir un jeune dattier, ce qui confirmerait l'assertion de Théophraste, que cet arbre peut porter des fruits n'étant éncore arrivé qu'à la hauteur d'homme.

2. Mémoires de la mission archéologique francaise au. Caire, t. V, liv. I, p. 166, pl. XXXVIII. 
vingt-huit dattiers ou doums, alternant entre eux; plus loin on voit une troisième et dernière rangée de 22 arbres, encore sans caractère bien défini: sycomores, perséas, myrobalans, grenadiers peut-ètre et arbres fruitiers semblables. De la villa le portique seul est représenté.

Sur une peinture d'un des to mbeaux de Tell-el-Amarna, celui d'Ai, gendre du pharaon Khouniaton, l'artiste pharaonique, au contraire, a représenté en son entier la résidence du défunt, avec son jardin et ses dépendances ${ }^{1}$. Entourée d'une enceinte murée, une porte principale, flanquée de deux portes plus petites, y donnait accès et conduisait dans une allée ombragée d'arbles, qui séparait les deux corps de bâtiments principaux; devant chacun d'eux et au midi se trourait un bassin, une rangée d'arbres régnait tout autour du baitiment de droite et au nord de la maison de gauche se trouvait le jardin avec son large bassin au milieu, une espèce de pavillon ou de kiosque à côté, une double rangée d'arbres en bas, ainsi qu'à droite et à gauche et une simple rangée en haut. Au-dessus du corps de bàtiment de droite et au delà de la rangée d'arbres qui le bordait au nord, s'étendait la maison d'exploitation agricole, avec la basse-cour, les écuries, les greniers, etc. Des deux corps de bàtiments celui de droite était de beaucoup le plus considérable, celui de gauche semble avoir été entouré d'une cour intérieure plantée d'arbres, et à droite et à gauche de laquelle se trouvaient les offices ou les caves, $\dot{a}$ en

1. Lepsius, Denkmäler, t. VI, pl. 95. - Wilkinson, op. lauü., t. I, p. 369, pl. 9. - Prisse d'Avennes, Histoire de l'art égyptien, pl. 45. - G. Maspero, L'Archiologie égyptienne. Paris, 1887, p. 17. 
juger par le grand nombre d'amphores et de vases divers qu'on y voit.

Quant aux arbres qui entourent les habitations, on en compte de cinquante à soixante; mais il est assez difficile d'en reconnaitre la nature; deux en bas paraissent être des figuiers, si l'on en juge d'après la forme de leurs feuilles, qu'on pourrait prendre aussi, il est vrai, pour des feuilles de vigne; les autres étaient probablement des sycomores, mais leur forme purement schématique ne permet guère de se prononcer avec certitude. Plusieurs des arbres du jardin, par contre, se reconnaissent sans peine; on $y$ voit un palmier doum, quatre dattiers, dix grenadiers, six arbres qu'on peut prendre comme ceux du bas pour des figuiers, quatre sans caractère défini, peut-être des sycomores, et un dont les rameaux dénudés ne permettent aucune identification.

On voit quelle quantité d'arbres étaient accumulés autour de la villa des tombes de Tell-el-Amarna; il y en avait un bien plus grand nombre encore dans le parterre de la villa d'un chef militaire, contem porain d'Amenhotpou II, septième roi de la XVIII ${ }^{\circ}$ dynastie, résidence dont Champollion ${ }^{1}$ et Rosellini ${ }^{2}$ ont donnéle dessin, reproduit dans les ouvrages sur l'agronomie, l'archéologie ou la botanique égyptiennes de Wilkinson ${ }^{3}$, Franz Wonig ${ }^{4}$, Charles Moldenke ${ }^{5}$ et Maspero ${ }^{6}$. Leur ensemble for-

1. Monuments de l'Égyple et de la Nubie, pl. 261.

2. Monumenti civili, texte, t. II, p. 382 ; atlas, t. II, pl. 69.

3. The manners and customs t. I, p. 377, pl. 150.

4. Die Pflanzen im alten Aegypten, p. 232.

5. Ueber die allägyptischen Büume, p. 41.

6. L'Archéologie égyptienne, p. 15. - Histoire ancienne, t. I, p. 291. 
mait un raste carré, entouré de murs crénelés et situé à l'ouest d'un canal dont il est séparé par une chaussée, que bordait une rangée de sycomores. Sur celte chaussée s'ourre la porte d'entrée; elle donne sur une cour intérieure, où se trouve une petite habitation; on dirait la loge du portier. Au delà de la cour s'étend le vignoble, vaste plant composé de vingt-quatre ceps, conduits sur des treillis, et formant un berceau impénétrable aux rayons du soleil. Plus loin et à l'extrémité opposée à la porte d'entrée s'élève la villa, séparée du mur de clòture de droite par une double rangée d'arbres, la première composée de quatre sycomores, la seconde de dattiers et de palmier's doums. De chaque côté de la cour qu'entourent des murs peu élevés sont creusés deux bassins rectangulaires; deux autres bassins presque carrés sont situés au-dessus et au-dessous de la vigne; à droite et à gauche de ceux-ci et à gauche des deux autres sont plantés des papyrus; des touffes de papyrus encore semblent pousser au milieu de ces bassins, sur les eaux desquels se jouent des viseaux aquatiques, des oies et des canards. Près des derniers bassins, et à còté des papyrus, s'élevaient deux espèces de kiosques, où l'on venait sans doute prendre le frais.

Le reste du jardin était rempli par des arbres à fruit. Des deux côtés de la vigne s'étendait une longue rangée de dattiers; au nord et au sud de la villa, étaient plantés des sycomores, huit au nord, quatre au sud; derrière eux s'alignaient huit dattiers; une rangée de douze sycomores, au contraire, se trouvait au delà des bassins du jardin; en haut et en bas de celui-ci courait, parallèlement au mur de clôture, une autre rangée de trente-cinq arbres, palmiers doums, sycomores et dattiers alternant entre eux. Une double rangée de pal- 
miers s'élevait aussi le long du mur de clôture de droite, tandis que trois sycomores se dressaient de chaque côté de la loge du garde, ainsi que huit sycomores au-dessous, huit palmiers au-dessus des bassins de droite. Enfin, entre les bassins et ceux du jardin se trouvait un petit enclos rempli de douze arbres, peutêtre des sycomores. Cela faisait en tout, y compris les vingt-quatre vignes, deux cent soixante dix-huit arbres. On voit par là quel aspect particulier offrait ce jardin ; les arbres y étaient multipliés, moins pour l'agrément qu'en vue de l'ombre et de la fraîcheur, et il faut ajouter de l'utilité, puisque presque tous étaient ou paraissent avoir été des arbres à fruits.

Autant qu'on en peut juger par une représentation souvent imparfaite ou conventionnelle, les jardins dont je viens de parler, si l'on fait abstraction des deux arbustes de la peinture du Musée britannique, ne renfermaient pas plus de six à sept espèces d'arbres; le jardin de Thèbes à obélisque n'en contenait même peutêtre que deux; c'est peu et sans doute il en était souvent dans la réalité tout autrement; nous connaissons au moins un jardin, celui du scribe Anna, qui en renfermait un nombre bien plus grand '. L'inscription funéraire de cet officier nous apprend qu'il avait planté dans son parc vingt espèces différentes d'arbres, parmi lesquelles d'ailleurs se trouvaient les diverses essences dont j'ai signalé l'existence dans les jardins que je viens de décrire ${ }^{2}$. Malheureusement sur ces vingt espèces,

1. Henri Brugsch, Recucil de monuments, pl. XXXVI. G. Maspero, Histoire ancienne, t. I, p. 201.

2. Charles E. Moldenke, op. laud., p. 20. - H. Boussac, Le tombeau d'Anna, pl. s. n. La peintụ'e du jardin d'Anna ne donne qu'une idée imparfaite du nombre - on en voit moins 
cinq ou six n'ont pu ètre identifiées. Restent quatorze à quinze espéces comnues; mais elles étaient loin d'être cultivées, nous venons de le roir, dans tous les jardins pharaoniques, et le nombre de celles qu'on y rencontrait variait d'ailleurs avec l'étendue de ces jardins, ainsi qu'arec le gout ou la richesse de leurs possesseur's. Ce n'étaient pas toutes non plus des essences à fruits; à còté d'elles prenaient place des arbres d'ornement, cultivés pour la gràce ou la beauté de leur feuillage, mais en bien moins grand nombre toutefois que les arbres fruitier's.

Les plus répandus parmi ces derniers étaient le dattier, le palmier doum et le sycomore; le jardin du scribe Anna renfermait cent soixante-dix dattiers, cent vingt palmiers doums et quatre-vingt-dix sycomores, ainsi que trente-un caroubiers ${ }^{1}$ et douze vignes; mais les autres arbres fruitiers n'y figuraient qu'en nombre presque insignifiant; on n'y comptait que cinq figuiers, cinq grenadiers, autant d'arbres nebs ${ }^{2}$, trois perséas, deux arbres à noix de ben ${ }^{3}$ et seulement un palmier argoun. Quant aux arbres d'ornement, ce même jardin

de cinquante - et des espèces d'arbres qu'il renfermait; la disposition en est d'ailleurs toute particulière; la maison se trouve avec les greniers dans la partie méridionale; au delà est le bassin avec ses lotus habituels, ayant à droite quatre, $\dot{a}$ gauche trois arbres, qui semblent appartenir à des essences différentes ; puis viennent trois rangées d'arbres, dont les dattiers, plantés au milieu de la première, sont seuls reconnaissables; enfin au fond et à gauche se trouve le kiosque, où Anna et sa femme prennent le frais et devant lequel s'étend un plant irrégulier de palmiers doums.

1. Victor Loret, Recherches sur plusieurs plantes, VII. Le caroubier. (Recueil de travaux, t. XV, p. 113.)

2. Voir le chapitre suivant, p. 125.

3. Victor Loret, Recherches, etc. I. L'olivier el le moringa. (Recueil de lravaux, t. VII, p. 101.) 
en renfermait trois espèces, en nombre restreint toutefois: dix tamaris, ainsi que dix saules safsaf et seulement trois acacias. Aucune de ces espèces ne se rencontrait, à ce qu'il semble, dans le verger de l'officier d'A menhotpou II, ainsi que dans les autres parterres des hypogées thébains. ${ }^{1}$. Ils ne paraissent aussi avoir renfermé ni caroubiers, ni arbres nebs ou à noix de ben, ni palmiers argouns; mais les autres arbres à fruits $y$ étaient plus ou moins complètement représentés : il y avait jusqu'à quatre-vingt-douze dattiers, vingt-deux palmiers doums, soixante-douze sycomores et vingtquatre vignes dans le parterre du chef militaire contemporain d'Amenhotpou II.

Telle était à peu près la composition d'un jardin égyptien à l'époque de la XVIII ${ }^{\circ}$ dynastie; il suffisait peut-être aux exigences de l'époque; mais il manquait de variété et on y eût cherché en vain quelquesuns des meilleurs fruits de l'Asie antérieure, ainsi que plusieurs des plus beaux arbres d'ormement de la région méditerranéenne, qu'il devait posséder plus tard. Il les acquit successivement, d'abord sous les grands princes du nouvel empire, plus tard à l'époque de la domination perse et surtout sous celle des Ptolémées. Les expéditions des Ahmessides et des Ramessides en Éthiopie au sud, en Syrie et jusqu'à l'Euphrate au nord, l'occupation de la terre de Qimit par les Perses, qui, partis du plateau de l'Iran, araient étendu leur domination d'un côté jusqu'à l'Indus et de l'autre jusqu'aux rivages de la Méditerranée, enfin la conquête d'Alexandre et l'établissement de dynasties

1. On apercoit, au contraire, un acacia ou un tamaris dans le jardin potager de la peinture d'Abd-el-Gournah. 
grecques en Syrie et sur le tròne d'Égypte, n'avaient pu manquer d'aroir leur contre-coup sur l'agronomie et l'horticulture, comme sur la civilisation, de l'Asie antérieure tout entière et en particulier de la vallée du Nil. Ces grands mouvements de peuples et d'armées, ainsi que les relations pacifiques et commerciales qui les accompagnèrent ou qui les suivirent, eurent pour conséquence naturelle de transporter, hors de leur pays d'origine, nombre d'espèces végétales; plusieurs pénétrèrent alors pour la première fois dans l'antique patrie des Pharaons.

Dès longtemps les princes égyptiens avaient aimé à enrichir leur pays des produits de l'étranger; l'expédition pacifique enroyée par la reine Hatshopsitou au pays de Pount chercher des arbres à encens, dont j'ai parlé plus haut, ne fut pas un fait isolé. Ramsès III rappelle dans le grand Papyrus Harris les arbres que, lui aussi, avait fait planter dans la cour du temple de Thèbes ${ }^{2}$. Leurs expéditions militaires au delà des frontières de l'Égypte offraient, d'ailleurs, aux Pharaons une occasion naturelle d'importer dans leur pays les régétaux utiles qu'ils araient remarqués à l'étranger. Ils n’y manquèrent pas. C'est sous les Ahmessides probablement, conquérants de la Syrie, que la flore horticole de l'Égypte s'enrichit de l'olivier ${ }^{3}$, cultivé depuis un temps immémorial au pays de Chanaan. Le pom-

1. Chapitre I, p. 18.

2. Zeitschrift für ägyplische Sprache, t. XI, p. 35.

3. M. IV. Pleyte (La couronne de la Justification, p. 13), suppose que l'olivier a été introduit en Égypte sous la XIX dynastie; il me semble plus conforme à la réalité d'en faire remonter l'introduction, soit aux Hycsos, soit aux princes de la XVIII dynastie. 
mier apparaît à l'époque de la XIX ${ }^{e}$ dynastie; Ramsés II fit planter des pommiers dans ses jardins du Delta ${ }^{1}$. L'importation de l'amandier semble aroir été plus tardive ${ }^{2}$, elle n'eut peut-être lieu que sous les Ptolémées. C'est sous ces princes encore, et vraisemblablement même sous les derniers d'entre eux, sinon à l'époque romaine, que le mûrier à fruits noirs, le pêcher, le cerisier, de même peut-être que le poirier ${ }^{3}$, furent importés dans la vallée du Nil.

En même temps qu'ils s'enrichissaient ainsi d'arbres fruitiers jusqu'alors inconnus, les parcs égyptiens s'embellirent également de nouveaux arbres d'ornement. L'introduction du peuplier blanc et du platane, que Théophraste montre croissant, bien qu'en petit nombre, sur les bords du Nil', doit remonter à une époque déjà reculée; celle du chêne, qui, d'après le mème naturaliste, se serait trouvé en abondance, arec des perséas et des oliviers, dans un bois des environs de Thèbes ${ }^{5}$, doit être également ancienne; en est-il de même de l'introduction du frêne, qu'il faitcroître aussi, de même que l'orme ${ }^{6}$, en Égypte? Je ne saurais rien en dire, pas plus qu'au sujet de l'importation dans la vallée du Nil du. tilleul, cet arbre de la région tempé-

1. Victor Loret, Recherches, etc. V. Le pommier. (Recueil de travaux, t. VII, p. 113.)

2. Victor Loret, La Flore pharaonique, p. 83.

3. M. Flinders Petrie a trouvé des fruits de ces divers arbres dans la nécropole gréco-romaine de Hawara, ce qui n'est pas toutefois une preuve absolue que ceux-ci fussent cultivés en Egypte; le poirier même n'y est pas planté aujourd'hui. G. Schweinfurth, Illustration de la Flore d'Égypte, s. v.

4. Historia plantarum, lib. IV, cap. VIII, 2.

5. Historia plantarum, lib. IV, cap. 2, 8. Théophraste se sert seulement du mot opous sans dire de quelle espèce il s'agit.

6. IIisloria planlarum, lib. IV, cap. viII, 2. 
rée de l'Europe, dont $M$. Flinders Petrie a retrouvé des restes dans la nécropole gréco-romaine de Hawara $^{1}$. Cette importation remonte sans doute assez peu haut. C'est probablement aussi à une date récente que le laurier ${ }^{2}$, originaire de la région méditerranéenne, a pénétré dans les jardins de l'Égypte. Si l'introduction de ces espèces nouvelles enleva à ces jardins le caractère intertropical qu'ils avaient eu jusque-lì et les rapprocha des jardins de l'Asie antérieure ou de l'Europe méridionale, c'est à des parcs et à des vergers bien plus qu'à des parterres qu'ils ressemblaient. Pour ètre de véritables parterres, il leur aurait fallu ce qui semble leur avoir longtemps manqué, des Heurs et une végétation herbacée.

On a admis, il est vrai, que les Égyptiens ont cultiré les fleurs de tout temps; mais rien ne tend à le prouver, et on peut dire qu'en réalité les choses se sont passées autrement. Tels qu'ils sont représentés, les jardins des hypogées pharaoniques ne renferment, un seul excepté, aucune autre fleur ou plante herbacée que les lotus qui en couvrent les bassins et les papyrus qui garnissent les bords de ceux-ci. Unger toutefois mentionne un jardin représenté, dit-il'3, sur les murs d'une tombe de Thèbes et où se trouveraient de véritables fleurs; après avoir remarqué qu'il était situé au

1. Kahun, Gurob and Hawara, p. 46, 2.

2. 'Trouvé par Flinders Petrie dans la nécropole de Hawara, Hawara, Biahmu and Arsinoë, p. 51.

3. Sitaungsberichte, t. XXXVIII (an. 1859), p. 105. Unger s'est contenté de citer la tombe $n^{0} 11$, indication vague que Franz Wœnig s'est borné à reproduire. Il s'agit ici évidemment des peintures de fantaisie qu'on voit sur les parois du tombeau de Ramsès II. 
bord d'un canal, à en juger par les hachures destinées à figurer l'eau : "Près de ce canal, ajoute-il, sont disposées par rang des plates-bandes de fleurs en forme de demi-lune, chacune d'elles couverte d'une espèce de lleur différente, mais d'une seule. Cependant, remarque-t-il encore, comme ces fleurs sont représentées d'une manière conventionnelle, il est à peu près impossible de les reconnaitre; j'inclinerais toutefois à voir dans l'une d'elles une corymbifère et j'en prendrais une autre, dont les feuilles seules sont figurées, pour une espèce de betterave. "Il est trop évident qu'on ne peut rien conclure d'une pareille peinture; pour l'artiste pharaonique elle n'a été qu'un motif de décoration et les plantes qu'il a représentées n'ont pas plus de réalité dans la nature que les quadrupèdes à tète d'oiseau qu'on aperçoit sur d'autres monuments.

La présence dans les sarcophages de débris de plantes ou de guirlandes de fleurs n'est pas non plus une preuve aussi concluante de la culture de ces dernières qu'on a souvent voulu le croire. Une partie des débris végétaux trouvés dans les tombes pharaoniques appartiennent à la flore des champs ou des marécages, tel que le coquelicot, la dauphinelle orientale, la centaurée déprimée, le chrysanthème à couronnes, l'épilobe, etc.'; les couronnes qui en sont faites ne prouvent pas plus que ces plantes fussent

1. G. Schweinfurth, Bulletin de l'Institut égyptien, no 3 (an. 1882), p. 72, suppose que le coquelicot et la centaurée déprimée ont été introduits en Égypte comme plantes d'ornement; mais si l'on n'y rencontre plus aujourd'hui cette espèce de centaurée à l'état sauvage, rien n'indique qu'elle ne s'y trouvât pas autrefois et le coquelicot se voit encore dans les moissons du Delta. 
cultivées par les anciens Égyptiens que les bouquets de bluets et de bruyère commune qu'on vend dans nos rues n'indiquent que ces fleur's croissent dans des jardins et non dans les champs ou dans les landes.

Il ne saurait y avoir de difficulté que pour les plantes qui n'existent plus aujourd'hui en Égypte, comme la dauphinelle orientale et la centaurée déprimée, on qui ne s'y rencontrent, croit-on, que cultivées ou naturalisées, telle que l'alcée ou guimauve à feuilles de figuier " mais les premières, introduites avec les cultures, ont pu finir par disparaitre, après avoir subsisté dans la vallée du Nil un temps plus ou moins long, disparition qui est moins surprenante que celle du papyrus, tout indigène qu'il était en Égypte. Quant aux secondes, c'est-àdire aux plantes qui sont cultivées de nos jours ou se sont naturalisées, il faudrait mieux en connaître l'histoire qu'on ne le fait pour se prononcer. Schweinfurth s'est demandé ${ }^{2}$ si l'alcée à feuilles de figuier, qu'on trouve à l'état sauvag'e dans le Liban, et la dauphinelle orientale, plante de la région méditerranéenne, ne pourraient pas se rencontrer quelque jour en Égypte, d'où elles ont disparu à l'état spontané. Ainsi les raisons qu'on a inroquées jusqu'ici en faveur de la culture des fleurs dans les jardins de l'Égypte pharaonique sont à peu près sans valeur.

Toutefois le moment vint où, dans la vallée du Nil, on cultiva les plantes à fleurs, comme on le faisait de temps immémorial pour les arbres à fruits. A quelle époque remonte cette culture particulière? Rien n'est

1. G. Schweinfurth, Bulletin de l'Instilut égyptien, $n^{\circ} 8$ (an. 1887), p. 318.

2. Bulletin de l'Institul égyptien, n०3 (an. 1882), p. 70. 
venu nous l'apprendre jusqu'ici; peut-être commençat-elle dès le temps des Ahmessides ou du moins des Ramessides. Un document officiel en constate du moins l'existence sous cette dernière dynastie. Dans la charte où Ramsès III énumère les dons qu'il avait faits aux divinités de Thèbes, de Memphis et d'Héliopolis, le Pharaon parle, à plusieurs reprises, des fleurs qu'il avait fait planter dans les bosquets sacrés. Ainsi, rappelant le domaine consacré au dieu de Thèbes et les vastes jardins dont il était embelli, il ajoute qu'il s'y troudes " serres - c'est ainsi que traduit Eisenlohr" - avec des fleurs de tout pays, des arbustes et des lotus ». Et parlant ailleurs des jardins immenses, ainsi que des bosquets, remplis d'arbres et de dattiers, qu'il avait consacrés au dieu d'Héliopolis, il dit encore qu'on y trouvait des bassins "couverts de lotus et de joncs, et des tleurs de tous pays, douces et parfumées".

Sans doute il faut faire la part de l'emphase habituelle aux documents officiels de l'ancienne Éypte, et ces mots "fleurs de tous pays» ne sauraient être l'expression de l'état véritable de la culture horticole au temps du nouvel empire. Mais sous ces exagérations ordinaires aux scribes pharaoniques, on ne saurait nier qu'il n'y ait un fond de vérité, et qu'on ait sans doute cultivé des fleurs, peut-être mème exotiques, dans les jardins des Ramessides. Il dut en ètre de même sous les dynasties suivantes; mais c'est après la conquête perse, sous les Ptolémées, et encore plus à l'époque gréco-romaine, que le culte des fleur's achera de se développer et prit une véritable importance en

1. Aus dem grossen Papyrus Harris, pl. VIII et XXVII. (Zeilschrift für ägyptische Sprache, t. Xl, p. 5's et 99). 
Egyte. Lhorticulture fit de grands progrès dans le monde hellénique '; les Égyptiens s'y livrèrent avec ar'deur et arec succès, ainsi qu'en témoigne Athénée "; désormais les plantes de leur pays ne leur suffirent plus; ils en demandèrent d'inconnues aux pays étrangers : arbustes et plantes herbacées d'ornement y pénétrèrent à la fois.

Les arbustes y prirent place, à ce qu'il semble, les premiers. La sesbanie d'Égypte (Sesbania aegyptiaca, Pers.), cet arbrisseau d'origine nubienne, dont on a trouvé des fleur's dans la tombe d'Ahmès I ${ }^{\text {er }}{ }^{3}$, était peutêtre dès lors cultivé dans les jardins de Thèbes; elle ne dut pas ètre le seul arbuste qui y fut planté. Sur la peinture $n^{0} 177$ de la galerie nord du British Museum, dont j'ai parlé plus haut, on voit, comme je l'ai remarqué, au bord du bassin central, à côté des touffes de papyrus, deux arbustes, l'un, à fieurs rouges, mais à peine distinctes, ainsi que les feuilles, et qu'il est dès

1. Dans son étude sur Les premiers établissements des Grecs en Égypte (Paris, 1893, in-4, p. 234), M. D. Mallet semble croire que le goût des fleurs, avec celui des couronnes, était venu d'Égypte en Grèce ; mais c'est là une pure supposition. "Nulle part, dit-il, les fleurs n'étaient plus variées et plus nombreuses que dans la vallée inférieure du Nil. „Quoi qu'en aient pu affirmer les poètes grecs, qui d'ailleurs n'en parlaient que par ouï dire, rien n'est moins conforme à la réalité, et la plupart des fleurs dont les Égyptiens des derniers temps faisaient des couronnes étaient exotiques et ont dû être importées dans leur pays. II faut ajouter que le témoignage d'Apulée qu'invoque $\mathbf{M}$. Mallet ne peut rien prouver pour l'époque pharaonique.

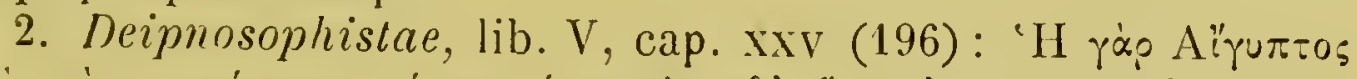

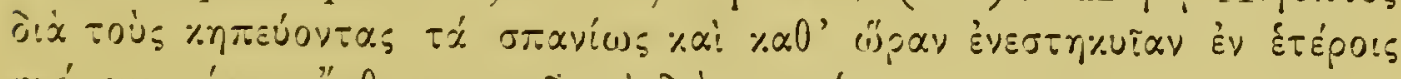
çứ

3. G. Schweinfurth, Bulletin de l'Institut égyplien, no 3 (an. 1882), p. 68. 
iors impossible d'identifier; l'autre, à fleurs blanches, en bouquets terminaux, à feuilles qui paraissent entières, dans lequel j'inclinerais à voir l'arbre au henné, s'il était permis de se prononcer en présence d'une peinture aussi confuse et si cet arbrisseau était réellement indigène dans l'Ethiopie orientale comme on l'a dit'. Recherché pour la belle couleur orange que donnent ses feuilles, l'arbre au henné ne devait pas l'être moins pour le parfum de ses fleurs, à une époque surtout où le jasmin était sans doute encore inconnu en Égypte. Il n'y aurait donc rien de surprenant à ce qu'il eût été importé de bonne heure et cultivé dans les jardins pharaoniques. C'eût été aussi naturel et plus facile que d'y planter les "sycomores à encens" que la reine Hatshopsitou fit apporter du pays de Pount à Thèbes.

Avec cet arbuste bien d'autres devaient pénétrer, mais beaucoup plus tard, dans les jardins égyptiens, tel que le myrte, la rose à cent feuilles, le jasmin sambảc, originaire de l'Hindoustan, qu'on a cru, peut-être par erreur, reconnaitre dans une tombe de Déir-elBahari ${ }^{2}$, mais dont M. Flinders Petrie a découvert des débris certains dans la nécropole gréco-romaine de Hawara ${ }^{3}$. Le savant égyptologue anglais y a aussi rencontré des fleurs de la rose sainte, variété cultivée de la rose d'Abyssinie ${ }^{4}$. S'il fallait en croire Pline ${ }^{5}$, par malheur si souvent inexact, les Ptolémées auraient également fait planter en Égypte le ladanum ou ciste

1. Voir plus haut, chap. II, p. 51 .

2. G. Schweinfurth, Berichte der botanischen Gesellschaft, t. II (an. 1884), p. 268.

3. Kahun. Gurob and Hawara, p. 47, 1.

4. Hawara, Biahmu and Arsinoë, p. 48 .

5. Itist. naturalis, lib. XII, cap. Xxxvrr. 
ladanifère. C'est encore sous ces princes, sinon seulement à l'époque romaine, que fut aussi introduit en Égypte le lierre ${ }^{1}$, cet arbuste grimpant de la région tempérée de l'Europe et de l'Asie antérieure.

Après ces arbrisseaux ou en même temps qu'eux prirent place dans les jardins égyptiens des fleurs herbacées; de temps immémorial, — nous le voyons par les direrses peintures des hypogées, - les plantes symboliques du papyrus et du lotus blanc, avaient figuré dans les réservoirs et les étangs; il en fut peut-être de mème du lotus bleu, indigène comme eux dans la vallée du Nil. Le lotus rose, exotique au contraire, n'y fut planté que beaucoup plus tard, seulement à l'époque perse, dit Schweinfurth ${ }^{2}$. Quant aux fleurs de jardin proprement dites on les voit apparaitre à l'époque des Ahmessides ou des Ramessides. Une peinture de la villa d'Apoui, contemporain de Ramsès II, est la première où l'on en aperçoive.

Sur une des parois du tombeau de ce personnage, " porteur de ciseau », estreprésenté, avec le portique de sa maison, deux bassins couverts de lotus et flanqués tous deux de papyrus ${ }^{3}$. Quatre jardiniers sont occupés à puiser de l'eau à l'aide de shadoufs, établis à l'ombre des arbres du jardin, un puissant sycomore et quatre autres arbres dont deux pourraient bien être des sidrs (Zizyphus spina-Christi W.), les deux autres des jujubiers communs (Zizyphus vulgaris Lam.), accompagnés chacun d'un tamaris ou d'un saule safsaf. Nous n'avons jusqu'ici sans doute rien qui distingue ce jardin des

1. Trouvé par Flinders Petrie dans la nécropole de Hawara.

2. Bulletin de l'Institut égypticn, no 3 (an. 1882), p. 65 .

3. Némoires publiés par les membres de la mission archéologique francaise au Caire, t. V, fasc. IV, p. 607, pl. 1. 
parterres pharaoniques que nous connaissons; ce qui est nouveau, c'est la présence de deux touffes de plantes d'ornement; l'une aux fleurs composées, terminales et de couleur bleue, parait être le bluet oriental (Centaurea depresse Bieb.), dont on a précisément découvert des restes dans une tombe de cette époque, l'autre aux feuilles radicales, lancéiformes et entières, aux fleurs également terminales, jsolées et blanches, portées sur un pédoncule, a tout l'aspect d'une liliacée ou d'une plante analogue 1 , mais sa représentation conventionelle rend difficile de dire à quelle espèce elle appartient.

Quoi qu'il en soit, il semble bien qu'il y ait là un commencement de culture florale; et à partir de cette époque, sinon plus tôt, à côté des arbres fruitiers ou d'ornement prendront place dans les jardins égyptiens quelques fleurs ou plantes d'agrément. Telles furent peut-être la dauphinelle orientale, l'alcée à feuilles de figuier, même l'iris de Sibérie, que Percy Newberry croit avoir reconnue sur une peinture de la tombe de Thoutmès III à Karnak ${ }^{2}$, la menthe poivrée, découverte en 1884 par M. Maspero, à Shéik Abd-el-Gournah ${ }^{3}$, plantes auxquelles il ne serait pas impossible que fussent venues se joindre quelques représentants de la flore indigène comme l'héliotrope de Nubie au parfum délicieux". Toutefois ce fut beaucoup plus tard, et

1. On serait tenté d'y voir un Arum colocasia. On retrouve d'ailleurs cette plante dans la planche où Prisse d'Avennes a figuré des "plantes et fleurs tirées des monuments ", mais il a donné à la corolle la couleur jaune. Iristoire de l'arl égyplien, t. II, pl. 62.

2. Finders Petrie, Kahun, Gurob and IIawara, p. 47, 1.

3. Berichte der deutschen bolanischen Gesellschaft, t. Il (an. 1884), p. 367. - Zeilschrift für Ethnologie, an. 1891, p. 666.

4. Flinders Petrie, Kahun, Gurob and Hawara, p. 47, 1. 11 
seulement depuis l'établissement de relations suivies avec la Grèce, surtout sous les Ptolémées et mème à l'époque de la domination romaine, que pénétrèrent en Égypte le convolvulus épineux, qu'on n'a trouvé ¿ l'état spontané que dans la Gédrosie et les déserts de la Perse méridionale ${ }^{1}$, la célosie argentée, gracieuse amaranthacée de la région soudanienne ${ }^{2}$, le réséda odorant, dont la patrie est incertaine ${ }^{3}$, ainsi que la marjolaine, qu'on croit originaire de la Grèce ${ }^{4}$, peut-être la douce-amère et le narcisse tazzette ", importé probablement de la Palestine, plantes dont M. Flinders Petrie a découvert des débris dans la nécropole de Hawara ${ }^{6}$.

faut dire toutefois que ni Schweinfurth, ni Boissier ne font mention de cette espèce en Égypte.

1. Flinders Petrie, Kahun, Gurob and IIawara, p. 47, 1.

2. Flinders Petrie, Hawar a, Biahmu and Arsinoë, p. 51.

3. Flora orientalis. Genève, 1876-83, in-8, t. I, p. 423. Kahun, Gurob and Arsinoë, p. 46, 2.

4. Percy E. Newberry, On some funeral wreaths discovered in the cemetery of Hawara. (The archaeological Journal, t. XLVI (an. 1889), p. 428.)

5. Les végétaux du musée égyptien du Louvre. (Recueil de travaux, t. XVII, p. 186).

6. Flinders Petrie, IIawara, Biahmu and Arsinoë, p. 51. Malheureusement l'étude que M. Percy E. Newberry a faite des plantes découvertes par M. Flinders Petrie est pleine de fautes d'impression ou de rédaction; c'est ainsi qu'il y est question d'une Matthiola librator, espèce de giroflée qui n'existe pas; qu'au lieu du myrte est mentionné le Lychnis coli-rosa L. etc. G. Schweinfurth, Flinder's Pelries Ausgrabungen im Fajum. (Petermann's Geographische Mittheilungen, t. XXXVI (an. 1890), p. 54.) 


\section{CHAPITRE IV.}

\section{LES ARBRES FRUITIERS ET LES ARBRES D'ORNEMENT LES PLANTES D'AGRÉMENT}

I.

Parmi les arbres cultivés dans les jardins ou les vergers égyptiens, les espèces à fruit occupaient la place la plus considérable; au premier rang y figura, depuis au moins vingt-cinq siècles avant notre ère ${ }^{1}$, le dattier (Phœnix dactylifera L.). Indigène dans la zone chaude et sèche, qui s'étend du Sénégal au bassin de l'Indus ${ }^{2}$, ce palmier a dû être acclimaté de bonne heure dans la vallée du Nil, s'il n'y a pas existé de tout temps. M. Moldenke ${ }^{3}$ a admis qu'à leur arrivée dans ce pays les ancêtres des Égyptiens l'y auraient trouvé déjà cultivé; cette circonstance que le nom bennou ou bounnou, benra, que le dattier porte dans la langue hiéroglyphique, est égyptien, lui a fait supposer du moins qu'ils n'avaient pas reçu cet arbre de l'étranger.

1. Th. Fischer, cité par Engler, ap. Victor Hehn, Kullurpflanzen, p. 273.

2. Car. Frid. Phil. de Nartius, Historia naturalis palmarum. Monachi, 1836-50, t III, p. 260. - A. de Candolle, Géographie bolanique raisonnée, p. 343. - Id., Origine des plantes cultivées, p. 240.

3. Ueber die altügyptischen Bäume, p. 31. 
Charles Pickering a, tout au contraire, considéré le dattier comme exotique et en plaçait, sans preuves d'ailleurs, l'importation en Égyte, au temps de la $\mathrm{XII}^{\circ}$ dynastie ${ }^{1}$. Ce qui est vrai, c'est que cet arbre n'apparait d'abord sur les monuments que vers cette époque. Une peinture de Béni-Hassan ${ }^{2}$, qui en est contemporaine, représente deux hommes, suivis par des chèrres, occupés à abattre des dattiers. Toutefois si les débris de feuilles de dattier, qui, d'après Unger ${ }^{3}$, auraient été trouvés dans les hypogées de Saqqarah, sont bien authentiques, il y aurait là un témoignage de l'existence, sinon de la culture, en Égypte, de ce palmier, à l'époque de la VI ${ }^{\mathrm{e}}$ dynastie.

Le mot bennou, suivant M. Moldenke, signifie " qui porte de-doux fruits "; il semble indiquer qu'à l'époque où les Égyptiens le donnèrent au Phœnix dactylifera, ce palmier était déjà anobli par la culture. Où cette culture a-t-elle pris naissance? A quel peuple revient le mérite d'avoir découvert la fécondation artificielle des dattiers? Carl Ritter a supposé ${ }^{4}$ que cet arbre précieux était resté longtemps à l'état sauvage et que l'espèce en avait été anoblie d'abord par les Nabatéens de la Babylonie, assertion répétée depuis par Victor Hehn"; mais n'est-il pas plus vraisemblable de penser que la culture et la fécondation artificielle du dattier furent découvertes simultanément dans plusieurs des contrées où il croît spontané-

1. The races of man, p. 373.

2. Tombe no 2. Lepsius, Denlimäler, t. II, pl. 40.

3. Silzungsberichte, t. XXXVIII, p. 105.

4. Die Erdlunde im Verhällniss zur Nalur und zur Ges. chichte des Menschen. Berlin, 1847, in-8, t. XIII, p. 775.

5. Die Kullurpflanzen, p. 263. 
ment? En tout cas on ne roit guère comment les Égyptiens du moyen empire, à plus forte raison leurs ancêtres, auraient pu les recevoir des habitants des bords de l'Euphrate.

Quoi qu'il en soit de l'époque où la culture du dattier s'établit dans la vallée du Nil ${ }^{1}$, elle finit par y prendre la plus grande extension; elle y trouvait d'ailleurs, surtout dans la Thébaïde et les oasis, les conditions les plus favorables: un sol sablonneux et humide, avec un climat sec et brûlant. Sous la XVIII ${ }^{\mathrm{C}}$ dynastie cette culture apparaît comme florissante et une des plus importantes de la contrée; le jardin d'Anna, nous l'avons vu, renfermait 170 dattiers; celui du chef militaire contemporain d'Amenhotpou II, dont j'ai donné plus haut la description, n'en contenait pas moins de 93. Le port élevé et élégant du palmier, la bonté de ses fruits, les nombreux usages auxquels on employait son bois et même les fibres de ses feuilles, suffisent pour expliquer la prédilection dont il était l'objet; aujourd'hui encore il est l'accompagnement indispensable des villages arabes, que sa couronne de feuilles domine, en les défendant contre les ardeurs du soleil, leurs humbles maisons en terre et en " créant pour les plantes non désertiques, qu'on y cultive, un milieu tempéré où elles peuvent vivre » ${ }^{2}$.

1. A côté du palmier cultivé subsista l'espèce sauvage; il semble bien que ce soit à cette espèce qu'appartiennent les dattes conservées au Musée du Louvre. Recueil de travaux, t. XVII, p. 183. - Schweinfurth, Zeitschrift für Ethnologie, an. 1891, p. 65 et 66 , remarque que l'on rencontre par place des dattiers qui ont conservé les caractères du type sauvage; il incline néanmoins à regarder le dattier cultivé comme dérivé du P. reclinata Jacq., qu'on rencontre dans les montagnes de l'Abyssinie et de l'Arabie méridionale.

2. Henri Schirmer, Le Sahara, p. 205. 
Fait surprenant, Hérodote', qui parle de la beauté et de la fécondité des dattiers de l'oasis d'Augilas, ne dit rien de ceux de l'Égypte; mais Théophraste, auquel on doit des renseignements si précis sur la culture de cet arbre et sur les régions où on le rencontrait, n'a pas oublié de mentionner les palmiers de la vallée du Nil; il nous apprend mème ${ }^{2}$ que dans ce pays, comme en Syrie d'ailleur's, il y en avait qui domnaient déjà des fruits au bout de cinq ans et n'étant encore arrivés quà la hauteur d'homme. Strabon fait aussi mention des dattiers d'Égypte ${ }^{3}$; mais, d'après lui, ils étaient de l'espèce la plus commune, et il va jusqu'à dire que dans le Delta les fruits en étaient presque immangeables; toutefois il en était autrement dans la Thébaïde et en particulier dans l'ile de Méroé, dont les palmiers, remarque-t-il, l'emportaient sur ceux de tous les autres pays. Aujourd'hui c'est encore dans la Haute-Égypte, ainsi que dans les oasis, que le dattier réussit le mieux et donne les meilleurs fruits.

A côté du dattier prenait place dans les parcs égyptiens le palmier à éventail, mama - le doum des Arabes - (Hyphaene thebaica Mart.): il y en avait 120 dans le jardin d'Anna. Originaire de l'Afrique tropicale" et croissant aujourd'hui encore spontanément dans les vallées de la Nubie et de la Haute-Égypte ${ }^{5}$, cet arbre, on le voit, était cultivé déjà dans la vallée

1. Hislorice, lib. IV, cap. 172.

2. II istoria plantarum, lib. II, cap. 6, 7 .

3. Geograplica, lib. XVII, cap. 1, 51.

4. E. Boissier, Flora orientalis, t. V, p. 46. - O. Drude, IIandbuch der Pfanzengeographie, p. 464.

5. Illustration de la Flore d'Égyple, p. 1'i7. 
moyenne du Nil au xviI ${ }^{\mathrm{e}}$ siècle avant notre ère. Si les fruits de ce palmier trouvés par M. Flinders Petrie à Kahoun n'étaient pas importés de l'Éthiopie, il faut même admettre qu'il fut planté en Égypte huit siècles plus tôt. Le palmier doum tirait son nom indigène mama, " divisé en deux parties »", de la bifurcation que sa tige présente dans sa partie supérieure. Théophraste, qui l'appelle cucifère (\%ou\%śsopos), en a fait son caractère distinctif :

L'arbre qu'on appelle cucifère, dit-il ${ }^{2}$, présente, dans son tronc et ses feuilles, une grande ressemblance avec le dattier, mais il en diffère en ce que, tandis que la tige du dattier est simple et entière, son tronc se bifurque en deux branches secondaires, dont chacune se divise elle-même en deux autres, qui portent des rameaux courts et peu nombreux.

C'est avec ce tronc bifurqué que le palmier doum est représenté d'ordinaire sur les peintures des hypogées égyptiens, en particulier sur celle du parc de l'officier d'Amenhotpou II $^{3}$. Sur une peinture de Tell elAmarna ${ }^{4}$, au contraire, l'artiste pharaonique l'a représenté avec une tige simple, mais arec des feuilles en éventail, leur forme caractéristique.

Les fruits du palmier doum, qu'on a trouvés en immense quantité dans les tombes pharaoniques et dont on peut voir des échantillons dans tous les musées égyptiens d'Europe ${ }^{5}$, sont remarquables par leur forme et leur grosseur.

1. Charles E. Moldenke, op. laud., p. 66.

2. II istoria plantarum, lib. IV, cap. II.

3. Voir plus haut, chap. III, p. 88.

4. Lepsius, Dentimäler, t. III, pl. 95.

5. Migliarini, op. laud., p. 74, no 3605. - S. Birch, Calalogue of the collection of Egyptian antiquities, at Alnwick Caslle. 
Ils diffèrent de la datte, dit Théophraste', par leur dimension, leur forme et la saveur ; assez volumineux pour remplir la main, ils sont ronds et non oblongs; de couleur jaunatre, ils renferment un suc doux et agréable au goùt. Ils ne sont pas, ajoute-t-il, disposés en grappes comme les dattes, mais croissent isolés les uns des autres. Le noyau en est gros et très dur.

Ces fruits portaient en égyptien le nom de qouqou ${ }^{2}$, origine évidente du radical \%ou\%, du mot \%sus:ópopos, et du mot $x^{\prime} \xi_{5}^{3}$, que, dans un autre passage ", Théophraste attribue à un palmier qui croit en Éthiopie, et qui n'est autre éridemment, sa description mème en fait foi, que le rovжśspopos ou cucifère. C'est le cuci de Pline ${ }^{5}$, lequel, dans ce qu'il en a dit, s'est borné à copier le naturaliste grec; toutefois il a ajouté, au sujet du fruit, qu'il renferme une amande douce, tant qu'elle est fraiche, mais qui, séchée, durcit au point de n'être plus mangeable qu'après une macération de plusieurs jours. Dans un hymne, où un poète pharaonique compare Thot à un doum haut de soixante coudées ${ }^{6}$, il est question de ces amandes et du suc qu'elles renfermaient. Les fruits du palmier doum ne pouvaient être comparés à ceux du dattier; ce n’était pas pour eux aussi, mais bien plutòt pour son port et pour la dureté

London, 1880, in-4, p. 18́, no 1437. - Les végélaux du musée égyplien du Louvre. (Recueil de travaux, t. XVII, p. 182).

1. Historic plantarum, lib. IV, cap. $2,7$.

2. Victor Loret, Recherches sur quelques plantes, I. Les Palmiers d'Égyple. (Recueil de travaux, t. II, p. 2't.)

3. Charles Joret, Des noms de palmier. (Revue des éludes grecques. Paris, in-8, an. 1892, p. 417.)

4. Hisloric plantarum, lib. II, cap. vi, 10.

5. IIistoria naluralis, lib. XIII, cap. 18.

6. Victor Loret, Les palmiers d'Égyple. (Recueil de travaux, t. II, p. 23.) - Charles Moldenke, op. lıud., p. 69. 
de son bois, que ce bel arbre était cultivé dans les parcs égyptiens, dont il était un des ornements.

On y plantait aussi, mais bien plus rarement, le palmier argoun (Hyphaene argun Mart.), - le dellah des Arabes; - originaire de l'Éthiopie, il croît encore aujourd'hui en Nubie, où le voyageur Kotschy l'a retrouvé en $1837^{\prime}$, dans la vallée formée par la courbe que décrit le Nil entre Korosko et Abou-Hamed; il avait été importé de cette région dans la moyenne Égypte, où il ne fut jamais commun et d'où il a disparu; il y avait un palmier argoun, mais un seul, dans le jardin du scribe Anna. Cet arbre était-il cultivé dans d'autres parcs égyptiens? On serait tenté de le supposer, d'après le grand nombre de fruits qu'on en a rencontrés dans les tombes pharanniques et qu'on voit dans les musées égyptiens ${ }^{2}$; mais il pourrait se faire aussi que ces fruits eussent été importés directement de leur lieu d'origine.

Quoi qu'il en soit, les fruits du palmier argoun, sinon cet arbre lui-même, furent connus en Égypte dès les temps du moyen empire; M. Flinders Petrie en a découvert plusieurs dans la nécropole de Kahoun ${ }^{3}$, contemporaine de la XII ${ }^{\mathrm{e}}$ dynastie. On en a également trouvé dans les tombes de Drah-Abou'l-Neggah, qui remontent à la même époque ${ }^{4}$. Le palmier argoun portait en ancien égyptien le nom de mama-n-Thanen,

1. Journal of Botany, feb. 1877.

2. Il y en a en particulier quatre au Musée du Lourre. (Recueil de travaux, t. XVII (an. 1895), 7, p. 182.

3. Kahun, Gurob and Hawara, p. 49, 2.

4. G. Schweinfurth, Ueber Pflanzenreste. (Berichle der bolanisihen Gesellscha/l, t. II (an. 1884), p. 369). 
" cucifère à noyaux " ", comme si les noyaux de ses fruits eussent été plus gros que ceux du palmier doum ou que, étant dépourrus de péricarpe charnu, ils eussent été composés presque exclusivement d'un noyau, ce qui en est le caractère réritable et les distingue des fruits du palmier doum ou mama ordinaire.

Si le dattier finit par prendre une place prédominante dans les cultures égyptiennes, plus ancien que lui dans la vallée moyenne du Nil, s'il n'y est pas indigène, le sycomore (Ficus sycomorus L.), en avait occupé à l'origine une bien plus considérable, mais qu'il ne conserva qu'en partie. Il était si répandu dans la vallée du Nil, au temps de l'ancien empire, que l'Égypte a parfois été appelée " la Terre des sycomores " ${ }^{2}$, et le nom de beaucoup de localités était tiré de celui de cet arbre. Le nom indigène du sycomore, neha ou nehi, copte nouhi, "qui protège ", est dérivé de l'ombre fournie par son épais feuillage; il remonte à l'antiquité la plus reculée; il était devenu synonyme d’arbre en général; aussi, avec l'adjonction d'un qualificatif, servait-il à désigner les arbres exotiques encore inconnus ${ }^{3}$.

Arant l'importation du figuier, les fruits du sycomore, quoique un peu douceàtres, devaient être très recherchés; son bois ne l'était pas moins; aussi ne cessa-ton de le planter dans les parcs et les places publiques

1. Ou mama en khanini. Victor Loret, Les palmiers d'Égypte. (Recueil, vol. II, p. 24.) - Charles Moldenke, op. laud., p. 71.

2. Inscription de Roselle, lig. 11, ap. Charles Moldenke, op. laud., p. 83, note.

3. Dans les inscriptions de Deir-el-Bahari, les arbres à encens, apportés à la reine Hatasou, sont appelés « sycomores à encens »: nehaou-il nl ânti. 
de l'Égyte; le scribe Anna, nous l'avons vu, avait 90 sycomores dans son jardin; on en comptait 92 dans celui de l'officier d'Amenhotpou II, et les vergers de toutes les villas représentées sur les murs des hypogées pharaoniques en l'enferment quelques-uns. Reposer et respirer le frais à l'ombre de ses sycomores passait pour la jouissance suprême, celle qui devait être le plus enviable pour les morts dans l'autre vie. "Il parcourt son domaine du couchant (sa nécropole), il prend le frais sous ses ombreux sycomores ", dit Anna de lui-même ${ }^{1}$ dans son inscription funéraire.

Le sycomore est un des arbres le plus anciennement représenté sur les monuments; une peinture des pyramides de Gizeh, contemporaine de la $\mathrm{V}^{\circ}$ dynastie, nous fait assister à la récolte de ses fruits ${ }^{2}$. Deux persomnages, montés chacun dans un sycomore, en cueillent les figues, que des serviteurs, debout au pied de ces arbres, reçoivent et rangent dans des corbeilles. Une peinture d'une tombe de Zaouiet-el-Maïétin ${ }^{3}$, qui remonte à la $\mathrm{VI}^{\mathrm{e}}$ dynastie, représente des hommes en train d'abattre dẹs sycomores, dont les chèvres s'empressent de venir brouter le feuillage. Il n'est presque aucune représentation de villa ou de jardin, où l'on n'aperçoive quelques sycomores, et souvent, on se le rappelle, en grand nombre ${ }^{4}$. D'ordinaire, ils étaient fi-

1. Brugsch, Recueil, t. I, pl. XXXVI, trad. Moldenke, op. laud., p. 19.

2. Lepsius, Denlimäler, t. III, pl. 53, tombe 16. Une scène analogue, mais où on ne voit que la partie inférieure des sycomores, est représentée sur la tombe 14 de Zaouïet-el-Maïétin.

3. Lepsius, Denkmäler, t. IV, pl. 111, t. 14.

4. Par exemple dans la peinture du jardin du chef militaire, contemporain d'Amenhotpou II. 
gurés d'une manière schématique et couverts de feuilles a peine distinctes'; plus tard les artistes pharaoniques les représentèrent arec des feuilles éparses et lancéolées et des fruits attachés au tronc par bouquets; parfois aussi ils supprimèrent les feuilles, mais en laissant les fruits ${ }^{2}$. Le sycomore jouait un rôle considérable dans le culte; ses fruits en particulier étaient une des offrandes les plus ordinaires qu'on faisait aux morts; aussi en trouve-t-on de nombreuses représentations dans les peintures des hypogées et de non moins nombreux spécimens dans les tombes pharaoniques et dans les musées égyptiens ${ }^{3}$.

De tous les arbres de l'Égypte, le sycomore fut un de ceux qui attira le plus l'attention des écrivains de l'antiquité classique:

Le sycomore, dit Théophraste 4 , $\dot{\eta}$ su»x́uvos, ressemble beaucoup à notre figuier, à la fois par le feuiliage, la hauteur et le port; mais ses fruits offrent ceci de particulier qu'ils poussent sur le tronc lui-même; ils ressemblent à ceux du figuier pour la forme et la grosseur; par le goût, ils rappellent les figues d'Olynthe, mais ils sont plus sucrés et n'ont pas de pépins. Ils ne peuvent arriver à maturité que quand on y pratique. une incision; mais cette incision, une fois faite, ils mûrissent en quatre jours. Quand ils ont été enlevés, d'autres repoussent à la même place et cela peut se répéter jusqu'à trois fois

1. Champollion, Description de l'Égyple, t. II, pl. 162. Memoires des membres de la mission française au Caire, t. Y, fasc. IV, p. $607, \mathrm{pl}$. 1. Tombeau d'Apoui. Ici les fruits sont
représentés isolés.

2. C'est le cas pour quelques-uns des sycomores de la peinture 177 du Musée britannique.

3. Il y en a plusieurs, en particulier, au Musée du Louvre. Recueil de travaux, t. XVII (an. 1895), 16, p. 188.

4. Hisloria plentarum, lib. IV, cap. II, 1. 
et davantage même, dit-on, chaque année. Cet arbre distille un suc laiteux; le bois en sert à une foule d'usages.

Pline ${ }^{1}$ n'a fait que répéter, en le traduisant, ce que Théophraste avait dit $\mathrm{du}$ sycomore, mais il s'est étendu plus que le naturaliste grec sur les nombreux emplois du bois de cet arbre, d'après lui aussi le sycomore aurait donné, non pas trois, mais quatre et même sept récoltes de fruits par an.

A côté du sycomore il faut placer le figuier commun (Ficus carica L.), l'un des arbres les plus communément cultivés dans l'ancienne Égypte, quoiqu'il ne le fût pas autant, il s'en faut, que le sycomore. Il n'y avait que 5 figuiers dans le jardin d'Anna contre 90 sycomores. Le figuier est souvent mentionné dans les textes pharaoniques et on le voit aussi fréquemment représenté sur les monuments, mais il y apparait moins anciennement que le sycomore. Une des peintures de la tombe $n^{0} 2$ de Béni-Hassan ${ }^{2}$, qui date de la XII ${ }^{\mathrm{e}}$ dynastie, représente un serviteur qui cueille les fruits d'un figuier et en remplit une corbeille de papyrus ou de jonc qu'il tient à la main; plus loin un autre se prépare à enlever, à l'aide de courroies, une corbeille déjà remplie, déposée sur le sol, tandis que trois singes cynocéphales, qui ont aidé à cueillir les figues, se paient de leur peine, en dévorant quelques-uns de ces fruits savoureux. La fidélité du dessin ne laisse place à aucun doute au sujet de l'arbre ou des fruits représentés par l'artiste pharaonique.

Cette peinture nous montre que le figuier existait

1. Hisloria naluralis, lib. XIII, cap. 14.

2. Côté occidental B. Lepsius, Denkmäler, t. IV, pl. 127. Rosellini, Monumenti civili, t. II, pl. 39, 2. 
en Égpte vingt-trois ou vingt-quatre siècles arant notre ère. Il y fut cultivé bien plus anciennement; des listes d'offrandes, qui datent de la $I V^{\mathrm{e}}$ et de la $V^{\mathrm{e}} \mathrm{dy}$ nastie, permettent de conclure à la présence du figuier - l'arbre teb ou tab - dans la vallée du Nil à cette époque reculée ${ }^{1}$, car il n'est guère possible de supposer que les figues mentionnées dans ces textes eussent été importées des pays voisins. D'ailleurs un document, qui remonte à 4000 ans avant notre ère, nous apprend que dans la villa d'Amten, officier d'un haut rang contemporain de la IV ${ }^{\circ}$ dynastie, se trouvait une plantation considérable de figuiers et de vignes ${ }^{2}$.

Mais de quelle contrée la culture de cet arbre, étranger à la flore indigène, avait-elle été importée dans la vallée du Nil? Le comte de Solms-Laubach, dans son étude sur "L'origine, la domestication et la diffusion du figuier commun $)^{3}$, incline à croire qu'elle a été découverte d'abord, au sud-est de la presqu'île arabique, chez le clan des Bahrâ de la tribu des Qouçâa, au dialecte desquels le nom du figuier - sém. tïn, héb. ta'nat parait emprunté ${ }^{4}$; cette culture aurait passé avec eux dans l'Idumée et de là dans la Célésyrie. En tout cas, c'est aux Sémites que l'Ancien monde en est rede-

1. Charles Moldenke, op. laud., p. 96.

2. Lepsius, Denkmüler, II, pl. 3-7. - Cf. Charles Moldenke, op. laud., p. 97.

3. Die IIerkunfl, Domesticalion und Verbreilung des gewöhnlichen Feigenbaums (Ficus carica L.), p. 77. Abhandlungen der Königlichen Gesellschaft zu Göltingen, t. XXVIII, an. 1881, no 2). Schweinfurth, Zeilschrift für Elhnologie, an. 1891, p. 657, croit le figuier originaire du pays de Pount.

4. Paul de Lagarde, Ueber die semilischen Namen des Feigenbaums und der Feigen. (Nachrichlen von der Kön. Gesellschafl der. Wissenschaften und der Universilül zu Göllingen, an. 1881, p. 378-382.) 
vable; c'est d'eux que l'Égypte l'a reçue vraisemblablement a travers l'isthme de.Suez ou la Mer Rouge'.

Dans le bois sacré du temple de Denderah se trouvait un arbre appelé kounth qui n'est mentionné, ni parmi ceux qui croissent dans le jardin d'Anna, ni parmi les arbres d'aucun autre parc connu : à quelle espèce végétale appartenait-il? La ressemblance de ce mot kounth avec le copte kente " ficus " a fait penser à Dümichen ${ }^{2}$ qu'il désigne l'espèce de figues appelées ^órzxy par les Grecs, nom que Pline ${ }^{3}$, sous la forme cottana, indique comme étant celui des petites figues de Syrie. Les Arabes donnent aujourd'hui encore aux figues sauvages du désert le nom de kottayn ${ }^{4}$, qui n'est autre que le cottana des Grecs et de Pline. Il n'y a donc point de doute sur l'identification faite par Dümichen; mais cette variété de figuier paraît avoir été peu commune en Égypte et n'y avoir été cultivée qu'assez tard.

Dans l'énumération des arbres qu'Anna, le scribe de Thoutmès I (XVIII ${ }^{\mathrm{e}}$ dynastie), avait fait planter

1. Toutefois, il faut dire que le figuier croît à l'état saurage dans toute la région qui s'étend de la Perse orientale et même du Gange supérieur aux rivages de l'Atlantique, entre le $25^{\mathrm{c}}$ et le 40-42e degré de latitude. (A. de Candolle, op. laud., p. 235. Engler, ap. V. Hehn, p. 98.) On a mème trouvé des restes fossiles de cet arbre au nord de cette dernière limite dans le terrain quaternaire du bassin de Paris. Gaston de Saporta, Sur l'existence constatie du figuier aux environs de Paris à l'époque quaternaire. (Bulletin de la Société géologique de France. Série III, vol. II (1873-74), p. 442.)

2. Dümichen, Baunrliunde des Denderatempels, pl. XIX. Cf. Charles Moldenke, op. laud., p. 18, note et p. 100.

3. II istoria naturalis, lib. XIII, cap. 10 (5).

4. Wilkinson, The manners of the ancient Egyptians, t. II, p. 408 . 
dans son parc, on trouve cinq anhmen; c'est là la plus ancienne mention que l'on connaisse de cet arbre, mais ce n'est pas la seule; sous la forme anhmāni ou arhmāmi, on le trouve également mentionné au nombre des arbres d'une villa de Ramsès II (XIX ${ }^{0}$ dynastie) ${ }^{1}$; parmi les dons en nature offerts aux temples d'Égypte par Ramsès III ( $\mathrm{XX}^{\mathrm{e}}$ dynastie), il y avait, avec des pommes et des figues, des raisins et des dattes, etc., des fruits d'ahrmāni ${ }^{2}$. Il est également question dans le Papyrus Ebers de l'écorce de l'ahrmāni ${ }^{3}$. MM. Charles Moldenke ${ }^{4}$ et Victor Loret ${ }^{3}$ ont presque en même temps et indépendamment l'un de l'autre, ce qui donne plus de force à leur démonstration, reconnu dans cet anamen, anhmàni ou arhimāni - ce ne sont pas là les seules formes que présente ce mot - le grenadier, en copte crman ou herman. Cet arbre était donc cultivé en Égypte dès l'époque de la XVII $\Gamma^{\mathrm{e}}$ dynastie; mais la diversité mème des noms qu'il y portait, non moins que leur forme exotique, montre qu'il n'y était pas indigène: de quel pays et à quelle époque y avait-il été importé?

De Candolle a rendu vraisemblable que le grenadier est originaire de la région située au sud du Caucase et de la Caspienne, jusqu'au golfe persique ${ }^{6}$; il paraît l'avoir été aussi dans tout l'Afghanistan et le Béloutchistan ${ }^{7}$. C'est de là qu'il se serait répandu dans toute l'Asie occidentale et dans le bassin de la Médi-

1. Papyrus Anastasi, IV, pl. II. 11, 3-5.

2. Papyrus Harris. K. Piehl, Dict., no 1, p. 6.

3. Pl. XVI, 15-18 et XIX, 19-22.

4. Ueber die allägyptischen Bäume, p. 115, note.

5. Recherches sur plusieurs planles, etc., III. Le Grenadier. (Recueil, vol. VII, p. 109.)

6. Origine des plantes cullivées, p. 184.

7. Engler, ap. Victor Hehn, Kulturp/lanzen, p. 239. Boissier 
terranée; avait-il pénétré en Égypte avant la XVIII ${ }^{e} d y$ nastie? Dans le désert, les Israélites, entre autres plaintes, reprochaient à Moïse de " les avoir conduits dans des lieux stériles, où ne croissaient ni figuiers, ni vignes, ni grenadiers ${ }^{\prime}$ ». Ils avaient donc vu le grenadier en Egypte; mais si ce fait confirme les témoignages qui l'y font croître, il ne nous apprend point si cet arbre y était cultivé longtemps avant l'exode des Hébreux; peut-être avait-il été importé par les Hyksos dans la vallée du Nil. En tout cas, c'est, il semble bien, par l'intermédiaire des Sémites que le grenadier a été connu des Égyptiens; le nom qu'il portait chez ce peuple rappelle l'hébreu rimmoun, arabe roumman, berbère armoun.

Le grenadier parait aroir été assez commun en Égypte à l'époque du nouvel Empire; on le rencontre fréquemment sur les peintures des anciens hypogées, et on a trouvé souvent aussi des fruits de cet arbre dans les tombes de la même époque. Dans le jardin de la villa d'Amenhotpou IV (XVIII dynastie), peints sur la muraille d'un tombeau de Tell-el-Amarna ${ }^{2}$, on voit dix grenadiers recomnaissables à la forme des fruits et des feuilles. C'est là la plus ancienne représentation que l'on connaisse du grenadier. Sur une peinture un peu postérieure d'une autre villa, cet arbre a été figuré sans feuilles, mais avec des fruits arrivés à leur complet développement ${ }^{3}$. Les grenades se re-

admettait aussi que le grenadier est indigène dans une partie de la péninsule des Balkans et des îles de l'Archipel.

1. Numeri, cap. xx, v. 5 .

2. Lepsius, Denlimëler, t. V, pl. 95.

3. Champollion, Momuments, vol. II. pl. 174. - Rosellini, Monumenti civili, vol. [I, pl. 78, 2. 
trouvent fréquemment, avec les figues et les grappes de raisin, dans les représentations des offrandes faites aux dieux; on en voit en particulier parmi les offrandes de Ramsès IV, pharaon de la $\mathrm{XX}^{\mathbf{c}}$ dynastie ${ }^{1}$. C'est à cette époque aussi que remontent les plus anciennes grenades découvertes dans les tombes ${ }^{2}$. Les fleurs de grenadier que M. Maspero a trouvées dans un hypogée de Shéik-Abd-el-Gournah paraissent plus récentes ${ }^{3}$; elles ne datent peut-être que de la XXVI ${ }^{0}$ dynastie.

Cet arbre semble s'être modifié sous le climat de l'Égypte; Théophraste ${ }^{\text {in }}$ dit que les fruits des grenadiers qu'on plantait ou semait dans cette contrée avaient quelque chose de sucré avec une saveur vineuse. Pline mentionne ${ }^{5}$ deux variétés de grenadiers d'Égypte, l'une à feuillage rouge et l'autre à feuillage. blanc. Les produits de cet arbre occupaient une grande place, nous le verrons, dans la médecine égyptienne, ainsi que dans la confiserie et l'art du liquoriste.

Si l'on ignore à quelle époque le grenadier a été importé en Égypte, on ne connait pas davantage la date à laquelle le Mimusops, exotique comme lui, fut transplanté dans la vallée du Nil; mais cet arbre dut y être cultivé dès les temps les plus reculés; on en a trouvé des restes dans les tombes des époques les plus diverses; celles de Drah-Abou'l-Neggah et de Gébéleïn

1. Franz Wœnig, op. laud., p. 326.

2. Victor Loret, La Flore pharaonique, p. 77, no 131. Elles paraissent plus petites que les grenades ordinaires; Schweinfurth les compare à celles de la presqu'île du Sinaï.

3. G. Schweinfurth, Berichte der deutschen botanischen Gesellschaft, t. II, p. 360.

4. II istoria plantarum, lib. II, cap. 2, 7.

5. II istoria naturalis, lib. XIII, cap. 36. 
en renfermaient des fruits ${ }^{1}$. Si ces fruits ne remontent point d'une manière certaine jusqu'à la XII dynastie, comme on l'a cru parfois ${ }^{2}$, il n'en est pas moins vrai que le Mimusops était connu dès cette époque en Égypte; la découverte faite par M. Flinders Petrie de fruits et de feuilles de cet arbre dans la nécropole de Kahoun ${ }^{3}$ prouve qu'on le connaissait et peut-être aussi qu'on le cultivait dans la vallée du Nil dès le temps des Ousirtesen.

Mais si l'existence, sous l'ancjen Empire thébain, du Mimusops dans la terre de Qimit parait incontestable, on a été longlemps avant de reconnaître à quelle espèce appartiennent les spécimens trouvés dans les hypogées - antiques. Le botaniste Kunth, qui a étudié les plantes de la collection Passalacqua ${ }^{4}$, et Migliarini, auteur du Catalogue du Músée de Florence ${ }^{5}$, avaientrapporté les débris de Mimusops de ces collections à la variété elengi L. Cette espèce étant originaire de l'Inde, il est par suite bien douteux qu'elle ait pu être connue en Égypte du temps de l'ancien Empire thébain. En étudiant de plus près, en 1877, les échantillons de Mimusops au Musée de Berlin, Braun a reconnu qu'ils appartenaient à une espèce différente, le $M$. Kummel Bruce ${ }^{6}$, plante abyssinienne, dont le fruit, de la forme et de la grosseur

1. G. Schweinfurth, Die letzlen bolanischen Entdecliungen. (Bolanische Jahrbücher, t. VIII (an. 1886), p. 7.)

2. Schweinfurth, ibid., les regarde comme étant de l'époque gréco-romaine.

3. Kahun, Gurob and IIavara, p. 49, 2.

4. Catalogue raisonné el historique des antiquilés découverles en Egyple. Paris, 1826, in-8, p. 22, 8.

5. Indicalion succincle des Monuments hisloriques, p. 7多, no 3613: "Noyau singulier du fruit, Mimusops elengi. »

6. Ueber PRanzenresle aus altägyplischen Grïbern. (Zeitschrifl für Elhnologie, t. IX (1877), p. 301. 
d'un cynosbatos, est assez agrëable à manger, à cause de son gout sucré; tandis que son noyau, relativement rolumineux, renferme une amande d'une sareur amère ${ }^{1}$.

C'est à cette espèce qu'Ascherson a également attribué les feuilles des couronnes du musée de Leyde ${ }^{2}$. Mais par une comparaison attentive de nombrevix échantillons provenant d'Abyssinie arec les feuilles des guirlandes anciennes, Schweinfurth a constaté l'identité des dernières avec le M. Schimperi. Hochst ${ }^{3}$, espèce dont les feuilles, portées sur des pétioles longs et minces, sont plus elliptiques et moins obtuses que celles du $\boldsymbol{M}$. liummel'. Lie $\boldsymbol{M}$. Schimperi indigène, comme le $\boldsymbol{M}$. liummel, en Abyssinie, ainsi que dans l'Arabie méridionale", fut importé en Égypte à une époque reculée et, à partir de la XXII ${ }^{\mathrm{e}}$ dynastie, ses feuilles furent employées dans la fabrication des couronnes funéraires.

Schweinfurth a regardé le Mimusops Schimperi comme le Perséa des Anciens Schreber ${ }^{6}$ et Sprengel ${ }^{7}$ avaient identifié cet arbre fameux avec le Cordia my $x a$.

1. Victor Loret, La Flore pharaonique, no 98, p. 61-62. M. Loret a en vue le fruit du M. Sehimperi H. ; mais ce qu'il en dit s'applique également à celui du $M$. liummel B.

2. W. Pleyte, La couronne de la justification. (Acles du sixieme Congrès international des Orientalistes, tenu en 1883 ì Leide. Leide, 1885, t. IV, p. 15.)

3. Bulletin de l'Institul égyptien, no 3, p. 67.

4. A. de Candolle, Prodromus, t. VIII, p. 203 et 672.

5. Schweinfurth, Zeilschrift für Elhnographie, an. 1891, p. 656.

6. De persea Aegyptiorum. (Iagazin fïr die Botanik, hergg. v. Römer u. Usteri. Lief. IV, 46 et V, 14.)

7. Theophrast's Naturgeschichte der Gewüchse, t. II, p. 130, ap. Franz Wœnig, op. laud., p. 320. 
E. Meyer ${ }^{1}$ y voyait le Diospyros mespiliformis Schimper. Delile ${ }^{2}$, suivi par Unger ${ }^{3}$, Brugsch, Piehl et d'autres, le Balanites aegyptiaca Del. Malheureusemerit rien n'a permis jusqu'ici de trancher la question. La description que Théophraste a donnée du perséa ${ }^{4}$ ne convient entièrement à aucun des arbres auxquels on l'a rapportée. Cet arbre toujours vert du naturaliste grec, dont le port, les feuilles et les fleurs sont ceux du pommier, ne ressemble à aucun d'eux. Il faut en dire autant du fruit, de la grosseur d'une pomme, d'après Théophraste, mais oblong et semblable à une amande, all noyau moins gros, mais plus tendre que celui du coccyméléa - le Cordia myxa -, à la chair d'une saveur douce et agréable, légère à l'estomac. Cepen. dant quelques-uns de ces derniers caractères peuvent se rapporter au fruit du Mimusops; ils excluent, au contraire, le Cordia myxa et ne peuvent s'appliquer au Balanites aegyptiaca, pas plus, quoi qu'en ait pensé E. Meyer, qu'au Diospyros méspiliformis, arbre dont il se borne à dire d'ailleurs "qu'il est peut-être le perséa »; le Diospyros ne parait pas au reste avoir été cultivé en Égypte. Il n'en fut pas de même du Cordia myxa et en particulier du Balanites aegyptiaca, dont on a retrouvé des débris dans les hypogées.

Indigène dans les vallées désertes de la Nubie méridionale et en Abyssinie, ainsi que dans le Soudan oriental et occidental et le territoire du Nil Blanc ${ }^{5}$, le Bala.

1. Botanische Erläuterungen $\approx$ Strabons Geographie. Königsberg, 1852, in-8, p. 116.

2. Description de l'Égyple. Paris, 1824, in 8, t. XIX, p. 273.

3. Silungsberichte, t. XXXVIII (an 1859), p. 125.

4. II isloria plantarum, lib. IV, cap. 2, 5.

5. A. de Candolle, Prodromus, t. I, p. 708. - Delile, op. 
nites aegyptiaca Del. (Nimenia aegyptiaca L.) - le lebalih des ancions auteurs arabes, le hegelig des Arabes d'aujourd'hui - dut pénétrer do bonne heure en Égypte ct y être fréquemment cultivé; des fruits de cet arbre ont été reconnus par Schwcinfurth dans des tombes antiques', et M. Flinders Petrie en a décourert aussi en abondance dans la nécropole de Kahoun, qui remonte ì la XII dynastie; Rohlfs a trouvé également des noyaux de Balanites dans uno ancienne tombe de l'oasis de Dakhel au désert de Libye ${ }^{2}$; les musées égyptiens de Florence ${ }^{3}$, de Berlin ${ }^{4}$ et de Paris ${ }^{5}$ en renferment aussi des fruits.

D'uné hauteur moyenne, aux ramcaux épineux, avec dos feuilles ovales, le Balanites porte des fruits allongés, de la grosscur d'une prune - les bas-reliefs leur domnent la forme d'une amande ou d'un ouf - ; ils renfermont un seul noyau; d'un vert sombre avant la maturité, ces fruits prennent, quand ils sont mûrs, une couleur brun-orangée; la chair, d'abord d'un goùt astringent, prend à la longue une saveur douccâtro et

laud., p. 269. - Th. von Heuglin, Reise im Gebiel des weissen Nil, p. 41 et 82, ap. Franz Wonig, op. laud., p. 319.

1. Bulletin de l'Instilut égyplien, no 3 (an. 1882), p. 66, no 5 (an. 1884), p. 5, no 6 (an. 1885), p. 268.

2. Verhandlungen der Berliner Gesellschaft für Anlhropologie, etc. Berlin, an. 1875 , p. 58.

3. Migliarini, Indicalion succincle, p. 74, no 3607 . Sous Ie no 2692, p. 56, est indiqué aussi un bâton recourbé en bois de Balanites aegypliaca.

4. Braun, Ueber Pflanzenresle. (Zeilschrift für Ethnologie, t. IX, p. 306.)

5. V. Loret et J. Poisson, Éludes de bolanique égyplienne. Les végétaux antiques du Louvre, 31. (Recueil de travaux t. XVII, p. 196.) Les fruits des échantillons du Louvre sont plus allongés que ceux du type moderne. 
légèrement amère; l'amande aussi est amère et on en retire une huile estimée. Cette olacince, décrite longuement par Delie, croit encore aujourd'hui en Égypte à l'état subspontané. M. Moldenke, qui l'identifie avec l'antique perséa ${ }^{1}$, a cru la reconnaître dans l'arbre shoub ou shuubou, dont il se trouvait 31 pieds dans le jardin d'Anna, mais M. Loret voit dans l'arbre shoub le lentisque ${ }^{2}$. M. Maspero, de son côté, a identifié le Balanites aegyptiaca avec l'arbre ashed ou ashdou des textes hiéroglyphiques ${ }^{3}$, arbre dans lequel M. Moldenke avait voulu voir ${ }^{4}$ le Cordia myxa ou sébestier.

Indigène peut-être en Nubie et en Abyssinie, ainsi que dans le territoire de la rivière des Gazelles, contrée où il est un des principaux représentants de la flore arborescente, le Cordia myxa L. croissait aussi dans l'ancienne Égypte et on le trouve encore fréquemment dans l'Égypte actuelle. On en voit des noyaux dans les Musées égyptiens de Florence ${ }^{3}$ et de Vienne ${ }^{6}$. et M. Flinders Petrie en a découvert des fruits dans la nécropole gréco-romaine de Hawara?. D'une hauteur d'environ quatre mètres, l'écorce grisàtre, couronnée d'une large cime, le sébestier a les feuilles presque aussi larges que longues et les fleurs en corymbe; il produit un fruit noirâtre et savoureux, à la chair visqueuse et de la grosseur d'un gland. Il semble être le

1. Leber die altägyplischen Bäume, p. 12ィ.

2. La Flore pharaonique, p. 103, no 169.

3. Procecdings of the sociely of biblical Archacology,

t. XIII (an. 1891), p. 498.

4. Op. laud., 9.107 , note.

5. Migliarini, op. laud., p. 74, no 3610 .

6. Unger, Silsungsberichle, t. XXXVIII, p. 113.

7. IIawara, Biahmu and Arsinoë, p. 48 . 
coccymelea de Théophraste', arbre qui, d'après le naturaliste grec, se rencontre en abondance dans la Thébaïde et dont les fruits desséchés et écrasés servaient aux habitants de cette contrée à faire des gaiteaux ${ }^{2}$. M. Moldenke a identifié le jujubier avec l'arbre ash ou ashdou ${ }^{3}$, qu'on rencontrait dans les bois sacrés de dix-sept des nomes égyptiens; M. Victor Loret, lui, y avait vu le sébestier"; mais il a renoncé à celte interprétation, depuis que M. Maspero a attribué au mot ashdou une signification différente.

Comme l'arbre ashdou, et plus souvent encore, on rencontre l'arbre nebs, nabas ou noubsou dans les textes hiéroglyphiques. M. Moldenke a cru pouvoir identifier cet arbre avec le sébestier ; Brugsch avait vu en lui d'abord le sycomore, puis le murier ${ }^{6}$; avec bien plus de raison M. Victor Loret $^{\top}$ y a reconnu le jujubier (Zizyphus lotus L. ou spina-Christi W., Rhamnus napeca Forsk.), identification que M. Maspero est venu confirmer par de nouvelles et toutes puissantes raisons ${ }^{8}$.

1. Historia plantarum, lib. IV, cap. 2, 10. Il faut remarquer toutefois que Théophraste attribue au coccymelea une hauteur considérable et compare son fruit à une nèfle, caractères qui ne conviennent guère au sébestier.

2. Ibn Baithar, Traité des simples, no 57. (Notices el extraits, t. XXV, 2, p. 236.)

3. Il avait d'abord vu dans l'arbre ashı le jujubier (Zizyphus spina Christi W.). Op. laud., p. 107, note 1.

4. Flore pharaonique, p. 64, no 101.

5. Op.laud., XI, p. 108. Toutefois, il faut dire que dans une note de la même page il incline à y voir le lotus (Zizyphus lolus W.)

6. Werterbuch, 750 , et Dictionnaire géographique, s. v.

7. La Flore pharaonique, p. $98, n^{\circ}$ 166. Il lui donne la forme plus complète nabas, mot qui rappelle le nom arabe nabak du fruit.

8. Noles au jour le jour, 12. (Proceedings of the society of biblical Archaeology, t. XllI, p. 496.) 
Cet arbre - le sidr des Arabes - appartient, suivant Schweinfurth ', à la flore indigère de l'Égypte; cultivé aujourd'hui dans les jardins et les parcs de cette contrée, on le rencontre aussi fréquemment à l'ètat sauvage dans la haute vallée du Nil, où Cailliaud l'a signalé dans ses voyages au delà de la seconde cataracte ${ }^{2}$.

Le jujubier porte un fruit, nabak, qui, arrivé à son complet développement, ressemble, dit A thénée ", pour la couleur à une datte, pour la grosseur à une olive, mais renferme un très petit noyau. M. Flinders Petrie a trouvé quatre de ces fruits dans une tombe de Kahoun ${ }^{4}$. Les fouilles de M. Maspero à Gébéleïn ont fait aussi découvrir des baies et des graines de cette rhamnée ${ }^{5}$, que Schweinfurth a pu étudier et reconnaître ${ }^{6}$. Il est d'ailleurs fait mention des fruits du nabas, c'est-à-dire du jujubier, dans la plupart des listes d'offrandes. Cet arbre avait donné son nom à la ville nubienne de Pnob. sou ${ }^{\top}$. Le nabas était ainsi connu et probablement aussi cultivé en Égypte dès les temps les plus reculés; on l'a identifié avec le lotos des écrivains grecss, et la

1. Bulletin de l'Institut égyptien, no 8 (an. 1887), p. 308.

2. Voyage ì Méroé, t. IV, p. 378.

3. Deipnosophistae, lib. XIV, 65.

4. Kahun, Gurob and Hawara, p. 42, 2.

5. Bulletin de l'Institut égyptien, no 6 (an. 1885), p. 260. Bolanische Jahrbücher, t. VIII (an. 1886), p. 3.

6. On a trouvé six fruits de cet arbuste parmi les plantes égyptiennes du Musée du Louvre. (Recueil de travaux, t. XVII, 27, p. 193.) Bonastre l'y avait déjà signalé, Journal de Chimie, t. XIV (an. 1828), p. 434.

7. G. Maspero, Proceedings of the sociely of biblical Archaeology, t. XIII, p. 497.

8. Fraas, Flora classica, p. 94. - O. Lenz, Botanit der Griechen, p. 549 . 
description qu'a domée Polybe ${ }^{1}$ de ce dernier ne peut guère convenir qu'au Zizyplus lotus ou spina-Christi. Théophraste, au contraire, parait plutòt désigner le micocoulier (Celtis australis L.) par le nom de lotos et le jujubier par celui de paliouros ${ }^{2}$; il en est de même de Pline ${ }^{3}$, qui au reste n'a guère fait que copier, en l'abrégeant, le naturaliste grec".

Parmi les arbres à fruits de l'ancienne Égypte, il faut encore ranger le caroubier (Ceratonia siliqua L.). M. Flinders Petrie a trouvé à Kahoun une gousse et six graines de cette légumincuse, répandue de nos jours sur tous les bords de la Méditerranée; il dit aussi en avoir découvert a Gourob deux gousses et sept graines, et M. Greville Chester en aurait rencontré également des restes dans les tombes de Thèbes ${ }^{5}$. Un fruit, qui figure parmi des offrandes dans la peinture d'un hypogée de Béni-Hassan, a été regardé par Unger comme une gousse de Ceratonia, et Kotschy a rapporté en Europe une canne trouvée à Thèbes dans le cercueil d'une momie et qu'on a reconnue, à l'cxa-

1. Athénée, Deipnosophistae, lib. XIV, cap. 65 (561 d). C'est aussi, il semble bien, de fruits du Zizyphus, que, d'après Hérodote (lib. IV, cap. 177), se nourrissaient les Lotophages.

2. Historia plantarum, lib. IV, cap. 3. Il faut dire toutefois que quelques caractères, entre autres la forme du fruit et la taille de l'arbre, conviennent bien peu au micocoulier.

3. II istoria naturalis, lib. XIII, cap. 32 (17).

4. On a trouvé, au Nusée du Louvre, un fragment de noyau qui a paru appartenir au jujubier commun (Zizyphus vulgaris L.) Recueil de travaux, t. XVII, n²8, p. 194); mais comme on ignore l'origine de la collection végétale de notre grand Musée, il est impossible de conclure de cette trouvaille, à moitié douteuse encore, à l'existence du jujubier commun en Égypte à l'époque pharaonique.

5. Kirlum, Gurol and IIawara, p. 48, 1 et 50, 1. 
men microscopique, comme étant en bois de caroubier $^{1}$. On ne peut guère douter dès lors que cet arbre n'ait été connu et probablement aussi cultivé en Égypte à une époque très reculée. Il se rencontre encore aujourd'hui, bien qu'il n'y soit pas commun, dans la vallée du Nil. Y est-il indigène ou y a-t-il été importé? Théophraste affirme que le caroubier -

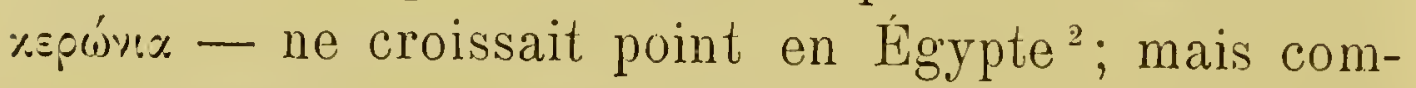
ment expliquer alors la dénomination de figuier d'Égypte qu'on lui donnait - à tort, remarque-t-il, il est vrai? - Il ajoute qu'il croissait en Syrie et en Ionie, ainsi qu'aux environs de Cnide et de Rhodes, c'est-àdire dans la région orientalc de la Méditerranée; c'est elle que de Candolle regarde comme son pays d'origine, ainsi peut-être que la Cyrénaïque ${ }^{3}$; il a dû se répandre de là dans tout le bassin méditerranéen. On vient de voir qu'il était connu, sinon cultivé, en Égypte, sous la XII dynastie. Strabon, qui l'appelle жeparía, le fait même croitre en Éthiopie ${ }^{4}$.

Dans le Papymus des signes, découvert à Tanis par M. Flinders Petrie ${ }^{5}$, après la figure de la gousse, on

1. Unger, Sitzungsberichte, t. XXXVIIl (an 1859), p. 132.

2. IIistoria plantamu, lib. IV, cap. 2, 4.

3. Origine des plantes cultivées, p. 268. On le trouve aussi dans le Yémen, où A. Deflers en a vu dans les gorges du Sabor, près Tâez, à 1,400 mètres d'altitude, des troncs de 1 à 2 mètres de circonférence et de 10 à 12 mètres de hauteur. Voyage au Yémen. Journal d'une excursion bolaniqne faile cn 1887 dans les montagnes de l'Arabie heureuse. Paris, 1889, in-8, p. 134.

4. Geographica, lib. XVIII, cap. 2. E. Neyer a cru que le géographe grec s'était trompé.

5. Two hieroglyphic Papyri from Tanis. London, 1889, p. XVII, $n^{\text {กs }} 2,3$. 
lit: " fruit de l'arbre noudjim "; M. Victor Loret" traduit par " fruit du caroubier », noudjim ou noutem étant, suivant lui, le nom du caroubier, et s'écrivant " au moyen d'une gousse " ${ }^{2}$. Il a cru aussi retrouver la mention des fruits du noudjim ou du caroubier dans une vicille recette de parfumerie, qui indique la préparation d'un parfum destiné à oindre les membres divins. Le signe hiéroglyphique de la gousse étant employé dans les inscriptions des plus anciennes pyramides $^{3}$, M. Victor Loret en conchut à l'antiquité du mot noudjim ${ }^{4}$ et par suite à celle du caroubier dans la vallée du Nil. M. Moldenlie ${ }^{5}$ a rencontré la mention d'un arbre not'em, mais il n'est pas parvenu à le déterminer; ce mot, qui est sans doute le même que nout'em ou noudjim, se présente, dit-il, après la chute du $t$, sous la forme abrégée nem; si ce dernier mot est identique pour la signification avec nout' $\mathrm{em}$ et noudjim, comme il y arait seize arbres nem dans le jardin d'Anna, le caroubier aurait été planté dans le parterre de l'officier de Thoutmès I. M. Moldenke n'a pas été plus heureux dans l'explication du mot sesnotem, que pour celle du vocable not' $\mathrm{em}^{6}$; il l'a expliqué par " bois de cèdre " ou de " cyprès ", tandis qu'il faut le traduire sans doute, arec M. Victor Loret, par "bois de caroubiel. ")

1. Victor Loret, Recherches sur plusieurs planles, VII, 1893, p. 10. (Recueil de travaux, t. XV.)

2. La Flore pharaonique, p. 83, no 116 .

3. M. Moldenke a vu dans cette gousse un fruit d'acacia.

4. Il faut dire toutefois que l'identité de la gousse et du mot noudjim ne prouve pas que celui-ci ait toujours nécessaire-
ment signifié caroubier.

5. Ueber die allägyptischen Bäume, XXIII, p. 139.

6. Op. laud., XX, p. 133. 
Le fruit du caroubier s'appelait en ancien égyptien darouga, autre forme, dit M. Victor Loret, de garouta, mot qui signifie gousse et répond au copte garaté; mais à còté de ce vocable, qu'on peut rapprocher de l'arabe kharob et qui parait d'origine sémitique, il y en avait deux autres purement égyptiens djaïri et ouhd ou houá; le premier, qui signifie "aigrelet ", ne s'est, d'après M. Victor Loret ${ }^{1}$, appliqué d'abord qu'à la pulpe seule du fruit; plus tard il a servi à désigner le fruit tout entier et s'est substitué au mot plus ancien ouhá. Celui-ci, qui signifie "être recourbé en forme de croissant ", servait à désigner la gousse du caroubier, surtout la gousse fraîche, tandis que garouta était plutôt le nom de la caroube sèche.

Parmi les arbres fruitiers anciennement cultivés en Égypte, il faut placer l'olivier et l'arbre à noix de ben, encore que les fruits en soient plutôt employés pour leurs propriétés oléifères que comme comestibles. L'olivier n'est point indigène en Égypte et l'on ignore à quelle époque il a été importé dans la vallée du Nil; mais croissant spontanément dans presque toute l'Asie antérieure, des steppes du Pendjab aux bords de la Méditerranée ${ }^{2}$, cultivé à l'époque la plus reculée en

1. Recherches sur plusieurs plantes, IX, p. 13. (Recucil de travaux, t. XV.) - La Flore pharaonique, p. 89.

2. D'après Engler, ap. Victor Hehn (Kullurpflansen, p, 117), l'olivier serait aussi indigène dans la presqu'ile des Balkans, ainsi qu'en Italie, en Sicile, en Espagne et dans toute la Mauritanie. A. de Candolle (Origine, p. 215), le croyait originaire de la région syrienne, Schweinfurth (Bulletin de l'Institut égyptien, an. 1887, p. 305) le supposait importé de l'Arabie heureuse; depuis (Zeitschrift für Ethnologie, an. 1891, p. 663) il s'est borné à dire qu'il a d'abord été cultivé en Syrie. 
Syrie, il dut pénétrer de bonne heure en Égyte. En tout cas, il existait dans la vallée du Nil au temps des Ramessides; s’il n'est point sir qu’il soit, comme l'ont cru Rosellini ${ }^{1}$ et Franz Wøonig ${ }^{2}$, représenté sur une peinture des hypogées de Tell-cl-Amarna, qui remontent à la XVIII ${ }^{\mathrm{e}}$ dynastie, un document, qui date de la $X^{\circ}$, le Papyrus Harris, en fait connaître la culture d'une manière certaine et on pourrait dire officielle. L'un des rois de cette dynastie, Ramsès III, rappelle lui-mème, dans ce papyrus, les plantations d'olivier's qu'il arait faites en l'honneur des Dieux".

Ainsi l'olivier était connu et fut même cultivé en Égypte sous la $\mathrm{XX}^{\mathrm{e}}$ dynastie; on a trouvé d'ailleurs dans les tombes de cette dynastie et des suivantes des couronnes faites de feuilles d'olivier. M. Maspero a aussi découvert dans une tombe de Shéikh Abd-el-Gournah des fragments de guirlandes faites en feuilles d'olivier " et le Musée de Leyde possède deux couronnes semblables, dont l'une est encore intacte. M. W. Pleyte a $\mathrm{cru}^{r}$, d'après ces faits ${ }^{\circ}$, pouvoir reporter l'introduction de l'olivier en Égypte à l'époque de la XIX ${ }^{\circ}$ dynastie. Elle remonterait bien plus haut, si les noyaux

1. Monumenti dell Egitlo, t. II, pl. 78 .

2. Die Pflrnzen im allen Aegyplen, p. 329.

3. Papyrus IIarris, von Eisenlohr, XXVII, XLIX. (Zeitschrift für acgyptische sprache, t. XI (an. 1873), p. 99, 155, etc.)

4. G. Schweinfurth, Ueber P/lansenreste (Berichte der botanischen Gesellschaft, t. II (1884), p. 368).

5. “Si les momies qui les portent (les couronnes d'olivier) appartiennent à la XX⿳亠口了 jusqu'a la XXVe dynastie, nous pouvons déduire de là, comme date de l'introduction de l'olivier en Égypte, à peu près la XIX e dynastie. " La couronne de la justification. (Actes du sixieme Congress international des Orientalistes, tenu en 1883 à Leide. 40 partie. Leide, 1885,
in-8, p. 13.) 
d'olive, découverts par Schiaparelli dans une tombe de Drah-Abou'l-Neggah, étaient bien contemporains de ce monument et provenaient de cultures égyptiennes. Ils ont paru à Schweinfurth appartenir à deux races distinctes ${ }^{1}$; peut-être se rapportent-elles à l'olivier ordinaire (Olea europea L.) et à sa variété de Nubie (Olea nubica Schw.), que Percy Newberry a reconnue parmi les restes végétaux trouvés à Hawara ${ }^{2}$. Migliarini a, dans son catalogue du Musée égyptien de Florence $^{3}$, attribué certaines feuilles de guirlandes funéraires à une autre espèce, l'olivier sauvage (Olea oleaster L.).

Suivant Théophraste ${ }^{4}$, l'olivier croissait en Égypte, mais seulement dans la Thébaïde, non près du Nil toutefois, mais à une certaine distance - trois cents stades - du fleure, "dans les terres qui n'étaient arrosées que par des sources "; le naturaliste grec avait ici sans doute en vue les oasis du désert libyque, où l'olivier pousse encore de nos jours d'une manière merveilleuse. Théophraste ajoute que l'huile d'olive égyptienne n'était pas inférieure à celle de la Grèce, mais l'odeur, dit-il, en était moins agréable. D'après Pline $^{5}$, les olives d'Égypte auraient été très charnues, mais auraient donné peu d'huile. Strabon ${ }^{6}$ vante la beauté et la fécondité des oliviers du nome d'Arsinoë - le Fayoum actuel, - et il dit que l'huile en était

1. Bulletin de l'Instilul égyptien, nn 6 (année 1885), p. 266.

2. Flinders Petrie, IIavara, Biahmu and Arsino"̈, p. 48.

3. Indicalion succincle des Honumenls igyptiens, p. 72, $11^{\circ} 2465$.

4. II isloria plantarum, lib. IV cap. 2, 9.

5. "In $\Lambda$ egypto carnosissimis olei exiguum ». Lib. XV, cap. 4.

6. Geographica, lib. XVII, cap. 1, 35. 
excellente, quand elle était faite avec soin; mais il ajoute que le reste de l'Égypte était dépourvu d'oliviers, a l'exception des jardins d'Alexandrie; on y voyait des oliviers qui produisaient des fruits, toutefois sans fournir de l'huile.

Brugsch' a attribué à l'olivier le nom bek, bek-t, belia. balia, qu'on trouve dans les textes hiéroglyphiques, comme celui d'un arbre dont on retire un breurage et un parfum; d'autres égyptologues ont cru qu'il était désigné par le mot t'ettu ; la forme authentique est djadi, radical qui se rencontre avec ce sens depuis l'époque des Ramessides ${ }^{3}$. Les textes où il apparait montrent qu'on faisait en Égypte une grande consommation d'olives, comme fruits comestibles, mais encore plus pour en extraire l'huile des lampes sacrées.

Le nom baq, qu'on a attribué parfois, je viens de le dire, à l'olivier, désigne en réalité un tout autre arbre, mais des fruits duquel on extrait également de l'huile,

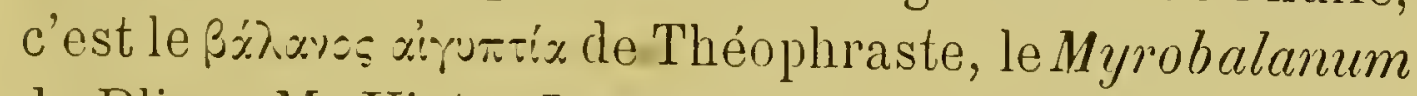
de Pline. M. Victor Loret avait cru pouvoir identifier cet arbre avec le Moringa oleifera Lam. ${ }^{4}$; mais Schweinfurth" ayant trouvé une graine de Moringa aptera Gaertner parmi les restes végétaux découverts par Schiaparelli à Drah-Abou'l-Neggah, le savant égyp-

1. Hierogl. demotisches Wherterbuch, vol. II, pl. 425-426.

2. Charles Moldenke, op. laud., p. 117 et 119.

3. Victor Loret, La Flore pharaonique, p. 59. Le signe hiéroglyphique pour les mots l'ellu et djadi est d'ailleurs le même.

4. La Flore pharaonique, p. 86, n 145.

5. Les dernières dicouverles bolaniques dans les anciens tombeaux de l'ígyple. (Bullelin de l'Institul égyplien, no 6 , 1886, p. 270.) - Bolanische Jahrbücher, t. VIII (an. 1886), p. 8. 
tologue a renoncé à sa première interprétation et admis que le baq des textes hiéroglyphiques est bien l'espèce de Moringa déterminée par le botaniste allemand ${ }^{1}$. Le Moringa aptera, nommé yesser (yesar) en arabe, se rencontre fréquemment dans les vallées du désert arabique, ainsi que dans l'oasis de Dakhléh ${ }^{2}$. Il en est fait mention dans les textes des plus anciennes dynasties.

Les arbres fruitiers dont je viens de parler sont ceux qui furent le plus anciennement cultivés dans les jardins de l'Égypte pharaonique; c'étaient ceux d'ailleurs que leur offrait la flore indigène ou des contrées voisines; mais ils ne suffirent pas toujours aux habitants de la vallée du Nil et quand ceux-ci furent en rapport avec l'Asie antérieure ou avec la Grèce, ils leur empruntèrent quelques-unes de leurs espèces indigènes; commencée dès le temps des Ramessides, cette importation nourelle se continua jusqu'aux premiers siècles de notre ère.

La plus importante des espèces qui ait été ainsi acclimatée en Égypte est le pommier (Pyrus malus L.); il y fut cultivé dès le temps des Ramessides ${ }^{3}$. Son nom hiéroglyphique dapih, qu'il faut rapprocher du mot arabe taffah, copte djepeh, hébreu tappoulh, se rencontre déja dans le papyrus Anastasi III. "Les grenades, les pommes, les [olives] et les figues du jardin fruitier ", comme traduit M. Victor Loret". Ramsès II, je l'ai

1. Recherches sur plusicurs plantes. I. (Recueil de travaux, t. VII, p. 101.)

2. Illustration de la Flore d'Égyple, p. 60.

3. Victor Loret, La Flore pharaonique. p. 82, no 137.

4. Recherches sur plusicur's plantes. V. "Le pommier." (Recueil de travaux, vol. VII, p. 113.) 
déji dit, fit planter des pommiers dans ses jardins du Delta. On voit Ramsès III donner aux prêtres de Thèbes, pour leurs offrandes, 348 paniers de pommes ${ }^{1}$. Le pommier était donc cultivé dans la vallée du Nil dès le temps de la $x \mathrm{xx}^{\mathrm{C}}$ dynastie, et son nom à forme sémitique montre qu'il y était renu sans doute de la Syrie; il avait dù être apporté dans cette dernière contrée de l'Asie Mineure ou de la région du Caucase, son vrai pays d'origine?

Si le pommier a été ainsi cultivé en Égypte à une époque reculée, en a-t-il été de mème du mûrier, de l'amandier, du pècher, du cerisier et du poirier dont M. Flinders Petrie a trouvé des fruits dans la nécropole de Hawara ${ }^{3}$. Cela est peu probable. Rien ne prouve d'abord que les fruits de Hawara fussent tous de provenance indigène, et supposé qu'ils l'aient été, on serait tout au plus en droit d'en conclure que les arbres qui les portaient étaient cultivés dans la vallée du Nil à l'époque gréco-romaine. Il n'est pas impossible toutefois que quelques-uns d'entre eux aient été importés en Égypte, sous la domination des premiers Ptolémées ou même plus tôt; mais la plupart ne purenty être transplantés que vers le commencement de notre ère $^{4}$. En tout cas on ne leur connaît pas de nom hiéroglyphique ${ }^{5}$, et la forme hellénique des noms coptes de

1. Grand Papyrus Harris, pl. XL, a, 14-15.

2. A. de Candolle, op. laud., p. 187.

3. IIawara, Biahmu and Arsinoë, p. 48 et 50. - Kahun, Gurob and Hawara, p. 47, 2.

4. Ces divers arbres sont aujourd'hui cultivés en Égypte, à l'exception du poirier, dont Schweinfurth ne fait point mention dans l'Illustration de la Flore d'Égyple.

5. C'est par erreur que Brugssch a attribué celui de net'à 
leurs fruits peut faire croire qu'ils n'ont été connus des Égyptiens que par l'intermédiaire des Grecs. Tels sont ceux de l'amande, leuke, de la pêche, ou-persi, de la cerise, tamaskion, de la poire, apidia'; quant au mîrier, M. Percy Newberry dit, il est vrai, qu'il est souvent mentionné dans les inscriptions hiéroglyphiques de la XIX ${ }^{0}$ dynastie ${ }^{2}$; mais il n'en donne aucun exemple. Il y a là une erreur évidente du botaniste anglais. Originaire du Pont et de la Médie, le Morus nigra L. ne paraît point aroir été connu des anciens Sémites; il a dû l'être encore moins de l'Égypte pharaonique ${ }^{3}$.

M. Flinders Petrie a trouvé aussi dans la nécropole de Hawara une noisette et des noix ${ }^{4}$; faut-il en conclure que le noyer et le noisetier étaient cultivés

l'amandier. Hier.-demolisches Woerterbuch, suppl. 713. Cf. Moldenke, op. laud., XXV, p. 143.

1. Victor Loret, La Flore pharaonique, p. 83 et 8 't, nos 138 , 139, 140, 141. Les pommes, les poires, les prunes, qu'on mange aujourd'hui en Égypte sont mauvaises et y sont pour la plupart importées, ainsi que les cerises, les noix et les noisettes, de la Grèce ou de la Syrie. P. Ascherson et Magnus, Ueber Pflanzenreste. (Zeitschrift für Ethnologie, t. IX, p. 29'.)

2. Flinders Petrie, Kahun, Gurob and Hawara, p. 48, 1.

3. M. Victor Loret (La Flore pharaonique, p. 46, n" 60), regarde le nom copte ou-mation du Morus nigra L. comme une dénomination populaire grecque, le mot ou-katmis. au contraire, par lequel est désigné le Morus alba L., lui parait être d'origine égyptienne; mais comment le murier blanc, indigène seulement dans la Chine et la Mongolie, aurait-il pu être connu en Égypte avant le mûrier noir, originaire de la région du Caucase? Le Morus alba ne parait pas avoir été importé dans l'Asie occidentale et par conséquent en Égypte avant le moyen âge. A. de Candolle, op. laud., p. 120 et 122. - V. Hehn, op. laud., p. 318.

4. Kahun, Gurob and Hawara, p. 47, 2. - IIavara, Bialimu and Arsinoë, p. 50. 
en Egypte? Rien de moins légitime; arbuste des pays froids ou tempérés, le noisetier ne parait pas avoir été planté dans la rallée du Nil, où il ne pourrait guère s'acclimater. Quant au noycr, il n'est aujourd'hui encore que rarement cultivé en Égyte'; il ne devail pas l'ètre davantage au I ${ }^{\text {er }}$ siècte de notre ère, si mème il l’était déjà. En tout cas il l'y était depuis peu. Le nom copte de la noix, loöri ou licüré, déformation évidente du pluriel grec \%xpus, montre bicn que le noyer a dû être importé de la Grèce en Égypte. Quant au nom copte de la noisette, pantoli, il semble une simple transformation de l'arabe boundouq ${ }^{2}$, ce qui témoigne encore en faveur d'une provonance exotique ${ }^{3}$.

Ce que je viens de dire du noisetier et du noyer s'applique aussi au cédratier; sans doute cet arbre a été anciennement cultivé en Eygpte; mais il n'a dû, quoique M. Victor Loret ait cru le contraire ${ }^{4}, y$ être importé qu'à une époque relativement récente, sinon postérieure à notre ère ${ }^{3}$. Il n’y a donc pas lieu

1. Illustration de la Flore d'Égypte. (Mémoires de l'Institul égyptien, vol. II (1889), lre partie), p. 141.

2. Victor Loret, La Flore pluarconique, p. 45. no 58.

3. "Hazel nut is likely to have reached Havara by means of trade », dit M. Percy Newberry, Kahun, Gurob and IIawar", p. 48, 1. Je parlerai plus loin des fruits de pin pignon trouvés à Drah-abou'l-Neggah. (Bullelin de l'Instilut égyptien, no 5 (188'). p.6), et dont il existe aussi un exemplaire dans la collection égyptienne du Musée du Louvre. (Recueil de rravaux, t. XVII, 14, p. 187).

4. Le cedralier dans l'antiquité. Paris, 1891, in-8. (Extrait des Innales de la Société bolanique de Lyon, vol. XVII). Il existe aussi un citron - il a dù même en exister deux au Musée du Louvre; mais la présence, mème dans cette collection, de ce fruit, originaire de l'Inde, suffit à fairc douter de son ancienneté.

5. Revue crilique, an. 1893, I, t. XXXV, p. 113. 
de parler ici de cet arbre; c'est quand j'étudierai la flore de l'Asie antérieure, où il apparait d'abord, qu'il conviendra d'en faire l'histoire, ainsi d'ailleurs que des cinq ou six derniers arbres fruitiers, dont il vient d'être question, et qui sont indigènes ou ont été cultivés depuis la plus haute antiquité dans cette région.

Mais il est un arbre à fruit - la vigne — dont il me faut parler maintenant, à cause de l'ancienneté de son importation dans la terre des Pharaons, et de l'extension qu'y prit sa culture. La vigne (Vitis vinifera L.), parait originaire de la région forestière, qui s'étend du Turkestan et du Caucase aux montagnes de la Thrace '; mais de lì elle s'est répandue dans toutes les parties du monde connu des Anciens. Les Sémites la cultivèrent dès les temps les plus éloignés. L'histoire de Noé a symbolisé la découverte des propriétés enivrantes du jus de raisin". L'offrande de pain et de vin, faite par Melchisédech en l'honneur d'Abraham ${ }^{3}$. montre l'usage des produits de la vigne connu en Syrie près de vingt siècles avant notre ère. On sait, par le récit du Livre des Nombres ${ }^{4}$, que, avant l'ètablissement des Hébreux, la vigne était, avec le figuier et l'olivier, cultivée dans le pays de Chanaan, et qu'elle y portait des fruits d'une merveilleuse grosseur.

Elle était aussi alors et depuis longtemps connue en

1. A. de Candolle, op. laud., p. 153. - A. Grisebach, Die Vegclation der Erde, t. I, p. 323. - O. Schrader, Thier- und Pflanzengeographic, p. 27. - Engler, ap. Victor Hehn, liullurpflanzen, p. 87.

2. Genesis, cap. $\mathrm{Ix}$, vers. 20 et 21.

3. Gencsis, cap. xiv, vers. 18.

4. Numeri, cap. Xill, vers. 24.

5. Franz Delitzsch, Dic Bibel und der Wein. Leipzig, 1885, in- 8, p. 8. 
Égyte. Osiris, preuve de son ancienneté, était regardé comme l'inventeur de sa culture'. Des peintures contemporaines de la $\mathrm{IV}^{\mathrm{c}}$, de la $\mathrm{VI}^{\mathrm{e}}$ et de la XII $\mathrm{I}^{\mathrm{c}}$ dynasties représentent la cueillette du raisin et la fabrication du vin $^{2}$. Il est souvent question de la vigne dans les textes hiéroglyphiques de l'époque des Ahmessides et des Ramessides; on en voit aussi de fréquentes représentations sur les monuments contemporains. Sur la table des offrandes d'une des peintures du tombeau de Rat'eserkasenb (XVII ${ }^{\circ}$ dydastie), on voit quatre jarres de vin enguirlandées de pampres couverts de raisins ${ }^{3}$. Le scribe Anna, nous l'avons vu, avait fait planter douze vignes dans son jardin et on en comptait vingtquatre dans le parterre de l'officier d'Amenhotpou II. Le papyrus Harris nous apprend que Ramsès III fit établir des vignobles en plusieurs lieux de la Haute et de la Basse-Egypte ${ }^{4}$ On voit par les peintures des hypogées de Béni-Hassan et d'El-Amarna que les anciens Egyptiens plantaient leurs vignes en rangs parallèles et les conduisaient sur des treillages, de manière à former des berceaux impénétrables aux rayons du soleil.

On a souvent rencontré des débris de vigne dans les hypogées. Schiaparelli a trouvé à Drah-Abou'l-Neggah

1. Franz Wønig, op. laud., p. 259.

2. Lepsius, Dentimäler, t. III, pl. 53, tombe no 6 de Gizeh (IVe dynastie); t. IV, pl. 111, tombe no 14 de Zaouiet-el-Maiétin (VIe dynastie); ibid., pl. 127, tombe 2 de Béni-Hassan (XIIc dynastie). - Rosellini, Monumenti civili, t. II, nos 37, 38 et 40 .

3. Mémoires de la mission archéologique au Caire, t. V, fasc. IV (an. 1893), pl. II, paroi F, p. 575.

4. P. VII, 1. 10. (Zeilschrift für ägyptische Sprache, t. XI (an. 1873), p. 35.) 
des paquets de feuilles, dont quelques-unes étaient encore en parfait état de conservation; après les avoir ramollies dans de l'eau tiède, Schweinfurth a pu les étaler dans l'herbier de Boulaq'; elles ressemblent aux feuilles de l'espèce encore aujourd'hui cultivée en Égyte; mais la face inférieure en est recouverte d'une espèce de feutre de poils blancs. Parmi les restes végétaux découverts par Mariette dans la même localité se trouvaient entre autres des raisins secs, qui 'appartiennent, d'après Schweinfurth ${ }^{2}$, à la variété noire à grosses baies recouvertes d'un duret bleuâtre. Dans les fouilles faites à Gébéleïn par M. Maspero, on a rencontré aussi des grains de raisin à peau épaisse, de 16 à 17 millimètres de longueur sur 10 à 11 de largeur, avec trois à quatre graines, abruptement atténuées en pointe tronquée et mesurant en longueur, largeur et épaisseur respectivement T, 4 et 3 millimètres ${ }^{3}$. On voit de ces raisins dans la plupart des musées égyptiens d'Europe ${ }^{4}$. Braun, qui a étudié, arec un soin particulier, ceux qui sont au Musée de Berlin", a trouvé qu'ils renfermaient chacun trois graines; quant à la dimension, ils se rapprochent des raisins de Corinthe, mais devaient ètre plus foncés. Enfin, dans les raisins rapportés de Hawara par. M. Flinders Petrie, M. Percy Newberry a reconnu la Vitis vinifera L. var. Corinthiaca ${ }^{6}$. Les Égyptiens

1. Bolanische Jahrbïcher, t. VIII (an. 1886), p. 8.

2. Bullelin de l'Inslilul égyptien, $\mathrm{n}^{\circ} 5$ (1884), p. 9.

3. Bulletin de l'Instilul égyplien, no 6 (an. 1886), p. 260.

4. Il en existe une trentaine de grains au Musée du Lourre, à peau épaisse et à grosses graines. Recueil de travaux, t. XVII, 29, p. 19 '.

5. Zeilschrift für Elhnologie, t. IX (an. 1877), p. 306.

6. IIawara, Biahmu and Arsinoë, p. 50. 
avaient d'ailleurs plusieurs espèces de raisins, tels que le thasien qui, d'après Pline ${ }^{1}$, était sucré et légèrement laxatif, l'ecbolos², qui aurait, d'après lui, provoqué les arortements.

Le nom hiéroglyphique de la vigne, ainsi que celui du raisin, était arouri - le copte aloli. - Le raisin rert s'appelait gangani, en copte shelshêli, le raisin séché au soleil ashep ou shep ${ }^{3}$. Quant au vin il portait le nom de arp.

II.

Les arbres à fruits n’étaient pas sculs cultivés dans les jardins égyptiens, le besoin d'ombre et de fraîcheur y fit aussi planter, ainsi que dans le voisinage des rilles, des arbres ou des arbustes d'ornement". La flore indigène en offrait un certain nombre, remarquables par la grice et l'élégance de leur feuillage, tels que les tamaris, les acacias et le saule safsaf; plusieurs d'entre eux, sinon tous, furent cultivés dans les parterres pharaoniques.

On compte en Egypte huit espèces de tamaris ${ }^{5}$, dont l'une, le $T$. arborea Bunge, est même spéciale à

1. Hisloria naluralis, lib. XIV, cap. 9 (7).

2. Hisloria naluralis, lib. XIV, cap. 24 (18).

3. Victor Loret, La Flore pharconique, p. 101, no 167.

4. “J'ai planté (autour de) la ville de Thèbes des arbres, des arbustes, des fleurs », dit Ramsès III dans le grand papyrus Harris, pl. VII. Zeilschrifl für aegyplische Sprache, t. XI, p. 54 .

5. Ce sont, d'après l'Illustration de la Flore d'Égyple, les T. lelragyna. Ehr., nilolica B., mannifera Ehr., arborea B., arliculala Vahl, amplexicaulis Ehr., passerinoides Del. et macrocarpa Ehr. 
cette contrée ; deux surtout sont représentées aujourd'hui dans les plantalions: le T. arliculata Vahl ar. athl ou acl, - dont on fait des avenues, et le $T$. nilotica Bunge, - ar. tarfah. - Ce dernier vocable est traduit dans les Scalae coptes par she-n-osi, tandis que açl répond à pi-nam ou pi-nom ${ }^{2}$; M. Victor Loret incline à reconnaitre dans l'osi copte l'équivalent du terme hiéroglyphique aser, qu'on rencontre dans l'inscription du tombeau du célèbre Khnoumhotpou à BéniHassan $^{3}$. Ce mot qu'il faut rapprocher du nom hébreu du tamaris ashel, doit donc être traduit aussi par tamaris; il servait sans doute à désigner plus particulièrement le $T$. nilotica; mais on le donnait probablement aussi à d'autres espèces du genre.

L'inscription du tombeau de Khnoumhotpou parle d'un bois de tamaris que possédait l'illustre mort; celle du tombeau d'Anna nous apprend que le scribe d'Amenhotpou II avait planté dix tamaris dans son jardin. Unger a découvert des fragments de $T$. nilotica dans une brique d'El-Kab"; la momie de Kent, personnage de la $\mathrm{XX}^{\mathrm{e}}$ dynastie, reposait sur un lit de branches, reliées par des fibres de dattier et que M. Schweinfurth a reconnues comme appartenant à la même espèce de tamaris ${ }^{5}$. M. Flinders Petrie a trouvé aussi des débris de ce bel arbre dans une tombe de Kahoun ${ }^{6}$.

1. Illustration de la Flore d'Égyple, p. 50.

2. Victor Loret, La Flore pharaonique, p. 79, no 133.

3. Lepsius, Denlimäler, t. II, pl. 124. Cf. Charles Moldenke, op. laud., p. 129.

4. Silzungsberichle, t. XLV (an. 1862), p. 81.

5. Bulletin de l'Instilul égyplien, no 6 (an. 1885), p. 282. Bolanische Jahrbücher, t. VIIII (an. 1886), p. 1'́.

6. Kahun, Gurob and IIavara, p. 50, 1. 
En comprenant le Prosopis Stephaniana Willd. ou Acacia heterocarpa Del., il y a en Egypte huit espèces d'acacias '; deux paraissent avoir été plus par'ticulièrement cultivées: I'Acacia nilotica For'sk., variété de l'A. arabica Willd., et l'Acacia seyal L. Au nord du $11^{\circ}$ degré de latitude, l'acacia du Nil ne se rencontre aujourd'hui que planté dans les jardins et les promenades, mais on l'y trouve fréquemment. Il ne devait pas ì une époque reculée ètre moins commun à l'état spontané, et on peut supposer qu'il formait un des principaux éléments des forèts qui durent à l'origine couvrir une partie de la vallée du Nil; suivant Théophraste $^{2}$, cet arbre était, de son temps, très répandu dans la Thébaïde.

Mais si l'Acacia nilotica croissait ì l'état spontané en Égypte, on le cultivait aussi, à l'époque pharaonique, comme on le fait de nos jours, dans les parcs et les jardins. Anna en avait trois dans le parterre de sa villa ${ }^{3}$; ce bel arbre figurait dans 24 des 42 bois sacrés des nomes égyptiens. Strabon parle d'un bocage d'acanthes thébaiques - acacias - qui se trouvait auprès du sanctuaire d'Acanthos en Lybie ${ }^{4}$. On a trouvé fréquemment des débris de cette mimosée dans les hypogées pharaoniques; M. Flinders Petrie a découvert dans la nécropole de Kahoun divers ustensiles en bois, ainsi que des gousses, d'acacia ${ }^{3}$. Unger a cru aussi reconnaitre des débris de gousses du mème arbre dans une brique d'El-

1. Illustration de la Flore d'Égypte, p. 71-72.

2. IIistoria plantarum, lib. IV, cap. 2, 8 .

3. Charles Moldenke, op. laud., IV, p. 74.

4. Dahshour. Geographica, lib. XVII, cap. 1, 35.

5. Kahun, Gurol and IIawar', p. 50, 1. 
Kab'. Quelques-unes des guirlandes qui ornaient les momies d'Ahmès I et d'Ahmenhotpou I étaient faites en fleurs d'acacia du $\mathrm{Nil}^{2}$.

L'Acacia nilotica est souvent mentionné dans les textes égyptiens; il portait, dans la langue hiéroglyphique, le nom de shent ou shant, - hébreu shett, ar. sant, copte shonte ou shanti ${ }^{3}$; - les Grecs lui don-

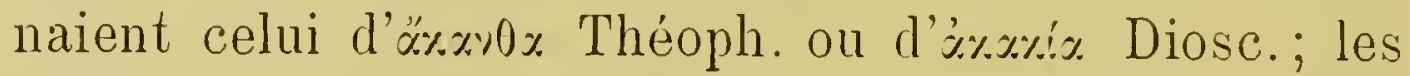
Latins l'appelaient acanthus ou spina aegypliaca. Son port, la beauté de ses fleurs, employées pour faire des couronnes, la dureté de son bois, qui le rendait propre aux usages les plus diver's, la multiplicité de ses épines, dont toute la plante, à l'exception du tronc, était couverte; enfin la gomme - lami", copte lomé, origine probable du grec xósu, lat. gummi, - qui sortait naturellement à travers l'écorce, mais coulait surtout en abondance des incisions faites à l'arbre, avaient frappé les Anciens; Théophraste ${ }^{3}$ et après lui Pline ${ }^{6}$ en ont longuement parlé.

On rencontre souvent dans les textes hiéroglyphiques une espèce d'arbre du nom de ash, dont le bois était, comme celui de l'acacia du Nil ou shent, employé dans les constructions, en particulier pour faire des bateaux, des portes ou des boiseries. C'est ainsi que sur une

1. Silsungsberiche, t. XLV (an. 1862), p. 81.

2. G. Schweinfurth, Ueber Pfansenreste. (Berichle der bolanischen Gesellschaft, t. II (188'), p. 363.)

3. Victor Loret, La Flore pharaonique, p. 84, no 142.

4. A. Viedemann, Sammlung allägyptischer Wörter, welche von kilassischen Auloren umschrieben oder überselst worden sind. Leipzig, 1883, in-8, p. 26, donne les formes kemui, kema.

5. IIistoria planlarum, lib. IV, cap. 2, 8 .

6. IIistoria naluralis, lib. XVII, cap. 19. 
inscription de l'obélisque de Thèbes, transportée aujourd'hui à Rome, Thoutmès III se vante d'avoir fait construire en bois ash une grande barque dédiće à Amon '. Dans l'inscription qui rappelle la restauration du grand portail du temple d'Edfou, il est dit aussi que les portes en ont été faites en bois de l'arbre $a s h^{2}$ : quel était cet arbre ash, dont le bois était ainsi employé dans l'industrie pharaonique? Depuis M. de Rougé on s'est généralement accordé à y voir une espèce d'acacia, sans doute l'A. seyal Del., espèce indigène en Égypte comme l'acacia du Nil, inais dont les feuilles sont composées d'un moindre nom bre de folioles ${ }^{3}$. Une inscription du temple de Hibé, qui nous apprend que les portes en avaient été faites en bois de l'arbre ash, ajoute que ce bois renait des contrées de l'ouest et que l'arbre qui l'avait fourni portait le surnom de pir-shen".

II. Victor Loret, qui mentionne ce vocable et lui attribue le sens de " grains chevelus », l'a donné d'abord comme le nom des fleurs de L'A. Farnesiana Willd ${ }^{5}$; mais cette espèce américaine n'ayant pu évidemment ètre connue dans l'ancienne Égypte, il s'est demandé depuis si l'arbre pir-shen, dont les fleurs portent dans les recettes de parfumerie le nom de sannâr, ne serait

1. J. de Horrack, Nolice sur le nom hiéroglyphique du cèdre. (Revue archéologique, t. X (an. 1864), p. 45.) Il faut dire que II. de Horrack traduit ash par cèdre.

2. H. Brugsch, Eine neue Baurrliunde des Tempels von Edfou. (Zeilschrifl fïl' aegyplische Sprache, t. XIII (an. 1875), p. 122. - Charles Moldenke, op. laud., VI, p. 89.

3. M. Victor Loret dit que cet acacia, appelé lalh en arabe, porte dans les Scalae le nom de pi-larinon; Kircher qu'il cite aussi traduit tout simplement pi-larinon par virgulnum.

4. Charles Moldenke, op. laud., VI, p. 91.

5. La Flore pharaonique, p. 86, no 144. 
point l'A. spirocarpa Hochst., en arabe sammor. C'est là une hypothèse ingénieuse, mais difficile à accepter; l'A. spirocarpa, arbre du désert arabique méridional, n'a guère pu être le pir-shen des contrées de l'ouest, dont parle l'inscription hiéroglyphique. En réalité, ce nom désigne l'ash ou $A$. seyal, comme nous le voyons par l'inscription du temple de Hibé ${ }^{\text {. }}$

Il n'est point certain que l'arbre ash eût été planté dans le jardin d'Anna, mais il se trouvait dans les bois sacrés de dix-sept nomes. Rencontrait-on dans ceux-ci d'autres espèces d'acacia? On l'ignore, mais le fait n'est pas improbable; on pourrait le croire en particulier pour les $A$. albida Del., Ehrenbergiana Hayne, tortilis H. et heterocarpa Del. (Prosopis Stephaniana. Willd), très répandus dans l'Égypte; on a du moins trouvé au Musée du Louvre un fragment de guirlande, faite de fleurs d'A. tortilis et cinq gousses d'A. heterocorpa ${ }^{2}$, ce qui montre que, s'ils ne les cultivaient pas, les anciens Égyptiens connaissaient et utilisaient ces espèces, comme les $A$. nilotica et seyal. Ils connaissaient aussi le Mimosa polyacantha L., dont " les feuilles ailées, dit Pline ${ }^{3}$, se replient quand on les touche ». Cet arbre, qu'on rencontre aujourd'hui encore dans la Haute-Égypte, croissait autrefois, suivant le polygraphe latin, aux environs de Memphis.

Il se trouvait dans le jardin d'Anna huit arbres

1. "Ses portes ont été faites en bois d'ash de la région de l'Occident, (arbre) dont le nom est pir-shen ». H. Brugsch, Ueber eine Bauurkunde von Edfu. (Zeitschrift für ägyptische Sprache, t. XIII (an. 1875), p. 123).

3. Recueil de travaux, t. XVII (an. 1895), 22 et 23, p. 191.

4. II istoria noturalis, lib. XIII, cap. 19. 
ther, ter ou lari ${ }^{1}$; ce nom - copte tôré ou thôri ${ }^{2}$ désignait le Salix safsaf Forsk., espèce de saule indigène dans la vallée du Nil, ainsi que dans les oasis, mais qu'on plantait également dans les parcs et en haies. Ses feuilles élégantes et flexibles servaient à faire des guirlandes. Les momies des pharaons de la XVIII ${ }^{\circ}$ dynastie, Ahmès I et Amenhotpou II, trouvées, en 1881, à Deir-el-Bahari, étaient ornées de couronnes faites de feuilles de safsaf $^{3}$; la momie de la princesse Nesi-Khonsou de la XXII dynastie en était également parée. Schiaparelli aussi a découvert dans une tombe de Shéil Abd-el-Gournah ", des débris d'une couronne faite de feuilles de saule et de fleurs de carthame.

Ces arbres ne durent pas être les seuls de la flore indigène qui furent cultivés dans les jardins ou les parcs pharaoniques; y planta-t-on aussi la Maerua uniflora Vahl - le mérou des Arabes, - arbre immense, qui est un des ornements du désert éthiopien; on en a retrouvé des fruits et des graines dans une tombe de Gébéleïn ${ }^{\circ}$; mais ce n'est nullement une preuve qu'il fût cultivé dans la vallée du Nil. On peut en dire autant du Cocculus leaeba D., arbrisseau rampant des déserts égyptiens, dont on a trouvé également des baies dans la tombe d'Ani à Gébéleïn ${ }^{6}$.

A-t-on cultivé dans l'ancienne Égypte le térébinthe (Pistacia terebinthus L.) et le lentisque (Pistacia

1. Charles Moldenke, op. laud., p. 126.

2. Kircher, Scala magna, p. 175, donne pi-thori.

3. G. Schweinfurth, Ueber Pflanzenresle. (Berichle der bolanischen Gesellschafı, t. II (an. 1884), p. 369.)

4. G. Schweinfurth, Die lelzlen bolanischen Enldeckungen. (Bolanische Jahrbücher, t VIII (an. 1886), p. 9.)

5. G. Schweinfurth (Bolanische Jahrbiucher, t. VIII, p. 4.

6. Bolanische Jahrbücher, t. VIII, p. 15. 
lentiscus L.), arbres qui appartiennent à la flore méditerranéenne? M. Moldenke a cru pouvoir identifier le térébinthe avec l'arbre $\hat{a} r u^{1}$, planté dans les bois sacrés de deux nomes; mais, suivant M. Victor Loret, le nom du térébinthe n'a pas encore été trouvé dans les textes égyptiens $^{2}$; seule la résine qu'on en tire, la térébenthine, y est mentionnée dès les temps de l'ancien empire; son nom hiéroglyphique paraît avoir été sounter, conservé dans le copte sonte ou sonti " résine ". Il est vrai que le sounter était parfois tiré, comme on le voit par les inscriptions de Deir-el-Bahari ${ }^{3}$, du pays de Pount, ce qui ferait supposer que l'arbre qui le produisait était exotique.

Quoi qu'il en soit, il est d'autant plus vraisemblable que le Pistacia terebinthus existait dans l'Égypte ancienne qu'un des nomes de cette contrée portait le nom de "Nome du térébinthe " ${ }^{4}$. Quant au lentisque, d'après Galien ${ }^{5}$, il croissait autrefois en Égypte, ce qui ne doit pas surprendre, car une espèce roisine, le Pistacia atlantica Desf., s'y rencontre encore aujourd'hui à l'état spontané ${ }^{6}$. Le lentisque s'appelait shoub, suivant M. Victor Loret ${ }^{7}$, dans la langue hiéroglyphique et la résine qu'on en retirait portait le nom de fatti; elle était d'un usage fréquent dans la parfumerie égyptienne.

S'il est probable seulement que le lentisque ait été

1. Ueber die allägyptischen Bäume, XXIV, p. 142.

2. La Flore pharaonique, p. 97, no 164.

3. Victor Loret, op. laud., p. 97.

4. G. Maspero, Hisloire ancienne, t. I, p. 72.

5. De facultatibus alimentorum, VII, 69.

6. P. Ascherson, dans le Supplément à l'Illustration de la Flore d'Égypte (Mémoires, t. II, p. 752), indique au lieu du $P$. allantica, le P. khinjuk, comme croissant en Égypte.

7. La Flore pharaonique, p. 97, no 165. 
cultivé en Égypte, il est certain que des " sycomores à encens "y furent plantés; la reine Hatshopsitou (XVIII dynastie) en envoya chercher, nous l'avons vu, au pays de Pount, et l'un des rois de la XIX dynastie, Ramsès III, en fit planter, comme elle, dans les jardins du dieu de Thèbes'. Il paraît que ce double essai d'acclimatation réussit. Abd-allatif affirmait avoir vu encore quelques-uns de ces arbres aromatiques et l'on rapporte, dit $M$. Victor Loret ${ }^{2}$, que le dernier périt en 1613. A quelle espèce végétale appartenaient-ils? Il est probable que c'étaient des Boswellia Carteri Franchet ${ }^{3}$, unc des espèces qui représentent, dans le pays des Somalis, la tribu des Burséracées, dont les précieux représentants donnent l'encens et la myrrhe.

On a trouré dans les tombes de Deir-el-Bahari et de Drah-Abou'l-Neggah des fruits du genévrier de Phénicie (Juniperus phoenicea L.) ${ }^{4}$, et M. Flinders Petrie en a mème découvert des rameaux entiers à Hawara, dans la momie d'un crocodile ${ }^{5}$; cet arbre ne parait pas avoir été indigène en Égyte, y était-il cultivé? Il portait dans la langue hiéroglyphique les noms de ouan, aoun, ouar, arou, dont la diversité même semble indiquer une origine étrangère ${ }^{6}$; le bois de genévrier, utilisé dans l'industrie, est désigné dans les textes comme " bois

1. Grand Papyrus Harris, (Zeilschrifl, t. XIII, p. 55.)

2. Le cédralier, p. 43.

3. Dr. Hamy, Étude sur les peinures ethniques d'un tombeau thébain de la YVIII dynastic. Paris, 1884, p. 23.

4. G. Schweinfurth, Ueber Pflanzenresle. (Berichle der bolanischen Gesellschaft, t. II, p. 369.) Il existe deux fragments d'un pareil fruit au Musée du Louvre. Recueil de travaux, t. XVII, 12, p. 186.

5. IIcuwara, Biahmu and Arsinoë, p. 48.

6. Victor Loret, La Flore pharaonique, p. 41, no 51. 
syrien ", dénomination qui en rappelle la provenance exotique. Les baies de genièvre, pershou, mot qui paraît à M. Victor Loret dériver d'un radical sémitique, étaient employées dans la médecine égyptienne; il n'est donc pas surprenant qu'on en ait trouvé dans les hypogées pharaoniques.

On rencontre dans un texte religieux de la pyramide du roi Pépi, de la VI ${ }^{e}$ dynastie, le mot sib ${ }^{1}$, qui serta désigner le cèdre; dans la tombe de $\mathrm{Ti}$ à Saqqarah sont représentés aussi deux ouvriers en train de travailler du bois de cet arbre; supposant qu'au temps de l'ancien Empire, les Égyptiens n'avaient pas de relations commerciales avec la Syrie, M. Victor Loret en a conclu que le bois de cèdre mis en œuvre par les charpentiers du roi Ti était de provenance indigène, encore qu'aucune conifère ne croisse spontanément en Égypte ${ }^{2}$. En tout cas, si l'on a jamais rencontré le cèdre dans la vallée du Nil, il y a disparu de bonne heure et on ne paraît pas avoir jamais songé à le planter dans les parcs ou les bois sacrés. Mais dès une époque reculée on importa en Égypte du bois de cèdres coupés sur le Liban ${ }^{3}$; on $y$ fit aussi usage de la résine quon retire de cet arbre. On peut croire que le cône de cèdre conservé au Louvre, loin d'ètre de provenance indigène, est un fruit apporté de la Syrie.

Les deux cônes de pin pignon découverts par Mariette dans une tombe de la XII ${ }^{\mathrm{e}}$ dynastie à Drah-Abou'l-

1. Victor Loret, La Flore pharaonique, p. $42, \mathrm{n}^{\circ} 52$.

2. Ed. Bonnet, Le pianle egiziane del Musco reale di Torino. (Nuovo Giornale Bolanico Ilaliano (Nuova serie), t. II (an. 1895), p. 82.)

3. G. Maspero, Récit de la campagne de Mageddo. (Recueil de lravaux, etc., t. II, p. 148 et 150.). 
Neggah, mais dont Schweinfur'th a mis en doute l'antiquité ${ }^{\prime}$, sont aussi sans doute des fruits importés de la région méditerranéenne; on peut en dire autant du còne de même espèce, qui existe au Musée du Louvre $^{2}$ et de ceux que M. Flinders Petrie a décourerts dans la nécropole ${ }^{3}$ gréco-romaine de Hawara ; ce sont des fruits importés d'un arbre exotique, non d'une espèce arborescente indigène; rien ne dit mème qu'on ait jamais cultivé le pin pignon à l'époque pharaonique, dans les parcs égyptiens".

On y planta, au contraire, des espèces exotiques, apportées de l'Asie antérieure ou mème de l'Europe, telles que le chène, le peuplier blanc, le platane même. On cultive de nos jour's trois espèces de chêne en Égypte, les Quercus Suber L., pedunculata Ehr. et lusitanica Lam. ${ }^{5}$ : les y rencontrait-on déjà avant notre ère? M. Flinders Petrie a trouvé une paire de semelles en liège à Hawara ${ }^{6}$; mais rien ne prouve qu'ils ne fussent pas d'importation étrangère ou que le chêne liège fùt alors planté en Égypte. D'après Migliarini ${ }^{7}$ une guirlande, dont les restes fragiles sont conservés au Musée de Florence, était composée de feuilles de Quercus aesculus $\mathrm{L}$. et de saule, mêlées à des pétales de lotus blanc; malheureusement Migliarini s'est trompé

1. Bulletin de l'Institut égyptien, no 5 (an. 1884), p. 6.

2. Victor Loret, Recueil de travaux, t. XVII (an. 1895), 1's, p. 187.

3. Hawara, Biahmu and Arsinoë, p. 50.

4. D'après Schweinfurth (Illustration, p. 179), il y est encore rare aujourd'hui.

5. Illustration de la Flore d'Égypte, p. 141.

6. IIawara, Biahmu and Arsinoë, p. 52.

7. Indication succincte des Monuments égyptiens du Musée de Florence, p. 72. Le Q. aesculus est étranger à la flore de l'Asie antérieure. 
si souvent dans ses déterminations qu'on est en droit de se demander si les feuilles qu'il a observées appartiennent bien au Q. aesculus. Etait-ce le Q. lusitanica ou le Q.pedunculata, variété du rouvre, qui se trouvait, avec des perséas et des oliviers, dans le bois de Thèbes, dont parle Théophraste ${ }^{1}$ ? Comme le naturaliste grec s'est servi ici du simple mot joüs, sans y ajouter aucune épithète, il est impossible de se prononcer à cet égard. Mais quoi qu'il en soit, les chênes de Thèbes avaient dû être importés de l'Asie antérieure ou de l'Europe, leur patrie.

C'est des mêmes régions aussi que fut tranplanté en Égypte le peuplier blanc qu'y fait croitre également Théophraste $^{2}$; la beauté de son feuillage arait sans doute frappé les habitants de la vallée du Nil; cette. circonstance explique qu'ils aient essayé d'acclimater dans leur pays cet arbre, ami du voisinage des eaux. Peut-être y plantèrent-ils aussi le peuplier de l'Euphrate (Populus euphratica Oliv.), qu'on rencontre aujourd'hui dans la petite oasis ${ }^{3}$ et dont on a trouvé un fragment de guirlande au Musée du Louvre". L'ombre qu'il donne et son large fenillage les engagèrent à $y$ planter le platane, objet, nous le verrons, d'une vénération si grande chez les Anciens. On pourrait supposer que le peuplier blanc et le platane furent introduits dans la vallée du Nil seulement sous la domination perse; il ne semble pas du moins que ce soient des Sémites qu’ils les aient reçus.

Il est probable que le tilleul, dont M. Flinders Pe-

1. Historia plantarum, lib. IV, cap. $2,8$.

2. Historia plantarum, lib. IV, cap. 8, 2.

3. Illustration de la Flore d'Égypte, p. 142.

4. Recueil de travaux, t. XVII (an. 1895), 15, p. 187. 
trie a trouvé des fragiments dans la nécropole de Hawara ${ }^{1}$, ne pénétra que beaucoup plus tard dans la patrie des Pharaons. Il dut y ètre importé d'Europe, . probablement de la Grèce. C'est aussi de ce pays, sinon de l'Asie occidentale, que le laurier fut introduit en. Égypte, ou depuis il n'a pas cessé d'être cultivé. D'après M. W. Pleyte ${ }^{2}$, plusieurs momies du Musée de Leyde, qui datent, il est rrai, de la basse époque, portent des couronnes en feuilles de laurier; M. Flinders Petrie a également découvert à Hawara des débris de guirlandes qui, suirant M. Percy Newberry, sont faites de fenilles du Laurus nobilis ${ }^{3}$.

Ce fut pour son feuillage odorant, mais peut-être encore plus pour l'emploi qu'on en faisait en couronnes, que cet arbre fut importé dans la vallée du Nil. L'usage des couronnes, qui apparait dès la plus haute antiquité et dont le goût alla toujours en augmentant, fit naturellement rechercher les plantes qui pouvaient les fournir. La flore égyptienne en offrait un nombre assez considérable à l'état sauvage; on dut songer, pour se les procurer plus aisément, à les cultiver dans les jardins, et comme ces plantes indigènes finirent par n'être plus suffisantes, on en importa des pays étrangers. C'est ainsi, nous le savons, que plusieurs espèces exotiques, arbres, arbustes ou plantes herbacées, pénétrèrent successivement dans la vallée du Nil, dont elles enrichirent et complétèrent la flore horticole.

Quels furent les arbustes à fleurs les plus anciennement cultivés dans les jardins égyptiens? Il est pro-

1. Kahun, Gurob and IIawara, p. 17, 1.

2. La couronne de la justification. (Actes du sixième Congrès international des Orientalistes, IVe partie, p. 6.)

3. IIcuara, Biahmu and Arsinö̈, p. 51. 
bable que la sesbanie (Sesbania aegyptiaca Pers.) fut un des premiers que l'on y planta. Cette papilionacée dut attirer de bonne heure, par la beauté de ses fleurs, l'attention des habitants de la vallée du Nil, où elle est indigène; j'ai rappelé plus haut qu'on en a trouvé des guirlandes qui ornaient la momie d'Ahmès I ${ }^{\mathrm{er}}{ }^{1}$; aujourd'hui encore on s'en sert pour faire des clôtures"; il n'est pas impossible qu'on l'ait plantée dans les jardins dès le temps des Pharaons, encore que son nom n'ait point été relevé dans les textes hiéroglyphiques.

Le nom de l'arbre au henné s'y rencontre, au contraire, mais rarement et seulement dans des textes de parfumerie; on a trouvé aussi des restes de cet arbuste dans une tombe du nouvel Empire, postérieure toutefois à la $\mathrm{XX}^{0}$ dynastie $^{3}$; il est probable qu'il fut importé en Égypte au plus tard sous les Ramessides. Le parfum de ses fleurs, non moins que la couleur qu'on retire de ses feuilles, devait également le faire rechercher et désormais il ne dut plus cesser de prendre place dans les cultures égyptiennes. M. Flinders Petrie en a trouvé des débris dans la nécropole gréco-romaine de Hawara ${ }^{4}$.

Pickering ${ }^{5}$ et Unger ${ }^{6}$ ont pensé que les rameaux verts que les chanteuses et les danseuses des peintures pha-

1. Schweinfurth, Bulletin de l'Institul égyptien, $\mathrm{n}^{\circ} 3$ (an. 188'), p. 68. - Ueber Pflanzenreste. (Berichte der bolanischen Gesellschaft, t. II, p. 363.)

2. A. Figari, Studii scientifici sull' Egillo, t. II, p. 226.

3. Schweinfurth, Ueber Pfanzenreste, etc. (Berichte der botanischen Gesellschaft, t. II, p. 360.)

4. Hawara, Biahmu and Arsinoë, p. 50.

5. The races of man, p. 375 .

6. Situngsberichte, t. XXXVIII, p. 131. 
raoniques tiemment parfois à la main étaient des branches de myrte; mais c'est là une simple supposition. Théophraste', il est vrai, parle du myrte d'Égypte et dit que dans ce pays il était particulièrement odorant. Pline $^{2}$ affirme aussi que cet arbuste était cultivé dans la rallée du Nil, et Figari ${ }^{3}$, ainsi que M. Flinders Petrie ${ }^{*}$, en ont trouvé des rameaux dans d'anciens hypogées, le premier à Bubastis, le second à Hawara; mais ces hypogées sont de l'époque gréco-romaine. Il est probable que le myrte ne fut introduit en Égypte que sous les Ptolémées et peut-être même sous les dernier's princes de cette dynastie.

C'est probablement aussi à cette époque qu'y fut importé le Jasminum sambac L.; M. Percy Newberry a reconnu cette espèce hindoustanique parmi les restes végétaux découverts à Hawara par M. Flinders Petrie $^{5}$; Schweinfurth a cru aussi reconnaitre des fleurs de cet arbuste ou d'une espèce voisine dans une couronne trouvée en 1881, à Deir-el-Bahari ${ }^{6}$. Ascherson, de son côté, dit' avoir vu au musée de Zurich une couronne de même origine dans la composition de laquelle se trouvaient des fleurs d'une espèce de jasmin semblable au sambac. Il faut ajouter que le nom copte asmi de la fleur du jasmin paraît bien d'origine égyptienne ${ }^{8}$, circonstance qui peut faire croire à une cul-

1. Historia plantarum, lib. VI, cap. 8, 5 .

2. Historia naturalis, lib. XV, cap. 29.

3. Studii scientifici sull' Egillo, t. II, p. 220.

4. Hawara, Biahmu and Arsinoë, p. 51.

5. Kahun, Gurob and Hawara, p. 47, 1.

6. Ueber Pflanzenreste.(Berichte der botanischen Gesellschaft, t. II, p. 368.)

7. Berichte der botanischen Gesellschaft, t. I, p. 546.

8. Victor Loret, La Flore pharaonique, p. 58, no 93. 
ture ancienne de cet arbuste au doux parfum dans la vallée du Nil.

Bien que Ebers l'ait donné à une de ses héroïnes ${ }^{1}$, le nom de la rose n'apparait sous la forme ouartou copte ouert - que dans des textes démotiques, et il est probable que la reine des fleurs, originaire de la région du Caucase, ne pénétra en Égypte qu'assez tard, peut-être même pas avant l'époque des Ptolémées; sa culture devait bientôt prendre une grande extension dans le nome d'Arsinoé; on l'y trouvait en abondance au commencement de notre ère. Ce fut l'espèce à cent feuilles qui y fut d'abord introduite; mais une autre espèce, forme voisine de la Rosa sancta Rich., laquelle paraît être une variété cultivée et acclimatée en Abyssinie de la Rosa gallica L. ${ }^{2}$, y fut aussi importée; M. Flinders Petrie en a trouvé des restes dans la nécropole gréco-romaine de Hawara ${ }^{3}$.

J'ai dit plus haut que cet égyptologue y a aussi trouvé le lierre, arbuste européen, importé au plus tôt, sans doute à cause de sa signification religieuse, sous les Ptolémées. Il portait en égyptien, dit Plutarque ${ }^{4}$, le nom de $\chi \varepsilon$ vóro!s " plante d'Osiris ». Quant aux autres arbustes ou arbres d'ornement, qui purent être introduits depuis cette époque dans la vallée du Nil, ils n'appartiennent pas à la flor'e pharaonique, c'est quand

1. Ouarda. Roman de l'ancienne Égypte, 1882.

2. Fr. Crépin, Sur les restes de roses découverts dans les tombeaux de la nécropole d'Arsinoé. (Bulletin de la Sociélé royale de botanique de Belgique, t. XXVIII (an. 1888), 2* partie, p. 184.)

3. Hawara, Biahmu and Arsinoë, p. 48.

4. De Iside el Osiride, cap. 37. C'est l'ègyptien khi-n-ousiri " arbre d'Osiris », Victor Loret, La Flore pharaonique, p. 69, $n^{0} 115$. 
il sera question de celle de leur pays d'origine qu'il conviendra d'en parler, ainsi que des arbres fruitiers qui furent importés en mème temps dans l'Égypte grecque ou romaine. Mais il me faut dire un mot des fleurs et des plantes d'ornement qui paraissent y avoir été anciennement cultivées.

III.

Si en Egypte, comme chez tous les peuples d'ailleurs, les fleurs ne furent à l'origine l'objet d'aucune culture, elles finirent cependant, quelques-unes au moins, par prendre place dans les jardins, et non contents de celles que leur offrait leur pays, les habitants de la vallée du Nil en importèrent plusieurs des pays roisins; les Pharaons qui enroyaient des expéditions lointaines pour leur rapporter des aromates ou les rares produits du Tonitru ou du Pount, qui faisaient représenter sur leurs édifices les végétaux ou les animaux inconnus des contrées qu'ils avaient subjug'uées ${ }^{1}$, ne pouvaient manquer d'importer dans leur patrie quelques-unes des plantes exotiques qu'ils avaient remarquées dans leurs expéditions. C'est ainsi que furent successivement cultivées dans les jardins égyptiens, à côté peut-être de quelques plantes indigènes, des plantes exotiques dont j'ai donné les noms dans le chapitre précédent, la célosie argentée, l'héliotrope de Nubie, l'iris de Sibérie, le bluet oriental, l'alcée à feuilles de guimauve, le réséda odorant, la menthe poivrée, l'immortelle Helichrysum staechas

1. Peintures de Karnak faites sous Thoutmès III. G. Maspero, L'Archéologie égyptienne. Paris. 1887, in-8, p. 90. 
L.) ${ }^{1}$, et d'autres encore. Toutefois ces plantes n'ont pas d'histoire et elles n'ont d'importance la plupart que par l'usage qu'on en fit pour les couronnes; c'est donc seulement quand il sera question de ces dernières, qu'il conviendra de parler de ces fleurs successivement acclimatées ou cultivées dans les parterres pharaoniques.

Il en est une cependant pour laquelle il me faut faire exception, parce qu'elle vient d'ètre l'objet d'une assez longue étude par MM. Poisson et Loret ${ }^{2}$ : c'est le lis blanc. On ne connaît point d'une manière certaine le pays d'origine de cette fleur ${ }^{3}$, qui joue, depuis deux mille ans, un si grand rôle dans la symbolique et la poésie des peuples de l'Asie antérieure et de l'Europe. Toutefois, quelle qu'en soit l'origine, le lis paraît avoịr été, comme la rose, cultivé assez tôt dans la partie occidentale du bassin de la Méditerranée, mais seulement sans doute après celle-ci; Théophraste, qui parle longuement de la rose, ne dit rien du lis.

Comme la rose aussi, le lis se répandit successivement dans les diverses contrées de la région méditerranéenne; il pénétra ainsi en Égypte. Il servait à préparer un parfum ou huile odorante célèbre, l'unguentum susinum. L'auteur du traité De la nature de la femme,

1. Au lieu de cette espèce méditerranéenne, mais étrangère à la flore de l'Égypte, M. Percy Nerwberry a indiqué le Gnaphalium luteo-album I., commun dans le Delta. Schweinfurth, Petermann's Mittheilungen, an. 1890, p. 54.

2. Recueil de travaux, t. XVII, no 10, p. 185.

3. "Habitat in regione montanî... sed ubique cultum et fere non semper spontaneum. "Boissier, Flora orientalis, t. V, p. 173. Le botaniste génevois croyait cependant en avoir un échantillon d'un pied indigène, récolté dans le Liban, auprès de Ghafir. 
attribué à Hippocrate, en fait déjà mention; mais il est douteux qu'il le considérât, ainsi qu'on l'a dit', comme propre à l'Égypte. Dioscoride ${ }^{2}$, qui s'est étendu longuement sur la préparation du sousinon, vante celui qu'on faisait en Phénicie et en Égpte, Galien parle aussi du sousinon blanc d'Égypte ${ }^{3}$. Dioscoride ${ }^{4}$ donne au lis les noms égyptiens de oup.ç:øoú et de rixhos - les symphaephos et tialos d'Apulée ${ }^{5}$. M. Victor Loret ne serait pas éloigné d'attribuer à ce dernier vocable une origine ancienne, et il nous apprend qu'une liliacée du Musée du Louvre, " trouvée, dit l'étiquette, sur la poitrine de la momie d'une jeune fille ", et qu'on avait regardée jusqu'ici comme une scille, serait, d'après M. Poisson, un lis blanc ${ }^{6}$. Quoi qu'il en soit de cette identification, que l'état incomplet de la plante permet de contester, on peut admettre que le lis a été cultivé en Égypte; mais il est vraisemblable qu'il n'y a pénétré qu'après la conquête perse, sinon seulement sous la domination grecque; il n'y a d'ailleurs joué aucun rôle ni dans le culte, ni dans l'ornementation. Il n'en a pas été de même des quatre plantes aquatiques, les trois premières indigènes et l'autre exotique: le papyrus, le lotus blanc, le lotus bleu et le lotus rose, dont l'utilité et le caractère religieux exigent que nous étudiions en détail chacune d'elles.

Indigène en Égypte, d'où il a disparu, et en Nubie, où

1. Littré, Euvres complètes d'Hippocrate, t. VII, p. 361, note 9.

2. De materia medica, lib. I, cap. 62, p. 63. Édit. Sprengel.

3. Steph. Lexicon graecum, s. v. бoústvov.

4. De materia medica, lib. III, cap. 106.

5. De herbarum virtutibus, cap. 108.

6. Recueil de travaux, t. XVII (an. 1895), 10, p. 185. 
il est encore abondant, le papyrus était autrefois commun dans le pays de Qimit; les nombreuses représentations qu'on rencontre de cette cypéracée sur les anciens monuments en sont une preuve, et témoignent de la place considérable qu'il occupait dans la vie des anciens Égyptiens. Son nom hiéroglyphique était $h a^{1}$, les Grecs lui donnèrent celui de $\beta j \beta \lambda o s^{2}$ ou de $\pi \dot{x} \pi j \rho o s^{3}$, en latin papyrus. Sa tige triquêtre, qui peut atteindre de quatre à cinq mètres de haut - Théophraste dit quatre coudées - garnie à la base de quelques feuilles, nue à sa partie supérieure, et couronnée par une panicule élégante, d'abord pyramidale, mais qui, lors de son complet développement, s'étale en forme de bouquet, en font déjà une plante ornementale; les divers usages auxquels il servait le rendaient encore plus précieux.

Choisi comme symbole du Delta, le papyrus croissait, en abondance, dans les marécages qui avoisinaient le Nil et dans les eaux calmes dont la profondeur ne dépassait guère un demi-mètre, le rhizome de la'grosseur du bras, rampant au-dessus de la vase, où il enfonçait de nombreuses radicules, avait parfois, suivant Théophraste ${ }^{4}$, jusqu'à dix coudées, et les stolons qui en surgissaient formaient à eux seuls une espèce de fourré inextricable; les plantations de papyrus servaient aussi de refuge aux innombrables oiseaux aquatiques, qui peuplent les marais de l'Égypte. Les peintures des hypogées nous représentent les chasseurs qui

1. Victor Loret, La Flore pharaonique, p. 29, no 28.

2. Hérodote, Historiae, lib. II, cap. 92.

3. Théophraste, IIisloria planlarum, lib. IV, cap. 3, 3 .

4. Hisloria planlarum, lib. IV, cap. 8, 3 . 
les poursuivent, montés sur des bateaux plats, au milieu de leurs humides retraites'.

Le papyrus ne croissait pas en Égyte seulement a l'état sauvage; on le plantait aussi dans les pares, au bord des pièces d'eau dont il conservait la fraicheur; c'est ainsi que dans le jardin de l'officier d'Amenhotpou II, dont jai donné plus haut la description, on voit, de chaque còté des quatre bassins qui y sont creusés, une rangée de papyrus. On en voit aussi des touffes à l'extrémité des bassins des jardins d'Apoui ${ }^{2}$. On ne cultivait pas seulement le papyrus a cause de son port élégant et gracieux, mais aussi à cause de son utilité; ses feuilles et ses tiges, ses racines mêmes, servaient à de nombreux usages et en faisaient une des plantes les plus précieuses de la vallée du Nil.

Les nymphéacées occupaient encore une place plus grande dans la vie des anciens Égyptiens. Plusieurs plantes de cette famille ont été cultivées ou croissaient spontanément en Égypte à l'époque pharaonique, la plus commune et celle qu'on trouve le plus anciennement représentée sur les monuments antiques est le Nymphaea lotus L., indigène, d'ailleurs, dans la vallée du Nil. On le reconnait sans peine ${ }^{3}$ à ses pétales blancs, parfois bordés de rouge, à ses sépales lancéiformes, au

1. Lepsius, Denlimäler, t. IV, pl. 106. Zaouïet-el-Naïetin (VIe dynastie), tombe 2.

2. Mémoires publiés par les membres de la Mission française au Caire. Le tombeau d'Apoui, paroi B, t. V, fasc. 4.

3. En particulier sur la peinture de la tombe II de Zaouietel-Maïetin. (Lepsius, Denlimäler, t. IV, pl. 105) et sur celle de la tornbe de Ti au musée Guimet, tableau 2, peintures qui représentent l'une et l'autre une joute sur l'eau, ainsi que dans la peinture 177 de la galerie égyptienne au Brilish Museum, dont j'ai déjả parlé et dans celle du jardin d'Apoui. 
nombre de quatre, à ses feuilles arrondies et profondément échancrées, à ses fruits en forme de capsule de pavot. On a trouvé dans la tombe de Ramsès II, pharaon de la $\mathrm{XIX}^{\mathrm{e}}$ dynastie, un grand nombre de pétales de ce lotus très bien conservés ${ }^{1}$. Il en existe cinq fleurs au Musée égyptien du Louvre ${ }^{2}$.

Hérodote a mentionné d'une manière expresse cette nymphéa parmi les plantes aquatiques de l'Égypte. " II pousse, dit-il ${ }^{3}$, dans les campagnes inondées par le Nil une quantité prodigieuse de lis que les Egyptiens appellent lotus. "Il parle ensuite de leurs graines, semblables à celles du pavot et dont on se servait comme aliment, ainsi que de leurs racines comestibles. Théophraste a décrit, en détail, le Nymphaea lotus", sa corolle blanche, composée de pétales étroits rappelant ceux du lis, mais qui, plus nombreux, sont appliqués les uns contre les autres, sa tige et ses feuilles, analogues à celles du Nelumbium, mais plus petites et plus faibles, sa capsule enfin, qui, divisée en compartiments, égale en dimension le plus gros pavot et renferme un grand nombre de graines, semblables à celles du millet. Le botaniste grec signale aussi cette proprièté du lotus blanc, commune à toutes les nymphéacées, qu'au coucher du soleil ses pédoncules se recourbent et cachent sous les eaux les fleurs qui se ferment alors, pour se rouvrir, en même temps que les pédoncules se dressent, quand le soleil reparait sur l'horizon.

Le lotus blanc portait en ancien égyptien le nom de

1. G. Schweinfurth, Ueber Pflanzenreste. (Berichte der botanischen Gesellschaft, t. II (an. 1884), p. 359.)

2. Recueil de travaux, t. XVII (an. 1895), 34, p. 199.

3. Historiae, lib. II, cap. 92.

4. Plantarum historia, lib. IV, cap. 8, 9. 
seshen ou soushin, copte shöshan, dont il faut rapprocher l'hébreu shưshan et l'arabe sousan, vocables qui, toutefois, désignent, non le lotus, mais le lis blanc'. Cette uymphéacée, qui apparaît sur les plus anciens monuments et était regardée comme l'emblême de la Haute-Egypte, parfois mème de l'Egypte tout entière, croit spontanément dans la vallée du Nil; on l'y rencontre aujourd'hui encore, comme au temps d'Hérodote et de Théophraste; lorsque le Nil inonde les campagrnes, elle se développe peu à peu et fleurit à l'époque de la plus haute crue des eaux, pour se faner et périr, quand celles-ci se retirent dans le lit du fleuve.

Le Nymphaea lotus n'était pas la seule nymphéacée qu'on trouvât dans les canaux, ainsi sans doute que dans les bassins des parcs égyptiens; avec lui y croissait le lotus bleu (Nymphaea caerulea Sav.), indigène, comme lui, dans la vallée du Nil, mais dont ni Hérodote, ni Théophraste n'ont parlé; Athénée ${ }^{2}$ est le premier auteur ancien qui en ait fait mention; mais ce nymphéa - le $\lambda$ ivicos, comme il l'appelle - est figuré souvent sur les monuments pharaoniques; Delile ${ }^{3}$ a été jusqu'à dire que les Égyptiens l'ont " peint et sculpté dans leurs temples plus fréquemment qu'aucune autre plante $"$. On le trouve représenté dès les temps de l'ancien empire. Un jeune veau blanc sur une peinture du tombeau de $\mathrm{Ti}$ ( $\mathrm{V}^{\mathrm{e}}$ dynastie) porte au cou, en guise

1. Victor Loret, La Flore pharcionique, p. 113, no 193. Cf. Sur les noms égyptiens des lotus. (Recueil de travaux, t. I, p. 190 et suivantes.) Outre le nom de soushin, Franz Wœnig, op. laud., p. 23, attribue aussi au lotus blanc les noms de nekheb, nesheb, neheb.

2. Deipnosophislae, lib. XV, cap. 21 (677).

3. Flore d'Egyple, p. 423. 
de clochette, un lotus bleu attaché par un ruban rose ${ }^{1}$. Sur la planche 74 du grand ouvrage de Rosellini ${ }^{2}$, on voit un lotus qui, d'après Unger ${ }^{3}$, est certainement le Nymphaea caerulea. Le même botaniste parle aussi d'un papyrus du musée égyptien de Londres où se verrait encore une représentation de cette nymphéacée. Schweinfurth ${ }^{4}$ a trouvé des fleurs entières de lotus bleu avec leurs longs pédoncules dans la tombe de Ramsès II restaurée sous la XXI ${ }^{\circ}$ dynastie; les pétales et les sépales de cette plante, remarque le royageur naturaliste, entrent pour une part considérable dans la composition des guirlandes de fleurs de cette époque, et on les rencontre également dans celles d'autres dynasties. M. Flinders Petrie a découvert des restes de cette nymphéacée dans la nécropole gréco-romaine de Hawara ${ }^{5}$.

La couleur des fleurs du Nymphaea caemulea le distingue tout d'abord du lotus blanc; mais il en diffère aussi par d'autres caractères : la corolle est plus arrondie, les pétales lancéolés, moins rayonnants, au nombre seulement de douze à quatorze, les feuilles sont plus petites, ovales, évasées en forme de cœur. Le nom

1. Musée Guimet, tab. VI. Cf. Victor Loret, L'Égypte au temps des Pharaons, p. 114 .

2. Monumenti dell' Egitto, t. II.

3. Bolanische Streifzüge. Silzungsberichte, etc., t. XXXVIII, p. 123.) On pourrait croire que c'est là tout simplement une figure de fantaisie, comme celle du $n^{0} 4$, qui représente des lotus jaunes.

4. Ueber Pflanzenreste. (Berichte der botanischen Gesellschaft, t. II, p. 357.) - Bulletin de l'Instilut égyptien, $\mathrm{n}^{\circ} 3$ (an. 1882), p. 60.

5. Hawara, Biahmu and Arsinoë. p. 52. 
hiéroglyphique du lotus bleu est sarpal ${ }^{1}$. Cette plante de l'ancienne Égypte, qui croissait encore dans la basse vallée du Nil, il y a moins d'un siècle, en a disparu de nos jours; on ne la trouve plus que dans le bassin supérieur du lleuve vers le $14^{\mathrm{e}}$ degré de latitude, mais plus au Sud il courre toutes les eaux stagnantes, en compagnie du lotus blanc ${ }^{2}$.

Dans le cercueil d'un nommé Kient, enseveli à Shéik Abd-el-Gournah sous la XX' dynastie, M. Schweinfurth ${ }^{3}$ a remarqué une guirlande formée en partie « de pétales et de fleurs naines et choisies exprès de lotus bleu »; dans cette forme plus petite du Nymphaea caerulea, M. Victor Loret" a cru reconnaitre la variété stellala Willd. de cette nymphéacée, variété reconnue par Deliles; mais dont on peut se demander si les contemporains des Pharaons l'avaient distinguée de la forme plus grande.

En mème temps que le lotus blanc et le lotus bleu arec sa variété, qui croissent spontanément dans la vallée du Nil, on cultivait dans l'ancienne Égypte une autre nymphéacée, exotique, elle, et plus remarquable, sinon plus célèbre: le lotus rose (Nelumbium speciosum Willd). A quelle époque ce lotus, que M. de Candolle a cru à tort indigène, mais qui est originaire de l'Inde, a-t-il été transplanté dans la patrie des Pharaons? Jessen ${ }^{6}$ parle de son importation, mais sans en fixer la date; Schweinfurth la croit récente; l'absence du

1. Victor Loret, La Filore pharaonique, p. 116, no 19't. Recueil de travaux, t. I, p. 194.

2. Franz Wœnig, op. laud., p. 34.

3. Les dernières découvertes. (Bulletin, no 6 (1885), p. 280.)

4. La Flore pharaonique, p. 118, no 195.

5. Flore d'Elypte, p. 422.

6. Botanik der Gegenwart und Vorzeit in culturhistorischer Entwickelung. Leipzig, 1864, in-8, p. 3. 
Nelumbium dans les peintures des hypogées pharaoniques, tandis qu'on le rencontre dans celles de l'époque gréco-romaine, lui a fait admettre que cette belle nymphéacée n'avait été apportée en Égypte qu'après la conquête perse ${ }^{1}$. M. Victor Loret est d'une autre opinion; il suppose que l'introduction du lotus rose remonte très haut dans le passé, mais que son caractère de plante sacrée l'a fait représenter sur les anciens monuments d'une manière conventionnelle, qui n'a pas permis de l'identifier jusqu'ici 2. Pour lui, il n'hésite pas, dans les fleurs des nymphéacées aux couleurs vives et variées, qu'on aperçoit sur certaines peintures du nouvel empire, à voir des fleurs de Nelumbium.

L'explication est ingénieuse; mais j'avoue qu'elle ne me convainc pas. Les nymphéacées des peintures auxquelles fait allusion M. Victor Loret sont de simples motifs de décoration sans caractère distinct; on ne peut rien en conclure. D'ailleurs, lc lotus blanc était, lui aussi, une plante sacrée, ce qui n'a pas empêché les artistes pharaoniques de le représenter avec une vérité qui le fait reconnaìtre à première vue. C'est lui qu'on voit sur tous les canaux et les réservoirs des jardins de l'ancien, comme du nouvel empire; pourquoi n'y aurait-on pas représenté également le Nelumbium, s'il y avait été réellement cultivé comme le lotus blanc? A l'époque des Plolémées, où il n'avait rien perdu de son caractère sacré, on n'a pas hésité à le peindre; il est difficile de comprendre pourquoi on ne l'aurait pas fait

1. Bulletin de l'Institut égyptien, no 3 (an. 1882), p. 64. Ueber Pfanzenreste. (Berichte der botanischen Gesellschaft, t. II, p. 357.) - Zeitschrift fïr Ethnologie, an. 1891, p. 659.

2. Recueil de travaux, t. I, p. 193. - La Flore pharaonique, p. 111, no 192. 
sous la XVIII et la XIXe dynastie, s'il avait été connu alors dans la vallée du Nil. Il existe au Musée britannique un paysage du temps des Ptolémées où l'on voit, parmi les plantes qui y figurent, des Nelumbium reconnaissables à leurs feuilles orbiculaires et à leurs fruits en pommes d'arrosoir ${ }^{1}$. Depuis cette èpoque, le lotus rose devint de plus en plus fréquent sur les monuments mème en dehor's de l'Égypte; le Musée de Naples possède une grande mosaïque venant de Pompeï, où les fruits de cette belle nymphéacée figurent parmi les autres produits caractéristiques du Nil? .

M. Victor Loret attribue au lotus rose le nom de neliheb ou nesheb, qui apparait dans les textes hiéroglyphiques dès la XVIII dynastie, mais qu'on a attribué aussi au lotus blanc ${ }^{3}$. Hérodote est l'auteur le plus ancien qui ait fait mention du Nelumbium; après avoir parlé du lotus blanc, auquel il donne le nom de lis:

Il nait encore dans le Nil, ajoute-t-il", d'autres lis, mais semblables à des roses; leurs fruits, qui ont la forme d'un nid de guêpes, renferment des graines nombreuses, de la grosseur d'un noyau d'olive.

Théophraste a complété le peu que nous apprend, Hérodote :

La fève (d'Égypte) - c'est le nom qu'il donne au Nelumbium - croit, dit-il ${ }^{5}$, dans les marais et les eaux stagnantes; sa tige, de la grosseur du doigt, analogue à celle du roseau,

1. Unger, Bolanische Streifzüge. (Silzungsberichte der Wiener Akademie, t. XXXVIII (an. 1859), p. 118.)

2. Schweinfurth, De la Flore pharaonique. (Bulletin de l'Inslilul égyplien, nº 3 (an. 1882), p. 65.)

3. II. H. E. Lüring, Die über die medicinischen Kemntnisse der alten Aegypter berichtenden Papyri, Leipzig, 1888, in-8, p. 162, attribue, lui, ce nom au lotus bleu.

4. Historiae, lib. II, cap. 92, 14.

5. Hisloria planlarum, lib. IV, cap. 8, 7. 
mais non noueuse, est remplie de cloisons, comme un rayon de miel; elle peut atteindre une longucur cle quatre coudées et se termine par une capsule qui a la forme d'un nid de guêpes ${ }^{1}$. Chacune des cellules dans lesquelles elle se divise renferme une espèce de fève qui fait saillic; elles sont au nombre de trente au plus. La fleur, deux fois plus grande que celle du pavot, est d'un rose pâle ; elle dépasse de beaucoup la surface de l'eau. Les feuilles qui l'entourent, portées sur des pétioles de mêrne longlicur que le support du fruit, sont grandes et rappellent par leur forme le chapeau thessalien. Quand on écrase la graine, on voit au-dedans un petit corps replié sur lui-même - le germe - duquel sort la tige. La racine est plus épaisse que celle du roseau et est cloisonnée comme la tige.

Pline $^{2}$ n'a rien ajouté d'essentiel à la description de Théophraste; pour la rendre plus exacte, voici quelques caractères complémentaires empruntés pour la plupart à Delile ${ }^{3}$. Les feuilles du lotus rose, entières, d'un rert gai et mollement velues, ont 45 a 50 centimètres de large; leurs pétioles qui s'élèrent de 4 à 7 décimètres au dessus de la surface des eaux sont garnis d'aiguillons recourbés, ce que Théophraste dit à tort des racines; celles-ci sont charnues, noueuses et traçantes; quant aux fleurs, elles ont jusqu'à 15 à 20 centimètres de large; elles dépassent de 1 mètre $1 / 2$ à 2 mètres le niveau de l'eau et ont une forte odeur d'anis ou de cannelle; les pétales nombreux, obovales aigus, sont disposés sur trois rangs, les intérieurs sont plus petits; ils sont couronnés d'une frange épaisse de filets d'étamines, qui environne l'ovaire; celui-ci, évasé à sa par. tie supérieure, est, à son complet développement, large environ comme la paume de la main; les graines ovoüdes

1. Il serait plus exact de dire d'une pomme d'arrosoir.

2. II istoria naturalis, lib. XVIII, cap. 30.

3. Flore d'Égypte, p. 427. 
et un peu saillantes ont l'écorce dure, noire et lisse et renferment une amande douce, blanchìtre et charnue, bonne à manger, pourvu qu'on en rejette le germe intérieur qui est amer.

Théophraste regardait le Nelumbium comme croissant spontanément en Égypte, mais, ajoute-t-il, on en sème aussi les graincs; on les entoure d'une couche de limon, mèlé de paille, pour qu'elles s'enfoncent plus surrement et qu'elles ne pourrissent pas. "On peut créer ainsi de réritables plantations de fèves (d'Égypte); car, une fois que les graines ont germé et pris racine, elles ne meurent plus ". Strabon " parle d'une de ces plantations de lotus roses, - il les appelle, comme Théophraste, "fères d'Égypte ", -- qu'on voyait, semblable à une forèt aquatique, aux environs d'Alexandrie; " elle offrait, dit-il, un aspect agréable et qui réjouissait la rue. On allait dans des barques garnies de cabines diner au milieu de ces lotus et à l'ombre de leurs larges feuilles $»$.

1. Geograplicu, lib. XVII, cap. 1, 15. 


\section{CHAPITRE V.}

LES PLANTES DANS L'ALIMENTATION ET DANS L'INDUSTRIE.

La flore indigène de l'Égypte et celle des pays voisins, qu'ils mirent - on en a ru de nombreux exemples - si souvent à contribution, offraient aux habitants de la vallée du Nil de quoi satisfaire à leurs besoins les plus pressants, comme aux exigences de l'industrie et des arts qu'ils poussèrent si loin; elle leur fournissait à la fois des aliments sains et fortifiants, des boissons rafraìchissantes et agréables, des tissus souples et légers pour se vêtir, des matériaux variés pour se construire des maisons et des embarcations, fabriquer leurs meubles ou les ustensiles indispensables à la vie; il faut ajouter, objet d'étude pour un autre chapitre, des aromates pour le culte ou la parfumerie, des remèdes dans leurs maladies.

I.

Si les premiers habitants de l'Égypte furent avant tout un peuple de chasseurs et de pêcheurs, ils trouvaient aussi une partie de leur nourriture dans les fruits sauvages et les plantes. bulbeuses qui croissent spontanément dans la terre de Kimit ${ }^{6}$. Les populations supérieures, qui les chassèrent ou les asservirent ne

1. Unger, Silzungsberichle, t. XXXVIII, p. 77. 
dédaignèrent point ces mets simples que leur offrait d'elle-mème la nature; mais ils y joignirent d'autres aliments végétaux plus nutritifs et plus variés qu'ils demandèrent à la culture des champs. Aussi loin que nous pourons remonter dans le passé, nous trouvons les habitants de l'Égypte en possession des céréales; les apportèrent-ils avec eux? Les reçurent-ils par l'intermédiaire du commerce? Nous l'ignorons; mais ce qui est hors de doute, c'est que dès les temps les plus reculés ils leur demandèrent une partie de leur alimentation.

On a ru plus haut ${ }^{1}$ comment ils cultivaient ces plantes précieuses; quand ils les avaient récoltées - orge, froment ou variétés d'épeautre - et que, en les faisant fouler aux pieds des boufs ${ }^{2}$ ou des înes ${ }^{3}$, ils avaient séparé de la paille le grain nourricier; ils écrasaient celui-ci entre deux pierres plates ou le broyaient dans une espèce de mortier ${ }^{4}$; plus tard peut-être, car les monuments ne nous renseignent pas à cet égard, ils eurent recours à des moulins à bras, composés de deux meules, dont l'une mobile et percée d'un trou pour y verser le grain tournait sur la seconde fixe et placée au-dessous ${ }^{5}$. La farine obtenue par ce procédé primitif était séparée du son, au moyen d'un tamis en papyrus ou en jonc tressé, puis transformée en pain.

1. Chapitre II, p. 24.

2. Rosellini, Monumenti, t. II, pl. xxxir et xxxIII. - Lepsius, Denkmäler, t. IV, pl. 106. Zaouïet-el-Maïétin, tombe 2, d.

3. Lepsius, Denkmüler, t. II, pl. 9.

4. C'est ce qu'a cru S. Birch. Cf. Wilkinson, op. laud., t. I, p. 359 , note 2 .

5. Wilkinson, op. laud., t. I, p. 359. 
Les peintures du tombeau de Ramsès III ${ }^{1}$ nous font assister aux divers procédés de la panification; nous y voyons des ouvriers pétrir, ici avec leurs mains, là avec leurs pieds ${ }^{2}$, la pâte dans une espèce d'auge ou de cuve en pierre; quand elle avait été suffisamment travaillée, on la passait au maitre boulanger, qui la façonnait en pains aux formes les plus diverses ${ }^{3}$. La farine d'orge et de froment servait indifféremment, ainsi que celle d'olyra et de zeia, à faire ces pains; la farine d'orge, Hérodote semble dire aussi celle de froment - mais il faut peut-être entendre par là la farine de gros blé, - donnait naturellement un pain inférieur et par là même dédaigné ${ }^{4}$; avec la farine d'olyra et de zeia on faisait un pain meilleur et plus léger, dont les Égyptiens se nourrissaient surtout, suivant l'historien grec.

Les anciens habitants de la vallée du Nil semblent avoir ignoré l'emploi de la levure; pour rendre leur pain plus agréable et plus digestif ils y mêlaient sans doute diverses plantes, en particulier, une lichénée, la Parmelia furfuracea Asch.; on a trouvé dans la cachette de Deir-el-Bahari une corbeille remplie de ce lichen, le sheba ou shibah des Arabes, qui s'en servent aujourd'hui encore comme de levure pour faire leur pain et lui

1. Tombeau de Ramsès III à Thèbes. Wilkinson, op. laul., t. II, p. 34 .

2. "Les Égyptiens, dit Hérodote, lib. II, cap. 36, pétrissent la pâte avec les pieds, l'argile avec les mains. »

3. Wilkinson, op. laud., t. II, p. 34. - Franz Wœnig, op. laud., p. 177.

4. Hérodote, lib. II, cap. 36, 2, va jusqu’à dire, ce qui doit être au moins en partie inexact, que les Égyptiens regardaient comme une honte de se nourrir d'orge et de froment. 
domner un goùt qu'ils recherchent'. La Parmelia furfuracea est étrangère ì l'Égypte; on l'importe des îles de l'Archipel oi elle croit en abondance. Forskål avait déji signalé cette coutume ${ }^{2}$, et il a mentionné l'impor. tation de ce lichen et d'une autre espèce du même genre, la Parmelia prunastri, dont il avait vu des bateaux chargés débarquer à, Alexandirie.

Si le pain était un des aliments les plus recherchés par les Egyptiens, le monde végétal leur en fournissait, parfois mème sans culture, d'autres encore, et en quantité telle qu'ils auraient pu, dit Pline ${ }^{3}$, se passer de céréales. Les habitants du-Delta trouvaient en particulier dans les plantes aquatiques, si abondantes dans cette région, une partie importante de leur alimentation; les racines de quelques-unes d'entre elles, les graines, seules ou avec les racines, de quelques autres leur offraient une nourriture salubre et peu coùteuse: tels étaient le papyrus, plusieurs espèces de souchets et les diverses nymphéacées.

Après avoir arraché les papyrus qui croissent en abondance dans les endroits marécageux, les habitants du Delta, dit Hérodote 4 , coupent la partie supérieure de la tige, qu'ils réservent pour différents usages; quant à la partie inférieure, ils la mangent ou la vendent. Ceux qui veulent la rendre plus délicate la font rôtir dans un four ardent.

Si l'on en croyait Théophraste, c'eût été surtout comme aliment que le papyrus était utilisé en Égypte.

. «Tous les habitants, dit-il ${ }^{5}$, mangent les rhizomes de cette

1. Schweinfurth, De la Flore pharaonique. (Bulletin de l'Institul égyptien, $\mathrm{n}^{\circ} 3$ (an. 1882), p. 74.

2. Flora aegyptico-arabica, p. 193.

3. Hisloria naturalis, lib. XXI, cap. 50 .

4. Historiae, lib. II, cap. 92, 5 et 6 .

5. II istoria planlarum, lib. IV, cap. 8, 4. 
plante, soit crus, soit bouillis ou grillés. Ils en sucent aussi les tiges en rejetant ensuite les fibres, après les avoir mâchées. "

Diodore fait également mention de l'usage que les Égyptiens faisaient du papyrus dans leur alimentation; parlant de la sobre éducation des enfants ${ }^{1}$ :

Ils ne leur coûtent presque rien à èlever et il les accoutument à la plus grande frugalité. Ils leur donnent à manger n'importe quel mets grossier et facile à préparer, tels que des rhizomes de papyrus cuits sous la cendre, ainsi que des racines d'autres plantes aquatiques, soit crues, soit bouillies ou grillées.

Parmi ces plantes il faut citer le souchet comestible (Cyperus esculentus L.) - la malinathalle de Théophraste $^{2}$, - autre cypéracée, dont les rhizomes servaient, ainsi que ceux du papyrus, à la nourriture des habitants du Delta, tout comme ils leur servent encore aujourd'hui. "Ils en recueillaient les bulbes, nous apprend le naturaliste grec, les faisaient cuire dans de la bière, ce qui leur donnait un goùt sucré et les mangeaient comme dessert. ") Ils màchaient, au contraire, les tiges du sari" - les saria - comme celles du papyrus, ainsi peut-être que les tiges du mnasion, plantes qui croissaient spontanément dans les lieux inondés et dans les flaques d'eau. J'incline à voir dans ces deux plantes les Cyperus alopecuroïdes Rottb. et auricomus Spr. ou dives Delile, espèces à racines tuber-

1. Bibliolheca historica, lib. I, cap. 80, 5 .

2. Hisloria plantarum, lib. IV, cap. 8, 12.

3. Historia planlarum, lib. IV, cap. 8, 5 et 6 . Théophraste se borne à remarquer que le mnasion servait à l'alimentation, sans dire comment on le mangeait. 
culeuses ', que l'on peut, je crois, identifier avec les plantes aquatiques sar et menh, dont il est question dans une inscription du temple d'Edfou ${ }^{2}$.

Malgré les ressources que leur offraient pour leur alimentation les divers Cyperus dont je viens de parler, les Egyptiens en trouvaient encore de plus abondantes dans la famille des nymphéacées. La racine du lotus blanc -- le corsion - "ronde et de la grosseur d'une pommen, suivant Hérodote ${ }^{3}$, d'un coing, d'après Théo-

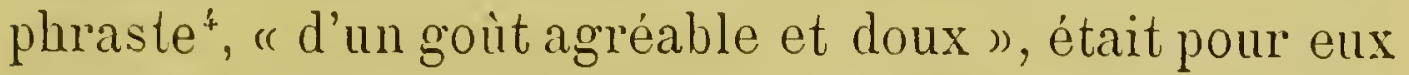
un mets facile à se procurer, puisque, après l'inondation, toute la partie de l'Égypte qu'elle atteignait se couvrait de ce nymphéa. Ils la mangeaient, nous apprend le naturaliste grec, bouillie et grillée. Il est probable que les racines du lotus bleu se mangeaient de la même manière.

L'acclimatation du Nelumbium ou lotus rose procura aux habitants de la vallée du Nil un nouvel aliment $^{5}$; sa racine, écrasée et bouillie, servait de pain, dit Théophraste ${ }^{6}$, aux habitants des marais; ils la mangeaient aussi crue, comme les rhizomes ou les tiges de souchets. Toutefois les graines des nymphéas en-

1. Boissier, Flora orientalis, t. V, p. 367 et 375. Théophraste n'ayant donné aucune description du mnasion, il est trop évident que cette identification n'est qu'hypothétique.

2. "Les sar, menh, serped, sesen, toutes plantes croissant dans le Nil. » Dümichen, Edfou, ap. Brugsch, Dict. hiérog., p. 659 et 1169. Les serped et les sesen sont les lotus bleus et blancs.

3. Historiae, lib. II, cap. 92, 3.

4. Fistoria plantarum, lib. $1 V$, cap. 8, 11.

5. Goodyear (Wm. H.) n'hésite pas à dire qu'il est probable que le lotus rose fut introduit en Égypte comme plante alimentaire. The grammar of the lotus. London, 1891, in-4, p. 35.

6. Historia plantarum, lib. [V, cap. 8, 11. 
traient encore plus que leur racine dans l'alimentation des Égyptiens.

Ils mangeaient vertes ou sèches, rapporte Hérodote ${ }^{1}$, les graines du lotus rose - les lis du Nil, comme il les appelle; - ils pilaient, au contraire, les graines du lotus blanc et en faisaient une espèce de pain, qu'ils cuisaient au feu. C'était le " pain de lis ", dont il est question dans un des Papyrus Anastasi ${ }^{2}$; il fut longtemps recherché des goumets et on le voit figurer encore sur la table des rois de la XIX ${ }^{e}$ dynastie $^{3}$. Théophraste, qui a décrit très exactement les fruits du Nelumbium, ne parle point de leur emploi dans l'alimentation; mais il s'est étendu longuement sur les usages que les habitants du Delta faisaient pour leur nourriture des graines du lotus blanc. Après avoir dit que les fruits de ce nymphéa ressemblent à des têtes de pavot:

“Les Égyptiens, ajoute-t-il'4, ramassent ces fruits et les mettent en tas pour qu'ils se désagrègent, puis quand l'écorce en est pourrie, ils les lavent et en enlèvent les graines: ils font sécher celles-ci, les écrasent et en fabriquent du pain, dont, ils se nourrissent. "

Pline, qui donne au lotus blanc le nom de lotometra, connaissait aussi très bien l'usage que les Égyptiens en faisaient dans leur alimentation :

"Avec la graine qui est semblable au millet, dit-il ", les

1. Historiae, lib. II, cap. 92,3 et 4 .

2. No IV, p. 14, l. 1.

3. Maspero, Histoire ancienne, t. I, p. 66.

4. II istoria plantarum, lib. IV, cap. 8, 11.

5. II istoria naturalis, lib. XXII, cap. 28. M. Franz Wonin, op. laud., p. 28, a supposé, mais sans en donner aucune raison, que, sous le nom de lolometra, Pline avait désigné le lotus bleu. 
habitants de l'Égypte, les bergers surtout, font un pain que l'on pétrit avec de l'eau et avec du lait. On prétend que rien n'est plus salutaire ni plus léger que ce pain. »

Si les rhizomes des souchets et des nymphéas, ainsi que les graines de ces dernières, entraient d'une manière toute particulière dans l'alimentation des anciens Egyptiens, ces plantes étaient loin d'ètre les scules, sans parler des céréales, qui servissent à les nourrir. La flore nilotique leur offrait bien d'autres aliments végétaux, et ils en avaient demandé un non moins grand nombre à celle des pays étrangers : légumes cultivés en grand nombre dans les jardins, plantes herbacées croissant à l'état sauvage, fruits des arbres indigènes ou acclimatés dans les jardins, complétaient et variaient leur alimentation.

On raconte que, quand Amrou s'empara d'Alexandrie, il n'y avait pas moins de quatre mille marchands occupés à vendre des légumes dans cette ville ${ }^{1}$; la consommation et la vente n'en devaient pas être moindres dans les villes de l'Égypte ancienne; les scènes de la vie privée, représentées sur les tombes pharaoniques et qui nous permettent de deviner ce qu'était alors la culture et l'usage des plantes potagères dans cette contrée, les offrandes si nombreuses de légumes et de fruits, que l'on voit sur les peintures des hypogées en sont une preuve indéniable. Hérodote rapporte ${ }^{2}$, avec une exagération manifeste, il est vrai, mais elle n'infirme pas le fait en lui-mème, qu'on paya la somme de 1,600 talents d'argent pour les radis, oignons et aulx, consommés par les ouvriers de la quatrième pyramide.

1. Wilkinson, op. laud., t. II, p. 25.

2. Historiae, lib. II, cap. 125, 5. 
Une peinture de Béni-Hassan, dont il a déjà été question, nous montre un potager, dont les plates-bandes soigneusement arrosées sont couvertes de légumes: oignons qu'on arrache et met en bottes, concombres qui débordent dans les allées. Sur une autre peinture de la même localité on voit deux ouvriers qui reviennent des champs en portant dans des paniers, soutenus par des courroies, des lotus et des légumes de diverses espèces ${ }^{1}$.

Les oignons, les aulx et les poireaux d'Égypte étaient, avec les pastèques et les melons chate, ceux que regrettaient le plus les Hébreux, errant dans le désert ${ }^{2}$. C'étaient là aussi les légumes les plus recherchés des habitants de la vallée du Nil. Au premier rang venait l'oignon, auquel le sol léger et gras de l'Égypte convient à merveille; il y acquérait des qualités toutes particulières et il y croissait en telle abondance qu'il ne devait pas être plus difficile de se procurer ce légume à l'époque pharaonique que de nos jours, où il est à vil prix. On portait les oignons au marché, comme le montrent les peintures des hypogées, par bottes de cinq à six pieds ${ }^{3}$. D'après Plutarque, les prêtres égyptiens s'abstenaient de l'oignon, ce légume ne convenant pas, dit-il ${ }^{4}$, à ceux qui font pénitence, pas plus qu'à ceux qui célèbrent des fètes, parce qu'il excite la soif et fait pleurer.

Suivant Hérodote, on vient de le voir, l'ail aurait

1. Champollion, Les Monuments de l'Égypte, t. IV, pl. 10 ' bis.

2. Numeri, cap. xI, vers. 5 .

3. Champollion, Monuments, t. IV, pl. 358, 2. Béni-Hassan. - Rosellini, Monumenli, t. II, pl. LI, 1. - Lepsius, Denkmäler, t. IV, pl. 127, etc.

4. De Iside el Osiride, cap. vin. Ed. Gustav Parthey. Berlin, 1850, in-8, p. 13. 
existé dans la vallée du Nil dès l'époque de la IV a dynastie; Pline dit que les habitants l'avaient divinisé ${ }^{1}$, ainsi que l'oignon : c'est là tout ce que les écrivains de l'antiquité nous ont appris sur l'emploi, dans l'alimentation des habitants le l'Égypte, de ce condiment si recherché des populations du Midi. Quant aux pastèques et aux qauta ou aggour - le melon chate la Bible seule en parle²; les peintures des hypogées et quelques feuilles de pastèques trouvées dans les tombes sont, avec le récit mosaïque, tout ce qui nous renseigne sur leur' culture; mais on ne peut douter qu'elle n’y fùt très développée, ni que l'usage que l'on faisait de ces cucurbitacées ne fût important, et leur présence dans presque toutes les représentations d'offrandes est la preuve manifeste du prix qu'on y attachait.

A côté de ces légumes qui jouent plutôt le rôle de condiments ou de dessert que d'aliments proprement dits, prennent place les graines si nutritives des papilionacées. Hérodote affirme ${ }^{3}$ qu'on ne cultivait pas les fèves en Égypte et qu'on ne mangeait ni crues, ni cuites, celles qui y poussaient par hasard. "Les prêtres, " ajoute-t-il - affirmation qu'il est impossible de prendre au pied de la lettre - «n'en supportent même pas la vue, dans la croyance que ce légume est impur. "La trouvaille, qu'on a faite, nous l'avons vu", de fèves dans une tombe de Drah-Abou'l-Neggah et dans la nécropole de Kahoun prouve que cette légumineuse servait dans les offrandes funéraires et par suite était connue et probablement aussi cultivée en

1. Historia naluralis, lib. XIX, cap. 32, 1.

2. Numeri, cap. XI, vers. 5.

3. Hisloriue, lib. II, cap, 37.

4. Voir chapitre III, 4, p. 5 ป. 
Égypte dès le temps de la XII ${ }^{e}$ dynastie; mais on ne sait rien du rôle qu'elle jouait en ce pays dans l'alimentation.

On n'est pas mieux renseigné au sujet de l'usage que les Égyptiens de l'époque pharaonique pouvaient faire des pois chiches et des lupins pour leur nourriture; tout ce qu'on peut en dire, c'est que ces légumes existaient en Égypte, les pois chiches du moins, depuis une époque reculée ${ }^{1}$. Il en fut de même du cajan des Indes, dont la culture dans la vallée du Nil, ainsi que celle des petits pois, remonte à la XII ${ }^{\circ}$ dynastie, sinon au delà. A cette époque, une découverte de M. Flinders Petrie l'a montré ${ }^{2}$, ces deux légumineuses étaient déjà connues des Égyptiens.

La culture des lentilles dans la terre de Kimit remonte au moins à une date aussi reculée et elle y a été plus répandue. Strabon rapporte ${ }^{3}$ que les habitants de Memphis regardaient les débris, de forme lenticulaire, des pierres employées à la construction de cette ville, comme les restes des mets dont se nourrissaient les ouvriers des pyramides : souvenir, conservé par la tradition, de l'usage ancien des lentilles dans l'alimentation des habitants de cette contrée. Les Égyptiens faisaient avec les lentilles une espèce de bouillie grossière; c'est sous cette forme sans doute qu'on les offrait aux morts, ainsi que le peuvent faire supposer les restes qui ont été trouvés à Drah-Abou'l-Neggah, dans une tombe de la XII dynastie ${ }^{4}$. Wilkinson a supposé que le serviteur qu'on voit, sur une peinture de Ramsès III

1. Voir plus haut, chap. III, p. 57.

2. Kahun, Gurob and Hawara, p. 30, 1.

3. Geographica, lib. XVII, cap. 1, 34 .

4. Bulletin de l'Institut égyption, no 5 (an. 1884), p. 7. 
à Thebes, accroupi devant une marmite, était occupé à faire cuire des lentilles ${ }^{1}$; à côté de lui se trouveraient deux corbeilles remplies de ces légumes. Loin de diminuer, la culture des lentilles ne fit, ce semble, qu'augmenter avec le temps. Un fait montre l'extension qu'elle avait prise, à l'époque gréco-romaine, dans la vallée du Nil. Le vaisseau qui transporta à Rome l'obélisque de Caligula, avait, rapporte Pline ${ }^{2}$, reçu, en guise de lest, 120,000 boisseaux de lentilles. Les lentilles de Péluse étaient renommées ${ }^{3}$ pour leur bonne qualité bien au delà des frontières de l'Égypte.

Si l'on en croit Hérodote, dès le temps des pyramides, les radis servaient d'aliment en Égypte, tout comme les aulx et les oignons; les fouilles de M. Flinders Petrie à Kahoun ont montré qu'ils y étaient certainement connus, au moins à l'époque de la XII $\mathrm{dy}$ nastie ${ }^{4}$. D'après Pline ${ }^{5}$ la culture en aurait eu une grande importance, surtout à cause de l'huile qu'on retirait des graines; le polygraphe latin va jusqu'à dire que les Égyptiens semaient les radis de préférence aux autres plantes, parce qu'ils en retiraient plus de profit que du blé et que ce produit payait moins d'impôts ${ }^{6}$. Les radis se mangeaient crus, comme les racines des souchets et des lotus. Ce n'étaient pas les seuls légumes que les Égyptiens consommassent de

1. The manners of the ancient Egyptians, t. II, p. 34.

2. Historia naluralis, lib. XVI, cap. 76, 5.

3. Virgile, Georgica, lib. 1, v. 228.

4. Kahun, Gurob and IIawara, p. 50, 1.

5. Historia naturalis, lib. XIX, cap. 16. Il est probable que le radis, dont on retirait de l'huile n'était pas le Raphanus sativus ordinaire, mais la variété olifer.

6. Il faut dire toutefois que la description de Pline ne parait s'appliquer qu'en partie au radis proprement dit. 
cette facon; ils en mangeaient bien d'autres sans être cuits, comme la chicorée, peut-être la laitue, le fenouil, le cresson alénois et le corchorus, ainsi, d'après Pline ${ }^{1}$, que la chondrille, l'hypochéride, etc.

A côté des légumes proprement dits, il faut ranger les plantes qui servaient de condiments, tels que le cumin, la coriandre, l'aneth et l'anis, le sésame même, qui furent successivement acclimatés et sans doute aussi employés dans la vallée du Nil ${ }^{2}$. Il est même probable que ce ne furent pas les seuls condiments en usage chez les Égyptiens; peut-être comnurent-ils, à l'époque des Ptolémées du moins, quelques-unes des épices qui croissent dans l'Hindoustan et la Malaisie; mais jusqu'ici aucun document ne nous renseigne sur ce point.

Les fruits occupaient, commé les légumes, une large place dans l'alimentation des anciens Égyptiens; ils y avaientservi dès les temps préhistoriques; ils y servirent encore plus à l'époque des Pharaons. Les plus anciennement connus furent les fruits indigènes ou demisauvagés du sycomore - neha - du jujubier - noubsou; plus tard vinrent les dattes, les raisins, les fruits de l'arbre ashdou (Balanites aegyptiaca L.), du perséa, ainsi que les figues ordinaires, les grenades, puis les pommes, les amandes, enfin les mûres, les cerises, peut-ètre même les poires et les noix.

Parmi ces fruits, ceux du sycomore figurèrentlongtemps au premier rang; on les mangeait frais ou

1. Hisloria naturalis, lib. XXI, cap. 52.

2. Cf. chapitre III, paragraphe 4, p. 75-78. 
secs; on en faisait aussi des gâteaux ${ }^{1}$. Mais si ces fruits ne cessèrent pas d'être recherchés et prirent toujours place dans les offrandes, ils ne vinrent dans la suite que bien après les figues proprement dites, qui leur sont si supérieures. Comme aujourd'hui, on mangeait ces dernières fraîches ou séchées.

Il en était de même des dattes, que leurs qualités nutritives firent mettre bientôt au premier rang des fruits égyptiens, et qui, comme de nos jours, devinrent, surtout dans les nasis et la Haute-Égypte ${ }^{2}$, un des principaux aliments des habitants de la vallée du Nil, et l'une des richesses du pays. Ils les mangeaient fraiches au moment de la maturité, ou le plus souvent ils les conservaient pour l'hiver. Dans ce dernier cas, ils les faisaient sécher à l'air libre, ou en faisaient des espèces de gâteaux - l'agoueh des Arabes - qu'on mangeait cuits, ou qu'on servait comme dessert. Dans le Pa. pyrus Harris il est question de dattes "pressées " " fertes par Ramsès III aux divinités de Thèbes. Wilkinson parle de gâteaux de dattes trouvées à Thèbes ${ }^{4}$; il en est fait mention dans la liste d'offrandes de la tombe de Rekhmara (XVIII dynastie) ${ }^{5}$. Les dattes étaient employées aussi pour la nourriture des animaux. Les fruits du palmier doum entraient, comme ceux du

1. Il est fait mention de gâteaux de figues de sycomores dans la liste des offrandes faites à Rekhmara (XVIIIe dynastie). Philippe Virey, Le lombeau de Rekhmara. (Némoires de la Mission archéologique au Caire, t. V, p. 104.)

2. Fr. Hasselqvist, Voyage en Palestine dans les années 1749 1752, 2e partie, p. 145.

3. Zeitschrifl fur aegyptische Sprache, t. XI (an. 1873), p. 70.

4. The manners of the ancient Egyptians, t. I, p. 398.

5. Philippe Virey, Le tombeau de Rekhmara. (IIémoires de la Mission archiologique au Caire, t. V, p. 104.) 
dattier, dans l'alimentation des anciens habitants de l'Égypte; les habitants actuels en font encore usage; ils enlèvent le noyau et en mêlent la pulpe à la farine de dourrah pour en faire du pain ${ }^{1}$.

Si l'on en juge par leur fréquence dans les tombes, les fruits du Balanites aegyptiaca ou ashdou servaient aussi, du temps des Pharaons, à l'alimentation en Egypte. On en voit des amas dans les peintures des hypogées de l'ancien et du nouvel empire. Ces fruits sont d'ordinaire peints en rouge ou jaune brun, parfois même en noir ${ }^{2}$. On les mangeait comme dessert. Il devait en être de même des fruits du Mimusops Schimperi ou perséa, qu'on rencontre aussi fréquemment dans les tombes pharaoniques. La présence des fruits du caroubier et du sébestier dans les tombes permet de supposer qu'ils entraient aussi dans l'alimentation; les baies du sébestier sont encore aujourd'hui employées contre la toux, et la pulpe des siliques du caroubier est comestible. Dans le Papyrus Anastasi IV il est fait mention de trente paniers de ouahh, nom hiéroglyphique de la caroube, et le grand Papyrus Harris mentionne un don, fait par Ramsès III aux divers temples d'Égypte, de 92,000 couffes de caroubes, une autre fois de 106,000 et de 21,000 mesures de darouga, autre nom, mais d'origine sémitique, du même fruit ${ }^{3}$. Il entrait donc en quantité considérable dans la consommation;

1. Franz Wœnig, op. laud., p. 316.

2. G. Maspero, Notes au jour le jour, III. (Proceedings of the Society of biblical Archaeology, t. XIII, p. 500.)

3. Victor Loret, Recherches sur plusieur's plantes, IX et X. (Recueil de travaux, t. XV.) 
mais il servait, ce semble, surtout à la nourriture des bestianx ${ }^{1}$.

Les jujubes - ar. nabaq, nabéca - jouaient encore un rôle plus grand dans l'alimentation des anciens Égyptiens. Ils les mangeaient fraîches ou sèches. On voit de véritables amas de ces fruits recherchés dans plusieurs peintures des mastabas de Saqqarah. Ils figuraient sur les tables funéraires, soit sous leur forme ordinaire, soit pétris en une espèce de gàteaux - tiou noubsou ou ta-nebs? ${ }^{2}$. - Pline dit que des fruits du prunier d'Égypte - le jujubier - on faisait une pàte estimée ${ }^{3}$. Athénée nous apprend comment on la préparait :

"Quand les fruits du jujubier, dit-il", sont arrivés à maturité, on les cueille; une partie écrasée avec de la farine est conservée dans des vases pour l'usage des serviteurs; le reste, dépouillé de son noyau, est préparé de la même manière et sert à la nourriture des maitres. On en retire aussi, ajoute le polygraphe grec, une espèce de vin. »

D'un usage non moins grand, sinon plus grand, que les derniers fruits dont je viens de parler, étaient ceux du grenadier. La culture de cet arbre prit une grande extension - les monuments en font foi - à partir de la dynastie des Ahmessides; la saveur agréable et les qualités rafraîchissantes des grenades expliquent l'importance de la consommation que l'on en faisait. Ces fruits figurent en quantité parmi les dons que Ram. sès III fit aux temples d'Égypte ${ }^{5}$. On mangeait d'ordi-

1. Franz Wœnig, op. laud., p. 334 et 345.

2. G. Naspero, Proceedings, t. XIII, p. 497.

3. Historia naturalis, lib. XIII, cap. 19.

4. Deipnosophistae, lib. XIV, cap. 65.

5. Grand Papyrus IIarris, pl. XVI, lig. 15 et 18. 
naire les grenades fraîches; mais on les faisait cuire aussi, et on fabriquait, dit-on, avec leur sirop épaissi, une épaisse de miel.

Les raisins, soit frais, soit séchés, entraient aussi en quantité considérable dans l'alimentation des Égyptiens. Sur les plus anciennes peintures on voit des corbeilles remplies de grappes de raisin. Ces fruits figurent souvent aussi parmi les offrandes funèbres ${ }^{1}$. Dans le Papyrus Harris ${ }^{2}$, il est fait également mention de dons nombreux de raisins offerts par Ramsès III aux trois grands sanctuaires pharaoniques.

Aux fruits dont je viens de parler, récoltés de temps immémorial dans la vallée du Nil, vinrent s'ajouter plus tard ceux du pommier, du mûrier, du noyer et de l'amandier, arbres acclimatés successivement dans l'Égypte ancienne, ainsi peut-être que le pêcher et le cerisier. Le pommier avait été importé déjà, nous le savons, à une époque reculée; les autres arbres n'y furent sans doute transplantés, s'ils le furent tous, - les noms même qu'ils portent en sont la preuve, qu'à l'époque grecque; j'incline à croire qu'on se borna à importer de la Syrie ou des iles de la Grèce les fruits du noisetier et du pin pignon, dont le premier même ne parait jamais avoir été cultivé en Égypte. Y importait-on aussi les fruits de la macre (Trapa natans L.); les récoltait-on aux bords du Nil, où d’après Pline ${ }^{3}$, croissait cette plante aquatique? Je ne saurais le dire; mais il existe $u$ fruit de macre au Musée du

1. Bulletin de l'Inslitut égyplien, $\mathrm{n}^{\circ} 5$ (an. 1884), p. 9 et no 6 (an. 1885), p. 260.

2. Zeilschrifl für aegyplische Sprache, t. Xl (an. 1873), p. $67,1.8$.

3. II sloria naluralis, lib. XXI, cap. 58. 
Lourre', ce qui montre que les Égyptiens commaissaient cette plante comestible et probablement se nourrissaient de ses fruits.

Ils connurent aussi, mais sans doute assez tard, le baobab (Adansonia digitala L.), cet arbre gigantesque des steppes de l'Afrique tropicale, dont les fruits atteignent la grosseur d'un petit melon. Ne dépassant pas le $14^{\circ}$ degré de latitude dans la valléc dı Bahr-el-Abiad, il s'élève dans celle du Bahrcl-Azrak jusqu'à Abou-Harras, à une quarantaine de lieues au sud de Kihartoum, et remonte vers l'est, jusqu’à Keren, presque à la hauteur du $16^{\mathrm{e}}$ degré, sans atteindre les bords de la mer Rouge ${ }^{2}$. Schweinfurth a signalé sa présence dans les jardins du Caire ${ }^{3}$; Prosper Alpin l'avait déjà rencontré en Égypte"; mais aucun écrivain de l'antiquité n'en a parlé. Il est peu probable aussi qu'il ait jamais été planté dans l'Égypte pharaonique. Il existe un fruit de baobab dans la collection égyptienne du Musée du Louvre $^{5}$ et trois, de diverse grosseur, dans celle du Musée de Turin ${ }^{6}$; mais on en ignore malheurcusement la provenance et l'époque. Il est probable qu'ils auront été importés par des caravanes venues d'Éthiopie en Égypte, où leur pulpe acidulée était peut-être re-

1. Recueil de travaux, t. XVII (an. 1895), p. 190.

2. Schweinfurth, Mittheilungen aus Justus Perthes' geographischer Anslall, an. 1868, p. 159 et pl. 9.

3. Illustration de la Flore d'Égyple, p. 53.

4. De plantis Aegypti liber, fol. 27.

5. Victor Loret et Jules Poisson, Études de holanique égyplienne. (Recueil de travaux, t. XVIl, p. 197.)

6. Dr. Ed. Bonnet, Le pianle egiziane del Museo reale di Torino. (Estrallo dal Nuovo giornale Bolanico Ilaliano, vol. II, no 1. Gennaio, 1895.) 
cherchée déjà autrefois, comme elle l'est aujourd'hui', à cause de ses propriétés alimentaires et médicinales.

Les anciens Égyptiens ne demandaient pas uniquement des aliments aux plantes; ils en retiraient encore des boissons variées: les principales étaient la bière, le vin de raisin, de dattes et de quelques autres fruits. La bière était faite avec de l'orge fermentée ${ }^{2}$ ou même germée ${ }^{3}$. Peut-être y ajoutait-on quelque amer, comme des lupins ou une espèce de siser - Sium sisarum L. ${ }^{4}$. - Cette boisson, don d'Osiris, ne le cédait, d'après Diodore $^{3}$, au vin ni en force ni en agrément. Il en est souvent question dans les offrandes; elle figure aussi au nombre des dons faits par Ramsès III aux temples des Dieux ${ }^{6}$.

La bière servait surtout de boisson aux basses classes; il en était de mème du vin de palmier. On préparait ce dernier, soit en faisant une incision dans le tronc du dattier, dont on recueillait dans un vase la sève qu'on laissait ensuite fermenter, soit, — procédé sans doute plus ordinaire, le premier entraînait la mort

1. Raffenau-Delile, Centurie de plantes d'Afrique, etc. (Fr. Cailliaud, Voyage à Méró, au fleuve blanc, etc. Paris, 1826, in-8, t. IV, p. 302.)

2. Hérodote, Historiae, lib. II, cap. 77, 4.

3. Bulletin de l'Institulégyptien, no 6 (an. 1885), p. 271 et 279.

4. Wilkinson, op. laud., t. I, p. 395.

5. Bibliotheca historica, lib. I, cap. 34, 10.

6. Papyrus Harris, pl. XVII, a, l. 14; pl. XVII, b, 1. 13; pl. XLIV, XLIX, etc. 
du dattier, - en faisant macérer dans l'eau les dattes arrivées à maturité et en exprimant ensuite le jus ${ }^{1}$.

Les anciens Egyptiens fabriquaient encore du vin ou des liqueurs avec d'autres fruits: grenades, jujubes, figues, caroubes, sébestes, etc. L'abus n'en paraissait pas moins funeste que celui du vin de raisin: "Abstiens-toi de liqueur de grenade, ne t'adonne pas à la liqueur de figue, ignore la liqueur de caroube ", lit-on dans le Papyrus Anastasi IV ${ }^{2}$. Il est fait mention, parmi les produits d'un jardin de Ramsès II, d'une espèce de sirop, appelé shedeh-it; M. Victor Loret croit qu'il était fait avec des grenades ${ }^{3}$. Une liqueur faite arec des figues ou des grenades figure dans la liste des offrandes, donnée par une inscription de la tombe de Rekhmara, préfet de Thoutmès III ${ }^{4}$. Athénée a donné la recette de la préparation du vin de jujubes $^{5}$. Pour le faire on laissait macérer ces fruits dans l'eau, puis on les écrasait et on exprimait le jus. Le vin ainsi obtenu, dit le polygraphe grec, était d'un goût aussi doux et agréable que le meilleur moût. On le buvait sans eau; mais il ne se conservait pas plus de dix jours. On le préparait aussi de temps à autre, suivantle besoin qu'on en avait. On faisait également, ajoute Athénée, du vinaigre avec ces fruits, comme on en fabriquait d'ailleurs avec les dattes ${ }^{6}$ et sans doute avec les grenades.

1. Pline, Historia naturalis, lib. XIV, cap. 19, 3.

2. Victor Loret, Recherches, etc., XII. La caroube. (Recueil de (ravaux, t. XV).

3. La Flore pharaonique, p. 77, no 131.

4. Philippe Virey, op. laud., p. 104.

5. Deipnosophistae, lib. XIV, cap. 65.

6. Xénophon, Anabasis, lib. II, cap. 3, 15, parle du vinaigre fait avec les dattes. 
A part la bière, les divers breuvages dont je viens de parler étaient sans grande importance dars la consommation égyptienne; il n'en était pas de même du vin de raisin. Les nombreuses représentations, que les peintures des hypogées de Béni-Hassan, Zaouïet-elMaïétin, Karnak, Abd-el-Gournah ${ }^{1}$, nous ont laissées de la fabrication du précieux liquide, sont la preuve évidente de l'extension qu'en avait pris l'usage dès l'époque la plus reculée et du prix qu'on y attachait. Elles nous permettent de suivre les diverses scènes de la vendange, telle qu'on la faisait il y a près de cinq mille ans.

Après les avoir cueillis, on portait les raisins dans une auge en bois, où ils étaient foulés aux pieds. Le jus qui en découlait était reçu dans des cures; pour extraire celui qui restait dans les grappes on pressait le marc dans une espèce d'outre ou de poche en fil de laine au moyen de leviers attachés aux deux extrémités. Lorsque le vin recueilli dans les cuves avait cessé de fermenter, on le rersait dans des jarres ou amphores que l'on bouchait et scellait; puis on les rangeait dans les celliers. Un serviteur spécial paraît avoir été chargé de cette importante fabrication; il est question dans le Papyrus Anastasi d'un chef des vignobles, dans les caves duquel se trouvaient de larges provisions de vin ${ }^{2}$.

Les Egyptiens distinguaient le vin blanc âbesh du

1. Champollion, Monuments, t. IV, pl. 380 et 389. - Rosellini, Monumenticivili, t. II, pl. XXXVII et XXXVIII.- Lepsius, Denkmüler, t. 1II, pl.111, a. Zaouiet-el-Maïétin, t. 14. - Wilkinson, op. laud., t. I, p. 385. - Franz Wœnig, op. laud., p. 263.

2. IV, pl. 7, 1. 3, ap. Franz Wwenig, op. laud., p. 270. 
vin rouge teshr, et ils avaient des crus très différents et quelques-uns renommés. Rosellini avait déjà mentiomé un certain nombre de vins égyptiens ; M. Victor Loret en a relevé dix espèces différentes, la plupart connues dès l’époque des pyramides ${ }^{2}$. On ne peut s'expliquer aussi qu'Hérodote ait dit que l'Égypte ne produisait point de vin et qu'avant Psammétique les habitants de ce pays n'en buvaient pas et n'en faisaient point d'offrandes ${ }^{3}$. Les écrivains postérieurs furent mieux renseignés. Suivant Strabon ${ }^{4}$, le nome d'Arsinoë produisait du vin en grande quantité; la région maréotique en produisait davantage encore et qui se conservait très longtemps. La couleur, dit A thénée ${ }^{5}$, en était pâle, la qualité excellente; il était doux et léger, avec un bouquet agréable; il n'était nullement astringent et ne portait point à la tête.

Le sophiste grec mettait néanmoins le vin de Ténia au-dessus de celui de la Maréotique; il était plus riche en alcool, son bouquet avait quelque chose d'aromatique et il était légèrement astringent. A en croire Clément d'Alexandrie ${ }^{6}$, le vin de Mendès était aussi renommé. Mais il y avait, remarque Apulée, bien d'autres crus dans la vallée du Nil, dont les produits réputés différaient à la fois par le goût et la couleur. Et il ajoute que le vin qu'on récoltait à Antylla était préféré à tous les autres. Quelques-uns des vins de la Thébaïde étaient recherchés pour leur légèreté, en

1. Monumenti civili, t. I, p. 377.

2. La Flore pharaonique, p. 101, no 167.

3. Historiae, lib. III, cap. 6.

4. Geographica, lib. XVII, cap. 1, 14.

5. Deipnosophislae, lib. I, cap. 60 (33).

6. Paedagogus, lib. II, cap. 2 (68). 
particulier celui de Coptos, "si salubre, dit Athénée, que les malades pouvaient en boire impunément, même pendant la fièvre. " Pline vante aussi le sebennytique, "fourni, dit-il ${ }^{1}$, par trois espèces de raisin, le thasien, l'æthale et le peucé. ".

Les Égyptiens faisaient une consommation importante de vin; l'État en accordait chaque jour une ration déterminée aux guerriers et aux prêtres ${ }^{2}$. On voit, preuve évidente du prix qu'on y attachait, Ramsès III en faire don de quantités énormes aux divinités de Thèbes, de Memphis et d'Héliopolis" ${ }^{3}$. Dans les réunions et les fêtes, on offrait, les monuments en font foi, du vin à tous les hôtes, hommes ou femmes. C'était surtout après le repas qu'on le buvait, et on ne parait pas en avoir usé toujour's avec modération. Une peinture de Béni-Hassan " nous montre des maitres que leurs esclaves rapportent ivres morts chez eux. A en juger par d'autres peintures de Thèbes, qui nous les montrent chancelantes et arrivées au dernier période de l'ivresse ${ }^{5}$, les dames elles-mêmes ne se faisaient pas faute de boire du vin avec excès.

\section{II.}

Outre les boissons proprement dites, les Égyptiens

1. Historia naturalis, lib. XIV, cap. 9 ( () .

2. Vilkinson, op. laud., t. I, p. 391.

3. Papyrus IIarris, pl. XVII, $b, 1.13$, tr. by Birch: "Wine amphorae 39510. " (Zeitschrift fïr aegyptische Sprache, t. XI (an. 1873), p. 65.)

4. Wilkinson, op. laud., t. I, p. 394, no 169. Cf. "Lettre au scribe Pentaour ", F. Chabas, Mélanges égyptologiques. $3^{\text {e }}$ série, t. II, p. 86.

5. Wilkinson, op. laud., t. I, p. 392 et 393, nos 167 et 168. 
demandaient aussi au règue végétal l'huile nécessaire non seulement à leur alimentation, mais à l'éclairage de leurs maisons et à la fabrication des parfums. J'ai parlé de la culture des principales plantes dont les fruits ou les graines leur servaient pour cet usage; celles auxquelles ils paraissent avoir eu recours le plus anciennement furent le sésame, le ricin ${ }^{1}$, ainsi peut-être que le carthame et le moringa; ils ne durent faire usage que plus tard de l'huile tirée des fruits de l'olivier, comme de celle des graines du radis, dont nous ne comnaissons d'ailleurs l'emploi que par le témoignage de Pline ${ }^{2}$; le polygraphe latin ${ }^{3}$ mentionne aussi l'huile de chortine que donnait une herbe dont il n'indique pas le nom, et celle de cnédine, extraite d'une espèce d'ortie.

Pour fabriquer l'huilé de sésame, on mettait d'abord les graines à macérer dans l'eau chaude *; on les étendait sur une table, on les frottait, puis on les plongeait dans l'eau froide pour faire surnager les pailles; on exposait alors les graines ainsi nettoyées au soleil et, une fois sèches, on les portait au moulin. L'huile de sésame était, il semble ${ }^{5}$, employée à la fois dans la cuisine et pour l'éclairage; inférieure comme assaisomnement, on la regardait cornme la meilleure huile à brûler ${ }^{6}$; elle était fort chère dans l'antiquité.

1. Pline, mais son autorité est ici, comme si souvent ailleurs, sans valeur aucune, dit que, l'huile de ricin n'était connue que depuis peu de temps en Égypte. Lib. XV, cap. 7.

2. Historia naturalis, lib. XIX, cap. 26.

3. Historia naturalis, lib. XV, cap. 7.

4. Pline, op. laud., lib. XVIII, cap. 23, 2.

5. Dioscoride, De maleria medica, lib. II, cap. 121, dit que les Egyptiens s'en servaient, mais sans indiquer au juste pour quel usage.

6. Wilkinson, The manners and cusloms, t. 11, p. 399. 
Les Égyptiens se servaient aussi beaucoup plus de l'huile de ricin, qui coûtait deux fois moins ${ }^{1}$. La préparation de cette dernière a été décrite par Hérodote. "Après avoir recueilli-les graines de ricin, dit-il" ${ }^{2}$, on les écrase et on exprime l'huile; d'autres fois aussion les fait bouillir et on recueille alors l'huile qui surnage ${ }^{3} »$. Cette huile est grasse, remarque-t-il encore, et non moins bonne pour l'éclairage que celle d'olive; mais elle répand une odeur désagréable; elle n'en était pas moins recherchée; Strabon rapporte que " presque tous les habitants en faisaient usage ", et il ajoute que "les hommes des classes pauvres et les ouvriers s'en oignaient même les membres ". On s'en servait aussi, on l'a vu par l'inscription d'Apriès mentionnée plus haut $^{5}$, pour l'éclairage des temples.

L'huile de sésame et celle de ricin étaient les deux espèces d'huile les plus communes à l'époque pharaonique; tout en restant très employées, elles cédèrent néanmoins par la suite en partie la place à d'autres huiles qui, quoique beaucoup moins répandues dans l'antiquité, finirent, dans les derniers temps de la domination égyptienne, par entrer dans la consommation.

1. Eugène Revillout, La valeur de l'huile. (Revue égyptologique, t. II (an. 1882), p. 162.)

2. Historiae, lib. II, cap. 94.

3. Pline, lib. XV, cap 7, 2, indique les deux procédés: suivant Dioscoride $(1,38)$, les Égyptiens, après avoir lavé les graines de ricin, Ies moulaient avec soin, puis ils en exprimaient l'huile à l'aide du pressoir.

4. Geographica, lib. XVII, cap. 2, 5.

5. Page 46: "En l'an 30, le 4 de Thot, dit une autre ins. cription, en la main du dieu Imouth et de la grande déesse
Astarté, don d'un kesro d'huile de tekem pour le Iuminaire. " Astarté, don d'un liesro d'huile de tekem pour le luminaire. "
Eugène Revillout, L'antigraphe des luminaires. (Revue égyptologique, t. II (an. 1882), p. 179.) 
Lor'sque l'olirier eut été importé en Égypte, il servit, malgré sa rareté relative, à faire une huile qui fut bien rite appréciée.

Plus importante encore peut-être fut la fabrication de l'huile fournie par l'arbre baq, et plus ancienne aussi, car il en est fait mention dans les documents de l'époque la plus reculée. Cette huile estimée était extraite des fruits du Moringa - les noix de ben; - elle portait le nom de baqi'. On en distinguait deux espèces, le báqi rouge et le baqi vert, ce qui concorde avec ce que Pline dit de l'huile du myrobalan ${ }^{2}$, laquelle, d'après lui, était rouge en Égyte, verte en Arabie.

Non moins recherchée parait avoir été l'huile de carthame; on cultivait surtout cette composée, dit Pline ${ }^{3}$, pour l'huile qu'on retirait de ses graines. Suivant Dioscoride 4 , on la préparait en les écrasant, après les avoir préalablement lavées. On procédait, sans doute, de même pour obtenir l'huile d'ortie ou cnédine, celle de chortine et l'huile de radis.

Outre l'huile, on demandait au règne végétal le tanin usité, comme aujourd'hui, pour la préparation des peaux, la gomme et les résines d'un emploi si fréquent, enfin les matières colorantes. La gomme était produite par diverses espèces d'acacias ${ }^{5}$, en particulier par celui du Nil; on se bornait le plus souvent à la recueillir sur le tronc de l'arbre; mais pour que la récolte füt plus abondante on $y$ pratiquait une incision ${ }^{6}$. Les 1. Victor Loret, Recherches sur quelques plantes, II. (Recueil
de travaux, t. VII.)

2. IIistoria naturalis, lib. XII, cap. 46.

3. Historia naturalis, lib. XXI, cap. 53.

4. De materia medica, lib. I, cap. 44.

5. Pline, IIstoria naluralis, lib. XXIV, cap.

6. Théophraste, IIisloria planlarum, lib. IV, cap. $2,8$. 
résines et gommes-résines étaient fournies par le lentisque, le térébinthe, le pin d'alep, le cèdre et diverses burséracées exotiques, dont il sera question dans le chapitre de la pharmacopée.

Les longues gousses de l'acacia du Nil fournissaient, ainsi que l'écorce de l'acacia seyal, un excellent tanin ${ }^{1}$. On retirait surtout les matières colorantes végétales du carthame et de l'indigo ${ }^{2}$; c'est avec ces substances qu'étaient presque exclusivement teintes les étoffes ${ }^{3}$; mais nous ignorons comment on les préparait. On parait avoir retiré aussi une manière colorante du genévrier", et les feuilles broyées du henné donnaient une couleur jaune orange recherchée; les Égyptiens s'en servaient, on l'a reconnu à l'examen de quelques momies, pour se rougir la paume des mains. Dioscoride nous apprend ${ }^{5}$ qu'à l'aide de la même poudre diluée dans du suc de saponaire, les dames égyptiennes se teignaient les cheveux en blond. Quant à l'encre, on la fabriquait avec diverses plantes et divers fruits - figues, dattes — réduits en charbon et délayés dans de l'eau avec de la myrrhe ${ }^{6}$.

Nous ne savons pas à l'aide de quels procédés les Égyptiens nettoyaient les tissus; la découverte, dans la

1. Wilkinson, op.laud., t. II, p. 417, dit, mais sans s'appuyer sur aucun témoignage authentique, que l'écorce du Rhus oxyocanlhoüdes L. servait au même usage.

2. Chapitre II, paragraphe III, p. 49-52.

3. Nois Riegl, Die ügyplischen Texlilfunde im l.k. öslerreich. Museum, Wien, 1889, p. Ix, ne parle pas du carthame, mais cite la garance, dont il n'est, à ma connaissance, fait mention dans aucun texte égyptien.

4. H. L. E. Lüring, Die über die medicinischen lienntnisse der allen Aegypter berichtenden Papyri, p. 16x.

5. De maleria medica, lib. I, cap. 124.

6. H. L. E. Lüring, op. laud., p. !02. 
collection Passalacqua', d'un fruit de Sapindus emarginatus Vahl, fruit qui sert dans l'Inde à transformer l'eau on une émulsion savonneuse et à nettoyer les éloffes précieuscs, pourrait faire croire que les Égyptiens des derniers temps l'importaient de son pays d'origine et s'en servaient aux mèmes usages.

III.

Le règne végétal ne fournissait pas seulement aux anciens liabitants de la vallée du Nil les aliments les plus indispensables, ainsi que des boissons salutaires ou l'huile nécessaire à leur usage, ils lui demandaient aussi des textiles pour confectionner les étoffes dont ils se rêtissaient, les matières fibreuses qui servaient à faire divers ustensiles de ménage, le bois avec lequel ils construisaient leurs bateaux et fabriquaient les meubles de leurs maisons, ainsi que leurs outils ou leurs instruments aratoires.

La plante textile par excellence des anciens Égyptiens était le lin; il était cultivé en grand dans la vallée du Nil, dont le sol léger et friable lui convenait at merveille. Les peintures de Kom-el-Ahmar et de Béni-Hassan ${ }^{2}$ nous font assister à sa récolte, ainsi qu'aux diverses transformations qu'il subissait avant d'ètre employé par l'industrie. L'artiste pharaonique nous montre d'abord des ouvriers occupés à arracher' celte précieus e plante et à la mettre en bottes; d'autres la portent dans des réservoirs préalablement remplis

1. A. Braun, Die Pflanzerreste. (Zeilschrift für E'lhnologie, t. IX, p. 307).

2. Rosellini, Monumenti civili, t. II, pl. 35. - Lepsius, Dentimäler, t. IV, pl. 127. 
d'eau, où on la rouit; on l'en retire, on la sèche, on la bat sur une table, avec des maillets en bois arrondis pour la désagréger ${ }^{1}$. Les tiges ainsi écrasées et débarrassées des fibres inutiles étaient remises à des femmes qui les filaient. Les fils simplement tordus ou, quand on voulait des qualités plus fines, "roulés " et "lissés» sur une large pierre, étaient mis en pelotons ou écheveaux ${ }^{2}$. On les livrait enfin aux hommes ou aux femmes, qui, au nombre de un à quatre par pièce, les tissaient sur un métier à main quelquefois vertical, le plus souvent horizontal, en poussant d'ordinaire la trame, remarque Hérodote ${ }^{3}$, non en haut, mais en bas.

Dès la plus haute antiquité, le tissage avait été porté en Égypte à une grande perfection. Pline ${ }^{4}$ attribue l'honneur de l'avoir inventé aux habitants mêmes de ce pays. Les étoffes trouvées dans les to mbes pharaoniques ont le tissu serré et ferme, en même temps que très élastique ${ }^{5}$; les fils en sont remarquablement unis, réguliers et d'une souplesse qui peut rivaliser avec la soie. Les toiles et les étoffes de lin d'Égypte étaient renommées dans l'antiquité et faisaient l'objet d'un commerce considérable avec l'étranger. Pour leur donner plus de force, on réunissait ensemble un grand nombre de brins. Pline rapporte ${ }^{6}$ que la cuirasse d'Amasis, conservée dans le temple de Minerve à Rhodes, était faite de fils composés de trois cent

1. Wilkinson, op. laud., t. II, p. 173.

2. Champollion, Monuments, t. IV, pl. 366. - Wilkinson, op. laud., t. II, p. 170.

3. Historiae, lib. II, cap. 35.

4. Historia naturalis, lib. VII, cap. 57 (56).

5. Wilkinson, op. laud., t. II, p. 161.

6. Historia naturalis, lib. XIX, cap. 2 (1). 
soixante-cinq fibres. On arrivait par ce moyen à fabriquer des étoffes, des toiles à voiles, des filets, etc., d'une très grande solidité.

Les étoffes de lin étaient souvent employées sans apprèt ou teinture; non moins souvent aussi on les teignait; le carthame fournissait toutes les nuances du rouge, depuis le rose jusqu'au rouge pourpre'; l'indigo la couleur bleue. L'analyse chimique a démontré l'origine végétale de ces deux conleur's ${ }^{2}$. Mais, grâce à l'action des mordants variés, les Égyptiens obtenaient, nous dit Pline $^{3}$, avec la mème matière colorante, les nuances les plus diverses.

On faisait avec le lin non seulement des étoffes et des toiles, mais encore des cordages. Ces derniers étaient parfois aussi fabriqués avec des fibres de papyrus. Hérodote nous apprendt que les Égyptiens attachèrent avec des câbles en papyrus les bateaux qui servirent à faire le pont gigantesque que Xerxès jeta sur l'Hellespont. Le papyrus servait également à divers travaux de sparterie. On en faisait en particulier des sandales; la plupart des musées égyptiens d'Europe en renferment quelques-unes ${ }^{5}$. Ramsès III fit don de 15,110 paires de sandales de papyrus aux divinités de Memphis ${ }^{6}$. On fabriquait aussi avec le papyrus des corbeilles, des nattes, des tapis, miême des toiles à

1. Bulletin de l'Institut égyptien, n० 3 (an. 1882), p. 70.

2. Wilkinson, op. laud., t. II, p. 163. - Unger, Sitzungsberichte, t. XXXVIII, p. 113.

3. IIisloria naturalis, lib. XXV, cap. 42.

4. Hisloriae, lib. VII, cap. 3't.

5. Wilkinson, op. laud., t. II, p. 335. - Franz Wœnig, op. laud., p. 82.

6. Papyrus Harris, tab. XIX b, 1. 3. (Zeitschrifl fur aegyptische Syrache, t. XI, p. 70.) 
voiles', ainsi que des barques, en particulier des barques funéraires.

D'après une tradition rapportée par Plutarque ${ }^{2}$, ce fut sur une barque de papyrus qu'Isis se mit à la recherche du corps d'Osiris au milieu des marécages de l’Égypte. La nacelle dans laquelle fut exposé Moïse était en papyrus enduit de poix ${ }^{3}$. Dans une de ses malédictions contre l'Egypte, Isaïe fait allusion à l'usage dans ce pays de barques en papyrus". La construction de ces barques est souvent représentée dans les peintures tombales de l'ancien et du moyen empire. On voit les ouvriers descendre vers le fleuve pour y ramasser les tiges de la précieuse plante ${ }^{5}$; une partie de ces tiges étaient réunies ensemble de manière à former une sorte de caisse pointue et recourbée aux deux bouts; c'était le corps du canot. Arec le reste, on fabriquait des cordes destinées à lier l'arant, l'arrière. et le milieu de la coque ${ }^{6}$.

Bien plus encore qu'à faire des ouvrages de sparterie ou de corderie, le papyrus servait à fabriquer du papier. La matière première était fournie par les pellicules concentriques très minces qui forment au-dessous de l'écorce la partie extérieure de la tige rectangulaire de cette cypéracée ${ }^{\top}$. On détachait ces pellicules

1. Odyssée, chant xxI, v. 392. - Théophraste, Hisloric plantarum, lib. IV, cap. 9.

2. De Iside el Osiride, cap. xvin, p. 29.

3. Victor Loret, La Flore pharaonique, p. 28, no 28. - Wilkinson, op. laud., t. Il, p. 206, dit qu'elle était en jonc.

4. Cap. xviII, vers. 2.

5. Lepsius, Denlimïler, t. III, pl. 12. - Franz Wonig, op. laud., p. 57. - G. Maspero, Éudes égypliennes, p. 105.

6. Wilkinson, op. laud., t. II, p. 208.

7. Pline, IIistoria naturalis, lib. XIII, cap. 23. 
en battant doucement la tige, puis on les étendait sur une table préalablement mouillée, en ayant soin de les couper par bandes de 20 i 30 centimètres de long sur 5 ì 6 de large; on rémissait ensuite, à l'aide de colle, dans le sens de la longueur, un certain nombre de ces bandes, on les faisait sécher et on avait ainsi une feuille de papier.

Si le papyrus servait seul à faire du papier, bicin d'autres plantes égyptiennes étaient employées dans la fabrication des différents objets de sparterie et de rannerie. Tel était l'Eragrostis cynosuroüdes R. (Leplochloa bipinnata $\mathrm{H}$.) dont on faisait des corbeilles. On a trouvé dans la tombe d'Ani ( $\mathrm{XX}^{\circ}$ dynastie) ${ }^{\prime}$ une soixantaine de petits paniers tressés avec cette graminée très commune en Ėgypte, où clle est désignée de.nos jours sous le nom de halfa. Tel était encore le jonc (Juncus acutus L.) - asir. - Il y a, d'après M. Maspero ${ }^{2}$, au Musée de Boulaq, un panier en jonc tressé de diverses couleurs, qui remonte à la $\mathrm{XI}^{\circ}$ dynastie. Il existe au Musée de Leyde des corbeilles et des mannes en jonc tressé ${ }^{3}$. "Fais donner aux fabricants de couffes, lit-on dans le Papyrus Anastasi IV ${ }^{4}$, la matière première, à savoir des roseaux - gasha et des joncs - asir. "

M. Victor Loret m'apprend que la plante gash, dont

1. Schweinfurth, Bulletin de l'Institut égyptien, no 7 (an. 1886), p. 419.

2. Guide du visiteur au Musée de Boulaq. Boulaq, 1883, in-16, p. 115.

3. C. Leemans, Aegyptische Monumenten van het nederlansche Iuseum van Oudheden te Leyden. Leyden, 18ł6, in-fol., pl. LXXVII.

4. Victor Loret, Pecherches sur quelques plantes, XII. (Recueil de lravaux, t. XVI.) 
il est ici question, est la canne à sucre d'Égypte (Saccharum aegyptiacum Willd.); cette graminée était, donc en usage dans la vannerie. Les feuilles du roseau proprement dit (Arundo donax L.) et sans doute aussi du roseau d'Isis (Phragmites Isiaca Del.) entraient dans la fabrication des nattes. On employait au même usage les chaumes fendus en deux et tressés ensemble d'une espèce de souchet à haute tige le Cyperus alopecuroïdes. - M. Maspero a trouvé à Gébéleïn, dans le cercueil de la princesse NesiKhonsou ${ }^{1}$, une sorte de natte ou plutôt de store, faite avec cette plante et qui " servait de remplissage pour arrondir les formes de la momie ». Avec les tiges du roseau commun - le nabi — on fabriquait aussi des flèches, des tuyaux, des treillages. Les tiges du roseau d'Isis, elles, servaient à faire des calames à écrire; on en faisait également avec les tiges de la canne à sucre d'Égypte ${ }^{2}$. Avec celles de la Ceruana pratensis Forsk. - le chédite des Arabes - plante de la famille des composées, caractéristique de la flore nilotique, elle croît exclusivement sur les bords du fleuve, - on fabriquait, comme aujourd'hui, à l'époque pharaonique, des balais destinés à des usages vulgaires; on en voit un au Musée de Boulaq, ainsi qu'au British Museum $^{3}$.

Les feuilles des différents palmiers n'étaient point d'un moindre usage dans l'industrie du vannier que les

1. G. Schweinfurth, Bulletin de l'Institut égyptien, no 7 (an. 1886), p. ${ }^{4} 26$.

2. Victor Loret, La Filore pharaonique, p. 19 et 24 , nos 6, 7 et 20 .

3. G. Schweinfurth, Bulletin de l'Institut égyptien, no 5 (an. 188́), p. 8. 
cypéracées ou les graminées. On faisait arec les feuilles du palmier doum - le mama des textes hiéroglyphiques, - Théophraste nous l'apprend ${ }^{1}$, diver's ouvrages de sparterie; il existe au muséc de Florence des sandales fabriquées avec les feuilles de cet arbre ${ }^{2}$. Celles du dattier étaient encore plus usitées; avec le rachis - bai - on faisait des cannes ${ }^{3}$; leurs folioles outou ou witou, en copte bî - servaient non seulement à confectionner des sandales ${ }^{4}$, mais encore des cordages, des nattes et des corbeilles, usage qui s'est conservé jusqu'i nos jours ${ }^{5}$. Les filaments qui naissent i la basc des fouilles ou palmes - les shou (nou) bennou " cheveux de dattier" - étaient employés, comme nos brosses de chiendent, pour nettoyer les objets peu fragiles, tels que les cornes et les sabots des taureaux, destinés à être sacrifiés. "Qu'on lui lave la tête, dit, en parlant de l'un deux, l'inscription d'Osiris à Dendérah ${ }^{6}$, et qu'on nettoie ses sabots avec les fibres du dattier. ")

Je n'en ai pas fini avec l'énumération des nombreux usages que l'industrie des habitants du pays de Kimit, faisait du règne végétal; il me reste à parler de l'em-

1. Historia plantarum, lib. IV, cap. 27.

2. Migliarini, Calalogue, p. 57, no 2703.

3. Grand Papyrus IIarris, 65, 9 et 74, 9.

4. Il en existe plusieurs au Musée de Leyde. C. Leemans, op. laud., pl. XXX, 19, 20, 22, 23.

5. Victor Loret, Recherches, XIV. "La fronde et la feuille du palmier. " (Recueil de travaux, t. XVI. )

6. Dümichen, Recucil, t. IV, p. 90, ap. Ch. Moldenke, op. laud., p. 47. 
ploi des diverses espèces de bois dans les différents arts ou métiers. Quoique peu variée et peu riche, la flore arborescente de la vallée du Nil offrait néanmoins aux habitants les matériaux dont ils avaient besoin pour leurs constructions. Leurs premières demeures furent probablement semblables à celles qu'on rencontre encore de nos jours en Nubie '; c'étaient des cabanes formées et recouvertes de branches ou de troncs d'arbres, reliés entre eux par des tiges de roseau ou d'autres graminées. Mais ces huttes grossières ne durent pas tarder à faire place à de réritables maisons.

Dès la plus haute antiquité, Jes Égyptiens eurent des habitations en pierres ou en briques, mais ils continuèrent en mème temps d'en avoir en bois ${ }^{2}$; malheureusement il ne nous reste rien de ces dernières, et nous ne pouvons que nous faire une idée approximative de ce qu'elles purent être. Ce n'étaient plus les huttes grossières des premiers âges, mais des maisons cons. truites suivant les règles de l'art du charpentier et du menuisier. Dès longtemps cet art était pratiqué en Égypte. Sur les monuments de l'ancien empire, on voit souvent représenté l'abattage d'arbres, sycomores ou palmiers, destinés aux travaux de charpente ou de menuiserie ${ }^{3}$. Une des peintures du tombean de $\mathrm{Ti}$ nous fait assister aux diverses scènes du travail du bois ${ }^{4}$. Sur un des bas-reliefs d'Abd-el-Gournah on voit deux

1. Perrot et Chipiez, Histoire de l'art dans l'antiquile, t. I, l'Égypte, p. 507.

2. Perrot et Chipiez, op. laul., p. 107 et 116.

3. Lepsius, Denlmäler, t. III, pl. III, tombe 146 . Zaouiet-elMaiétin, VIe dynastie.

4. Musée Guimet, tableau V. 
ouvrier's, dont l'un est occupé à fendre un trone d'arbre i l'aide d'une scie, tandis que l'autre débite une bille de bois'. Une peinture de l'époque de Thoutmès III nous per'met de pénétrer dans un atelier d'ouvriers en bois et de nous rendre compte du degré de perfection quarait atteint le travail de l'ébénisterie sous les Pharaons du nouvel empire?

Il ne s'agit plus seulement de débiter des poutres et des solives pour les maisons en bois ou en briques, des planches pour les cercueils, véritables monuments, " dont la construction mettait en branle une escouade d'ourriers ${ }^{3}$ "; ce qu'on demande surtout désormais, ce sont des coffres, des lits, des tables, des fauteuils, des divans, des. statues mêmes qui doivent meubler les palais ou les tombes; car les morts avaient leur mobilier comme les vivants. Il fallait du bois pour ces meubles si variés; il en fallait encore pour les ustensiles de ménage et les instruments agricoles, pour la fabrication des char's de guerre ou la construction des vaisseaux de transport, qu'on ne pouvait songer à faire en tiges de papyrus, enfin pour les statues, qui n'étaient pas en pierre ou en métal. Naturellement on ne se servait pas des mèmes essences végétales pour tous ces travaux et on n'en employait pas d'exclusivement indigènes; dans la suite du temps on eut recours à des espèces exotiques ou devenues étrangères à la flore nilotique, comme le cèdre et l'ébène; parmi les arbres indigènes, le sycomore, l'acacia du

1. Champollion, Monuments, t. Il, pl. 164. - Rosellini, Monumenti, t. II, pl. 43.

2. Prisse d'Avennes, IIisloire de l'art égyplien d'après les Monumenls, texte de P. Marchandon de la Faye, p. 321.

3. G. Maspero, L'Archéologie égypticnne, p. 270. 
Nil et l'acacia seyal, puis le dattier et le mama, fournissaient les hois les plus usités.

Théophraste regardait le bois du sycomore comme un des plus utiles; mais il fallait, avant de l'employer, le soumettre à une préparation prarticulière. "On le coupe vert, dit-il ${ }^{1}$, et, fait digne de remarque, on le plonge alors dans une pièce d'eau ou réservoir pour le faire sécher; il va d'abord au fond, mais lorsqu'il a perdu sa sève, il surnage et on peut alors s'en servir. » Le bois de sycomore était noueux et difficile à trarailler; mais il n'en était pas moins recherché en Egypte et, gràce à sa dureté et à son peu de corruptibilité, ainsi qu'à sa fréquence dans ce pays, il rendait les plus grands services à l'industrie. Charpentiers, menuisiers, ébénistes, tourneurs s'en servaient à l'envi: stèles $^{2}$, images des dieux, statues funéraires, figures d'animaux, amulettes, ustensiles de ménage et outils divers, instruments aratoires, coffrets, cercueils surtout, étaient fabriqués avec le bois de cet arbre utile. "Le cercueil d'Osiris Khent-Ament est fait en sycomore ", lit-on au paragraphe 43 d'une inscription du temple d'Osiris à Dendérah ${ }^{3}$. Et de mème aux paragraphes 44 et 45 : " Le coffre de Khent-Ament... Le coffre des bassins de Sep est l'ait en sycomore. "

La durée naturelle du bois d'acacia, la gomme qui en découle et le rend presque incorruptible, lui donnaient

1. Historia plantarum, lib. IV, cap. 2, 2.

2. La stèle de la princesse Nesi-Khonsou à Deir-el-Bahari était en bois de sycomore. Am. B. Edwards, Relics from the tombs of the priest-kings at Dayr-el-Baharee. (Recueil de travaux, t. IV (an. 1882), p. 81.)

3. Victor Loret, Les fêtes d'Osiris au mois de Khoiak. (Recucil de travaux, t. III (an. 1882), p. 56.) 
un prix particulier et le faisaient employer de préférence pour les travaux qui demandaient un bois résistant, en particulier pour la construction des bateaux.

"Les barques des Égyptiens, dit Hérodote ${ }^{1}$, celles du moins qu'ils emploient pour porter les fardeaux, sont faites avec un arbre épineux qui ressemble beaucoup au lotus de Cyrène ${ }^{2}$, et dont il sort une larme qui se condense en gomme. Ils tirent de cet arbre des planches d'environ deux coudées; ils les agencent de la même manière qu'on dispose les briques et les attachent avec des chevilles fortes et longues; ils placent sur leur surface des solives, sans se servir de varangues, ni de côtés; mais ils affermissent en dedans cet assemblage avec des liens de papyrus; ils font ensuite un gouvernail qu'ils passent ì travers la carène, puis ụn mât avec l'arbre et des voiles avec le papyrus. »

Tous les bateaux égyptiens n'étaient pas faits de cette facon; " le plus souvent mème ${ }^{3}$, les planches longues et minces comme celles dont on se sert aujourd'hui étaient assemblées selon les procédés que nous employons à la fabrication de nos navires. » Mais cela importe peu ici, ou il n'est question que de la matière qui entrait dans leur construction. C'était, on le voit, le bois d'acacia qu'on employait au temps d'Hérodote, dans la fabrication des bateaux de transport ou des vaisseaux de guerre. Les monuments montrent qu'il en fut ainsi depuis l'époque des Pyramides jusqu'à celle des Ptolémées et au delà. Dans un document publié d'abord par M. de Rougét, Ouna, officier des rois Téti, Pépi I et Pépi II, nous apprend qu'il

1. Historiae, lib. II, cap. 96, 1.

2. Le Rhamnus spina-Christi.

3. G. Maspero, Eludes égyptiennes, t. I, 2e fascicule, p. 102.

4. Recherches sur les monuments qu'on peut altribuer aux six premières dynasties de Manéthon. Paris, 1866, in-4, p. 117 et suivantes. - Charles Moldenke, op. laud., p. 77. 
avait construit pour son maître, en dix-sept jours, "un grand bateau en bois de shent qui avait soixante toises de long sur trente de largeur ".

La consommation que l'on faisait du bois d'acacia pour les constructions navales était telle que les forêts égyptiennes finirent par ne plus suffire et on fut obligé d'en demander à l'étranger. "Le roi, dit le mème Ouna que je viens de citer ', m'a envoyé essarter cinq districts pour construire cinq gros vaisseaux et quatre plus petits en bois de shent du pays d'Ouaoua ${ }^{2}$. "Le Papyrus Anastasi IV, contemporain des Ramessides, nous fait connaitre l'ordre donné pour réparer une barque sacrée, dont plusieurs planches d'acacia s'étaient pourries par un séjour trop prolongé dans l'eau et pour les remplacer par des ais plus longs ${ }^{3}$. Et le Papyrus Harris fait mention à plusieurs reprises de grands et de petits bateaux fabriqués en bois de shent4, ainsi que de livraisons importantes de ce bois de charpente précieux.

Le bois de shent ou d'acacia n'était pas utilisé seulement pour les constructions navales; on l'employait a bien d'autres usages; il servait en particulier à faire les pylones des temples et les portes des édifices privés, des poutres et des piliers, des meubles précieux, des cassettes sacrées, des cercueils et des statues, ainsi que des chevilles et même des peignes. "Les pilier's

1. Charles Moldenke, op. laud., p. 78.

2. La partie de la Nubie située à l'est du Nil.

3. Charles Moldenke, op. laud., p. 79.

4. P1. 12 b, 1. 11. "Acacia barges. " Ibid., 1. 13 : "Total of cedar and acacia boats 92 », etc. Zeitschrift für aegyptische Spracke, t. XI (an. 1873), p. 38. Au lieu de "cedar ", il faut lire " ash" (Acaciu seyal.) 
(des pylones), dit la charte de fondation du temple d'Edfou', sont en bois d'acacia. Ils touchent au ciel et sont garnis de cuirre". Une inscription du tombeau de Ti, ¿ Saqqarah, nous apprend qu'une des statues de ce personnage, qui vivait sous la $\mathrm{V}^{\circ}$ dynastie, était faite en bois de shent². Et dans l'inscription du temple d'Osiris, à Dendérah, il est fait mention d'une cassette d'acacia, "coffre mystérieux, naos, dans lequel on ne sait ce qu'il $\mathrm{y} \mathrm{a}^{3}$. »)

Le bois de l'acacia seyal - le bois d'ash - était employé aux mèmes usages que le bois de shent ou d'acacia du Nil. Dans le P'apyrus Harris il est fait mention de bateaux en bois d'ash, expression que Birch a traduite ì tort par bois de cèdre. Il est fait également mention du bois d'ash pour la construction de bateaux dans un des Papyrus Anastasi et dans le Papyrus de Turin. Les barques qui servaient a porter les images des Dieux sur le Nil en particulier étaient faites en bois d'ash. L'inscription de l'obélisque que Thoutmès III avait fait dresser, au xvir siècle, devant le temple d'Amon à Thèbes, et qui se troure maintenant à Rome, fait mention de " la grande barque, nommée Amonouserha et construite en bois d'ash, que sa majesté avait fait couper dans le pays des Rokbet" ».

Comme le bois de shent, le bois d'ash servait en particulier à faire les portes des édifices sacrés. L’ins-

1. H. Brugsch, Eine neue Baururkunde des Tempels von Ed/u. (Zeilschrifl fï aegyplische Sprache, t. XIII (an. 1875), p. 122.)

2. Charles Moldenke, op. laud., p. 80.

3. Victor Loret, Les fêles d'Osiris au mois de khoiak, no 65. (Recueil de travaux, t. IV (an. 1883), p. 25.)

4. Revue archéologique, t. X (an. 1864), p. 45. - M. Moldenke, op. laud., p. 88, lit Rokhet à la place de Rotekh. 
cription du temple d'Edfou nous apprend que " les battants des diverses portes étaient faites en vrai bois d'ash ${ }^{1}$ ». Il est également question dans la même inscription de " portes faites en bois d'au ", dernier mot dans lequel il faut voir une expression synonyme de la première, mais qui désigne le bois d'ash, non par son nom générique, mais par une épithète qualificative, celle de " bois beau et excellent $)^{2}$, preuve manifeste du prix que l'on attachait au bois de l'acacia seyal. C'est avec le bois précieux de cet arbre qu'avaient été faites aussi les portes du temple de Hibé, situé dans l'oasis de Khargeh ${ }^{3}$. Le bois d'ash servait encore, comme celui de shent, moins rare et moins précieux, à fabriquer des statues et des objets destinés au culte. Les épines de ces deux arbres étaient employées en guise d'aiguilles.

La nature fibreuse du palmier ne permettait pas de tirer un aussi grand parti du bois de cet arbre que de celui de l'acacia ou du sycomore; les troncs servaient néanmoins à faire des poutres, des planchers et des linteaux de portes ${ }^{4}$. Mais c'étaient surtout les diverses parties des feuilles qui étaient employées dans l'industrie; c'était avec les rachis - ba, bai - dépouillés de leurs folioles qu'étaient fabriqués les divers objets dits en bois de palmier, cannes, cages et en particulier ces espèces de sièges de forme quadrangulaire, mais plus large que haute, - les qafas des Arabes -

1. H. Brugsch, Zeilschrifl für aegyptische Sprache, t. XIII (an. 1875), p. 122.

2. Charles Moldenke, op. laud., p. 89.

3. R. Lepsius, Zcitschrift für aegyptische Sprache, t. XII (an. 1874), p. 73.

4. Wilkinson, op. laud., t. I, p. 357. 
qui pouvaient indifféremment servir de chaise, de banc, de table ou d'escabeau, et dont il est souvent question dans les textes ${ }^{\prime}$. Ainsi l'inscription de Dendérah nous apprend que le pròtre, chargé d'officier devant la déesse Rirt, prenait place sur un siège en bois de palmier ${ }^{2}$. Les pétioles des feuilles de palmier servaient aussi à faire des cannes légères et flexibles; c'est avec elles qu'on domnait la baitonnade, sorte de chàtiment, on le roit par les peintures des hypogées, fréquemment usité dans l'ancienne Égypte ${ }^{3}$. Si l'on en croit ce que dit Hérodote dans le dénombrement de l'armée de Xerxès ${ }^{4}$, les habitants de l'Éthiopie confectionnaient mème leurs arcs arec les pétioles des feuilles du dattier.

Bien plus recherché était le bois du palmier doum ou mama; plus dense, plus dur et beaucoup plus résistant, comme le remarquait Théophraste ${ }^{5}$, il était naturellement préféré à celui du dattier. La taille considérable de cet arbre présentait un autre avantage; de son tronc gigantesque on faisait des mâts de vaisseau, ainsi que ces espèces de mâts vénitiens, dressés auprès des pylones des temples et auxquels on attachait les étendards sacrés. Les noyaux gros et durs servaient, nous apprend Théophraste, à faire des anneaux de rideaux. On en faisait aussi des têtes de forets ${ }^{6}$.

Il y avait encore beaucoup d'autres arbres dont le

1. Wilkinson, op. laud., t. I, p. 420. - Charles Moldenke, op. laud., p. 45.

2. Dümichen, Recueil, IV, pl. XXI, 121, ap. Charles Moldenke, op. laud., p. 44.

3. Wilkinson, op. laud., t. I, p. 305-308.

4. Historiae, lib. VII, cap. 69.

5. Hisloria planlarum, lib. IV, cap. 2, 7.

6. Wilkinson, op. laud., t. I, p. 409. 
bois était employé dans l'industrie; Théophraste nous apprend ${ }^{1}$ que celui de perséa, résistant et d'une belle couleur noire, servait à faire des statues, des lits, des tables et toute espèce de meubles. Il est fait mention dans le Papyrus Harris de livraisons de bois de perséa ${ }^{2}$. Suivant Théophraste ${ }^{3}$, le bois de myrobalan, ou sébestier, - l'arbre baq des textes hiéroglyphiques était dur et propre aux constructions navales et à nombre d'autres travaux. Le bâton de jet qui figurait dans certaines cérémonies était en bois de myrobalan. "Il saisit le bâton en bois d'arbre baq pour lancer la balle (symbole) de la défaite de ses ennemis (d'Hàthor), " liton dans un texte hiéroglyphique ${ }^{4}$.

On employait aussi le bois de tamaris et celui de jujubier ou arbre noubsou pour fabriquer différents meubles ou ustensiles. Dans une inscription du temple de Dendérah $^{5}$, il est dit que la charrue dont on se servait pout labourer le champ d'Osiris était faite en bois de tamaris. Le chant des Huit Dieux dans le temple de Hibé était gravé sur une table en bois de noubsou ${ }^{6}$. Quoique Théophraste n'en parle pas, les Egyptiens tiraient également grand parti du bois de caroubier -

1. Historia plantarum, lib. IV, cap. 2, 5. - Cf. Pline, IIistoria naturalis, lib. XIII, cap. 17 (9).

2. Pl. $216,1.16$. "Persea wood ten 4415. " Zeitschrift für acgyptische Sprache, t. XI (an. 1873), p. 72.

3. Historia plantarum, lib. IV, cap. 2, 6. Pline, ibid., qui paraphrase, parfois en le faussant, le texte de Théophraste, dit que le bois de balan, "tordu dans la plus grande partie, " est moins estimé que celui de perséa.

4. Dümichen, Resultate ciner archaclogischen Expedition, t. I, p. 46. - Charles Moldenke, op. laud., p. 117.

5. Dümichen, Recucil, t. IV, p. 11, 61. - Charles Moldenke, op. laud., p. 129.

6. II. L. E. Lüring, op. laud., p. 157. 
le sisi-noudjim. - Rayé de reines d'un rouge sombre, d'une dureté et d'une densité incomparables, il était non moins recherché par l'ébénisterie que le bois indigène de l'acacia ou le bois exotique de l'ébène. On l'employait concurremment avec eux et avec l'ivoire. Une scène du tombeau de Rekhmara, qui représente des ébénistes confectionnant des lits, des coffres, des chaises, etc, est accompagnée de la légende: "Fabrication de meubles en ivoire, ébène, caroubier, acacia1. " "Il s'assit, lit-on dans le Papyrus Westcar ${ }^{2}$, sur une chaise d'ébène dont les pieds étaient en caroubier. " Kotschy, on l'a vu plus haut, a trouvé dans une tombe un baton en bois de caroubier ${ }^{3}$. Il existe aussi, preuve qu'on faisait usage du bois de cet arbre̊, un biton de Balanites aegyptiaca au Musée de Florence ${ }^{4}$.

Sur la tombe de $\mathrm{Ti}$ à Saqqarah on voit deux ouvriers occupés à travailler du bois de sib; le mème mot sib se retrouve dans les pyramides d'Ounas, et de Pépi I ${ }^{\text {or }}$ Miriri ${ }^{5}$. A la ligne 565 de l'inscription d'Ounas, M. Maspero traduit sib par cèdre, toutefois avec un point d'interrogation ${ }^{6}$; et à la ligne 589 il voit dans sib un nom d'homme ${ }^{\top}$; M. Victor Loret y

1. Philippe Virey, Mémoires de la mission française au Caire,

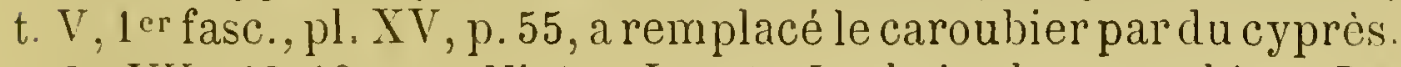

2. VII, 12-13, ap. Victor Loret, Le bois de caroubier. Recherches sur plusieurs planles, VIII. Recueil de travanx, t. XV.

3. Sitzungsberichte (Recueil de travaux, t. XV), t. XXXVIII.

4. Migliarini, Indicalion succincte, p. 56, no 2692.

5. Victor Loret, La Flore pharaonique, p. 42, no 52.

6. "Tu pénètres dans tes bois d'acacia? (de cèdres?)" Recueil de travaux. t. IV (an. 1883), p. 68.

7. "C'est Toum qui a rendu son arrêt [avec] Sib. " Ibid., p. 72. 
voit partout le nom d'un arbre - celui du cèdre - et il en conclut que cet arbre était indigène en Égypte, qu'on l'y a exploité et employé jusqu'à ce qu'il ait disparu. Ce qui est certain, c'est que les Ramessides importaient ce bois du Liban. Dans le “Récit de la campagne de Mageddo sous Thoutmès III », il est question à plusieurs reprises de bois de cèdre rapporté comme butin du pays des vaincus ${ }^{1}$. De ce bois une partie était déjà ouvrée, puisqu'il en est fait mention avec l'épithète "orné d'or "; mais une autre partie parait avoir encore été à l'état brut et ne dut être travaillée qu'en Égypte. On sait d'ailleurs que les Pharaons, rainqueurs de la Syrie, envoyèrent plus d'une fois couper des cèdres sur les hauteurs du Liban, pour les employer dans leurs constructions ${ }^{2}$. Il n'est donc pas surprenant que cet arbre soit souvent mentionné dans les textes hiéroglyphiques, ni qu'on en ait trouvé de la sciure dans les tombes anciennes ${ }^{3}$.

Le cèdre ne fut pas le seul arbre exotique ou devenu étranger à l'Égypte, dont les habitants de cette contrée utilisèrent le bois. Ils employèrent celui de plusieurs autres espèces, comme le cyprès, l'if même, mais surtout l'ébène. En examinant au microscope des débris de cercueils trourés à Méir et remontant au moins à la XII ${ }^{\circ}$ dynastie, M. le professeur Beaurisage

1. Traduction de M. Maspero. (Recueil de travaux, etc., t. II, p. 148 et 150 .)

2. Une inscription fait mention du bois de cèdre que le roi Râmenkheperou " fait couper dans le pays de Rotekh pour la construction d'un vaisseau. "Zeilschrift fur aegyplische Sprache, t. II (an. 1864), p. 37.

3. Franz Woenig, op. laud., p. 387. 
de Lyon a reconnu qu'ils étaient faits en bois d'if ', arbre étranger à la flore égyptienne et dont le bois avait dù ètre importé de l'Asie antérieure dans la vallée du Nil. Parmi les tributs que les chefs des Rotenou enroyèrent, en 40 , à Thoutmès III, figurent des troncs de cyprès, à côté de troncs de cèdre ${ }^{2}$. Dans la peinture, qui représente Rekhmara, officier du même pharaon, recerant les offrandes des chefs du Midi (la Nubie et l'Éthiopie), se trouvent, avec des dents d'éléphant, des blocs entier's d'ébène ${ }^{3}$. Originaire de cette région, l'ébénier" (Dalbergia melanoxylon G. P. R) égyptien habin, sans doute son nom vernaculaire, d'où le

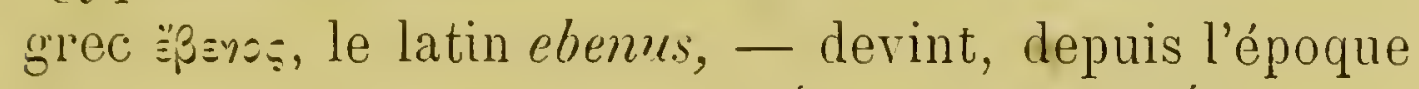
des premières relations de l'Égyte avec l'Éthiopie, l'objet d'une importation, qui alla toujours croissant; c'était une des redevances ordinaires que les Pharaons imposaient à leur's tributaires du Midi. Depuis lors aussi ce bois prit dans l'ébénisierie et dans l'art pharaonique une place de plus en plus grande. Une statue du tombeau de $\mathrm{Ti}$ à Saqqarah ( $\mathrm{V}^{\mathrm{e}}$ dynastie) représente des personnages qui transportent une statue du défunt, de couleur noire et au-dessous de laquelle est écrite la légende: "Statue d'ébène" ». Dans une autre partie du même tombeau sont figurés des ouvriers polissant un

1. Le bois d'if. Recherches sur quelques bois pharaoniques, I. (Recueil de travaux, t. XVIII, an. 1896).

2. Ph. Tirey, Le tombeau de Rekhmara. (Mémoires de la mission francaise au Caire, t. V, $1 \mathrm{er}$ fasc., p. 37.)

3. Lepsius, Dentimïler, t. III, pl. 39, a, b. J. Lieblein, Handel und Schiffahrt auf dem rothen Meere in alten Zeilen, p. 38.

4. Strabon, Geographica, lib. XVII, cap. 2, 2. - Pline, lib. XII, cap. 8, dit que "l'ébénier est rare depuis Syène jusqu'à Méroë ».

5. H. Brugsch, Die allägyplische Gräberwelt, no 90. 
lit de repos également de couleur noire; l'inscription porte arec une forme particulière du mot habinn": " polissage d'un lit de repos en ébène par les polisseurs de la maison d'éternité ". Sur les parois du tombeau de Horhotpou (XI' dynastie) à Thèbes, sont peintes une tablette et une palette de scribe en ébène, et on lit sur une inscription de la stèle $G$, au Lourre, qui date de la $\mathrm{XII}^{\circ}$ dynastie ${ }^{2}$, l'inscription: " J'ai vu la perfection de tes bras dans ton travail en tontes matières précieuses, depuis l'argent et l'or, jusqu'à l'ivoire et l'ébène ».

Désormais l'ivoire et l'ébène, importés des mêmes régions, sont associés également dans les mèmes œuvres d'art, non seulement en Égypte, mais dans toute l'Asie antérieure. Thoutmès III rapporta, de sa campagne contre Mageddo ${ }^{3}$, dans son immense butin, six petits autels ou sièges en iroire, ébène et acacia. Les statues du roi, blanches et noires, qui figurent sur le tombeau d'Amenhotpou II, sont accompagnées de la légende: "Statues en ivoire et en ébène ${ }^{4}$ ). Parmi les dons nombreux offerts par Ramsès III aux dieux d'Égypte se trouvaient 407 cannes en acacia et en ébène". Dans les textes de l'époque des Ptolémées il est plus d'une fois fait mention de meubles en ébène; qu'il suffise de citer le tabernacle en ébène orné d'or, dans lequel on enfermait le moule de Sokari, pendant les fètes d'Osiris à Tentyris'.

1. Victor Loret, L'ébène chez les anciens Égyptiens. (Recueil de travaux, t. Vl, p. 127.)

2. Victor Loret, L'ébène, ibid., p. 127.

3. G. Maspero, Récil de la campagne de Mageddo. (Recuéil de travaux, t. II, p. 148.)

4. Champollion, Description de l'Égypte, t. I, p. 500.

5. Grand Papyrus Harris, pl. XXXIV a, 1. 13, LXXI $a, 5$.

6. Victor Loret, Les fétes d'Osiris au mois de Khoiak: $\S 67$. (Recueil de travaux, t. IV, p. 26.) 
On voit par là combien était varié l'usage de l'ébène; aussi les quantités de ce bois qu'on employait étaient-elles énormes. Dans un gigantesque cortège triomphal qu'on vit à Alexandrie sous le règne de Ptolémée Philadelphe V figuraient " des Éthiopiens portant les uns six cents défenses d'éléphant, d'autres deux mille troncs d'ébène ${ }^{1}$ ". Ce fait peut donner une idée de la masse énorme d’ébène livrée par là au commerce ou à l’industrie égyptienne. Dès longtemps il en avait été de mème.

Dans les peintures de tous les tombeaux des gouverneurs égyptiens d'Éthiopie, conservés jusqu'à nos jours, ceux entre autres de Houi, de Merimès, d'Amenhotpou, sont représentés des bateaux venant du HautNil chargés de poutres d'ébène, de défenses d'ivoire et d'autres produits de la région². Aussi le nombre d'objets et d'ustensiles en ébène qui nous ont èté conservés est-il considérable; on voit dans les divers musces égyptiens d'Europe des chaises, des coffrets, des statues, des figurines funéraircs, des palettes de scribes, des manches de cuillors et de miroir, des bàtons en ébène, le plus souvent incrusté d'ivoire, et même un pilon dont l'extrémité est formée d'un noud de ce bois dur et recherché.

1. Athénée, Deipnosophistae, lib. V, cap. 32.

2. Victor Loret, L'ébène, p. 128. 


\section{CHAPITRE VI.}

LES PLANTES DANS L'ART ET DANS LA POÉSIE.

Non seulement les anciens Égyptiens demandèrent au règne végétal des matériaux, pour construire leurs demeures et les temples des Dieux, fabriquer leurs meubles et les outils les plus nécessaires; mais ils lui empruntèrent quelques-unes des formes architecturales les plus originales, ainsi que les plus beaux motifs de décoration, et ce monde charmant fournit à leurs poètes des comparaisons et des images pour embellir et orner leurs fictions.

\section{I.}

Les premières demeures des habitants de la vallée du Nil furent, sans doute, comme je l'ai dit plus haut ${ }^{1}$, faites de roseaux et de branchages, plus tard de troncs frustes d'arbres et en particulier de palmiers, grossièrement assemblés entre eux; l'architecture égyptienne garda toujours quelque chose de cette origine; quand la pierre se fut depuis longtemps substituée au bois dans les constructions pharaoniques, on n'en conserva pas moins quelques-unes des formes employées à l'époque

1. Voir chapitre v, p. 204. 
où celui-ci surtout servait de matière première aux artistes. "C'est ainsi que le sarcoplnage trouvé dans la pyramide du roi Menkérî̀, bien qu'il soit en basalte, nous présente des ornements qui dérivent essentiellement de l'architecture en bois. ")

D'ailleurs, alors mème qu'ils faisaient déjà usage de la pierre et du granit, les Égyptiens ne dérlaignèrent pas l'emploi du bois. Il existe, faites en cette matière, des colonnettes qui appartiennent a toutes les époques. Telle est celle du tombeau de Ti à Saqqarah, reproduite par Prisse d'Aremnes². Le chapiteau représente une Heur de lotus entr'ouvert, flanqué de deux boutons plus petits, reliés ensemble par des bandelettes; quant au fuit, il est légèrement conique et strié, "comme pour figurer plusieurs tiges». On dirait un de ces bouquets composés de deux ou trois lotus liés ensemble, qu'on voit si sourent à la main des personnages figurés dans les peintures des hypogéés.

Ce type une fois trouvé devait se conserver en se modifiant. Dans une colonnette d'un hypogée de Zaouietel-Maiétin (XVII ${ }^{\circ}$ dynastie), par exemple ${ }^{3}$, le fût a perdu ses cannelures, mais le chapitean est toujours composé d'un bouton — plus petit, il est vrai - et d'une fleur plus ouverte de lotus. Une autre colonnette du temple de Semneh, reconstruit par Thoutmès III, nous montre encore la mème fleur de lotus, mais plus ornée, sinon mieux comprise". De plus la

1. Prisse d'Avennes, IIistoire de l'art égyptien, texte par Marchandon et la Faye, p. 153.

2. IIistoire de l'art égyptien, texte, p. 462, atlas, t. I, pl. 17,1 .

3. Prisse d'Avennes, op. laud., texte, p. 362 ; atlas, t. I, pl. $17,2$.

4. Prisse d'Avennes, op. laud., atlas, t. I, pl. 17, 3. 
base du füt est encadrée de feuilles qui rappellent celles qui entourent la tige du papyrus. Ce dernier motif de décoration a été par la suite employé dans la plupart des fûts de colonnes. Quant aux lotus qui parent le haut de la colonne, on les retrouve aussi, mais modifiés dans leur disposition, comme s'ils étaient indépendants de la colonne et n'étaient qu'un ornement d'emprunt, ils sont représentés attachés par des liens au sommet du fût, que leurs tiges accompagnent sur une partie plus ou moins considérable de sa longueur.

Le bouquet formé d'un lotus et de deux boutons ne fut plus ì lui seul, dans la suite, jugé suffisant pour embellir le sommet de la colonnette, on y ajouta d'autres ornements. Ainsi dans les colonnettes de Tell-elAmarna ${ }^{1}$, le chapiteau, formé d'un bouquet de trois fleurs de lotus, est garni d'un double collier, au-dessous duquel pend un groupe d'oies. En outre le füt est. orné d'une triple rangée ou guirlande de feuilles ou de pétales renversés. Les colonnettes de l'hypogée du scribe Horemheb, contemporain de Thoutmès IV², ont un triple chapiteau, composé d'abord d'un bouquet formé de deux boutons et d'une fleur à moitié épanouie de lotus, du milieu de laquelle sort un lotus héraldique, accompagné de deux boutons de forme conventionnelle; an-dessus enfin s'élève une large fleur de papyrus, flanquée de deux petites fleurs de lotus, munies de leur pédoncule. Dans une colonnette du naos de Ramsès IX ${ }^{3}$, on retrouve le lotus du chapiteau, mais sans boutons, le lotus en fer de lance qui le sur-

1. Prisse d'Avennes, texte, p. 362 ; atlas, t. I, pl. 18, 1.

2. Prisse d'Avennes, texte, p. 363 ; atlas, t. I, pl. 19, 3.

3. Prisse d'Avennes, atlas, t. I, pl. 19, 1. 
monte est, au contraire, accompagné i sa base de deux fleurons, qui reposent sur celui du chapitean, et andessus se dresse me fleur de papyrus flanquée de deux uraeus. Dans le chapiteau d'une colonnette provenant d'un tombeau de Thèbes (XVIII dynastie)', on retrouve encore une fleur conventionnelle de lotus, un lotus héraldique, et une fleur de papyrus superposées; mais au-dessous de la fleur de lotus qu'accompagnent deux boutons, s’étale une espèce de collier et le lotus héraldique est tlanqué de deux têtes d'ibex, tandis que la fleur de papyrus est ornée de chaque còté d'une marguerite ou d'un fleuron blanc.

On voit combien a été variée et riche dans le chapiteau de la colonnette en bois l'ornementation empruntée au monde des fleurs, on ne trouve rien de semblable i l'origine dans la colonne en pierre ou en granit; mais elle ne tarda pas, en se transformant, à se rapprocher de l'élégance de la colonnette en bois, et elle ell imita les décorations empruntées au règne végétal. C'est ainsi que le pilier quadrangulaire primitif des antiques hypogées perdit d'abord ses quatre angles et se transforma en colonne octogonale ${ }^{2}$, dont les huit angles abattus donnèrent ensuite naissance au pilier élégant à seize pans, qu'on évida encore plus tard pour rendre sensible à l'ceil cette division deux fois octogonale du fùt. D'autrefois, au lieu d'être cannelé, celui-ci fut arrondi et prit ainsi la forme d'un tronc d'arbre ou d'une tige de roseaux. Pour que le soutien monolithe des anciens temples se transformât

1. Prisse d'Avennes, atlas, t. I, pl. 19, 2.

2. Perrot et Chipiez, Histoire de l'art dans l'antiquité. Paris, in-8, t. I. L'Égypte (1882), p. 549. 
en colonne complète, il ne restait qu'à le surmonter d'un chapiteau. L'architecture légère en offrait le modèle depuis de longs siècles. Ainsi dans un édicule de la $V^{0}$ dynastie, on voit deux colonnettes, dont le füt, en se terminant par deux trones de pyramides réunis par leurs bases, simule, dans son ensemble, un bouton de lotus tronqué ${ }^{~}$. Il y avait là un type très simple de chapiteau; on l'adopta tout d'abord. On le trouve dans les colonnes des ruines de Béni-Hassan, qui remontent à la XII ${ }^{e}$ dynastie ${ }^{2}$. "De robustes rudentures en découpent le fût, quatre minces baguettes occupent dans la partie, supérieure les angles rentrants, auxquels cet arrangement donne naissance. Les mêmes courbes se montrent sur le chapiteau, qui déborde sur le fût aminci, puis bientôt se contracte et se replie sur soi. ${ }^{3}$ ")

On retrouve ici, mais en partie dissimulée, l'imitation d'un bouquet de lotus, que j'ai signalée dans les colonnettes en bois; toutefois au lieu des trois tiges de lotus que nous avions dans celles-ci, il y en a maintenant quatre; la fleur ouverte a été aussi remplacée par un bouton; et si les quatre tiges sont toujours serrées, au-dessous de la naissance des calices, par un lien que représente les annelets qui tournent autour de la colonne, les quatre boutons ne sont plus libres; ils sont soudés entre eux et tronqués, comme pour mieux supporter l'abaque.

Le chapiteau lotiforme ne fut pas le seul que connut l'architecture égyptienne; à còté dè celui-ci, dérivé d'un lotus fermé ou entr'ouvert, entier ou tronqué, il

1. Perrot et Chipiez, op. laud., t. I, p. 540.

2. Lepsius, Denkmäler, t. I, pl.60.- Wilkinson, op. laud., t. II, p. 292.

3. Perrot et Chipiez, op. laud., p. 553. 
existe, depuis le second empire thébain, un autre chapiteau que l'on a appelé campaniforme, mais auquel on derrait domner le nom de papyriforme, parce qu'il représente en réalité une fleur conventionnelle de papyrus ${ }^{1}$. Cette plante symbolique ne pouvait manquer de prendre place dans la décoration égyptienne, et, comme le lotus, elle y apparait d'abord dans son entier. L'un des deux piliers que l'on voit dans les " appartements de granit " de Karnak est décoré sur la face intérieure et extérieure de trois tiges de papyrus faisant saillie et qui diffèrent d'une face à l'autre ?. Accompagnées à leur base de feuilles rudimentaires, clles sont terminées par la fleur conventionnelle que cette plante affecte sur toutes les peintures pharaoniques. Il n'y avait qu'à détacher ces tiges, à les transformer en support isolé, et un nouveau type de colonne était créé.

Nous arons rencontré la fleur conventionnelle du papyrus associée au lotus dans les chapiteaux des colonnettes en bois, nous la trouvons formant à elle seule le chapiteau des colonnes en granit de l'époque des Ramessides et des Ptolémées. Le papyrus avait déjà fourni à l'antique colonne plusieurs des ornements qui

1. Pour Alois Riegl, Grundlegungen zu einer Geschichte der Ornamentik, Berlin, 1893, in-8, p. 56, qui ne fait d'ailleurs que suivre ici W. H. Goodyear, l'auteur de The Grammar of the Lotus, p. 68, c'est encore là un chapiteau lotiforme, seulement d'une espèce particulière.

2. Prisse d'Avennes, op. laud., t. I, pl. 14, 2. - Ebers, L'Égyple, trad. Maspero. Paris, 1881, in-fol., p. 193. - Perrot et Chipiez, op. laud., p. 548. Sur l'autre pilier, se dressent, au contraire, trois tiges de lotus à fleurs en forme de fer de lance. Inutile de dire que pour Goodyear, ce sont des lotus différents de forme seulement qu'on voit sur les deux piliers. 
la décorent; ce sont ses feuilles radicales et rudimentaires', qui en enveloppent si souvent la base arrondie et renflée, comme le collet de ses racines; ce sont ses tiges triquètres qui apparaissent dans les huit cannelures trouvées dans le voisinage du labyrinthe et qui remontent à l'époque d'Amenemhat III, " le roi de l'inondation ${ }^{2}$ ». Sa fleur maintenant s'étale au sommet du fût évasé et en forme le chapiteau. Telles sont les colonnes de Médinet-Abou, lourdes, trapues et sans autres ornements que les feuilles aiguës de cette cypéracée, transportées de la base du füt au chapiteau qu'elles entourent ${ }^{3}$. Telles encore les colonnes massives de la salle hypostyle de Karnak ${ }^{4}$, au füt recouvert de figures et d'emblèmes, et dont les chapiteaux, outre les feujlles qui. les encadrent, sont ornés, comme pour rappeler leur origine, le premier, de deux rangs de tiges inégales de papyrus en fleurs, flanqués, dans le rang inférieur, de deux boutons, et, au rang supérieur, de deux lotus héraldiques ${ }^{5}$, le second, d'une seule rangée de fleurs de papyrus accompagnées de deux boutons du milieu desquels s'élance encore un lotus héraldique ${ }^{6}$. Le chapiteau des colonnes de la salle hypostyle du Ramesséum estlui aussi orné de feuilles triangulaires et de deux rangées de papyrus entremêlés de

1. Goodyear y voit une transformation des sépales de la fleur du lotus.

2. Ebers, L'Égyple, p. 185.

3. Perrot et Chipiez, op. laud., t. I, p. 556. - Description. de l'Égypte, t. II, p. 6.

4. Perrot et Chipiez, op. laud., t. I, p. 557, fig. 335, d'apres la Description de l'Égyple, t. III, p. 30. - Lepsius, Denlimäler. t. II, p. 77.

5. Prisse d'Avennes, atlas, t. I, pl. 43.

b. Lepsius, Denkmäler, t. II, pl. $81 a$ et $b$. 
lotus plus petits en forme de fer de lance', en même temps que la base du füt est encadrée de ces mêmes feuilles décoratives.

Il y avait encore dans la flore indigène de l'Égypte un arbre commun et vénéré entre tous, qui ne pouvait manquer d'entrer dans l'ornementation architecturale; c'est le dattier, dont le tronc élancé avait peut-être, avec la tige élégante du papyrus ${ }^{2}$, contribué à donner l'idée de la colonne. A l'époque du nouvel empire, il prèta au chapiteau, comme le papyrus et le lotus, la forme gracieuse de ses feuilles; on les rencontre déjà dans la colonne de Soleb (XVIII ${ }^{\mathrm{e}}$ dynastie) ${ }^{3}$; disposées en bouquet autour d'me corbeille ronde, elles se dressent au-dessus du filet supérieur du füt, en s'étalant, de manière que l'extrémité tronquée de chaque palme forme une sorte de lobe.

Les chapiteaux du temple de Sesebi, bâti par Seti I $^{\text {er }}$ en Nubie, présentent la même disposition ${ }^{4}$; mais 'le travail est plus fini; "chaque tige est accusée par une saillie plus marquée et chaque palme, à son sommet, figure un lobe d'un dessin plus franc et plus accentué ${ }^{3} »$. Toutefois la forme de ces palmes n'est qu'indiquée par leur contour général; plus tard on s'attacha à

1. Prisse d'Avennes, atlas, t. I, pl. 23. Le rang inférieur se compose d'un papyrus flanqué de deux lotus héraldiques, le rang supérieur d'un lotus héraldique entre deux tiges de papyrus.

2. "Le chapiteau des colonnes a la forme de fleurs, le fût la forme de dattiers et de papyrus. " H. Brugsch, Bau und Marsse des Tempels von Edfu. (Zeilschrift für aegyplische Sprache, t. I (an. 1871), p. 141.)

3. Lepsius, Denlimäler, t. II, pl. 117, col. x. - Prisse d'Avennes, texte, p. 392.

4. Lepsius, Denlimäler, t. II, pl. 118 et 119.

5. Perrot et Chipiez, op. laud., t. I, p. 568.

I. 
en reproduire tous les détails. On l'a tenté dans les colonnes du palais de Tell-el-Amarna, en imitant les feuilles à l'aide d'incrustations coloriées'. A Philae, dans le temple de Nectanébo, on a procédé autrement; cinq chapiteaux des colonnes du Dromos représentent, finement sculptées, les nervures des palmes ${ }^{2}$; à la base de l'un d'eux on a même figuré des régimes de dattes ${ }^{3}$; sur un autre les palmes sont mêlées à des tiges et à des fleurs de papyrus". On ne s'arrêta pas là: sur un chapiteau du temple d'Esneh, monument de l'époque romaine, il est vrai, on a sculpté des palmiers entiers ${ }^{\circ}$. Prisse d'Avennes a même reproduit un chapiteau où des pampres, couverts de raisins, alternent avec les palmes ${ }^{6}$.

On ne se contenta pas d'embellir ainsi la base et le haut des colonnes d'ornements empruntés au monde végétal, on en décora également le fût; plusieurs colonnes du palais de Tell-el-Amarna ${ }^{7}$ sont, genre d'ornementation unique en Égypte, entourées de tiges grimpantes garnies de feuilles triangulaires, qui les enveloppent, sans souci de la symétrie et dans une confusion qui témoigne de l'indépendance de l'artiste.

Ce n'est pas à la décoration seule de la colonne qu'ont servi les plantes; on en retrouve l'imitation dans les sculptures ou les peintures de presque tontes

1. W. M. Flinders Petrie, Tell-el-Amarna. London, Methuen, 1894, in-4, p. 10, 1 .

2. Lepsius, Denkmüler, t. II, pl. 106 et 107.

3. Lepsius, Denkmüler, t. II, pl. 168, III. - Prisse d'Avennes, atlas, t. I, pl. 27.

4. Lepsius, Denkmëler, t. II, pl. 108, virr.

5. Lepsius, Denlimäler, t. II, pl. 98.

6. IIistoire de l'arl, atlas, t. I, pl. 61.

7. Flinders Petrie, Tell-el-Amama, p. 10, 2, pl. VIII. 
les autres parties les édifices, ainsi que dans les différents objets d'art et mème souvent dans les ustensiles les plus vulgaires en bois ou en métal.

"Ce qui touchait au sol, dit M. Maspero", se revêtait de végétation... Le pied des murs sc garnissait de longues tiges de lotus ou de paprrus... Des bouquets de plantes fluviales, émergeant de l'eau, égayaient les soubassements de certaines chambres. Aillours, c'étaient des fleur's épanouics, entremèlées de boutons isolés ou reliées par des cordes. "

Ces ornements étaient parfois sculptés, d'autres fois inscrustés dans la pierre, le bois ou le métal, plus sourent peints, ou à la fois peints et sculptés. Ils variaient d'ailleurs suivant le monument ou la partie du monument qu'on voulait orner, et ils sont très différents suivant les époques auxquelles elles remontent.

Le premier objet qui s'imposait à la décoration, c'étaient les soubassements; l'ornementation en était indiquée par la situation qu'ils occupaient dans le monument; on les couvrit, à Karmak et à Dendérah, par exemple, de tiges de papyrus lotiformes, dressées en rangs serrés, comme dans les marécages des bords du $\mathrm{Nil}^{2}$, ou bien réunies en bouquets, composés soit de fleurs idéalisées ${ }^{3}$, soit de gerbes faites de fleurs et de boutons gracieusement étalés ${ }^{4}$, parfois mème, à l'époque ptolémaïque, accompagnés de fleurs en fer de lance ${ }^{5}$ ou mème de fleurons ou de rosettes ${ }^{6}$. D'autres fois ce furent des rangées alternantes de fleurs ouvertes et de

1. L'Archéologie égyptienne, p. 89.

2. Prisse d'Avennes, atlas, t. I, pl. 56, 7 et 57, 4, 5, 6 .

3. Prisse d'Avennes, atlas, t. I, pl. 57, 3.

4. Prisse d'Avennes, atlas, t. I, pl. 56, 4, ว et 57, 7.

5. Prisse d'Avennes, atlas, t. I, pl. 56, 8 et $57,8$.

6. Prisse d'Avennes, atlas, t. I, pl. 57, 9, 10. 
boutons de lotus ${ }^{1}$, ou de lotus épanouis accompagnés d'un double pédoncule recourbé et terminé par un bouton $^{2}$. A Karnak la décoration a pris un caractère plus grandiose et plus artistique; sur le soubassement du temple élevé par Thoutmès III, le statuaire a gravé, avec les animaux, les fleurs et les plantes des pays subjugués par le pharaon ${ }^{3}$.

Les frises furent, à l'instar des soubassements, ornées de décorations empruntées au règne végétal; elles apparaissent, placées comme appendices des architraves, au bas desquelles ces ornements pendent semblables à des guirlandes de fleurs". Telles on les voit sur les parois intérieures du gynécée de Ramsès III. Sur l'une de celles-ci des fleurs renversées alternent avec des boutons de lotus, sur l'autre avec des fleurs de papyrus ${ }^{3}$. Ces frises fleuronnées sont rares toutefois dans les palais; on les rencontre, au contraire, fréquemment dans les hypogées et les naos des dieux et des hommes. Là encore elles sont le plus souvent ornées de fleurs renversées de lotus, auxquelles sont parfois mêlés des fleurons, des grappes de raisin ou encore des espèces de boutons ou d'ovaires cordiformes ${ }^{6}$, que l'artiste a peints soit isolés, soit émergeant des fleurs du lotus ou du papyrus. M. Goodyear,

1. Prisse d'Avennes, atlas, t. I, 52, 2.

2. Prisse d'Avennes, atlas, t. I, pl. 52, 1.

3. G. Maspero, L'Archéologie égyptienne, p. 90.

4. Prise d'Avennes, atlas, t. I, pl. 54, texte, p. 385 . Il faudraitajouter: et de fruits, car sur l'une des frises de l'hypogée du grand prêtre Aïkhesi (Ibid., no 8), de chaque côté des lotus, on voit des grappes de raisin.

5. Prisse d'Avennes, atlas, t. I, pl. 45.

6. Prisse d'Avennes, atlas, t. I, pl. 54, texte, p. 385. 
dans son parti pris, veut y voir des feuilles transformées de lotus ${ }^{1}$.

Comme sur les soubassements et les frises, les plantes ont pris place dans la décoration des plafonds des édifices; mais dans ces derniers, plus que partout ailleurs peut-ètre, les artistes égyptiens ont laissé libre cours à leur fantaisie. Les fleurs n'aspirent plus ici à une exacte ressemblance; "le motif a été suggéré, mais non fourni par la nature », dit avec beaucoup de vérité M. Perrot". "Méandres, dans lesquels s'encadrent d'élégantes rosaces; lignes ondoyantes formées par des cordes qui s'enroulent en volutes ou se déroulent en spirales; postes qui se croiscnt ou se contrarient et qui renferment dans les espaces qu'elles limitent, ici des lotus, là des rosaces ».

Ces décorations, qu'on rencontre dès l'époque des pyramides, non seulement sur les plafonds, mais sur les tentures ou fonds de sparterie, n'étaient d'abord que des carrés composés de losanges, des chevrons ou spirales; plus tard apparaissent des volutes et des guillochis, qui encadrent des fleurons de diverses couleurs; sous la XVIII dynastie s'y mêlent, dans de gracieuses combinaisons, les fleurs de lotus et parfois aussi des tiges de papyrus. Nous rencontrons d'abord ces fleurs symboliques dans les plafonds de l'hypogée du "Père divin Nofirhotpou ${ }^{3}$ "; ici le lotus idéalisé

1. The grammar of the Lotus, p. 50. Ce seraient plutôt des boutons, puisqu'ils sont d'ordinaire entourés de trois sépales à leur base.

2. Histoire de l'art, t. I, p. 811. On peut en dire autant des fleurs qu'on voit sur les frises.

3. Prisse d'Avennes, op. laud., atlas, t. I, pl. 30, 1 et $2 ;$ pl. 33, 1, texte, p. 367 et 369 . 
s'épanouit dans tous les espaces laissés vides par les spirales et par les fleurons, tandis que sur les côtés se dressent au-dessus d'un ornement de fantaisie trois tiges de papyrus, accompagnées de leurs boutons conventionnels. Ailleurs des lignes ondoyantes, formées par des cordes, s'enroulent en volutes ou se déroulent en spirales autour de fleurs ouvertes de lotus ${ }^{1}$, comme dans le plafond du bel hypogée d'Aïkhesi, œuvre d'un artisan au goût délicat et original; d'autres fois ces fleurs de lotus sont opposées à des anthémions de convention ${ }^{2}$. Sur d'autres plafonds on voit tantôt quatre Heurs de lotus s'étaler entre autant de losanges réunis a angle droit, ou des grappes de raisin alterner arec des corolles à quatre pétales dans les carrós de la voûte ${ }^{3}$, ou bien encore des fleurs de papyrus ou de lotus se dresser sur leurs tigew cn alternant avec leurs boutons idéalisés, ici entre des rangées de fleurons, là dans des niches en arcades ${ }^{4}$.

Les plantes entraient aussi dans la décoration des briques émaillées et par suite du dallage des édifices. On en connait peu d'exemples, il est vrai, mais quelques-unes des représentations que nous possédons témoignent d'une imitation attentive et exacte de la na. ture végétale bien faite pour étonner. Les murs d'une chambre de Saqqarah avaient déjà été recouverts d'une parure de faïence, conservée jusqu'au commencement

1. Prisse d'Avennes, atlas, pl. 31, 1, 2, 3, texte, p. 3668 .

2. Prisse d'Avennes, atlas, t. I, pl. 31, 8.

3. Tombe d'Aïkhesi à Thèbes (XXe dynastie). - Prisse d'Avennes, atlas, t. I, pl. 32, 4 et 5 .

4. Plafonds des fenêtres du gynécée de Ramsès III et compartiment du plafond de la grande salle de Bekensanef à Saqqarah. Prisse d'Avennes, atlas, t. I, pl. 32, 7, 9 et pl. 34, 5, texte, p. 369 et 370 . 
de ce siècle'. Deux mille ans plus tard, à l'imitation pent-itre de ce qu'on royait dans les palais de Ninive, Ramsès III reprit ce genre d'ornementation et l'appliqua au temple qu'il fit bitir à T'ell-el-Yahoudi près Memphis²; les murs en sont revètus de briques, la plupart ornées d'élégantes rosaces ou de fleurons encadrés de dessins géométriques.

On se servit encore de ces carreaux de faïence, les récentes trouvailles de M. Flinders Petrie nous l'ont appris, pour orner les planchers. Le savant égyptologue a découvert à Tell-el-Amarna un dallage en partie conservé, qui est orné, non plus de simples motifs de décoration empruntés au monde végétal, mais d'un paysage entier ${ }^{3}$. Au centre se trouve un réservoir, couvert de lotus, au milieu desquels se jonent des poissons; tout autour l'artiste a représenté avec une fidélité jusque là inconnue des plantes, parmi lesquelles paissent des génisses et des bouvillons, dont l'approche fait fuir une bande d'oies domestiques : c'est en haut une plante à fleurs rouges dont les feuilles ressemblent à celles du coquelicot, puis une touffe de papyrus sous sa forme schématique traditionnelle, un souchet - peut-être le Cyperus alopecuroüdes Rottb. - un coquelicot, une composée à fleurs bleues, probablement la centaurée déprimée $e^{4}$ un papyrus, une touffe de roseaux - l'Arundo isiaca Del., à ce qu'il semble - , un souchet encore et une touffe de papyrus. Ces mèmes plantes se retrouvent au-dessous du

1. G. Maspero, L'Archéologie égyptienne, p. 257.

2. Perrot et Chipiez, op. laud., t. I, p. 824.

3. Tell-el-Amarna, p. 13, pl. II.

4. C'est aussi l'opinion du docteur Bonnet, à qui j'ai montré cette peinture. 
bassin, ainsi qu'à droite et à gauche; mais on en voit de plus une petite à fleurs obovales, opposées et infléchies, avec une fleur à calice bleu et ì style peint en rouge; je n'ai pu l'identifier.

La décoration primitive s'était alors, on le voit, singulièrement élargie et transformée, ce ne sont plus les simples ornements des anciens plafonds, mais un vrai paysage que nous avons ici. C'étaient aussi le plus souvent de véritables tableaux que l'on voyait sur les murs des édifices : palais, temples ou hypogées. Les scènes qu'on y trouvait représentées étaient le plus souvent destinées à rappeler les événements de la vie publique ou privée des personnages qui les avaient fait peindre ou sculpter. Ici, par exemple, est représentée une chasse à l'hippopotame ${ }^{1}$; là, nous voyons un contemporain de la XII dynastie se livrer dans les marais au plaisir de la chasse aux oiseaux ${ }^{2}$ ou de la pêche ${ }^{3}$; ailleurs nous assistons à une lutte de marinier's sur l'eau". Dans ces peintures, on le comprend, les artistes se sont surtout préoccupés de la représentation des personnages qu'ils mettent sous nos yeux; toutefois l'amour de la couleur locale les a portés aussi à dessiner les plantes qui croissaient dans les lieux ou se passent les scènes représentées; s'ils l'ont fait sou-

1. Tombeau de Ti. Musée Guimet, tableau 4. - Nemphis, VI dynastie et XVIIe dynastie. Prisse d'Avennes, atlas, t. II, pl. 10 et 61 .

2. Gizeh, IVe dynastie et Béni-Hassan, XIIe dynastie. Lepsius, op. laud., t. IV, pl. 60 et 130. - XVIII dynastie. Prisse d'Avennes, atlas, t. II, pl. 6, 2.

3. Lepsius, op.laud., t. IV, pl. 130.

4. Musée Guimet, tableau 2. - Kom-el-Amarna. Prisse d'Avennes, Monuments égyptiens, bas-reliefs, peintures, Paris, 1847, in-fol., pl. XXXVII. 
rent d'une manière conventionnelle, comme dans le tableau de la joute des marinier's, où " les lotus qui parsèment l'eau semblent des Heurs et des feuilles détachées de leurs tiges ${ }^{\prime}$ ", ils ne les ont pas moins figurées arec une grande vérité relative; ainsi a leurs corolles blanches légèrement bordées de rose, surtout a la forme échancrée de leurs feuilles, il est impossicle de ne pas reconnaitre dans les nymphéas, le lotus blanc, indigène en Égypte.

Dans les diver's tableaux de chasse aux oiseaux, la scène se passe au milieu des fourrés de papyrus; la représentation de cette cypéracée à l'ombelle étalée et dirergente présentait des difficultés particulières; l'artiste de la $I^{\mathrm{e}}$ dynastie, pour y échapper, a peint le papyrus arant son complet développement, au moment où les fleurons, renfermés dans les stipules, forment une espèce de fleur évasée et le type qu'il a adopté a été depuis fidelement conservéz ${ }^{2}$ parfois seulement, comme dans la peinture de la chasse aux oiseaux, les tiges ont été gracieusement courbées, les boutons oroïdes habilement mêlés aux ombelles des fleurs, de manière à en former un ensemble harmonieux.

A còté de ces scènes privées ou domestiques les artistes du nouvel empire en ont peint d'autres d'un caractère historique et général : vastes tableaux de batailles et de guerres, ou les plantes n'avaient point à figurer. Il est toutefois une de ces peintures où elles ont pris place : c'est celle du temple de Deir-el-Bahari, où la reine Hatshosipou a fait représenter l'expédition

1. Prisse d'Avennes, op. laud., texte, p. 399.

2. Charles Joret, De la représentation du papyrus sur les monuments égyptiens. (Iélanges Wahlund, 1895, in-8.) 
qu'elle envoya au pays de Pount chercher des " sycomores à encens ». Ici les plantes avaient leur place marquée, car il importait le mettre sous les yeux du spectateur quelques-uns des produits de cette " terre divine "; l'artiste contemporain n'y a pas manqué.

Les représentations semi-conventionnelles, mais facilement reconnaissables du lotus et du papyrus se retrouvent dans les peintures des nombreuses tables d'offrandes, qu'on voit sur les murs des hypogées ou des temples; on y rencontre aussi d'autres végétaux, Heurs, légumes, fruits, offerts aux dieux ou aux morts; mais, j'ai eu plus d'une occasion de le remarquer, il n'est pas toujours possible de reconnaître ces derniers, du moins pour nous autres modernes. Il n'est guère plus aisé souvent d'identifier les arbres et les plantes des jardins peints sur les hypogées ${ }^{1}$. Il est vrai que l'artiste pharaonique tenait moins à les faire reconnaitre qu'a nous donner une vue d'ensemble du parc ou du jardin dans lequel leur ombre et leurs fruits servaient à l'agrément du maitre. Il faut toujours faire exception pour les papyrus, qu'on reconnait à première vue sous leur forme schématique, et pour les lotus, dont l'artiste a toujours fidèlement respecté le caractère distinctif: les feuilles échancrées ${ }^{2}$, les sépales verditres et les pétales étalés, blancs et bordés de rose.

Il n'en était plus ainsi, lorsque ces fleurs, et à plus forte raison les autres plantes, au lieu de figurer dans une espèce de tableau, servaient de motifs de décoration; leurs formes se modifiaient alors selon le caprice

1. Vör à cet égard le paragraphe 2 du chapitre III.

2. C'est ainsi qu'il apparait sur tous les bassins ou réservoirs et les canaux. 
de l'artiste. Les papyrus prennent les couleurs les plus diverses, leur ombelle s'étale ou se rétrécit à volonté. Il en est de mème des lotus; leurs fleur's se transforment au point le se confondre parfois avec les ombelles conventionnelles du papyrus'; le nombre de leur's pétales varie; ils sont colorés indifféremment ou mème ì la fois en blanc, en bleu et en rose ${ }^{2}$. On traita arec la mème liberté les autres fleurs qui, à partir de la $\mathrm{XX}^{\mathrm{e}}$ dynastie, prirent à còté des lotus et des papyrus une place de plus en plus grande dans l'ornementation ${ }^{3}$.

Mais les transformations du lotus ne se bornèrent pas à quelques modifications de la fleur; de bonne heure leur pédoncule assez court fut allongé, ct on le courrit tantôt de feuilles triangulaires, analogues dès lor's à celles qui partent du collet de la racine, tantôt orales, rappelant ainsi la forme embryonnaire des boutons. Ce ne fut pas tout; au lieu de représenter la fleur de côté, on s'efforça de la reproduire de haut en bas, et elle apparait alors à l'artiste sous l'aspect d'une marguerite à nombre de fleurons variable; telle serait d'après Goodyear ${ }^{5}$ l'origine de la rosette.

1. C'est le cas pour des bouquets qu'on voit dans Prisse d'Avennes, atlas, t. II, pl. 62, 4 et 5 .

2. Par exemple dans un hypogée de la nécropole de Thèbes (XIXe dynastie) ou dans les bouquets des hypogées de la XIX et de la $\mathrm{XX}^{\mathrm{e}}$ dynasties, reproduits par Prisse d'Avennes. Atlas. t. II, pl. 67 et 68 .

3. W. II. Flinders Petrie, The arts of ancient Egypt. London, 1884, in-8, p. 23.

4. Goodyear, The grammar of the lotus, p. 56 et 57.

5. The grammar of the lotus, p. 99-105. Cf. Alois Riegl, Stilfragen, p. 52. Goodyear admet aussi pour la rosette deux autres origines; elle serait la représentation de l'ovaire du lotus vu d'en haut ou de la réunion de boutons assemblés par la base. 
D'autrefois ce furent les sépales qu'on s'attacha à reproduire de préférence, en en exagérant les dimensions; on alla même jusqu'à recourber en volute les deux latéraux qui, avec le médial plus ou moins arrondi, donnèrent naissance au lotus en forme de fer de lance ou lotus héraldique. Les artistes du nouvel empire ne s'en tinrent pas là; ils entourèrent de feuilles triangulaires la base des sépales et divisèrent en segments arrondis celui du milieu; la fleur primitive se trouva ainsi transformée en palmette ${ }^{1}$. Tels sont les ornements variés à l'infini que mit en curre l'art égyptien dans ses diverses applications.

Le monde végétal n'entrait pas seulement dans la décoration sculpturale ou pittoresque des édifices, les arts industriels: ébénisterie, verrerie, poterie, joaillerie, etc., lui avaient aussi emprunté de nombreuses formes ornementales. Si les meubles n'étaient pas nombreux dans l'ancienne Égypte, l'art du menuisier n'y avait pas moins, dès les temps les plus reculés, été porté à un haut degré de perfection ${ }^{2}$. Les coffrets étaient souvent revêtus de peintures, où des plantes, lotus et papyrus en particulier, étaient représentées. On en dessinait aussi sur les lits, les fauteuils et même, à l'époque gréco-romaine, sur les cercueils des momies. Sur les côtés d'un fauteuil trouvé dans la tombe de Khamhati, intendant d'Amenhotpou, sont peintes, avec un épervier et un sphinx, des fleurs de lotus $^{3}$. Le trône, trouvé dans la tombe de Ramsès III,

1. Goodyear, op.laud., p. 109. - Alois Riegl, Stilfragen. p. 64-66.

2. G. Maspero, L'Archéologie égyptienne, p. 268.

3. Descriplion de l'Égypte, t. II, pl. 89. - Perrot et Chipiez, op. laud., t. I, p. 843. 
est orné sur ses deux còtés de rosaces et de tiges fleuronnées ${ }^{1}$. Des tiges de papyrus décorent aussi, de leurs fleurs conventionnelles et de leurs boutons, deux fauteuils de la mème époque reproduits par Prisse d'Avennes".

Mais ce sont surtout les objets de bimbeloterie ou de toilette: boites, cuillers à parfums, qui sont riches en décorations végétales rariées et gracieuses. Ici c'est un bouquet formé de trois tiges de lotus, nouées ensemble, avec deux boutons et une fleur, dont l'ovaire saillant forme la boìte ou la cuiller ${ }^{3}$. Là un triple bouquet s'étage le long du manche et se termine par' une boite ornée d'un lotus". Ailleurs le fond d'une cuiller, dont le manche est formé par un élégant bouquet de lotus, est orné de deux poissons, qui cherchent à saisir cette plante sacrée ${ }^{5}$. Ou bien c'est une joueuse de guitare, debout entre deux tiges de lotus ou de papyrus, qui supportent une boite ovoïde ou quadrangulaire $^{6}$. Le manche d'une autre boite est formé par une porteuse d'offrandes, tenant dans ses mains des bouquets de lotus, tandis qu'un double faisceau de ces mêmes plantes, posé sur sa tête, sert de support à la cuiller ${ }^{\top}$. Dans une boite souvent reproduite, on re-*

1. Prisse d'Avennes, atlas, t. II, pl. 89, 1.

2. Atlas, t. II, pl. $89,2,3$ et 4 .

3. Prisse d'Avennes, atlas, t. II, pl. 93. - Perrot et Chipiez, op. laud., p. 844.

4. Musée britannique. Wilkinson, op. laud., t. II, p. 16.

5. Prisse d'Avennes, atlas, t. II, pl. 95.

6. Prisse d'Avennes, atlas, t. II, pl. 92, 6. - Perrot et Chipiez, op. laud., p. 845. Dans le fond d'une de ces boîtes, aujourd'hui au musée de Leyde, on voit un poisson qui cherche à saisir une fleur de lotus. C. Leemans, op. laud., pl. LXXIX, 387.

7. Prisse d'Avennes, atlas, pl. 92, 3. - Maspero, L'Archíologic égyplienne, p. 267. 
trouve encore le double faisceau de lotus; mais cette fois il repose sur la tête d'une jeune fille comme perdue au milieu d'un fourré de ces nymphéacées, dont elle cherche, gracieusement inclinée, à arracher la tige de l'une d'elles avec effort'.

Les plantes n'ont pas fourni moins de motifs de décoration à l'art du verrier ou du potier en Égypte, qu'à celui du menuisier ou de l'ébéniste. Le verre a été connu dans la vallée du Nil dès la plus haute antiquité; la fabrication en est déjà représentée sur une peinture tombale de Béni-Hassan ${ }^{2}$. Les artisans pharaoniques préféraient les verres de couleur aux verres blancs; la variété de nuances qu'ils leur donnaient devait les inviter à les orner de figures géométriques ou de dessins empruntés le plus souvent au monde végétal. C'est ainsi que deux coupes en verre opaque, l'une provenant de la tombe du père nourricier d'A menhotpou II, l'autre d'un hypogée du voisinage, ont la surface extérieure enveloppée d'une fleur de lotus ${ }^{3}$. Une petite œnochoé en verre bleu clair, qui porte l'inscription de Thoutmès III, présente sur ses flancs, tracée en jaune, une décoration végétale, qui rappelle l'arbre sacré des Assyriens, tandis qu'une ampoule lenticulaire de la même époque nous offre des entrelacs de palmes ou de feuillages qui s'enlèvent en jaune sur un fond bleu marin ${ }^{4}$.

Les Égyptiens aimaient à émailler les faïences, les poteries et, nous l'avons vu, les briques mêmes, ainsi

1. Prisse d'Avennes, atlas, t. II, pl. 92, 4. - Perrot et Chipiez, op. laud., p. 844.

2. Wilkinson, op. laud., t. II, p. 140.

3. Prisse d'Avennes, atlas, t. II, pl. 78. 2 et 3.

4. G. Maspero, L'Archiologie égyplienne. p. 251. 
qu'à les orner de motifs empruntés à la faune et à la Hore de leur pays. Les flancs d'une œnochoé en "faience égyptienne, " par exemple, reproduite par Willinson ', sont ornés dans toute leur partie inférieure de feuillages palmiformes. l)es amphores, provenant de la nécropole de Thèbes (XVIII ${ }^{\circ}$ dynastie) ${ }^{2}$, sont, elles aussi, ornées à leur partie supérieure ou sur le goulot de pétales renversés de lotus; la panse de la seconde est en mème temps ornée de branches fleuries renrersées d'une plante conventionnelle; on retroure des fleurs de cette même plante au-dessous du goulot de la première, contemporaine de Thoutmès III, tandis que la panse en est décorée par des bovillons, qui gambadent au milieu des Heur's. Sur des jarres de la mème époque ${ }^{3}$, on voit ici des pampres couverts de raisins, la des tleurs ou des boutons de lotus neêlés de grappes de raisin. On retrouve une décoration analogue sur une lampe en terre cuite du Musée de Leyde, qui est ornée de quatre feuilles et de quatre grappes de raisin". On voit également des palmes multicolores se dresser sur tout le pourtour d'un gobelet trouvé ì Deir-el-Bahari, dans la tombe de la princesse Nesi-Khonsou ". Parfois aussi les artistes pharaoniques appliquaient sur les vases des fleurs ou des boutons de lotus, dont les pédoncules les entouraient gracieusement ${ }^{6}$.

Deux tasses à pied d'une nécropole de Thèbes sont

1. The manners and customs, t. II, p. 12, no 280, 2 .

2. Prisse d'Avennes, atlas, t. II, pl. 81, 1 et 2 .

3. Prisse d'Avennes, atlas, t. II, pl. 82, nos 5 et 8,6 et 7 .

4. C. Leemans, op. laud., pl. I,XVII, no 441.

5. Maspero, L'Archcologie égyplienne, p. 252.

6. Prisse d'Avennes; atlas, t. II, p. 81, nos i et 7. 
entourées à leur base de pétales de lotus, en même temps qu'une fleur renversée de cette plante sacrée en forme de couvercle'. Sur le fond d'une patère on voit trois poissons affrontés, entre lesquels s'étalent trois fleurs de lotus ${ }^{2}$. Ailleurs des lotus accompagnés de l'œil mystique se dressent sur les parois intérieures d'un verre de forme analogue, conservé au Musée britannique. Dans le creux d'une espèce de patère du mème musée on voit, disposés aux quatre angles d'un rectangle ornemental, qui en occupe le centre, des bouquets de papyrus aux tiges gracieusement infléchies $^{3}$. Un vase à parfums en terre émaillée, du Musée de Leyde, dont le fond est orné de pétales de lotus, offre sur son pourtour deux bandes décoratives dont la seconde nous montre quatre léopards courant dans un bois de palmiers ${ }^{4}$. Sur un hippopotame du Musée de Boulaq, le potier, qui l'a représenté debout, a dessiné sur son corps à l'encre noire des pousses de roseaux et de lotus, " manière naïve de représenter la bête dans son milieu naturel ${ }^{5}$ ").

Les ornements empruntés à la nature végétale se retrouvent aussi sur les étoffes, les objets en cuir, ainsi que sur les meubles ou vases et les bijoux en métal. Ce sont surtout des feuilles et des tiges rampantes, mais souvent aussi des arbustes, que l'on voit sur les étoffes, sans qu'on puisse, il est vrai, dire à quelles espèces végétales se rapportent au juste ces motifs de

1. Prisse d'Avennes, op. laud., t. II, pl. 78, 4 et 5.

2. S. Birch, IIistory of ancient potery. London, 1873, in-8, p. 55 .

3. Perrot et Chipiez, op. laud., p. $822, n^{\circ} 531$ et 532.

4. C. Leemans, op.laud.. pl. LIX, 265.

5. G. Maspero, L'Archéologie égyptienne, p. 253, fig. 224. 
décoration; on ne peut guère douter toutefois que la feuille de lotus n'ait servi de modèle aux formes variées qu'ont fini par adopter les artistes pharaoniques, mais ils ne se sont astreints à reproduire aucune espèce particulière; sur des étoffes de Saqqarah on rencontre déjà des feuilles de vigne stylisées; on les trouve sous leur forme naturelle à Akhmim'. Parmi les nombrcux restes de tissus du musée égyptien de Vienne, étudiées par M. Alois Riegl, beaucoup sont remarquables à cause de leur décoration empruntée au règne végétal; tel est un fragment d'étoffe jaune en laine avec une garniture, dit l'auteur ${ }^{2}$, en forme de feuille, - on pourrait presque dire aussi en forme de sycomore -, brodée en pourpre sur fond blanc, et couverte de dessins en spirales de nature plus ou moins végétale. Tel encore plus le gatlon d'une manche en toile ${ }^{3}$, semé de feuilles arboriformes de grandeur diverse, couvertes elles-mêmes d'ornements végétaux.

Mais les plus beaux modèles d'ornementation végétale sur des tissus égyptiens me paraissent offerts par deux morceaux d'étoffe rouge en laine; sur la bande de l'un on voit, brodé en bleu sur fond blanc, un enfant ailé, qui tient un canard, planant entre deux larges feuilles arboriformes à nervures fortement marquées, au delà desquelles s'étale une espèce d'arbuste à trois branches terminées par un bouquet de feuilles ou de fleurs ${ }^{4}$. Au centre de l'autre morceau se trouve un carré, dans lequel on voit un génie agenouillé, tout autour sont des formes végétales, analogues à celles du premier, et

1. Alois Riegl, Die ägyptischen Textilfunde, p. XxI.

2. Page 1, col. 2, pl. I, no 202.

3. Pl. II, no 210, p. $23,2$.

4. Pl. IV, no 371, p. 37, 2. Cet arbuste ressemble à un doum. I. 
placées le long des bords d'un carré concentrique avec celui qui encadre le génie ailé; aux quatre angles, un arbuste à quatre branches recourbées, puis au milieu des quatre côtés une prétendue feuille, ou plutôt un sycomore couvert de son feuillage ${ }^{1}$. Le bord de ce carré est orné d'espèces de croix qui pourraient bien avoir, dans la pensée de l'artiste, représenté une bordure d'arbres.

Cette ornementation végétale n'a pas été employée seulement sur les étoffes, on s'en est servi également ainsi que de dessins géométriques sur les objets en cuir souple dont l'industrie devint florissante depuis l'époque des Ahmessides. Le dais de Deir-el-Bahari, conservé au Musée de Boulaq, en offre le modèle le plus parfait. A gauche, une touffe de lotus, flanquée des cartouches royaux, occupe le centre; viennent ensuite deux antilopes agenouillées, avec une fleur de lotus au cou, puis deux bouquets de papyrus ${ }^{2}$, enfin deux scarabées.

Le travail des métaux, par sa diversité, rendait la décoration plus variée et plus facile; elle n'a pas toujours, il s'en faut, été empruntée au monde végétal, mais il lui a fourni de nombreux motifs. Le Musée du Louvre possède une coupe d'or, présent de Thoutmès III à l'un de ses généraux Thoutii, dont le fond plat est orné, au centre, d'une large rosace, autour de laquelle nagent en cercle six poissons, entourés eux-

1. Pl. IV, no 372, p. 38,1 . Le tout simule un jardin analogue à celui qu'on voit sur la peinture 177 de la galerie égyptienne du Musée britannique; la bordure, garnie de croix arboriformes, achève la ressemblance.

2. G. Maspero, L'Archéologie égypticnne, p. 283, fig. 264. Il m'a été impossible de reconnaitre la nature de ces bouquets. 
mèmes d'une ceinture de fleurs de papyrus, reliées par une ligne sinueuse. Les vases de Thmouïs, conserrés à Boulaq, sont aussi décorés au repoussé de boutons et de fleurs épanouies de lotus ${ }^{1}$.

Les grands d'Égypte, au temps des Ahmessides et des Ramessides, possédaient, pour le service de leur table, une riche argenterie: plats, aiguières à pied, coupes, gobelets, corbeilles, ainsi que de grands vases décoratifs, qu'on remplissait de fleurs aux jours de fète. Quelques-uns étaient d'une richesse extraordinaire. Telle une coupe, dont le fond est orné et le pied formé par une fleur épanouie de lotus; deux boutons de cette plante forment avec leurs tiges les anses, que supportent deux esclaves asiatiques somptueusement vêtus ${ }^{2}$. Telle encore une espèce d'hydre aux anses formées par deux tiges de papyrus et qui a pour couvercle une fleur de lotus renversée, accompagnée de deux têtes de gazelles. La panse, divisée en zones horizontales, figure, dans celle du milieu, un fourré de papyrus que traverse en courant un antilope ${ }^{3}$. Deux burettes ont aussi pour anse des tiges recourbées de lotus en fleurs, dont la courbure est occupée par une élégante rosace ${ }^{4}$. Enfin sur un étendard en bronze de Mouth ou de Neith on voit, au-dessous de l'image de la déesse, des fleurs et des boutons de lotus ${ }^{5}$.

Les ornements tirés de la nature végétale occupent

1. G. Naspero, L'Archéologie égyptienne, p. 300, fig. 275 et 276 .

2. Prisse d'Avennes, op. laud., t. II, pl. 95. - G. Maspero, op. laud., p. 302, fig. 279.

3. Prisse d'Avennes, op. laud., t. II, pl. 93, 5. - G. Maspero, op. laud., p. 302, fig. 280.

4. G. Maspero, op. laud., p. 303, fig. 281 et 282.

5. C. Leemans, op. laud., pl. LXXXIII, $71 \mathrm{c}$. 
encore une place plus grande dans divers surtouts en or; l'un, offert à Amenhotpou III par un vice-roi d'Éthiopie, se compose de deux coupes à pied bas, surmontées, la première d'une pyramide, la seconde d'une espèce de mastaba, et entourées l'une et l'autre par des palmiers doums ${ }^{1}$; un autre, de même date et de même provenance, représente la cueillette des fruits dans un bois de palmiers doums; on voit des singes et des hommes grimpés sur les arbres, au pied desquels deux indigènes tiennent en laisse des girafes apprivoisées, tandis que d'autres habitants agenouillés à la lisière du bois lèvent les mains, comme s'ils imploraient la pitié des troupes égyptiennes ${ }^{2}$. Sur d'autres surtouts présentés à Ramsès II, dans le temple d'Ibsamboul, les girafes ont fait place à des buffles courant à travers les palmiers ${ }^{3}$.

Les Orientaux sont grands amateurs de bijoux, les Égyptiens ne faisaient pas exception à cette règle; l'on a trouvé d'énormes quantités de joyaux dans les tombes pharaoniques; mais les ornements qui les embellissent ne sont qu'exceptionnellement empruntés au monde des plantes; il n'y a que plus d'intérêt à signaler ceux qui en sont tirés. Le Musée de Leyde possède un diadème en argent doré, trouvé dans la tombe du pharaon Entouf IV, dont l'agrafe est ornée de deux fleurs de lotus émaillées ${ }^{4}$. On y voit aussi un collier formé de corolles de lotus en émail et de grains

1. Gournet-Mourrai. Lepsius, Denkmäler, t. VI, pl. 118.

2. Lepsius, op laud., t. VI, pl. 118. - G. Maspero, L'Archéologie égyptienne, p. 303, fig. 283.

3. G. Maspero, op. laud., p. 303.

4. C. Leemans, op. laud., pl..XXXIV. 
de corail ${ }^{1}$. Une égide contemporaine de la XXII ${ }^{\circ} \mathrm{dy}-$ nastie et maintenant au Louvre offre, autour d'une figure assise et ailée, des bandes concentriques remplies d'une riche ornementation ${ }^{2}$; les deux extérieures sont formées par des fleur's épanouies de lotus, séparées dans l'une, par de petits cercles, dans l'autre, par des cercles et des boutons.

\section{II.}

Le monde des plantes n'occupait guère moins de place dans la poésie que dans l'art pharaonique. Il n'en pouvait pas être autrement; le règne végétal était trop intimement mêlé à la vie des habitants de la vallée du Nil, pour qu'il ne fût pas associé à toutes les manifestations de leur pensée. Il faut voir avec quel amour Penbisit, scribe de Ramsès II, décrit les richesses végétales d'Aanakhtou, " la ville de Ramsès", fondée par le pharaon ${ }^{3}$ :

"Ses campagnes, s'écrie-t-il, sont pleines de toutes les choses délicieuses... ses prés foisonnent d'herbages; la plante ades en touffes, la plante aden-roga, aussi douce que le miel (croît) dans ses champs bien arrosés. Ses greniers sont pleins de blé et d'orge... Les joncs et les plantes aciqer de l'enclos, les fleurs $\grave{a} b \hat{u}$ du jardin fruitier... les grenades, les fruits tephou et $t e b$, de la pépinière, les vins doux de Kahêmê (y abondent). »

Il était inévitable que les poètes d'un peuple qui ai-

1. C. Leemans, op. laud., pl. XXXVII, 112.

2. Perrot et Chipiez, op. laud., p. 834 .

3. G. Maspero, Du genre épistolaire chez les anciens Égyptiens. Paris, 1872, in-8, p. 103. Cf. Letter of Panbesa, transl. by. C. W. Goodwin. (Records of the past, t. VI, p. 13.) 
mait avec une telle passion les choses de la nature végétale, ne s'en inspirassent pas dans leurs fictions et ne lui empruntassent pas quelques-unes de leurs images et de leurs comparaisons les plus habituelles.

L'un d'eux, on le comprend, place au milieu d'un jardin fleuri le récit de ses aventures et de son rendezrous avec son amie ${ }^{1}$ :

" Elle me prit par la main et nous entrâmes dans son jardin... Les buissons (en) étaient verdoyants et tous étaient en fleurs. Il y avait des fruits kaiou et tipau ${ }^{2}$ plus rouges que le rubis, ceux du perséa y ressemblaient au bronze et les bosquets avaient le lustre de la pierre nasheni ${ }^{3}$, les menni ${ }^{4}$ entrouverts nous livraient leurs amandes; leur ombre, fraiche et bien aérée, était douce pour le repos de l'amour. "

Dans un conte célèbre, celui des deux Frères, Bitiou, après avoir révélé à Anoupou la perfidie de sa femme, refuse de revenir au domicile commun et se retire au Val de l'Acacia ${ }^{5}$. Là, il se construit une maison, dépose son cœur sur le sommet de la fleur de l'arbre et passe désormais ses jours dans la solitude. Mais les Dieux, " descendus en visite sur la terre, " ont pitié de son isolement, et Khnoum lui fabrique une belle femme. Bitiou en devient amoureux fou. Un jour cependant il la quitte pour aller à la chasse, non sans lui avoir auparavant confié le secret de sa vie et recommandé de

1. The tale of the garden of flowers, transl. by Fr. Chabas. (Records of the Past, t. VI, p. 153.)

2. Chabas traduit par groseillier et cerises tout en avouant que ces fruits n'avaient probablement rien de commun que la couleur avec les kaiou et tipau.

3. Feldspath de couleur verte.

4. Fruit d'espèce inconnue.

5. G. Maspero, Les contes populaires de l'Égypte ancienne. Paris, 1882, in-12, p. 1'1. 
ne pas quitter la maison, car le fleuve, qui traverse la vallée, pourrait l'enlever. Bitiou parti, la jeune femme va se promener sous l'Acacia, qui était près de leur demeure; bientôt elle aperçoit le fleuve qui venait à elle, elle s'enfuit et rentre dans la maison. Pour la saurer, l'Acacia livre une boucle de ses chereux. La boucle, portée en Égypte, est remise à Pharaon; sur le conseil de ses scribes, celui-ci envoie des messagers à la recherche de celle à qui elle avait appartenu; il se troure qu'elle est la fille de Phrâ-Harmakhouti, et qu'elle " a en elle l'essence de tous les Dieux. » Bitiou tue les messagers, à l'exception d'un seul, lequel informe le roi de ce qui était arrivé. Alors le Pharaon expédie une véritable armée, et cette fois on ramène la fille des Dieux, que Pharaon « salue grande favorite ${ }^{1}$ ». Quand il connut l'histoire de Bitiou, il envoya des hommes couper l'acacia. Lorsqu'ils eurent tranché la fleur sur laquelle était le cœur de Bitiou, celui-ci tomba mort sur l'heure.

Averti par un présage, Anoupou se rend au Val de l'Acacia; il y trouve son frère étendu sans vie; mais ce ne fut qu'au bout de trois ans qu'il put découvrir son cœur. Bitiou ressuscité se change alors en taureau et se rend avec son frère à la cour du roi pour reprocher son inconstance à celle qui l'a abandonné. Irritée, celle-ci obtient qu'on mette à mort le taureau accusateur; mais de son sang naissent deux perséas, qui trouvent une voix pour reprocher à l'épouse infidèle sa perfidie. Elle obtient qu'on les abatte, qu'on en façonne des planches, et pour être certaine de sa vengeance, elle assiste à l'opération; mais un copeau

1. Le conte des deux frires, p. 20. 
roulant sous l'herminette des menuisiers lui entre dans la bouche ${ }^{1}$; elle conçoit et met au monde, incarnation de Bitiou, un fils, qui devient roi d'Égypte.

On voit par ce conte à quel point le monde des plantes pouvait être mêlé aux conceptions égyptiennes de la vie humaine; pour le conteur pharaonique rien de plus naturel que la société entre l'acacia et Bitiou, que l'apparition soudaine des perséas sortis de son sang. Ceux-ci sans doute ne sont que l'incarnation de Bitiou et l'on concoit dès lors qu'ils puissent parler. Dans le dialogue suivant ${ }^{2}$, ce sont de vrais arbres, qui entrent en scène, mais ils n'en sont pas moins capables de sentiment et de raison. Il n'est pas difficile, il est vrai, de trouver, cachés derrière eux, le poète et ses amis, qui ne semblent avoir pris ce costume d'emprunt que pour exprimer arec moins de gêne leurs vrais sentiments.

"Mes graines sont l'image de ses dents, dit le premier; mon port ressemble à ses deux seins... Tous [les arbres] passent, sauf moi, dans le verger... je suis le premier '[et pourtant] ils ne me considèrent plus que cornme étant au second rang. S'ils me traitent encore de la sorte, je ne me tairai point à leur sujet... et quand on saura le crime, on châtiera la bien-aimée, qui ne retrouvera plus de bouquets de lotus blanc ${ }^{3}$ et de boutons [d'offrandes] de lotus bleus et de parfums...

On ne sait quel est l'arbre qui parle ainsi; M. Maspero suppose que c'était peut-être un vieux sycomore;

1. G. Maspero, op. laud., p. IX et 26.

2. G. Maspero, Les chants d'amour du papyrus de Turin et du papyrus Harris, no 500. (Études égyptiennes. Paris, 1879, in-8, p. 217 et suivantes.)

3. J'ai substitué au mot " lis " de la traduction de M. Maspero celui de "lotus blanc. " 
on peut le croire facilement à ses prétentions, aux plaintes qu'il fait entendre d'ètre négligé pour un autre et relégué au second rang. Tout autre est le langage des deux autres arbres, jeunes et satisfaits, le figuier et le petit sycomore; on ne peut nier que pour des arbres, ils ne parlent fort bien.

"Le figuier ouvre sa bouche, et son feuillage vient dire ${ }^{1}$ : [Apprends de moi] ce qu'on [me] fait. Je [viens] vers une maitresse, qui certes est une reine comme moi et n'est pas une esclave. Moi donc, je suis le serviteur [apporté de Syrie] prisonnier de la bien-aimée; elle m'a fait mettre dans son parc, elle ne ma pas donné [un breuvage commun]; le jour où je bois, mon ventre ne s'emplit point de vulgaire eau d'outre. On a trouvé réjouissant [que le jardinier] ne me donne plus à boire. Par mon double, ô bien-aimée, puisse-t-il être amené en ta présence! »

Plus disert encore est le troisième arbre; mais son langage ne mérite pas seul de nous arrêter; les comparaisons dont s'est servi le poète pour nous le peindre ne sont pas moins dignes de fixer l'attention.

“ Le petit sycomor'e qu'elle a planté de sa main ouvre sa [bouche] pour parler ${ }^{2}$. [Ses] accents sont [doux comme] une liqueur miellée d'un miel excellent; ses touffes sont gracieuses, fleuries... chargées de baies et de graines, plus rouges que la cornaline; ses feuilles sont drues et bariolées comme l'agate, son bois est de la couleur du jaspe vert; ses graines sont comme les tamaris... son ombre est fraîche et éventée de brise; il [a fait] sa missive par la main d'une autre personne, la fille du chef jardinier qui le soigne et celle-ci l'a transmise à la bien-aimée. "Viens... Le verger est dans son beau jour... les gouverneurs de tes domaines se réjouissent... Que tes esclaves défilent devant moi, armés de leurs outils, grisés par

1. Éludes égyptiennes, p. 224.

2. Études égypliennes, p. 225. - A. Erman, Aegyplen und aegyptisches Leben im Alterlum. Tubingen, 1885, in-8, p. 272273. 
leur ardeur à courir vers toi... Quo tes domestiques viennent, apportant de la bière, toute sorte de pains, des plantes nombreuses, tous les fruits plaisants. Allons passe chaque jour dans le bonheur... assise à mon ombre, ton maître à ta droite; enivre-le, obéis à ce qu'il dit. Si la salle où l'on boit la bière est bouleversée par l'ivresse... si elle soulève son voile pendant sa promenade, moi j'ai le sein fermé et je ne dis point ce que je vois, non plus que ce qu'ils disent. "

Quel arbre discret et comme il est digne de celle qui l'a planté! Il devait fleurir à une époque cultivée, ou plutôt raffinée, et son langage peut nous donner une idée assez exacte du style en honneur dans les premiers temps de la $\mathrm{XX}^{0}$ dynastie ${ }^{1}$. Les fragments des chansons récréatives, qui nous ont été conservées par le même papyrus que le dialogue des trois arbres, nous en révèlent un autre aspect. Ici les plantes ne sont pas prises comme porte-parole, mais comme témoins de la sincérité du langage du poète, en même temps qu'elles lui fournissent les comparaisons les plus gra. cieuses et les plus variées:

"O pourpiers", mon cœur est en suspens quand tu fais ce qu'on recherche... O maitre de mon cœur, qu'elle est belle mon heure!

“ O armoises de mon frère, devantqui l'on se sent plus grand, je suis ta sœur favorite et je te suis comme le champ où j'ai fait pousser des fleurs et toute espèce de plantes odorantes...

“ O marjolaines de mon frère, j'ai pris tes guirlandes, quand ivre tu viens à moi... »

Dans un chant d'amour du mème papyrus, ce sont surtout les comparaisons que le poète a accumulées;

1. C'est à ceite époque que M. Maspero, place la rédaction de ce dialogue poétique.

2. Études égyptiennes, p. 253. J'ai conservé les noms de plantes tels que les donne M. Maspero, encore que quelquesunes, n'étant pas indigènes en Égypte, y aient dù être difficilement naturalisées à l'époque de la XX $X^{\mathrm{e}}$ dynastie. 
tel est en particulier ce passage d'une prière adressée au "(dieu) juste "!

"Que ma sœur soit pendant la nuit comme la source vive dont les myrtes sont semblables à Phtah, les nymphaeas semblables à Solihit, les lotus bleus semblables à Aditi. ”

Dans l'inscription d'une stèle du Louvre, dédiće par un Pharaon à sa fille Montiritis ${ }^{2}$, les comparaisons, empruntécs au règne régétal, devicnnent des métaphores ingénieuses, qui servent au roi à exalter la beauté do la princesse :

"Une palme d'amour. la prètresse d'Hâthor Montiritis, une palme d'amour, auprès du roi Menkhopriri! C'est une palme auprès de tous les hommes, un amour auprès de toutes les femmes. Noire est sa chevelure plus que le noir de la nuit, plus que les baies du prunellier (?), [rouge] sa [joue] plus que les grains du jaspe rouge, plus que l'entame d'un régime de dattes. ")

Ces comparaisons tirées du monde des plantes apparaissent à toutes les époques connues de la langue pharaonique. "Douce comme la palme, dans son amour pour son époux », lit-on ${ }^{3}$ dans l'épitaphe de Noufirhotpous, femme de Ti. " Je me parai des fines étoffes de mon palais pour paraitre aux yeux comme une des plantes de mon jardin", dit Amenemhat dans ses instructions à son fils Osourtisen". "O palmier mama, grand de soixante coudées ", s'écrie encore un poète ${ }^{3}$, s'adressant au dieu Thot, dont il voulait ainsi peindre ia puissance.

1. Éludes égyptiennes, p. 237.

2. Traduite par N. Naspero, Études égyptiennes, p. 257.

3. Dümichen, Resullate, tab. III, p. 1, 2.

4. G. Maspero, Hisloire ancicnne, éd. in-12, p. 96.

5. Victor Loret, Recherches sur quelques plantes, I. (Recueil de travaux, t. II, p. 23.) 


\title{
CHAPITRE VII
}

\author{
LES PLANTES DANS LES LÁGENDES DIVINES \\ ET DANS LES CÉRÉMONIES PROFANES ET RELIGIEUSES.
}

I.

Comme tous les peuples primitifs, les anciens Égyptiens vénérèrent d'abord les forces de la nature; c'étaient pour eux des divinités bienfaisantes ou funestes, dont il fallait obtenir la faveur ou apaiser la colère ${ }^{1}$. Créés par un effort d'abstraction, chacun de ces dieux vit ses attributs varier avec le temps et le lieu où il était en honneur; des rapports arbitraires de parenté ou de dépendance furent établis entre eux; ils se groupèrent et se hiérarchisèrent, en vertu de conceptions particulières et formèrent le panthéon le plus complexe et le plus nombreux que l'on connaisse.

C'est ainsi que Nou, l'océan ou l'abîme céleste ${ }^{2}$, dieu, source de la vie et longtemps unique, forma plus

1. Il faut être égyptologue pour entreprendre un exposé de la théogonie égyptienne; on comprendra que je ne l'aie point essayé ; je me suis borné ici à faire connaitre les principales divinités du Panthéon égyptien, qui figurent dans les légendes des plantes.

2. Victor von Strauss und Torney, Entstehung und Geschichte des allügyptischen Götterglaubens. Heidelberg, 1891, in-8, p. 108, ne voit dans Nou que le ciel divinisé. 
tard, avec Nounet, la matière primordiale, Kek, l'Érèbe égyptien, et Keket, personnification des ténèbres, Heh et Hehet, le temps et l'esprit, et les personnifications obscures de Nenou et de Nenout, un premier groupe de huit divinités, abstractions confuses des forces primordiales de la nature, qui s'effacèrent, à l'exception de Nou, pour faire place à une ennéade de divinités plus saisissables, issue du Dieu solaire Toum. ou Atoum ${ }^{1}$ : Shou, l'atmosphère déifiée, qui sépare le ciel de la terre ${ }^{2}$, et Tafnout, la déesse de la gràce et de la beauté suprêmes, puis Sibou, le "père des dieux », regardé à l'origine comme une émanation de Nou, plus tard comme fils de Shou et de Tafnout, et Nouit, la "mère des Dieux ", personnification du ciel étoilé, puis encore Ousiri — Osiris - - issu de Sibou et de Nouit, le dominateur du monde suprême et le juge des enfers, et Ousit ou Isit - Isis — sa sœur et son épouse, la bienfaitrice des hommes, Har ou Hor - Horus - leur fils, le successeur et le vengeur d'Osiris. Encore enfant et resté près d'Isis, c'était Horpikhroud ou Harpekhroudou, "Horus l'enfant ", Harpocrate - ; considéré comme l'égal d'Osiris, c'était Horus l'ainé ou Haroïri. Horus fut aussi envisagé comme la personnification du ciel ou de la lumière céleste et comme tel il remontait aux premiers âges de la religion égyptienne. Outre Osiris et Isis, naquirent

1. H. Brugsch, Religion und Mythologie der alten Aegypter nach den Denkmälern. Leipzig, 1888, in-8, p. 128-145, 231
et 281 .

2. N. Paul Pierret le regarde comme la force cosmogonique du soleil personnifiée. Le Panthion égyptien. Paris, 1881, in-8, p. 17. - A. Wiedemann, Die Religion der alten Aegypter. Münster i. W., 1890, in-8, p. 18, en fait un fils de Râ et de Hâthor. 
de Sibou et de Nouit, Sit, rival d'abord, puis l'ennemi et le meurtrier de son père, et Nebthat - Nephthys mère d'Anoupou-Anubis -, sœur de Sit et son épouse, avant qu'il fût devenu le génie du mal ${ }^{1}$.

Comme les autres forces de la nature, le soleil et la lune furent personnifiés; ils avaient à l'origine fait partie du ciel; alors que Hor en était regardé comme l'incarnation, ils étaient les deux yeux du Dieu; mais ils prirent bientôt une existence individuelle et Je soleil devint un Dieu et même le plus puissant des Dieux ${ }^{2}$. Sorti du sein de Nou, il s'est engendré luimême, ainsi que le monde; mais il s'est manifesté sous les formes les plus diverses. Dans la BasseÉgypte il prit le nom de Toum ou Atoum, le maître des Dieux de On - Héliopolis - force génératrice de la lumière, édificateur du monde dont les membres, créés par lui, composent la grande ennéade ${ }^{3}$.

Toum fut surtout le dieu des théologiens; pour le peuple la vraie personnification du soleil était et resta Rà ${ }^{4}$, dieu suprême, tout puissant, sans égal, élevé au-dessus des autres dieux et leur père, comme celui des hommes ${ }^{5}$. Toutefois, par une de ces alliances si communes dans la théogonie égyptienne, Râ et Toum ne restèrent pas isolés dans leur grandeur abstraite; ils s'unirent pour former un seul dieu Râ-Toum ou

1. H. Brugsch, op. laud., p. 408-422.

2. Pour A. Wiedemann, op. laud., le culte du soleil remonterait aux temps préhistoriquos.

3. H. Brugsch, op. laud., p. 281-282.

4. Maspero, Histoire ancienne, t. I, p. 136 et 140. - Les hypogées royaux de Thèbes. (Études de mylhologie et d'archiologie égyptiennes, t. II, p. 7.)

5. V. von Strauss und Torney, Die altügyptischen Götter und Güttcrsagen. Heidelberg, 1889, in-8, p. 240-253. 
Atoum-Rì, qui hérita de leur's prérogatives respectives. Après avoir été "le soleil antérieur au monde", Toum représenta surtout le soleil à son coucher; Ǩhepra ou Kíhopri, "le dieu qui nait ", le personnifia à son lever'; la lumière de midi s'incarna dans $R \hat{i}^{\prime}$ ou dans la déesse Mentou, et Sovkou, "le crocodile", qui veille sur la montagne d'où sort le soleil, personnifia les rayons redoutables de l'astre ${ }^{2}$. Il y eut aussi non pas union, mais une confusion véritable entre Rì et Horus ; Rà s'empara de toutes les épithètes d'Horus et Horus de tous les rôles de Rà ; Harmakhouti, "l'Horus des deux horizons ", désigne à la fois l'Horus, c'esta-dire le soleil, qui sort de la montagne do l'est au matin - le soleil levant - et l'Horus, qui le soir s'enfonce dans la montagne de l'ouest - le soleil couchant ${ }^{3}$.

L'évolution de la pensée religieuse des anciens Égyptiens ne s'arrèta pas lì et le caractère de leurs dieux était trop peu stable pour qu'ils ne dussent pas subir sans cesse de nouvelles transformations. C'est ce qui arriva à Shou en particulier; il devint tour à tour ou suivant les lieux Phtah, Khnoum, Khonsou mème, Haroïri - Aroêris - d'autres encore ${ }^{4}$. Phtah, le dieu de Memphis "demeure de Phtah »- Hà-kou-Phtah —, le grand dicu, auquel les divinités des demeures supérieures donnent le vivre et le manger, le distribu-

1. "Je suis Khepra le matin, Râ à midi et Toum le soir », dit un papyrus de Turin. Lanzone, Dizionario di elimologia egiziana. Torino. 1887-88, in-4, p. 930.

2. V. von Strauss und Torney, op. laud., p. 3330-336. Paul Pierret, op. laud., s. v.

3. Naspero, Histoire ancienne, t. I, p. 100.

4. II. Brugsch, op laud., p. ${ }_{422-532 .}$ 
teur des vivres dans le monde souterrain, s'est transformé à son tour en Tanen ou Tatounen, dieu suprême, qui a formé les hommes et fait les Dieux. Comme tel, il eût pour femme Sokhit, "la gaie ", assise à l'occident du ciel ", et pour fils Imhotpou, " le paisible ", dieu guérisseur, l'Asklepios égyptien.

Tandis que le dieu suprème était Phtah à Memphis, à Éléphantine c'était Khnoum, à la tête de bélier; créateur des hommes, père des pères et mère des mères, on le trouve associé aux "fées des cataractes" Satit - Satis - , qui veille au seuil du Nil et Anouqit, l'Hestia égyptienne ${ }^{2}$. De Khnoum et de Satit naquit un dieu populaire, Khem, le Pan des Grecs, force vivifiante de la divinité; uni à Hor, il devint Khem-Hor, à Rà, Khem-Rầ .

De Phtah il faut rapprocher Nefertoum ou Noufirtoum, " la belle fleur de Toum ", honoré comme lui à Memphis, une des personnifications du soleil levant. Le soleil à midi, lui, trouvait une nouvelle personnification dans Haroïri, autre divinité de la famille de Shou. A cette famille Brugsch rattache encore $^{4}$, Mât ou Maït, déesse de la vérité et de la justice et Thot ou Thout, " le secrétaire des dieux, seigneur des paroles divines, calculateur du ciel et de ses $\operatorname{astres}$ ". On peut lui associer Safekh, "la déesse des livres ", et en rapprocher Anoupou ou Apherou, dieu

1. V. von Strauss und Torney, op. laud., p. 426 et 431.

2. G. Maspero, La mythologie égyptienne. (Études de mythologie et d'archéologie égyptiennes. Paris, 1893, in-8, t. II, p. 273.) - Brugsch, op. lcud., p. 299-302.

3. V. von Strauss und Torney, op. laud., p. 271.

4. Op. laud., par. 178 et 181, p. 477 et 484 .

5. Paul Pierret, op. laud., p. 13 et 15. 
bienveillant qui, à la mort, prenait sous sa garde le corps du défunt".

A còté de Toum se plaçait Hiithor, l'Aphrodite égyptienne, "la belle et la bonne ", comme elle est appelée dans une inscription de Merenra, déesse de l'amour et de la joie, "la dame du ciel "; devenue épouse de Hor, elle en eut pour fils Ahi. Son culte, à l'époque de la décadence égyptienne, prit une importance consid̉érable; l'érection du temple de Dendérah en son homneur on est un témoignage éclatant?2.

En face de Rì se trouve Amon ou Amen, "le caché ", dernier terme et suprême degré du développement mythique de l'ancienne Égypte; uni à Râ, il est devenu Amon-Rà, qui domine sur la double terre, seigneur du ciel, roi des Dieux, maitre de l'éternité $^{3}$. On lui donna pour épouse Mout ou Maut, "la mère ", qui devịnt ainsi la " maîtresse du ciel ", la "reine des Dieux " ". D'Amon-Râ et de Maut naquit Khonsou, " le maitre des sacrifices ", dieu ancien, regardé aussi comme une transformation de la divinité primordiale Shou", admis maintenant au rang des dieux solaires.

Telles étaient les principales divinités du Panthéon égyptien, les autres prirent naissance plus tard ou sont sans importance pour le sujet qui nous occupe; inutile dès lors d'en faire mention. Il est encore un dieu pharaonique cependant dont il me faut parler, c'est Hapi, personnification du Nil, ce fleuve bienfaiteur de

1. V. von Strauss und Torney, op. laud., p. 201.

2. V. von Strauss und Torney, op. laud., p. 166-172.

3. Paul Pierret, op. laud., p. 95.

4. V. von Strauss und Torney, cp. laud., p. 407.

5. H. Brugsch, op. laud., p. 493. 
l'Égypte, sorti d'Osiris et dont les flots gonflés descendent de l'Océan céleste ${ }^{1}$. De serviteur des dieux, il fut, sous le nouvel empire, élevé à leur rang; on le compara à Khnoum; il devint le symbole cosmique du principe surnaturel Nou, le créateur de ce qui est nécessaire à la vie des hommes, un dieu mystérieux, objet de la vénération des nouvelles générations.

II.

Comme le reste de l'univers, le monde végétal était soumis aux dieux, arbitres de tous les êtres. Râ ou Amon-Rà en était le créateur. Les plantes, avec tout ce qui a vie, avaient jailli de ses prunelles, quand le dieu, apercevant, à son premier lever, la terre nue et déserte, l'avait inondée de ses rayons, comme d'un. flot de larmes. " "Dieu unique, créateur des ètres, formateur des choses », dit un hymne en son homneur, qui remonte à la XIX dynastie $^{3}$, " il a créé les plantes qui nourrissent le bétail et les arbres à fruits pour les hommes ".

Une légende " attribuait l'origine de diverses plantes aux larmes tombées des yeux ou à la salive sortie de la bouche de certains Dieux. Ainsi lorsque Horus pleure, ses larmes donnent naissance à de suaves

1. H. Brugsch, op. laud:, p. 638. - Maspero, Histoire ancienne, t. I, p. 19.

2. G. Maspero, Hisloire ancienne, t. I, p. 156.

3. Grébaut, Hymne à Ammon-Râ du papyrus égyplien du Musie de Boulaq. Paris, 1875, in-8, pl. VI, p. 16 et pl. XXIII, p. 27. - G. Maspero, op. laud., p. 285. - H. Brugsch, Religion und Mylhologie, p. 693.

4. Magical Texts. (Recorls of the past, t. VI, p. 115-116.) 
parfums. Les larmes tombées des yeux de Shou, " le fils, " et de Tefnout, " la fille du soleil', " se changent en arbres qui produisent l'encens. Quand le soleil faiblit et qu'il transpire, la salive, qui de sa bouche dégoutte sur la terre, fait naitre autant de papyrus. La sueur de Nephthys donne de même naissance à la plante tas.2. Baba - Typhon - au contraire, saigne-t-il du nez, son sang se change en une plante qui devient un cèdre et produit l'essence de térébenthine.

Osiris présidait à la propagation du monde végétal ; c'était lui qui fécondait le sol de l'Égypte. "Osiris de l'Cuest, dit un hymne sacré ${ }^{3}$, toutes les plantes croissent à ton approche; ce qui était mort repousse et les papyrus verdoient sous l'humidité. "Aussi ce dieu fut-il considéré comme le père de l'agriculture. On lui attribuait l'invention de la charrue; c'était lui qui avait enseigné aux hommes à faconner la terre et récolter le blé et l'orge".

Isis avait des attributs analogues; on la regardait comme la " créatrice des vertes moissons ${ }^{5}$ ". C'était elle qui avait apporté aux hommes les céréales qui les nourrissent. "Je suis Isis, lui fait dire une inscription mentionnée par Diodore ${ }^{6}$. qui la première enseigna aux hommes l'usage du blé. " Et le texte, qui accompagne son image sur une peinture du temple d'Osiris

1. Wilkinson, op. laud., t. III, p. 172 et 192, pl. XXXIX.

2. Peut-être le cinnamone. Victor Loret, Le Kyphi, parfum sacré des anciens Égyptiens. Paris, 1887, in-8, p. 46.

3. H. Brugsch, op. laud., p. 613 et 626 .

4. Diodore, Bibliolheca, lib. I, cap. 14, 1.

5. H. Brugsch, op. laud., p. 647.

6. Bibliotheca, lib. I, cap. 27, 4. 
à Dendérah", la représente comme " celle qui fait grandir le blé par son action, qui donne au blé son éclat depuis le soir jusqu'au matin ».

Ainsi qu'Osiris, Khem, " le laboureur ${ }^{2}$ ", présidait au monde des plantes; c'était le dieu des jardins et des champs; une peinture pharaonique le montre debout, la main droite levée ${ }^{3}$; derrière lui, sur une espèce d'autel, se dressent deux sycomores, tandis que devant lui un roi retourne, avec le hoyau, le sol auquel sera confié la semence nourricière. Sur une peinture de Médinet-Habou, qui représente une procession faite en l'honneur de Khem à l'époque de Ramsès III ${ }^{4}$, on voit le pharaon, assisté de la reine, couper, emblême de la moisson, des épis de blé que lui présente un prêtre.

Le Nil - Hàpi -, " créateur de toutes les choses bonnes ", présidait, lui aussi, au monde végétal; dans un hymne que j'ai déjà cité ${ }^{5}$, il est célébré comme " faiseur de blé, producteur d'orge ", comme celui qui " donne le fourrage des bestiaux » et remplit les greniers.

Créées par les dieux, soumises à leur influence féconde, les plantes prirent place dans leurs légendes. Avant la création, Râ, le soleil, encore plongé dans le Nou, l'Océan primordial, tenait son disque emprisonné dans un bouton de lotus, dont les pétales repliés l'avaient préservé; mais lorsqu'au matin du premier

1. A. Mariette, Dendérah. Description générale du grand temple de celle ville. Paris, 1875, in-4, p. 283 ; atlas, t. IV, pl. 58.

2. V. von Strauss und Torney, op. laud., p. 372.

3. Wilkinson, op. laud., t. I, p. 40' et t. III, p. 22-24.

4. Wilkinson, op. laud., t. III, pl. LX.

5. Chapitre I, p. 5. 
jour Toum lança du fond du gosier le mot créateur : "Viens à moi », le lotus s'ouvrit et le dieu en surgit brusquement, semblable à un enfant coiffé du disque solaire '. "Le soleil, qui existe dès le commencement, dit une inscription du temple d'Hiithor à Dendérah ", en faisant allusion à cet acte créateur, s'élève, semblable à un faucon, du milieu de son bouton de lotus, lorsque s'ouvrent, dans leur éclat de saphir, les portes de ses feuilles, il sépare la nuit du jour. "s Sur une peinture du temple d'Edfou, qui représente les divinités primordiales, on voit le soleil enfant au-dessus d'une fleur ouverte de lotus, qui se dresse au milieu d'un bassin rempli d'eau, emblême de Nou ${ }^{3}$. "En ouvrant les yeux, dit l'inscription qui s'y rapporte ${ }^{4}$, il éclaire le monde et sépare la nuit du jour... Quand il s'élève brillant du milieu du lotus, tous les êtres prennent vie ».

Lorsque le coffre, dans lequel Sit, aidé de ses complices, avait traitreusement enfermé Osiris, eut été jeté dans le Nil, les flots le déposèrent au pied ou, suivant une autre version, au milieu des branches d'un tamaris gigantesque". Cet arbre figure aussi souvent dans les représentations d'Osiris. Dans la chambre du temple de Philae, consacrée à ce dieu, ainsi que sur une tombe de Hou - Diospolis parva - était peint

1. G. Maspero, Histoire ancienne, t. I, p. 137 et 140.

2. H. Brugsch, Die mylhologic und Religion, p. 103.

3. G. Maspero, op. laud., t. I, p. 136.

4. H. Brugsch, op. laud., p. 164.

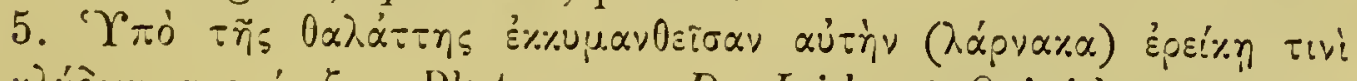

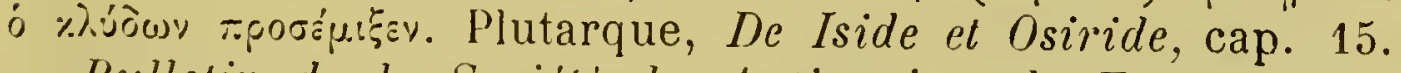
- Bullelin de la Sociélé des Antiquaires de France, 1858, p. 133. 
un tamaris ${ }^{1}$. Sur les rameaux de l'arbre sacré, dans la peinture de cette dernière localité, on voit planer l'oiseau bennou, espèce de héron (Ardea garzetta L.), symbole de l'âme du dieu.

Une peinture de la chambre hypostyle du Memnonium² représente Ramsès II, revêtu des insignes du pouvoir souverain, assis sur un trône à l'ombre d'un perséa, pendant que le dieu suprême Râ-Toum, la déesse de la science Safekh et Thot écrivent sur les fruits de l'arbre le nom du pharaon. A Médinet-Habou ${ }^{3}$, on voit Thoutmès III, amené par Hảthor et Thot devant l'arbre de vie, sur les fruits duquel le dieu Amon-Rì écrit une formule sacrée. A Derri ${ }^{4}$, Ramsès III est représenté debout au pied du même arbre entre Thot, Phtah et Safekh ou Pakht.

Mais les anciens Égyptiens n'associèrent pas seulement les arbres à la vie des dieux, ils en firent des dieux eux-mêmes et les honorèrent comme tels. Ce fut surtout le sycomore qui devint pour eux un objet de vénération; poussant jusqu'à la limite du désert, où il prospère, comme par miracle, sur son lit de sable, et défiant, par sa ramure impénétrable, les rayons du soleil du midi, cet arbre revêtit à leurs yeux un caractère divin; on se le représentait comme animé par un esprit qui se cachait en lui, mais se manifestait en certaines occasions, sortait du tronc la tête ou le corps

1. Wilkinson, op. laud., t. III, p. 349 et 350 .

2. Champollion, op. laud., t. IV, pl. CCCXXXIV. - Lepsius, Denkmäler, t. VI, pl. CLXIX. - Wilkinson, op. laud., t. III, p. 203, pl. XLIII.

3. Lepsius, Denkmäler, t. V, pl. 37.

4. Champollion, op. laud., t. I, pl. XLIII. - Rosellini, op. laud., t. IlI, pl. VIII, 3. 
tout entier, puis y rentrait, pour être résorbé ou mangé de nouveau ${ }^{1}$. Sur une peinture du tombeau de Rat'eserliasenb, on voit, à la lisière d'un champ, dont il semble protéger la récolte, se dresser un sycomore, au pied duquel sont placées, à droite, de la vaisselle, à gauche, une grande jarre entourée d'une tige végétale. Plus loin sont disposés trois vases environnés de tiges semblables. Devant l'arbre symbolique, un personnage fait une révérence en levant une main à la hauteur de la bouche et baissant l'autre au niveau des genoux ${ }^{2}$. C'est la représentation fidèle de ce qui se faisait chaque jour dans une partie de l'Égypte. Les nomes Memphitite et Létopolite, en particulier, renfermaient plusieur's sycomores, où des doubles de Nouit et d'Hâthor habitaientau su de tous. Le plus célèbre d'entre eux, le sycomore du Sud - nouhit risit —, était, dit M. Maspero ${ }^{3}$, " comme le corps vivant d'Hâthor sur notre terre $"$.

Ces arbres vénérés prirent place avec les autres végétaux dans le monde mythique; le portail immense que traversait le soleil, en sortant des régions où il avait voyagé la nuit, était encadré de deux sycomores, tout en pierres précieuses de couleur verte ${ }^{4}$. Dans leur voyage périlleux aux "Champs des Souchets ) - Sokhit Ialou - le paradis des Égyptiens, les àmes des "suivants d'Horus " — Shosouou.Horou-

1. G. Maspero, Les hypogées royaux de Thèbes. (Études de mythologie et d'archéologie égyptiennes, t. II, p. 104.)

2. V. Scheil, Le tombeau de Ral'eserliasenb. (Mémoires de la Mission archioiogique au Caire, t. V, fasc. 4, p. 578, pl. IV.)

3. G. Naspero, Histoire ancienne, t. I, p. 122. - Études, t. II, p. 226.

4. G. Maspero, Le livre des morts. (Études de mythologie et d'archiologie égyplicmnes, t. I, p. 333.) 
rencontraient dans le désert où ils s'enfonçaient, après avoir gravi la montagne de l'Occident, un sycomore toujours vert; sortant du milieu du feuillage, la déesse Nouit ou Hàthor leur tendait, d'une main, du pain et des fruits et, de l'autre, leur versait une eau rafraîchissante ${ }^{1}$.

Cet acte mystique est souvent représenté sur les monuments, et en particulier dans le Livre des Morts. Il préparait le défunt à recevoir la couronne de la justification 2. Le Papyrus d'Ani, par exemple, nous montre l'àme et le double du défunt qui, après s'être désaltéré avec sa femme de l'ean d'un réservoir ombragé d'un palmier et de divers arbres's, prie agenouillé devant le sycomore de la déesse : "O Sycomore de Nouit, donne-moi l'eau et le souffle de vie qui procède de toi. " Et la déesse, en lui versant l'eau fortifiante, et lui tendant des fruits de l'arbre sacré, lui répond: “ O Osiris, père divin, reçois la libération de ma propre main *.) Dans un passage du Livre des Morts de Turin, on trouve une prière semblable, mais adressée en faveur du double, non par lui ${ }^{5}$ :

"O sycomore de Nout, toi qui rafraîchis ceux qui sont dans le royaume des morts, étends tes bras sur ses membres, protège-le contre la chaleur, rafraîchis l'Osiris N. à l'ornbre des arbres qui apportent les brises du nord. »

1. G. Maspero, Histoire ancienne, p. 18'.

2. W. Pleyte, La couronne de la justification. (Actes du sixième Congrès international des Orientalistes, t. IV, p. 9.)

3. The book of the dead. Facsimile of the papyrus of Ani. London, 1890, in-fol., pl. XVI, p. 14.

4. Paul Pierret, Libation vase of Osor-ur. Ilusée du Louvre, no 988. (Records of the Past, t. XIl, p. 77-78).

5. Turiner Todtenbuch, cap. 152, 7, 8, ap. V. von Strauss und Torney, op. laud., p. 63. 
Une peinture du tombeau de la reine Thiti ${ }^{1}$ représente cette princesse debout en prières devant le sycomore de vie, du sommet duquel la déesse Häthor semble lui tendre la nourriture purifiée qui doit la fortifier. De l'autre côté de l'arbre, la vache d'Hâthor l'attend au pied du Manou, "la montagne de l'Occident ", pour la guider dans son voyage vers l'autre vie. Dans le Livre des Morts de Turin², le bras seul de la déesse sort du sycomore, qui se dresse auprès d'un réservoir, pour verser à l'Osiris agenouillé l'eau qui doit étancher sa soif :

"Elle conforte et rafraîchit les esprits du couchant, dit l'inscription qui accompagne cette représentation symbolique "; elle enlace les nembres de N. N. de ses bras; elle le protège contre la chaleur; elle donne de la fraicheur à ce mort sous le couvert et à l'ombre de ses feuilles, qui font entendre de doux murmures au défunt assis, le cœur calme, sur son siège dans l'éternité. »

Sur une peinture d'Abd-el-Gournah ${ }^{4}$ on voit Nouit surgissant du milieu d'un sycomore et tenant un plateau couvert de pains et de fruits d'une main, del'autre une jarre, d'où se répand l'eau salutaire que boivent avidement six personnages, assis autour de l'arbre sacré : "Je te verse cette eau ", dit la déesse, en

1. G. Bénédite, Le tombeau de la reine Thiti. (Mémoires de la Mission archéologique au Caire, t. V, p. 406, fasc. 3, pl. VII.)

2. Das aegyptische Todtenbuch der XVIII bis XX Dynaslie. Aus verschiedenen Urkunden hgg. v. Edouard Naville. Berlin, 1886, in-fol., pl. LXXIII.

3. Je me sers de la traduction donnée par Franz Woenig, Die Pflanzen im alten Aegyplen, p. 284.

4. Champollion, Monuments de l'Égypte, t. II, pl. CLXXXIV, 1. - Rosellini, Monumenti civili, atlas, pl. CXXXIV, 1. Maspero, Histoire ancienne, t. I, p. 185. 
s'adressant au premier personnage, le chef de la famille, dont l'artiste pharaonique a représenté, outre le double, l'âme sous forme d'un oiseau. Au pied du sycomore s'étend un réservoir couvert de lotus, au milieu desquels nagent des poissons et des oiseaux aquatiques; deux touffes de papyrus se dressent sur ses bords, où se promènent des hérons ${ }^{1}$. Rosellini a reproduit une autre peinture de la même scène, provenant de la même région, mais d'une époque postérieure; elle représente encore Nouit versant l'eau, qui doit les désaltérer, à un seigneur égyptien et à sa femrne, assis, de magnifiques bouquets de fleurs à la main, devant l'arbre sacré.

Dans une peinture reproduite par Wilkinson ${ }^{3}$, c'est l'âme seule du mort, épervier à tête humaine, qui reçoit, dans ses mains avides, l"eau versée par Nouit du milieu de son sycomore. Le perséa se substituait parfois à ce dernier arbre, comme Hâthor à Nouit, dans ce rite funéraire; une peinture, reproduite encore par Wilkinson, mais sans indication d'origine ${ }^{4}$, nous montre cette déesse versant, du milieu des rameaux d'un perséa, à une âme altérée l'eau qui lui rendra sa force et sa vigueur premières.

Si les défunts avaient déjà rencontré pendant leur voyage aux champs d'Ialou des arbres sacrës et bienfaisants, le monde végétal tout entier les attendait dans ce séjour délectable, véritable paradis ${ }^{3}$, où Osi-

1. Rosellini, Monumenti civili, texte, t. III, p. 45 .

2. Monumenti, pl. CXXXIV, 3.

3. The manners, t. III, pl. XXIV, p. 65.

4. The manners, t. III, pl. XXVIII, p. 118.

5. F.-J. Lauth l'appelle “ l'Élysée ». Aus Aeqyptens Vorzeit. Berlin, 1836 , in -8 , p. 52 et suiv. 
lis, rappelé à la vie par les arts magiques d'Isis et l'adresse d'Anubis, avait fixé sa résidence et réservait une place aux suivants d'Horus. Le soleil et la lune éclairaient sans cesse cette région fortunée, le vent du nord y tempérait de son souflle les ardeurs du jour; les moissons poussaient vigoureuses et abondantes dans ses champs immenses; des canaux y entretenaient la fécondité; des jardins délicieux offraient la fraîcheur de leur ombre aux àmes fatiguées des travaux de la moisson ${ }^{1}$. Il y avait lid, comme le dit M. Maspero ${ }^{2}$, une " sorte d'Égypte céleste, d'une fertilité inépuisable "; j’ajouterai que la flore de cette région mythique ne différait pas de celle de l'Égypte terrestre.

Si les plantes de la vallée du Nil avaient pénétré dans les champs d'Ialou - l'Élysée pharaonique quelques-unes aussi se retrouvaient dans l'Amenti l'Hadès égyptien -; au pied de la montagne, qui le borne à l'occident, croissaient de gigantesques papyrus; la planche XXXVII du Papyrus d'Ani ${ }^{3}$ nous montre la vache Mirit, personnification d'Hâthor, s'avançant, la tête couronnée du disque solaire, par une fente de la montagne au milieu d'un fourré de ces cypéracées.

Ainsi associées aux légendes sacrées et aux croyances inythiques, les plantes devinrent l'emblême des divinités du Panthéon égyptien. Le tamaris était l'apanage ordinaire d'Osiris; un tamaris, qui dépassait, dit Plutarque ${ }^{4}$, un olivier en hauteur, ombrageait le tombeau

1. G. Maspero, Histoire ancienne, t. I, p. 181.

2. Le livre des morts. (Études de mylhologie et d'archíologie égyptiennes, t. I, p. 348.)

3. Cf. Naville, Das Todtenbuch, cap. 186, pl. CCXII, 2 et 3.

4. De Iside el Osiride, cap. 21. 
du Dieu à Philae. Un tamaris est aussi représenté sur les bas-reliefs du grand temple de Dendérah près du cercueil de l'Osiris de l'ouest '. Parfois aussi on regardait le palmier comme l'apanage de ce Dieu². Le perséa était consacré à Hâthor ${ }^{3}$. D'après Plutarque ${ }^{4}$, cet arbre était aussi consacré à Isis, ainsi qu'à Osiris ; l'écrivain. grec en donne pour raison singulière que son fruit ressemble au cour et sa feuille à la langue. Le sycomore était à la fois consacré à Isis, à Nouit et à Hâthor; cette dernière est appelée la "dame du sycomore " sur une inscription ${ }^{5}$, et Nouit se donne à elle-même le titre de “ nourrice du sycomore $~^{6}$. Arbre d'Hâthor, le sycomore était cher aux amoureux ${ }^{\top}$; ils aimaient à reposer à son ombre. D'après Horapollon ${ }^{8}$, l'année avait pour emblême une branche de palmier ; on représentait Toth, ainsi parfois que Safekh et Khonsou ${ }^{3}$, marquant sur une palme, qu'ils tiennent à la main, le nombre des années de la vie humaine. Le jujubier noubsou - avait donné son nom à la ville nubienne de Pnoubsou. Thot de Pnoubsou estreprésenté sur un basrelief de Dakleh assis à l'ombre de cet arbre, sous la forme du singe Ostanès ${ }^{10}$.

1. A. Mariette, Dendérah, atlas, t. IV, pl. LXXII. - Champollion, Monuments, t. I, pl. 88.

2. Magical texts. (Records of the past, t. VI, p. 117.)

3. G. Schweinfurth, De la Flore pharaonique. (Bulletin de l'Institut égyptien, $\mathrm{n}^{\circ} 3$ (an. 1882), p, 68.)

4. De Iside el Osiride, cap. 68.

5. Wilkinson, op. laud., t. III, p. 118, pl. XXXIII,

6. Rosellini, Monumenti civili, t. III, p. 458.

7. Franz Woenig, Dic Pflanzen im alten Aegypten, p. 287.

8. Hieroglyphica, lib. I, cap. 8.

9. Wilkinson, op. laud., t. III, p. 164, 174 et 203.

10. G. Maspero, Notes au jour le jour. (Proceedings of the sociely of biblical Archaeology, t. XIII, p. 497.) Cf. Goodwin, 
Les arbres ne furent pas seuls revètus d'un caractère divin et consacrés aux Dieux, bien d'autres plantes jouirent du mème privilège. Tels furent en particulier le lotus et le papyrus, emblèmes le premier de la Haute et le second de la Basse-Égypte'. Sur un bas-relief de Dendérah on voit Osiris accroupi sur une colonne entre deux bouquets de papyrus et de lotus, personnification de ces deux régions ${ }^{2}$. Ces plantes étaient aussi l'emblême du Nil - le dieu Hapi - ; une statue du British Museum le représente tenant à la main un plateau d'où pendent, avec des oies et des grives, des épis et des fleurs de lotus, tandis qu'à ses pieds se dressent des tiges de papyrus ${ }^{3}$. Sur un bas-relief du temple de Séti I, à Abydos, on voit les Nils agenouillés qui apportent la richesse à chaque nome de l'Égypte, tenant chacun à la main gauche un faisceau de papyrus, et de la droite un plateau chargé de fruits, de divers mets et de jarres pleines d'eau, ainsi que de bouquets faits de fleurs de lotus 4 .

Une peinture du Musée de Berlin nous montre le pharaon Amenhotpou II offrant des fleurs à Khnoum, qu'accompagnent Satit et Anouqit, pendant que Hapi, la tète couronnée de papyrus, tend un plateau chargé de jarres et de tiges de lotus ${ }^{5}$. Le premier des dix

On the name Astennu. (Zeitschrift für aegyptische Sprache t. X (an. 1872), p. 108.)

1. Unger, Sitzungsberichte, t. XXXVIII, p. 77. - Victor Loret, L'Égyple au temps des Pharaons. Paris, 1889, in-12, p. 106.

2. Nariette, Denderah, t. II, pl. 86.

3. G. Maspero, Histoire ancienne, t. I, p. 38.

4. G. Maspero, IIistoire ancienne, t. I, p. 41.

5. Lepsius, Königliche Museen. Aegyptische Wandgemälde. Berlin, 1881, in-fol., p. 16, pl. XXI. 
Nils, qui figurent dans la procession, représentée sur les parois de la tombe de Ramsès II ${ }^{1}$, a la tête couronnée de papyrus et il fait, ainsi que six des autres Nils, une offrande de fruits et de lotus. On roit, sur une peinture du temple de Philae, Ptolémée-Evergète II et la reine Cléopâtre faisant des offrandes, suivis des Nils de la Haute et de la Basse-Égypte, la tête couronnée le premier de papyrus, le second de lotus héraldiques ${ }^{2}$; plus loin la déesse Égypte, au milieu de fourrés de papyrus, tient à la main un bouquet de lotus. Sur les bas-reliefs du temple de Dendérah les papyrus ont disparu $^{3}$; les Nils portent tous sur la tête un bouquet de lotus et ils ne tiennent à la main que des vases à libations, symboles de l'inondation.

Le lotus était regardé comme l'emblème du soleil, " qu'il paraît honorer, dit Proclus ${ }^{4}$, en étendant ou en contractant ses fleurs ». C'est là la raison pourquoi l'épervier, emblème de Horus ou de Rà - le soleil - est représenté posé sur une fleur de lotus. Au centre de la barque solaire Mehit, peinte sur les murs du temple de Dendérah se dresse un lotus épanoui, d'où s'élance un serpent, figure symbolique de Hor ${ }^{5}$. On voit sur une des peintures du Livre des Morts les quatre génies de l'Amenti ou des Morts debout sur cette fleur ${ }^{6}$. Un lotus, faisant face à une oie, image

1. Rosellini, Monumenti, t. III, pl. LXXIV, 1.

2. Rosellini, Monumenti, t. III, pl. XII, 1 et 2.

3. A. Mariette, Dendérah, t. III, pl. 25 et 26.

4. Ap. Wilkinson, op. laud., t. III, p. 350.

5. Mariette, Dendérah, t. II, pl. 48 et 49 , III, pl. $9 a$ et 44 ; texte, p. 176.

6. The papyrus of Ani, pl. XXX. - Naville, Das aegyptische Todlenbuch, chap. CXXv, pl. CXXXVI. 
symbolique de Sibou, est gravé sur une pierre de la collection d'Abbott'. La déesse Noufirtoum est représentée avec une fleur de lotus sur la tête ${ }^{2}$.

Quelques grains de blé, semés dans deux vases et dont les tiges se dressent au dehors, personnifient sur une peinture de Dendérah Osiris mort qu'on a confié à la terre et qui ressuscite ${ }^{3}$. Et à Philae, la momie du Dieu est représentée couchée sur un crocodile vert, emblème du Nil, au milieu d'un fourré de papyrus, mêlés de quelques lọtus ${ }^{4}$. On y voit également Isis, venant de mettre Horus au monde au milieu des papyrus de la rive ${ }^{5}$. Montée sur une nacelle en papyrus, la déesse partit à la recherche du corps d'Osiris, mis en pièces par Sitt ${ }^{6}$. Depuis lors, d'après une légende égyptienne, les crocodiles ont une sainte horreur pour les barques de papyrus. En souvenir de ce fait, une peinture de Dendérah représente la vache, qui personnifie Isis, ainsi qu'Hàthor, voguant, au milieu de fourrés de papyrus, sur une barque faite en papyrus et dont une fleur de papyrus forme la proue et la poupe ${ }^{7}$. La barque, qui portait la momie au temps du moyen et du nouvel empire, avait aussi à la poupe et à la proue une Hleur de papyrus et des tiges de cette plante se dressaient de chaque côté du cercueil ${ }^{\mathrm{s}}$. Mirit, la déesse du

1. Goodyear, The grammar of the Lotus, p. 22, pl. II, 10.

2. Wilkinson; op. laud., t. III, p. 180.

3. A. Nariette, Dendérah, p. 283 ; atlas, t. IV, pl. 58.

4. Champollion, Monuments, t. I, p. XCIII. - Rosellini, Ionumenti, t. III, pl. XVI, 1.

5. Rosellini, Monumenti, t. III, pl. XIX, 2.

6. Plutarque, De Iside et Osiride, cap. 15

7. Rosellini, Monumenti, t. III, pl. XV, 1.

8. Wilkinson, op. laud., t. III, pl. 67. 
Nord et du Sud, comme l'appelle une inscription ", portait sur sa tête un bouquet de papyrus ${ }^{2}$. Le dieu Sovkou est peint aussi debout devant un autel, tenant $\dot{a}$ la main une tige de papyrus ${ }^{3}$.

III .

Les arbres et les plantes aquatiques dont je viens de parler n'étaient pas seulement regardés comme des emblèmes religieux, ils figuraient avec les autres représentants de la flore égyptienne dans le culte et dans les cérémonies sacrées. Les fêtes religieuses occupaient une place considérable dans la vie des anciens Égyptiens; c'était la conséquence naturelle de l'idée qu'ils se faisaient de l'existence future et de la nature des dieux et de l'homme.

La haute conception d'un dieu suprême et unique, créateur de tous les êtres, ne fut jamais générale ni populaire chez eux, elle fit place à une idée moins élevée et tout anthropomorphiste, celle qu'on eut de la triade osirienne; désormais les dieux ne furent plus considérés que comme des hommes, dont le corps, " pétri d'une substance plus ténue, il est vrai, et invisible à l'ordinaire, était doué des mêmes qualités et atteint des mêmes imperfections que les rootres ${ }^{4}$ )).

Comme les hommes aussi, l'histoire d'Osiris le prou-

1. Wilkinson, op. laud., t. III, p. 231, pl. 53.

2. Mirit était surtout la personnification de la berge du Nil; aussi y en avait-il deux : Mirit Qimait, la berge méridionale et Mirit Mihit, la berge septentrionale. Maspero, Histoive ancienne, t. I, p. 37.

3. Goodyear, op. laud., p. 60.

4. G. Maspero, IIstoire ancienne, t. I, p. 109. 
vait, les dieux pouvaient perdre la rie avec le sentiment et connaitre les horreurs de la tombe; leur ombre alor's se détachait de leur corps et, animée d'une existence indépendante, elle pouvait se mouvoir et sortir du tombeau; leur double, image visible de leur corps, était monacé de la mort par la ruine de celui-ci; leur ìme clle-mème, qui, malgré sa nature spirituelle, dépendait du corps, perdait quelque chose d'elle-même à mesure que son associé dépérissait.

Il n'y avait qu'un moyen efficace pour mettre un terme à cet état de misère, c'était d'empêcher la destruction du corps et d'en assurer la conservation. C'est ce qui arait été fait à la mort d'Osiris; son corps, embaumé par Anubis, avait été préservé de la pourriture du tombeau, et les puissantes incantations d'Isis et d'Horus, les pratiques savantes de-Thot lui avaient rendu le sentiment, avaient de nouveau animé ses membres, et son âme, affranchie de toute crainte, s'était élancée vers la Voie lactée et retirée aux champs d'Ialou ${ }^{1}$.

Comme Osiris, l'homme pouvait atteindre ces demeu. res paradisiaques, mais c'était à la condition que son corps pût échapper à la destruction et que son âme n'eùt point à craindre de se voir amoindrie par la destruction graduelle de celui-ci. L'embaumement était un premier moyen de remédier à cet état de choses; transformé en momie, déposé dans le sable du désert ou dans un sarcophage de pierre dure, le corps pouvait, dans sa gaine noire et rigide, durer presque indéfiniment ${ }^{2}$. Sans inquiétude désormais sur le sort

1. G. Maspero, IIisloire ancienne, t. I, p. 178-181.

2. G. Maspero, IIisloire ancienne, t. I, p. 112.

I. 
du corps, auquel elle avait été unie, l'âme pouvait poursuivre tranquillement son existence, et même, si elle en était jugée digne, être reçue, elle aussi, dans les jardins d'Ialou. Quant au double, il suivait le corps au tombeau et y vivait à côté de lui; toutefois il avait la faculté d'accomplir toutes les fonctions de la vie animale; il se inouvait, allait, venait, accueillait les hommages des dévòts; mais ses organes voulaient être nourris, comme ceux du corps l'avaient été jadis, et de lui-même, il ne possédait que "la faim pour nourriture, la soif pour boisson ${ }^{1}$ »; le besoin aussi l'arrachait à sa retraite; la nuit, chassé par la faim et la soif, il sortait de sa tombe et errait au milieu des habjtations des hommes, ramassant avec avidité, pour s'en nourrir, tout ce qu'il pouvait trouver ${ }^{2}$. Il ne souffrait pas que les siens l'oubliassent et il se rappelait par tous les movens à leur souvenir. La seule manière efficace de s'affranchir de ses visites importunes était de lui porter au tombeau, ce qu'il renait chercher dans les demeures des vivants, les provisions qui lui étaient nécessaires ${ }^{3}$. Telle fut l'origine des sacrifices et des offrandes funéraires.

Quoique d'une nature supérieure, les dieux n'échappaient pas plus que les hommes aux angoisses du tombeau, leur corps s'altérait et était menacé de destruction, si, comme celui d'Osiris, il n'était embaumé et transformé en momie. L'embaumement assurait l'existence de leur âme; mais leur double craignait à la

1. G. Maspero, Histoire ancienne, t. I, p. 114.

2. G. Maspero, Études relalives à la mylhologie el à l'archiologie égyptiennes, t. I, p. 155.

3. G. Naspero, IIisloire ancienne, t. I, p. 115. 
fois et regrettait la lumière; il était soumis à tous les maux de la vie; la faim et la soif dont il souffrait étouffaient en lui tout sentiment de pitié et les vivants et les morts n'échappaient aux effets de son humeur farouche qu'à la condition de lui payer tribut et de le nourrir comme un simple double humain ${ }^{1}$. Les vivants s'acquittaient envers lui par des fètes et des sacrifices, répétés chaque année à des intervalles réguliers; les morts aussi étaient tenus de l'apaiser; il lui fallait sa part des offrandes qu'on leur apportait, et il se la faisait lui-mème; c'était à lui qu'on devait les présenter d'abord, et c'était seulement, quand il avait prélevé ce qui lui convenait, qu'il abandonnait le reste au destinataire.

Cette conception montre comment les fètes religieuses et funéraires prirent sur les bords du Nil une importance exceptionnelle; si, comme le remarque Hérodote ${ }^{2}$, les Égyptiens étaient le plus religieux des peuples et s'ils "surpassaient tous les autres hommes dans les honneurs qu'ils rendaient aux dieux ", ils le durent avant tout, on le voit, à la nature de leurs croyances; mais le caractère particulier que prirent chez eux les fêtes religieuses, le nombre et la diversité de leurs dieux, le goût national pour les cérémonies, entretenu peut-être par leurs prêtres, contribuèrent à augmenter le nombre et l'importance de ces solennités. Dans le seul temple de Dendérah on célébrait chaque année trente-cinq fêtes ${ }^{3}$, dont quelques-unes duraient

1. G. Naspero, Hisloire ancienne, t. I, p. 116-117.

2. IIistoriae, lib. II, cap. 37. 3. A. Mariette, Denderah. Descriplion générale du temple
de celle ville. Paris, 1875, in- 4, p. 96 . 
des semaines entières. On n'en célébrait guère moins, ni de moins importantes dans les autres sanctuaires.

La première des grandes fètes égyptiennes et l'une des plus solennelles était la "Panégyrie de tous les Dieux et de toutes les Déesses " ou "Panégyrie du commencement de l'année ${ }^{1}$ "; célébrée au mois de Toth, cette "fète de la fondation des mois " durait trois semaines; elle commençait " en la bonne nuit" de la veille du premier de l'an par des libations "d'eau de rajeunissement " ${ }^{2}$; le 2 , avait lieu la procession du grand Lotus, la fête particulière d'Ahi, le 10, celle d'Horus et le 20, celle de Rà. Une autre fète non moins solennelle était célébrée au mois de Khoïak en souvenir de la mort d'Osiris, dans seize villes d'Égypte; elle commençait le 12 et ne finissait que le $30^{3}$. Il y avait aussi la fête du. Nil — le Niloa - ; cette fête, une des plus grandes de l'Égypte, était célébrée vers le solstice d'été, au moment où les eaux du fleuve commençaient à monter ${ }^{4}$. Avant elle prenait place la double fête d'Isis, la première célébrée surtout à Busiris, et qui se confondait avec la fête d'Osiris; la seconde, qui avait lieu à l'époque de la moisson; les Égyptiens offraient alors à la déesse les prémices des fruits de la terre ${ }^{5}$.

A la fin de la saison des récoltes, dans le mois de Pharmouti, des offrandes étaient faites aussi à la déesse

1. A. Mariette, Dendérah, p. 101.

2. Georges Bénédite, Tombeau de Neferhotpou. (Mémoires de la mission archéologique au Caire, t. V, fasc. 3, p. 518.)

3. Victor Loret, Les fêtes d'Osiris an mois de Kihö̈ak. (Recueil de travaux, t. V (an. 1883), p. 98.)

4. Héliodore, Aethiopica, lib. IX, cap. 2. - Wilkinson, op. laud., t. III, p. 369.

5. Wilkinson, op. laud., t. III, p. 378. 
de la moisson, Rannoutit; une peinture du tombeau de Rat'eserkasenb r'eprésente cette déesse, "dame des greniers ", dressée debout sur sa quene". Derrière elle est une fleur de Lotus, à tige feuillue, et devant elle sont placés des pains de formes diverses, des jarres ì paroi poreuse, des fleurs de lotus, des concombres et des victuailles.

Parmi les grandes fètes égyptiennes, Hérodote mentionne encore la fète de la divinité qu'il appelle Diane — Bast, Maut ou Sòkhit². - En réalité c'était moins une fète arrivant à des époques régulières que la commémoration d'un événement historique particulier, instituée par Osorkon II, et à laquelle étaient conviés tous les dieux de l'Égypte ${ }^{3}$. Outre cette fête, la plus solennelle, d'après Hérodote, de toute l'Égypte, l'historien grec en comptait encore cinq autres principales; parmi celles-ci, il faut mentionner celle de Minerve Neit - à Saïs, célébrée elle aussi avec une grande pompe dans le temple de cette ville'.

Si c'étaient là les fêtes ordinaires, les plus importantes de l'Égypte, il y en avait d'autres qui n'arrivaient point à des époques fixes, mais qui n'étaient pas célébrées avec moins de pompe; telle était la fête de la dédicace d'un temple ou du couronnement d'un roi, le triomphe célébré par un pharaon au retour d'une

1. V. Scheil, Tombeaux thébains. (Mémoires de la mission archéologique au Caire, t. V, fasc. 4, p. 577, pl. IV.)

2. Hislorice, lib. II, cap. 59-60.

3. Édouard Naville, Bubastis (1887-89). (Eighth Memoir of the Egypl exploration fund. London, 1891, in-4, p, 51, 1.)

4. Edouard Naville, The festival hall of Osorkon II in the great Temple of Bubastis (1887-1889.) (Tenth Memoir of the Egypl exploration fund.)

5. Nallet, Les fêles de Neit à Saïs. Paris, 1888, in-8, p. 37. 
expédition glorieuse contre les nations voisines, enfin les cérémonies des funérailles'. Toutes ces fêtes commencaicnt par le sacrifice des victimes ; c'était Rì luimème, qui l'avait institué, avant de remonter au ciel. La victime inmolée, on en offrait une partie aux dieux ou aux morts, en même temps que d'autres mets tirés du règne végétal.

Dans aucun culte, les offrandes n'ont eu une importance comparable à celle qu'elles prirent dans les fêtes religieuses et dans les cérémonies funèbres de l'ancienne Égypte. Les dieux du Panthéon pharaonique acceptaient avec empressement les dons que leur faisaient leurs fidèles et ils ne dédaignaient pas de les provoquer $^{2}$; c'était d'ailleurs le seul moyen qu'eussent ceux-ci de gagner leurs bonnes grâces et d'éviter leur mécontenternent ou leur courroux; aussi s'attachait-on à les combler de présents et d'hommages. Un document précieux, le Papyrus Harris, qui contient l'énumération des dons faits par Ramsès III aux divinités de Thèbes, d'Héliopolis et de Memphis, nous montre jusqu'où pouvait aller la générosité des pharaons à l'égard de leurs dieux ${ }^{3}$. Même en faisant la part de l'exagération naturelle à ce genre de documents, il faut avouer que la pieuse libéralité de Ramsès III dépasse tout ce qu'on peut imaginer. C'est par milliers que l'on compte, pour ne parler que des produits du règne végétal, les amphores de vin, de bière, d'huile, les boisseaux ou sacs de blé, de farine, de sésame, d'oignons, de dattes fraiches ou "pressées ", de

1. Wilkinson, op. laued., t. III, p. 358-366.

2. Naspero, Hisloire ancienne, t. I, p. 125.

3. Sam. Birch, Papyrus IIarris. (Zeilschrift für aegyplische Sprache, t. XI (an. 1873), p. 9-12, 34-39, 65-72.) 
grappes de raisin et d'autres fruits, d'encens et de baume, ainsi que les bouquets de lotus' et de papyrus ${ }^{2}$, les bouquets et les couronmes de fleurs diverses ${ }^{3}$ et mème d'épis de blé,' les feuilles de palmier, les bottes de roseaux, etc.

Moins importantes, mais non moins précieuses, sont les listes, gravées sur le pylone antérieur du temple de Médinet-Habou, des offrandes faites dans ce temple, sous le règne de Ramsès III - Rampsinit -, à l'occasion des fètes annuelles, instituées par son prédécesseur Ramsès II, en l'honneur des dieux vénérés dans le sanctuaire; sur ces listes, publiées par Dümichen", figurent, entre autres offrandes d'origine végétale, d'énormes quantités de farine, de froment et d'orge, de gâteaux, de bière et de vin, de l'encens, des raisins, des jujubes et diverses autres espèces de fruits, que le savant égyptologue n'a pu identifier.

Les peintures et les sculptures des temples nous donnent déjà l'idée la plus haute des nombreuses offrandes, empruntées au règne végétal, qu'on faisait aux dieux égyptiens sous le nouvel empire. C'est ainsi que sur un bas-relief du temple de Ramsès II à Abydos, qui nous fait assister à une fête donnée en l'honneur dú pharaon victorieux ${ }^{5}$, nous voyons le long défilé des officiants, qui précèdent la victime, portant les uns des

1. Il est fait mention une fois de 110,000 et l'autre de 144,720 poignées de lotus. Pl. XXI, ligne 10.

2. Pl. XXI, ligne 13 , il est fait mention de 68,800 bouquets de papyrus.

3. A la ligne 5 de la pl. XXI sont mentionnées 60,450 couronnes de fleurs.

4. Die kalendarischen Opferlisten im Tempel von MedinetHabu. Leipzig, 1881, in-fol. pl. V-IX.

5. A. Nariette, Alydos. Paris, 1869, in-fol., t. II, pl. 47. 
corbeilles de fruits, les autres des pains; l'un d'eux, qui mène en laisse deux gazelles, tient à la main un faisceau de tiges de papyrus, tandis que les autres portent des bouquets de lotus.

Une peinture de la XIX ${ }^{0}$ dynastie, reproduite par Rosellini ${ }^{1}$, représente Minephtah I faisant à Khem et à Hâthor une offrande de pains et de fleurs de lotus. Sur une peinture du tombeau ${ }^{2}$ de Ramsès IV (XX ${ }^{\mathrm{c}} d y-$ nastie), on voit un des Nils portant des figues, des grenades et des raisins artistement disposés audessous d'un plateau, que recouvrent des fleurs de lotus. Un bas-relief du temple d'Edfou ${ }^{3}$ nous montre Ptolémée-Alexandre offrant à Horus un faisceau d'épis de blé. Mieux que tous les autres monuments peut-être, les bas-reliefs du temple de Dendérah nous montrent. quelle profusion on portait dans les présents qu'on faisait aux Dieux: fruits des espèces les plus diverses, pains aux formes variées, fleurs en particulier de lotus, branches de feuillage même, on voit le roi en faire successivement offrande aux trois divinités du sanctuaire", à Hor-Sam-Taoui, "la divinité qui sort du grand lotus », à Hor-Hout et surtout à Hâthor, la déesse "qui apporte la rie à tous les hommes, la maitresse de la terre, la maitresse du pain, l'inventrice de la bière ". Ces dieux se montrent reconnaissants; ils promettent au roi, en retour de ses dons, d'abondantes récoltes;

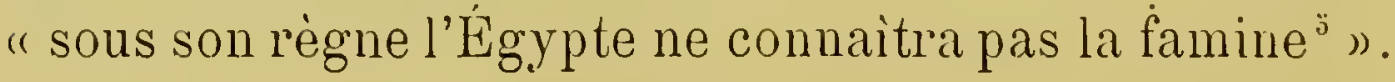

1. Honumenti del culto, t. III, pl. 54, 1.

2. Champollion, Monuments, t. III, pl. CCLIII. - Rosellini, t. III, pI. LXXIV.

3. Franz Woenig, op. laud., p. 161.

4. A. Mariette, Denderah. Atlas, t. I, pl. 55 a et $55 b$; t. II, pl. 47 a et $47 b$; t. III, pl. 54.

5. A. Mariette, Dendérah, p. 255. 
Comme dans le culte des dieux, les offrandes tirées du monde des plantes occupaient une place considérable dans les cérémonies funèbres. Une des parties les plus importantes du culte des défunts consistait dans la consécration des "vivres donnés au mort pour la vallée céleste ", au $K a$, "habitant de l'Amenti " . Ces " vivres ", les peintures des hypogées le montrent, étaient empruntés en partie au règne végétal: fruits, légumes et racines - ou aux produits qu'on en retire - pain, vin, bière, liqueurs diverses.

Sous l'ancien empire le nombre des fruits offerts aux dieux ou aux morts était encore peu considérable; il augmente à partir de la XII dynastie et devient considérable surtout depuis la XVIII', époque où l'horticulture égyptienne prit une extension chaque jour grandissante. Les premiers fruits, les seuls même, qu'on aperçoive parmi les offrandes de l'ancien empire sont les figues du sycomore; on en voit également sur les bas-reliefs ou les peintures de Gizeh ${ }^{2}$, de Saqqarah ${ }^{3}$ (IV et $V^{\mathrm{e}}$ dynasties), de Béni-Hassan ${ }^{4}$ (XII dynastie). Plus tard on continue de rencontrer ces figues, mais le plus souvent en même temps que d'autres fruits. Sul les peintures de Béni-Hassan on voit déja avec elles des dattes ou des fruits du jujubier. Sur celles de la XVII ${ }^{\circ}$ dynastie et des dynasties suivantes, on aperçoit à la fois des tigues, des grappes

1. V. Scheil, Le tombeau des graveurs. (Mémoires de la mission archéologique au Caire, t. V, fasc. 4, p. 559.)

2. Lepsius, Dentimüler, t. III, pl. 22, tombe 24; pl. 41, tombe 89 ; pl. $5:$, tombe $16 ;$ pl. 57 , tombe 15 .

3. Lepsius, op.. laud., t. III, pl. 46 ; tome XV, pl. 67, tombe 17 ; t. IV, pl. 6'.

1. Lepsius, op. laud., t. IV, pl. 129, tombe 2. 
de raisin, des grenades, des fruits de perséa, de nabas, etc ${ }^{1}$.

Les inscriptions de la tombe de Rekhmara, préfet de Thèbes sous Thoutmès II, à Abd-el-Gournah² mentionnent parmi les mets, que le défunt pouvait demander "durant ses séjours à l'Occident et au Nord ", des gàteaux de dattes, des raisins, des fruits de perséa, des figues de sycomore. Et parmi les offrandes que lui présentent les prêtres et les membres de sa famille, " en se tommant vers l'Orient ", figurent encore des dattes, des figues de sycomore, des fruits de perséa, des grappes de raisin. Sur une peinture du tombeau d'Harmhabi ${ }^{3}$, de la même époque, un des officiants tient d'une main un paquet de grenades attachées ensemble et de l'autre un bouquet de fleurs de lotus et de papyrus. Au nombre des offrandes représentées sur les peintures du temple de Karnak (XIX ${ }^{\mathrm{e}}$ dynastie)", se trouvaient aussi, avec des figues de sycomore, des grappes de raisin, des grenades, ainsi que des fruits de perséa et peut-être de nabas.

Les fruits n'étaient pas seulement offerts cueillis et séparés de l'arbre, on les offrait également attachés encore à la branche qui les portait. Sur la peinture du tombeau de Rat'eserkasenb", qui le représente, dans un kiosque, avec sa femme Uat-ronpit " pour faire fète,

1. Tombeau de Ramsès IV (XX• dynastie) à Bab-el-Molouk. Champollion, Monuments, t. III, pl. 253.

2. Philippe Virey, Tombeau de Relihmara, (Hémoires, t. V, fasc. 1, pl. IlI et IV, p. 102-103, ct 121.)

3. Bouriant, Le tombeau d'llarmhabi. (Mémoires, t. V, fasc. 3 , pl. III.)

4. Lepsius, op laud., t. VIII, pl. 2'1.

5. V. Scheil, Tombeau de Rat'eserkasenb. (Mémoires. t. V, fasc. 4, pl. II, p. 575. 
au gré de leur cœur ", on aperçoit quatre jarres it vin, enguirlandées de ceps chargés de raisins. Sur une stèle du tombeau de Nakhti' se roient deux serviteur's qui apportent des guéridons couverts de dattes, de figues et de lotus; au-dessous de l'un de ces guéridons pend un large chapelet de grenades mires, audessous de l'autre un faisceau de grappes de raisin. A droite et à gauche de la table d'offrandes, deux femmes, la tète surmontée d'un petit sycomore chargé de feuilles et de fruits, tiennent d'une main un bouquet formé de trois tiges de lotus ou de papyrus, de l'autre un plateau chargé de pains, d'oignons et autres mets, d'ou pend une longue branche de vigne, garnie de grappes de raisin. Deux serviteurs agenouillés au haut de la mème stèle offrent aussi des pampres courerts de grappes.

Une des porteuses d'offrandes d'une peinture thébaine $\left(\mathrm{XIX}^{\circ}\right.$ dynastie), reproduite par Prisse d'Arennes ${ }^{2}$, présente d'une main un bouquet, de l'autre un faisceau formé de plusieurs grappes de raisin liées ensemble. Et au-dessous de la table d'offrandes, placée devant l'image d'Osiris, qui se voít sur une stèle peinte sur un cercueil de momie de la nécropole de Thèbes (XX $^{\text {e dynastie) }}{ }^{3}$, pend un pampre couvert de raisins, tandis que des grappes et des fruits de l'arbre ashdou, arec des lotus, recourrent cette table.

En même temıs que les fruits et au même titre qu'eux, les légumes prenaient place parmi les offrandes funèbres; les oignons y figurèrent dès les temps les

1. G. Maspero, Tombeau de Nalhli. (Memoires, t. V, fasc. 3 , pl. I, p. 475 .

2. Hisloire de l'art égyplien. Atlas, t. II, pl. 67.

3. Prisse d'Avennes, op. laud. Atlas, t. II, pl. 71. 
plus reculés et y ont toujours figuré au premier rang; on en voit déjà parmi les offrandes des tombes de Gizeh ${ }^{1}$, de Saqqarah", de Béni-Hassan ${ }^{3}$, etc.; ils sont d'ordinaire réunis en bottes d'un nombre variable de pieds; quelquefois aussi ils sont attachés autour d'une espèce de cerceau. A côté des oignons, mais plus tard, prirent place les concombres, les pastèques et melons chate". La liste des offrandes de la tombe de Rekhmara, dont j'ai déjà parlé, ne fait mention que d'oignons et de concombres; toutefois ce n'est pas à dire que, à l'époque de Rekhmara et, à plus forte raison, aux époques postérieures, on n'offrit que ces légumes seuls dans les cérémonies funèbres, on en apercoit sur les peintures pharaoniques bien d'autres dont il n'est pas toujours possible de reconnaître la vraie nature; mais les trouvailles faites dans les anciennes tombes depuis un demi-siècle, et surtout en ces dernières années, nous en ont fait connaitre plusieurs, ainsi que quelques-uns des fruits offerts aux morts, dont les peintures des hypogées ne domnent qu'une idée incomplète.

Si le double, condamné à errer sur terre par l'oubli des siens, ne se montrait pas difficile dans le choix des aliments avec lesquels il cherchait à apaiser sa faim, les vivants ne croyaient pas bien souvent deroir être plus difficiles que lui pour ceux qu'ils lui offraient, et à côté des mets et des fruits dont ils se nourrissaient, ils lui en offraient qu'ils n'auraient pu ou voulu man-

1. Lepsius, Denkmäler, t. III, pl. 36; Gizeh, tombe 90, t. IV, pl. 53. Gizeh, tombe 16 .

2. Lepsius, op. laud., t. IV, pl. 65, tombe 17.

3. Lepsius, op. laud., t. IV, pl. 129, tombe 2.

4. Lepsius, op. laud. Saqqarah, tombe 16, t. IV, pl. 46; tombe $15, \mathrm{pl}$. 64, tombe 16 . 
ger; grains de cèréales, fruits encore verts ou de rebut, tout sembla bon pour les oflrandes funèbres ${ }^{1}$; cela en explique la variété et parfois la singularité. C'est ainsi quon a trouvé dans les tombes égyptiennes avec des épis ou des grains d'orge ${ }^{2}$ et de froment amidonnier ${ }^{3}$, de la pite, faite avec des rognures de grains d'orge" ou des lentilles cuites", des rhizomes de souchet comestible ${ }^{6}$, du malt ou des grains d'orge germés $^{7}$, des calebasses ${ }^{8}$, des fèves ${ }^{9}$, des graines de cajan $^{17}$, de gesse ${ }^{11}$ et de vesce cultivées ${ }^{12}$, de lupin ${ }^{13}$ et mème de lin ${ }^{14}$, des tiges d'ail ${ }^{13}$, ainsi que de la $P$ armelia furfuracea ${ }^{16}$.

Les espèces des fruits rencontrés dans les tombes pharaoniques ne sont ni moins nombreuses, ni moins variées; on y a trouvé, outre des figues de sycomore ${ }^{17}$,

1. Victor Loret, Le Cédratier, p. 45.

2. Bulletin no 5, p. 4. - Bulletin no 7, p. 420.

3. Bulletin $n^{\circ} 5$, p. 4. - Bulletin n० 7, p. 420 .

4. Drah-Abou'l-Neggah. Bulletin no 5, p. 4.

5. Drah-Abou'l-Neggah. Bulletin no 5, p. 7.

6. Bulletin no 3, p.75. - Bulletin no 5, p. 5. -.. Bulletin no 6, p. 260. - Bulletin $n^{\circ} 7$, p. 420 .

7. Shéikh Abd-el-Gournah. Bulletin no 6, p. 279.

8. Drah-Abou'l-Neggah. Bulletin no 3, p. 10.

9. Drah-Abou'l-Neggah. Bulletin no 5, p. 7.

10. Drah-Abou'l-Neggah. Bulletin no 5, p. 7.

11. Gébéleïn. Bulletin no 6, p. 260.

12. Drah-Abou'l-Neggah. Bulletin no 5, p. 10.

13. Bulletin no 6, p. 260. - Bulletin no 7, p. 420.

14. Bulletin n० 5, p. 8. - Bulletin no 6, p. 277.

15. L’Assassif près Thèbes. Bulletin no 6, p. 272 .

16. Bulletin no 3, p. 74. M. Schiaparelli a également trouvé à Drah-Abou'l-Neggah des bulbes d'oignon et d'ail et des graines de melon et de ricin; mais Schweinfurth doute de leur antiquité. Bulletin no 6, p. 265.

17. Gébéleïn. Bulletin n ${ }^{\circ}$ 6, p. 260. - Bulletin no 7, p. 420 . S. Birch, Calalogue of the antiquities at Almwick Castle, p. 183. 
des grenades ${ }^{1}$, des fruits ou des noyaux de nabaq ${ }^{2}$, de perséa ${ }^{3}$ et de l'hégélig ", des raisins" ${ }^{3}$, des dattes ${ }^{6}$, des fruits de palmier doum ${ }^{7}$ et de palmier argoun ${ }^{8}$, des olives ${ }^{9}$ et mème des baies de genévrier ${ }^{10}$, sans parler des baies de Cocculus leaeba ${ }^{11}$ et de Moerua uniflor ${ }^{12}$, trouvées à Gébéleïn, et des pommes de pin ${ }^{13}$, découvertes à Drah-Abou'l-Neggah.

Mais les fruits et les légumes n'étaient pas les seuls présents d'origine végétale que l'on faisait aux dieux et aux morts; on leur faisait hommage aussi, comme de l'offrande la plus agréable, de fleurs et de bouquets. Les Égyptiens se représentaient les dieux comme aimant à en être sans cesse parés ${ }^{14}$.

"Merenra s'en approche et tu courbes ta tête, tu inclines tes bras, grande Eau, devant les divins enfants de Nouit. Tous, ils voguent vers toi; ils posent leurs couronnes sur leurs têtes, ils posent autour de leur cou des couronnes de feuillage et de fleurs de lotus; ce sont les couronnes des étangs et des champs de paix de la grande Nouit, entourés de fleurs et de la verdure de la plante de miel. »

1. Drah-Abou'l-Neggah. Bulletin n० 5, p. 5. - Bulletin no 6, p. 268.

2. Bulletin no 6, p. 263. - Bulletin n० 7, p. $\{20$.

3. Drah-Abou'l-Neggah. Bulletin no 5, p. 5 .

4. Bulletin no 5, p. 5. - Bulletin n० 6, p. 260 et 268 .

5. Bulletin no 5, p. 9. - Bullelin $n^{0}$ 6, p. 260.

6. Bulletin n० 5, p. 10. - Bulletin no 6, p. 260.

7. Bulletin no 5, p. 5. - Bulletin no 6, p. 268. - Naspero, Les momics royales de Deir-el-Bahari. (Mémoires, note 8, t. I, p. 189).

8. Bulletin no 6, p. 6. - Bullelin no 6, p. 268.

9. Thèbes. Bulletin no 6, p. 266.

10. Bulletin no 3, p. 75. - Bulletin n० 6, p. 268.

11. Gébéleïn. Bulletin no 7, p. 420.

12. Gébéleïn. Bulletin no 6, p. 262.

13. Drah-Abou'l-Neggah. Bulletin no 5, p. 6.

14. V. von Strauss und Torney, op. laud., p. 120. 
On comprend d'après cela ${ }^{1}$ que, dès les temps les plus reculés, les fleurs eurent leur place marquée dans toutes les cérémonies religieuses; les peintures el'les sculptures des temples et des hypogées nous en fournissent de nombreux exemples. Dans les processions, on voit les officiants porter à la main une fleur de lotus ou un faisceau de tiges de papyrus². Quand il voulait contempler la divinité, le sacrificateur tenait des Heurs à la main, en pénétrant dans le sanctuaire". Aux funérailles, les enfants et les parents du défunt accompagnaient sa dépouille des palmes à la main". Des branches de feuillage décoraient la barque dans laquelle était transportée la momie du mort ${ }^{5}$; lor'squ'on l'avait placée debout à l'entrée du sépulcre, on déposait dessus une fleur de lotus et parfois aussi des rameaux couverts de feuilles ${ }^{6}$. Dans le cortège de la fète du nouvel an, représenté sur les parois du temple de Dendérah ${ }^{7}$, on voit les solihil - officiantes - répandre des fleurs sur le chemin de la procession.

Mais c'était plus particulièrement à titre d'offrandes que les fleurs figuraient dans le culte égyptien. Dans les processions, l'hiérophante, prêtre ou roi, présentait une fleur sacrée de lotus à l'image du dieu vénéré ${ }^{8}$ ou

1. $V$. von Strauss und Torney, op. laued., p. 329 et 353.

2. Lepsius, Denkmäler. Gizeh, t. III, pl. 30 et 35 ; t. IV, pl. 50. - Saqqarah, t. IV, pl. 46 et 65 . - El-Kab; t. V, pl. 11 , etc. - A. Mariette, Abydos, t. II, pl. 47.

3. A. Wiedemann, Die Religion der alten Aegypter, p. 13.

4. Wilkinson, op. laud., t. III, pl. LXIX.

5. Wilkinson, op. laud., t. III, pl. LXVII.

6. Wilkinson, op. laud., t. III, pl. LXIX.

7. A. Nariette, Dendérah, p. 97 et 317.

8. Lepsius, Denkmäler. Gournet-Mourrai, t. VI, pl. 119. Karnak, t. VIII, pl. 247. - Offrande d'Amenhotpou III à Amon. Prisse d'Avennes, op. laud. Atlas, II, pl. 18. 
au double du mort ${ }^{1}$. Un bas-relief, reproduit par Rosellini², nous montre Ramsès III offrant des lotus et des papyrus au dieu de Silsilis. Une peinture du temple de Dendérah représente le pharaon offrant au soleil du nouvel an, "le bel enfant qui brille au sein du lotus", une fleur de cette plante sacrée. Parmi les offrandes que le roi, dans les bas-reliefs du temple de Dendérah, fait aux divinités de ce sanctuaire célèbre, les fleurs occupent une grande place. En échange de celles qu'il présente à Hâthor, la déesse lui promet “ l'abondance et la fertilité du sol ». Et en récompense des bouquets qu'il offre à Hor-Hout, " l'Égypte, lui dit le dieu, sera, sous son règne, un jardin couvert de fleurs odoriférantes ${ }^{3}$ ". Ailleurs on le voit offrir à Hor-Sam-Taoui, ici un lotus, là une branche de saule ${ }^{4}$.

Ces dernières représentations d'offrandes de fleurs sont récentes, mais nous en avons vu beaucoup d'autres de date ancienne; dès l’époque des premières dynasties les tables d'offrandes sont couronnées de lotus, blancs d'abord, bleus dans la suite ${ }^{5}$. C'est plus tard cependant, avec l'extension que prit leur culture, que les fleurs occupérent dans le culte des dieux et surtout dans le culte des morts une place vraiment considérable. La signification symbolique qu'on leur attribuait explique le rôle qu'elles jouaient dans les cérémonies funèbres; considérées comme l'emblème

1. Lepsius, Denkmäler. Gizeh, t. IV, pl. 53 et 57.

2. Monumenti del culto, t. III, pl. 32, 2.

3. A. Mariette, Dendérah, p. 254 et 256.

4. A. Mariette, Denderchh, p. 173; atlas, t. II, pl. 47. L.e savant égyptologue a pris la branche de saule pour des feuilles d'acacia.

5. Lepsius, Denkmäler. El-Kab, t. V, pl. 11. - Wilkinson, op. laud., t. III, pl. LX et LXVI. 
du "mystère de la vie ", le symbole de l'existence ronouvelée qui succède ì la mort, comment n'y auraientelles pas pris une large place? Les peintures du tombeau d'Amenemhabi, officier de Thoutmès III, mettent en évidence cetto vérité ${ }^{1}$. Elles nous montrent ce scigneur assis, couvert du pagne ot d'une longue robe claire, arec un collier et des bracelets bleus. Sa fomme Beki, portant aussi un collier bleu et couronnée de fleurs, est assise à ses côtés. Devant lui se tient son fils Amou; de la main droite il présente un bouquet à son père; de la gauche il tient un autre bouquet.

"Il arrive en bienvenu avec le symbole de vie, dit l'inscription $^{2}$, son fils qu'il aime, la demeure de son cœur, aimé du Seigneur de la double terre, Amou, qui dit: "A ta personne le symbole de vie. "De son côté, il se réjouit à voir les bonnes fleurs, à en respirer [le parfum], le noble seigneur qui suit le roi du midi [le soleil] dans ses voyages aux régions du midi et du nord... Amenemhabi, makhérou ${ }^{3}$. ”

Ce n'est pas un simple lotus, c'est tout un bouquet que le fils d'Amenemhabi lui offre; l'hommage d'une fleur unique de lotus ou de papyrus ne tarda pas à paraitre trop humble; on en réunit deux ou trois ensemble $^{4}$. Mais ce ne fut bientót pas assez; on y joignit quelques-unes des fleurs qui croissaient spontanément dans la vallée du Nil ou qui furent cultivées dans les jardins égyptiens, surtout à l'époque des Ptolémées,

1. Philippe Virey. Tombeau d'Amenemheb. (Mémoires, t. V, fasc. 2, p. 251.

2. Philippe Virey, op. laud., p. 251.

3. Le "justifié ".

4. Champollion, Monuments, t. II, pl. CIX, 1. - Lepsius, Ientimëler, t. VII, pl. 235. Abd-el-Gournah, tombe 18.

I. 
tel que le perséa et l'olivier" ${ }^{1}$, le laurier, le myrte et le jasmin, le coquelicot, la giroflée, la mauve à feuilles de figuier, l'épilobe velu, le chrysanthème à couronnes, le bluet oriental, la conyse de Dioscoride, la cresse de Crète, des convolvulus, l'héliotrope de Nubie, la marjolaine, la célosie argentée, le narcisse tazzette, l'iris de Sibérie, etc. ${ }^{2}$

On eut ainsi des bouquets remarquables par l'éclat et la variété des couleurs, non moins que par l'art avec lequel ils étaient composés. Tels sont ceux qu'on voit sur les peintures d'Abd-el-Gournah ou du temple de Khonsou à Karnak (XXI ${ }^{\mathrm{e}}$ dynastie) ${ }^{3}$; tels sont en particulier les bouquets que Prisse d'Avennes a reproduits" d'après la peinture d'un hypogée thébain contemporain de la XIX dynastie.

Le premier se compose de trois papyrus, dont les ombelles épanouies forment le sommet et dont les tiges traversent des corolles de fleurs de paparéracées alternativement purpurines et bleues, accompagnées de fleurs de composées, elles aussi rouges ou bleues peut-être des bluets et des fleurs de carthame - ainsi que de ces boutons ou ovaires qu'on rencontre fréquemment à l'extrémité de tiges nues, émergeant de touffes de feuilles longuement lancéolées. Le second est formé d'une ombelle de papyrus, du centre de laquelle sortent deux fleurs et un bouton idéalisé de lotus, et sa tige traverse huit fleurs de lotus, aux pétales

1. G. Schweinfurth, Botanische Jahrbücher, t. VIII (an. 1886), p. 7. T'ombeaux de Gébéleïn et de Drah-Abou'l-Neggah.

2. Flinders Petrie, IIawara, Biahmu and Arsinoë, p. 57.Kahun, Gurob and Hawara, p. 46-47.

3. Lepsius, Denkmüler, t. IV, p. 69 et t. VIII, pl. 247.

4. Histoire de l'art égyptien. Atlas, t. II, pl. 68, nos 1 et 2 . 
bleus et blancs, accompagnées de fleur's composées bleues etrouges et de boutons ovoïdes apointis, comme dans le bouquet précédent.

Ce n'étaient pas seulement des fleurs et des bouquets qu'on offrait aux morts, on leur offrait aussi des couronnes et des guirlandes. On en trouve dans les tombes des époques les plus diverses, mais on les a rencontrées surtout à partir de la XVIII dynastie. La découverte de guirlandes funéraires dans les tombes des pharaons de cette dynastie, Ramsès II, Amenhotpou I ${ }^{\text {er }}$ et Ahmès I ${ }^{\text {er }}$, a Deir-el-Bahari, a permis de connaitre mieux qu'on ne le savait cet usage des anciens Égyptiens ${ }^{1}$. Les guirlandes de Ramsès paraissent avoir été renouvelées sous la XX ${ }^{\mathrm{e}}$ dynastie, alors qu'un pharaon de cette époque fit refaire à son grand prédécesseur un cercueil nouveau ${ }^{2}$; elles sontformées de feuilles pliées, entières ou déchirées en deux parties, de Mimusops Schimperi Hochst. " ; elles servent comme d'agrafes, dit M. Schweinfurth, aux pétales et aux sépales du lotus bleu et le tout est disposé autour de fibres tirées des feuilles du dattier, fendues et cousues ensemble. Les pétales du lotus bleu ont, dans quelques-unes de ces guirlandes, fait place à celles du lotus blanc. Outre ces guirlandes, il y avait, soit à côté de la momie, soit fixées entre les bandelettes extérieures qui l'enserraient, des fleurs entières de Nymphaea caerulea avec leurs pédoncules long's de

1. G. Schweinfurth, De la Flore pharaonique. (Bulletin de l'Instilul égyptien, no 3 (an. 1882), p. 60-75.)

2. G. Naspero, Rapport sur la trouvaille de Deir-el-Bahari. (Bulletin de l'Instilul égyptien, no 1 (an. 1880), p. 14.

3. M. Maspero a également trouvé à Gébéleïn des fragments d'anciennes couronnes formées de rameaux du même arbuste, mêlées à des branches d'olivier. Bulletin no 6 (an. 1885), p. 263. 
cinquante centimètres. Les guirlandes se croisaient sur. la poitrine en plusieurs rangs semi-concentriques.

Les guirlandes du cercueil d'Ahmès I er étaient d'une composition tout autre que celles de Ramsès II ${ }^{1}$; elles étaient formées, les unes de feuilles de saule avec des fleurs de pied d'alouette oriental et de la sesbanie d'Égypte, les autres de pétales de lotus et de mauve à fenilles de figuier, retenus par des feuilles de saule soigneusement cousues ensemble. Dans quelques-unes des guirlandes d'Amenhotpou, des fleurs d'acacia du Nil et de carthame ont été substituées à celles de mauve et de dauphinelle ${ }^{2}$. Théophraste avait déjà mentionné ${ }^{3}$ l'emploi des fleurs d'acacia dans la confection des couronnes.

Dans un autre cercueil de la nécropole de Deir-elBahari, celui de la princesse Nesi-Khonsou de la XXI ${ }^{\circ}$ dynastie, se trouvaient des guirlandes d'une composition différente" ; des feuilles de saule en faisaient encore la base, mais les fleurs qui composaient les guirlandes des pharaons de la XVIII dynastie ont fait place aux fleurs de la picride à feuilles de corne de cerf, du coquelicot et de la centaurée déprimée - le bluet oriental. - Sur les yeux et la bouche de la momie étaient collées des pelures du bulbe d'une espèce de Crinum, peut-être le juccaefolium, belle plante d'Abyssinie à fleurs blanches rayées de rouge ${ }^{5}$.

M. Schiaparelli a découvert aussi, à Drah-Abou'lNeggah, des guirlandes composées de feuilles de Mimu-

1. Bulletin no 3 (an. 1882), p. 68.

2. Bulletin no 3 (an. 1882), p. 70.

3. IIistoria planlarum, lib. IV, cap. 2,8 .

4. Bulletin nc 3 (an. 1882), p. 72.

5. G. Schweinfurth, Sur les dernières trouvailles botaniques. (Bulletin no 7 (an. 1886), p. 427-29.) 
sops et de capitules de bluet oriental, et il a trouvé, dans une tombe déjà ouverte de Sheikh Abd-el-Gournah, un fragment de guirlande faite de feuilles du saule égyptien et de fleurs de carthame. A Drah-Abou'l-Neggah il a rencontré également plusieurs capitules du chrysanthème à couronnes, plante méditerranéenne, qu'on retrouve dans les guirlandes de l'époque grécoromaine, ainsi que cinq fleurs de Sphaeranthes suaveolens DC., composée commune dans les lieux humides de la Basse-Égypte ${ }^{1}$. L'égyptologue italien a découvert encore à Drah-Abou'l-Neggah dans un puits de tombe, de l'époque ptolémaïque, il est vrai, des rameaux de patience à feuilles dentées (Rumex dentatus L.) ${ }^{2}$.

Il est vraisemblable que cette plante avait servi à envelopper une momie; celle de Kent, contemporain de la $\mathrm{XX}^{\mathrm{e}}$ dynastie, découverte par $\mathrm{M}$. Maspero ${ }^{3}$, était complétement entourée de branches de sycomore; autour du cou se trouvait aussi une espèce de guirlande composée de grains d'orge germés, réunis ensemble à l'aide de leurs racines. Sur la poitrine était en même temps placée en demi-cercle une guirlande faite de feuilles et de rameaux fleuris de céleri sauvage, avec des pétales et des fleurs naines de lotus bleu, tressés ensemble à l'aide de fibres de papyrus. La momie reposait couchée sur un lit de branches de tamaris du Nil. Il existe au musée de Leyde des débris de couronnes contemporaines, peut-être de la XXVe ou de la XXVI

1. G. Schweinfurth, Les dernières decouvertes botaniques. (Bulletin no 6 (an. 1885), p. 270, 271 et 272.)

2. Bulletin no 6 (an. 1885), p. 272.

3. Bulletin no 6 (an. 1885), p. 278-283. - G. Schweinfurth, Die lelzlen botanischen Entdeckungen. (Bolanische Jahrbïcher, t. VIII (an. 1886), p. 13.) 
dynastie et qui, au lieu d'être, comme les guirlandes de Gébéleïn et de Drah-Abou'l-Neggah, formées de feuilles de saule et de Mimusops, ont été faites avec des feuilles d'olivier et d'une espèce de laurier ${ }^{1}$.

Ainsi les végétaux les plus divers entraient successivement dans la composition des couronnes égyptiennes; mais la manière de les faire était toujours à peu près la même. On coupait, dit $\cdot \mathrm{M}$. Pleyte ${ }^{2}$, dans une feuille de palmier doum, une petite bande d'environ trois millimètres de largeur, mais aussi longue que possible; on prenait ensuite une feuille de mimusops, de saule ou d'olivier, que l'on pliait en deux, puis on posait dessus un pétale de nymphéa ou, suivant le cas, une fleur d'acacia, de chrysanthème, de pied d'alouette, de bluet ou de mauve; on agrafait le tout a la bande de feuille de palmier, et on réunissait les deux bouts de celle-ci au moyen d'un bouton ou d'un nœud, orné parfois d'une fleur de lotus bleu, qui retom. bait sur le front de la momie.

Ces couronnes étaient l'emblème de la justification du défunt ${ }^{3}$; on les plaçait sur sa tête, quand Osiris l'avait jugé digne d'entrer dans les champs d'Ialou.

" Ton frère Toum a tressé pour toi cette belle couronne de la justice", ce fronteau rivant, aimé de tous les dieux; tu vis pour l'éternité. Osiris, résident de l'ouest, a proclamé ta parole comme vérité contre tes ennemis... Osiris, le résident de l'ouest, a réuni les dieux du monde inférieur et supérieur... pour proclamer juste Osiris le défunt, devant ses ennemis. ”

Ainsi les plantes, sous des formes diverses, figurent

1. W. Pleyte, La couronne de la justification, p. 5-6.

2. Op. laud., p. 17.

3. W. Pleyte, np. laud., p. 7-8.

4. Le livre des morts, chap. xix. 
à chaque instant dans le culte des morts; elles les accompagnaient dans l'autre vie, image de la vie d'icibas; leur double les retrouvait dans les champs d'Ialou, et pour qu'il ne füt pas privé de ce voisinage charmant, un jardin était parfois, semble-t-il, disposé près de la tombe ${ }^{1}$; d'ailleurs il lui était loisible de venir, dans son ancien jardin, se divertir " à regarder tous les végétaux », prendre le frais à l'ombre de ses arbres ou sur le réservoir qui s'y trouvait ${ }^{2}$. C'est ainsi qu'une peinture du tombeau de Rekhmara nous montre, symbole de ces réjouissances surnaturelles, le seigneur égyptien se reposant, à l'abri du soleil, dans la cabine d'une barque de plaisance sur les eaux tranquilles d'un étang couvert de lotus ${ }^{3}$. Et quand, plus loin, " afin de faire un jour heureux, pendant qu'il est sur terre ", il préside au festin préparé pour sa visite parmi les vivants, ce sont encore les plantes et les fleurs qui font sa principale joie.

"On te présente, lui dit son fils, ainsi qu'à sa mère Mirit", les fleurs de lotas qui surgissent sur l'étang de ton jardin... C'est pour toi qu'il porte en présent tous les fruits et tous les légumes qui sont en lui; sois approvisionné de ses produits; sois dans l'abondance avec ses provisions. Jouis de sa verdure; rafraîchis-toi à l'ombre de ses arbres; fais-y ce qu'aime ton double pour le temps et pour l'éternité. »

Le rôle joué dans la vie religieuse des Égyptiens par les fleurs et les jardins, le caractère sacré des

1. Perrot et Chipiez, Histoire de l'art, t. I, p. 306.

2. Philippe Virey, Le tombeau de Khem. (Mémoires, t. V, fasc. 2, p. 367.)

3. Philippe Virey, Le lombeau de Rekhmara. (Mémoires, t. V, p. 156, fasc. 1, pl. XXXVIII.)

4. Philippe Virey, op. laud., p. 160. 
arbres, expliquent le soin qu'ils mettaient à en entourer leurs villas et les temples. Parmi les dons, si riches et vraiment innombrables faits par Ramsès III aux dieux de Thèbes, d'Héliopolis et de Memphis, figurent, outre des vignes, des domaines entiers, avec leurs viviers et leurs champs, qui pouvaient servir à l'entretien des prêtres et des temples, des plantations d'arbres, faites en l'honneur de ces divinités et des jardins qu'il leur abandonnait par un véritable contrat.

"J'ai planté, dit le pharaon dans la charte où il énumère les dons faits au dieu de Thèbes ${ }^{1}$, ta ville d'arbres, d'arbustes et de fleurs agréables à respirer, un vaste domaine dans la ville du nord... [il est] pourvu de vastes jardins, de promenades avec des arbres de toutes sortes, des dattiers avec leurs fruits. "

Et ailleurs dans l'énumération des dons faits au dieu d'Héliopolis ${ }^{2}$ :

" J'ai relevé les murs de la maison d'Horus... J'ai fait alranger lo magnifique bosquet qui est à l'intérieur. »

Et s'adressant au dieu de Memphis ${ }^{3}$ :

“ J'ai planté dans ta grande et superbe demeure des oliviers, des arbres à encens... que mon bras a rapporté de la terre sainte - le Pount - pour les consacrer chaque matin à ta magnificence. »

Tous les temples étaient accompagnés de bois sacrés, remplis d'un certain nombre d'espèces arborescentes.

1. Aug. Eisenlohr, Aus dem grossen Papyrus Harris. pl. VIII. (Zeilschrifl fïr aegyptische Sprache, t. XI (an. 1873), p. 54.)

2. Ibid., pl. XXIX. Zeilschrift, t. XI, p. 100.

3. Ibid., pl. XLIX. Zeilschrift, t. XI, p. 155. 
Hérodote "parle d'un bois de palmiers, qui se trouvait auprès du temple de Persée - Khem — dans la ville de Kihemnis. Il avait dì en voir d'autres. Il y avait un bois sacré en particulier dans l'enceinte du sanctuaire de chacun des quarante-deux nomes entre lesquels était divisée l'Égypte.

Un texte précieux, gravé sur la base des murailles extérieures du sanctuaire d'Edfou, mentionne avec le nom de la divinité principale de chacun des nomes, de leur capitale, de leur relique particulière, etc., les noms des arbres sacrés plantés dans le bocage qui les entourait ${ }^{2}$. Il résulte de la comparaison de ces listes deux ont été détruites - que l'arbre shent - Acacia nilotica - était planté dans vingt-quatre nomes, l'arbre asht - le Balanites - dans dix-sept, le nebs ou noubsou - le sébestier —, dans seize, l'arbre inconnu qebs, dans trois seulement, l'incertain am, le neha le sycomore - et l'indéterminé arou — dans deux, enfin l'aser - le tamaris - le douteux tem et le shen.a " le grand arbre ", rien que dans un.

La charte de fondation du temple d'Hâthor à Dendérah ${ }^{3}$ fait également mention de quatre jardins sacrés ou d'un seul jardin divisé en quatre sections, désignées chacune par l'arbre ou les arbres qui y étaient plantés; c'était ${ }^{4}$ l'am, l'asht, le shent — l'acacia du Nil - , le ther - le saule safsaf, — le ncha — le sycomore, - le qebs, dans lequel Mariette a vu à tort un

1. IIistoriae, Iib, II, cap. 91.

2. Charles Moldenke, Die altïgyptischen Bäume, p. 9-16.

3. Aug. Nariette, Dendérah. Description générale, p. 89-90.

4. Dümichen, Bauurkunde von den Tempelanlagen von Dendera, pl. VIII, ap. Moldenke, op. laud., p. 17. 
palmier ${ }^{1}$, le mama ou doum et le tem, avec l'arbre kunth, espèce ou variété de figuier, qui était à Dendérah l'objet d'un culte particulier. Il en était de même du saule; sur un des bas-reliefs du même temple, on voit le roi consacrer un saule à Hâthor et à Hor-Hout ${ }^{2}$.

IV́.

On vient de voir quelle place considérable les offrandes tirées du règne végétal occupaient dans le culte égyptien; mais les plantes ne figuraient pas uniquement, sous leur forme naturelle, dans les cérémonies religieuses; leurs produits y jouèrent un rôle non moins important, les peintures pharaoniques nous le montrent ${ }^{3}$, sous forme de libations et de fumigations.

On faisait les libations avec du vin, de la bière, des huiles ou des essences parfumées, tel que " l'extrait surfin de styrax ", fabriqué " pour parfumer Hàthor de l'odeur que donne son eau " $"$. On répandait d'ordinaire ces liquides sur les offrandes elles-mêmes, parfois aussi, sur une espèce d'autel en pierre, en granit ou en basalte, destiné à cet effet. On versait encore de l'huile ou des parfums sur la tête de la momie, avant de la placer dans la niche où elle était conservée ${ }^{3}$. C'était à la fois un hommage rendu au mort et un moyen de conservation de sa dépouille dernière, sinon un symbole de vie.

1. Denderah. Description générale, p. 90.

2. A. Mariette, Dendérah, p. 136. Atlas, t. I, pl. 24.

3. Wilkinson, op. laud., t. III, pl. LXVI et LXVII.

4. Victor Loret, Études de drogueric égyptienne. Paris, 189', in-4, I, p. 2.

5. Wilkinson, op. laud., t. III, p. 423, 429, 430 et 431 . 
Les onctions paraissent avoir eu la même signification; on les faisait arec de l'huile renfermée dans des vases d'une matière précieusc et parfumée avec des aromates particuliers. Une inscription du temple d'Edfou parle des 14 aromates ant et des 8 aromates $a b$, "sortis des yeux de Rì et d'Horus, et qui servaient ¿ préparer l'huile sacrée, dont le parfum donne aux membres des dicux une force nouvelle ${ }^{1}$ ". Le moment venu, le prêtre ou le roi trempait l'annulaire ou le petit doigt dans une de ces huiles saintes et le passait ensuite respectueusement sur l'effigie du dieu ou du mort qu'on voulait honorer. C'est ainsi qu'une sculpture nous montre Séti Ior oignant la tête du dieu Khem. ${ }^{2}$. "Il répand l'huile ", dit l'inscription qui l'accompagne, " afin qu'il devienne un dispensateur de la vie ". D'autres fois les libations et les onctions semblent avoir été un emblème de purification. Sur une peinture de Derri $^{3}$, qui représente Horus et Thot de Hat, faisant une libation sur la tête d'Amenhotpou II, on lit ces mots: "Tu es pur et pur tu resteras ". "Je t'ai oint les yeux, fait dire à l'Horus défunt une inscription du tombeau de Pétaménap", je t'ai oint le front avec l'huile préparée par la déesse Sokhit.... Tu triompheras, tu ceindras, au milieu des Dieux, la couronne souveraine ».

Après les libations, ou en même temps qu'elles, prenaient place les fumigations; une des pratiques les plus

1. Dümichen, Der Grabpalast des Paluamenap in der Thebanischen Neliropolis. Leipzig, 1885, in-fol. t. II, p. 13.

2. Wilkinson, op. laud., t. III, pl. LXII, 2.

3. Champollion, Monuments, t. I, pl. XLII. - Rosellini, Monumenti, t. III, pl. VII. - IVilkinson, op. laud., t. III, pl. LVII, 1.

4. Dümichen, op. laud., t. II, p. 19. 
anciennes du culte égyptien, elles s'arlressaient également à toutes les divinités et sans elles il n'y avait point de cérémonie religieuse complète. Dans les représentations des fètes données en l'honneur des dieux ou des morts, qu'on rencontre si souvent sur les murs des temples ou des hypogées ${ }^{1}$, on voit l'hiérophante, tenant de la main gauche le brûle-parfum, et jetant de la droite, sur le feu qu'il renferme, des balles ou des pastilles d'encens. Au lieu du chef des prêtres, c'était le roi parfois qui remplissait cet office, debout devant la statue du dieu qu'il invoquait ou dont il réclamait une faveur. Quelquefois aussi, il faisait en même temps une libation, soit seul, soit en compagnie de la reine ou de son fils. C'est ainsi que Ramsès II et son fils sont représentés deux fois sur une peinture du Sérapeum, faisant des libations derant un Apis de forme humaine ${ }^{2}$. Sur un bas-relief du temple d'Ibsamboul on voit le pharaon qui brûle de l'encens, tout en faisant une libation, tandis que la reine agite les sistres sacrés ${ }^{3}$.

Si les peintures pharaoniques nous montrent comment se faisaient les fumigations sacrées, elles ne nous apprennent pas quelle était la nature des balles ou des pastilles qu'on y brûlait. Sans doute elles étaient de nature variable. Tantòt elles étaient formées d'encens - anti - et de ses nombreuses variétés, d'autres fois de quelque autre gomme-résine, telle que la myrrhe, ou peut-être le mastic, enfin d'un aromate

1. Wilkinson, op. laud., t. I, p. 183 et t. III, pl. LX et LXVII, p. 398 et 399 .

2. A. Mariette, Le Sérapeum de Memphis. Paris, 1857, in-fol., p. 13 et pl. 8 .

3. Wilkinson, op. laud., t. III, pl. LXV, 8. 
composé. Plutarque dit ${ }^{1}$ que les Égyptiens brîlaient de la résine au lever du jour, do la myrrhe ì midi, et, au coucher du soleil, du kyphli, aromate dans la composition duquel entraient seize ingrédients divers.

\section{V.}

L'emploi des fleur's et des aromates n'était pas exclusivement réservé au culte des dieux ou des morts, on s'en servait également dans les fêtes profanes et dans les usages ordinaires de la vie. Pour les Égyptiens, comme pour tous les Orientaux, les parfums étaient à la fois une satisfaction et un besoin. Ils brûlaient du kyphi pour parfumer leurs maisons et leurs vètements; ils aimaient à s'oindre le corps et en particulier la tête d'huiles parfumèes. "Ils se réjouissaient, dit un ancien texte ${ }^{3}$, quand leur main était pleine des fleurs de leurs jardins"; ils en aimaient la vue et en recherchaient l'agréable senteur. Les femmes se paraient de fleurs la tête et le sein.

Les peintures pharaoniques" nous montrent les dames égyptiennes se faisant oindre par leurs femmes la chevelure d'essences précieuses et couronner de guirlandes de fleurs. Une inscription du tombeau de Thoutmès III ${ }^{5}$ parle de "guerriers, qui s'oignent d'huiles, comme on a coutume de le faire les jours

1. De Iside et Osiride, cap. 80 et 81.

2. Dümichen, Papyrus Ebers, p. 19.

3. Papyrus de Boulaq, 19, 3.

4. Hypogées thébains. Prisse d'Avennes, Monuments égyptiens, bas-relie/s, peintures, inscriptions. Paris, 1847, in-fol., pl. XLV.

5. Brugsch-Bey, Geschichte des alten Aegyptens, p. 308. 
de fête dans la terre d'Égypte ". Cet usage n'était pas nouveau; il remontait à une haute antiquité. "Je me parfumai d'essences aussi libéralement que si je versais l'eau de mes citernes », dit Amenemhat I, le fondateur de la XII dynastie, dans ses Instructions à son fils". "Fais un jour heureux ", chante le joueur de harpe ${ }^{2}$, "qu'il y ait toujours des parfums et des essences pour ton nez, des guirlandes et des lotus pour les épaules et la gorge de ta sœur chérie ". Dans les conseils et les reproches adressés par le chef des archivistes Amen-em-ant au scribe Pentaour, celui-ci est représenté se livrant à l'indolence, " assis, oint d'essence, une guirlande de fleurs au $\operatorname{cou}^{3}$ ". Et un scribe égyptien, dans la description qu'il fait de la ville de Ramsès-Aanakhtou, fondée par Rhamsès II à l'Orient du Delta", nous en montre les habitants " de l'huile parfumée sur la tête, debout sur leurs portes, les mains chargées de bouquets, de rameaux verts du bourg de Pà-Hâthor, de guirlandes du bourg de Pahour, au jour d'entrée de Pharaon ".

Les fleurs, on le voit, étaient un symbole de joie et une marque d'allégresse; aussi figuraient-elles, avec les parfums, dans toutes les réunions. Après les avoir oints d'essences et d'huiles de senteur, on parait de guirlandes de fleurs tous les invités. On offrait des bouquets aux dames à leur arrivée et, au moment où

1. Papyrus Sallier, no 2, pl. I, ap. Naspero, Hisloire ancienne, t. I, p. 466.

2. G. Naspero, Études égyptiennes, p. 174. - Ludw. Stern, Das Lied des Harfners. (Zeitschrift, t. XI (an. 1873), p. 60).

3. G. Maspero, Du genre épistolcive chez les Égyptiens de l'époque pharcaonique. Paris, 1872, in-8, p. 33.

4. G. Maspero, IIistoire ancienne, éd. in-12, p. 229. Du genre épislolaire, p. 105. 
elles entraient dans la salle des fètes, elles recevaient une fleur de lotus qu'elles devaient garder à la main durant toute la réunion'; on leur donnait aussi des couronnes de lotus bleus ou blancs, entremêlés de boutons dressés, avec une fleur ouverte ì chacun des deux nœuds ${ }^{2}$, celle de devant, disposée de manière à pendre sur le front. D'autres fois elles avaient pour coiffure un bandeau orné de pétales de lotus avec un bouton de cette fleur attaché par derrière et retombant sur le front.

Une des peintures du tombeau de Rekhmara nous fait assister à une réception de ce genre. On y voit ${ }^{3}$ trois dames assises sur une natte, une fleur de lotus à la main; une servante les pare de colliers, tandis qu'une autre leur offre des rafraìchissements; plus loin deux autres servantes sont également occupées à la parure d'une quatrième dame; l'une soulève sa chevelure, pour que la seconde puisse lui attacher autour du cou une guirlande de fleurs, qu'elle vient de prendre sur une table placée derrière elle. Ailleurs deux autres servantes encore apportent des colliers de fleurs pour deux dames déjà parées de couronnes.

1. Champollion, Monuments, t. II, pl. 187. - Wilkinson, op. laud., t. 1, p. 424, 426 et 427 , nos 190,201 et 204.

2. Couronnes des filles de Tahoutihotpou sur une peinture du tombeau de ce prince. Archaeological Survey of Egypt. El Berseh. The tomb of Tehuli-IIelep by Percy E. Newberry. London, 1894, in-4. D'autres fois, les dames égyptiennes avaient pour coiffure un bandeau orné de pétales de lotus avec un bouton de cette fleur attaché par derrière et retombant sur le front. Nécropole de Thèbes. Prisse d'Avennes, Monuments égypliens, pl. XLIV.

3. Philippe Virey, Mémoires de la mission francaise au Caire. t. V, fasc. 1, p. 160-161, pl. XLI. - Champollion, Monuments. t. II, pl. 187. 


\section{CHAPITRE VIII.}

LES PLANTES DANS LA PHARMACOPÉE ET LA PARFUMERIE ÉGYPTIENNES. - LES AROMATES ET LEURS USAGES.

I.

Pour les Egyptiens comme pour les autres nations de l'ancien Orient - il en est encore de même chez tous les peuples primitifs - les maladies n'étaient pas la conséquence fatale de la fragilité même de la nature humaine; elles avaient une cause tout autre et surnaturelle; elles étaient l'œuvre de génies malfaisants; aussi ne pouvait-on les guérir qu'avec le secours des Dieux ${ }^{1}$. Les prières que leur adressait le patient étaient le moyen le plus sûr de triompher du màl dont il souffrait, et les remèdes qui lui étaient prescrits ne devaient leur efficacitéqu'aux incantations magiques qui avaient accompagné leur préparation et qu'on récitait au moment où ils lui étaient administrés. Cette conception explique le caractère surnaturel que prit la médecine dans l'Égypte ancienne ${ }^{2}$;

1. Salvatore di Renzi, Sloria della medicina. Napoli, 1849, in-8, t. I, p. 46. - G. Maspero, Hisloive ancienne, t. I, p. 212.

2. Aux pages 46 et 47 du Papyrus Ebers, sont mentionnés cinq remèdes, deux que Râ et Shou avaient faits pour eux- 
quelques-unes de ses recettes étaient attribuées aux plus grands dieux du Panthéon pharaonique; Thot, l'inventeur des arts, en avait domné les premiers préceptes, el c'étaient ses disciples - prètres et magiciens - qui en étaient les dépositaires et avaient pour mission de les appliquer.

L'origine dirine, qui lui était assignée, montre déjà l’antiquité de la thérapeutique en Égypte; la tradition la faisait remonter aux premiers temps de son histoire. Manéthon rapporte ${ }^{1}$ que le fils de Ménès, T'éti - l'Athòtis des Grecs —, qui "était médecin ", avait écrit un traité d'anatomie; aussi, parfois, ce prince a-t-il été représenté sous la figure d'un ibis, emblème du dieu Thot. Un autre pharaon de la troisième dynastie, Nibka - Tosorthros —, mérita par ses connaissances médicales d'être identifié avec Esculape ${ }^{2}$. Le nombre et la variété des procédés thérapeutiques des Égyptiens, l'emploi qu'ils faisaient des incantations magiques, frappèrent d'étonnement les peuples étrangers, qui entrèrent en relations avec eux. L'Égypte apparut aux Grecs, en particulier, comme unc terre des merveilles, remplie de plantes vénéneuses ou salutaires et dont chaque habitant, rapporte Homère, était un médecin habile, " car ils étaient de la race de Péan ${ }^{3}$. " Hérodote fut frappé du soin que les Égyptiens prenaient de leur

mêmes et trois autres que Sibou, Nouit et Isis avaient composés pour Râ.

1. Éd. Car. Müller. (Fragmenta historicorum graecorum. Parisiis, 1853, t. II, p. 539.)

2. H. Brugsch, Ueber die medicinischen Kenntnisse der alten Aegypler. (Allgemeine Monatschrift für Wissenschaft und Lileratur. Braunschweig, 1853, p. 44.)

3. Odyssée, chant IV, v. 229-232. 
santé et du grand nombre de médecins, "qui abondaient en tous lieux ${ }^{1}$ ». Ces témoignages, qu'elle qu'en soit l'incertitude, n'en sont pas moins une preuve du développement que l'art de guérir avait pris dès longtemps dans la vallée du $\mathrm{Nil}^{2}$; aussi ne doit-on pàs être surpris que les Grecs aient emprunté plus d'un remède à la pharmacopée égyptienne ${ }^{3}$.

Les papyrus médicaux découverts ou étudiés depuis un demi-siècle nous permettent de nous faire une idée de ce qu'était la médecine des sujets des pharaons et des procédés auxquels elle avait recours. Les deux plus considérables qui soient connus ${ }^{4}$, - un troisième non moins important, le papyrus médical du British Museum, n'a point encore été publié ${ }^{5}$ - sont le Papyrus Ebers et le Papyrus de Berlin. Ce dernier, découvert à Saqqarah par l'égyptologue Passalacqua et le premier qui ait été publié, date de la XIX $\mathrm{X}^{0} \mathrm{dy-}$ nastie et paraît avoir été écrit sous le règne de Ramsès II, vers l'an 1350 avant notre ère ; mais certaines parties sont beaucoup plus anciennes et remontent peut-ètre à l'époque des Pharaons constructeurs des pyramides ${ }^{6}$. Remarquable par son ancien-

1. Historiae, lib. II, cap. 84.

2. Clément d'Alexandrie, Stromata, lib. VI, cap. 4, 37, parle d'un traité hermétique de médecine égyptienne en six livres, dont malheureusement nous ne connaissons rien.

3. M. S. Houdart, Hisloire de la médecine grecque depuis Esculape jusqu'à Hippocrate exclusivement. Paris, 1856, in-8, p. 89. - J. Berendes, Die Pharmacie bei den alten Cullurvölkern. Halle, 1890, in-8, t. I, p. 60.

4. Je ne parle pas des fragments d'un papyrus médical de Leyde, à cause de leur peu d'importance.

5. S. Birch, Medical Papyrus with the name of Cheops. (Zeitschrift für aegyptische Sprache, t. IX (an. 1871), p. 61-64.)

6. H. Brugsch, Ueber die medicinischen Kennlnisse der 
neté, ce recueil l'est encore par le grand nombre de recettes qu'il donne pour les maladies les plus diverses. M. Chabas n'en a pas compté moins de cent soixantedix ${ }^{1}$; mais les noms de la plupart des remèdes végétaux qu'elles prescrivent nous sont inconnus ${ }^{2}$ et le traité manque souvent de clarté; aussi, malgré son étendue, ne nous initie-t-il qu'imparfaitement à la comnaissance de la pharmacopée égyptienne. I] n'en est pas de mème du Papyrus Ebers dont la composition offre, du reste, la plus grande ressemblance avec celle du Papyrus de Berlin.

Rapporté d'Égypte, pendant l'hiver 1872-73, par l'égyptologue dont jl porte le nom, annoncé aussitôt au monde savant ${ }^{3}$ et publié in extenso peu après ", le Papyrus Ebers nous offre le manuel le plus complet de la thérapeutique égyptienne. C'est, comme le titre l'indique, un lirre de la préparation des remèdes pour toutes les parties du corps. Il fut écrit au milieu du xvI ${ }^{\mathrm{e}}$ siècle avant notre ère ${ }^{5}$; mais plusieurs des traités

alten Aegypter, p. 49. - Id., Notice raisonnée d'un trailé médical datant du XIV siècle avant notre ère. Leipzig, 1863, p. 2 et 13.

1. La médecine des anciens Égyptiens. (Mélanges égyptologiques. Châlon-sur-Saône, 1861, in-8, t. I, p. 56.) - Brugsch, Notice raisonnée, p. 5 , parle d'une cinquantaine d'herbes et de neuf espèces de différents arbres.

2. Tel que le bois de shel, l'herbe aau, l'herbe ashl, l'herbe haka; mais on y retrouve aussi les fruits du jujubier, l'huile de moringa, non identifiés d'ailleurs par Chabas.

3. Zeitschrift für aegyptische Sprache, t. XI (an. 1873), p. $41-46$.

4. Georg Ebers, Das hermetische Buch über die Arsneimittel der alten Aegypter in hieratischer Schrift. Leipzig, 1875, 2 vol. in-fol.

5. L'an 1552. G. Ebers, Das hermetische Buch, p. 9, 2.- 
qui y sont résumés sont d'une date bien autrement reculée; il peut donc être considéré dans son ensemble comme le manuel de thérapeutique le plus ancien et le plus complet que l'on connaisse; grâce à lui, nous savons ce qu'était l'art de guérir dans le pays des Pharaons et ce qu'il y resta jusqu'à la conquête grecque.

Trouvé dans une cassette à Sekhem, aux pieds d'une statue d'Anubis, comme on lit à la page $103^{1}$, et porté au roi de la Haute et de la Basse-Égypte, Sa Majesté Housapaiti - Ousaphaïs - ce précieux et antique papyrus s'ouvre par un préambule solennel sur son origine prétendue divine et sur l'utilité des recettes qu'il renferme, recettes dictées par le " maitre de l'univers lui-même pour écarter des dieux et des hommes toute espèce de maladie. "

" Il a été pris de compassion Râ et il a dit²: "Je le protège contre ses ennemis; son guide est Thot, qui lui a donné la parole; il inspire les livres, il donne aux amis de l'étude et aux médecins qui le suivent la science de guérir. Qui aime Dieu, Dieu le fera vivre. Je suis un homme qui aime Dieu, Dieu me fera vivre. "

Puis vient une incantation, qui, prononcée pendant la préparation des médicaments, devait assurer la guérison du malade :

“Qu'Isis me guérisse ${ }^{3}$, comme elle guérit Horus du mal

H. Joachim, Papyros Ebers. Das älteste Buch über Heillunde, aus dem Aegyptischen übersetzt. Berlin, 1890, in-8, p. x.

1. G. Ebers, Das hermetische Buch, p. 5. Une origine semblable est attribuée au Papyrus de Berlin et une encore plus mystérieuse à celui du British Museum.

2. Papyros Ebers, p. I, 1. 7-10.

3. Papyros Ebers, p. I, 1. 12-20. 
quil souffrit, quand Sit tua son père ! O Isis, grande magicienne, délivre-moi ; affranchis-moi de toutes les choses mauvaises, funcstes, infernales, du Dicu et de la déesse des maladies meurtrières, ainsi que des impuretés de toutc cspèce, qui fondent sur moi, comme tu as délivré, comme tu as affranchi ton fils Horus.... Que Rà et Osiris me délivrent de toute chose mauvaise, funeste, infernalc, du Dieu ct de la déesse du mal. »

Après ce préambule, commence le "Livre des médecines ", contenant l'énumération des remèdes appropriés aux diverses maladies dont peuvent être atteintes les différentes parties du corps humain ${ }^{1}$ : remèdes pour les maladies des intestins, du foie, de l'estomac ou de la vessie, contre les vers intestinaux et pour les maux de tête, les nausées, les maladies des yeux, les ulcères, blessures ou piqûres, les maladies de la langue, du nez et des oreilles, des dents ou de la chevelure, enfin pour les maladies des femmes, $y$ sont longuement indiqués. Il y en a même pour délivrer de la vermine et des insectes si redoutables dans les pays chauds.

La préparation des médicaments avait été portée en Égypte à un haut degré de perfection; elle était entre les mains d'une classe particulière de prêtres, qui tenaient leurs procédés secrets; les diverses recettes pharmaceutiques qui ont déjà été publiées, en particulier celles des Papyrus Ebers et de Berlin, nous montrent combien leurs procédés étaient ingénieux et quelle habileté ils y déployaient ${ }^{2}$. Les ingré-

1. G. Ebers, Das hermetische Buch, p. 24-26.

2. H.-L. Lüring, Die über die medicinischen Kenntnisse der alten Aegypler berichlenden Papyri verglichen mil den medicinischen Schriften griechischer und römischer Autoren. Leipzig, 1888, in-8, p. 8. 
dients des médicaments composés étaient soit purement mélangés entre eux, soit bouillis ensemble, parfois après avoir été préalablement écrasés. Le mode de cuisson était lui-même réglé avec un soin religieux; dans certains cas, on devait se servir de bois ou de charbon d'acacia, dans d'autres, le feu était fait arec du bois de sycomore. Après la cuisson, les médicaments solides étaient pressés, puis filtrés à travers un tamis. Les remèdes étaient d'ailleurs pris comme aujourd'hui, soit en pilules ou en poudres, soit en décoctions ou en potions, le matin, le soir ou à des heures fixes. Du vin de palmier, du miel ou de la bière douce servaient à édulcorer ceux qui avaient un goût désagréable. A l'extérieur, on faisait usage de cataplasmes, d'emplâtres, de collyres, de frictions et de pessaires. Les médecins égyptiens employaient aussi les inhalations, les clystères et les instillations de liquides dans les organes ${ }^{1}$. Enfin, on prononçait, en administrant les remèdes, diverses formules magiques, destinées ì en augmenter l'efficacité ${ }^{2}$.

Les divers ingrédients dont la pharmacopée pharaonique se servait - on en compte plus de sept cents - étaient empruntés au règne minéral, comme au règne animal, mais surtout au règne régétal. Les médecins de l'Égypte ancienne avaient une connaissance étendue, non seulement des plantes de leur propre pays, mais encore des plantes de toutes les contrées avec lesquelles les sujets des Pharaons entrèrent en relation ${ }^{3}$; on rencontre dans leurs

1. Lüring, op. laud., p. 165-170.

2. Lüring, op. laud., p. 57.

3. G. Ebers, Ein Kyphirecept aus dem Papyrus Ebers. (Zeilschrift für aegyptische Sprache, t. XII (an. 1874), p. 106.) 
recettes des produits végétaux de la Syrie, de la côte orientale d'Afrique, de l'Arabie et même de l'Inde. Mais, de quelque nature que fussent ces produits, ils étaient rarement employés seuls; les médicaments égyptiens étaient de nature essentiellement complexes ; on comptait dans la plupart d'entre eux jusqu'à dix à douze substances différentes ${ }^{1}$; à cet égard, la pharmacopée pharaonique a servi de modèle à celle des Grecs et a devancé celle des Arabes.

Il est très difficile d'identifier les divers végétaux, qui, par eux-mêmes ou par leurs produits, entraient dans la composition des remèdes usités dans l'Égypte pharaonique; les signes hiéroglyphiques qui les représentent sont loin d'être tous expliqués et les noms égyptiens qu'on rencontre dans Dioscoride, parfois aussi dans Pline et dans Apulée, ne peuvent que bien exceptionnellement prétendre à l'authenticité ${ }^{2}$. Aussi ne connait-on qu'une faible partie des plantes qui entraient dans la pharmacopée des Égyptiens. M. Franz Woenig n'en cite qu'une dizaine ${ }^{3}$, et sur ce petit nombre il y en a plusieurs qu'il ne mentionne que sur le témoignage douteux des écrivains grecs ou latins; M. Ludwig Stern n'en a pas identifié d'une manière certaine plus de douze à quinze "; le traducteur allemand du Papyrus Ebers, M. Joachim, en a nommé un plus grand nombre ${ }^{\ddot{\prime}}$, mais ses déterminations, bien souvent aventurées, ne peuvent entrer en ligne de

1. Lüring, op. laud., p. 56.

2. Lüring, op. laud., p. 143.

3. Die Pflarsen im alten Aegyplen, p. 392-396.

4. Glossarium hieroglyphicum, t. II, p. 1-63 du Papyros Ebers.

5. Papyros Ebers, p. 196-207. 
compte; M. Lüring, à côté de quelques-unes qu'il n'a point cherché à identifier, en énumère une trentaine qu'il donne comme connues', mais sur ce nombre plusieurs encore sont contestables ou non suffisarnment déterminées. On voit combien la solution du problème est peu avancée.

Les principaux produits d'origine végétale étaient les huiles de lin, de ricin, de carthame, de sésame et de noix de ben, les liqueurs fermentées: bière, vin de raisin et vin de palmier, les fruits du sycomore et du figuier commun, du dattier, du sébestier, du caroubier, du jujubier, les raisins, les pastèques, les baies du genévrier et peut-être du Cocculus leaeba ${ }^{2}$, les graines de coriandre, d'anis, de sésame, de cumin, de lin, le blé; l'écorce du grenadier, ainsi que la gomme de l'acacia, la myrrhe et l'encens même. Les feuilles, les tiges ou les racines d'autres plantes: oignon, menthe poivrée, sauge d'Égypte, aneth, fenouil, laitue, ache, indigo, etc., servaient à faire des décoctions ou entraient dans la préparation de divers médicaments composés ${ }^{3}$. D’autres plantes n'étaient pas employées comme remèdes, mais comme

1. Die medicinischen Kenntnisse, p. 143-164.

2. "Il n'y a pas de doute, dit Schweinfurth, que cette plante (dont l'écorce et la graine renferment un principe amer narcotique) jouait un ròle important dans la pharmacopée des anciens Égyptiens. "Bulletin de i'Iustitul egyptien, no 7 (an. 1886), p. 421 .

3. M. Joachim mentionne également, dans sa traduction du Papyrus Ebers, l'absinthe, le cèdre, le crocus, le dourah, la jusquiame, la mandragore, le mélilot, le nasturtium, le pavot et la Pistia stratioles. La pharmacopée égypticnne aurait encore employé, d'après M. Lüring, le palmier nain (Chamaerops humilis L.) la coloquinte, les produits du cotonnier, etc.; mais ces désignations n'ont rien de certain. 
moyens magiques', tols les grains de blé et d'orge qu'on faisait tremper dans l'urine d'une femme pour constater si elle était grosse ou non ${ }^{2}$. Germaient-ils, c'étail un signe de grossesse, s’ils ne germaient pas, c'est qu'elle n'était pas enceinte. Si c'était l'orge qui germait, elle était grosse d'un garcon; le froment, au contraire, germait-il, elle devait mettre une fille au monde.

Je n'ai point l'intention de passer en revue toutes les maladies dont les Papyrus Eber's et de Berlin donnent le traitement; je me bornerai à indiquer les remèdes connus d'origine végétale employés dans la. médicamentation de quelques-unes des plus répandues en Égypte. Commençons par les maladies d'estomac et des intestins qui y étaient fort communes. S'agissait-il d'une pléthore de l'estomac, on prescrivait au malade une espèce de bouillie faite avec de l'huile et dans laquelle on avait mis du miel et des baies de genièrre avec du sam et du shasha ${ }^{3}$. Pour un simple embarias gastrique on lui administrait de la farine de dattes déliée arec des graines de ricin dans de la petite bière. Des fruits du Cordia myxa ou sébestier, des figues, des raisins cuits avec de la pàte de froment, de l'en-

1. Il en existait 36 , d'après Pamphile, qui prétendait en avoir vu énumérées les propriétés merveilleuses dans un des livres hermétiques; mais Galien affirme que ce n'était que

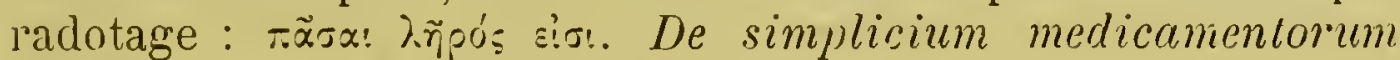
facullalibus, t. XI, p. 798, éd. Kuhn.

2. Papyı'us de Berlin, verso 2, 1. 2-5. - Lüring, op. laud., p. 138. - Le Page-Renouf a rapproché cette recette ėgyptienne de celle qu'on trouve dans l'Experienced midhife et que ce vieil ouvrage attribue à Aristote. Zeilschrift für acgyplische Sprache, t. XI (an. 1873), p. 123.

3. Lüring, op. laud., p. 23. 
cens, des oignons, du vin et de la bière douce servaient à ouvrir l'appétit. La plupart de ces ingrédients, en particulier le vin, la bière, le miel, les figues, les fruits du Cordia myxa et les raisins étaient employés aussi comme tonifiants ${ }^{1}$.

- De nombreux remèdes servaient à combattre les maladies de la vessie; ainsi, dans une recette contre les impuretés de l'urine, on trouve à la fois du miel, de l'encens, des baies de genévrier, des rhizomes de souchet, de l'écorce de sycomore, de la racine de qaqa et de lihazit, des dattes fraîches et de la pâte, le tout mélangé à chaud et passé au tamis ${ }^{2}$. Contre les vers intestinaux, en particulier contre le ténia, maladie fréquente en Égypte, les Papyrus Ebers et de Berlin prescrivent l'écorce de la racine de grenadier, remède encore usité aujourd'hui; on écrasait cette écorce dans de la bière, puis on mettait le résidu avec de l'eau dans un vase et on le laissait reposer jusqu'au lendemain, après quoi on le buvait ${ }^{3}$.

On calmait les fluxions de dents avec des dattes ramollies dans du lait. Des emplàtres de jeunes pousses de ricin, écrasées dans de l'eau, guérissaient "instantanément " du mal de tête, " comme si on n'avait rien eu $"{ }^{4}$. Des frictions à l'huile de ricin étaient prescrites pour les ulcères de mauvaise nature; le latex de sycomore était également préconisé contre le même mal. Un onguent fait avec des graines de tekhou, du miel ou du mastic et du vin servait à guérir les tumeurs des

1. Lüring, op. laud., p. 30 .

2. Lüring, op. laud., p. 26-27.

3. Joachim, Papyros Ebers, p. 11, no 16.

4. Lüring, op. laud., p. 25. Il existait un traité particulier des vertus du ricin - l'arbre teqem. 
jambes'. Contre les éruptions, en particulicr contre le "bouton du Nil" ", on prenait pendant quatre jours un remède composé d'un mélange de lait d'ânesse, d'at, d'acacia du Nil, d'indigo, de fruits de térébinthe et de miel, le tout cuit ensemble et passé au tamis. Sur les cicatrices on mettait des emplâtres dans lesquels entraient entro autres ingrédients végétaux des dattes et des endives. Au nombre des substances qui servaient a la confection d'un onguent employé pour guérir les morsures se trouvent aussi des oignons cuits avec de l'encens et la plante nouter. Parmi les remèdes en usage dans les affections de la langue, il y en avait un composé d'un mélange d'encens, de cumin, de miel et de graisse délayés dans de l'eau ${ }^{3}$. On combattait l'éternuement ou le coryza, en mettant sur le nez un emplâtre fait de menthe poivrée et de dattes pilées.

C'est dans le traitement des maladies d'yeux que la thérapeutique égyptienne paraît avoir déployé le plus d'invention ". Les ophtalmies, conséquence de la nature mème du climat, sont fréquentes dans la vallée du Nil ${ }^{5}$;

1. Pap. médlic., XI, 2, ap. Victor Loret, Études de droguerie égyptienne. Paris, 1894, in-4, p. 21. (Recueil de travaux, t. XVI.) M. V. Loret regarde la plante tekhou comme étant la violette, identification qu'il me parait difficile d'admettre sans hésiter.

2. Furoncle qui atteint les habitants pendant la crue du Nil. Bulletin de l'Institut égyptien, no 10 (an. 1889), p. 350.

3. Lüring, op. laud., p. 35-39.

4. G. Ebers, Das Kapilel über die Augenkrankheiten im Papyrus Eber's. Leipzig, 1889, in-4.

5. G. Haspero, Histoire ancienne, in-12, p. 75. "L'ophtalmie d'Égypte " a été l'objet de nombreux travaux. Cf. J. Hischberg, Aegypten. Geschichtliche Studien eines Augenarztes. Leipzig, 1890 , p. 76 et 94 , qui, tout en croyant à l'ancienneté des inflammations d'yeux en Égypte, ne pense pas que l'ophtalmie endémique remonte à l'époque des l'haraons. 
les médecins du pays s'appliquèrent à les guérir, et l'habileté qu'ils y déployèrent les rendit célèbres dans l'antiquité. Si l'on en juge par le nombre de collyres et les remèdes divers dont ils faisaient usage, cette réputation aurait été méritée. La myrrhe et la plupart "des espèces d'anti ou encens, ainsi que le sounter, que M. Lüring regarde ${ }^{1}$, avec M. Krall, comme une espèce d'encens, mais qui semble bien plutôt avoir été la gomme résine du lentisque ou du pin d'Alep, la sciure de bois d'ébène, les rhizomes charnus et arrondis du souchet $(C \text {. rotundus })^{2}$, le mati - l'ache ${ }^{3}$ - d'autres plantes encore non identifiées, comme l'intérieur du fruit de l'arbre kesb, le suc et les fruits frais du tarl de l'oasis, servaient à combattre les inflammations, conjonctivites, pustules, ainsi que l'affaiblissement de la vue.

Les Égyptiens ne paraissent pas avoir pris moins de soin de leur chevelure que de leurs yeux; la tradition attribuait à la reine Sesha, mère du roi Téti", une recette poul faire pousser les cheveux, preuve de l'antiquité de la médicamentation dont ils étaient l'objet. Un cosmétique célèbre dans l'antiquité hellénique était mis sous le nom de la reine Cléopâtre, dernière souveraine de l'Égypte ${ }^{5}$; mais plusieurs des ingrédients

1. Op. laud., p. 107. Krall, Das Land Punt, iVien, 1890, in-8, p. 27.

2. M. Lüring dit des rhizomes de souchet comestible ( $C y$ perus esculentus $\mathrm{L}$.

3. Avec M. Victor Loret, j'admets que le mot égyptien mati, auquel M. Lüring attribue, après Stern, le nom de crocus, désigne en réalité l'Apium graveolens L. II. Hirschberg, lui, Aegypten, p. 68, a vu dans cette plante le Chelidonium majus L, le rooro de Dioscoride.

4. G. Ebers, Das hermetische Buch, p. 42. Cf. G. Maspero, Revue crilique, 8 avril 1876, t. $\mathrm{X}, 1, \mathrm{p} .233$.

5. Lüring, op. laud., p. 123. 
qui y entraicnt: myrrhe ou encens pour combattre la teigne, ladanum écrasé dans de l'huile el du vin doux pour faire épaissir les cheveux, racine de lotus broyée pour les empècher de tomber, remontent à un passé reculé.

II.

Les dernières recettes dont je viens de parler sont du ressort de la parfumerie, bien plus que de la pharmacopée; il en était de mème des pommades dontles dames égyptiennes se servaient pour entretenir la fraìcheur ou la souplesse de la peau, effacer les rides, des substances avec lesquelles elles se teignaient les sourcils ou les mains, des essences et des huiles dont on faisait usage pour oindre les statues des dieux ou les momies des morts, se parfumer les cheveux et les diverses parties du corps, ainsi que des aromates employés dans les fumigations. C'étaient des ingrédients minéraux surtout qui servaient à conserver la souplesse de la peau ou à teindre les sourcils; les substances régétales: grains d'encens, rhizomes de souchet, écrasés dans du lait, reparaissaient dans la préparation de la pommade employée pour effacer les rides du visage ${ }^{1}$. On a vu qu'un autre produit végétal, l'extrait de feuilles de henné, servait à colorer la peau des mains et des pieds, comme à teindre les cheveux.

Les huiles ou essences parfumées, en usage dans le culte et dans les cérémonies funèbres étaient de nature très diverse; il y en eut d'abord sept, puis huit ou

1. Papylos Eber's, p. 87. Cf. Lüring, op. laud., p. 40. 
neuf, et enfin dix espèces différentes ${ }^{1}$, employées pour " la cérémonie de l'ouverture de la bouche "; mais il y en avait bien d'autres, par exemple l'huile teshep et le " parfum divin ", dont Dümichen a donné les formules compliquées ${ }^{2}$. Au lieu de les reproduire ici, je préfère donner la composition d'un autre parfum, "l'extrait surfin de styrax ", qui vient d'être l'objet d'une étude pénétrante de M. Victor Loret ${ }^{3}$. Cette essence, dont la recette est gravée sur une des parois du temple d'Edfou, se composait de huit ingrédients d'origine végétale, combinés savamment entre eux : suc de caroube, encens de première qualité, écorce de styrax, calame aromatique, aspalathe, mastic, graines de la plante tekhou et vin très alcoolique. Cela peut donner une idée de la composition des essences usitées dans l'Égypte ancienne.

La composition des aromates employés dans les fumigations n'était pas moins savante ou complexe, ni le nombre de ces aromates moins considérable que celui des essences en usage dans les onctions. La grande quantité des gommes-résines, quatorze résines ant et huit résines $a b$, malheurement non identifiées, qui servaientsurtoutà préparer ces substances - ces kyphi*,

1. Dümichen, Der Grabpalast des Paluamenap, p. 12-13 et 28. L'une d'elles, qui porte le nom d'essence baq, était sans doute préparée avec de l'huile de moringa.

2. Die Oasen der libyschen Wüste. Strasburg, 1877, in-4, p. 3-6. - Der Grabpalast des Patuamenap, p. 26. Dümichen a également donné dans ce dernier ouvrage, p. 29, la composition de l'huile parfumée matet et dans la Zeitschrift fïr. aegyplische Sprache, t. XVII (an. 1879), p. 97-128, la formule de l'huile heken.

3. Études de droguerie égyptienne, I, p. 1-25. (Recueil de travaux, t. XVI.)

4. D'un radical kep "fumiger ». Lüring, op. laud., p. 46. 
comme on les appelait - peut déjà domner une idée de leur variété. Celui qui portait le nom de sti-heb, par exemple, contenait huit ingrédients végétaux ${ }^{1}$ : poix, graines de tekhou, colophane, graines d'acacia, encens frais, plantes as et ham. Le kyphi proprement dit était autrement compliqué; il renfermait d'ordinaire le nombre symbolique de seize substances ${ }^{2}$, combinées entre elles avec un soin infini. Deux recettes, gravées sur les murs du laboratoire du temple d'Edfou, ont été publiées et étudiées par Dümichen ${ }^{3}$; en les étudiant à son tour et en les comparant avec celle qui se trouve sur les murs du temple de Philae, M. Victor Loret a pu donner la, formule définitive et complète de ce parfum celèbre ${ }^{4}$.

Les premiers ingrédients qui y entraient étaient le lianen, "roseau odorant " (Acorus calamus L.), le shou-ament, " jonc d'éthiopie ) (Andropugon schonanthus L.), la résine sheb ou fit (Pistacia lentiscus L.), l'écorce de bois de qat (Laurus cassia L.), le tas, " bois odorant" (Laurus cinnamomum L.), l'alcaï (Mentha piperita L.) et le djalma ou djabi, aspalathe ou " bois de rose " (Convolvulus scoparius L.); on pilait dans un mortier ces sept aromates de manière à les réduire en poudre très fine; puis on en séparait la partie la plus ténue, et on ajoutait au reste quatre ingrédients nouveaux, du pershou ou "grains d'ouan " (Juniperus phoenicea L.), du sannar, "graines chevelues " (Acacia seyal Del. ou tortilis Schw.) ${ }^{5}$, du

1. Victor Loret, Études de droguerie, I, p. 21.

2. Plutarque, De Iside et Osiride, cap. 80.

3. Der Grabpalast des Patuamenap, p. 20-25.

4. Le kyphi, parfum sacré des anciens Égyptiens. Paris, 1887, in-8.

5. M. Victor Loret, Le Kyphi, p. 53, suppose qu'il s'agit de 
pouqer (Lawsonia inermis L.) et du shbin ou kaiou, nom, d'après M. Victor Loret, de la racine du Cyperus longus L.; on humectait le mélange avec du vin; on le laissait ensuite reposer jusqu'au matin, " afin qu'il se tasse "; puis, après avoir ajouté des shep, " raisins d'oasis "; et de l'ar-hor, " œil d'Horus-vert », nom mystique du vin, on écrasait le tout ét on le laissait reposer jusqu'au cinquième jour. On mêlait alors à l'espèce de pâte ainsi obtenue de la résine fraìche, sans doute de la térébenthine ${ }^{1}$, et du miel, " œil d'Horus-doux "; puis on mettait le tout dans une marmite et on le faisait cuire jusqu'à un degré convenable d'épaississement. L'ad dition à la masse d'une certaine quantité de myrrhe achevait la préparation du lyphii, " deux fois bon pour l'usage du culte ".

Quand, au lieu d'être employé dans le culte, le kyphi était simplement destiné à parfumer les appartements ou les vêtements, on n'y faisait, d'après le Papyrus Ebers ${ }^{2}$, entrer que dix ingrédients qu'on combinait d'ailleurs comme ceux du kyphi sacré. De ces dix ingrédients, auxquels on ajoutait du miel, on faisait des pastilles destinées aux fumigations; on les màchait aussi pour donner à l'haleine une odeur agréable ${ }^{3}$. Le kyphi décrit par Dioscoride ne contenait aussi que dix ingrédients ; mais Galien, comme Plutarque, en attribue seize à ce parfum ${ }^{4}$.

l'A. Farnesiana Wild.; mais cette espèce, étant originaire d'Amérique, n'a pu entrer avant notre ère dans la composition d'un parfum égyptien. Cf. plus haut, p. 145.

1. TEqu:v0:

2. P. 98. Cf. Lüring, op. laud., p. 48.

3. G. Ebers, Ein Kuphirecept. (Zeilschrift fïr aegyptische Sproche, t. XII (an. 1874), p. 109.

4. Plus tard, ce nombre augmenta; Paul Éginète connais- 
Les formules des huiles parfumées et des aromates destinés au culte étaient fixées par la tradition religieuse; elles étaient inscrites sur les parois des laboratoires sacrés, où ces parfums se préparaient. Une inscription de Dendérah, après avoir dit que son labora toire " était pourvu des produits du pays de Pount", de tout ce qui venait du pays des Fekker ${ }^{2}$ et de ce qui se rencontrait de précieux dans la Terre divine, » ajoute "qu'on y trouvait entassées, comme le sable [du désert], les résines hat et nehel, qu'on y voyait mille plantes aux douces senteurs, la résine $a b$ à son état natif et telle que la fournit son pays d'origine, les résines t'ser et aham en quantité incommensurable, avec l'huile aber et le mystérieux composé hekermu ". La plupart de ces aromates étaient étrangers à la terre de Qimit; je n'ai donc pu en parler, quand j’ai passé en revue les arbres et les plantes utiles de l'Égypte; je n'en parlerai pas davantage ici; je remets à faire connaître ceux qu'il a été possible d'identifier: encens, myrrhe, calame aromatique, aspalathe, mastic, térébenthine, cassie, cinnamome, etc., aux chapitres ou je traiterai de la flore des divers pays qui les produisent. Mais avant de finir celui-ci, je dois dire encore quelques mots de l'emploi des aromates dans les embaumements.

sait déjà un kyphi composé de vingt-huit ingrédients; Nicolas Myrepsus, au Xin ${ }^{\circ}$ siècle, en mentionne un dans lequel entraient cinquante substances diverses. G. Parthey, Ueber Isis und Osiris. Berlin, 1850, in-8, p. 278.

1. Dümichen, qui a identifié le Pount avec l'Arabie, traduit naturellement par le nom de cette contrée. Der Grabpalast, p. 28.

2. Un des pays d'où les F́gyptiens tiraient les aromates. 
III.

Aucun peuple de l'antiquité n'a donné aux funérailles une importance comparable à celles qu'elles prenaient chez les Égyptiens. Avant que le défunt fût conduit solennellement à sa demeure dernière et que son corps fût déposé dans le mastaba ou le sépulcre destiné à le recevoir, de longues pratiques devaient assurer la conservation de sa dépouille mortelle; leur ensemble constituait l'embaumement dont les écrivains de l'antiquité et les textes démotiques nous font connaitre les diverses phases.

Aussitôt après la mort, le corps du défunt était livré aux paraschistes ou taricheutes ${ }^{1}$; ils commençaient par faire sortir à l'aide d'un crochet en fer la cervelle à travers les marines et ils y substituaient des substances antiseptiques ${ }^{2}$. L'un d'eux faisait alors avec une pierre d'Éthiopie une incision dans le côté, et, par cette ouverture, retirait les intestins, à l'exception des reins et du cour ${ }^{3}$; on lavait ensuite la cavité abdominale avec du vin de palme et d'autres substances aromatiques; puis on déposait le corps, d'après Hérodote, dans un bain de natron où il restait trente-cinq jours. C'était là la première phase de la momification. La seconde, mèlée de prières et de libations ou d'onctions sacrées et complétée par l'emmaillottement de la mo-

1. Eug. Revillout, Une famille de paraschisles ou laricheules thébains. (Zeilschrift. fïr aegyplische Sprache, t. XVII (an. 1879), p. 8't.)

2. Hérodote, Hisloriae, lib. II, cap. 86.

3. Diodore, Bibliollece, lib. I, cap. 91. 
mie, était surtout l'aurre des coachytes ' ; mais comme la première, on feignait qu'elle fût accomplie par les Dieux :

"Isis la grande, mère des Dieux, arrive, dit un texte démotique ${ }^{2}$, pour ton embaumement... Tu as été oint de l'huile basli par les mains d'Horus, seigneur du as (laboratoire) et de l'enveloppe de sechem par ses doigts. Le vêtement d'étoffe de byssus, destiné à ton liesau (emmaillottement), est comme les vêtements des Dieux et des Déesses. C'est Anubis qui, comme kiherheb (paraschiste), a rempli ta tète de sel, de résine syrienne, de poix. Ta chair est liuilée. Elle est entourée d'étolfes magnifiques, pour que tu puisses apparaître à l'horizon et adorer le soleil dans son disque. »

La cérémonie de l'onction et de l'emmaillottement des diverses parties du corps est décrite longueinent dans le Papyrus 558 du Louvre et le Papyrus $n^{\circ} 3$ de Boulaq ${ }^{3}$; chacun des actes qui la constituaient ètait accompagné de prières et de conjurations:

"Le voici pour toi le parfum d'Arabie, disait le « ministre divin " en oignant la tête". O Osiris, tu as reçu un parfum de fête qui rend tes membres parfaits... Te voilà oint... Elle vient à toi l'huile pour huiler tes membres... Elle vient à toi la résine de Phénicie, la poix de Byblos; elles rendent parfait ton ensevelissement... Elles viennent à toi, les plantes vertes sorties de la terre, les guirlandes des prés d'Ialou, les herbages excellents des champs de $H^{‘}$ ââ. »

Après avoir oint la tête du défunt, on l'enveloppait de bandelettes, puis on " faisait dessous un semis de

1. Eug. Revillout, Taricheules el choachyles. (Zeitschrift, t. XVIII (an. 1880), p. 78.)

2. Papylus Rhind. (Zeilschrift, t. XVII (an. 1879), p. 91.)

3. G. Maspero, Mémoire sur quelques Papyrus du Lounre. II. Riluel de l'em'saumement. (Nolices el extrails des manuscrits, t. XXIV, p. 14-51.)

4. G. Maspero, Riluel de l'embaumement, p. 18-21. 
grains de myrrhe et de résine ». On procédait ensuite à l'embaumement et à l'emmaillottement des nains et des doigts, puis des cuisses, des jambes et des pieds, sur lesquels on mettait des fleurs ankhimu, du natron et de la résine. Chacme de ces diverses opérations sacrées ètait accompagnée de nouvelles invocations. L'embaumement n'était définitivement terniné qu'au bout de soixante-dix jours; alor's seulement la momification était considérée comme complète, et le corps, dont la conservation était désormais assurée par tant d'onctions saintes, était enfin placé avec des amulettes, sur lesquelles étaient gravées des formules magiques ${ }^{1}$, et des fleurs dans le cercueil préparé pour lui.

Les substances végétales employées dans la longue cérémonie de l'embaumement sont loin d'être toutes connues; les huiles employées pour les onctions étaient sans doute quelques-uns des parfums préparés dans le laboratoire des temples et dont j'ai parlé plus haut; on peut l'affirmer de " l'huile sainte ", et de "l'huile détachée des choses divines ». M. Maspero" mentionne l'huile de cèdre et l'huile extraite d'olivier, mais celle-ci me paraît devoir ètre plutôt celle de baq (Moringa aptera $)^{3}$. De quelle espèce aussi était la poix renue de Byblos, la résine apportée de Phénicie et les dix parfums, dunt on enduisait tout le corps a l'exception de la tête? On l'ignore, mais dans les fleurs ankhamu, M. Victor Loret voit des fleurs d'acacia, qu'on mettait, d'après le Rituel de l'embaumement,

1. G. Maspero, Le chapilie de la boucle, (Nolices el extraits, t. XXIV, p. 1.

2. Le Riluel de l'embaumemenl, p. 5'́ .

3. Victor Loret, L'Olivier el le Moringa. (Recueil de travaux, t. VII, p. 10'.) 
avec du natron el des grains de résine à l'extrémité des jambes, en les fixant au moyen d'eau de gomme d'ébénier. La gomme de cet arbre exotique figurait dans l'embaumement ${ }^{1}$, comme son écorce macérée, dans la pharmacopée. Il est probable qu'on employait également dans la momification la résine de cèdre, ainsi peut-ètre que celle du pin d'Alep ${ }^{2}$; on a trouvé du moins de la sciure de bois de cèdre dans une momie du Musée de Berlin ${ }^{3}$, comme on a cru reconnaitre des fragments de bois de santal mélangés à du natron dans une autre". On faisait d'ailleurs avec de la résine de cèdre et du naphte un vernis jaunatre qui serrait à conserver les peintures des sarcophages.

1. L'ébine ches les anciens Égyptions. (Recucil de travaux, t. VI, p. 129.)

2. Victor Loret, La Flore pharaonique, no 5's, p. 43.

3. Franz Woenig, op. laud., p. 387.

4. Calalogue de Passalacqua, p. 286. 



\section{LIVRE SEGOND}

\section{LES PLANTES CHEZ LES SÉMITES.}

\section{CHAPITRE PREMIER}

\section{LA FLORE DE L'ASIE ANTÉRIEURE.}

La vaste contrée, qui s'ëtend de l'Indus à la Méditerranée, se divise en trois régions naturelles: c'est, à l'est, le plateau de l'Iran, compris entre les montagnes qui forment la limite occidentale du bassin de l'Indus et celles qui servent de frontière orientale au bassin du Tigre, l'Océan indien et la chaîne des hauteurs qui courent de l'Hindou-Koush - l'ancien Paropamise - à l'Elbourz au sud de la Caspienne. Au centre, la région comprise entre la chaîne bordière du Zagros à l'est, le Taurus et les monts qui bornent les bassins de l'Oronte et du Jourdain à l'ouest; elle comprend le double bassin du Tigre et de l'Euphrate - la Mésopotamie, - séparé de celui de l'Oronte et du Jourdain par le désert de Syrie, prolongement septentrional de l'Arabie, immense presqu'île, que bornent des trois autres còtés le golfe Persique, l'Océan indien ct la mer Rouge. Enfin l'Asie Mineure, autre presqu'ile moins 
vaste que l'Arabie, mais non sans analogie avec elle, limitée à l'est par la chaîne de l'Amamus et du Taurus, qui la séparent de la Syrie et du massif arménien, au nord par le Pont-Euxin, à l'ouest par la Propontide et la mer Égée et au sud par la Méditerranée.

S'étendant du $24^{\circ}$ au $70^{\circ}$ degré de longitude orientale et du $12^{\circ}$ au $42^{\circ}$ degré de latitude septentrionale, l'Asie antérieure présente les différences les plus grandes dans son climat et sa constitution géologique; elle n'en offre pas de moins considérables dans sa flore. Sillonnée ici par des montagnes, dont beaucoup dépassent en hauteur la limite des neiges éternelles et renferment des vallées profondes et bien arrosées, couvertes là de plateaux élevés ou de déserts sablonneux et arides, ailleurs de plaines basses et brûlantes, on y trouve à la fois les plantes des steppes ou du Sahara, la végétation forestière des hauteurs alpestres, ainsi que celle des plaines cultivées et des grasses alluvions; néanmoins, malgré la diversité des espèces végétales qui les représentent, les flores de ces diverses contrées offrent des traits communs qui permet. tent de les réunir en une seule, la "Flore de l'Orient ${ }^{2}$ ", caractérisée par certaines formes typiques de la région des steppes, comme les 74 espèces du genre Acantholimon, et la presque totalité des 760 espèces du genre Astragalus ${ }^{3}$.

Toutefois la végétation change singulièrement quand

1. H. Kiepert, Lehrbuch der alten Geographie. Berlin, 1878, in-8, p. $17-49$.

2. Edm. Boissier, Flora orientalis. Genevæ, 1867-1882, 5 vol. in -8 .

3. Oscar Drude, Die Florenreiche der Erde. (Pelcrmann's Millhcilungen, an. 1884, Ergänzungsheft, no 73, p. 57.) 
on descend du plateau de l'Asie Nineure ou des hauteur's de l'Arménie sur les côtes de la Caspiemne ou du Pont-Euxin, de la mer Égée ou de celle de Chypre, ou bien qu'on s'avance dans la plaine de la Mésopotamie ou dans les déserts de la Syrie et de l'Arabie. Sur les còtes du Pont et de la Caspienne la végétation se rapproche de celle de l'Europe centrale, en même temps que de la région méditerranéenne; c'est la flore de cette dernière région que l'on rencontre, cela se comprend, sur les cótes de la mer Égée et de la mer de Chypre, en partie aussi "dans la Syrie occidentale. Au sud et au sudouest de cette contrée, au contraire, ainsi que dans l'Arabie septentrionale et centrale, apparait une autre flore, celle du Sahara, laquelle fait place à son tour; sur les côtes méridionales de la Péninsule, à la flore semi-tropicale de l'Yémen et de l'Hadramaout. Mais mème dans la région des steppes, qui s'étend de l'extrémité occidentale de l'Anatolie à la frontière orientale du plateau iranien, la végétation varie singulièrement, lorsqu'on passe d'une contrée à une autre; la flore de chacune de celles dont la réunion compose l'Asie antérieure: Anatolie et Arménie, Syrie, Arabie et Mésopotamie - je laisse pour le moment de còté l'Iran — doit par suite être étudiée à part.

\section{I.}

Limitée à l'est par l'Anti-Taurus, qui la rattache au massif arménien, l'Anatolie ou Asie Mineure est un vaste plateau d'une hauteur moyenne de 1,000 mètres',

1. P. de Tchihatchef, Une page sur l'Orient. L'Asie Mineure. Paris, 1868 , in-12, p. 37. 
incliné vers le Pont-Euxin, qui le borne au nord, ouvert à l'ouest par de nombreuses vallées sur la mer Égée et séparé, au sud, de la mer Méditerranée par la chaìne du Taurus, doṇt le pic culminant s'élève à près de·3,500 mètres. Sillonnée par des montagnes secondaires, qui limitent les bassins de l'Halys, du Sangharios, de l'Hermos et du Méandre, ses principaux cours d'eau, l'Asie Mineure offre à la végétation les conditions les plus variées: aussi sa flore est.elle une des plus riches que l'on connaisse; en 1866, le voyageur russe de Tchihatchef y comptait 6,800 espèces ${ }^{1}$, dont plus de 2,000 exclusivement orientales. Ces plantes sont d'ailleurs très diversement répandues sur le territoire de l'Anatolie.

Le plateau central de cette presqu'ile, avec ses hivers rigoureux et ses étés secs et brûlants, qui arrétent le développement de la végétation forestière, est aussi dépourvu d'arbres qu'abondamment courert de plantes vivaces, le plus souvent épineuses, qui caractérisent la flore des steppes: caryophyllées, légumineuses, composées, plumbaginées, salsolacées ${ }^{2}$. Elles s'étagent sur les flancs des montagnes en touffes gazonnantes, d'autant plus épaisses qu'on s'élève plus haut et mêlées alors d'arbustes: nerpruns, cytiṣes, épines, sorbiers, troènes, buis, genévriers et d'autres espèces, dont quelques-unes atteignent parfois aux proportions d'arbres véritables, comme le Pistacia mulica et le Juniperus excelsa.

Mais c'est surtout dans les montagnes élerées, qui

1. Asie Mineure. Description physique, statistique el archiologique de celle contrée. $3^{*}$ partie, botanique. Paris, 1860, in-8, préf., p. Xvi.

2. Edm. Boissier, Flora arientalis, t. I, p. 9 de la préface. 
forment la ceinture du plateau anatolien ou qui en bornent les divers bassins, que la végétation prend un caractère arborescent; les forêts y ont dù être immenses dans les temps anciens et sur bien des points les grands arbres abondent encore: chênes au feuillage toujours rert ou caduc ${ }^{1}$, charmes, saules, peupliers, érables, frènes, aulnes, bouleaux, bien d'autres encore. Les conifères n'y comptent pas de moins nombreux représentants; pins, sapins, piceas, genévriers, ifs, s'y rencontrent sur les points les plus divers. La " mer d'arbres ", qui s'étend à l'est de l'ancien Sangharios dans les montagnes de Boli ${ }^{2}$, peut donner une idée de ce qu'étaient les forèts de l'Asie Mineure, arant les déboisements qui l'ont dévastée depuis l'époque romaine.

Le massif arménien, qui se dresse, véritable "îlemontagne ", entre l'Asie Mineure et le plateau de l'Iran, à une hauteur moyenne de 1,500 mètres, appartient tout entier avec son climat extrême à la région des steppes; les hivers sont aussi froids que les étés brùlants dans ses vallées abruptes; mais la végétation s'y déreloppe arec une singulière intensité, aussitôt après la fonte des neiges ${ }^{3}$, et les montagnes, aujourd'hui dénudées, étaient autrefois couvertes d'épaisses forêts. Là où les arbres n'ont pas encore entièrement

1. Tandis que la France ne possède que douze espèces de chênes, l'Anatolie en renferme avec l'Arménie vingt-deux espèces d'après Boissier, quarante-neuf d'après 'T'chihatchef, donit huit lui sont particulières.

2. Élisée Reclus, Nouvelle géographie universelle, t. IX, p. 528. La zone boisée s'étend, sur une profondeur de vingt à trente lieues, tout le long de la mer Noire, de l'Ida phrygien aux montagnes du Lazistan. G. Perrot, Souvenirs d'un voyage en Asie Mineure. Paris, 1864, in-8, p. 218.

3. A. Grisebach, Die Vegetation der Erde, 2e éd. Leipzig, $188^{4}$, in 8 , t. I, p. 396. 
disparu, on retrouve les essences de l'Anatolie. C'est aussi sa végétation herbacée qu'on rencontre presque exclusivement dans les parties basses de l'Arménie; mais les choses changent quand on descend des hauteurs du plateau sur les bords orientaux du PontEuxin ou les côtes méridionales de la Caspienne.

Protégées par le Caucase contre les vents des steppes de la Russie et du Turkestan, arrosées par les pluies qu'amènent les courants qui traversent la mer Noire, la côte pontique et la vallée du Phase, en particulier, sont couvertes d'une végétation forestière, qui rappelle, par quelques-unes de ses espèces, celle de l'Europe centrale. La région qui s'étend au pied du Caucase oriental et de l'Elbourz, quoique moins abritée contre les vents du nord, n'est pas moins favorisée au point de vue de la végétation; les vapeurs, qui s'élèvent sans cesse de la Caspienne, se précipitent pendant l'été en pluie sur les flancs des montagnes, et y entretiennent durant l'hiver une température élevée ${ }^{1}$; grâce à ces conditions climatériques, le Mazandéran, le Ghilan et le Lenkoran jouissent à la fois, suivant la remarque de Grisebach ${ }^{2}$, des étés de l'Andalousie et des hivers de l'Irlande, et leur climat doux et égal convient à la fois aux produits de l'Europe centrale et à ceux de l'Asie Mineure. C'est ainsi qu'à còté d'espèces qui leur sont propres, comme les chènes d'Arménie et du Pont, l'érable “ insigne » et le curieux Ptcrocarya caucasica ${ }^{3}$, on rencontre, dans ces provinces

1. II. Binder, Au Kurdislan, en ILésopolamie el en Perse. Paris, 1887, in-8, p. 484.

2. Opus laud., t. I, p. 393.

3. G. Radde, Die Fauna und Flora des suduesllichen CaspiGebiets. Leipzig, 1886, p. 410. 
et dans l'Imérétic of le Lazistan, les arbres de l'Anatolie el de l'Europe tempérée, tel que le hètre, qui atteint sa limite orientale dans le Mazandéran '. La végétation alpestre de ces contrées offre encore dautres formes de la flore européenne, les Rhododendrons ${ }^{2}$ et les myrtilles.

En mème temps que ces plantes de l'Europe centrale ou du plateau anatolien, apparaissent sur les cotes orientales du Pont ou dans les provinces voisines de la Caspienne méridionale quelques-uns des représentants de la flore méditerranéenne, que l'humidité persistante de cette région n'empêche pas de se développer, tol que le micocoulier et le laurier-cerise. C'est cette flore que nous rencontrons exclusivement sur la còte occidentale et méridionale de l'Asie Mineure; le climat teimpéré par le voisinage de la mer, protégé, surtorit au sud, contre les vents du nord, avec ses étés secs et ses hivers cléments et humides, offre toutes les conditions qu'elle réclame; la végétation perd le caractère de la flore des steppes qu'elle avait sur le plateau anatolien; les arbres ou arbustes au feuillage persistant se multiplient à mesure qu'on se rapproche du littoral ${ }^{3}$; cistes, myrtes, arbousiers, genêts, lentisques, pistachiers, lauriers, oléandres, bruyères, chênes verts, pin d'Alep et pin maritime, pin pignon, genévriers de Phénicie et "fétide, » cyprès, enfin, sur les croupes du Taurus, où il forme de véritables forêts, le cèdre du Liban.

1. Drude, Handbuch der Pflanzengeographie. Stuttgart, 1890, in-8, p. 402.

2. Rhodendron caucasisum, ponticum et flavum (Azalea pontica).

3. Grisebach, op. luud., t. I, p. 271-30't. 
A ces arbustes et arbres, qui embellissent de leur éternelle verdure les paysages méditerranéens, se mèlent les plantes herbacées, qui, au printemps, émaillent de leurs fleurs les champs et les prairies ${ }^{1}$; renoncules, malvacées, géraniacées, légumineuses, composées, labiées, iridacées, liliacées surtout, réjouissent la vue de leurs couleurs variées, en même temps que de gracieux arbrisseaux la charment de leur port élégant; j'en ai déjà cité quelques-uns; il faut. ajouter comme caractéristiques de la région, le vitex agnus castus, qu'on rencontre sur tout le littoral anatolien, l'aliboufier (Styrax officinalis L.), dont la gomme-résine odoriférante était si recherchée dans l'antiquité, des chèrrefeuilles aux fleurs parfumées, etc. Quelques-uns de ces arbustes s'élèvent plus ou moins haut dans la montagne et atteignent aux dimensions d'arbres véritables; tel le Liquidambar orientalis. ${ }^{2}$, dont l'écorce exsude le storax liquide. On le rencontre dans le Taurus cilicien en compagnie du platane oriental, parfois à une hauteur considérable, ainsi que le pin de Cilicie, le chène à feuilles de chàtaignier, le chêne du Liban, le frêne de Syrie et d'autres essences arborescentes.

Comme sur le Taurus, le chêne du Liban, son nom l'indique, croit sur. les montagnes de la Syrie; ce n'est pas la seule plante du bassin de la Méditerranée qu'on rencontre dans cette contrée; la flore de sa

1. Grisebach, op. laud., t. I, p. 310.

2. Grisebach, op. laud., t. I, p. 362. 
région occidentale offre le plus grand rapport avec celle toute méditcrranéenne de la Cilicie, tandis que dans la partie méridionale on rencontre déjà les produits du Sahara'. La constitution géologique et la situation géngraphique de la Syrie explique sans peine la diversité que présente sa végétation; s'étendant sur une longueur de plus de six degrés, du mont Amamus, qui la sépare de l'Anatolie, jusqu'à la presqu'ile du Sinaï, sillonnée de montagnes, dont plusieurs conservent la neige pendant une grande partie de l'année, entrecoupée de vallées profondes et bien arrosées, bornée enfin à l'est et an sud par le désert, la végétation de cette contrée offre nécessairement les contrastes les plus grands. Sur les côtes de la mer et sur les premières pentes des montagnes qui la dominent, on rencontre la plupart des plantes de la flore méditerranéenne. Ces mèmes végétaux se retrouvent en partie, arec quelques autres, propres à cette région, dans le bassin de l'Oronte et dans la fertile vallée que laissent entre eux le Liban et l'Antiliban - la Coelé-Syrie ainsi que dans la vallée de la Nazana, qui s'étend entre le Liban et l'Hermon, dans la Syrie-Damascène, située à l'est de cette dernière montagne, et dans la haute vallée du Jourdain. Mais à mesure que l'on descend dans la gorge étroite où s'enfonce ce fleuve et qu'on s'approche de la dépression an fond de laquelle s'étend la mer Morte, la végétation prend un caractère différent ${ }^{2}$. Il en est de même encore plus quand on s'avance

1. W. H. Groser, Scripture natural history. The trees and plants mentioned in the Bible. London, 1888, in-8, p. 4-19.

2. H.-B. Tristram, The Fauna and Flora of Palesline. Préface, p. XIr. (The survey of western Palesline. London, 1884, in-4.) 
dans le pays d'Ammon ou de Moab, ou qu'on pénètre dans la presqu'ile du Sinaï; ce sont alors les plantes de la flore saharienne que l'on rencontre presque exclusivement. On retrouve, au contraire, sur les hauteurs du Liban, du Carmel et de l'Hermon la végétation forestière de l'Asie Mineure et de l'Europe centrale, mêlée de quelques espèces particulières à la Syrie.

M. Tristram compte dans la flore de la seule Pales. tine plus de 3,000 espèces; si quelques familles de plantes de la flore anatolienne manquent dans cette longue liste, les plus importantes y sont représentées et souvent par leurs espèces les plus belles ou des espèces nouvelles ${ }^{1}$. Les vallées et les coteaux offrent et devaient encore plus autrefois offrir au printemps le spectacle le plus charmant, alors qu'elles sont émaillées des milliers de fleurs qu'on y rencontre: anémones, renoncules, nigelles, pieds d'alouette, hélianthèmes, œillets, silénés, malvacées, géraniums, coronilles, trigonelles, gesses, achillées, armoises ${ }^{2}$, centaurées, campanules et convolvulus, solanées, en particulier la curieuse mandragore, héliotropes, véroniques, thyms et lavandes, sauges, phlomis, arums, orchis, Pancratium, colchiques, fritillaires, crocus et tulipes, ornithogales, hyacinthes et asphodèles ${ }^{3}$.

S'il est autre, le spectacle n'est pas moins séduisant quand on gravit les pentes des collines et des mon-

1. Des soixante-quatorze espèces d'astragales que Tristram mentionne dans sa Flore, plus du quart sont particulières à la Palestine.

2. Arlemisia herba albr, arborescens, monosperma, chritmifolia, etc.

3. G. Diener, Libanon. Grundlinien der physischen Geographie und Geologie von Millelsyrien. Wien, 1886, in-8, p. 175. 
tagnes, les arbustes verts qu'on rencontre déjà dans la plaine deviemnent plus nombreux et bientôt se mèlent aux grands arbres, qui les remplacent presque seuls sur les dernières hauteurs: thymélées - l'hirsute et la tartomraire - myrtes du littoral, lauriers-roses et tamaris, gattiliers ct roseaux gigantesques aux bords des eaux' ${ }^{1}$, berberis, cistes, lentisques, pistachiers de Palestine - le térébinthe des auteurs ${ }^{2}$ - jujubiers, dictamnes, genêts d'Espagne, d'Orient et du Liban, cytises de Syrie, arbres de Judée, églantiers, amandiers, cerisiers nains, poiriers de Syrie, sorbiers, néfliers, azarolier's, chèvrefenilles arborescents ${ }^{3}$, aliboufiers, lauriers, chalefs, et à côté, ou plus haut dans la montagne, érables, ornes, frènes, micocouliers, saules, peupliers blancs et noirs avec le peuplier de l'Euphrate, chênes verts, kermès et faux-kermès, tinctorial ou de Lusitanie, cerris, aegilops et du Liban, rouvres", cyprès, genévriers, pins pignon et pins d'Alep, pins des Pyrénées et enfin le cèdre, dont il ne reste aujourd'hui que quelques groupes isolés, mais qui, dans les anciens temps, couvrait toutes les croupes neigeuses du Liban, végétation arborescente à laquelle se mêlent des astragales épineuses et des ombellifères frutes-

1. Victor Guérin, La Terre-Sainle. Son hisloire, ses souvenirs, ses siles, ses monuments. Paris, 1882-188', in-fol., t. I, p. 192 et 361 .

2. Tristram, op. laud., p. 263.

3. I. Lortet, (La Syrie d'aujourd'hui. Voyages dans la Phinicie, le Liban el la Judée (1875-1880). Paris, 1884, in-4) mentionne entre autres, p. 631, dans la région du mont Thabor, le Lonicera nummularifolia, ainsi que le Cralaegus orientalis et le Berberis cretica.

4. Frédéric Hamilton, La Bolanique de la Bible. Nice, 1871, in-8, p. 28, no 6. - Boissier, Flor'a, t. IV, p. 1164-1173. 
centes, et qui semblait "obscurcir le ciel » aux yeux des Égyptiens étonnés ${ }^{1}$.

Une flore différente, celle du Sahara, s'offre au voyageur sur les bords de la mer Morte et sur les plateaux brûlés qui l'environnent, ainsi que dans la plaine aride qui s'étend entre la Syrie et l'Égypte; crucifères du désert ou des coteaux stériles, telles que l'Anastatica hierochuntina, la Mathiola oxyceras; malvacées, comme l'abutilon frutescent et mutique; géraniacées hirsutes, par exemple l'Erodium hirtum ; zygophyllacées, tels que le Zygophyllum dumosum² et diverses Fagonia; le faux-lotier - Zizyphus-spina Christi,que Victor Guérin a pris pour un acacia3. - Légumineuses frutescentes, tel que le rétem, qui donne aux rochers qu'il revêt de ses tiges fleuries des teintes ravissantes $^{4}$; mimosées, comme les acacias tordu, seyal, du Nil et a feuilles blanchitres, avec le Prosopis Stephaniana, arbuste haut d'un à deux pieds, que nous avons déjà rencontré dans les oasis égyptiens et que nous retrouverons en Mésopotanie et en Perse ; Boissier le fait croitre auprès de Joppé, de Sidon, à l'embouchure de l'Oronte et dans les environs de Damas. Tamaris du Nil, du Jourdain et de Syrie, ainsi que le Moringa aptera et le Cordia myxa. Composées, telles que l'absinthe de Judée, la centaurée du Sinaï et d’Égypte, la rose de Jéricho -- Astericus pygmaeus, - la Conyza Dioscoridis, la Scorzonera papposa, etc. La Calotropis procera, asclépiadée au feuillage sombre

1. F. Chabas, Voyage d'un égyptien en Syrie, en Phénicie, etc., an xive siecle avant notre ère. Paris, 1867, in-4, p. 312.

2. Lortet, op. laud., p. 400.

3. La Terre Sainte, t. II, p. 197.

4. Tristram, op. land., p. 264. 
et au suc laiteux, répandue du Soudan jusquaux frontières de l'Inde'. La Saluadora persica - le sénevé de l'Erangile, - le Solanum Sodomeum, la pomme de Sodome - Leimoun louth, - dont les fruits desséchés laissent échapper, quand on les presse, une poussière noiraitre $\stackrel{?}{ }$, en même temps que le Solcmum coagulans, arborescent et hispide. Labiées aromatiques, comme les Salvia graveolens, controversa, Hierosolymitana; l'Achyranthes argentea; diverses chénopodées et salsolacées - Atriplex halimus, Arthrocnemum fruticosum, Suaeda asphaltica; - l'Ephedra alata et des graminées rigides et piquantes - Stipa, Aristida, Pennisetum.

Les plantes sahariennes, dont je viens de parler, se rencontrent en partie dans le désert, d'ailleurs peu connu, qui sépare la Syrie de la Mésopotamie et où les tribus arabes mènent paître leurs troupeaux; quelques-unes croissent encore dans le double bassin du Tigre et de l'Euphrate; mais elles sont loin de caractériser à elles seules la flore de cette région, et la végétation varie singulièrement, quand on descend du massif arménien, oi les deux grands fleuves prennent leur source, dans la plaine basse qu'ils inondent de leurs eaux débordées:3. Au nord, c'est la flore des

1. Lortet, op. laud., p. 411, lui donne le nom d'orange de Sodome.

2. Victor Guérin, op. laud., t. I, p. 200. Lortet donne à la pormme de Sodome le nom de $S$. melongena.

3. G. A. Olivier, Voyage dans l'empire othoman, l'Égypte et la Perse. Paris, 180', in-4, t. II, p. 416-422. - Ainsworth, Rescarches in Assyria, Balyglonia and Chaldeca. London, 1838, 
steppes qui domine avec la végétation forestière des montagnes, mais représentée par quelques espèces particulières, comme le chêne à glands énormes du Kourdistan (Quercus oophora ou Brantii ${ }^{1}$ ), en compagnie du chêne de Perse ou du Liban. Plus au sud, à la flore des steppes se mêlent les plantes de la flore méditerranéenne : anémones, glaïeuls ${ }^{2}$, asphodèles, hyacinthes, tulipes, crucifères; elles émaillent dès les premiers jours du printemps de leurs fleurs brillantes l'uniformité de la plaine et les hautes herbes des prairies $^{3}$. Bientôt leur succèdent des astragales et d'autres légumineuses, des composées velues et épineuses, des labiées aromatiques. Parmi les composées figure cette absinthe " très odorante ", dont l'abondance frappa Xénophon*; à côté d'elle prend place le Prosopis Stephaniana", qui envahit tous les lieux stériles de la Mésopotamie au nord de Bagdad, tandis que les oasis et les bords de l'Euphrate et du Tigre sont couverts de fourrés épais de tamaris ${ }^{6}$, qui servent

in-8,.p. 217-37. - F. Hoefer, Chaldée, Assyrie, Médie, Babylonie, Mésopolamie, etc. Paris, 1852, in-8, p. 172-182.

1. P. Müller-Simonis, Du Caucase au golfe Persique ì travers l'Arménie, le Kurdistan el la Mésopolamie. Paris, 1892, in-8, p. 356.

2. Probablement le Gladiolus Aleppicus signalé par Boissier en Mésopotamie.

3. Tavernier, Les six voyages, t. I, p. 188. - Comte de Cholet, Voyage en Turquie d'Asie, Arménie, Kurdistan el Misopolamie. Paris, 1892, in-18, p. 272, 34 et et 365 . - W. Kennet Loftus, Travels and researches in Chaldaea and Susiana. Londres, 1857 , in-8, p. 5.

4. Anabasis, lib. I, cap. 5.

5. Ainsworth, op. laud., p. 48, lui donne le nom de mimosa agrestis, ce qu'á répété Hoefer; Olivier l'appelle simplement mimosa.

6. Ainsworth l'appelle $T$. orientalis, c'est probablement le $T(\ell$ marix passerinoïdes Del. ou la variété Tigrensis du T. Pallasii. 
de retraite aux lièvres et aux antilopes et sont peuplés d'oiseaux'. On y rencontre également le peuplier de l'Euphrate - le saule de Babylone ${ }^{2}$. - Sur les derniers monts qui dominent la plaine on voit aussi quelques arbres rabougris, chènes, poiriers ou amandiers sauvages.

Ils disparaissent avec les dernières collines, qui viennent mourir au-dessous de Hit ${ }^{3}$, là où, après un long circuit, l'Euphrate se rapproche du Tigre, pour s'en éloigner bientôt de nouveau, puis s'en rapprocher, jusqu'à ce que, aujourd'hui réunis, ils roulent ensemble vers le golfe Persique leurs flots confondus. Sur la plaine d'alluvions, qu'ils recourrent pendant de longs mois de leurs eaux débordées, les dernières plantes des steppes ont disparu, à l'exception des salsolacées ${ }^{4}$, dont le sol imprégné de nitre favorise la végétation; quelques buissons isolés de tamaris et les dattiers, qui se dressent de toutes parts dans la plaine, sont les derniers arbres indigènes que l'on aperçoive; mais après l'inondation la terre se couvre de graminées, mèlées aux fleurs les plus diverses, que la chaleur brûlante de l'été fait bientòt périr; seules les lagunes, formées par le débordement des deux fleuves, restent

1. Eduard Sachau, Reise in Syrien und Mesopolamien. Leipzig, 1883, in-8, p. 245 et 258. - J. Cernik, Technische StudienExploration durch die Gebiele des Euphral und Tigris. (Geographische Nillheilungen. Ergänzungsheft 4 4́ (an. 1875), p. 13,17 , etc.).

2. Ainsworth, op. laud., p. 34 et 48 . Il y fait croître aussi le Rhus coriaria, que Boissier ne mentionne pas dans cette région, mais qu'on trouve en Syrie avec le Rhus cotinus.

3. J. Baillie-Fraser, Mesopolamia and Assyria. London, 1842, in-8, p. 27.

4. Ainsworth, op. laud., p. 49. 
couvertes de plantes aquatiques toujours vertes, mêlées à ces immenses roseaux, que les bas-reliefs assyriens nous montrent dépassant la tête des cavaliers qui défilent au milieu de leurs fourrés ${ }^{1}$. Au-dessous du confluent actuel du Tigre et de l'Euphrate, à gauche surtout du Shat-el-Arab, la plaine n'est plus qu'un vaste marécage, où quelques cypéracées et le dattier seuls peuvent croitre.

Mais tout change quand on sort des lagunes du Shat-el-Arab, où se perdent le Kíaroun - l'ancien Pasatigris - et la Kerka - le Choaspès des Grecs - et qu'on pénètre dans la contrée montueuse - l'Élam ou la Susiane - qu'ils traversent dans leur cours moyen et supérieur; sur le premier plateau, qui domine les marécages de la plaine, croissent la plupart des plantes de la Mésopotamie méridionale; plus haut on rencontre successivement la flore des collines, puis celle des montagnes, avec leurs essences particulières, acacias, tamaris, peupliers, chênes et autres bois de charpente $\%$. Ces espèces forestières se retrouvent dans le Zagros septentrional avec le noyer, le frêne à feuilles aiguës et le pistachier mutique.

La flore des steppes, qui n'a plus que de rares représentants dans les plaines basses de la Mésopotamie, domine, avec celle du Sahara, presque exclusivement dans la péninsule arabique; mais elle y est bien diver-

1. Koyoundjik. A. H. Layard, Discoveries in the ruins of Niniveh and Babylon. Londres, 1853. in-8, p. 585.

2. Rawlinson, The five great monarchies of the ancient castern world. London, 1879, in-8, 4e éd., t. II, p. 289. 
sement représentée, et, sur la côte méridionale, elle fait place en partie a la flore des tropiques. Les contrastes que présente la végétation de l'Arabie dépendent de la constitution géologique et du climat de cette vaste contrée. Entourée d'une ceinture de montagnes presque ininterrompue, l'Arabie forme un plateau incliné rers le golfe Persique et le bassin de l'Euphrate et sillonné par une double chaine de hauteurs, le Shammar et le Tooueik, qui se rattachent aux montagnes de la mer Rouge; au pied de celles-ci se déroule la bande étroite du Téhamah, "le pays chaud ", tandis qu'au sud-est du Tooueik, entre cette chaine, l'Hadramaout et l'Oman, s'étend le désert inexploré et stérile de Dahna, auquel correspond au nord du Shammar, le désert de Néfoud ${ }^{1}$.

Arec ses côtes montueuses au climat brûlant, sa région rocheuse du nord-ouest et son double désert de sable, l'Arabie offre de grandes différences dans sa végétation. Daus la presquìle du Sinaï, on rencontre la plupart des plantes de la Syrie méridionale et du désert libyque, arec quelques espèces indigènes particulières: graminées rigides, tel que l'alfa - Imperata cylindrica, - borraginées relues, labiées aromatiques, par exemple la sauge du désert, composées odoriférantes, comme l'absinthe de Judée, des arbres mêmes, tel que le faux-sycomore - le hamad ainsi que le Cralaegus aronia et la rose rubigineuse de l'Horeb².

La flore du plateau central est beaucoup moins

1. Élisée Reclus, Géographie universelle, t. IX, p. - Houdas, art. Arcabie dans la Grande Encyclopédie.

2. Louis Crié, art. Arabie dans la Grande Encyclopédlie. 
variée, mais dans les oasis du Nedjed, compris entre le Shammar et le Tooueik, et dans les vallées arrosées de cette dernière chaine on rencontre, avec le dattier, plusieurs plantes caractéristiques de la région saharienne, comme l'ilhcl, espèce de mélèze, dit Palgrave', propre à l'Arabie, mais dans lequel j'inclinerais à voir un tamaris ${ }^{2}$; le lahl, qui, remarque-t-il, a pour fruit une baie, mais qu'il n'a pas identifié; le sidr ou $n a b a q^{3}$, - le faux-jujubier. - Le voyageur anglais fait aussi mention du kharta aux larges feuilles, arbuste qu'on emploie d'après lui pour le tannage, mais qu'il ne fait pas autrement connaitre. Il ne nous apprend pas davantage ce qu'est le markh, arbrisseau aux longues branches, "semblables à celles du chêne ", qui croît dans le Tooueik 4 . Parmi les plantes du BasNedjed, Palgrave mentionne, aussi une euphorbe, qu'il appelle ghada, sans en dire davantage, - probablement l'Euphorbia cornuta ${ }^{5}$-, ainsi que le katad épineux, " si fort recherché des chameaux ", sans doute la Comulaca monacantha, salsolacée que nous avons rencontrée, sous le nom de had, dans le désert égyptien.

1. Narrative of a year's Journey through central and eastern Arabia. London, 2e édit., 1865, in-8, t. I, p. 232, 253 et 338. 'Trad. d'E. Jonveaux. Paris, 1866, in-8, t. I, p. 205, 222 et 299.

2. Le Tamaris articulata porte en arabe le nom d'athel; mais il y a, parait-il, dans le Sahara un pin nommé elhel.

3. Palgrave écrit nebaa' et en fait un arbre différent du sidr, qui, d’après lui, serait un acacia.

4. C'est sans doute la Lepladenia ou Sarcostemma pyrolechnica, asclépiadée décrite par Forskill, p. 53, et indiquée par Boissier, Flora orienlalis, t. IV, p. 63, dans l'Arabie Pétrée.

5. Celte euphorbe, l'E. reluśa de Forsk. est indiquée par Boissier, t. IV, p. 1093, dans l'A rabie Pétrée. 
Le royageur anglais n'a rien dit des graminées et des autres plantes herbacées de l'Arabie centrale; ce sont sans doute pour la plupart celles du Sahara. On les retroure, en partie du moins, dans le Hedjaz; toutefois la flore de cette région montagneuse et voisine de la mer offre bien plus de diversité que celle du plateau central de l'Arabic et se rapproche de la flore du désert oriental de l'Égypte, mais elle n'est qu'imparfaitement connue. Il n'en est pas de même de la flore de l'Yémen ou Arabie heureuse, avec laquelle elle offre plus d'une ressemblance, et qui, décrite au siècle dernier par le botaniste danois Forskål, a été depuis lors étudiée par plusieurs naturalistes contemporains.

Sans ètre très riche, la flore de l'Yémen offre un intérèt particulier par la nature de ses produits et son caractère tropical, caractère qu'elle partage d'ailleurs arec celle de l'Hadramaout et de l'Oman. Parmi les plantes les plus remarquables qu'elle renferme, sont des capparidées ${ }^{1}$, en particulier deux Moerua arborescents, l'Oncoba spinosa, plante rampante que nous arons rencontrée déjà dans le désert libyque, des polygalées, des malvacées et des tiliacées particulières, la Fagonia arabica, le faux-jujubier, le côt-Catha edulis, - dont les bourgeons exercent sur le système nerveux une action stimulante analogue à celle du thé, plusieurs espèces d'indigotier et d'acacias gommifères ${ }^{2}$, le caféier, des élichryses, l'abrotanon et d'autres composées épineuses et velues ${ }^{3}$, la Salvadora persica

1. Entre autres la Capparis sodada et les Clcome arabica et viscosa. A. Deflers, Voyage au Yémen, p. 110 et suivantes.

2. Acacia arabica, lacla, nubica, verrugera, etc.

3. Par exemple, l'Echinops spinosus et la Centaurea robuslu. 
et des asclépiadées frutescentes ${ }^{1}$, des borraginées ${ }^{2}$ et des convolvulacées, des labiées aromatiques, comme la sauge de Nubie et d'Egypte, de nombreuses amaranthacées et euphorbiacées, des figuiers - entre autres le sycomore, les figuiers à feuilles de saule et de peuplier, celui de Socotra, les figuiers à feuilles palmées et crénelées, - des amaryllidées, des liliacées arborescentes, comme l'aloès officinal et le Dracaena draco - le dragonnier - ; quelques cypéracées et ces graminées aux tiges rigides et velues, habitantes ordinaires du Sahara, - Pennisetum, Eleusine, Andropogon, etc., - en même temps que le panicaut de Ténériffe et le panicaut à tiges renflées, qui servent à la fois de fourrage et de textiles.

Le dragonnier croit aussi dans l'Hadramaout; l'aloès s'y rencontre également, ainsi que dans l'Oman ${ }^{3}$.

- Dans cette dernière province on trouve aussi en abondance le tarfa - Tamarix articulata - et le cassie Cassia lanceolata. - On rencontre encore dans les montagnes de l'Oman des bois d'acacias avec des fourrés d'euphorbes, diverses espèces de rue, des labiées odoriférantes et près de la mer la coloquinte. Sur tout le littoral de la Péninsule enfin croissent ces prétendus lauriers et oliviers, dont parlent les anciens ${ }^{4}$, lesquels, " à marée basse, émergent en entier hors de l'eau, et se trouvent quelquefois, à marée haute, complètement

1. Tel que le Cynanchum arboreum.

2. En particulier les Ileliotropium persicum, strigosum, etc., l'Alkanna orienlalis, etc.

3. J. R. Wellsted, Travels in Arabia. London, 1838, in-8, t. I, p. 283-286 et t. Il, p. 448.

4. Théophraste, IIistoric planlarum, lib. IV, cap. 7. Strabon, Geographia, lib. XVI, cap. 3,6. 
submergés. "Ces oliviers, qui, d'après Diodore', portent des fruits semblables it des chitaignes, ne peuvent éridemment ètre que des mangliers, probablement l'Aviccmia officinalis L. Quant aux soi-disant lauriers, E. Never ${ }^{2}$ y roit un palétuvier, le Rhizophora candelaria $\mathrm{L}$.

On voit par ce qui précède combien riche est la flore de l'Asie antérieure ot de quelle variété d'espèces elle se compose, mème sans y comprendre les plantes de l'Iran dont il sera question plus loin. Pourtant je n’ai point encore mentionné les céréales et les légumes les plus recherchés pour l'alimentation, qu'on y trouve à l'état spontané, ainsi que les arbres fruitiers et quelques-unes des plantes oléagineuses et textiles les plus précieuses, sans parler des aromates qui ont joué de tout temps un si grand rôle dans la vie des Orientaux. On comprend qu'avec cette richesse de plantes alimentaires et industrielles l'Asie antérieure ait été, arec l'Égypte, le berceau de la civilisation antique.

Base, depuis un temps immémorial, de l'alimentation des peuples de l'ancien monde, le froment est originaire de la région qui s'étend de la Caspienne et de la Perse à la Méditerranée; Bérose affirmait déjà que le blé, ainsi que l'orge, croissaient à l'état sauvage en Mésopotamie ${ }^{3}$; une variété de froment, l'engrain, a été trouvée par Balansa sur le mont Sipyle;

1. Bibliotheca historica, lib. III, cap. 19, 3 .

2. Bolanische Erläuterungen su Strabo's Geographie. Königsberg; 1852, in-8, p. 97.

3. Babylonica. E libro primo, 2. (Fragmenla hislor. greecorum, éd. Müller-Didot, t. II, p. 496.) 
Olivier a découvert aussi aux environs d'Anah, non loin de l'Euphrate, "dans une sorte de ravin ", le blé, l'orge et l'épeautre, qu'il avait déjà vus, dit-il ${ }^{1}$, plusieurs fois en Mésopotamie. Il semble d'après cela qu'il considérait ces céréales comme indigènes dans cette contrée.

On n'a rencontré nulle part ailleurs le froment à l'état supposé spontané; mais l'orge à deux rangs a été trouvé aux environs de la mer Caspienne entre Lenkoran et Bakou et dans le désert de Shirvan, ainsi qu'auprès du Sinaï et dans l'Arabie Pétrée ${ }^{2}$; toutefois on n'a point rencontré à l'état sauvage l'orge à quatre et à six rangs; ces formes pourraient bien dès lors n'ètre que des variétés de l'orge à deux rangs, produites par la culture à une époque très reculée, car on a trouvé des débris de la première dans des tombes égyptiennes de la XII dynastie et dans les palafittes de la Suisse.

Pays d'origine du froment et de l'orge, l'Asie antérieure l'est aussi de plusieurs des légumineuses alimentaires; on a trouvé sur divers points de l'Anatolie une forme voisine de la lentille cultivée ${ }^{3}$. Il est probable que le petit pois, ainsi que la fève, sont également originaires de cette contrée, encore qu'on ne les y ait rencontrés nulle part à l'état spontané; ils ont au moins dù y être cultivés dès une haute antiquité, puisqu'on a trouvé des petits pois et des fèves dans les fouilles d'Hissarlick en Troade ${ }^{4}$.

1. Voyage dans l'empire othoman, ctc., t. III, p. 360.

2. A. de Candolle, Origine des plantes cullivées, p. 295.

3. Cf plus haut, p. 56 .

4. L. Wittmack, Unsere jelzige Kenntniss vorgeschichllicher. Samen. (Berichle der deulschen bolanischen Gesellschaft, t. IV (an. 1886), XXXIII.) 
Le lin, lui, n’a pas été seulement cultivé à une époque reculée dans l'Asie antérieure, il y croìt aussi spontanément; on rencontre le lin á feuilles étroites, dont le lin cultivé n'est peut-ètre qu'une variété ou une race particulière, depuis le Caucase jusqu'au Liban" ; le lin " usité " lui-mème aurait, dit-on", été trouvé à l'état sauvage sur la côte orientale de la mer Noire.

Mais ce sont surtout les arbres à fruits dont l'Asie antérieure est la vraie patrie. On y rencontre sur les points les plus différents plusieurs espèces de poiriers ${ }^{3}$; le poirier commun, souche de toutes les variétés cultivées, se trouve à la fois, à l'état spontané, en Mysie, dans le Pont, le Lazistan, la Transcaucasie et la Perse septentrionale. Le pommier commun croìt de même dans le Pont, la Cis et la Transcaucasie, la Colchide et l'Abkhasie ${ }^{4}$. On a trouvé également à l'état sauvage le cognassier dans la Transcaucasie, les provinces de Talysch et d'Asterabad, l'Arménie ${ }^{5}$, etc. Plusieurs espèces de prunier croissent aussi spontanément dans l'Asie antérieure; le prunier domestique en particulier se rencontre dans la région du Caucase, où il s'élève jusqu'à une hauteur de 4,000 pieds, surtout dans le Lazistan et l'Abkhasie, comme dans le Pont. Le prunier propre-

1. Boissier, Flora orientalis, t. I, p. 861.

2. A. de Candolle, op. laud., p. 97.

3. Boissier, op. laud., t. II, p. 653-655. - Fr. Th. Köppen, Geographische Verbreitung der Holzgewächse des europäischen Russlands und des Kaukasus. St. Petersburg, 1888, in-8, t. I, p. 400-404.

4. Boissier, op. laud., t. II, p. 657. - Köppen, op. laud., t. I, $\mathrm{p}-409$.

5. A. de Candolle, op. laud., p. 188. - Köppen, op. laud., t. I, p. 420 . 
ment dit - Prumus insilicic L. - se trouve aussi dāns cette régrion, ainsi que dans la province de Talysch, en Arménie et en Cilicie ${ }^{1}$. Diverses espèces de cerisiers croissent également à l'état spontané dans la même contrée; on rencontre le cerisier des oiseaux et le cerisier à fruits acides - Cerasus caproniana L. - dans l'Ibérie et les provinces de Lenkoran, de Talysch et de Ghilan, dans le Pont et l'Arménie ${ }^{2}$.

Le mûrier à fruits noirs paraît bien être aussi indigène dans les montagnes de l'Arménie, où $\mathrm{M}$. de Tchihatchef l'a rencontré à une hauteur de 1,000 à près de 1,800 pieds ${ }^{3}$. On l'a trouvé encore dans la Transcaucasie, en particulier dans la province de Talysch; Koch l'a vu croitre dans le Shirvan jusqu'à la hauteur de 2,500 pieds; on l'a signalé aussi dans le territoire de Batoum et de Kars ${ }^{4}$. Le grenadier parait également spontané dans l'Abkhasie, la Mingrélie et sur tout le littoral de la mer Caspienne ${ }^{5}$, comme il l'est certainement, nous le verrons, sur presque tout le plateau de l'Iran. Le domaine de l'amandier n'est pas moins étendu; de nombreuses espèces de cette rosacée sont répandues dans l'Asie antérieure tout entière; le type de l'espèce cultivée a été trouvé, semble-t-il, à l'état sauvage par Medwedew dans les provinces méridionales et orientales du Caucase jusqu'à une hauteur de 4,000 pieds; on l'a signalé aussi dans l'Aderbaïdjan et le Kíour'-

1. A. de Candolle, op. laud., p. 169-170. - Köppen, op. laud., t. I, p. 261-265.

2. Köppen, op. laud., t. I, p. 280-292. - Boissier, op. laud., t. II, p. 6ł6-650.

3. Asie Mineure. Bolanique, t. II, p. 461.

4. Köppen, op. laud., t. II, p. 14.

5. Köppen, op. laud.,. t. I, p. 420. - Engler, ap. V. IIehn, liullurpflanzen. p. 386. 
distan, dans la Mésopotamie au-dessus de Mardin et de T'érek ì 3,000 pieds d'altitude et sur l'Antiliban'.

Croissant avant lépoque glaciaire dans toute la région tempérée de l'ancien monde, la Chine exceptée, le noyer vient encore spontanément aujourd'hui dans la Transcaucasie, la province de Talysch, la Géorgie, le Pont, l'Arménie et la Perse septentrionale 2. Le châtaignier qui, à l'époque tertiaire, était répandu sur une étendue de pays bien plus vaste, se rencontre de nos jours encore à l'état sauvage dans la Transcaucasie, en compagnie du hètre et de diver's chênes, ainsi que dans la région subalpine de l'Anatolie septentrionale et occidentale ${ }^{3}$. Le coudrier se trouve aussi dans toute la région du Caucase, ainsi que dans le Pont, la Cappadoce, la Cilicie et même la Syrie septentrionale ${ }^{*}$.

On rencontre également le caroubier dans ces dernières contrées; arbre méditerranéen, il croît spontanément sur la còte méridionale de l'Anatolie, ainsi que sur celle de la Syrie; on l'a même trouvé dans une gorge du mont Sabor, près de Tìez dans l'Arabie heureuse ${ }^{5}$. Le faux-jujubier ne croît que dans la vallée du Jourdain et dans l'Arabie; mais le jujubier ordinaire, que Boissier dit n'avoir pas rencontré en Syrie, où on l'a cru indigène, se trouve à l'état spontané dans l'Anatolie,

1. Boissier, Flora orienlalis, t.II, p. 640-645. - Engler, Ibid:, p. 386 .

2. A. de Candolle, op. laud.,p. 342. - Boissier, op. laud., t. IV, p. 1160.

3. A. de Candolle, op. laud., p. 283. - köppen, op. laud., t. II, p. 142. - Engler, ap. V. Hehn, p. 386.

4. Boissier, op. laud., t. IV, p. 1176.

5. Boissier, op. laud., t. II, p. 632. - Deflers, Voyage dans l'Yémen, p. 13'. 
l'Arménie, les provinces de la Transcancasie '. Quoique le pistachier ait été trouvé en Syrie dans l'Antiliban, il n'est point certain que cet arbre y soit indigène, sa patrie parait plus orientale ${ }^{2}$. Avec l'olivier et la vigne nous retrouvons deux des représentants les plus incontestables de la flore de l'Asie occidentale.

Répandue dans toute la région tempéréc de l'ancien monde avant l'époque glaciaire, la vigne est restée spontanée dans la Transcaucasie, où elle atteint d'énormes proportions, surtout dans la partie voisine de la mer Noire et dans la province de Talysch; on l'a observée aussi dans la vallée d'Argouri, sur le versant septentrional de l'Ararat, jusqu'à une altitude de 5,000 pieds $^{3}$. De là elle s'est répandue dans les contrées de l'ancien monde habitées par les Sémites, qui paraissent avoir découvert la culture de cet arbuste, avec la fabrication du vin. L'olivier a un domaine plus étendu et plus méridional; on le rencontre à l'état spontané au sud de l'Asie Mineure, "où il forme de véritables forêts », dans la Mésopotamie, la Syrie, la Palestine, la presqu’ile du Sinaï et dans l'Arabie méridionale jusqu'à Mascate ${ }^{4}$. Cette région, non seule toutefois, est aussi la patrie du figuier. Cet arbre croît à l'état spontané dans la Transcaucasie, en particulier dans l'Abkhasie, l'Ibérie, la Mingrélie et dans la province de Talysch, ainsi que dans l'Anatolie occidentale et méridionale, la Syrie et la Mésopotamie septentrio-

1. Boissier, op. laud., t. II, p. 12-13.

2. A. de Candolle, op. laud., p. 252. - Köppen, op. laud.,

t. I, p. 16'́

3. Köppen, op. laud., t. I, p. 97.

4. A. de Candolle, op. laud., p. 233. - Engler, ap. X. Hehn, p. 117. 
nale'. Le sycomore, espéce semi-tropicale, ne vient spontanément qu'au sud de la P'alestine ct dans l'Arabie. Là se trouve aussi le dattier; arbre de la flore saharionne, on le rencontre dans toute cette région, de la presqu’ile du Sinaï an golfe Persique; il apparait aussi plus au nord, dans la Mésopotamie méridionale, oì sa culture, nous le verrons, avait pris dès la plus haute antiquité une grande importance.

Les céréales, les légumincuses et antres plantès alimentaires, ainsi que les arbres fruitiers, n'étaient pas les seuls représentants du règne régétal que la flore indigène offrait aux habitants de l'Asie antérieure; elle leur fournissait aussi la plupart des aromates dont ils se servaient dans les usages ordinaires de la vie et surtout dans le culte: baume, myrrhe, encens, calame ou roscau aromatique, galbanum, jonc odorant, ladanum, mastic, storax et téébenthine, ainsi peut-ôtre que l'opopanax. La Carie, la Lycie et les montagnes de la Cilicie, d'où il somble avoir été importé dans l'île de Cypre, produisaient le Liquidambar orientalis ${ }^{2}$, bel arbre qui fournit le storax liquide. Dans l'Anatolie presque entière, en particulier dans la Cilicie, à Cypre, dans la Syrie septentrionale, Ja région inférieure du Liban et de l'Antiliban, ainsi que dans la Palestine, croissait le Styrax officinalis ${ }^{3}$, dont l'écorce exsude le storax proprement dit. On trouvait dans l'Anatolic méridionale et occidentale, los iles de l'Archipel, à Cypre et sur le littoral de la Syrie, le Pistacia lentis-

1. A. de Candolle, op. laud., p. 223. - Köppen, op. laud., t. II, p. 20.

2. Grisebach, op. laud., t. I, p. 362. - Boissier, op. laud., t. II, p. 849 .

3. Boissier, op. laud., t. IV, p. 35.

I. 
cus, dont on retire le mastic. Le Pistacia terebinthus et le Pistacia Palaestina, qui produisent l'un et l'autre la térébenthine, se rencontraient le premier en Bithynie et à Cypre, le second. dans le Pont, la Paphlagonie et la Pamphylie, en Cilicie, en Syrie, sur les contreforts du Liban et de l'Antiliban, ainsi que dans la Galilée ${ }^{1}$. Les divers cistes, qui produisent le ladanum, croissent dans presque toute l'Anatolie, à Cypre, dans la Syrie septentrionale et la Palestine ${ }^{2}$.

D'après Théophraste ${ }^{3}$, la férule, qui fournissait le galbanum, aurait crì aussi en Syrie; mais on ignore l'espèce véritable dont on le retirait; peut être était-ce la Ferula Ilermonis et la Ferulago cassia, indigènes dans ce pays ${ }^{4}$; nous rencontrerons dans la flore de l'Iran deux espèces de férules - la $F$. galbaniflua et la $F$.gommosa, erubescens ou rubricaulis Boiss., - qui fournissent certainement cette résine ${ }^{5}$. Théophraste ${ }^{6}$ fait également croitre en Syrie la plante qui donne l'opopanax; mais les ombellifères de cette famille, qui passent pour le fournir, ne sont point indiquées par Boissier dans cette contrée; nous les rencontrerons, quand nous étudierons la flore de l'Iran. On trouvait, au contraire, si l'on en croit 'Théophraste ${ }^{\top}$, en Syrie, au delà du Liban, aux bords desséchés d'un

1. Boissier, op. laud., t. II, p. 6-8.

2. Boissier, op. laud., t. I, p. 436-441.

3. Historia plantarum, lib. IX, cap. 2.

4. Boissier, op. laud., t. II, p. 985 et 999.

5. F.-A. Flückiger et Dan. Hanbury, IIistoire des drogues d'origine végélale, trad. par J.-L. de Lanessan. Paris, 1878, in-8, t. I, p. 563. - Boissier, t. II, p. $988-989$ et 995 .

6. IIistoria plantarum, lib. IX, cap. 11.

7. IIistoria plantarum, lib. IV, cap. 8, \& et IX, cap. 7, 1.-Pline, op. laud., lib. XII, cap. 22. 
lac, le calame et le Schocus ou choin. On s'accorde génèralement ì voir dans le premier l'Acorus calamus L.; quant au second ou jonc odorant, Sprengel ' et Dierbach ${ }^{2}$, suivis par R. Sigismond ${ }^{3}$, l'ont regardé comme l'Andropogon schocnanthus, plante indienne; c'est en réalité l'Andropogon laniger Desf., que Boissier" n'indique pas, il est vrai, en Syrie, mais qui est indigène dans les régions arides de la Mésopotamie et de l'Iran oriental, ainsi qu'en Arabie.

C'est en Arabie aussi et sur les côtes de l'Afrique orientale que croissent des arbustes de la famille des burséracées qui exsudent la myrrhe, lo baume et l'encens. Le Balsamodendron myrrha Nees - B. Kataf Kunth, Amyris liataf Forsk. - qui fournit la myrrhe, se rencontre dans l'Yémen ", ainsi que dans l'Hadramaout et dans le pays des Somalis. Schweinfurth a trouvé dans le pays des Bisharris, en Abyssinie, le baumier, Balsamodendron opobalsamum Kunth ${ }^{6}$, espèce qui ne semble pas différente du Balsamodendron Ehrenbergianum Bergō, découvert en Arabie, à Ghizan dans le Téhàma; on l'a réunie aussi au Balsamodendron gileadense DC., nom donné à cette burséracée, en sou-

1. Geschichte der Bolanik. Leipzig, 1817, in-8, t. I, p. 138.

2. Die Arsneimiltel des Hippolirates. Heidelberg, 1842, in8, p. 160.

3. Die Aromala in ihrer Bedeulung für Religion, Sillen, Gein-8, p. 34 .

4. Flora orienlalis, t. V, p. 463. Boissier indique en Syrie l'A. hirtus, var. pubescens.

5. Forshål, Flora agypliaco-arabica, p. 80. - A. Deflers, op. laud., p. 120.

6. Petermann's geographische Mittheilungen, an. 1868, p. 127. 276.

7. F-A. Flückiger et D. Hanbury, op. laud., t. I, p. 269 et 
venir d'un pays oi les anciens la faisaient croitre ${ }^{1}$. Des diverses espèces d'arbres à encens, le Boswellia papyrifera, dont l'écorce s'cnlève par feuilles et exsudc unc résine d'un parfum très agréable ${ }^{2}$, se rencontre dans le Sennaar et en Abyssinic; le Bosuellic Frereana - lo louban matti - vient dans le pays des Somalis; plusieurs variétés du B. Carterii Fr., le véritable encens ou oliban des Anciens, croissent également dans la mème contréc; enfin une autre variété de cette espèce, le $B$. sacra de Fliickiger ${ }^{3}$, est indigène dans l'Hadramaout.

Ainsi, on le roit par ce qui précède, la flore de l'Asie antérieurc renferme les plantes les plus utiles et les plus propres à satisfaire à tous les besoins de l'homme; mais, avant de rechercher quelles ressources en ont tirées les habitants de cette vaste région, il importe de rappeler quels peuples l'ont occupée dans l'antiquité.

Depuis l'époque la plus reculée les contrées occidentales de l'Asie ont été successivement traversées par les peuplades les plus diverses d'origine. Après avoir été habité d'abord, plus ou moins complètement, par des tribus de race altaïque, le plateau de l'Iran a

1. Pline. Hist. naturalis, lib. XV], cap. 32.

2. A. Richard, Tentamen florae abyssinicae. Paris, 1851, in8, t. I, p. 148.

3. F.-A. Flückiger et D. Hanbury, op. laud., t. I, p. 260 et 266-268. Il y a aussi dans l'Inde une autre espèce, le $B$. serrala Roxb., B. thurifera Colebr., dont je parlerai ailleurs. 
été colonisé presque en entier par un peuple de la grande famille indo-européenne, les Iraniens, dont nous étudierons dans le livre suivant les mours et la cirilisation. Ce sont des tribus indo-européennes aussi qu’on retrouve sur les bords asiatiques de la mer Égée, occupés par des colonies grecques.

Les populations, d'origine thrace, de la Bithy'nie, de la Mýsie, de la Lydie et de la Phrygie, qui habitaient dans l'angle nord-ouest de la Péninsule, leur étaient étroitement apparentées ' ; mais au sud et à l'est les peuplades primitives de la Lycie, de la Carie, de la Cilicie et de la Paphlagonie paraissent aroir appartenu à une race brachycéphale et par suite toute différente, répandue des bords de la mer Noire au nord de la Syrie. Les premiers habitants de l'Arménie se rattachent probablement au mème type et n'ont été indogermanisés qu'à la suite des invasions iraniennes du ril siècle arant notre ère ${ }^{2}$. Il faut peut-être voir dans ces diverses nations les débris d'un même peuple, les Hétéens ou Hittites - les Kheta ou Khiti des Égyptiens, les Khatti des monuments assyriens. - Cantonnés d'abord dans la région du Taurus, les Hétéens en sortirent vers le $\mathrm{xvI}^{\mathrm{e}}$ siècle avant notre ère, et s'avançant d'un côté jusqu'à l'extrémité occidentale de l'Asie Mineure, de l'autre jusqu'au Liban et a l'Euphrate

1. Hérodote, Historice, lib. I, cap. 171 et lib. VIII, cap. 138. - Strabon, Geographia, lib. XII, cap. 4.4. - Edmond T'exier, Asie Mineure. Paris, 1862, in-8, p. 46, 152, 232 et 377.

2. Felix von Luschan, Reisen in Lyliien, Milyas und Kibyratis. Wien, 1889, in-fol. p. 205. - Fr. Hommel, Neue Werlie über dic ältesle Bevöllierung Kleinasiens. (Archiv. für Anthropologie, an. 1891, p, 251-260.)

3. William Wright, The Empire of the Hittites. London, 188', in-8, p. 12-63. - W. Max Müller, Asien und Europa nuch altüg!plischen Dentimülern. Leipzig, 1893, in-8, p. 324. 
fondèrent sur cette vaste étendue de territoire un grand empire militaire, qui tint un instant en échec. les forces des Ramessides et des rois d'Assyrie'. Dépouillés par ces derniers de leurs possessions d'au delà de l'Amamus, ils subsistèrent longtemps encore, comme nation indépendante, dans la Cappadoce, berceau de leur puissance, jusqu'au jour où, vaincus déjà par Crésus, ils furent définitivement subjugués par les Perses et absorbés dans leur monarchie.

Au sud de l'Amamus, les Hétéens se trouvaient en contact avec les populations sémitiques de la Syrie et de la Phénicie, établies sur les deux versants du Liban et de l'Antiliban, dans les bassins de l'Oronte et du Jourdain, dont ils avaient asservi ou exterminé les premiers habitants. Des tribus sémiliques occupaient aussi dans toute son étendue la presqu'ile arabique $^{2}$, qu'on a considérée même parfois comme le berceau de leur race $^{3}$; enfin on retrouvait encore des Sémites dans le double bassin du Tigre et de l'Euphrate, mais au sud, mêlés avec des peuples d'origine et de langues différentes, les Sumériens ${ }^{4}$. Des

1. A. H. Sayce, Les IIéléens. IIisloire d'un empire oublié. Paris, 1891, in-16, p. 79, 86, 91 et 103-108. - Léon de Lantsheere, De la race el de la langue des Hilliles. Bruxelles, 1891, in-8, p. 89 .

2. M. J. IJalévy toutefois la fait occuper d’abord par les Couschites, "peuple principal de la race hamitique ", puis par les sémites Yoqtanides et enfin par les Abrahamides. Les anciennes populations de l'Arabie. (Mélanges de crilique "l d'histoire relalifs aux peuples similiques. Paris, 1883, in-8, p. 74-93.)

3. Eberh. Schrader, Die Abstammung der Chaldiier und die Ursilze der Semilen. (Zeilschrift der deulschen morgenlündischen Gesellschuft, t. XVII (an 1873), p. 397-424.)

1. J. Oppert, art. Balyylone dans la Grande Encyclopédie. Fritz Hommel, Geschichte Babyloniens und Assyriens. Berlin, 
peuplades de race non sémitique, les Élamites, les Kishshou - les Cosséens on Cassii des auteurs grecs et latins - et les Namri occupaient toute la région, qui s'étend il l'est de la vallée du Tigre, de la plaine que trarerse son cours impétueux aux sommets neigeux de la chaine du Zagros: negritos dans la contrée marécageuse des bords du fleuve, tribus de race altaïque, au contraire. peut-ètre apparentées aux Sumériens de la Basse-Mésopotamie ${ }^{2}$, dans le massif montagneux, dont la partie la plus élevée a été plus tard occupée par les Iraniens.

A quelle époque ces différents peuples pénétrèrentils dans les pays oi nous les trouvons établis au début de leur histoire? Nous l'ignorons et nous savons encore moins à quel dêgré de civilisation ils étaient arrivés, quand ils s'y fixèrent. Ils menaient sans doute alors la vie nomade, à laquelle sont restés fidèles une partie des Sémites; mais plus de quatre mille ans avant notre ère plusieurs d'entre eux y avaient renoncé et avaient formé des états, qui témoignent déjà d'une culture arancée. Tels étaient ceux que nous trouvons dans la Basse-Mésopotamie - la Chaldée - , où Sumériens et Sémites, déjà si intimement mêlés les uns aux autres qu'on a voulu n'en faire qu'un seul peuple ${ }^{3}$, avaient fondé plusieurs villes bientôt florissantes.

1885, in-8, p. 237. - Hugo Winckler, Geschichle Babyloniens und Assyriens. Leipzig, 1892. in-8. p. 20.

1. Fritz Hommel, op. laud., p. 276.

2. Fréd. Houssay, Les races humaines de la Perse. Lyon, 1887, in 8, p.4-30. - A. Billerbeck, Susa. Eine Studie zur alten Geschichte Westusiens. Leipzign, 1893, in-8, p. 22-25.

3. J. Halèvy, Recherches criliques sur l'origine de la civilisation bab!lonienne. Paris, 1876, in-8, p. 1 et suiv. - Revue crilique, 1880, no 8, p. 150-151. - Les lois sumériennes. (Mé- 
Vers l'an 3800 régnait sur l'une d'elles, Lagash Sirpourla ou Sirgoulla --, Ourou-Kaghina, le monarque peut-être le plus ancien dont l'histoire ait conservé le souvenir ${ }^{1}$. Le plus illustre de ses successeurs, le patési Goudéa, apparait déjà comme un prince puissant, en relation arec les contrées lointaines de l'Occident. Une autre cité mésopotamienne, la seule qui soit située sur la rive droite de l'Euphrate, Ourou, ne joua pas, aux environs de l'an 2900, un rôle moins considérable, sous le gouvernement d'Ourbaou; les villes voisines, Lagaslı elle-même, reconnurent sa suzeraineté. Dounghi, fils d'Ourbaou, accrut encore la puissance du nouvel état, et Ourou resta la capitale du "Pays de Soumir et d'Akliad ", jusqu'au jour oì la prédominance passa d'abord aux princes de Nishin, puis aux rois de Nipour, auxquels une famille de Larsam derait à leur tour enlever la suprématie ${ }^{2}$.

Celle-ci ne derait pas la garder longtemps. Après aroir réuni sous sa domination les tribus jusque-là indépendantes de l'Élam, le roi Koutournakhouta envahit la Mésopotamie méridionale et renversa le patési de Larsam. Son fils Rimsin continua ses conquètes et il avait soumis à son pouroir tout Soumir et Akkad, quand il entra en lutte arec Hammourabi, roi de Ba-

langes de critique el d'hisloire, p. 38-45.) - Les nouvelles inscriptions chaldéennes el la question de Sumer et d'Accad. (Ibid., p. 389-409.)

1. M. E. Henzey (Gencialogie de Sirpourla, dans la Revuce d'Assypiologie, t. II, p. 78-8') met en tête des rois de cette ville Our-Nina; M. Hommel (Geschichte, p. 291) ne place ce prince qu'au troisième rang et le fait vivre vers 4200 avant notre ère. Cf. P. Jensen, Inschriften der Könige von Lagash. (Keilinschrifliche Bibliothek, t. 11I, 1, p. 8 et 11).

2. II. Winckler, op. laud., p. 23-36. - Maspero, IIisloire ancienne, t. I, p. 617-619. 
bylone (2394-2379), le sixième seulement, mais le plus illustre des princes de l'antique cité'. Le moment élait renu oi l'hégémonie politique allait passer dus ville sumériemnes de la Chaldée méridionale aux villes exclusivement sémitiques du nord de cette province. Plusieurs siècles avant Hammourabi, Sharroukin et son fils Naramsin, rois de l’inccrtaine Agadé, avaient régné, dit-on", sur " les quatre maisons " - Agadé, Babylone, Sippara el Nipour, - et étendu leur domination jusqu'au golfe Persique et i la mer de Cypre. La puissance de Hammourabi fut moins grande, mais elle est plus certaine. Il affranchit le pay's de Soumir de la suzeraineté de l'Élam, et, en soumetlant toute la Basse-Mésopotamie à ses lois, il fonda le premier royaume babylonien ${ }^{3}$.

Les successeur's d'Hammourabi ne gardèrent pas longtemps le pouvoir, et l'état puissant qu'il avait constitué fut mème quelque temps asservi par des étrangers, les Kashshout. Mais comme les Élamites, ceux-ci furent subjugués par leurs vainqueur's; l'élément indigène reprit vite le dessus, et d'me petite ville roisine de Babylone, Pashi, sortit une dynastie nouvelle, dont Naboukodorossor - Nabuchodonosor fut le premier roi. Tainqueur des Kashshou et des Elamites, redevenus indépendants, ce prince fut moins heureux dans la lutte qu'il engagea contre le royaume naissant d'Assyrie, et dut lui abandomner' la

1. Hugo Winckler, op. laud., p. 58.

2. Maspero, op. laud., t. I, p. 596-602.

3. C. P. Tiele, Bubylonisch-Assyrische Geschichle. Gotha, 1886, in-8, p. 12'

4. Winckler, op.laud., p. 77 et 106. - Naspero, op. laud., t. II, p. 116-120. 
suprématie, que Babylone exerçait depuis longtemps sur le nord de la Mésopotamie. Les successeurs de Nabuchodonosor étaient trop faibles pour la ressaisir; ils furent même impuissants à arrêter l'invasion de la tribu sémite des Kashdi - les Khaldi des Babyloniens et des Grecs, les Khasdim des Hébreux ${ }^{1}$. - Partis des bords du golfe Persique, cette peuplade s'établit dans le pays de Soumir et d'Akkad, auquel elle donna son nom.

D'autres Sémites, de la famille araméenne, vinrent encore au siècle suivant se fixer dans cette contrée; affaiblis par des discordes intestines, les rois de Babylone non seulement ne purent empêcher ce nouvel établissement, mais ils tombèrent sous la suzeraineté de l'Assyrie, et, après le meurtre du dernier d'entre eux, Tiglathphalasar I s'empara de leur capitale et mit fin au premier royaume babylonien ${ }^{2}$. L'hégémonie que la Basse-Mésopotamie avait exercée pendant de longs siècles lui échappait pour passer à l'Assyrie.

Cantonnés d'abord dans le triangle formé par le petit Zab, le Tigre et la chaine du Zagros, les Assyriens appartenaient à la même race que les Babyloniens et, comme eux, ils paraissent être descendus du nord de la Mésopotamie dans la contrée qu'ils occupaient à l'aurore des temps historiques. Ashshour, leur capitale, était vers 1800 le siège d'un patési probablement vassal de Babylone ${ }^{3}$. Mais cette situation inférieure devait cesser. Fiers de leurs conquêtes dans le nord

1. II. Winckler, op. laud., p. 112.

2. II. Winckler, op. lauul., p. 125-135. - Tiele, op. laud., p. $138-147$.

3. Ilommel, op. laud., p. 490. - Winckler, op. laud., p. 145 et 152. - Jiele, op. laud., p. 138. 
de la Mésopotamie restée jusque-la indépendante, quoique soumise a l'influence de la civilisation babylonienne, on roil cinq siècles plus tard les patésis assyriens prendre le titre de " rois du monde ", que Salmanasar I porta le premier. Ce prince guerrier poursuivit l'œurre de ses prédécesseurs et poussa ses conquètes jusqu'au delà de l'Euphrate; afin d'ètre plus rapproche de ses nouvelles possessions, il fixa sa résidence près du confluent du Tigre et du grand Zab, à Kalakh - Nimroud, - qui resta jusqu'à Sargon la capitale de l'Assyrie'.

Après un siècle d'arrêt, l'Assyrie reprit avec Tiglathphalasar I le cours de ses victoires; ce prince porta tour à tour ses armes à l'ouest dans le Naharina et dans la Syrie septentrionale, au nord dans le pays des Moushki et de Koumouk, situé entre l'Euphrate et l'Halys, à l'est jusqu'en Médie, enfin au sud en Babylonie ${ }^{2}$. Ses successeurs se montrèrent bientôt incapables de conserver tant de conquêtes; mais Ashshournazirpal mit un terme à la décadence; non seulement il recouvra toutes les anciennes possessions de Tiglathphalasar I, mais il en acquit de nouvelles. Les guerres continuelles de Salmanasar II et de Tiglathphalasar II d'abord, puis celles de Sargon, d'Assarhaddon et d'Ashshourbanipal reculèrent encore les limites de l'empire assyrien. L'Arménie reconnut, au moins un temps, sa suzeraineté; la Syrie, la Phénicie et la Judée furent asservies ou rendues tributaires, la Cilicie orientale annexée; le Taurus et l'Antitaurus devinrent au nord-

1. Oppert, art. Assyrie dans la Grande Encyclopédic. T'iele, op. laud., p.111. - Winckler, op. laud., p. 161.

2. Naspero, op. laud., éd. in-12, p. 295-300. - Tiele, op. laud., p. 151-155. - Winckler, op. laud., p. 170-182. 
ouest la frontière de l'empire, au nord-est le Zagros fut franchi et la Médie dut payer tribut, enfin l'Arabie et l'Egypte furent envahies ${ }^{1}$.

La chute de Ninive, en 606, ne mit pas fin à la domination des Sémites dans l'Asie antérieure; seulement ce fut la Chaldée qui, pendant deux tiers de siècle, y exerça la suprématie à la place de l'Assyrie. Sous Nabopolassar, Nabuchodonosor II, et Nériglissor, le pays des Khaldi reprit les traditions glorieuses de l'antique Babylone ${ }^{2}$; ses mœurs, ses institutions firent loi et devaient survivre mème à la perte de son indépendance (538). L'histoire de la civilisation de l'Asie antérieure se confond durant des siècles avec celle de l'Assyrie et de la Chaldée ${ }^{3}$. Cependant il est un pays qui, gràce à sa longue indépendance, conserva, encore qu'il ait, lui aussi, subi l'influence des grands empires de la Mésopotamie, ses mœurs, ses institutions propres et qui, par suite, demande à ètre étudié à part, c'est la Syrie et en particulier la Phénicie et la Judée.

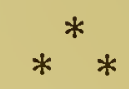

Si la population primitive de la Syrie, celle qui fut contemporaine de l'age de pierre, nous est inconnue, nous sarons qu'à l'exception du bassin inférieur de l'Oronte, occupé par les Hétéens ou Hittites et du pays des Philistins, peuple d'origine inconnue ${ }^{4}$, tout le reste de la contrée était, vers l'an 2000 avant notre ère,

1. Oppert, art. Assyrie. - Tiele, op.laud., p. 186.

2. Oppert, art. Babylone dans la Grande Encyclopédie.

3. H. Winckler, op. laud., p. 320-325.

4. R. Pietschmann, Geschiche der Phenisier. Berlin, 1889, in -8, p. 93 . 
habité par des tribus sémitiques; mais cette communauté d'origine était le seul lien qui les unit; en tout le reste elles étaient divisées entre elles et finirent par devenir étrangères les unes aux autres; elles s'étaicnt d'ailleurs établies dans la Syrie à des époques différentes. Parmi les premières peuplarles qui s'y fixèrent furent les Amorrhéens et les Cananéens, qu'on trouve cantonnés d'abord dans la région montagneuse qui s'étend du Liban septentrional au sud de la mer Morte. En mème temps qu'eux, les Phéniciens s'étaient établis dans la région còtière, à laquelle ils ont donné leur nom. Une tradition place leur berceau sur les bords du golfe persique; chassés de cette région par un tremblement de terro, ils seraient allés d'abord se fixer près du « lac de Syrie » — peut-être la mer Morte et auraient de lá poussé jusque sur la côte située au pied occidental du Liban.

Ce ne fut que longtemps après que les maitres futurs de la terre de Canaan, les Hébreux, arrivèrent en Syrie. Suivant une tradition nationale, la grande famille à laquelle ils appartenaient aurait eu pour berceau le pays de Paddan-Aram, dans la Mésopotamie septentrionale au pied du mont Masios; mais Térakh, l'ancètre direct des Beni-Israël, abandonnant cette contrée, se serait établi à Our-KKhashdìm - l'Ourou des Chaldéens ${ }^{1}$; - il aurait toutefois bientôt quitté cette ville et se serait rendu au sud du Jourdain, où Abraham, son fils, aurait fixé un temps sa résidence. De là le patriarche serait allé camper à l'ouest de ce fleuve, contrée qu'au bout de trois générations ses descendants abandonnèrent pour se retirer en Égyte.

1. Maspero, Ilistoire ancienne, t. II, p. 6't. 
Plusieurs siècles après, on le sait, ils revinrent s'établir à l'est du Jourdain, au nord de la contrée occupée par les Edonites, les Ammonites et leurs proches parents les Moabites ${ }^{1}$; c'est de là qu'ils marchèrent à la conquête du pays de Canaan, cette " terre Promise » à l'ancêtre de leur race.

Désormais les Hébreux prennent place dans l'histoire générale et, quand les différentes tribus, dont se composait leur nation, eurent été réunies sous le sceptre de David et de Salomon, ils formèrent un royaume un instant puissant; mais des divisions intestines ne tardèrent pas à l'affaiblir et le livrèrent sans défense anx armes des rois d'Assyrie et de Babylone. La Judée fut asservie et cessa d'exister comme nation indépendante; mais pendant les siècles qui avaient précédé sa conquête, elle avait joué, comme la Phénicie, quoique à un degré moindre et à d'autres égards, un rôle considérable dans l'histoire de l'Asie antérieure et en particulier des peuples sémitiques. La littérature qu'elle nous a laissée nous fait non seulement connaitre ses mœurs, son industrie, son art et ses croyances religieuses mieux que ne le sont ceux d'aucun autre peuple de l'Orient; mais elle nous permet de pénétrer danș la vie intime et dans l'àme des anciens Sémites. Aussi la Judée doit-elle, avec la Phénicie, trouver place dans cette étude après la Chaldée et l'Assyrie, dont elles ont l'une et l'autre subi l'influence, aussi bien que celle de l'Égypte. On comprendra, au contraire, que je ne parle point de l'Alménie ou de lempire des Hétécns. Il ne saurait

1. B. Stade, Geschichle des Volkes Israel. Berlin, 1887, in-8, t. I, p. 113-115. 
davantage ètre question ici de l'Arabie, habitée surtout par des nomades et qui n'a pas d'histoire avant Mahomet; tout au plus aurai-je à mentionner quelquesuns des produits que lui demandaient les nations roisines. Quant à l'Asie Mineure occidentale occupée par des peuplades indo-européennes ou par des colonies helléniques, elle est par là même, pour le moment, en dehors de mes recherches; j'en parlerai dans le second volume, lorsque je m'occuperai de la Grèce ancienne. 


\section{CHAPITRE II}

L'AGRICULTURE ET L'HORTICULTURE DANS L'ASIE ANTÉRieure. - les plantes dans l'alimentation et DANS L'INDUSTRIE dES NATIONS SÉMITIQUES.

La culture du sol est le premier trait qui marque le passage de la vie nomade à la vie sédentaire; dès que les habitants des déserts et des steppes en quiltent les régions inclémentes et stériles pour se fixer dans des contrées au sol fertile et au climat régulier, ils renoncent à la première pour se livrer à la seconde et se font agriculteurs. Les tribus sémitiques, qui sont restées en Arabie ou dans le désert de Syrie, ont gardé une existence nomade, celles qui s'établirent dans la Méso potamie méridionale ou dans les bassins de l'Oronte et du Jourdain, ainsi que dans la région du Liban, sont devenues depuis la plus haute antiquité sédentaires et agricoles. Tels nous apparaissent, aussi loin que nous pouvons remonter dans leur histoire, les Sumériens et les Sémites de la Chaldée, ou les Assyriens des bords du Tigre. La fertilité du pays où ils étaient venus se fixer les invitait a cette vie nouvelle; soumise ì des inondations longues et périodiques, la Basse-Mésopotamie d'ailleurs ne peut convenir à l'existence nomade du pasteur, tandis que ses grasses et fécondes alluvions se prètent à merveille aux traraux de l'agriculture. Populations sumériennes et sémitiques s'y lirrèrent 
arec ardeur et, graice ì leurs efforts, la contrée qu'elles étaient venues habiter devint un véritable jardin.

Nulle part, si ce n'est on Egypte, la culture était plus facile; il suffisait de retourner avec une espèce de looyau, trainé par des boufs', les alluvions molles et profondes de la plaine et de leur confier la semence, qui rendait au centuple, là où elle trouvait l'humidité nécessaire et n'était pas exposée à ètre emportée par les débordements du Tigre ou de l'Euphrate. Les habitants de la Basse-Mésopotamie nont jamais pu, comme le faisaient ceux de l'Égypte pour le Nil, laisser aux deux fleuves de lemr pays le soin d'arroser leurs terres; le sol est trop peu consistant, les crues sont trop subites ot leur débit trop variable pour qu'il soit possible de s'en rapporter à elles de ce rôle bienfaiteur ${ }^{2}$. Commençant d'ailleurs en mars pour se terminer au mois de mai, loin d'être favorables à la végétation, si elles n'araient été réglées, clles n'auraient pu que lui nuire; aussi, loin de les laisser venir à eux, les habitants maintenaient les eaux à distance pendant les mois d’inondation. Des canaux creusés de main d'homme recevaient alors le trop plein des deux fleuves; "subdirisés en fossés, les uns grands, les suivants moindres et finissant par n'être que de simples rigoles $)^{3}$, ils sillonnaient toute la Babylonie et y répandaient l'eau nécessaire à l'arrosage du sol; on l'y

1. Layard, Introduction à lhistoire du culte de Mithra, pl. XXXIV, no 5. - Rawlinson, op. laud., t. III, p. 18. - Maspero, Hisloire ancienne, t. I, p. 761.

2. Strabon, Geographia, lib. XVII, cap. I, 9. - Olivier, Voyage, t. II, p. 243.

3. Xénophon, Anubasis, lib. II, cap. Iv, 13. 
puisait, dit Hérodote ${ }^{1}$, à la main ou à l'aide de shadoufs.

Co système de canaux, dont la disposition frappa d'admiration les écrivains grecs, n'était pas de création récente; ils étaient l'œuvre des premiers rois historiques de la Chaldée et leurs successeurs les entretinrent soigneusement.

"Sinidinna, le puissant pourvoyeur sacré du pays d'Ourou, roi de Larsam, roi des pays de Soumir et d'Akkad, dit une inscription de ce prince ${ }^{2}$, le canal de délices qui fournit l'arrosage de la région, il le creusa. Il donna une eau perpétuelle, richesse permanente à sa capitale, à son peuple. »

Une autre roi de Larsam, Rimsin, suivit l'exemple de Sinidimna et " creusa le canal des dieux jusqu'au bord de la mer". Mais Hammourabi de Babylone surtout s'est rendu célèbre par ses travaux hydrauliques et son soin pour l'agriculture.

Je suis Hammourabi, dit-il dans une inscription ${ }^{3}$, le roi qui soumet à son obéissance les quatre régions... Lorsque les dieux Anou et Bel... eurent mis dans ma main le pouvoir, je creusai pour la population de Soumir et d'Akkad le canal de Hanmourabi, la richesse de la population, le véhicule des eaux d'abondance. Je rendis partout ses rives propres à l'agri-

1. Ilislorice, lib. I, cap. 193,1 . On voit sur un bas-relief de Koyoundjik une de ces shadoufs, auxquelles Hérodote donne le nom de «shavrirs. Layard, The monuments of $\mathrm{Ni}$ neveh, $2^{\mathrm{c}}$ série, p]. 15.

2. Fr. Lenormant, Éludes accadiennes, t. II, p. 340. - A. Delattre, Les travaux hydrauliques en Babylonie. (Revue des questions scientifiques, t. XXIV (an. 1888), p. 481.)

3. J. Menant, Inscriplions de Hammourabi, roi de Babylone. Paris, 1863 , in-8, p. 6 et 21 . - Id., Manuel de la langue asiygrienne. Paris, 1886, in-8, p. 306. - Friedrich Delitsch, Wo lag das Paradies. Leipzig, 1881, in-8, p. 191. - A. Delattre, op. laud., p. 482. 
culture. J'y élevai une paire de digues. Je fournis des eaux perpétuelles à la population de Soumir et d'Akkad... Je lui procurai la boisson et les aliments. Je l'établis dans la richesse et l'abondance. Je fis de sa demeure un séjour paisible.

Le monarque babylonien ne se borna pas à cette œurre d'irrigation générale; soucieux d'accroitre sans cesse le bien-ètre de ses sujets, il construisit encore un autre canal, destiné plus particulièrement à Sippara'; aussi se vante-t-il avec un juste orgueil d'" avoir fait pour le dieu Shamash ce que, de temps immémorial, nul roi parmi les rois de sa ville n'avait fait ». Il n'était pas le premier prince de Soumir et d'Akkad toutefois, qui eût cherché par des travaux de canalisation à favoriser l'a griculture dans son pays; il ne fut pas non plus le dernier ; vers la même époque un autre roi, Samisouïlouna, fit, lui aussi, creuser un canal, "sonrce de richesse ", auquel son nom resta attaché ${ }^{2}$. Deux documents, qui remontent au règne de Mardoukidinakhi, roi de Babylone, contemporain de Tiglathphalasar, parlent, l'un du Nir Zalmani, l'autre du Nìr Mì-Dandan; ce dernier, " canal des eaux puissantes, qui vivifient tout être animé ", fertilisait, à ce qu'il semble, la partie de la rive gauche du Tigre, en aval du Diyalah ${ }^{3}$.

Après la conquète de la Babylonie par les rois de Ninive, les entreprises nationales de canalisation furent arrêtées dans ce pays; les vainqueurs toutefois ne laissèrent pas tomber en ruines ce qui existait; on

1. H. Winckler, Einige neuverö/fentl. Texte, etc. (Zeitschrift für Assyriologie, t. II (an. 1887), p. 119-120.)

2. A. Delattre, op. laud., p. 485.

3. J. Oppert et J. Henant, Documents juridiques de l'Assyrrie et de la Chuldée. Paris, 1877, in-8, p. 83 et $92 .-$ A. Delattre, op. lauud., p. 486-487. 
voit Sargon, en particulier, faire rétablir le canal à moitié comblé de Borsippa'. Mais après l'affranchissement de Babylone, les princes de la nouvelle dynastie qui régna en Chaldée renouèrent la tradition interrompue des premiers rois; ils restaurèrent les anciens canaux et.en créèrent de nouveaux. C'est ainsi que le premier d'entre eux, Nabopolassar, rappelle, dans une inscription ${ }^{2}$, qu'il avait fait creuser à nouveau le canal de Sippara, œuvre de Hammourabi, et " y arait amené l'eau en abondance ». Les successeurs de Nabopolassar suivirent son exemple; " le fossé oriental de Babylone en mauvais état depuis des jours reculés " fut réparé et consolidé tour à tour par Nabuchodonosor et Nériglissor, et "des eaux perpétuelles furent établies pour le pays ". Babylone et sa banlieue étaient, nous le voyons d'après les actes de Nabonide, arrosées par six canaux, sans parler des fossés et des rigoles secondaires, qui s'y rattachaient et portaient partout l'abondance et la salubrité ${ }^{3}$.

Pourvues ainsi de l'eau nécessaires à la végétation, jouissant d'un climat privilégié, les campagnes de la Babylonie, avec leur sol gras et profond, étaient d'une fertilité qu'ont célébrée à l'envi tous les écrivains de l'antiquité.

De tous les pays que nous connaissons, remarque Héro. dote ${ }^{4}$, la Babylonie est, sans contredit, le meilleur et le plus fertile en fruits de Cérès... Le sol est si propre à toutes sortes de grains, qu'il rapporte toujours deux cents fois autant qu'on

1. A. Delattre, op. laud., p. 488.

2. H. Winckler, Ein Texl Nabopolassars. (Ibid., p. 69-75.)

3. A. Delattre, op. laukl., p. 490-496.

4. Hisloric", lib. I, cap. 193, 3. "Ager totius Asice fertilissimus", clit de son côté Pline, IIisloria naluralis, lib. VI, cap. 26. 
a semé, et que, dans les années où il se surpasse lui-mème, il rend trois cents fois autant qu'il a recu. Les feuilles du froment et de l'orge y ont bien quatre doigts de large'. Quoique je n'ignore pas a quelle hauteur y viennent les tiges de millet et de sésame, je n'en ferai point mention, persuadé que ceux qui n'ont point été dans la Babylonie ne pourraient ajouter foi à ce que jai rapporté des grains de ce pays.

En Babylonie, dit de son còté Théophraste ${ }^{2}$, on coupe deux fois les moissons, et on les fait paître par les troupeaux pour en arrèter la croissance excessive et permettre aux chaumes de se développer ; après cela, ajoute-t-il, " on récolte encore dans les terres mal travaillées cinquante pour un et cent pour un dans les terres bien cultivées ". Strabon affirme de son côté ${ }^{3}$, ce qui confirme et dépasse même le dire d'Hérodote, que " le rendement d'un champ d'orge en Babylonie était de trois cents pour un ". Les récits des voyageurs modernes ne donnent pas une idée moins haute de la fertilité de la Basse-Mésopotamie ${ }^{4}$. Mais quels grains produisait ce pays ainsi favorisé du ciel? Outre le froment et l'orge, qui semblent bien avoir été, depuis les temps les plus reculés, les cultures principales de la Babylonie, Hérodote y fait croitre aussi le millet et le sésame. Si ces plantes ont été, comme on n'en peut guère douter, cultivées dans cette contrée, vu leur origine étrangère, il est probable que leur culture y

1. Un cylindre du IIusée du Louvre représente des tiges de froment remarquables par leurs larges feuilles et dont les épis dépassent la tête des bœufs qui se trouvent à côté. J. Menant, Les pierres gravies de la IIaute-Asie. Recherches sur la glyptique orientale. Paris, 1883, in-8, p. 208.

2. IIistoria plentarum, lib. VIII, cap. 7. 4.

3. Geograplia, lib. XVI, cap. 1, 1'.

i. Cf. Rawlinson, op. laued., t. I, p. 32. 
élait relativement plus récente; celle du sésame, en particulier, ne peut remonter qu'à l'époque où les monarques assyriens et chaldéens entrèrent en relation avec l'Inde; des échanges commerciaux, il est vrai, ont dû avoir lieu entre la péninsule hindoustanique et le bassin de l'Euphrate dès les temps les plus reculés ${ }^{1}$.

Les habitants de la Babylonie cultivaient-ils d'autres plantes alimentaires ou industrielles que celles dont parle Hérodote? Cela est vraisemblable, mais les historiens anciens ne nous l'apprennent pas et les inscriptions ne nous renseignent pas davantage à cet égard ${ }^{2}$; Bérose sans doute fait mention de l'ochros parmi les plantes cultivées en Chaldée ${ }^{3}$; mais la nature véritable de l'ochros n'est pas connue d'une manière certaine, et il semble être bien plutôt une plante fourragère probablement une gesse ${ }^{4}$, - qu'une plante industrielle ou alimentaire. Quant " aux fruits de toute sorte " qu'on aurait, d'après l'historien de Babylone, trouvés aussi en Chaldée, ils ne pouvaient, en supposant celte assertion exacte, qu'y aroir été importés à une date peu ancienne, et l'on doit croire même qu'ils étaient plus rares et moins nombreux que ne le reut dire Bérose; sinon on ne s'expliquerait pas qu'Hérodote eût pu affirmer " qu'on n'essayait même pas on Babylonie de faire porter a la terre des figuiers, des vignes et des oliviers ". Il faut remarquer cependant que sur un cylindre chaldéen du Musée de la Haye", qui représente un jardin dans lequel se trourent debout trois

1. A. de Candolle, op. laud., p. 339. Cf liv. I, ch. II., p 48.

2. Hommel, op. laud., p. 191, note.

3. Fragmenta, lib. I, 2. Ed. Müller-Didot.

4. Le Lalhyrus cicera ou l'Ervum ervilia.

5. J. Nenant, Les pierres graveies de la IIaute-Asie, p. 191. 
personnages auprès d'un dattier, on voit, outre cet arbre et un autre dattier ou palmier plus petit, un arbuste qui ressemble à un figuicr, ainsi qu'un arbre à triple tige, mais sans caractère distinctif. Cette représentation n'en pronve pas moins que des arbres a fruits autres que le dattier croissaient autrefois, comme aujourdhui, en Babylonie, bien que peut-ìtre en petit nombre.

Le dattier, au contraire, y abondait; toute la plaine de la Basse-Mésopotamie, nous apprend encore Hérodote ${ }^{1}$, en était plantée, et Ammien Marcellin parle de rastes forèts de dattiers, qui s'étendaient du nord de cette contrée aux bords du golfe Persique ${ }^{2}$. Nous rerrons à combien d'usages variés servait cet arbre précieux. Le climat chaud de la Babylonie et l'humidité, que le sol lui fournissait en abondance, favorisaient sa régétation et il y domnait les fruits les plus savoureux. Le dattier est-il indigène dans la Chaldée, comme il l'est en Arabie et dans la Carmanie? On peut le croire, mais il y fut aussi cultivé de temps immémorial. Des préceptes d'agriculture rédigés en accadien imposent au fermier l'obligation de planter des dattiers et de les arroser $^{3}$. Des tablettes découvertes par M. de Sarzec a Telloh font mention d'un sanctuaire que le patési de Lagash, Entéména, éleva à la déesse Nina, "qui fait croitre les dattes" ". Les mèmes tablettes nous apprennent que ce chef religieux avait fait deux

1. Iislorice, lib. 1, cap. 193.

2. Res gestue, lib. ХXIV, cap. 3. 12.

3. G. Bertin, Ancienl Babylonian argriculural precepts. (Records of the Past, t. III, p. 96.)

4. L. Heuzey, Une villa royale en Chaldie. (Comples rendus de l'Acadimic des Inscriplions el Belles-Lellres, 8 nov. 189'.) 
grandes plantations qui, suivant toute apparence, étaient composées de palmiers. On obtenait d'urdinaire les dattiers de semis; mais on les propageait aussi a l'aide des drageons, qui poussent autour du collet de la racine ${ }^{1}$. S'ils ne l'ont pas inventée, comme on l'a prétend ${ }^{2}$, les habitants de la Basse-Mésopotamie ont connu de bonne heure la fécondation des fleurs femelles de cet arbre si utile. On la trouve représentée sur des cylindres chaldéens ainsi que sur des bas-reliefs de l'Assyrie ${ }^{3}$.

La partie de l'Élam ou Susiane voisine du Tigre et de la mer ressemblait à la Chaldée par ses produits agricoles, aussi bien que par sa constitution géologique, et sa fertilité n'était pas moins grande que celle de cette contrée; le froment et l'orge, rapporte Strabon $^{4}$, y rendaient cent et même deux cents pour un. Les dattiers y croissaient aussi en abondance". Quand on quittait la basse plaine, pour s'élever dans la région montagneuse traversée par le Pasatigris et le Choaspès, les produits du sol changaient, arec sa nature, et ils se rapprochaient de ceux de la Perse et de l'Assyrie.

Les produits de cette dernière contrée différaient beaucoup de ceux que nous avons rencontrés dans la Chaldée; le climat y était d'ailleur's moins chaud, surtout dans la partie montagneuse du nord-est et le sol tout autre; lá plus de ces alluvions grasses de la Basse-Mésopotamie, mais un terrain de formation ter-

1. Théophraste, IIistoria planlarum, lib. II, cap. 6, 1 et 2.

2. Cf. plus haut, liv. l, chap. IV, p. 105.

3. L. B. Tylor, The winged figures of the Assyrian and other. ancient monuments. (Proceedings of the society of Biblical archarology, t. XII (an. 1890), p. 383-393).

4. Geographia, lib. XV, cap. 3, 11.

5. Ibid., lib. XVI, cap. 1, 5. 
tiaire, que l'absence de pluies pendant plusieurs mois de l'année rendait impropre à la culture, si on ne lui fournissait l'eau que le ciel lui refusait. Aussi l'arrosage artificiel y était-il une nécessité bien plus encore qu'en Chaldée; les rois d'Assyrie le comprirent. Tout guerriers quils étaient, ces princes portaient un rif intérèt à l'agriculture.

Sargon le Grand se représente, dans une inscription", comme " un roi qui a mis son soin à rendre à la culture les territoires voisins, à faire des plantations de roseaux sippatu, à faire produire des collines rocailleuses où de temps infini ne poussait aucune plante, qui s'est attaché à faire porter des moissons à maint lieu désert, lequel n'avait point eu de canal d'arrosage sous les rois précédents, à ouvrir les lits comblés des cours d'eau et à abreuver le pays d'en bas et d'en haut d'une eau abondante, semblable à la masse des flots de la mer ", enfin comme " un roi qui, devenu grand dans le conseil et par la sagesse et plein de prudence, s'attache à remplir les greniers du raste pays d'Ashshour de provisions et de vivres en abondance... à ne pas laisser renchérir l'huile, la vie (?) des hommes, à fixer le prix du sésame comme du blé."

On voit Ashshourbanipal rappeler aussi, dans une inscription ${ }^{2}$, comme un de ses titres de gloire que, "les moissons réussirent et que l'abondance fut grande sous son règne ». "Le froment, ajoute-t-ii, s'élera de cinq coudées sur sa racine, l'épi atteignit cinq sixièmes de

1. Inscription du cylindre, 34-41. David-Gordon Lyon, lieilschriflexle Sargon's Königs von Assyrien (722-705 v. Chr.), p. 35. (Assyriologische Bibliollet, t. 1 , an 1883.)

2. A. Delattre, op.laud., p. 499. 
coudées. L'orge (?) fut abondant. Les arbres à fruit ne cessèrent de produire. Los oliviers prospérèrent. " Rhapsacès, le général de Salmanasar, vantait aux Juifs l'Assyrie comme une terre de froment, d'huile et de vin'.

Pour obtenir cette fortilité il fallait procurer au sol l'humidité qui lui faisait défaut. Afin de remédier a cette indigence, les monarques assyriens, comme les rois de Babylone, couvrirent leur pays de canaux qui portaient partout l'eau nécessaire à la végétation. Dès le commencement du xul siècle avant notre ère Ashshourdanan avait fait creuser un canal sur la rive droite du Tigre, pour arroser les campagnes de la ville d'Ashshour, comme nous l'apprend une inscription de son petit-fils 'Tiglathphalasar $I^{2}$.

Le canal qu'Ashshourdanan, roi du pays d'Ashshour, avait creusé - l'ouverture de ce canal n'existait plus. Pendant trente ans, les eaux n'y avaient plus coulé. Je fis renouveler et creuser l'ouverture de ce canal, j'y jetai l'eau et je plantai des jardins.

Trois siècles plus tard, Ashshournazirpal, voulant relever la prospérité de Kalakh, qui tombait en ruines, ne trouva pas de meilleur moyen que de donner à cette ville et à sa banlieue un canal, "véhicule de fécondité ". Sennachérib poursuivit avec zèle ces travaux de canalisation et d'irrigation. "Pour féconder les champs, dit-il dans une inscription", je dérivai du Khonsour le canal de Kharrou. Je fis couler des eaux perpétuelles dans son lit ". Et dans une autre ins. cription *, il se vante d'avoir " abreuvé la campagne de

1. 2 Regna, cap. XVIII, vers. 32.

2. A. Delattre, op. laud., p. 501.

3. Cylindre de Bellino, ap. A. Delattre, op. laud., p. 502.

4. H. Pognon, L'inscriplion de Bavian. Paris, 1879, in-8, p. 9. 
Ninive, qui dépérissait par le manque d'eau ", et il rappelle les dix-huit canaux, dont il avait "dirigé les eaux ver's le fleuve Khonsour ", ainsi que le fossé qu'il avait fait " creuser depuis le territoire de Kisiri jusqu'à Ninive ".

Ces textes peuvent nous donner une idée des travaux considerables de canalisation entrepris par les rois d'Assyrie pour fournir à leur pays l'cau nécessaire à l'agriculture. Ainsi arrosée, l'Assyrie devint, comme la Chaldée, d'une merveilleuse fertilité. On y faisait deux récoltes par an, une au printemps et l'autre à l'automne'; mais la culture de la terre y demandait, ce semble, plus de travail qu'en Babylonie; la charrue dont on se servait pour retourner le sol, quoique simple encore ${ }^{2}$, est plus solidement construite que celle des entailles chaldéennes. Mais quelles espèces végétales cultivait-on en Assyrie? Nous avons peu de renseignements à ce sujet. On a supposé, non sans raison ${ }^{3}$, qu'on y trouvait les céréales cultivées en Babylonie d'après Hérodote, qui ne parait pas distinguer très nettement l'agriculture des deux pays, c'est-idire le froment, l'orge et le millet. M. Layard parle d'un champ de millet qu'on verrait près d'une forteresse sur un bas-relief de Koyoundjik; mais on ignore oi se trouvait cette forteresse. Plusieurs inscriptions assyriennes font expressément mention de la culture du

1. J. Oppert et J. Nenant, Documents juridiques, no 31, p. 219.

2. Ph. Henry Gosse, Assyria; her manners and cusloms, ar's and arms. London, 1852, in-8, p. 567. - Rawlinson, op. laud., t. 1, p. 567 .

3. Rawlinson, op. laud., t, I, p. 272.

4. Layard, Niniveh and its remains. London, 18's, in-8, t. II, p. 140 . 
froment et probablement de l'orge ${ }^{1}$; celles de Sargon et de Sennachérib parlent aussi de la culture du sésame.

Ce ne sont pas là les seules plantes alimentaires ou industrielles dont on a jusqu'ici rencontré la mention dans les textes cunéiformes; mais on n'en a encore identifié qu'un bien petit nombre. Curieux, comme ils l'étaient, des produits de l'étranger, que plusieurs de leurs rois se vantent d'avoir importés dans leur pays, les Assyriens ne purent manquer, quand ils eurent étendu leurs relations commerciales et leurs conquêtes dans presque toute l'Asie antérieure, d'acclimater chez eux quelques-unes des plantes utiles à l'alimentation ou à l'industrie, cultivées dans les pays, visités par leurs armées ou par leurs marchands; tels'que l'oignon, l'ail, le poireau, diverses légumineuses et cucurbitacées -- laitue, bette, rave, radis, concombre, coloquinte des condiments comme l'aneth, la coriandre, l'assa fotida; enfin le lin, la luzerne, le safran et peut-être le carthamme? .

Ils acclimatèrent surtout aussi, les textes nous l'apprennent, les arbres à fruits ou d'ornement des pays qu'ils parcoururent.

Des cèdres, des ourlarinu, des allakani, disait déjà Tiglathphalasar I à la fin du x.ı siecle $^{3}$, arbres des contrées que j'avais soumises, que personne parmi les rois mes pères n'avait

1. Par exemple l'inscription de Sennachérib à Bavian et celle d'Ashshourbanipal.

2. B. Meissner, Babylonisshe Pflanzennamen. (Zeitschrift f. Assyriologie, t. VI (an. 1891), p. 289-298.)

3. F. Lenormant et E. Babelon, Histoire ancienne. Paris, 1889, in-', t, V, p. 2'. - A. II. Sayce, Inscription of Tiglath-Pileser. I, liing of Assyria. (Records of the past, t. I, p. 115.) - A. Delattre, op. laud., p. 500. Lenormant traduit ourliarimu et allukani par" "pins et lentisques ". - Keilschrifliche Bibliothek, t. I, (an. 1889), p. 41. 
plantés, je les pris et les plantai dans les jardins de mon pays. Des plantes de jardin que mon pays ne prorluisait point, je les pris et les plantai dans les pares de mon pays.

Ashshournazirpal parle également de plantations " d'arbres fruitiers de toute sorte et de vignes " qu'il arait faites sur les bords du canal de Kalakh ${ }^{1}$. Sennachérib sétend aussi avec complaisance, dans l’inscription malheureusement mutilée de Bavian, sur les plantations de rignes, de "plantes de tous lieux ", de forèts (?) faites par lui dans les environs de Ninive?. Il est aussi question, sur le cylindre de Bellino ${ }^{3}$, probablement de plants d'oliviers, qui se trouvaient dans la banlieue de la capitale.

Sur les bas-reliefs de l'époque des Sargonides on voit sourent des vignes, soit isolées, comme une vigne de Koyoundjik qui grimpe sur un pin ${ }^{4}$, soit formant un berceau de verdure, tel que celui à l'ombre duquel l'artiste assyrien a représenté Aslıshourbanipal couché ${ }^{5}$. On y roit aussi des palmiers; le dattier fut, en effet, cultiré en Assyrie, comme il l'est encore aujourd'hui; mais ses fruits, nous le savons par le témoignage des anciens ${ }^{6}, y$ étaient d'une qualité inférieure; ils ne mùrissent mème plus au nord du confluent du petit Zab et du Tigre ${ }^{\top}$. Des bas-reliefs, reproduits par

1. A. Delattre, op. laud., p. 501.

2. Ii. Pognon, op. laud., p. 11.

3. Ap. A. Delattre, op. laud., p. 502

4. Rawlinson, op. laud., t. 1, p. 353. On voit aussi deux vignes couvertes de raisins sur un bas-relief reproduit par Layard. Nineveh and Babylon, p. 341.

5. Musće britannique. Rawlinson, op. laud., t. I, p. 493. Perrot et Chipiez, Hisloire de l'arl, t. II, p. 107 et 652.

6. Pline, II istoria naturalis, lib. XIII, cap. 9.

7. A. H. Layard, Nineveh and its remains, t. II, p. 423. Layard ajoute, il est vrai, que cela tient au manque de soin. 
Layard ', représentent des grenadiers; on voit aussi des fruits de cet arbre portés par des serviteur's sur divers bas-reliefs de Koyoundjik; on ne peut guère douter d'ailleurs que cet arbre, indigène dans les contrées voisines de l'Assyrie, n'y ait été cultivé de bonne heure. Il faut en dire autant du figuier, qui croît spontanément dans les mêmes pays. Il a été représenté souvent sur les bas-reliefs assyriens ${ }^{2}$. On peut admettre encore que l'amandier, le mûrier à fruits noirs, ainsi pent-être que le caroubier, le pistachier, qui vient à l'état saurage dans la région montagneuse d'Orfah à Térek ${ }^{3}$, et le noyer furent également plantés dans les jardins ou dans les parcs assyriens. L'olivier y était cultivé dès longtemps; le cédratier, au contraire, n'y prit vraisemblablement place qu'après la conquète perse, et l'abricotier, ainsi que le pècher, encore plus tard.

Y cultivait-on aussi des arbres d'ornement? Il est impossible d'en douter; l'inscription de Tiglathphalasar, citée plus haut, en mentionne trois espèces, malheureusement non identifiées avec certitude. Dans une de ses inscriptions ${ }^{4}$, Sargon nous apprend qu'il " créa un grand parc " et qu'il " y fit planter toute espèce d'arbres du pays des Khatti et toutes les plantes de la montagne ". Quels étaient ces arbres et ces

1. The monuments of Nineveh, ser. II, pl. 15. - E. Bonavia, The flora of the Assyrian monuments, p. 12.

2. Layard, The monuments of Nineveh, ser. II, pl. 15, 20, 22. - Bonavia, The flora, p. 14. On voit aussi un figuier sur une brique émaillée de Khorsabad. Perrot et Chipiez, op. laud., t. II, pl. XV.

3. Boissier, Flora orientalis, t. II, p. 6. - Rawlinson, op. laud., t. I, p. 217.

4. Inscription du laurean, 10. D. G. Lyon, op. laud., p. 43. 
plantes? Le monarque assyrien n'a pas cru devoir le dire; matis les bas-reliefs assyriens, encore que représentant sourent des paysages non mésopotamiens, nous en font comnaître quelques-uns; il y avait d'abord les cèdres, dont parle Tiglathphalasar, des pins, dont il parait aussi faire mention, et qu'ont si souvent représentés les artistes assyriens soit alternant avec des palmier's, soit formant des espèces de bosquets ${ }^{1}$. M. Bonaria a supposé qu'ils appartenaient à l'espèce Pimus brutic ${ }^{2}$; mais la forme qu'ils affectent n'a rien de caractéristique, et tous n'ont pas d'ailleurs le mème aspect $^{3}$. On ne peut douter non plus que le cyprès, si répandu dès la plus haute antiquité dans toute l'Asie. antérieure, fut également cultiré dans les parcs assyrienst. On y plantait aussi sans aucun doute des arbres fruilicrs en même temps que des arbres purement d'ornement.

Si nous ne pourons dire au juste quels étaient les arbres cultivés dans les jardins mésopotamiens, un basrelief de Koyoundjik ${ }^{\circ}$, qui représente un parc du temps cies Sargonides, nous donne une idée assez juste de ce qu'était à cette époque un jardin de plaisance assyrien. Les arbres du parc de Koyoundjik nous apparaissent, comme dans les jardins pharaoniques, disposés en

1. Botta et Fiandin, Monument de Ninive. Paris, 1849, infol., t. II. p. 108-111, salle VII.

2. The fora of the Assyrian monuments, p. 29.

3. Les conifères des planches 46 et 81 , en particulier, des Monuments of Nineveh de Layard ne sont pas de la même espè‘e, ni peut-être dı mème genre.

4. Les conifères du jardin d'Ashshourbanipal, entre autres, me paraissent être des cyprès. Rawlinșon, t. I, p. 493.

5. Layard, Nincvele and Babıllon, j. 232. - Rawlinson, op. laud., t. I, p. 229. 
rangs, composés les uns d'arbres de mème taille et probablement de même espèce, les autres d'arbres alternativement grands et petits, de port et on peut ajouter d'espèce différents; des canaux sillonnent ce parc, au milieu duquel est aménagée une vaste pièce d'eau remplie de poissons.

Quand l'espace manquait pour faire ces parcs étendus, on se contentait de " jardins suspendus », établis sur des espèces de terrasses, supportées par des colonnes ou des arcades ${ }^{1}$. Sur un bas-relief du palais de Sennachérib à Koyoundjik on voit à côté d'un temple une rangéo de pins ou de cyprès, alternant avec des arbres qui ressemblent à des grenadiers, se dresser sur une terrasse que supporte une série d'arcs aigus². Cette invention devait passer en Babylonie, après que cette province eut recouvré son indépendance. Lorsque Nabuchodonosor le Grand eut rebàti sa capitale et l'eut entourée de ces murs, flanqués de tours, qu'on considéra comme une des sept merveilles du monde, il y construisit également un jardin suspendu non moins admiré. “C'est, dit Strabon" ${ }^{3}$ un immense carré de quatre plèthres de côté, composé de plusieurs étages de terrasses supportées par des arcades, dont les voûtes reposent sur des piliers de forme cubique. Ces piliers sont creux et remplis de terre, ce qui a permis d'y faire venir les plus grands arbres. " Malheureusement, le géographe grec ne nous apprend pas de quelle espèce étaient ces arbres. On ne peut guère douter qu'il ne s'y trouvât des pal-

1. Rawlinson, op. laud., t. I, p. 585. - Perrot et Chipiez, op. laud., t. II, p. 445.

2. Musée britannique. Rawlinson, op. lauel., t. I, p. 310. Perrot et Chipiez, op. lanul., t. II, p. 153.

3. Geogreaphia, lib. XVI, cap. I, 5. 
miers, qu'on roit d'ailleurs si sourent dans les pares, représentés sur les bas-reliefs. Une tradition veut aussi qu'un athel ou tamaris, qui se dresse au bord septentrional des ruines de Babylone et au tronc duquel, diton. Ali attacha son cheral après la défaite des ennemis du Prophète à Hillah, ait été un des arbres du jardin suspendu de l'antique cité .

Cultivait-on aussi des fleurs dans les jardins et les parcs de l'Assyrie ou de la Chaldéc? On peut le croire, il en fut ainsi du moins dans les derniers temps; un contrat contemporain d'Ashshourbanipal fait mention d'un jardin portant des fleurs tous les ans . Il semble mème y ètre question d'un champ de parfums ${ }^{3}$. Dans un autre acte, contemporain d'Ashshournirar et par conséquent plus ancien, il est déjà parlé aussi de la vente d'un champ produisant neuf' apha de parfums." Il semble donc bien que les Assyriens et sans doute aussi les Chaldéens du nourel Empire aient cultivé des fleurs d'agrément ou aromatiques. Tels furent, nous le savons", l'hysope et le thym; tel fut sans doute aussi, à l'époque assyrienne, le lotus; sur les bas-reliefs de Ninive, nous royons le roi, ici sacrifiant, là se rendant à la chasse, une tige de lotus à la main ${ }^{6}$; telles durent être probablement encore ces fleurs placées dans des vases,

1. Layard, Discoveries, p. 507.

2. Kiru Labriru shushu. J. Oppert et J. Menant, Documenls juridiques, p. 198.

3. Ekil al-ru. MM. Oppert et Menant toutefois remarquent, p. 201, que le mot al'u pourrait encore s'expliquer par troupeau.

4. Bit IXY lia at-ru. Ibid., p. 150. MN. Oppert et Menant rapprochent, p. 153, le mot atri de l'arabe atar et se demandents'il ne s'agit point de «roses, de safran ou d'autres produits semblables».

5. B. Meissner, op. laud. (Zeilschrift /ür'Assyriologie, t. VI, p. 292).

6. Botta, Monument de Ninive, t. II, pl. 105 et 113, salle 7 . I. 
que des serviteurs, sur les mêmes bas-reliefs, portent dans la salle du festin ${ }^{1}$. Mais quoi qu'il en soit, on peut affirmer que les plantes d'ornement cultivées par les Assyriens et les Chaldéens furent toujours en petit nombre. Comme tous les peuples anciens, ils durent se contenter avant tout de celles qui, en si grande abondance, croissaient au printemps dans lem pays.

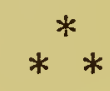

Si la connaissance que nous avons de la culture des champs et des jardins dans la Chaldée et l'Assyrie est à bien des égards incomplète, nous sommes un peu mieux renseignés sur ce qu'elle était en Syrie ou du moins dans une partie de cette contrée, la Palestine. J'ai dit plus haut quelles étaient la varièté et la richesse de la flore syrienne. Si l'on excepte la région aride du sud et de l'est limitrophe du désert et la région boisée et couverte de pâturages des montagnes, tout le pays était de la plus grande fécondité et cultivé avec le plus grand soin; la fertile vallée de l'Oronte, le littoral de la Phénicie aux jardins s'élevant en étages sur les coteaux, la plaine si bien arrosée de Damas, la Cœlé-Syrie, le plateau de Giléad, également propre, avec ses magnifiques forèts et ses riches prairies, à l'élevage du bétail et au labourage ${ }^{2}$; la plaine fécondée par les eaux du Kishon, celle de Sharon au sud du Carmel, pour me borner aux plus célèbres, invitaient les peuples qui s'y établirent à la vie sédentaire et agricole. Nous ne savons rien du parti que les popu-

1. Layard, The monuments of Nineveh, ser. II, pl. 8, 1.

2. B. Stade, Geschichte des Vollies Israël. Berlin, 1887, in-8, p. 107. 
lations primitives tirèrent du sol; mais les tribus cananéennes et amorbéennes, qui les asservirent ou les remplacèrent, le cultivèrent avec le plus grand soin.

Si l'on admet avec Hommel ${ }^{1}$, que les Héroushâ, contre lesquels Ouna, général de Pépi, pharaon de la $V I^{\mathrm{e}}$ dynastie, fit la guerre et dont il se vante d'avoir coupé les figuiers et les vignes, habitaient le sud de la Palestine, on voit que ces arbres à fruit auraient été cultivés dans cette contrée trois mille ans et plus avant notre ère, ce qui n'aurait d'ailleurs rien qui doive surprendre. Ils l'y étaient certainement du moins

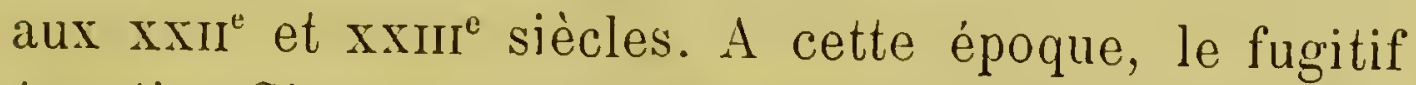
égyptien Sinouhit parle des vignes et des figuiers qu'il avait vus en Kadouma - le pays d'Édom. - "Le vin $y$ est en plus grande quantité que l'eau, dit-il ${ }^{2}$; nombreux y sont les fruits de moringa et toutes les productions des arbres; on y a de l'orge et du froment en abondance.»

Quand un peuple, qui habitait à la limite du désert, s'adonnait ainsi déjà à l'agriculture, on doit penser que les tribus qui occupaient les plaines et les vallées fertiles du pays de Canaan s'y livraient bien plus encore. Le Deutéronome ${ }^{3}$ représente cette contrée comme une terre de blé - khittah - et d'orge - coorah. - La culture de ces deux céréales y devait remonter à la plus haute antiquité. Celle de l'épeautre - koussemeth - et du millet - dokhan - y est peut-être moins

1. Die semitischen Völker und Sprachen. Leipzig, 1883, in-8, p. 105. - Maspero, Hisloire ancienne, t. I, p. 420.

2. Maspero, Histoire ancienne, t. I, p. 471. M.Maspero a traduit par olives, au lieu de noix de moringa.

3. Cap. v, verset 8 . 
ancienne. A côté de la culture des céréales avait pris place celle des plantes alimentaires et industrielles. Les lentilles - adashim - y étaient connues dès l'époque la plus reculée'; les fèves - pol - ne durent guère y être cultivées moins anciennement. Si les Hébreux regrettaient dans le désert les oignons, l'ail et les poireaux, les concombres et les pastèques d'Égypte, on peut affirmer qu'ils les retrouvèrent dans le pays de Canaan. La culture du lin, nous le savons, existait aussi déjà dans cette contrée. Ce fut sous des tiges de cette plante, entassées sur son toit, que Rahab cacha les deux espions envoyés par Josué à Jéricho et que le roi de cette ville voulait faire périr ${ }^{2}$.

Reçues par les Hébreux, ces diverses cultures furent soigneusement conservées et développées par eux. Sous leur domination la Judée devint-un vrai grenier d'abondance. Salomon fournissait par année ḋ son allié Hiram, roi de Tyr, 2,000 cors ou mesures de froment, et il donna 20,000 cors de froment et autant d'orge aux ouvriers phéniciens chargés de couper pour lui des cèdres sur le Liban ${ }^{3}$. La Judée resta longtemps le grenier où Tyr s'approvisionnait". Les pays des Philistins et des Ammonites n'étaient pas moins féconds en céréales. On voit le roi des Ammonites payer ì Joatham un tribut annuel de 10,000 cors de blé et d'orge ". Les Hébreux semaient le froment et l'épeautre, ainsi que l'orge, avant l'hiver; au mois d'arril ils

1. Il en est question déjà dans la Genèse, chap. Xxy: vers. 32.

2. Jesus Nave, cap. II, vers. 6.

3. 2 Paralipomena, cap. II, vers. 10.

4. Ezechicl, cap. xxvi, vers. 17.

5. 2 Paralipomera, cap. xivir, vers. 5. 
faisaicnt la récolte de l'orge, celle du froment et de lépeautre cinq ou six semaines plus tard. Les gerbes étaient aussitôt après portées sur l'aire et foulées aux pieds des boufs et des ànes '. Arec ces céréales on semait encore en automne d'autres plantes, telles que la nielle ou carri - qezalih - et le cumin -- kammon - " Quand le laboureur a aplani le champ, dit Ézéchiel ${ }^{z}$, ne ra-t-il pas semer la nielle, répandre le cumin, planter le froment en lignes, l'orge au lieu désigné, l'épeautre tout autour? "

S'il n'est point question du millet dans ce passage d'Ézéchiel, c'est que cette céréale se semait probablement à une époque différente; mais la culture de cette graminée, si elle était moins importante que celle des autres céréales, n'en était pas moins, nous le sarons par le témoignage du mème prophète ${ }^{3}$, pratiquée en Judée. L'usage considérable du lin - pishtah - dans l'industrie doit faire supposer que les Hébreux n’avaient fait que développer la culture de ce précieux textile. Se lirraient-ils aussi à celle du carthame, du sésame et du ricin? Cela n'est pas impossible; mais aucun texte ancien ne nous renseigne à cet égard, d'où on pourrait inférer que cette culture, si elle exista en Judée, n'y prit peut-être naissance qu'assez tard. Il semble bien, au contraire, qu'on y ait assez tôt, ainsi qu'en Assyrie, cultivé le safran - héb. karkom, ass. Karkouma" - dont on retirait une couleur jaune très recherchée.

Les oignons - betsalim, - l'ail - shoumim, - le

1. B. Stade, op. laud., t. I, p. 369.

2. Cap. xxvir, vers. 25-27, trad. d'Ed. Reuss.

3. Cap. IV, vers. 9.

4. Muss-Arnolt, Transaclions, t. XXIII (an. 189 ), p. 116. 
poireau - khatsir, - les pastèques - abattikhim, ainsi que les concombres ou melons chate-lisshouim, - durent être l'objet. d'une culture assidue en Judée, comme en Égypte, mais les anciens textes ne nous en font pas connaitre la nature et l'importance; ils ne nous apprennent rien non plus de celle des lentilles, des fèves et des autres légumes. Les Hébreux connaissaient dès longtemps la coriandre, à laquelle font allusion l'Exode et le livre des Nombres ${ }^{1}$; ils durent cultiver de bonne heure ce condiment, ainsi que l'aneth, la menthe et la rue, dont on voit les Pharisiens payer la dime, avec celle des autres plantes de leurs jardins ${ }^{2}$.

Il est probable que les plantes fourragères ne furent pas plus inconnues aux habitants de la Syrie qu'elles ne l'étaient aux Égyptiens et probablement aux habitants de la Mésopotamie; mais nous ne connaissons rien de leur, culture. Nous ne savons rien non plus de celle du roseau, qui, șil croit à l'état spontané en Syrie ${ }^{3}$, devait y ètre cultivé aussi. Nous ne sommes pas mieux renseignés au sujet du papyrus - gome, qui y fut probablement importé, mais qui aussi s'acclimata sur les bords marécageux des lacs de Tibériade et de Mérom, auprès de Jaffa et sur les rives du Nahrel-Aoudja, où on le trouve encore aujourd'hui'.

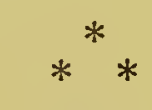

Les arbres à fruits furent dès longtemps cultivés

1. Cap. Xvı, vers. 31. - Cap. XI, vers. 7.

2. Mathias, cap. xxiII, vers. 23. - Lucas, cap. XI, vers. 42.

3. Boissier, Flora, t. V, p. 564 .

4. Boissier, Flora, t. V, p. 375. 
dans le pays de Canaan et des Amorrhéens; plusieurs y étaient indigènes, comme l'amandier, le poirier ${ }^{1}$, le caroubier et probablement le figuier et l'olivier; d'autres, qui croissaient dans les contrées voisines, y pénétrèrent de bonne heure; les Hittites en importèrent probablement quelques-uns avec eux, quand ils s'ètablirent dans le bassin de l'Oronte; les Araméens durent aussi en introduire plusieurs dans le Hauran, lor'squ'ils vinrent s'y fixer. Aussi la plupart des arbres fruitier's de l'Asie antérieure que nous arons vus cultivés dans la vallée du Nil, l'ont été, et sans doute plus anciennement, dans le pays de Canaan. Quand les Égyptiens envahirent la Syrie, ils furent frappés de la beauté des fruits de cette contrée, dont la plupart leur étaient encore inconnus; on le voit au soin que, au retour de son expédition, Thoutmès III prit de les faire peindre sur les murailles du temple de Karnak².

Parmi ceux que le Pharaon semble avoir le plus admirés sont les raisins et les grenades, tant ils sont représentés de fois sur les bas-reliefs du monument égyptien. On y voit ${ }^{3}$ trois vignes entières, couvertes de grappes de raisin et dont les feuilles trilobées étonnent, mais qu'on recomnait aux vrilles, qui terminent les rameaux. Des grappes énormes ont été en outre représentées à part pour donner une idée de leur grosseur aux habitants de la capitale égyptienne. Les grenades, qui devaient être encore rares à cette époque

1. Boissier, Flora, t. II, p. 642 et 655 . - Rich. E. Burton and Ch. F. Tyrrwhit Drake, Unexplored Syria, t. I, p. 68. Il y a deux espèces de poiriers indigènes en Syrie, le $P$. Syriaca et le $P$. Boveance.

2. A. Mariette-Bey, Karnak. Élude lopographique et archélogique. Leipzig-Paris, 1875, in-fol.

3. Karnak, pl. 31 a. 
en Égypte, y sont aussi reproduites en grand nombre ${ }^{1}$. A côté d'elles je serais tenté de voir des olives ${ }^{2}$. L'olivier a existé de temps immémorial en Syrie, qu'on a voulu regarder comme son pays d'origine ou du moins celui où il a été d'abord cultivé. Mais à l'époque de Thoutmès III, cet arbre n'avait pas encore probablement été importé en Égypte ${ }^{3}$; il n'en devait que davantage attirer l'attention des conquérants.

Il y a bien d'autres plantes et d'autres fruits représentés sur les bas-reliefs de Karnak; mais à part ceux que je viens de nommer et du lotus, il en est peu qu'on puisse reconnaître avec certitude. J'inclinerais cependant à voir des poires dans deux fruits de la planche $28 a$; un fruit de la planche $29 b$ pourrait, lui, passer pour un melon et divers autres ressemblent à des concombres; si ce ne sont là que des conjectures, il ne saurait guère, au contraire, y avoir de doute au sujet d'une plante figurée au haut de la planche $31 a$; MM. Bonnet et Poisson du Muséum ont été unanimes à l'identifier avec la colocase; cette aroïdée aurait donc déjà pénétré en Syrie 1500 ans avant notre èré.

Les renseignements fournis par les peintures de Karnak sont complétés et confirmés par ceuy que nous donne l'Ancien Testament. Les émissaires enroyés par Moïse dans le pays de Canaan coupèrent, dit le texte sacré ${ }^{5}$, dans la vallée de la Grappe, ainsi nommée des vignes qui y croissaient, un pampre, garni d'une énorme

1. Karnali, pl. $28 a, 30 a$ et $b, 31 a$.

2. Karnak, pl. 30 a.

3. Voir pl. haut liv. I, chap. Iv, p. 131.

4. Quant aux fleurs de la même planche 31 a et $b$, il m'a été impossible d'en identifier aucune.

5. Numeri, cap. xill, vers. 2's. 
grappe de raisin, ot ils le rapportèrent à leur's compatriotes arec des grenades et des figues. On roit par lit que la vigne, le figuier el le grenadier étaient alors les principaux arbres fruitiers du pays de Canaan et mobahlement de toute la Syrie. La rigne - gephen qui y produisait dejà de si beaux fruits, y était cultivée sans doute des longtemps, et olle continua de l'ètre encore plus sous la domination hébraïque. Isaïe parte des nombreux rignobles de l'ancien pays de Moab' et lc Cantique des Cantiques vante ceux d'Fngaldi ${ }^{2}$. La culture du figuier - teenah - que la Bible associe sans cesse a celle de la vigne, n'était pas moins ancienne en Syrie. La Terre Promise était, d'après le Deutéronome ${ }^{3}$, un pays riche en figuiers, et elle resta telle après l'occupation des Hébreux; sa prospérité était liée ¿ la culture du figuier et de la vigne. Les ennemis les détruisent pour punir Israël'; ces arbres prodiguent de noureau leurs richesses, quand Israèl est ¡ardonné. Le grenadier n’était ğuère moins répandu en Judée que le figuier; un grand nombre de localités de cette contrée tiraient leur nom de celui de cet arbuste, rimmon ${ }^{5}$.

La mention si fréquente dans l'ancien Tcstament de lolivier et de l'huile que fournissent ses fruits, fait pressentir l'importance qu'en avait prise la culture

1. Cap. Xvi, verset 8 .

2. Cap. I, verset 13 .

3. Cap. vili, ver's. 8.

4. IIosea, cap. II, vers. 12. - Joel, cap. I, vers. 7.

5. Olaus Celsius, Hierobolanicon sive de planlis sacrae scripurae dissertaliones breves. Upsaliae, 1745, in-8, t. I, p. 272.Rosenmüller, Biblische Nalurgeschichle, p. 275. Il y avait entre autres, un Rimmon dans les tribus de Juda, de Benjamin et de Zabulon. 
dans la Palestine. Sous la domination des Hébreux, comme avant leur conquête, le pays de Canaan fut véritablement une " terre d'huile et de miel ${ }^{1}$ ». Les vallées du Liban et de la Galilée, les flancs du Carmel et du Thabor, les collines d'Éphraïm et de Garizim, la plaine de Jéricho et de Sharon, étaient couvertes d'oliviers; David avait nommé un intendant, chargé de veiller sur les plantations qu'il avait faites de cet arbre précieux ${ }^{2}$.

Si c'étaient là les principaux arbres à fruits de la la Syrie, ce n'étaient pas les seuls, les bas-reliefs de Kiarnak nous l'ont montré, qu'on y rencontrât; malheureusement les textes ne nous donnent que peu de renseignements sur la nature de ceux qui y pouvaient ètre cultivés. On a traduit parfois par poiriers le mot bekaim, qu'on trouve dans un passage des livres de Samuel ${ }^{3}$; $^{3}$ mais cette interprétation a été contestée et avec non moins de raison la signification de muiriers, attribuée à ce mot ${ }^{4}$. Il n'est guère douteux toutefois que le poirier, dont deux espèces y sont indigènes, et le mùrier à fruits noirs n'aient été, ainsi que le pommier, cultivés en Syrie. Importé en Égypte dès la XVIII ${ }^{e}$ dynastie, ce dernier, indigène en Asie Mineure et dans la région du Caucase, dut l'ètre plus tòt encore dans les vergers de la Phénicie et du pays de Canaan. Joël en parle en mème temps que de la vigne, du figuier, du grenadier et du dattier ${ }^{5}$.

1. Deuleronomium, cap. vilI, vers. 8 .

2. Paralipomena, cap. xxvi, vers. 28.

3. 2. cap. ximl, vers. 2́.

4. W. H. Groser, The trees and plants mentioned in the Bible, p. 63.

5. Cap. I, ver's. 12. 
On a parfois roulu trouver dans le Cantique des Cantiques une mention du cognassier', mais le mot lappouakh, qui le représenterait, semble bien n'avoir d'autre signification que " pommier" ". Si on a cru que le vocable lhoakh désignait en hébreu le prunier saurage, on ne connait point dans cette langue de nom pour le prunier cultivé; mais on ne peut en conclure que cet arbre n'existait pas en Syrie, ainsi que le cerisier. Deux espèces comestibles indigènes de cerisier et de prunier ${ }^{3}$ se rencontrent d'ailleurs dans cette contrée, arec l'azarolier. Quant au prunier et au cerisier cultivés, ils n'ont dù y être importés qu'assez tard; l'abricotier et le pècher le furent à une époque encore postérieure, probablement pas avant celle de la domination romaine.

On traduit d'ordinaire le mothébreu egoz par noyer et shaqed par amandier"; ces deux arbres, on n'en pent douter, étaient cultivés dans la Syrie ancienne; mais l'amandier, indigène dans le Liban, a dû l'être sans doute plus anciennement que le noyer, espèce exotique. Des amandes figuraient parmi les présents que les fils de Jacob portèrent à Joseph en Égypte ${ }^{6}$. S'il

1. Cap. II, vers. 3 et 5. - Celsius, op. laud., t. I, p. 254. Rosenmüller, op. laud., p. 309.

2. W. H. Groser, op. laud., p. 89.

3. Les Prunus ursina et monticola et les Cerasus mahaleb et prostrala. Boissier, Flora, t. II, p. 6ł8-652 et 662.

4. Rosenmüller, op. laud., p. 224. - Fr. Hamilton, op.laud., no 15. - IV. H. Groser, op. laud., p. 108.

5. O. Celsius, op. laud., t. I, p. 297. - W. IH. Groser, op. laud., p. 87. L'amandier portait aussi le nom de louz. O. Celsius, op. laud., t. I, p. 253. Genesis, cap. xxx, vers. 37.

6. Genesis, cap. XumI, vers. 11. On leur fait aussi parfois y porter des noix, botnim, mais ce mot signifie plutôl " pistaches $»$. 
n'est pas indigène en Syrie, le pistachier a dî néanmoins y ètre cultivè à une époque reculée. Il en est de nème peut-ètre du caroubier, si commun en Galilée sur les collines d'au delà du Jourdain et dans les gorges du Liban'; mais il n'en est fait mention que dans le Nouveau Testament. Le sycomore - héb. sliqqmah s'était, des bords méridionaux de la mer Morte, réprandu dans toute la région occidentale de la Syrie, en particulier en Phénicie ${ }^{2}$, où on le plantait, il est vrai, plus à cause de l'usage que l'on faisait de son bois que pour ses fruits. Cet arbre n'était pas moins fréquent dans les plaines de la Judée", et David avait nommé un intendant chargé de veiller à sa conservation".

Le dattier - tamar - indigène, comme le sycomore, au sud de la mer Morte, fut cultivé dans toute la région avoisinante. Jéricho est appelée dans le Deutéronome $^{5}$ la ville des palmiers. Dans la plaine, au milieu de laquelle elle est située, se trouvait, dit Strabon ${ }^{6}$, le Phœnicón, verger de cent stades de long, arrosé d'eau courante et rempli d'arbres fruitiers de toute espèce, mais principalement de dattiers, qui fournissaient une abondante récolte. C'était là seulement et en Babylonie que l'on trouvait les célèbres dattes caryotes. Des confins de la mer Morte, le dattier fut importé sur tout le littoral syrien ${ }^{7}$, particulière-

1. IV. II. Groser, op. laud., p. 107.

2. Pietschmann, Geschichle der Phrenisier, p. 22.

3. 1 Regna, cap. x, vers. 27. - 2 Paralipomena, cap. 1. vers. 15 , et cap. IX, vers. 27.

4. 1. Paralipomena, cap. Xxvir, ver's. 28.

5. Cap. xxsiv, vers. 3.

6. Geographia, lib. XVI, cap. 2, í1.

7. C'est de lá qu'il a été porté dans les îles de l'Archipel. 
ment en Phénicie, encore que ses fruits n'y pussent mùrir.

Près du bois de palmiers de Jéricho se trourait aussi, d'après Strabon, un chàteau royal avec un parc appelé le " Jardin du baumier», du nom d'un "arbuste odoriférant assez semblable au cytise et au térébinthe $"{ }^{\prime}$. Grisebach a supposé, évidemment it tort, que cet arbuste était le chalcf ${ }^{2}$; il s'agit du Balsamodendron opobalsamm, qui avait èté importé d'Arabie aux enrirons de Jéricho. Une tradition, rapportée par Josèphe ${ }^{3}$, voulait que le premier picd de cet arbuste précieux ent été donné par la reine de Saba ì Salomon.

Le jardin du baumier est le seul verger de l'ancienne Syrie sur lequel nous ayons quelques renseignements précis; mais il est fait, dans, le Cantiquè des Cantiques, allusion à un jardin planté d'arbres à fruits exquis et d'aromates et arrosé d'eaux vives ${ }^{4}$. L'Ecclésiaste parle aussi de jardins et de parcs, où il avait planté toutes sortes d'arbres fruitiers ${ }^{5}$; mais il ne dit pas quels étaient ces arbres. Dans le "jardin clos",

1. Pline, qui dit, lib. XII, cap. 54 (25), que le baumier « ressemble plutôt à la vigne qu'au myrte", le fait croitre seulement en Judéc et dans les deux jardins royaux. Justin, lib. XXXYI, cap. 3, prétend que cet arbuste ressemble à un pin de petite taille, mais qu'on le cultive comme la vigne.

2. Die Vegelation der Erde, t. 1, p. 403. Le chalcf (Elaeagnus auguslifolius L.) est répand u dans toute la Judée.

3. Antiquilates Judaicae, lib. VIII, cap. 6, 6.

4. Cap. 15 , vers. 13 et 1'́.

5. Cap. II, vers. 5. 
auquel l'auteur du Cantique des Cantiques compare son épouse, croissent, en même temps que des grenadiers, l'arbre au henné - kopher —, le nard et le safran, la cannelle et le cinnamome, avec tous les arbres qui donnent l'encens, la myrrhe, le santal ou l'aloès et les plus excellents aromates ${ }^{1}$.

Le henné étant, il semble, indigène au sud de la mer Morte ${ }^{2}$, put bien être cultivé dans les jardins de Salomon; il en fut de même du baumier, compris pent-être parmi les aromates ou arbres à encens du Cantique; mais il est trop évident que le cinnamome et l'aloès ou le santal, produits de la presqu'ile hindoustanique, sont mis ici d'une manière figurée et n'ont pu être plantés dans les parterres juifs. Il faut en dire autant du nard, autre plante de l'Inde; le safran, au contraire, a bien pu y trouver place, ainsi que le calame aromatique kaneh - , si l'on entend par là l'Acorus calamus.

Rencontrait-on dans les jardins de la Syrie - hébreux ou phéniciens - d'autres arbres ou d'autres fleurs d'agrément? On peut supposer qu'il y avait au moins un certain nombre d'arbres. Le cyprès, en particulier, si intimement associé au culte d'Astarté, et comme tel planté dans les bois sacrés, qui environnaient ses temples, le fut probablement aussi dans les jardins des princes et des grands; on y plantait sans doute également le pin pignon à cause de son port majestueux et de ses fruits comestibles, ainsi peutêtre que le genévrier thurifère, le cèdre, le lentisque et quelques espèces de chênes verts. A l'époque des Séleucides on y dut joindre le laurier - ezrakh — et

1. Cap. IV, vers. 13 et 14.

2. Boissier, Flora, t. II, p. 744. 
peut-ètre aussi le myrte - hadas -, tout répandu qu’il était dans la contrée. A cette époque aussi le rosier prit vraisemblablement place dans les jardins syriens; la comparaison de la sagesse, par l'auteur de l'Ecclésiastique', à un rosier de Jéricho pourrait faire croire que cet arbuste était, au second siècle avant notre ère, cultivé dans les jardins de la ville biblique. Quant à la mention de la rose, qu'on a cru trouver dans Isaïe ${ }^{2}$ et dans le Cantique des Cantiques ${ }^{3}$, elle est au moins douteuse; le sens du mot khabalseleth, qui la représenterait, n'est point certain, et les Septante l'ont traduit par lis - rpivoy; - les traducteurs arabes ont, de leur côté, rendu ce mot par narcisse". Quoi qu'il en soit, la rose véritable finit par être cultivée en Judée et sans aucun doute dans toute la Syrie; le traité de la Maaseroth, qui remonte au commencement de notre ère, parle d'un jardin situé près de Jérusalem, où croissait la reine des fleurs ${ }^{5}$.

On n'est pas mieux fixé sur l'introduction de la culture du lis - shoshan - que sur celle de la rose; non seulement le mot shoshan n'a point de sens bien déterminé - tout au plus peut-on dire qu'il désigne une liliacée ou une plante analogue —; mais là où il est employé, il ne s'agit pas d'une Heur des jardins, mais d'une fleur des champs: "Je suis le lis des vallées ». - "Comme un lis au milieu des épines, telle est mon

1. Cap. xxiv, vers. 18.

2. Cap. xxxy, vers. 1.

3. Cap. II, vers. 1. "Je suis la rose de Saron ".

4. W. H. Groser, op. laud., p. 184. C'est aussi le sens adopté par Reuss ainsi que par M. Renan, Le Cantique des cantiques traduit de l'hébreu. Paris, 1870, in-8, p. 155.

5. Charles Joret, La rose dans l'antiquilé et au moyen age, p. 124-125. 
amie au milieu des jeunes filles '. "Aussi a-t-on parfois identifié le shoshan de l'Ancien Testament avec un iris ou un glaieul ${ }^{2}$. Quant au lis blanc - Litium candidum L. - que Boissier a cru indigène dans le Liban, rien n’indique qu'il ait été cultivé en Syrie arant notre ère et même beaucoup plus tard. Mais y cultiva-t-on d'autres plantes d'agrément. On voit des lotus sur les bas-reliefs de Karnak, ce qui pourrait faire supposer que cette plante était du temps de Thoutmès III cultivée en Syrie; mais peut-ètre ces lotus ne sont-ils rien autres que des nymphéas blancs. L'origan, au contraire, si c'est bien l'hysope de la Bible ${ }^{3}$, parait avoir pris place dans les jardins de la Judée, comme dans ceux de l'Assyrie. Toutefois on peut affirmer que, jusqu'à l'époque de la conquète grecque, on ne connut guère en Syrie d'autres plantes d'ornement que celles des champs; ce fut seulement sous la domination hellénique, quand le goùt des fleurs se fut répandu dans cette contrée, comme dans les autres parties du monde grec, que la culture des plantes d'agrément y prit réritablement naissance.

Comme chez les Égyptiens, les végétaux jouaient chez les Sémites de la Mésopotamie et de la Syrie un rôle considérable dans l'alimentation; les Chaldéens

1. Canticum, cap.11, vers. 1.

2. Il faut ajouter que le mot shoshan désigne aussi le lotus, et que son dérivé shoshannah est employé comme nom propre.

3. Rosenmüller. op.laud., p. 108. - IV. H. Groser, op. land.. p. 180. 
et les Assyriens se nourrissaient de blé et d'orge, mais plus particulièrement de blé, à en juger par les documents contemporains, ou il est surtout question de champs de froment ${ }^{1}$. Le blé et l'orge entraientégalement dans l'alimentation des anciens Hébreux; il est souvent fait mention de pain d'orge dans la Bible ${ }^{2}$, mais il était réputé plus grossier ${ }^{3}$; ce peuple se nourrissait aussi d'épeautre. Les Sémites de la Mésopotamie ct de la Syrie faisaicnt encore usage du millet comme aliment, mais peut-ètre seulement depuis une époque récente ${ }^{4}$.

Quel qu'il füt, le grain était sans doute écrasé entre deux pierres, comme on le fait encore aujourd'hui en Orient; la farine passée au tamis, puis mélangée d'eau était pétrie sur une table ou dans une auge; ensuite la pàte, façonnée en pains plats ct ronds, était portéc au four ${ }^{3}$. Il semble que le plus souvent chez les Chaldéens, parfois aussi chez les Hébreux, l'eau était remplacée par de l'huile, dans la fabrication du pain. Dans les "Comptes de dépenses " pour l'entretien des serviteurs de la cour des patési chaldéens, on voit qu'on remettait à chacun d'eux en mème temps de la farine et de l'huile ${ }^{6}$. Parmi les oblations, prescrites aux Israélites,

1. Caillou de Michaux, Contrat de Ada, Contrat de Hankas, Inscription de Mardouk-Abal-Idin, Actes assyriens, XIX, XXV, etc. J. Oppert et J. Menant, Documents juridiques, p. 92, 106, $122,136,185,200$, etc.

2. Judices, VII, 17. - 2 Regna, IV, 42. - Johannes, cap. VI, vers. 9 .

3. Rosenmüller, op.laud., p. 87.

4. La première mention qu'en fasse l'Ancien Testament se trouve dans Ézéchiel, cap. IV, vers. 9. Cf. A. de Candolle, op. laud., p. 302.

5. B. Stade, op. laud., t. I, p. 367.

6. Fr. Thureau-Dangin, La complabilité agricole en Chaldée 
figurait aussi en premier lieu de "la fleur de farine pétrie avec de l'huile et sans levain '" .

Les grains de froment et probablement d'orge servaient aussi d'aliment aux populations sémitiques, sans avoir été réduits en farine. Dans les "Comptes de dépenses " que je viens de citer, au lieu de farine, qimou, il est question de gar, remis aux serviteurs royaux. M. Fr. Thureau-Dangin attribue à ce mot, qui apparait plus tard sous la forme gar-zoun, la signification de "grains rôtis " ${ }^{2}$. On devait les préparer avec de l'huile, qui était donnée en même temps qu'eux. Dans quel état ces grains étaient-ils employés en Chaldée? Nous l'ignorons; mais chez les Hébreux on n'attendait pas leur entier développement. Les épis, coupés avant la complète maturité, étaient séchés et légèrement grillés; après cette simple préparation on mangeait les grains - on en apporta de pareils à David dans sa fuite ${ }^{3}$, ou bien on les écrasait et on les faisait cuire avec la viande".

L'orge n'entrait pas seulement dans l'alimentation des hoinmes, elle servait aussi à la nourriture des animaux. Les chevaux de Salomon étaient nourris avec de l'orge ${ }^{3}$. Les Comptes de dépenses étudiés par M. Fr. ThureauDangin font rnention de 900 qa d'orge pour la nourriture des ânes ${ }^{6}$. Il y est aussi question de 280 qa de

au troisième millénaire. (Revue d'Assyriologie el d'Archéologie orientale, t. lII, fasc. 4, p. 133-135.)

1. Leviticus, cap. II, vers. 5.

2. La comptabilité agricole. (Ibid., p. 137.)

3. 2 Samuel, cap. xvir, vers. 20.

4. Rosenmüller, op. laud., p. 81.

5. 1 Regna, cap. IV, vers. 28.

6. La complabilité agricole. (Ibidl., p. 135.) 
grains pour celle des canards; on pourrait supposer qu'il s'agit ici de millet.

Outre les céréales, les plantes herbacées et les légumes proprement dits entraient aussi, avec les fruits, pour une part considérable dans l'alimentation des nations sémitiques; malheureusement nous ne possédons sur leur emploi que des rensignements incomplets. Bérose dit ${ }^{1}$ qu'il croissait dans les marécages de la Chaldée des plantes aquatiques appelées gong, dont les racines, propres à la nourriture, avaient le gout du pain d'orge; il est probable qu'il désignait ainsi le souchet esculent, indigène dans la Basse-Mésopotamie, comme en Syrie, où l'on en faisait aussi sans doute usage. On mangeait encore en Chaldée et peut-être en Judée la moelle ou le cour du palmier, mets dont la douceur surprit agréablement les soldats de Xénophon². La colocase, que j'ai cru reconnaitre parmi les plantes syriennes figurées dans un bas-relief de Karnak, entrait peut-être aussi dans l'alimentation des habitants de la Syrie. Ce n'est là toutefois qu'une conjecture.

Des documents contemporains, au contraire, nous apprennent que les lentilles étaient un aliment recherché des populations sémitiques. On sait qu'Ésaï vendit pour un plat de lentilles son droit d'aînesse ${ }^{3}$. Les Hébreux connurent aussi très anciennement les fères; elles figurent, avec des lentilles et des pois grillés, au nombre des aliments qu'on apporta à David, obligé de fuir devant son fils Absalon* Les Hébreux

1. Fragmenta, lib. I, cap. 2.

2. Anabasis, lib. II, cap. 3,16 .

3. Genesis, cap. xxv, vers. 32-34.

4. 2 Samuel, cap. xvil, vers. 28. 
les mêlaient, ainsi que les lentilles, avec de la farine de froment, d'orge, d'épeautre et de millet, et en faisaient une espèce de pain ${ }^{1}$. Il est impossible de douter que les Assyriens n'aient pas aussi fait usage pour leur alimentation des lentilles, des fèves et de ces pois apportés grillés à David.

$\mathrm{Ce}$ ne furent pas les seuls légumes qui entrassent dans l'alimentation des Sémites de la Mésopotamie et de la Syrie; tous ceux qui, originaires de l'Asie antérieure, pénétrèrent dans la vallée du Nil deraient servir aussi à la nourriture des habitants de la Syrie et probablement des Chaldéo-Assyriens : comment les Égyptiens les auraient-ils connus, si ces peuples, qui seuls purent les leur transmettre, n'en avaient fait usage eux-mêmes? Oignons, ail, poireaux, concombres, pastèques, melons chate, cultivés, au moins pour la plupart, dans leurs jardins, figurèrent, dès la plus haute antiquité, au nombre des mets des nations sémitiques. Il en fut de même des principaux condiments et légumes verts en usage chez les Égyptiens, qui avaient dù les leur emprunter en partie avec les précédents, tel que le cumin, le carvi ${ }^{2}$, la coriandre et l'aneth; il faut ajouter les légumes verts — oroth - et les endires ou laitues sauvages — merorim ${ }^{3}$ - dont parle l'Ancien Testament, et sans doute plusieurs autres plantes alimentaires dont il ne fait pas mention.

Les fruits occupaient avec les légumes une place importante dans l'alimentation des peuples sémitiques. Les dattes constituaient en grande partie celle des Chal-

1. Ezechiel, cap. IV, vers. 9.

2. Esaias, cap. xxviII, vers. 25.

3. 2 Regna, cap. IV, vers. 39. - Exodus, cap. xir, vers. 8. - Numeri, cap. $1 \mathrm{x}$, vers. 11. 
déens; ils les mangeaient fraìches ou sèches, en guise de pain. Secs, ces fruits à la couleur ambrée, dont la beauté fit l'admiration de Xénophon', servaient plutôt de dessert. Les Assyriens et les Hébreux en mangeaient aussi; mais chez ceux-ci les dattes étaient plutòt une friandise, comme chez nous, qu'un aliment véritable. Des autres fruits, les raisins, les figues et les grenades furent connus des Sémites de la Mésopotamie septentrionale et de la Syrie dès l'antiquité la plus reculée; leur mention fréquente dans l'Ancien Testament prouve à la fois l'emploi qu'on en faisait en Judée et le prix qu'on y attachait. On mangeait les figues et les raisins, non seulement frais, mais encore et surtout conservés en gàteaux. Pour apaiser David, réfugié dans le désert, Abigaïl lui offrit, outre cinq mesures de grains grillés, cent gâteaux de raisins et deux cents gàteaux de figues. Les compagnons du roi prophète donnent à un égyptien, qu'ils avaient trouvé abandonné dans les champs par les Amalécites, du pain à manger, avec une tranche de gàteau de figues et deux gàteaux de raisins ${ }^{2}$. Parmi les vivres, que les habitants du voisinage apportèrent aux guerriers réunis à Hébron, afin d'établir David roi sur tout Israël, figuraient aussi, arec de la farine, des gâteaux de figues et des raisins $\operatorname{secs}^{3}$. Les bas-reliefs de Ninive et de Koyoundjik représentent souvent des vignes chargées de raisins ${ }^{4}$. D'autres bas-reliefs montrent des servi-

1. "Pulcritudine... admirabiles, specie ab electro nihil differebant. " Anabasis, lib. II, cap. 3, 13.

2. 1 Samuel, cap. xxv, vers. 18 et cap. xxx, vers. 11-12.

3. 1 Paralipomena, cap. XII, vers. 40.

4. Rawlinson, op. laud., t. I, p. 349 et 353 . On voit même sur un fragment d'ivoire assyrien du Musée britannique des femmes qui cueillent des raisins. Ibid., t. II, p. 205. 
teurs qui portent sur la table royale des plateaux chargés de grappes de raisin, de grenades et de figues ou même, il semble, de cônes de pin ${ }^{1}$. Si nous trouvons là les principaux fruits connus des Assyriens et des habitants de la Syrie, nous sommes loin de les avoir tous; ces peuples en connaissaient bien d'autres, telles que les pistaches - hébr. botnim ${ }^{2}$, ass. boutnou ${ }^{3}$, - les amandes et les noix,

Les Sémites occidentaux mangeaient probablement aussi les graines du pin pignon, de même peutètre, au moins en Galilée, que les glands du chêne égilops, comme les Arabes le font aujourd'hui ${ }^{4}$. Ils durent faire également usage des caroubes; mais elles servaient surtout, comme nous le montre la parabole de l'Enfant prodigue ${ }^{5}$, à la nourriture des animaux. On consommait aussi sans doute en Palestine les figues du sycomore. Lês poires et les pommes, sinon les coings $^{6}$, durent figurer de bonne heure sur les tables syriennes et mésopotamiennes ${ }^{7}$. On y vit peut-être aussi, mais beaucoup plus tard, des fruits du prunier et du cerisier cultivés; ils ne durent pas y paraitre avant

1. Layard, The monuments of Nineveh, ser. II, pl. 8.

2. Bosenmüller, op. laud., p. 246.

3. E. Schrader, Monatsberichte der Akademie der Wissenschaften zu Berlin, (an. 1881), p. 419.

4. W. H. Groser, op. laud., p. 58 et 68.

5. Lucas, cap. Xv, vers. 16.

6. "Dieser Baum scheint dem aegyptisch-semitischen Kulturkreis ursprünglich gefehlt zu haben ». Schrader, ap. V, Hehn, p. 243. Il faut dire toutefois que Celsius, I, 254, et Rosenmüller, 308, voient dans le tappouakh le cognassier et non le pommier. Cf. W. Houghton, The tree and the fruit represented by the Tapuakh of the ITebrew Scriptures. (Proceedings, t. XII, p. 42-48.)

7. Nous avons vu que les pommes furent connues en Égypte dès la XVIIIe dynastie. 
l'époque de la domination grecque ${ }^{1}$; les abricots et les pèches y figurèrent plus tard encore.

Aux substances alimentaires dont je viens de parler il faut ajouter la manne, qui pendant quarante ans servit de nourriture aux Hébreux dans le désert. "Quand la rosée était tombée", une graine petite et comme pilée, ressemblant à la gelée blanche, apparaissait sur la terre. " La manne se rencontre encore de nos jours en Arabie, en Syrie et en Mésopotamie; elle est produite par diverses espèces d'arbres ou d'arbustes, en particulier par le chêne ballout, qui croit en abondance aux environs de Mardin et dans le Zagros, ainsi que par une espèce de tamaris, le tarfal, - Tamaris nilotica -, très commun aux environs du Sinaï, et mème par l'aqoul - l'Alhagi Maurorum DC. ou mannifera Desv. ${ }^{3}$. - Au mois de mai ou de juillet, suivant la région, surtout par un temps humide ou de brouillard, la manne se forme sur ces divers arbres; on la recueille en secouant les branches. Dès que le soleil a lui, elle fond et coule sur le sol. Elle n'est point perdue pour cela; les habitants la ramassent avec les détritus auxquels elle adhère, la font bouillir et la passent à traver's un tamis. Elle peut se garder alors

1. Il est à remarquer que Théophraste ne parle pas des fruits du prunier et que, s'il fait mention, lib. III, cap. 13, des fruits du cerisier, il parle de celui-ci comme d'un arbre forestier.

2. Exodus, cap. xvi, vers. 14 .

3. Rosenmüller, op. laud., p. 317, fait aussi mention de el hadsch; mais cette plante ne' parait autre que l'aqoul. D'après Burckhardt, Travels in Syria, London, 1822, in-4, p. 393, il y aurait aussi dans la vallée du Jourdain un arbre à manne, appelé gharrab, qui, de la grandeur d'un olivier, a les feuilles semblables à celles du peuplier: serait-ce le gharqad, doṇt il sera question plus loin? 
pendant des mois. On la mange, comme le micl, en l'étendantsur le pain.

Nous n'avons que des renseignements incomplets sur la nature des substances liquides: boissons, huiles ou éssences, que les Sémites demandaient au règne végétal. Les documents nous apprennent que les Chaldéens connaissaient quatre espèces de boissons fermentées, "faites avec des éléments différents ou mélangés dans des proportions diver'ses " ${ }^{1}$. L'une d'elles, qui passait, ce semble, pour être d'mne qualité inférieure, était probablement fabriquée avec de l'orge et analogue dès lors à la bière des Égyptiens. Mais la plus ordinaire était le vin de palmier, le kash ou shikarou. On ignore jusqu'à présent ce qu'étaient les deux autres. Les boissons fermentées étaient d'un grand usage en Chaldée; on les trouve constamment mentionnées avec la farine ou les grains rôtis, qui formaient la base de l'alimentation du peuple.

La fabrication du vin de palmier en Chaldée est mentionnée par les écrivains grecs et latins ${ }^{2}$; elle remonte à la plus haute antiquité. Il est question dans une inscription de Nabonide de 6 gour de dattes remis à un personnage du nom de Shamashsharousour "pour en faire du shikarou $)^{3}$. Dans la ville royale d'Entéména, patési de Telloh, on a découvert des celliers, dont les murs sont creusés de cavités bitumineuses en

1. Fr. Thureau-Dangin, op. laud., p. 135.

2. Hérodote, lib. I, cap. 193. - Strabon, lib. XVI, cap. 1, 14. - Pline, lib. $\mathrm{L}$, cap. 9.

3. Fr. Thureau-Dangin, Op. laud., p. 136, note 3. 
forme d'amphores; c'étaient lih, on n'en peut douter, des réservoirs où se conservait ce précieux liquide ${ }^{1}$. Comment le faisait-on? Rawlinson a $\mathrm{cru}^{2}$ que ce n'était autre chose que la sève fermentée du palmier, assertion rien moins que vraisemblable. Les documents indigènes nous apprennent que ce vin était fabriqué avec des dattes soumises à une espèce de cuisson. Il était agréable au goùt, mais portait à la tête ${ }^{3}$. Exposé à l'air, il donnait le vinaigre dont parlent Hérodote et Strabon. En poussant plus loin sans doute la cuisson, on obtenait le miel, dont ces historiens font également mention, ainsi que Xénophon.

Arec les grenades on fabriquait aussi, en Assyrie et en Syrie, une liqueur fermentée; il en est question dans le Cantique des Cantiques". Mais c'était le raisin qui servait dans ces deux pays à faire la boisson la plus recherchée et le plus en usage. S'ils n'ont pas inrenté la fabrication du vin de raisin, les Sémites la connurent dès l'époque la plus reculée; la légende de l'ivresse de Noé, qui l'a en quelque sorte symbolisée, la reporte aux premiers temps de leur histoire. Melkiçédeq, roi de Salem, offre déjà du pain et du vin". La Judée et la Palestine avaient des crus renommés. Damas exportait à Tyr le vin de Khelbôn ${ }^{6}$.

Outre ces diverses boissons, les Sémites tiraient du règne végétal les huiles dont ils avaient besoin pour

1. L. Heuzey, Une villa royale chaldéenne, environ 6,000 ans avant nolre ère. (Revue d'Assyriologie, t. III, fasc. 2, p. 62.)

2. The five greal monarchies, t. I, p. 35.

3. Anabasis, lib. II, cap. 3, 15.

4. Cap. vili, vers. 2.

5. Genesis, cap. Ix, vers. 20-21, et cap. xıv, vers. 18.

6. Ezechiel, cap. Xxin, vers. 18. Cf. Franz Delitzsch, Die Bibel und der Wein, p. 12. 
l'assaisonnement des mets et la préparation des parfums. Le sésame fournissait exclusivement aux Chaldéens l'huile dont ils se servaient' ${ }^{1}$. Les Assyriens, au contraire, et surtout les habitants de la Syrie demandièrent de temps immémorial aux fruits de l'olivier celle qui leur était nécessaire. La Terre promise est représentée comme un pays où l'huile abondait ${ }^{2}$. Après l'invasion de la Syrie par les Égyptiens, on voit les villes conquises offrir ou obligées de donner en gage de paix de l'huile et du vin aux vainqueurs ${ }^{3}$. Salomon fournit 20,000 mesures d'huile aux ouvriers que le roi Hiram lui avait envoyés pour couper du bois sur le Liban ${ }^{4}$. Cette denrée était une de celles dont les Israélites approvisionnaient les marchés de Tyr". L'huile d'olive jouait un rôle considérable dans les préparations alimentaires des Sémites. Elle servait, nous l'avons vu, à pétrir la farine ou à arroser les grains rôtis, dont se nourrissaient les Chaldéens. Les Hébreux aussi en faisaient usage. Quand Moïse consacra Aaron et ses fils, il offrit à Dieu des gâteaux sans levain pétris à l'huile et des pâtes également sans levain, arrosées de ce même liquide ${ }^{6}$.

Les habitants de l'Asie antérieure firent-ils aussi usage, comme les Égyptiens, de l'huile de ricin? Cela est probable; mais ce ne dut ètre qu'assez tard; du moins les anciens textes n'en font pas mention, pas

1. Hérodote, lib. I, cap. 193-4.

2. Deuteronium, cap. VIII, vers. 8 .

3. A. H. Sayce, Correspondance between Palestine and Egypt in the fifteenth century B. C. (Records of the Past, t. V, p. 74 et 75.)

4. 2 Paralipomena, cap. II, vers. 10.

5. Ezechiel, cap. xxvit, vers. 17.

6. Exodus, cap xxix, vers. 2. 
plus d'ailleur's que de l'huile de carthame, en usage en Egypte, comme celle de ricin.

L'industrie des Sémites, comme celle des Égyptiens, tirait du règne végétal les produits les plus divers. C'était lui qui leur fournissait en particulier le combustible, le tanin et quelques-unes des matières colorantes dont ils faisaient usage. Les essences forestières les plus diverses, cèdres, pins, cyprès, chênes, bien d'autres encore, étaient, partout où on les rencontrait, " destinées au feu de l'homme pour préparer sa nourriture "'. En Chaldée, où les arbres manquaient, on employait à cet usage, daprès Hérodote ${ }^{2}$, les noyaux de dattes, ainsi sans doute que les broussailles et les racines.

Nous sommes mal renseignés sur les espèces de tanin, qui servaient pour l'apprêt des cuirs chez les nations sémitiques. En Mésopotamie, on parait avoir extrait cette substance du sumac - Rhus cotinus ou coriaria -; en Syrie et en particulier en Phénicie, on la retirait, il semble ${ }^{3}$, de l'écorce du grenadier, qui la renferme en grande quantité. Il est probable que l'écorce et même les glands de chêne la fournissaient également, comme aujourd'hui. Nous ne savons que fort peu de chose de l'emploi, dans l'Asie antérieure, des matières colorantes tirées du règne végétal. On a cru qu'une inscription d'Ashshournazirpal faisait mention

1. Esaias, cap. XLIV, vers. 15.

2. IIistorice, lib. I, cap. 193.

3. Pietschmann, Geschichte der Phœnizier, p. 22. On la retirait aussi probablement de l'écorce du sumac. Pline, lib. XIII, cap. 13. 
" d'étoffes teintes en berom et en safran "), données comme tribut par les habitants de la ville de Sour, voisine de l'Euphrate ${ }^{\prime}$; en réalité il s'agit de robes bariolées de $\operatorname{lin}^{2}$. Toutefois, elles pouvaient bien être teintes avec du safran, puisqu'il était cultivé en Mésopotamie, et nous savons par le témoignage des anciens écrivains quel usage fréquent on faisait dans la teinture des étoffes de cette plante asiatique. On donne à Bacchus, le dieu oriental, un vètement couleur de safran $^{3}$ - le \%po\%wós —; Virgile montre les Phrygiens fiers de leurs robes teintes en safran et en pourpre $^{4}$. Les Sémites faisaient-ils, comme les Égyptiens, usage de l'indigo et du carthame dans la teinture? Cela est probable, au moins pour le carthame; mais aucun texte ne nous renseigne à cet égard.

Mais c'étaient surtout des matières textiles et des bois de construction que les Sémites de la Mésopotamie et de la Syrie demandaient aux essences végétales de leur pays ou des contrées voisines. L'emploi du textile le plus précieux de l'antiquité, le lin, fut connu chez les Israélites et sans doute aussi chez les Chaldéens et les Assyriens dès l'époque la plus reculée. Les Araméens en fournissaient la ville de $\mathrm{Tyr}^{5}$. On en faisait des tissus et les objets les plus divers. Les draperies du tabernacle étaient de lin fin ${ }^{6}$. C'était en

1. J. Oppert, Histoire des empires de Chaldée el d'Assyrie d'après les monuments. Versailles, 1865, in-8, p. 79.

2. "Lubulti bir-mi. » Eb. Schrader, Inschriften von Ashurnâzirabal. (Keilinschriftliche Bibliothek, t. I, p. 65.)

3. V. Hehn, Kulturpflanzen, p. 256.

4. Aeneis, lib. IX, vers. 614.

5. Ezechicl, cap. xxvil, vers. 16.

6. Exodus, cap. xxvi, vers. 1. 
lin aussi qu'étaient faites les voiles des vaisseaux phéniciens'.

Les anciens Chaldéens portaient une courte tunique probablement de $\operatorname{lin}^{2}$; sous le nouvel empire babylonien, elle fut remplacée, dans les classes aisées, par une longue robe de même étoffe ${ }^{3}$. Le lin entrait aussi dans la composition du costume des Hébreux" ; les Proverbes nous montrent la "fermme forte " vêtue d'étoffes de lin qu'elle a fabriquées elle-même ".

Lorsque les lévites franchissaient les portes du sanctuaire, ils devaient ètre vêtus de robes de lin; des bandelettes de lin étaient sur leurs têtes, et ils avaient des ceintures de lin autour des reins ${ }^{6}$. Le rational, l'éphod, la robe, la tunique et la tiare du grand prètre Aaron étaient de fin lin retors, et tissus de fils de diverses couleurs; et pour entrer dans le tabernacle, il se revêtait, avec ses fils, de vêtements de lin depuis les reins jusqu'aux cuisses ${ }^{\top}$.

Il est souvent question dans l'Ancien Testament de ceintures de $\operatorname{lin}^{8}$; on faisait également avec ce textile des liens et des cordages. On les fabriquait aussi en Chaldée avec des fibres de palmier. On faisait encore avec les feuilles de cet arbre et avec les tiges de diverses plantes aquatiques, joncs, cypéracées et roseaux, des nattes, des treillages, des corbeilles ${ }^{9}$. Il est probable

1. Ezechiel, cap. xxyı, vers. 7.

2. Rawlinson, op. laud., t. I, p. 105.

3. Hérodote, Hislorice, lib. I, cap. 195.

4. Lucas, cap. XVI, vers. 19.

5. Cap. xxxi, vers. 22 et 24.

6. Ezechiel, cap. XuIv, vers. 17-18.

7. Exodus, cap. xxviII, vers. $4,8,39$ et 42.

8. Celles de Jérusalem et d'Ascalon étaient renommées. Movers, Das phönizische Alterthum, t. III, 1, p. 218.

9. Strabon, Geographica, lib. XVII, cap. I, 9. 
qu'on employait encore à ces derniers usages les rameaux flexibles du saule et du peuplier, ainsi que diverses plantes sarmenteuses. Les roseaux et les feuilles de palmier entrelacés durent aussi servir aux premiers habitants de la Basse-Mésopotamie à faire les huttes sous lesquelles ils vécurent d'abord '. Quand ils les dédaignèrent pour des maisons en briques, ils demandèrent au règne végétal les matériaux nécessaires pour faire la toiture et les portes de leurs nouvelles demeures.

Dans la Chaldée, où les espèces arborescentes manquaient, à l'exception du palmier, ce fut cet arbre qui fournit d'abord aux habitants tout le bois dont ils avaient besoin pour les travaux de charpente et la fabrication des objets les plus divers. Théophraste dit ${ }^{2} q u$ 'ils en faisaient même des idoles. Mais ils ne s'en contentèrent pas longtemps. Lorsque leur's rois eurentétendu au loin leurs conquètes et leurs relations, ils firent venir des contrées étrangères des bois plus résistants ou plus précieux. On voit déjà Nianâour, patési de Sirpourla, importer " toutes sortes de bois de la montagne de Mâgan ${ }^{3}$ ». Goudéa, un autre patési de la même ville, pour construire le temple Eninnoû, fait couper dans l'Amamus, "la montagne des cèdres ", des poutres et des madriers, longs de 50 et de 70 empans, de ce bois précieux, ainsi que des solives d'ourkarinou" d'une longueur de 25 empans. Le même

1. C'est dans de pareilles huttes que vivent encore les Arabes de la Basse-Mésopotamie.

2. Historia plantarum, lib. V, cap. 3,6 .

3. Arthur Amiaud, The inscriptions of Telloh. (Records of the Past, t. I, p. 65.)

4. Die Inschrift B von Gudêa. (Keilinschrift. Bibliothek, t. III, p. 33.) Le révérend C.-J. Ball croit que le mot ourkarinou 
patési fit aussi abattre sur le territoire d'Ibla ou de Tilla des solives de zabanoum, des grands arbres shaliou - ass. ashouishou-, de toulouboum et de gin. Enfin il importa de la contrée de Mélouggha des arbres liala - ass. oushou - et de Goubin, " pays de l'arbre ghaloution ", du bois de cet arbre, tandis que ses vaisseaux lui apportaient du pays de Nitouk - peut-être Tihnoum sur le golfe Persique - les essences de bois les plus diverses ${ }^{1}$. Ces matériaux servirent non seulement à faire les portes et les planchers du temple et sans doute du palais construit par le célèbre patési, mais encore à en lambrisser les murs et à construire, dans le sanctuaire même de Nin-Ghirsou, le grand dieu de Sirpourla, la chambre du " lieu du jugement ", chambre en bois de cèdre, où se rendaient les "arrêts divins " ".

Les rois du nouvel empire chaldéen ne firent pas, on le comprend, moins usage des bois les plus variés dans leurs constructions. "Le temple Esagil, que mon père n'avait pas achevé, dit Shamashshoumoukîn dans une inscription ${ }^{3}$, je l'ai mené à fin; je l'ai cóuvert de longues poutres de cèdres et de cyprès du mont Amamus et du mont Liban; j'ai fait faire et dresser

désigne une espèce de buis, probablement le $B$. balearica; mais ce n'est là qu'une ingènieuse hypothèse. Proceedings of the Sociely of Biblical Archaelogy, t. XI, p. 143. L'ourkarinou servait en particulier dans les travaux d'ébénisterie; il est question dans une hymne d'un trône en bois de cet arbre. A. R. E. Brunnow, Assyrian hymns. (Zeilschr. f. Assyr., t. V, p. 70.)

1. Amiaud, op. laud. (Recorls of the Past, t. II, p. 79-91.) II. Sayce croit qu'au lieu de ghaloukou on peut lire ghaloup- ass. houlouppou.

2. L. Heuzey, Un palais chaldéen d'après les dernières découverles de MI. de Sarzec, Paris, 1888, in-18, p. 53.

3. C. J. Lehmann, Shamashshumukin, König von Babylonien (666-648). Leipzig, 1892, in-4, 2e partie, p. 17. 
les portes en bois de palmier, de cyprès et de cèdre ". Nabuchodonosor le Grand, à son tour, dans l'énumération des travaux exécutés à Babylone ${ }^{1}$, dit qu'il avait rapporté pour ses constructions les plus beaux cèdres des forêts du Liban. C'était avec leurs énormes poutres recouvertes d'or qu'il avait fait faire le toit d'Ékoua, sanctuaire de Mardouk, " le maitre des Dieux ". C'était aussi arec des solives de cèdre et de cyprès qu'était façonné le toit du palais qu'il s'était élevé et les portes étaient en bois de palmier - mismakanna-, de cèdre, de cyprès et d'oushoù. Et Nabonide nous apprend qu'il fit amener tout ce qui était nécessaire pour la construction de l'Ébabbara: cèdres superbes, pins (?) énormes, hauts palmiers ${ }^{2}$.

Encore que l'Assyrie ne fùt pas aussi dénuée de bois de charpente que la Chaldée, les rois de ce pays ne se contentèrent pas de ceux qu'ils y trouvaient et ils firent venir de l'étranger la plupart des bois nécessaires à leurs constructions. Ashshournazirpal imposa au roi Akhouni, qu'il avait vaincu, un tribut de poutres de cèdre, et les rois de la Phénicie durent lui envoyer, de leur côté, du bois d'oushoú.

Étant monté aux montagnes de Khamani, dit-il ${ }^{3}$, j’y fis couper des solives de cèdre, de sherbin - J.oxycedrus -, de genévrier et de cyprès. Je fis transporter

1. C. J. Ball, The India House inscriptions of Nebuchadrezzar the Great. (Records of the Past, t. III, p. 107-120.)

2. Fr. V. Scheil, Inscription de Nabonide. (Zeitschrift fïr. Assyriologie, t. V, p. 403.) M. Scheil donne ici au palmier le nom de mussukianna; dans l'inscription de Salmanasar, il l'appelle muslianna.

3. A. H. Sayce, The inscription of Assurnatzirpal. (Records of the Past, t. I, p. 168-173.) Cf. S. Arthur Strong, A votive inscription of Assurnatsirpal. (Records, t. IV, p. 85.) 
du mont Amamus à E-Sarra des solives de cèdre, matériaux destinés au temple que j’y élevai... J'allai dans la contrée des Pins... J'y coupai des solives de pin... Je les transportai à Ninive. Je les offris à Ishtar, dame de Ninive.

Ashshournazirpal parle aussi ${ }^{1}$ des " palais en bois de cèdre, de cyprès, de genévrier, d'ourkarinou, de palmier, de pistachier et de tarpi ", qu'il avait élevés, et de leurs portes en bois de cèdre, de cyprès, de genérrier, de palmier. Salmanasar II rappelle également et les solives de cèdre et de genévrier, qu'il avait fait couper lui-même sur l'Amamus, et les nombreuses bûches de cèdre, qu'il avait reçues du roi Khayânou, qui habitait au pied de cette montagne, ainsi que le bois de palmier rapporté de la Chaldée? Tiglathphalasar II, au milieu de ses expéditions, n'oublie pas de monter sur l'Amamus pour y couper des poutres de cèdre $^{3}$, et parlant du palais qu'il avait bâti à Kalakh " avec un portique à la mode hittite ", énumère longuement les poutres de cèdre, " produits du Khamani, du Liban et de l'Amamus ", qu'il y avait employées, et les portes en bois d'oushou, d'ourkarinou, de palmier et de pin - douprân, - incrusté d'ivoire, ainsi que de cèdre et de cyprès, " dont l'odeur réjouit le cœur $\left.{ }^{4}\right)$.

1. Inscription ap. Hommel, Geschichte Babyloniens und Assyriens, p. 481, note 1 .

2. Scheil, Inscription of Shalmaneser 1I. - The monolithinscription of Sh. II. - Inscription of Balawat. (Records, t. IV, p. 39 et 44,63 et 79.)

3. J. Oppert, II istoire des empires de Chaldee et d'Assyrie, p. 114 et 121. Die Thoninschrift von Nimrûd. (Keilinschriftl.
Bibliothel, t. II, p. 23-25.)

4. S. Arthur Strong, The Nimiroud inscription of Teglithpileser III. (Records, t. V, p. 126-127.)

I. 
Les Sargonides, qui furent aussi grands constructeurs que conquérants, importèrent également d'immenses quantités de bois de l'étranger, pour les édifices qu’ils élevèrent. Le fondateur de la dynastie, Sargon le Grand, s'étend avec complaisance sur les palais en ivoire et en bois d'oushoú, d'ourkarinou, de palmier, de cèdre, de cyprès, de genévrier, de pin et de pistachier" "qu'il construisit sur l'ordre auguste des dieux ". Il énumère longuement les immenses poutres de cèdre, avec lesquelles il les couvrit, les battants de portes en bois de cyprès et de palmier, revêtu de bronze brillant, ainsi que les quatre colonnes en bois de cèdre, hautes de douze empans, qu'il y fit placer. Assarhaddon parle dans les mêmes termes des diverses espèces de bois que le roi de Syrie, " serviteur de șon règne ", lui envoya des montagnes de Sirar et du Liban pour la construction de ses palais, des poutres de cèdre dont il les couvrit et du bois de cyprès, plaqué d'argent et de cuivre, qui servit à en faire les portes ${ }^{2}$.

Ces bois importés de la Syrie, dont les monarques chaldéens et assyriens faisaient usage dans leurs constructions, les habitants de ce pays s'en servaient aussi depuis longtemps pour leurs travaux de char-

1. J. Oppert, Les inscriplions assyriennes des Sargonides et les fastes de Ninive. Versailles, 1862, in-8, p. 32. - D.-G. Lyon, Keilschriflexte Sargon's, p. 37, 45 et 53. - H. Winckler, Die Keilinschrifllexle Sargons. Leipzig, 1889, in-8, p. 91, $129,141,153$ et 161 .

2. J. Oppert, op. laud., p. 58. - B. Meissner u. P. Rost, Die Inschriften Asarhaddons. (Beitrïge zur Assyriologie $u$. semilischen Sprachen, t. III, 2, p. 199.) Les bois débités en longues pićces étaient charriés à travers le désert jusqu'aux bords de l'Euphrate, d'où les bas-reliefs de Khorsabad nous les montrent transportés sur le fleuve à Ninive.Botta et Flandin, Monument de Ninive. Paris, 1869, in-fol., t. I, pl. 34 et 35. 
pentage, de charronnage et d'ébénisterie. Les bois de cèdre et de cyprès en particulier durent entrer dans la construction des palais phéniciens ${ }^{1}$, comme dans celle des palais chaldéens et assyriens. Les habitants de la Plénicie les employaient aussi; avec plusieurs autres essences, dans la construction de leurs flottes. "Les flancs de tes vaissaux, dit Ézéchiel s'adressant à Tyr ${ }^{2}$, sont construits avec les cyprès de Sénîr; les cèdres du Liban en ont formé les mâts; les chênes de Bashan, les rames; les bancs en sont faits de bois incrusté d'ivoire. "

A l'exemple des Phéniciens, les Hébreux employèrent ces divers bois dans leurs édifices. "La parure du Liban viendra vers toi, dit Isaïe s'adressant à Jérusalem $^{3}$, le cyprès, le platane, le sapin orneront le lieu qui m'est consacré. " Lorsque David résolut de s'élever un palais, le roi de Tyr, Hiram, lui envoya du bois de cèdre avec des charpentiers et des tailleurs de pierres, pour le bàtir ${ }^{4}$. Ce furent aussi des ouvriers phéniciens qui dirigèrent les travaux du temple, que Salomon éleva à Jahveh, et les bois de cèdre et de cyprès, qu'on employa dans sa construction ou pour le meubler, lui furent donnés par le même roi Hiram et coupés sur le Liban ${ }^{5}$.

Cet édifice fut couvert de poutres et de planches en bois de cèdre; des lambris de cèdre en revêtirent

1. Ezechiel, cap; xxvil, vers. 5-6. Les Phéniciens devaient aussi, comme les Egyptions, se servir du bois de sycomore.

2. Cap. xxvir, vers. 5-6.

3. Esaias, cap. LX, vers. 13. 4. 2 Samuel, cap. v, vers. 11. - Josèphe, Antiq. Judaicae,
lib. VII, cap. 4, 2. 5. 1 Regna, cap. v, vers. 3-10. - Josèphe, op. laud., lib.VIII,
cap. II, 6 et 9 . 
les parois intérieures; le Saint des Saints fut également fait avec ce même bois. "Tout ainsi à l'intéricur était en cèdre. " L'autel aussi en était fait. Mais le sol était recouvert de planches de cyprès; les battants des portes étaient encore faits de ce même bois ${ }^{1}$. Dans le palais de Salomon - la maison du Bois-Liban —, les bois de la montagne, dont ce palais avait pris le nom, étaient également prodigués. Il était bâti sur quatre rangs de colonnes de cèdre, sur lesquelles reposaient des poutres également de cèdre; ce bois en formait aussi les plafonds ${ }^{2}$.

Le cèdre et le cyprès, qui jouaient, le premier surtout, un rôle si important dans les constructions, étaient loin d'être, avec le pin, le chêne et le buis, les seuls bois dont fissent usage les Sémites; ces peuples employaient bien d'autres essences indigènes ou exotiques dans leurs travaux de charpentage, d'ébénisterie ou de sculpture. Les inscriptions des patési de la Basse-Mésopotamie et des Sargonides nous ont montré de combien d'espèces de bois, la plupart restés inconnus, se servaient les Chaldéens et les Assyriens. Les habitants de la région syrienne n'en utilisaient pas un moins grand nombre.

Moïse avait fait faire en bois d'acacia - shittim l'arche d'alliance ${ }^{3}$. Salomon placa dans le temple deux kéroubs en bois d'olivier sauvage - ets shemen - A l'entrée de l'arrière-pièce se trouvait une porte à deux battants en bois du même arbre ${ }^{4}$. Le bois de caroubier était encore très employé dans l'ébénisterie. D'après

1. 1 Regnu, cap. vi, vers. 9-33.

2. 1 Regna, cap. vir, vers. 2-7.

3. Exodus, cap. xxv, vers. 10.

4. 1 Regna, cap. vi, vers. 21 et 33 . 
M. Victor Loret', dans le récit de la bataille de Mageddo, il est question de six grands coffres en bois de caroubier "orné d'or et d'émaux diver's".

Le caroubier et l'olivier sont des bois indigènes en Syrie; on se servait également dans ce pays, ainsi qu'en Assyrie, de bois venus des régions les plus lointaines du Midi et de l'Est. Les balustrades du temple et du palais de Salomon étaient faites en bois de santal, rapporté d'Ophir par la flotte de Hiram² ${ }^{2}$ Ce bois toutefois, s'il n'y a pas erreur sur son identification, était mne rareté, et l'écrivain sacré dit lui-même qu'on n'en vit plus depuis lor's. Il n'en est pas de même de l'ébène, importé dans l'Asie occidentale par les vaisseaux des Phéniciens ${ }^{3}$, il fut dès la plus haute antiquité employé dans l'ébénisterie de toutes les nations de cette vaste région. On le plaquait et on l'incrustait, ainsi que l'ivoire", sur le cèdre, le cyprès, le caroubier, l'acacia, etc. Dans le butin rapporté de Syrie par Thoutmès III, se trouvaient, entre autres, " six sièges du chef de Mageddo avec les six tabourets qui leur appartenaient, (en) ivoire, ébène et caroubier avec ornements d'or ${ }^{5}$ ").

1. Recherches sur plusieurs plantes. VIII. Le bois de caroubier. (Recucil de travaux, t. XV, p. 14.) - M. Maspero, Récit cle la campagne contre Mageddo. (Recueil de travaux, t. II, p. 148), a substitué le bois de cèdre au bois de caroubier.

2. 1 Regna, cap. x, 11 12. - 2 Paralipomena, cap. Ix, vers. 10.

3. Ezechiel, cap. xxvir, vers. 15.

4. Dans les palais assyriens, des “ plaques d'ivoire ciselèes, émaillées et dorées s'encastraient dans les lambris de cèdre ou de cyprès ». Perrot et Chipiez, Histoire de l'art, t. II, p. 315.

5. Victor Loret, Le buis de caroubier, p. 14. 


\section{CHAPITRE III.}

\section{LES PLANTES DANS L'ART ET DANS LA POÉSIE UES SÉMITES.}

De mème que l'industrie des peuples sémitiques trouva, nous venons de le voir, dans le règne végétal les matières premières les plus indispensables, leurs arts, comme ceux des Égyptiens, lui empruntèrent de nombreux motifs de décoration. L'absence de pierres en Chaldée et par suite la nécessité d'employer exclusivement la brique dans les constructions empêchèrent longtemps les architectes de cette contrée d'emprunter au monde des plantes les formes d'où sont sortis la stèle et le pylone égyptiens. Des murs massifs, percés de voûtes énormes, telles furent les formes caractéristiques de leurs premiers édifices. Ils n'ignorèrent pas entièrement toutefois la colonne, ou du moins le pilier, s'ils ne s'en servirent qu'exceptionnellement. M. de Sarzec a trouvé à Telloh les débris d'un énorme pilastre formé de quatre colonnes en briques juxtaposées ${ }^{1}$; mais il est peu vraisemblable que ce pilastre pût se terminer par un chapiteau fait en forme de fleur, comme les chapiteaux latiformes ou papyriformes des édifices égyptiens. En Assyrie mème, où la pierre

1. L. Heuzey, Un palais chaldien. Paris, 1888, in-18, p. 3749. - Id., Découvertes en Chaldée par Mr. de Sarzec. Paris. 1884, in-fol., p. 52. 
n'était pas inconnue comme en Chaldée, la colonne fut toujours d'un emploi assez rare', au moins dans les palais royaux, et les chapiteaux, quand il y en a, sont ornés de dessins géométriques, non de formes empruntées au monde des plantes ${ }^{2}$.

Il existe toutefois des colonnes ou l'artiste semble avoir voulu reproduire des formes végétales, ce sont celles du sanctuaire chaldéen de Shamash, qu'on voit sur une tablette découverte à Sippara et qui représente l'hommage rendu au dieu par le roi Nabon-abal-iddin ${ }^{3}$; l'espèce de chapelle où se trouve Shamash repose sur deux colonnes élancées, dont le fìt est garni d'imbrications semblables à celles d'un tronc de palmier; ces colonnes étaient sans doute en bois recouvert d'une feuille métallique, dont les saillies voulues imitent les rugosités de la tige déponillée de ses frondes. Un autre exemple de formes empruntées au monde des plantes, cette fois par des architectes assyriens, est fourni par une stèle que M. Place a découverte à Khorsabad $^{4}$; cette stèle dont on ignore la vraie destination est cannelée dans toute sa longueur et se termine par une palmette. Toutefois ces imitations de la nature vé-

1. A.-H. Layard, Nineveh and ils remains. London, 1849, in-8, t. II, p. 274.

2. Victor Place, Ninive el l'Assyrie. Paris, 1867, in-fol., t. III, pl. 35. - Perrot, Hisloire de l'art, t. II, p. 216. On voit, au contraire, des formes décoratives empruntées au règne animal, comme dans une colonne du temple de Koyoundjik, dont le chapiteau est orné de deux paires de cornes d'ibex superposées. Rawlinson, op. laud., t. I, p. 333. - Perrot, op. laud., t. II, p. 219.

3. Perrot, op. laud., t. II, p. 211, pl. 71. - Maspero, Hisloire anciemne, t. I, p. 657.

4. Ninive el l'Assyrie, t. I, pl. 96; t. II, p. 71-73. - Perrot, op. laud., t. II, p. 270. 
gétale étaient en quelque sorte accidentelles dans l'architecture chaldéenne ou assyrienne; on ne les rencontre d'ordinaire sur les édifices de la Mésopotamie que dans les ornements employés par la sculpture des bas-reliefs, dans l'émaillerie, ainsi que dans la bijouterie et l'orfèvrerie.

Les architectes de la Chaldée et de l'Assyrie durent ètre frappés de bonne heure de la nudité déplaisante de leurs larges murs de briques, aussi s'appliquèrentils à la dissimuler aux yeux en la faisant disparaître sous une couche d'enduit ou de peinture ${ }^{1}$; mais si cet enduit était suffisant pour les parties élevées, il eût été trop fragile pour les parties basses de l'édifice; on garnit celles-ci de pierres plates calcaires ou même basaltiques d'une hauteur variant de un à trois mètres, de deux à quatre de largeur. Ce revêtement, destiné à consolider le mur de brique, autant qu'à le dissimuler, reçut bientôt une autre destination plus artistique; elle servit à transmettre à la postérité la mémoire du souverain dont il entourait le palais ${ }^{2}$. Des sculpteurs furent chargés de cette besogne délicate, et ainsi prirent naissance ces bas-reliefs, dont les représentations parlantes nous font assister aux guerres des conquérants assyriens et à quelques-uns de leur's divertissements favoris.

Dans ces tableaux variés et d'un réalisme si saisissant les plantes avaient leur place marquée; dans les scènes de chasse des bas-reliefs de Koyoundjik ${ }^{3}$, par exemple, les artistes assyriens ont représenté les ar-

1- Rawlinson, op. laud., t. I, p. 104. - Perrot, op. laud., t. II, p. 285.

2. Perrot, op. laud., t. II, p. 280.

3. Victor Place, op. laud., t. III, pl. 48,50 bis, 51,53 et 56 . 
bres divers, au milieu desquels passent les chasseur's ou fuient les faures effrayés. Ils les ont également figurés dans les scènes guerrières: batailles, sièges de villes, défilés de troupes ou de prisonniers, qu'ils ont si souvent sculptées. C'est ainsi qu'on voit sur un basrelief de Koyoundjik, les captifs de la ville de Lakhish défiler au milieu des vignes, des figuiers et probablement des grenadiers qui l'entouraient', qu'un autre bas-relief qui représente Sennachérib subjuguant une peuplade établie au milieu des marécages ${ }^{2}$, nous montre les immenses roseaux qui les couvrent et les dattiers qui en garnissent les bords. Des dattier's se dressent aussi sur les bords d'une rivière qui coule au pied d'une ville dont un autre bas-relief représente le siège ${ }^{3}$. Sur d'autres bas-reliefs encore on voit ici la conquète d'une contrée montueuse plantée de conifères, de vignes et d'arbres sans caractère précis, lá le siège d'une ville entourée des mêmes arbres ${ }^{4}$. Un bas-relief de Koyoundjik, dont j'ai déjà parlé, représente ${ }^{5}$ Asshourbanipal se livrant, sous une treille, dans un jardin planté de conifères et de palmiers, aux joies d'un festin avec la reine favorite. Il faut encore mentionner un autre bas-relief, également de Koyoundjik, aujourd'hui au Musée britannique ${ }^{6}$, qui représente un lion et une lionne, couchés au pied d'un palmier et

1. Layard, The monuments, ser. II. pl. 22.

2. Layard, The monuments, ser. II, pl. 25-27.

3. Layard, The monuments, ser. II, pl. 42-43.

4. Layard, The monumenls, ser. II, pl. 37-39.

5. Victor Place, op. laud., t. III, pl. 57, 2. - Babelon, Mamuel d'archéologic orientale. Paris, s. d., in-8, p. 113.

6. Botta, Monument de Ninive, t. I, pl. 52 bis. - Victor Place, op. laud., t. III, pl. 52 bis. - Perrot, op. laud., t. II, p. 567. 
d'un conifère - pin oll cyprès, - sur lequel grimpe une vigne, tandis que derrière eux se dresse une composée corymbifère à tige nue et à feuilles radicales entières.

Si l'idée de ces bas-reliefs a été inspirée aux artistes chaldéens par les peintures des temples égyptiens, ils n'en ont pas moins un incontestable caractère d'originalité et sont des œuvres essentiellement nationales par l'inspiration et par l'exécution; on trouve, au contraire, l'imitation évidente du style égyptien dans un fragment d'un seuil de Koyoundjik ${ }^{1}$, dont la décoration consiste en lotus réunis par quatre dans des espèces de carrés que séparent des rangées de fleurcns; tout antour règne une bordure composée de fleurs et de boutons de lotus alternant entre eux, non isolés toutefois, comme dans les peintures pharaoniques, mais reliés pal' une tige commune. Un seuil de Khorsabad ${ }^{2}$ présente également une bordure de fleurs et de boutons de lotus, mais la partie centrale qu'entoure aussi une rangée de marguerites est occupée par des Heurons étoilés à six pétales, ingénieusement combinés entre eux.

Comme sur les bas-reliefs, les formes végétales se rencontrent sur les sculptures des cylindres; elles y apparaissent dès l'époque chaldéenne, moins comme motifs de décoration toutefois que comme éléments des scènes mises sous nos yeux. Sur un cylindre de la collection de Clercq ${ }^{3}$, on voit, il est vrai, une rosace ornementale à neuf rayons, mais M. Menant incline à le croire d'origine phénicienne; ce motif de décoration

1. Layard, t. II, pl. 56. - Rawlinson, op. laud., t. I, p. 350.

- Perrot et Chipiez, op. laud., t. II, p. 316.

2. Perrot et Chipiez, op. laurl., t. II, p. 251 et 319.

3. Calulogue méthodique. Paris, 1888, in-fol., fig. 27, p. 41. 
ne peut donc être attribué avec certitude ì l'art chaldéen. Il n'en est pas de mème des trois arbres, dont un palmier, aux frondes arquées et marquées en dessous par un simple zigzag, représentés sur un autre cylindre de la mème collection ${ }^{1}$; mais ils ont un caractère symbolique, non ornemental. Tel encore le palmier, qu'on voit sur deux autres cylindres chaldéens souvent reproduits; le palmier du premier cylindre ${ }^{2}$ s'élève entre deux personnages assis, qui étendent la main pour en saisir les fruits ou les spathes pendants, tandis qu'un serpent se dresse derrière le personnage de gauche, scène qu'on a eu le tort de regarder comme représentant la tentation dans le Paradis terrestre ${ }^{3}$. Sur le second cylindre ${ }^{4}$, qui symbolise la fécondation du dattier, on voit encore deux personnages, probablement féminins, cueillant les spathes d'un palmier, que l'un de ces personnages offre à un troisième. Un autre cylindre ${ }^{3}$ représente, lui, deux cherreaux, qui semblent vouloir brouter le feuillage d'un arbre sans caractère distinctif, placé derrière chacun d'eux. Sur un cylindre babylonien, d'origine récente - il est peut-ètre contemporain des Achéménides et qui représente un roi luttant contre deux ètres fantastiques ${ }^{6}$, on roit aussi se dresser de chaque côté de ces derniers un dattier à huit frondes, au-dessous des-

1. No 26, p. 40.

2. Lajard, Le culle de Milhra, pl. XVI, no 4. - Fr. Lenormant, Histoire ancienne, t. I, p. 35.

3. G. Smith, The Chaldean account of Genesis. London, 1876, in-8, p. 91. - J. Menant, Les pierres gravées de la Haule-Asie. Cylindres de la Chaldée. Paris, 1883, in-4, fig. 120, p. 190.

4. J. Menant, Les pierres gravées, fig. 121, p. 191.

5. Collection de Clereq. fig. 312.

6. Collection de Clerc', fig. 377. 
quelles pendent quatre régimes de dattes ou quatre spathes. Sur un cylindre de Sennachérib', au contraire, sont gravées, simple motif de décoration, deux fleurs de lis ou de lotus.

Les ornements tirés du règne végétal se retrouvent sur les enduits coloriés des murs en briques, ainsi que sur les briques émaillées qu'on appliquait souvent, au lieu d'enduit, sur ces murs. Telle est la double ligne de fleurons, qui encadre la porte nord du palais de Koyoundjik ${ }^{2}$, ainsi que la bande horizontale de boutons et de fleurs de lotus qui la surmonte et les rangées de marguerites dont toute la muraille est couverte. Telle encore la bordure de fleurons qu'on voit sur une archivolte émaillée de Khorsabad ${ }^{3}$, dont le champ offre une large rosace entre deux personnages ailés. On peut mentionner aussi les palmettes qui se dressent sur les murs du palais nord-ouest de Nimroud entre des cônes ou des ornements de fantaisie ${ }^{4}$. Il faut ajouter la décoration murale de diverses chambres du mème palais, formée de demi-cercles, soutenant, par leurs extrémités réunies, un ornement arrondi simulant une grenade ${ }^{5}$. La palmette, qui semble bien n'avoir été pour les artistes assyriens qu'une reproduction plus ou moins conventionnelle de la cime du dattier, se rencontre, ingénieusement combinée avec la grenade, sur des briques émaillées quadrangulaires ou ovales pro-

1. Rawlinson, op. laud., t. I, p. 475.

2. Rawlinson, op. laud., t. I, p. 335. -- Perrot, op. laud., t. II, p. 326 .

3. Victor Place, t. III, op. laud., pl. 15. - Perrot, op. laud., p. 308.

4. Layard, The monuments, ser. I, pl. 86, plan 3.

5. Layard, The monuments, ser. I, pl. 86, ch. C, plan 4, et pl. 87, ch. B, plan 4. 
renant du palais d'Ashshournazirpal '. Au lieu de simples motifs de décoration, les artistes représentèrent aussi, sur les briques émaillées, des scènes étendues et rariées; telle est cette chasse de Sémiramis, dont parle Diodore d'après Ctésias ${ }^{2}$; le monde des plantes y avait naturellement trouvé sa place, ainsi que sur les sculptures des bas-reliefs assyriens.

Tandis que les plantes occupent une si grande place dans la sculpture et la peinture décoratives, elles ne jouent qu'un rôle exceptionnel dans la céramique; les rares ornements dont se servaient d'ordinaire les poliers chaldéens et assyriens sont empruntés à la géométrie $^{3}$, non aux formes végétales. M. G. Smith, toutefois, a trouré à Koyoundjik des poteries", qui sont ornées de figures, dont quelques-unes sont tirées du monde des plantes. Les formes végétales, qui ne sont ici qu'exceptionnelles, apparaissent, au contraire, constamment dans la sculpture sur bois ou sur ivoire, ainsi que dans les ouvrages de bijouterie ou d'orfèvrerie.

Les Assyriens et tous les Sémites septentrionaux avaient poussé fort loin le luxe de l'ameublement et ils y ont montré le goût le plus délicat ${ }^{5}$; ils employaient dans la fabrication de leur mobilier les bois les plus précieux ouvrés avec soin, et pour en rehausser la valeur, ils les incrustaient d'ivoire, nous l'avons vu,

1. Perrot, op. laud., t. II, p. 310, fig. 127 et 128.

2. Bibliolheca, lib. II, cap. 8, 6. Sur les murs du harem de Khorsabad, Perrot, t. II, pl. XV, p. 707, on voit une vigne ou un figuier.

3. Layard, The monuments, ser. I, pl. 85. - Rawlinson, op. laud., t. I, p. 479-481. - Birch, History of ancient pollery, p. 85 et 19 .

4. Assyrian discoveries. London, 1875, in-8, p. 141.

5. Layard, Nineveh and its remains, t. II, p. 293 et suiv. 
ou les plaquaient en bronze ou en or. Ces différentes matières se prêtaient au travail des artistes et à l'ornementation végétale. Layard a reproduit un fragment de meuble en bronze, qui a la forme d'une palmette ${ }^{1}$. Un panneau d'ivoire, conservé au Musée britannique, présente dans sa partie inférieure une double rangée de fleurons, séparés par une espéce de balustrade formée de palmettes et de grenades alternant entre elles ${ }^{2}$, genre d'ornementation que nous avons déja rencontré sur les briques émaillées.

On voit aussi des palmettes et des grenades sur la garniture d'un sceau en métal, reproduit par Layard ${ }^{3}$. Mais c'est sur les coupes surtout que les artistes àssyriens ont prodigué l'ornementation végétale et ils en ont tiré les plus beaux effets. Ici nous avons un champ orné de fleurons à six pétales aigus, entourés d'une quadruple rangée de palmettes, reliées entre elles par des courbes gracieuses ${ }^{4}$. Là sont figurées en relief des montagnes, sur lesquelles le burin a gravé, motif bien indigène, des arbres et des cerfs ${ }^{5}$. On a encore, ce semble bien, une œuvre d'inspiration exclusivement assyrienne dans une coupe du Musée britannique, qui représente, sur des montagnes que le marteau a fait saillir en léger relief, des arbres et des animaux gravés

1. The monuments, ser. I, pl. 96,6 .

2. Perrot et Chipiez, op. laud., t. II, p. 730.

3. The monuments, ser. I, pl. 51 .

4. Layard, The monuments, ser. II, pl. $62 \mathrm{~A}$.

5. Iayard, The monuments, ser. II, pl. 66. Sur une autre coupe, pl. $61 \mathrm{~B}$, on voit au centre quatre figures coiffées it l'égyptienne, en même temps que dans la bordure se trouvent des personnages dont le costume et la pose semblent bien aussi empruntés à une peinture pharaonique, d'où on peut conclure à une influence étrangère. 
au trait', et entourés d'une bordure alternative d'ibex et d'arbres.

Les molifs de décoration fournis par le règne végétal se retrouvent sur les parties les plus diver'ses du costume; mais ils consistent presque exclusivement en fleurons ou rosaces et en palmettes. On aperçoit également des fleurons sur les bracelets, les tiares royales ou les bandeaux des grands, les ccinturons et les baudriers, les boucliers, les carquois, les manches de poignards, mème sur les masses d'armes et le harnachement des chevaux ${ }^{2}$. Sur le haut d'une bride figurée dans Rawlinson ${ }^{3}$, sont aussi dessinées deux fleurs, qui ressemblent à un lotus ou à une liliacée. Enfin on rencontre les formes si décoratives du règne régétal brodées sur les étoffes " : palmettes et rosaces ou fleurons émaillaient les vètements et les tapis; la robe de Sennachérib et son tròne sont couverts de rangées innombrables de rosaces, il en est de même de la robe du roi babylonien Mardouk-iddin-akhii ${ }^{5}$; mais sur le manteau royal et le pectoral, les rosaces sont en grande partie remplacées par des palmettes de formes diverses; les unes d'aspect ordinaire se dressent ici entre deux ibex, là entre deux taureaux affrontés ${ }^{6}$, les autres laissent passer entre leurs feuilles

1. Perrot et Chipiez, op. laud., t. II, p. 751, fig. 408 .

2. Botta, Monumeni, t. II, pl. 118, 160, 161, 18'. - Rawlinson, op. laud., t. I, p. 447, 452, 487, 490 .

3. The five greal monarchies, t. I, p. 417.

4. Layard, The momments, ser. I, pl. 8. - Franz Reber, Ueber altchaldäische Kunst. (Zeitschrift für Assyriologie, t. I, p. 292.) - Rawlinson, t. I, p. 486.

5. Rawlinson, op. laud., t. I, p. 398 et t. II, p. 560 .

6. Layard, The monuments, ser. I, pl. 43, 2 et 4 . - Perrot, op. laud., t. II, p. 321 et 322 . 
arrondies des tiges recourbées, que terminent des boutons ou des espèces de cônes ${ }^{1}$. Sur le pectoral et le manteau royal, a pris place aussi l'arbre de vie luimême ${ }^{2}$, qui n'est, nous le verrons, qu'une transformation de la cime du palmier.

Les plantes figurées sur les monuments chaldéoassyriens ou employées dans la décoration n'étaient pas nombreuses et n'ont pas toujours èté reproduites avec une fidélité qui permette de les reconnaître sans peine. A l'époque accadienne en Chaldée et en Assyrie avant les Sargonides la représentation en était fruste et conventionnelle; c'est ainsi que, sur les cylindres chaldéens et même encore sur les bas-reliefs assyriens de Nimroud, le palmier est représenté sous la forme d'un tronc droit et massif avec deux ou quatre appen. dices de forme ovale, destinés à figurer les spathes ou les régimes de dattes, et trois ou quatre paires de frondes, grossièrement simulées par des arcs de cercle, unis d'un côté, mais pourvus de l'autre de dentelures en scie pour représenter les folioles ${ }^{3}$. On voit aussi sur un bas-relief de Nimroud un arbre au tronc énorme, divisé en trois maîtresses branches, dont chacune est garnie de rameaux entiers, légèrement renflés à l'extrémité ${ }^{4}$, représentation informe dans laquelle l'imagination trop complaisante de M. Bonavia a voulu voir un baobab ${ }^{5}$. Mais tout change à l'époque suivante;

1. Layard, The monumenls, ser. I, pl. 47, 1.

2. Layard, The monumenls, ser. I, pl. 8 et 9. - Rawlinson, op. laud., t. II, p. 399. - Perrot, op. laud., t. II, p. 443-445.

3. J. Nenant, Les pierres gravees, pl. III, fig. 5. - Layard, The monumenls, ser. I, pl. 33.

4. Ravilinson, op. laud., t. I, p. 348.

5. The flora of the Assyrian monuments. London, 1894, in-8, p. 37. 
les bas-reliefs de Koyoundjik nous offrent des palmiers, des vignes et des figuier's d'une ressemblance saisissante; les conifères sont aussi sculptés avec un grand soin; la convention toutefois a pris place dans leur représentation; aussi est-il difficile de dire à quelle espèce ou mème à quel genre ils appartiennent ${ }^{1}$. Quant aux arbres, dont les rameaux étalés offrent l'aspect de longues feuilles pinnatifides, à en juger par ceux d'un bas-relief de Koyoundjik ${ }^{2}$, auxquels l'artiste assyrien a donné des fruits, ils doivent être des grenadiers.

La flore herbacée des monuments assyriens est peu riche; on n'y voit guère que trois ou quatre plantes; l'une, une fleur de six à dix pétales, dont les serviteurs royaux portent, dans des vases, des rameaux dans la salle du banquet, m'est inconnue ${ }^{3}$; l'autre, corymbifère à tige nue et à feuilles radicales entières, dont j'ai déjà parlé, a le facies d'un doronic *; une troisième, figurée sur la façade septentrionale du palais de Koyoundjik ${ }^{5}$, à la tige feuillée et aux fleurs peu nombreuses en forme de fer de lance, est évidemment une liliacée; mais il est impossible de dire au juste à quelle espèce elle appartient ${ }^{6}$.

1. M. Bonavia, op. laud., p. 28, les regarde comme appartenant à l'espèce Pinus Brutia.

2. Layard, The monuments, ser. II, pl. 14. 3. Layard, The monuments, ser. II, pl. 8. - Rawlinson, op.
laud., t. I, p. 581 .

4. M. Bonavia, qui n'hésite jamais dans ses identifications, dit, lui, p. 34, que "c'est sans aucun doute l'Iieracium pannosum », encore que cette plante soit une flosculeuse, non une
corymbifère.

5. Rawlinson, op. laud., t. I, p. 354.

6. M. Bonavia, p. 32, affirme, après Rawlinson d'ailleurs, que c'est le Lilium candidum L. 
Les arts ne prirent naissance chez les Sémites de la Syrie qu'à une époque relativement récente et il ne nous en reste que peu de monuments; mais ces rares débris et le témoignage des Anciens nous montrent qu'ils s'y développèrent sous l'influence de l'Égypte et de la Mésopotamie; cela n'a rien qui doive surprendre, puisque au moment où la Syrie prit place dans l'histoire générale elle subissait la suprématie politique de ces deux puissants états. Il faut donc nous attendre à rencontrer sur les monuments ou les objets d'art des Sémites de la région du Liban et du Jourdain des motifs de décoration végétale analogues à ceux que nous ont offerts les monuments ou l'industrie artistique de l'Égypte ou de la Mésopotamie.

Quelques-unes des populations syriennes vivaient encore de la vie nomade et les autres étaient à peine sorties de la barbarie, quand elles entrèrent en relation arec les habitants du bassin du Tigre et de l'Euphrate et du bassin du Nil, arrivés depuis longtemps déjà au plus haut degré de civilisation; elles subirent leur double ascendant, et de même qu'elles s'inclinèrent devant leur puissance militaire, elles acceptèrent leur influence artistique.

Nous ignorons jusqu'où avait pu être porté le goùt des arts dans le pays de Canaan avant la conquête des Hébreux; pour ceux-ci, restés nomades jusque-li, ils y étaient encore étrangers et ils ne s'y livrèrent même que beaucoup plus tard; mais les Phéniciens les cultivaient sans doute déjà depuis longtemps, nous ne pouvons dire toutefois jusqu'à quel degré de perfection 
ils en araient poussé la pratique; les rares débris qui nous restent de leurs monuments civils ou religieux sont trop récents et sont trop mutilés pour que nous puissions nous en faire une idée complète et exacte. Heureusement les ruines considérables, éparses sur le sol des nombreuses colonies, que les navigateurs intrépides de Tyr et de Sidon fondèrent sur presque tous les rivages de la Méditerranée, en particulier dans l'ile de Cypre et en Mauritanie, nous permettent d'entrevoir quels motifs de décoration étaient employés par les architectes phéniciens. A en juger par ce qui nous reste des monuments de la Phénicie proprement dite, ils ne connurent à l'origine d'autres ornements que les formes géométriques; les fûts des colonnes étaient lisses, les chapiteaux font pressentir ceux de l'ordre toscan, seulement avec un galbe et des proportions différentes ${ }^{1}$. Il en est tout autrement dans les monuments des colonies. A Cypre, en particulier, les lignes droites des chapiteaux phéniciens font place aux volutes savamment combinées, en même temps que des tiges et des fleurs de lotus remplissent l'espace libre laissé par leurs courbes élégantes ${ }^{2}$; sur le calathos d'une colonne de même origine on voit des tiges feuillées ou des palmes à folioles ovales, ornement qui semble bien indigène ${ }^{3}$.

Les colonnes elles-mèmes empruntèrent dès lors leurs formes au règne végétal; les plates-formes du célèbre temple de Paphos, représenté sur une mon-

1. Perrot et Chipiez, op. laud., t. III, p. 115.

2. Chapiteaux cypriotes du Musée du Louvre. Perrot, op. laud., t. III, p. 116.

3. G. Colonna-Ceccaldi, Monuments antiques de Cypre. Paris, 1882, in-8, p. 43. - Perrot, t. III, p. 118. 
naie de Cypre ', étaient soutenues par des colonnes simulant un tronc de palmier. Les chapiteaux des colonnes du temple de Byblos étaient, elles, formées par deux rangs de pétales de lotus, et l'arc de cercle qui réunissait les deux parties de l'entablement, supportait une palmette semblable à celle des monuments assyriens ${ }^{2}$. Une palmette analogue, mais formée de quatre feuilles régulières, reposant sur une triple volute qu'enveloppe une espèce de demi-cercle aux extrémités arrondies et recourbées, ce qui lui enlève presque tout caractère végétal, se rencontre aussi sur plusieurs dalles phéniciennes ${ }^{3}$. On voit encore trois palmettes superposées se dresser entre deux sphynx affrontés sur le chapiteau de deux stèles, qui se faisaient pendant aux extrémités d'un beau sarcophage d'Athiénau'. On retrouve, au contraire, la vraie palmette assyrienne à la base des anses d'un vase d'Amathonte ${ }^{5}$.

Les rosaces et les fleurons étaient souvent employés, ainsi que les palmettes, comme motifs de décoration par les artistes phéniciens; au bord d'une dalle d'albâtre au Musée du Louvre, on voit en particulier des fleurons à seize rayons alternant avec des rosaces, à quatre rayons à angle droit, séparés par des palmettes à cinq folioles ${ }^{6}$. Ces ornements décoratifs se rencontrent sur les monuments les plus divers; tels ces Heu-

1. T. L. Donaldson, Architechura numismatica. London, 1859, in-4 pl. 91. - Perrot, t. III, p. 120.

2. Donaldson, op. laud., pl. 20. - Perrot, op. laud., t. IJI, p. 126.

3. Musée du Louvre. Perrot, op. laul., t. III, p. 129, 131 et 133 .

4. Perrot: op. laul., t. III, p. 217, pl. 152.

5. Musée du Louvre. Perrot, t. III, p. 280 et 281.

6. Musée du Louvre. Perrot, t. HI, p. 132 et 133. 
rons à douze pétales, placés de chaque còté d'un liou sur un marchepied cypriote ${ }^{1}$ en pierre, ou la rosace à cinq rayons, sculptée au-dessus de la porte d'un caveau giblite? Sur un sarcophage de mème origine sont ciselées aussi des guirlandes, des palmes, une couronne et un arbre, indiqué sous la forme d'une large feuille ${ }^{3}$. On voit encore sur un bas-relief d'Ascalon, peut-être, il est vrai, postérieur à l'ère chrétienne, représentés assez grossièrement deux ceps de vigne, qui ombragent deux femmes accroupies".

Les stèles rotives de Carthag'e étaient souvent aussi, comme les colonnes phéniciennes, ornécs de motifs végétanx; c'est, ici une fleur de lotus, là un grenadicr chargé de fruits. Ailleurs on reconnait un tamaris à ses branches élancées et grêles, des fleurs de courge et dos épis de blè ${ }^{5}$. Le palmier aussi est représenté avec son tronc massif, ses feuilles en volutes et ses spathes ${ }^{6}$. Sur une stèle d'Hadrumète, deux colonnes, dont le füt cannelé sort d'un bouquet de feuilles et se termine par un buste de femme, supportent un entablement très riche et très compliqué; il se compose en bas d'une rangée de fleurons et de boutons alternatifs de lotus renversés; au-dessus s'étale le disque solaire; puis vient un rang d'uræus '. Nous avons là des motifs de

1. Louis Palma di Cesnola, Cyprus: its ancient cilies, tombs and lemples. London, 1877, in-4. p. 159. - Perrot, t. III,
p. 284 .

2. E. Renan, Mission de Phénicie. Paris, 1864, in-fol., pl. 27. - Perrot, t. III, p. 169.

3. Renan, op. laud., pl. 29. - Perrot, t. III, p. 175.

4. Nusée du Louvre. Perrot, t. III, p. 441.

5. Ph. Berger, Les ex-volo du temple de Tanil à Carlliage. Paris, 1877, in-4o, p. 20-22.

6. Bibliothèque nationale. Perrot, t. III, p. 460 et 461 .

7. Ilusée du Louvre, Perrot, t. III, p. 461. 
décoration presque exclusivement égyptiens ; on les retrouve aussi dans les monuments cypriotes, mais mêlés à l'ornementation assyrienne, ce qui ne doit pas surprendre, puisque l'ile de Cypre fut au vin ${ }^{\mathrm{e}}$ et au $\mathrm{VII}^{\mathrm{c}}$ siècle avant notre ère tributaire de Ninive. C'est ainsi que sur un sarcophage d'Amathonte on voit, de chaque côté des faces principales, trois palmettes assyriennes superposées, tandis qu'au-dessous de l'entablement règne un cordon de fleurs et de boutons de lotus ${ }^{1}$.

Les motifs de décoration, empruntés au règne végétal, que nous venons de rencontrer sur les monuments de l'architecture et de la sculpture de la Phénicie, de Cypre et de Carthage, se retrouvent aussi dans les produits des arts industriels de ces diverses contrées: fleurs et boutons de lotus, palmettes, rosaces y apparaissent dans les combinaisons les plus ingénieuses et parfois les plus fantaisistes. Telles sont les palmettes que l'on voit sur un disque sarde en terre cuite, surgissant d'une espèce de bourrelet entouré d'un croissant, tandis que des fleurs conventionnelles de lotus se dressent entre chacune d'elles et qu'une rosace en occupe le centre; telles sont encore les deux palmettes opposées d'un autre disque de mème origine arec leurs volutes recourbées ${ }^{2}$. Ici une double rangée de rosaces orne le goulot d'un vase d'Ormidia, tandis qu'une guirlande de fleurs et de boutons de lotus

1. Perrot, op. laud., t. III, p. 608 et 609. Un sarcophage d'Athiénau, Pcrrot, t. III, p. 613, n'offre, au contraire, aucun motif de décoration végétale, mais on voit plusieurs arbres dans la scène de chasse qui est représentée sur sa face principale.

2. Crespi, Calalogo, pl. E, fig. 1 et 2. - Perrot, t. III. p. 672. 
entoure le haut de la panse ${ }^{\prime}$. Là encore, des lleurs de lotus se dressent rerticalement sur les flancs d'un cratère. On roit également, sur une coupe cypriote, les mèmes lotus surgissant, flanqués de volutes et agrèmentés d'une rosace, entre des oiseaux de fantaisie?. Il faut citer encore les tiges couvertes de feuilles et de boutons de la mème nymphéacée, qu'un personnage, debout sur une œnochoé du Musée de New-York ${ }^{3}$, semble défendre contre un oiseau de fantaisie, ainsi que les guirlandes de feuillages qui ornent les parties renflées d'un autre vase cypriote, avec les tiges fleuries, qui se dressent de chaque côté d'un personnage, tenant lui-même une fleur à la main ".

Quoique la décoration des verres et des émaux phéniciens consiste surtout en ornements de forme géométrique, on y rencontrait aussi des motifs empruntés à la nature végétale: telles ces tiges feuillées ou ces palmes qu'offre un verre antique de la collection Gréau; telles encore ces rosaces que présentent des pàtes de rerre dessinées par Elson ${ }^{5}$. Mais c'est dans l'orfèvrerie phénicienne que la décoration végétale a joué le rôle le plus considérable: fleurons et rosaces, palmettes, fruits, tiges de papyrus, fleurs ou boutons de lotus, isolés ou groupés artistement entre eux, servent également à orner les coupes, les bracelets, les anneaux, les pendants d'oreilles ${ }^{6}$. Des paysages entiers avec des

1. Perrot, op. laud., t. III, p. 699 et 711.

2. Calalogue Barre, pl. 1, ap. Perrot, t. III, p. 700, fig. 508 et 509 .

3. Perrot, op. laud., t. III, p. 709.

4. Cesnola, Cyprus, p. 394. - Perrot, t. III, p. 710.

5. Perrot, op. laud., t. III, pl. VII, 1 et 2, et p. 746, fig. 539-5亿1.

6. Cesnola, Cypirus, p. 311, 312, 317, 319. - Perrot, t. III p. $798,817,819,823,833,834,835$ et 836 . 
scènes de guerre ou de chasse ont même été gravés sur le pourtour ou au fond des coupes. Tel est le tableau gracieux de la "Journée du chasseur ", représenté dans la seconde zone d'une coupe de Préneste ${ }^{1}$, avec l'arbre derrière lequel il se cache pour abattre sa proie, et le bois de palmiers et de grenadiers (?), où il se repose après sa chasse.

Tels encore la chasse au lion qu'on voit sur un plat d'argent de Céré, le siège d'une ville gravé sur une patère d'Amathonte ou la marche triomphale figurée au pourtour d'une patère de Dali. L'artiste phénicien ne s'est pas borné à représenter les scènes de la chasse, du siège ou de la marche triomphale, il y a joint des motifs divers empruntés au monde végétal, ainsi qu'à la mythologie assyrienne ou égyptienne. C'est ainsi que, sur le plat d'argent de Céré ${ }^{2}$, des arbres, dattiers ou conifères, séparent les différents épisodes de la chasse. Des palmiers et autres arbres, que les assiégeants abattent, se voient dans la zone extérieure de la patère d'Amathonte ${ }^{3}$, tandis que sur la seconde zone sont représentés entre autres l'arbre de vie, que viennent adorer deux génies ou divinités, ainsi qu'Horus, assis sur le lotus symbolique, et accompagné par Isis des lotus à la main, en même temps qu'une large rosace occupe le centre de la patère. Sur la zone extérieure de la patère de Dali ${ }^{4}$, quelques arbres sans ca-

1. Clermont-Ganneau, La coupe de Palestrina. (Éludes d'archéologie orienlale. L'Imagerie phénicienne. Paris, 1880. in-8, p. 16-39, et pl. I.) - Perrot, t. III, p. 759.

2. Grifi, Monumenti di Cere antica, pl. V, 1, ap. Perrot, t. III, p. 769.

3. Ceccaldi, Monuments, pl. VIII. - Perrot, t. III, p. 775.

4. Musée du Louvre. Perrot, t. III, p. 779. 
ractère défini s'élèvent au milieu du terrain sur lequel défilent les troupes; la seconde zone, au contraire, ou quelques régétaux apparaissent encore, est surtout occupée par des arbres de vie, qui alternent avec des génies en lutte contre des lions ou des guerriers qui égorgent un animal fantastique; quant au fond de la patère, il est rempli par un entrelacs de fleurons à six pétales, qu'entoure une double rangée de fleurs et de boutons de lotus reliés par un cordon continu.

La zone extérieure d'une patère de Curium ${ }^{1}$ nous offre une scène analogue à celle de la patère de Dali; mais les lions sont remplacés par des griffons, et l'on voit ici des spliynx, là des ibex, ailleurs encore des griffons venir se rafraichir à l'arbre de vie; dans la seconde zone alternent, séparés par des espèces de conifères, des lions, des taureaux et des chevaux, et au centre est sculpté le dieu Ashshour, perçant un lion de son glaive. Enfin le médaillon d'une coupe de Céré représente un fourré de papyrus au milieu desquels se cache une génisse et deux bouvillons ${ }^{2}$, scène qui en rappelle unc semblable que nous avons vue sur un dallage de Tell-el-Amarna. Des motifs décoratifs de nature végétale, fleurons et rosaces, se voient aussi sur des tablettes d'ivoire ${ }^{3}$; on rencontre miême des lotus et des palmettes de fantaisie gravés sur des œufs d'autruche ${ }^{4}$.

Ces motifs de décoration, qu'on retrouve sur les mo-

1. Ceccaldi, Monuments, pl. X. - Cesnola, Cyprus, p. 329. - Perrot, t. III, p. 789.

2. Grifi, Monumenli, pl. X, 1, ap. Perrot, t. III, p. 790.

3. Renan, Missions de Phénicie, p.'500. - Perrot, t. III, p. 847.

4. Perrot, op. laud., t. III, p. 856 et 857. 
numents de toutes les colonies fondées par les Phéniciens, furent importés en Jurlée par les artistes chargés de construile le palais de David et le temple de Salumon. En interdisant de faire des images taillées ou des représentations des êtres qui existent ${ }^{1}$, le Décalogue avait en quelque sorte condamné les Israélites a rester étrangers aux arts plastiques; aussi quand David voulut se batir un palais, ce furent des ouvriers phéniciens, envoyés par Hiram, qui l'édifièrent. Salomon fut également obligé d'avoir recours à des artisans phéniciens pour la construction du Temple et de la "Maison du bois du Liban ". On ne doit dès lors pas être surpris que les motifs de décoration propre aux monuments phéniciens se retrourassent dans les édifices des deux rois juifs. Tels étaient les palmiers et les fleurs épanouies sculptés, avec des figures de Kéroubs, sur les deux battants en bois d'olivier sauvage de la porte du sanctuaire ${ }^{2}$. Tels étaient encore les ornements des chapiteaux des deux colonnes de bronze, Iakin et Bo'az, fondues par Hiram, le fameux toreuticien de Tyr, et placées dans le vestibule du temple. Haut de cinq coudées, chaque chapiteau avait la forme d'une fleur de lotus épanonie; la partie inférieure renflée en était décorée par un ornement réticulé, compris entre deux rangées, chacune de cent grenades ${ }^{3}$. MM. Perrot et Chipiez supposent que quatre de ces grenades, " sans doute plus grosses que les autres " ", étaient fixées aux points où se rencontrent les lignes qui dessinent les différentes faces.

1. Exodus, cap. xx, vers. 4.

2. 1 Regna, cap. Ir, vers. 32 .

3. 1 Regna, cap. vil, vers. 18-20.

4. Ilisloire de l'art, t. IV, p. 318 et pl. VII. 
Les sculptures qu'on voyait sur le bord de la mer d'airain offraient sans doute des motifs de décoration analogues. On retrouvait ces mèmes motifs dans les divers produits des arts industriels et les monuments des derniers siècles de l'histoire juive. C'est ainsi qu'un vase en terre trouvé à Jérusalem, mais qui est peut-ètre de l'époque gréco-romaine, offre en saillie sur sa panse des grenades et des grappes de raisin alternant entre elles'. On voit aussi des grappes de raisin, des fleurons ou rosaces de fantaisie, des rameaux d'olivier, des feuilles et des fruits divers, mais sans caractère déterminé, sur l'entablement d'un sarcophage juif, dit le Tombeau des Rois, mais probablement peu ancien, ainsi que des rosaces de forme variée sur la convexité du couvercle et un fleuron entre deux lotus héraldiques ì son extrémité ${ }^{2}$. La surface de ce sarcophage offre une décoration végétale encore plus riche; c'est d'abord deux longues bandes, ornées chacune d'un double rang de feuilles d'olivier réunies par trois, avec deux fruits longuement pédonculés; puis, à droite et à gauche, un enguirlandement de fantaisie, composé de fleurs ouvertes et de boutons de lotus, de fruits et de feuilles de cucurbitacée, de glands de chêne et de grappes de raisin; enfin, au milieu, des entrelacs composés de feuilles de vigne, de grappes de raisin, de fleurons, de cônes de pin, de glands, etc. Les monuaies juives, postérieures également à l'époque de la conquète grecque, portent aussi des emblèmes tirés de la nature végétale: dattiers avec leurs fruits,

1. Musée du Louvre. Perrot et Chipiez, t. IV, p. 461.

2. F. De Saulcy, Voyage aulour de la mer Morle. Paris, 1853. Atlas pl. XXX, XXXII et XXXIII. - Cf. Perrot, t. IV, p. 389. 
palmes, feuilles de vigne, grappes de raisin, grenades, épis de froment ${ }^{1}$. On voit également un palmier qui se dresse derrière un cheval, sur une monnaie carthaginoise ${ }^{2}$.

L'influence de l'Assyrie et de l'Égypte ne se fit pas sentir seulement sur l'art des Phéniciens et des Juifs, les Hétéens, pour ne pas parler des Grecs et des autres populations indo-européennes de l'Asie-Mineure, la subirent aussi; on en trouve la preuve dans l'ornementation des rares monuments, que nous ont laissés cette nation. C'est ainsi que sur un bas-relief de Saktchégheuksou en Syrie, qui représente une chasse au lion, l'artiste hétéen a sculpté des rosaces, motif do décoration assyrien, dans le champ resté libre du paysage $^{3}$. On voit également des rosaces sur deux des quatre faces d'un cachet, probablement cappadocien, tandis que sur une autre face sont gravés deux taureaux, debout de chaque côté d'un arbre de vie ${ }^{4}$. Il est un monument hétéen de l'Asie Mineure qu'il faut encore mentionner ici, moins pour son ornementation qu'à cause du caractère symbolique des végétaux qu'on y trouve figurés; c'est la sculpture rupestre d'Ibriz ou Ivriz dans l'ancienne Lycaonie ", qui représente un

1. J.-J. Scheuchier, Physique sacrèe ou Histoire naturelle de la Bible. Amsterdam, 1732, in-fol., t. I, p. 99. - De Saulcy, Recherches sur la numismatique judaique. Paris, 1854, in-4, pl. I, 6; II, 1, 2; III, 9, 10, 11, 12, 13; VII, 5, 6, 8; VIII, 1, 2, $3,4,5,7,10 ;$ IX, 5, 11, 12. - Babelon, Calalogue des monnaies grecques de la Bibliotheque nationale. Les rois de Syrie. Paris, 1890, in-4, p. 75, 99, 10' 109, 110, 120, 161, 164, 168171, etc.

2. Pietschmann, op. laud., p. 14.

3. Perrot et Chipiez, op. laud., t. IV, p. 555, pl. 279.

Ł. Nusée du Louvre. Perrot, t. IV, p. 772.

5. E. J. Davis, On a new Hemathite inscription at lbreez. 
persomnage, prètre ou roi, en adoration devant un lieu champètre, lequel tient d'une main un faisceau d'épis, tandis que l'autre soutient les rameaux, chargés de raisins, d'un cep de vigne qui sort du sol derrière lui.

\section{II.}

Les arts du dessin n'ont pas seuls, chez les Sémites, emprunté au monde des plantes les formes et les motifs de décoration les plus gracieux, la poésie de ces peuples en a tiré aussi de nombreuses comparaisons et elle y a puisé d'ingénieuses fictions. Dans l'épopée d'Izdoubar, le poète chaldéen raconte un débat entre deux arbres, le cyprès et le laurier (?) ${ }^{1}$ : " Tes racines ne sont pas assez robustes, dit le premier au second; ton ombre n'est pas assez fraîche, ton écorce pas assez forte. " Le laurier s'irrite, mais le fragment mutilé de ce curieux dialogue ne renferme pas malheureusement sa réponse. "Semblable à l'herbe fauchée, Ishtar est descendue; d'une lèvre semblable au roseau languissant, elle implore (les eaux de vie) ", dit Allat de la déesse ${ }^{2}$, dont le portier des Enfers vient de lui annoncer l'arrivée; et dans l'hymne

(Transactions, t. IV, 2 (an. 1876), p. 336-346). - W.-M. Ramsay, Basrelief of Ibriz. (Archaeologische Zeilung, t. XLIII (an. 1885), p. 203-208, pl. 13. - Perrot, t. IV, p. 725.

1. A. Jeremias, Isdubar-Nimrod. Eine altbabylonische IIeldensage. Leipzig, 1891, in-4, p. 28.

2. A. H. Sayce, Lectures on the origin and growth of religion as illustrated by the religion of the ancient Babylonians. $3^{\text {e }}$ éd. London, 1891, in-8, p. 222. - M. J. Oppert, Fragmen/s mythologiques, Paris, 1882, in-8, p. 9, traduit: "Nous sommes comme l'herbe coupée, nous sommes comme la plante fanée. » 
désolé qu'il composa à l'occasion de l'enlèvement de la statue de Nanâ par le roi élamite Koutournakhounta, au xxIII siècle arant notre ère, un prêtre d'Ourouk s'écrie ${ }^{1}$ : "L'impie tout puissant m'a brisé comme un roseau, comme une roselière je gémis nuit et jour. " "Que par le charme d'Éa le sortilège soit enlevé comme la peau d'une gousse d'ail, coupé comme un régime de dattes, arraché comme une branche d'arbre ", dit le père des dieux à son fils Mardouk $^{2}$, en lui enseignant le moyen de guérir un homme frappé par une malédiction funeste.

Mais c'est surtout dans la Bible que les images et les comparaisons tirées du monde végétal occupent une place considérable. La fable des arbres par laquelle Joatham voulait montrer aux habitants de Sékem la faute qu'ils avaient commise en nommant roi $\mathrm{Abi}$ mélek, est célèbre.

Un jour, leur dit-il ${ }^{3}$, les arbres voulurent oindre l'un d'eux pour être leur roi et ils dirent à l'olivier : Sois notre roi! Mais I'olivier leur dit: Cesserais-je de produire mon huile, que les dieux et les hommes prisent en moi, pour aller planer audessus des arbres? Alors les arbres dirent au figuier: Eh bien, toi, sois notre roi! Nais le figuier leur répondit: Cesserais-je de produire mon doux et bon fruit, pour aller planer au-dessus des arbres? Et les arbres dirent à la vigne: Eh bien, toi, sois notre roi! Mais la vigne leur répondit: Cesserais-je de produire mon jus, qui réjouit les dieux et les hommes pour aller planer au-dessus des arbres! Alors tous les arbres dirent au buisson épineux: Eh bien, toi, sois notre roi! Et le buisson répondit

1. Hommel, Geschichte Babyloniens und Assypiens, p. 345. - Naspero, Histoire ancienne, t. II, p. 37.

2. Jensen, De incantamentorum sumerico-assyriorum seriei quae dicilur shurbu tabula VI. (Zeitschrift für Keilschriftforschung, t, I, p. 280.) - Sayce, Lectures, p. 472.

3. Judices, cap. $\mathrm{I}$, vers. 8-15, trad. d'E. Reuss: 
aux arbres: Si c'est de bonne foi que vous voulez moindre pour être votre roi, venez donc vous abriter sous mon ombre; si non, un feu sortira du buisson pour consumer les cèdres du Liban.

Dans la vision souvent citée que lui envoya l'Éternel, pour lui réréler le sort différent qui attendait les Israélites, emmenés par Nabuchodonosor à Babylone et ceux qui étaient restés en Judée avec Sédécias, Jérémie roit deux corbeilles pleines de figues placées devant le temple ${ }^{1}$; l'une renfermait de très bonnes figues, comme le sont celles de la première saison; l'autre des figues très mauraises et qui n’étaient même pas mangeables. Et l'Éternel lui dit: "Tels que ces bonnes figues, je recomnaitrai à bonnes fins les déportés de Juda, que j’ai chassés de ce pays-ci dans la terre des Chaldéens; je les ferai revenir; je les replanterai et ne les arracherai point. Et tels que les figues mauvaises, qui ne sont pas mangeables, je ferai de Sédécias et des restes de Jérusalem demeurés en ce pays ou établis en Égypte, un objet d'horreur pour tous les royaumes de la terre. ")

Non moins célèbre est l'allégorie dans laquelle le prophète Ézéchiel représente sous la figure de deux aigles Nabuchodonosor et le roi d'Égypte, sous celle d'un cèdre Jéchonias et d'une vigne Sédécias ${ }^{2}$. Le premier aigle l'arait mise dans un sol fertile, " pour qu'elle poussât et derînt une vigne plantureuse, humble de taille, de manière que ses branches se tournassent vers lui. " Mais après avoir poussé ses rameaux et s'ètre couverte de feuillage, cette vigne recourbe ses racines vers le se-

1. Cap. xxiv, vers. 1-9.

2. Cap. xvil, vers. 3-10. 
cond aigle et étend vers lui ses branches. Aussi au lieu de pousser de vigoureux sarments et de porter des fruits, elle est coupée, son feuillage se fane et elle se sèche complètement.

C'est aussi par des images empruntées au règne végétal, qu'Isaïe dépeint la ruine qui allait fondre sur Moab ${ }^{1}$ :

Elles languissent les campagnes de Heshbôn, les vignes de Sibmah, dont les nobles ceps enivraient les rois, qui poussaient jusqu'à Jazer, erraient à travers la savane, et dorst les pampres s'étendaient au-delà de la mer. Je pleure les vignes de Sibmah avec les larmes de Jazer.; je vous arrose de mes larmes, Heshbôn et Éléaleh, car le cri de guerre est venu fondre sur votre récolte, votre vendange. La joie et l'allégresse sont bannies des vergers; dans les vignes pius de chants, plus de cris! Personne ne foulera plus le vin dans les cuves.

Zacharie a recours aux mêmes images pour annoncer la désolation qui menace Israël ${ }^{2}$ :

Ouvre tes portes, ô Liban, pour que le feu dévore tes cèdres! Lamentez-vous, cyprès, de ce que les cèdres tombent, de ce que les puissants sont abattus! Lamentez-vous, chênes de Bashan, de ce que la forêt inaccessible est miseà bas !...Écoutez! les jeunes lions rugissent, car elle est détruite, la parure du Jourdain.

Dans Osée ${ }^{3}$, une allégorie semblable montre ce qu'Israël, qui, avant sa faute, était " une vigne couronnée de pampres et chargée de raisins ", redeviendra une fois pardonné par Dieu :

Je serai pour Israël comme la rosée; il fleurira comme le lis et jettera des racines comme celles du Liban; ses rejetons s'étendront, sa beauté sera comme celle de l'olivier; son par-

1. Cap. Xvi, vers. 8-10, trad. d'Ed. Reuss.

2. Cap. xi, vers. 1-2.

3. Cap. $\mathrm{x}$, vers. 1 et cap. xIv, vers. 6-8. 
fum sera pareil à celui du Liban. Ceux qui s'assiéront sous son ombre produiront de nouveau du blé, ils fleuriront comme la vigne et leur renom cela celui du vin du Liban.

Olivier verdoyant, beau et couvert de fruits magnifiques, tel était le nom que te donnait l'Éternel, s'écrie Jérémie ${ }^{1}$ en parlant d'Israël coupable; mais au milieu d'un grand fracas sa foudre est tombée sur toi et tes branches sont brisées.

Isaïe en particulier affectionne ces images tirées du monde végétal ; c'est ainsi que réprimandant Israël au sujet de son polythéisme :

En ce jour, dit-il ${ }^{2}$, la gloire de Jacob sera obscurcie... : il sera, comme le champ de la plaine de Réfaïm, dont le moissonneur a ramassé les chaumes, après avoir coupé les épis, et oì vient glaner misérablement le glaneur. Il sera comme l'olivier, où, après la récolte des olives, il reste à peine à grapiller deux ou trois fruits dans les hautes branches... Il a oublié le dieu de son salut, il a planté un jardin à sa guise, y a mis des ceps étrangers, et les a entourés d'une haie; de bonne heure il a vu fleurir ses plants, mais la récolte lui échappe au jour du malheur.

Et opposant à la fragilité des choses humaines l'immutabilité de la parole divine, le même prophète s'écrie $^{3}$ : "Tous les mortels sont comme l'herbe et toute leur beauté comme la fleur des champs: l'herbe se dessèche, la fleur se fane; mais la parole de notre Dieu subsiste à jamais. " "Les hommes, dit de même le Psalmiste ${ }^{4}$, croissent comme l'herbe; elle fleurit et pousse au matin, le soir on la coupe et elle sèche." Pour caractériser la conduite du dieu des armées et

1. Cap. xi, vers. 16 .

2. Cap. xvil, vers. $4-6$ et 10-11.

3. Esaias, cap. xL, vers 6-7.

4. Psalmus Lxxxix, vers 5-6. 
la prudence de ses conseils, le premier Isaïe le compare' au laboureur qui, après avoir aplani son champ, y sème, au temps voulu, la nielle et le cumin, le blé, le millet et l'orge. L'Écriture, voulant peindre la paix dont jouissait le peuple juif " durant les jours de Salomon ", dit que " chacun reposait sous sa vigne et sous son figuier depuis Dan jusqu'à Bersabée "; et afin de donner une idée de la prospérité qui régnait alors, elle ajoute ${ }^{3}$ que " les cèdres étaient devenus aussi communs que les sycomores, qui croissent dans les champs. "Pour montrer qu'il lui a pardonné, le Seigneur dans Joël ${ }^{4}$ promet à Israël de lui envoyer du blé, du vin et de l'huile. " Les prairies, ajoute-t-il, vont reprendre leur éclat, les arbres porteront leurs fruits, le figuier et la vigne prodigueront leurs richesses. "C'est dans le même sens que l'Éternel dit dans le second Isaïe" : "Je mettrai dans la steppe le cèdre et le cassie, le myrte et l'olivier; je planterai dans la lande le cyprès, le platane et le sapin. " Et ailleurs: "Les cyprès s'éleveront à la place des ronces, le inyrte croitra à la place de la bruyère. " "Leur vigne ", dit Moïse ${ }^{6}$, parlant des peuples idolàtres, " provient de celle de Sodome et des campagnes d'Amorah; leurs raisins sont des raisins rénéneux, ils ont des grains amers. Leur vin, c'est du fiel de serpent."

Jacob mourant compare Joseph à un "sarment fé-

1. Cap. xxvill, vers. 24-25.

2. 1 Regna, cap. IV, vers. 25. - 2 Regna, cap. xvill, vers. 31. - Esaias, cap. Xxxvi, vers. 16. - Mich., cap. Ir, vers.4.

3. 1 Regna, cap. x, vers. 27. - 1 Paralipomena, cap. 1, vers. 15.

4. Cap. II, vers. 19 et 22.

5. Cap. XI.I, vers. 19 ; cap. I.v, vers. 13.

6. Deuteronomium, cap. xixil, vers. 32. 
cond, planté près de la fontaine et dont les branches couvrent la muraille " , et il dit de Juda qu' "il liera son ànon à la vigne, et lavera sa robe dans le vin, son manteau dans le sang de la vigne. " Pour le Psalmiste ${ }^{2}$ Israël est " un cep de vigne, qui, apporté d'Égypte, a poussé des racines, remplit la terre et couvre les montagnes de son ombre "; et l'homme juste est " comme l'arbre planté près du cours des eaux. " " Il croit, dit-il encore, comme le palmier" il s'élève comme le cèdre du Liban. " Le poète sacré dit de luimème qu'il est semblable à un "olivier, qui se couvre de feuilles dans la maison de Dieu ${ }^{3}$. " Jahveh, dans Osée ${ }^{4}$, se compare à un "cyprès toujours vert ". "Je suis la vraie vigne ", dit Jésus", "et mon père est vigneron. " Pour Ézéchiel ${ }^{6}$ Ashshour est " un cèdre du Liban, aux branches belles et touffues, magnifique de hauteur et élevant sa cime jusqu'aux nues. "

On retrouve ces mêmes images, mais développées, dans la belle allégorie du livre de Daniel ${ }^{7}$, qui peint la grandeur et la puissance de Nabuchodonosor, puissance auquel un lamentable abaissement devait mettre un terme:

C'était un arbre d'une grandeur prodigieuse au milieu de la terre, arbre grand et fort, dont la cime touchait aux cieux et était visible jusqu'aux extrémités de la terre. Son feuillage était beau et ses fruits abondants; il y avait là de quoi nourrir le monde entier. Les animaux de la campagne s'abritaient

1. Genesis, cap. XLIX, vers. 22 et 11.

2. Psclmi Lxxx, vers. 8; I, vers. 3 ; xcII, vers. 15.

3. Psalmus Li, vers. 8.

4. Cap. Xiv, vers. 9.

5. Johannes, cap. $\mathrm{xV}$, vers. 1.

6. Cap. xxxi, vers. 3.

7. Cap. IV, vers. 7-9. 
sous son ombre, dans ses branclies se logeaient les oiseaux du ciel et tout ce qui vit s'y rassassiait.

Il faut rapprocher de ce portrait celui que la $\mathrm{Sa}-$ gesse trace d'elle-même dans l'Ecclésiastique ${ }^{1}$ :

Je m'élevai comme le cèdre du Liban, comme le cyprès sur les hauteurs de l'Hermon. Je grandis comme le palmier sur le rivage, comme les rosiers de Jéricho, comme un bel olivier dans la plaine, comme un platane aux bords des eaux. J'ai répandu l'odeur du cinnamome et de l'aspalathe; j'ai exhalé les parfums de la myrrhe.

Je ne puis m'empêcher de citer encore cette belle apostrophe du mème écrivain s'adressant aux hommes pieux $^{2}$ : "Croissez comme la rose au bord du ruisseau; répandez une suave odeur comme l'encens ; faites éclore des fleurs comme le lis. "

La Bible est remplie de ces comparaisons et de ces allégories empruntées au monde des plantes et partant bien faites pour frapper des populations agricoles ou pastorales. Pour annoncer la venue du Messie, Isaïe dit ${ }^{3}$ qu'un "rejeton sortira de la tige de Jessé et qu'une fleur s'élèvera de ses racines. " Les tentes d'Israël apparaissent à Biléam " comme des aloès plantés par Jahveh, comme des cèdres sur le bord des eaux 4 . "Voulant rappeler à quel point étaient redoutables les Amorrhéens, Amos dit "que " leur taille égalait celle des cèdres et leur force celle des cyprès. " Parlant du

1. Cap. Xxiv, vers. 17-23.

2. Cap. ixxis, vers. 17-19.

3. Cap. XI, vers. 1. Et parlant du "serviteur de Dieu " opprimé: «ll poussait, dit le second Isaïe, cap. L.II, vers. 2, comme un arbrisseau languissant, comme un rejeton, qui sort d'un sol aride. „Cf. Je portrait de Simon dans l'Ecclésiastique, L. 8-11.

4. Numeri, cap. xxiv, vers. 6 .

5. Cap. II, vers. 9. 
jour où Dieu humiliera la fierté des mortels, "Jahveh, dit le premier Isaïe, aura son jour... sur tous les cèdres du Liban, hauts et élevés, et sur tous les chènes de Bashan. " Ailleurs il compare ceux qui abandonnent l'Eternel à des " térébinthes dépouillés de leurs feuilles ${ }^{1}$. "Le second Isaïe, par une figure analogue, appelle les Israélites affranchis des " térébinthes de justice, plantation de l'Éternel. " Et, dernier trait au tableau de la béatitude future qu'il leur promet, il ajoute: "Comme la terre fait pousser ses germes, comme un jardin fait germer ses graines, ainsi le Seigneur fera germer le bonheur et la gloire 2. "D'après les Proverbes ${ }^{3}$, la femme étrangère devient à la fin "amère comme l'absinthe ". La hàte avec laquelle l'amandier fleurit est dans Jérémie " le symbole de la promptitude que l'Éternel met à accomplir ses menaces. Leur prètant du sentiment, Isaïe dit que " les cyprès et les cèdres du Liban se réjouissent de la chute de Babylone ". Ailleurs montrant les montagnes et les collines qui éclatent d'allégresse au retour des exilés, " tous les arbres de la campagne, ajoute-t-il, tressailleront de joie. Les cyprès s élèveront à la place des broussailles et à la place des ronces croîtra le myrte ${ }^{\circ}$. ")

1. Cap. II, vers. 11-12 et cap. I, vers. 30 .

2. Cap. Lxi, vers. 9 et 11.

3. Cap. v, vers. 4.

4. Cap. I, vers. 11-12.

5. Cap. xiv, vers. 8 et cap. Lv, vers. 12-13. 


\section{CHAPITRE IV.}

LES PLANTES DANS LES LÉGENDES DIVINES. DANS LES CÉRÉMONIES PROFANES ET RELIGIEUSES ET DANS LA MÉDECINE.

Au temps où en haut il n'y avait rien qui s'appelât ciel, où en bas rien n'avait reçu le nom de terre ${ }^{1}$, Apsou, l'Océan, qui le premier fut leur père, et Tiâmat, le chaos, qui les enfanta tous, mêlaient ensemble leurs eaux... Rien n'existait encore; enfin les dieux se manifestèrent. Loukhmou et Lakhamou parurent les premiers, puis Anshar et Kishar se produisirent. Après une longue suite de-jours Anou, Bel, Éa, naquirent à leur tour, car Anshal et Kishar les avaient enfantés.

C'esten ces termes que les tablettes du palais d'Ashshourbanipal racontent la lente genèse des dieux. Avec le temps leurs formes, confuses et incertaines tout d'abord, devinrent plus précises et leur individualité s'accrut avec leur fécondité. Anou, " le père des dieux " et le ciel divinisé, Bel - Inhil, - " le roi de la terre ", Éa, le souverain des eaux et le sage par excellence, se dédoublèrent, Anou en Anat, Bel en Bélit - la Beltis des Grecs —, Éa en Damkina, et s'unissant aux épouses qu'ils avaient déduites d'eux-

1. Fr. Lenormant, Les origines de l'histoire d'après la Bible. Paris, 1880, in-12, t. I, p. 495. - A.-H. Sayce, Lectures on the origin and growth of religion as illustrated by the religion of the ancient Babylonians. London, 1891, in 8, p. 384. - Naspero, Histoire ancienne, t. I, p. 537. 
mèmes, donnère̊nt naissance à d'autres divinités; le monde entier fut bientòt peuplé de leur descendance. Ce fut d'abord Sin, qui préside à la lune, Shamash, le dieu du soleil, le "messager des dieux ", Ramman, sourerain de l'atmosphère, Adar, le "dieu puissant", puis Ninib, Mardouk " l'interprète des esprits du ciel et de la terre ", Nergal " le roi de la bataille ", Ishtar "la première née du ciel et de la terre "1, et Nébo " seigneurs des planètes ": Saturne, Jupiter, Mars, Vénus et Mercure, enfin l'armée des « 65,000 dieux du ciel et de la terre, .) dont parle Ashshournazirbal².

Ce vaste panthéon ne prit pas naissance en une fois ; formé de la réunion des divinités sumériennes et sémitiques, il n'arriva à sa constitution définitive qu'après de longs siècles, pendant lesquels plus d'une divinité primitive se transforma, se confondit avec une autre plus jeune ou s'effaça devant elle. Chaque ville avait son dieu tutélaire, qui pour elle était aussi le dieu suprème. Ourouk honorait surtout Anou; Bel était le suzerain de Nipour; Éa, le protecteur d'Éridou; Sin trônait dans Our, Shamash à Larsa, Nergal était le dieu de Koutha, Nébo celui de Borsippa. Anou, Bel et Éa formaient une première triade de dieux; une seconde était composée de Sin, de Shamash et de Ramman. Ishtar, la " dame des dieux ", finit par se substituer à ce dernier; Éa la chargea avec Sin et Shamash de régir le firmament. A la fois "l'étoile du soir, qui précède l'apparition de la lune et l'étoile du matin, qui présage la venue du soleil ", le double caractère de

1. H. Winckler, Inschriften Salmanassar's II. (Keilinschriftliche Bibliolhek, t. I (an. 1889), p. 131.)

2. Sayce, Lectures, p. 216. 
l'astre reparaît dans sa nature divine; comme étoile du soir et sous le nom de Bélit-Ilanit - la Mylitta d'Hérodote, - elle était la déesse de l'amour et de la fécondité; comme étoile du matin et confondue avec Anounit, elle présidait, froide et chaste, aux combats. Cette seconde qualité devait la rendre chère à la race guerrière des Assyriens; elle prit place avec les principaux dieux chaldéens dans leur panthéon présidé par Ashshour", "le maitre des dieux ", dont le globe ailé, avec la figure auguste, se voit sur tous les monuments de Ninive, menaçant de son arc vainqueur les ennemis de sa nation ${ }^{2}$.

Seule des déesses du panthéon chaldéen, Ishtar n'était point, comme Bélit ou Damkina, l'incarnation féminine d'un dieu plus grand; mais elle ne vécut pas renfermée dans un farouche isolement; elle s'était éprise du berger Doumouzi, fils d'Éa et de Damkina, tandis qu'il paissait ses troupeaux sous l'arbre mystérieux d'Éridou, qui couvre la terre de son ombre. Son bonheur fut de courte durée; un sanglier blessa mortellement le doux pâtre et le précipita au royaume d'Allat, la déesse implacable des Enfers ${ }^{3}$. Ishtar ne craignit pas de pénétrer dans ces sombres demeures et elle ramena son époux à la lumière du jour.

Nous n'avons ici qu'une face, la meilleure de la mythologie chaldéenne et assyrienne; à côté des dieux

1. Ashshour, que MM. Peiser et Winckler appellent Ashour, avait épousé Nin-lil. Hymne K 100. Sayce, Lectures, p. 128.

2. Rawlinson, op. laud., t. II, p. 3 et 4 . Une inscription de Salmanassar If fait aussi mention de Nouskou, "le dieu sage ".

3. A. Jeremias, Die IIöllenfahrl der Istar. (Die babylonischassyrischen Vorstellungen vom Leben nach dem Tode. Leipzig, 1887, in-8, p. 11-23.) 
bienfaisants et promoteurs des choses bonnes, les Sémites de la Mésopotamie, comme les Sumériens avant eux, croyaient aussi à l'existence de génies malfaisants et instigateurs des choses mauvaises ${ }^{1}$, les Anounaki ou esprits de l'Enfer ${ }^{2}$. Jalouse des dieux, dont l'activité créatrice restreignait son domaine, Tiâmat les avait appelés ì la vie, et, à leur tête, elle était partie en guerre contre ses redoutables ennemis. Les habitants du ciel s'effrayèrent à son approche; seul Mardouk - le Mérodakh de la Bible, - fils d'Éa et de Damkina, ne craignit pas d'affronter la reine du chaos; armé par son père de la foudre et de la harpé, il l'attaqua, la vainquit et dispersa son armée ${ }^{3}$; puis de son couteau il fendit le cadarre du monstre, "suspendit en haut une des moitiés qui forma le ciel, étendit l'autre sous ses pieds pour en faire la terre et constitua l'univers, tel que les hommes l'ont connu depuis lors ${ }^{4}$."

La victoire de Mardouk en fit le représentant le plus auguste du panthéon chaldéen, "le roi de la terre, le seigneur du monde $n^{5}$; sous le nom de Bel-Mardouk, il devint le dieu suprême de Babylone ${ }^{6}$; on le regardait comme l'organisateur du ciel, le dieu qui en avait réglé les mourements et avait frayé leur route aux

1. Fr. Lenormant, Les Dieux de Babylone et de l'Assyrie. Paris, 1877, in-8, p. 13.

2. Les esprits du ciel, eux, s'appelaient Igigi. - C.-P. Tiele, Histoire comparée des anciennes religions de l'Égypte et des peuples símiliques, trad. G. Collins. Paris, 1882, in-8. p. 169.

3. Lenormant et Babelon, Hisloire ancienne, t. V, p. 243245. - Sayce, Lectures, p. 102.

4. Naspero, op. laud., t. 1, p. 542.

5. IIymne, vers. 1. Sayce, Bel-Merodach of Babylon. (Lec(ures, p. 99.)

6. Sa femme était Zarpanit et Nébo son fils. 
astres; c'est lui aussi qui aurait songé d'abord à peupler la terre; mais pour cette dernière cuvre, les autres dieux lui prêtèrent leur concours; ils couvrirent le sol de verdure, et tous ensemble fabriquèrent les êtres de toute espèce qui habitent le monde: animaux domestiques, bêtes sauvages, reptiles, hoinmes enfin. Ces derniers menèrent d'abord une existence assez misérable et "vécurent sans règle, à la manière des bêtes ", jusqu'au jour où, sorti de la mer Érythrée, un monstre doué de raison, Oannès, entreprit de les civiliser. Il leur enseigna les principes des lois, la pratique des sciences et des lettres avec l'art de bâtir les villes, et leur apprit à semer et à récolter, ainsi que tout ce qui peut contribuer à la douceur de la vie ${ }^{1}$.

Mais séduits par Tiâmat, les hommes se corrompirent et cessèrent de sacrifier aux dieux, à qui ils devaient leur heureuse existence. Mécontent de leur indifférence, Bel résolut de les exterminer; mais Éa, mû de pitié, avertit le vertueux Shamashnapishtim le Xisouthros de Bérose - du péril qui menaçait ses semblables. Sur l'avis du dieu bienfaisant, le héros construisit une arche de 140 coudées de long et y entra avec sa famille, ses serviteurs et des bètes de toute sorte. Bientôt la pluie commença à tomber; quand elle eut cessé et que l'inondation diminua, l'ar'che s'arrèta sur' le mont de Nisir. Le septième jour Shamashnapishtim licha une colombe, puis une hirondelle, qui revinrent, ne sachant où se poser; un corbeau, qu'il licha ensuite, revint aussi vers le navire, en battant de l'aile, mais il n'y rentra pas ${ }^{2}$. Le déluge était fini. Shamash-

1. Bérose, Frag. I, 3. (Fragmenta historisorum grecorum, éd. Car. et Th. Müilleri, t. II, p. 496).

2. George Smith, The Chaldean account of genesis, con- 
napishtim sortit alors de l'arche et offrit un sacrifice que les dieux acceptèrent. Bel lui-même, qui avait juré la perte du genre humain, s'apaisa et il récompensa le pieux patriarclie, en lui accordant, ainsi qu'à sa femme, l’immortalité. Shamashnapishtim, enlevé par le dieu du milieu des siens, "fut transporté au loin, à l'embouchure des fleuves ${ }^{1}$. "

Les dieux et les légendes de la théogonie babyloniemne n'étaient pas particuliers à la Chaldée et à l'Assyrie; on les retrouve en partie chez toutes les nations sémitiques. Là toutefois les mythes ne s'étaient pas combinés savamment, comme dans les sanctuaires de la Chaldée, et si la croyance au chaos et aux divinités qui en sont sorties y exista également, on ne trouve plus, à l'époque où nous reportent les monuments, que la notion d'un dieu suprême, dont le nom, sinon les attributs, varie quand on passe d'un peuple à l'autre; "monolatrie "locale, qui ne devait arriver à la forme pure et vraiment élevée du monothéisme hébreu, qu'avec le Yaveh des prophètes, "le dieu qui trône au milieu des éclairs et des tonnerres ${ }^{2}$. "Appelé El “ le Fort ", dénomination qui n'est qu'une autre forme du nom chaldéen Il ou Ilou, chez les Sabéens et dans une partie de la Syrie, Allah dans le Hedjaz et Hadad chez les Araméens, ce dieu suprême portait le nom de Baal

laining the description of the creation, the deluge, etc., éd. by. A.-II. Sayce. London, 1880, in-8, p. 279 et suiv. - Id., übers. v. Herm. Delitzsch nebst Erläuterungen v. Fr. Delitzsch. Leipzig, 1876, in-8, p. 227 et suiv. - A. Jeremias, IzduburNimrod, p. 35.

1. Paul Haupt, Der leilinschriflliche Sinlfulberichl, dans Eberh. Schrader, Die Keilinschriften und das alle Teslament. Giessen, 1883, in-8, p. 64.

2. Tiele, IIsioire des anciennes religions, p. 343. 
chez les Cananéens et dans quelqués villes de la Phénicie, tandis que les Moabites lui donnaient celui de Kémosh et que les Ammonites l'appelaient Milkom '. Chaque ville ajoutait d'ailleurs au nom de son " seigneur " particulier une épithète, qui le distinguait des dieux des autres villes; ainsi à Tyr Baal prenait le surnom de Melqarth "le roi de la ville », á Éqrôn celui de Zébout, à Gébal, le surnom d'Adôn "le maitre ".

De même qu'à côté de chaque dieu chaldéen se trouvait une déesse issue de lui, auprès de Baal avait pris place également sa sœur germaine et son épouse Astarit - l'Ashtoret des Hébreux, l'Astarté des Grecs, l'Ishtar assyrienne. - Comme la déesse tutélaire d'Ashshour s'était éprise du berger Doumouzi, l'Astarté phénicienne s'unit, au chasseur Tammouz Adonis - qui, lui aussi, fut mortellement blessé par un sanglier ${ }^{2}$. Enfin, autre trait de ressemblance avec le panthéon chaldéen, la mythologie phénicienne connaissait aussi des espèces de triades; à Sidon, il y en avait une composée d'Astarit, de Baal et d'Eshmoun; la triade de Carthage était formée de cette dernière divinité, de Baal-Hamon et de Tanit.

Créateurs du monde des plantes, comme de tout l'univers, les dieux du Panthéon sémitique en étaient aussi les protecteurs ${ }^{3}$; ils leur avaient communiqué quelque chose de leur nature auguste et les avaient

1. Lenormant et Babelon, IIistoire ancienne, t. VI, p. 566 . - Stade, Geschichte des Volkes Israel, t. I, p. 429. - Tiele, op. laud., p. 285-287.

2. Sayce, Lectures, p. 227. - Maspero, IIisloire ancienne, t. II, p. 175.

3. Il est question dans plusieurs incantations du "Dieu des herbes ». Sayce, Magical lexts. (Leclures, p. 468 et 476. .) 
associés à leur existence et à leurs pensées. Un ancien hymne salue Sin, le dieu-lune, comme faisant germer la terre par son ordre et la couvrant de verdure'. I)ans un " psaume " Shamash, le Dieu-Soleil, est représenté comme habitant à l'ombre des cèdres, les pieds reposant sur la brillante verdure de l'herbe ${ }^{2}$. On le regardait parfois aussi comme le patron et le maître des plantes marécageuses, qui croissaient dans le voisinage de Telloh ${ }^{3}$. Le nom d'Éa passait pour être inscrit sur le cône du cèdre ${ }^{4}$. Quand ce dien voulut faire connaitre à Shamashnapishtim l'approche du déluge, que Bel allait déchaîner sur la terre, il confia aux r'oseaux, qui devaient le lui révéler, le redoutable secret ${ }^{5}$.

Dans leur expédition contre Khoumbaba, roi élamite de la Babylonie ${ }^{6}$, Izdoubar - Gilgamès - et Éabani arrivent à un bois sacré, qui environne la ville du conquérant et dont l'étendue et les arbres magnifiques les remplissent d'admiration; une colline couverte de cèdres, séjour des dieux, sanctuaire d'Irnini, les frappe surtout; au pied se dresse un cèdre majestueux, à l'ombre puissante et plaisante, arbre dont ils célèbrent à l'envi la beauté, le bois odorant et l'imposante grandeur. C'est là le Tin-tir "bocage de vie", voisin du lieu où Delitzsch a placé le Paradis terrestre ${ }^{7}$, et qui pourrait bien aussi avoir été un de ces bois de la Babylonie,

1. Lenormant et Babelon, Histoire ancienne, t. V, p. 249.

2. A.-H. Sayce, Lectures, p. 173.

3. Sayce, op. laud., p. 24 '.

4. Sayce, op. laud., p. 242.

5. Jensen, Die Kosmologie der Babylonier. Strassburg, 1890, in-8, p. 369. - Maspero, IIistoire ancienne, t. I, p. 567.

6. A. Jeremias, Izdubar-Nimrod, p. 23.

7. Fr. Delitzsch, Wo lag das Paradies?, p. 141. 
dont Alexandre, au rapport d'Arrien ${ }^{1}$, fit couper les cyprès pour construire une flotte!

Une légende donnait, pour premier séjour à Doumouzi, le jardin d'Édin, que la tradition babylonienne plaçait dans le voisinage d'Éridou, et au centre de la terre. Dans ce jardin se trouvait un arbre merveilleux aux racines de cristal blanc, qui s'étendaient jusque dans l'abime, et dont la tête répandait une ombre semblable à celle d'une forêt; là aimaient à se reposer la déesse puissante, qui vogue à travers le ciel - Ishtar - et Doumouzi ${ }^{2}$.

La légende de l'arbre d'Éridou est comme le symbole du culte que les Sémites-Mésopotamiens avaient pour le monde végétal et en particulier pour les arbres. Dans la Chaldée, surtout dans la Chaldée méridionale, le palmier, cet arbre si précieux pour cette contrée, était devenu un objet général de vénération; aussi le voit-on souvent sur les cylindres, surmonté de l'emblème du dieu suprême et placé entre des génies qui l'adorent. Dans l'Assyrie, au contraire, c'est le cyprès qui semble avoir revêtu ce caractère sacré. On le rencontre au milieu de scènes d'initiation, et Lajard a voulu en faire un emblême de la déesse Mylitta ${ }^{4}$. Dans la plante vénérée sur un bas-relief de Ninive ${ }^{5}$, on a même cru reconnaìtre, bien qu'ì tort, l'Asclepias acida dont on extrayait dans l'Inde le breurage divin du soma ${ }^{6}$.

1. Anabasis, lib. VII, cap. 19,4 .

2. Sayce, Lechures, p. 238 et 471.

3. Lenormant, Les origines de l'histoire, t. I, p. 82.

4. Recherches sur le culte du cypres myramidal. (Hémoires de l'Academie des Inscriptions, t. XX, $2^{\mathrm{e}}$ partie, p. 62.)

5. Botta, Monuments de Ninive, t. II, pl. 150.

6. Lajard, Culle de Mithra, pl. LXI, no 6. 
Mais l'arbre sacré des Chaldéo-Assyriens offrait le plus souvent un aspect purement conventionnel et décoratif; la forme la plus ordinaire qu'il présente est celle d'une tige surgissant du milieu des cornes d'un ibex et projetant autour d'elle une série de rameaux plus ou moins simples, droits ou recourbés, dont chacun se termine, soit par une espèce de cône végétal ${ }^{\prime}$, soit par des grenades ${ }^{2}$ ou mème par un triple bouton ou ovaire $^{3}$. D'autres fois encore les rameaux de l'arbre sacré s'entrecroisentet se terminent par des palmettes de fantaisie". Surmonté d'ordinaire de l'image du dieu suprême, il apparaît tantôt entre deux génies le plus souvent ailés et ayant parfois une tête d'aigle, tantôt placé entre un génie ou un dieu - par exemple Dagon, le dieu-poisson ${ }^{\circ}$ - un roi, un pontife ou un simple adorateur. Quant au còne que les dieux et les génies des bas-reliefs tiennent à la main, on a voulu y voir une pomme mystique de pin ou de cèdre; on s'accorde aujourd'hui à la regarder comme le spathe mâle du paimier ${ }^{6}$; les cônes végétaux, qui terminent les branches de l'arbre sacré sont, eux, les spathes femelles et la représentation générale n'est autre chose que le symbole de la fécondation artificielle du dattier, opération dont l'importance explique qu'on l'ait repro-

1. Layard, The monuments, ser. I, pl. 9.

2. Cylindre du Musée britannique de l'époque des Sargonides. Perrot et Chipiez, op. laud., t. II, p. 685.

3. Musée du Louvre, Bas-relief de Sargon.

4. E. Bonavia, The sacred trees of the Assyrian monuments. (The Babylonian and oriental Rerord, t. III (an. 1888-89), p. 7, $35,56$.$) - Id., The flora of the Assyrian monuments, p. 41-$ 60. - Rawlinson, op. laud., t. II, p. 7-8.

5. Layard, Discoveries, p. 313.

6. Edw. B. Tylor, The winged figures of the Assyrian monuments. (Proceedings, t. XII (an. 1890), p. 388.) 
duite si souvent et qu'elle soit devenue un emblème sacré.

Les plantes n'occupaient pas une place moins considérable dans les traditions et les croyances religieuses des Sémites occidentaux que dans celles des Chaldéens et des Assyriens. La Genèse nous montre l'Éternel plantant un " jardin de délices » — l'Éden — vers l'Orient, ế y mettant l'homme qu'il venait de former ". Et parmi les arbres " agréables à la vue et bons pour la nourriture ", qui s'y trouvaient d'après le récit biblique, s'élevaient " l'arbre de vie et l'arbre de la science du bien et du mal », du fruit desquels il lui était interdit de manger. C'est de feuilles de figuier ${ }^{2}$ qu'Adam et Eve, après leur désobéissance, couvrirent leur nudité, et un rameau verdoyant d'olivier, rapporté par la colombe à Noé, annonça au patriarche la fin du déluge ${ }^{3}$. Quand l'Éternel se manifesta à Abrahain dans la vallée de Mambré, ce fut sous un arbre, un chêne ou un térébinthe, que le patriarche recut les envoyés du Seigneur. Après s'ètre séparé d'Abimélek à Bersabèe, Abraham planta un tamaris en ce lieu ${ }^{3}$. C'est sous le térébinthe de Sékem que le fondateur du peuple d'Israël cacha ses idoles ${ }^{6}$. Ainsi les lieux les plus vénérés de l'histoire des patriarches étaient marqués par des arbres.

1. Cap. II, vers. 8-9.

2. Genesis, cap. III, vers. 7.

3. Genesis, cap. Vilr, vers. 11.

4. Genesis, cap. Xvili, vers. 1 et 4 .

5. Genesis, cap. xxi, vers. 33.

6. Genesis, cap. xxxy, vers. 4. 
Le ròle que les arbres jonèrent ainsi dans l'histoire ancienne des Hébreux survécut à l'époque des patriarches; c'est sous un chène près de Sékem que Josué dresse la pierre, signe de l'alliance, qu'il renouvela arec le Seigneur, après l'entrée des Hébreux dans le pays de Canaan ${ }^{1}$. C'est sous un térébinthe, près d'Ofrah, que l'ange de Jahreh apparut à Gédéon, et celui-ci, ì l'exemple des patriarches, éleva en ce lieu un autel ${ }^{2}$. C'est encore sous le térébinthe de Sékem que les habitants de cette ville proclament Abimélek roi ${ }^{3}$. On voit la prophétesse Déborah rendre la justice sous un palmier entre Ramah et Bêt-El ${ }^{4}$, comme si c'eult été un lieu saint et consacré; le livre de Samuel nous montre également Saül se reposant sous un grenadier à Gabaa ${ }^{\text {s }}$, ou délibérant sous un tamaris à Ramah ${ }^{6}$, et les habitants de Jabès enterrèrent les restes de ce prince et de son fils sous un tamaris ${ }^{7}$ ou un térébinthe ${ }^{8}$, voisin de leur ville.

Si les arbres occupent une place aussi considérable dans l'histoire des Hébreux, c'est que ce peuple leur accorda longtemps un caractère mystérieux et surnaturel. Il en était de mème chez tous les Sémites de la Syrie et de l'Arabie; c'était une tradition chez ces peuples que les àmes des défunts aimaient à se reposer au milieu de leurs rameaux; dans les arbres aussi,

1. Jesus Nave, cap. xxiv, vers. 26.

2. Judices, cap. vi, vers. 11 et 19.

3. Judices, cap. $\mathrm{Ix}$, vers. 6.

4. Judices, cap. IV, vers. 5.

5. 1 Samuel, cap. xiv, vers. 2.

6. 1 Samuel, cap. xxir, vers. 6 .

7. 1 Samuel, cap. xxix, vers. 12.

8. 1 Paralipomena, cap. $x$, vers. 12. Les Septante ont traduit par chêne.

I. 
pensaient-ils, résidaient des esprits secourables aux mortels qui les imploraient. Ils allèrent plus loin, et les regardèrent comme des symboles de vie, des ashéras, animés par une divinité et doués d'un esprit prophétique ${ }^{\prime}$. Les Nabatéens croyaient entendre des voix annonçant l'avenir sortir des buissons appelés gharqad?. Jahveh reproche aux Hébreux, par la bouche d'Osée ${ }^{3}$ " d'interroger le bois pour qu'il leur prédise l'avenir ». Ils pratiquaient la divination en interprétant le murmure du vent dans les branches; un chêne de Sékem, qui servait à cet usage, portait le nom de Chêne des voyants ${ }^{4}$. "Le bruit de pas lans la cime des peupliers " annonce a David que l'Éternel marche contre les Philistins".

On comprend que, doués de telles prérogatives, les arbres aient pris place dans le culte des peuples sémitiques. Les habitants du pays de Canaan adoraient leurs dieux sous des arbres touffus, comme sur les hauts lieux ${ }^{6}$, et les Hébreux eux-mêmes y sacrifièrent longtemps. Abraham invoque le nom de Jahreh sous le tamaris, qu'il avait planté à l'endroit où il s'était rencontré avec Abimélek ${ }^{7}$, et plus tard Jacob y fit un

1. F.-K. Movers, Das phœnizische Alterthum, t. I, p. 562 et suiv. - Stade, Geschichte des Volkes Israel, t. I, p. 454. Pietschmann, op. laud., p. 203.

2. Lenormant, Les origines de l'histoire, t. I, p. 87. Le gharqad est, d'après Ascherson et Schweinfurth, la Nitraria retusa, plante de la famille des zygophyllacées. Mémoires de l'Institut du Caire, t. II, p. 57.

3. Cap. $1 \mathrm{v}$, vers. 12.

4. Judices, cap. Ix, vers. 37. Ed. Reuss traduit par "chêne aux sorciers $)$.

5. 2 Samuel, cap. v, vers. 2'́t. Le mot bekaim, que je traduis par peupliers, n’a point été identifié avec certitude.

6. Deuteronomium, cap. xII, vers. 2-3.

7. Genesis, cap. xili, vers. 33. 
sacrifice au dieu de son père Isaac. L'Éternel dans Osée" reproche aux Israélites de sacrịicr " sous le chêne, le peuplier et le térébinthe, parce que l'ombre en est agréable "; Salmanassar cnvahit le royaume de Samarie, sous le règne d'Osée, pour punir les habitants d'aroir éleré des Astartés sur les collines et à l'ombre des arbres touffus et d'y avoir brûlé de l'encens ?

Les arbres toujours verts on particulier: palmiers, cyprès, yeuses, térébinthes, étaient chez les nations sémitiques un objet de vénération. Chez les Phéniciens le palmier surtout était en honneur ${ }^{3}$; il parait avoir été consacré à Astarté; peut-être aussi figurait-il dans le cultc de Baal; dans le Hedjaz il était, en beaucoup d'endroits, l'objet d'un culte réritable ${ }^{4}$. Les Qoreyshites adorèrent, jusqu'au temps de Mahomet, la déesse Allat, dans le dattier Dhait-anwât ${ }^{5}$. Les habitants de Nadjran, dans le Yémen, avant leur conversion au christianisme, rénéraient comme un fétiche divin un immense dattier, qui se trouvait près de leur ville; ils l'ornaient de colliers et d'étoffes précieuses; chaque année, après avoir déposé leurs idoles sous son ombre, ils faisaient tout autour une procession solennelle, accompagnée de chants et de prières et ils croyaient

1. Cap. IV, vers. 13.

2. 2 Regna, cap. xvil, vers. 10-11.

3. IV. Baudissin, Studien zur semitischen Religionsgeschichte. Leipzig, 1876, in-8, t. II, p. 201 et 212.

4. Osiander, Sludien über die vorislümische Religion der Araber. (Zeitschrift der deulschen morgenländischen Gesellschafl, t. VII (an. 1853), p. 481.)

5. L. Krehl, Ueber die Religion der vorislamischen Araber. Leipzig, 1863, in-4, p. 73. - Fr. Lenormant, Les origines de lhisloire, t. I, p. 82, note 2. 
alors entendre du milieu des branches un esprit leur parler ${ }^{1}$.

Les Beni.Ghatafân vénéraient un samourah - Acacia arabica Willd. - comme l'image de la déesse El-Ouzzà ${ }^{2}$. Cet arbre était également sacré pour les Nabatéens, qui le regardaient comme l'arbre de Bel, et ils en portaient des épines en guise de talisman ${ }^{3}$. Mais nul arbre ne joua un rôle plus considérable que le cyprès dans la croyance des Sémites occidentaux. Emblème d'Astarté $^{4}$, on le rencontre fréquemment sur les monuments de la Phénicie et de la Syrie. C'est ainsi que, sur la face postérieure de l'autel d'Hiéropolis au Musée capitolin, on voit représenté un cyprès couvert de fruits; le monument de Palmyre du mème musée nous montre cet arbre se dressant entre les divinités Aglibol et Malakhbel, qui se tendent la main; enfin un tròne votif en bronze d'origine syrienne z sur sa face antérieure trois cyprès pyramidaux en relief, symboles vraisemblables d'Astarté, du soleil et de la lune". On rencontre également le cyprès sur les monnaies d'Héliopolis, entre un taureau et un cheval, et sur des médailles phéniciennes du temple d'Arados, entre un taureau et un lion, symboles encore du soleil et de la

1. Caussin de Perceval, Essaisur l'histoire des Arabes avant l'islamisme. Paris, 1847, in-8, t. I, p. 125. - Sir William Ouseley, Travels in various countries of the East. London, 1809 , in-4, t. I, p. 365.

2. Osiander, op.laud., p. 486. - Krehl, op. laud., p. 75. Fr. Lenormant, Les origines de l'histoire, t. I, p. 86.

3. Félix Lajard, Recherches sur le culte du cyprès pyramidal. (Hémoires de l'Académie des Inscriptions, t. XX, 2 (an. 1854), p. 8).

4. W. Baudissin, op. laud., t. II, p. 193.

5. F. Lajard, Recherches sur le culte du cypres pyramidal, p. 19 et 43. - Baudissin, op. laud., t. II, p. 195. 
lune, ainsi que sur des médailles de Damas, où il est représenté se dressant à l'entrée d'un temple '.

Les Phéniciens portèrent le culte du cyprès dans leur's colonies de l'Occident; en Crète il y avait un bois de cyprès près de Gnosse ${ }^{2}$; on en voyait un aussi à Corinthe auprès du sanctuaire de Bellérophon et du temple d'Aphrodite ${ }^{3}$. Le myrte et le grenadier prirent place aussi, quoique moins souvent, dans les pratiques ou les croyances religieuses des Sémites occidentaux. Le myrte, attribut d'Aphrodite en Grèce, semble l'avoir été aussi d'Astarté, sa sœur phénicienne. Le caractère sacré que les anciens Grecs attribuaient au grenadier avait vraisemblablement une origine sémitique, comme cet arbre lui-même, dont la culture fut probablement importée par les Phéniciens et les Carthaginois en Grèce et en Italie.

Mais les arbres isolés n'étaient pas seuls un objet de rénération pour les Sémites, les bois aussi et surtout étaient sacrés à leurs yeux; ils les regardaient comme le séjour favori des Dieux; aussi leur en consacraientils partout où ils s'établissaient. Ils abondaient en Arabie, en Mésopotamie, en Syrie, dans les pays colonisés par les Phéniciens. Nous verrons les rois de Chaldée et d'Assyrie en consacrer à leurs dieux ${ }^{4}$. Nous arons, dans la légende d'Izdoubar, rencontré un bocage sacré près de Babylone; un bois de cyprès se trouvait aussi dans le voisinage d'Arbelles ${ }^{\circ}$; les Hébreux eurent jusqu'à l'époque de la conquête assyrienne

1. Lajard, Le Culle ducyprès pypramidal, p. 52, 57, 61, 83 et 85 .

2. Diodore, Bibliolhec. lib. V, cap. 66, 1.

3. 'ausanias, Descriplio Graeciae, lib. II, cap. 2, 4.

4. Keilinschriftiche Bibliothek, t. III, p. 191.

5. Lajard, Le culte du cyprès pyramidal, p. 58. 
des bosquets sacrés ${ }^{1}$; Eshmoun, l'Asklépios phénicien, avait près de Béryte un bois qui fut longtemps célèbre; celui d'Aphaka dans le Liban n'était pas unoins connu; dans l'île de Cypre des bois nombreux étaient consacrés à Aphrodite-Astarté, et Rékhouf, l'Apollon phénicien, était vénéré dans un bois voisin de Curium ${ }^{2}$. Il y avait dans le voisinage du temple de Kronos, c'est-à-dire de Baal, à Carthage, des arbres sacrés auxquels, nous apprend Tertullien ${ }^{3}$, Tibère fit pendre les prêtres du dieu, et l'on parle d'un bois de pins et d'ifs, qui se trouvait dans le voisinage de la mème ville ${ }^{4}$ et où des sacrifices humains étaient offerts à Didon.

Les plantes ne furent pas seulement revètuës, aux yeux des Sémites, d'un caractère religieux, elles prirent place dans leur culte, comme dans celui des Égyptiens. C'était là une conséquence naturelle de l'anthropomorphisme, qui était au fond des croyances religieuses de ces peuples. Regardant les dieux comme semblables à eux, leur attribuant les mèmes besoins qu'ils avaient eux-mêmes, il n'est point suiprenant qu'ils aient songé à leur offrir les mêmes mets dont ils se nourrissaient, les breuvages qui servaient à les désaltérer, ainsi que les aromates dont le parfum les charmait. C'est ainsi que tout acte d'adoration fut ac-

1. Esaias, cap. 1, vers. 29."

2. Pietschmann, op. laud., p. 213, note 2.

3. Apologelica, cap. $\mathrm{Ix}, 73$ (315).

4. "Taxi circum et piceae squalentibus umbris. "

Silius Italicus, Punica, lib. I, vers. 83. 
compagné d'une offrande, de libations, d'encens brùlé sur l'autel du dieu que l'on vénérait.

Ces pratiques remontaient à la plus haute antiquité. Après ètre échappé au déluge, Shamashnapishtim fit une libation sur le sommet de la montagne où l'arche s'était arrètée $^{i}$, y dressa sept vases et y plaça du jonc odorant, avec du bois de cèdre et de genévrier. Pour assurer le succès de leur entreprise contre Khoumbaba, Éabani, le campagnon de Gilgamès, offre à Shamash un sacrifice d'encens et une libation ${ }^{2}$. Sur une intaille chaldéenne on roit, debout devant un autel, un persomnage tenant, de la main droite, une tige a trois branches qu'il semble présenter à deux autres personnes en adoration. "A Mardouk, roi de la ville de Sirpourla, dit la légende, pour la conservation de Dounghi, le héros puissant, roi de Our, fils de Our-Gour, j'ai fait une offrande ${ }^{3}$. "Et sur une autre pierre gravée de la mème époque, qui représente Ourou, patési de Nipour sacrifiant, on lit: "A Nouskou, l’intelligence suprème, notre roi, pour le salut et la vie de Dounghi... Ourou, patési de Nipour, a consacré ceci". ")

L'inscription G de Goudéa, patési de Sirpourla, nous le montre, le premier jour de l'année, fète de Baou, faisant des offrandes à cette déesse ; il en faisait également d'abondantes au dieu Ningirsou ${ }^{5}$. Il

1. Le mont de Nisir. P. Jensen, Die Kosmologie der Babylonier. Strassburg, 1890, in-8, p. 381. - Maspero, IIistoire ancienne, t. I, p. 570.

2. A. Jeremias, Izdubar-Nimrod, p. 21.

3. Menant, Les pierres graveses, p. 140, fip. 86.

4. Menant, Les picrres gravées, p. 1'ł2, fig. 87.

5. A. Amiaud, L'inscription G de Goudéa. (Zcitschrift für Assyriologie, t. III, p. 31.) - A. Amiand, Inscription de la slatue $B$ de Goudia, ap. E. De Sarzec, Découvertes en Chaldée, 
en institua même, ce qui peut surprendre, en l'honneur de sa propre statue, qu'il avait fait faire en pierre de Màgan - la presqu'ile de Sinaï - et placer dans le temple de Ningirsou. L'inscription B de cette statue nous apprend qu'on lui faisait des offrandes, ainsi qu'à celle de Dounghi, pour la néoménie de janvier ${ }^{1}$. Il est question dans la table liturgique de Sippara, qui ne date, il est vrai, que du $\mathrm{Ix}^{\mathrm{e}}$ siècle, d'offrandes destinées à Shamash par le roi de Babylone Éoulbarshàkinshoum ${ }^{2}$. Son successeur Naboupaliddin institua aussi, en l'honneur du mème Shamash et des autres grands dieux ${ }^{3}$, ainsi que pour l'entretien de leurs prêtres, de nombreuses et riches offrandes.

Les bas-reliefs assyriens représentent souvent les dévôts, prêtres ou rois, faisant des offrandes ou des libations aux dieux ${ }^{4}$, et les inscriptions en font aussi fréquemment mention. Ashshournazirbal parle des fruits et du vin qu'il offrit à Ashshour, son maitre ${ }^{3}$. Une inscription de Salmanassar II rappelle les présents

3o livr., p. vir. - P. Jensen, Inschriften aus der Regierungszeil Gudeas. (Keilinschriflliche Bibliolhek, t. III, 1, p. 27, 61 et 63.)

1. P. Scheil, Le culte de Gudia sous la rve dynastie d'Ur. (Recueil de travaux, t. XVIII (an. 1896), p. 64-69.)

2. Joh. Jeremias. Die Cullustafel von Sippar. (Beitrïge sur Assyriologie und vergleichenden semitischen Sprachwissenschaft, t. I (an. 1890), p. 271.)

3. Jeremias, Die Cullustafel, p. 274. - Fr.-V. Scheil, Inscriplion de Nabu-abil-iddin. (Zeitschrift für Assyriologie, t. IV (an. 1889), p. 330). - F.-E. Peiser, Inschrift von Nabuabaliddin. (Keilinschriftliche Bibliolhek, t. III, 1, p. 173.)

4. Botta et Flandin, Le monument de Ninive, t. I, pl. 43, 57, 75 ; t. II, pl. 105, 150, etc.

5. F.-E. Peiser, Inschriften Ashournasirpal's. (Keilinschriftliche Bibliothel, t. I (an. 1889), p. 117.) 
qu'il institua en l'honneur du grand Dieu ${ }^{1}$. Sargon mentionne également les nombreuses offrandes qu'il fit à Bel, Zarpanit, Nébo et à tous les dieux, "qui habitent les rilles de Soumir et d'Akkad " ${ }^{2}$. Les rois du nouvel empire babylonien se signalèrent surtout par leurs riches offrandes enver's leurs dieux nationaux. Dans l'inscription du Cylindre de Grotefend, Nabuchodonosor, le plus célèbre d'entre eux, énumère avec complaisance celles quill faisait tous les jours en leur honneur ${ }^{3}$ :

Chaque jour jai prodigué ail, piloû, parure des prairies, huile épurèe, vin épicé, lourarnou, sirash, ashpa, boisson de la montagne, vin clair, vin des cantons d'Izalla, Touimou, Sisimounini, Hilbouni, Aranabani, Souham, Bitkoubati, Bitati, comme l'eau du fleuve, sur la table de Nardouk et de Zerpanit, mes maitres.

Plus loin ${ }^{4}$ Nabuchodonosor rappelle les offrandes qu'il avait faites sur l'autel de Nébo et de Nana et sa constante fidélité envers les temples Ésagila et Izida, où il sacrifiait tous les jours de fêtes. Un des temples de Babylone recerait de Nabonide un don annuel de six boisseaux de dattes ${ }^{5}$. Ce n'était pas seulement toutefois en des occasions solennelles qu'on faisait des offrandes, les dieux babyloniens en recevaient chaque semaine et mème chaque jour. Le livre de Daniel nous

1. H. Winckler, Inschriften Salmanassar's II. (Keilinschriftliche Bibiolleek, t. I, p. 139.)

2. F.-E. Peiser, Inschriften Sargon's. (Keilinschriftliche Bibliolhek, t. II (an. 1890), p. 73.)

3. Lenormant et Babelon, IIistoire ancienne, t. V, p. 309. - H. Winckler, Inschriften Nebukadnezar's. (Keilinschriftliche Bibliothek, t. III, 2 (an. 1891), p. 33.)

4. H. Winckler, ibid., p. 37, 38.

5. A.-H. Sayce, Social life among the Assyrians and Baby. lonians. London, 1893, in-16, p. 12. 
apprend ${ }^{1}$ qu'on offrait tous les jours au dieu Bel douze mesures de fleur de farine et six amphores de vin avec quarante brebis.

Les produits végétaux les plus divers figuraient dans les offrandes sacrées, qui étaient faites aux dieux de la Chaldée et de l'Assyrie; mais la richesse et la variété en augmenta avec les siècles. Goudéa offre à la déesse Baou sept pats de dattes et sept cœurs de palmier ${ }^{2}$. Sur une pierre gravée, qui représente Beltis assise sur un trône devant un autel, sur lequel sont déposés les objets destinés au sacrifice, on voit un pontife présenter de la main droite à la déesse un rameau couvert de fleurs". Sur un bas-relief de Ninive un génie ailé, qui tient du bras gauche un chevreau, semble offrir de la main droite une palme ${ }^{4}$. Éoulbarshâkinshoum octroie à Shamash un qa de vivres et un qa de boisson fermentée; à son exemple Naboupaliddin son successeur institue en l'honneur du dieu et pour l'entretien de ses prètres des offrandes considérables de viandes, de bière de Nideâ et de légumes ${ }^{5}$. Ashshournazirpal offre à Bel des fruits et du vin; Sargon fait don aux dieux d'Ashshour de bois d'ourkarinou, de cèdre et de cyprès, ainsi que des " parfums de toute sorte, produits du mont Amamus ". Quant aux offrandes de Nabuchodonosor, on a vu quelle en était la variété et la richesse ${ }^{6}$. Na-

1. Cap. xiv, vers. 2.

2. Amiaud, ap. De Sarzec, Déconverles en Chaldée, p. x.x. - Keilinschriflliche Bibliolhek, t. III, 1, p. 61.

3. J. Menant, Les pierres gravées, t. I, p. 168, fig. 100.

4. Layard, The monumenls, ser. I. pl. 35 a.

5. Zeilschrift f. Assyriologie, t. IV, p. 330. - Keilinschrift. Bibliolhek, t. III, I, p. 177.

6. Ke eilinschrifliche Bibliollek, 1. I, p. 117; t. II, p. 73 et t. III, 2, p. 33, 37 et 38 . 
bonide, renchérissant sur lui, souhaite de faire entrer chaque année à l'Ésagil, " temple des cieux et de la terre ", le tribut des quatre régions, l'abondance des mer's, les produits des monts et des plaines ${ }^{1}$. Mais ce n'étaient pas seulement des produits végétaux qu'on offrait aux dieux et à leurs prêtres, on leur offrait également en don des vergers, des plantations d'arbres a fruits. On voit Êoulbarshâkinshoûm donner en l'honneur de Shamash, un jardin au grand prêtre de Sippar, Êkourshoumoushabshi ${ }^{2}$, et Mardouk-Baliddin offrir au même dieu un bois de palmiers".

Comme les offrandes, les libations en l'honneur des dieux occupaient une place considérable dans la liturgie chaldéenne et assyrienne. Les pierres gravées et les bas-reliefs de Khorsabad, de Nimroud et de Koyoundjik nous offrent de nombreuses scènes où elles sont représentées; ici c'est une libation que Ourou répand sur l'autel de Nouskou pour le salut de Dounghi"; la c'est Ashshournazirpal qui offre aux dieux une libation pour les remercier de la victoire qu'il a remportée sur un taureau sauvage". Ailleurs Sennachérib, une coupe à la main, fait une libation sur le corps des lions qu'il a tués à la chasse ${ }^{6}$. Sur un obélisque de Koyoun-

1. Fr.-V. Scheil, Inscription de Nabonide. (Zeitschrift fïr Assyriologie, t. V (an. 1890), p. 405.)

2. Fr.-V. Scheil, Inscription de Nabi-abil-iddin. (Zeitschrift fiir Assyriologic, t. IV (an. 1889), p. 330.) - F.-E. Peiser, Inschrift von Nabu-abal-iddin. (Keilinschr. Bibliothek, t. III, 1, p. 179.)

3. Peiser u. Winckler, Inschrift Merodach-Baladan's II. (Keilinschr. Bibliothek, t. III, 1, p. 191.)

4. Menant, Les pierres gravées, t. I, p. 142, fig. 87.

5. Musée britannique. Perrot et Chipiez, op. laud., t. II, p. ' $5^{\prime}$ ', pl. 205.

6. Botta, Monumenl de Ninive, t. I, pl. 57. - Victor 
djik on voit le mème roi verser une libation dans une large jatte, placée sur un support, qui se dresse devant un autel, d'où s'élèvent des flammes, tandis que les prêtres amènent la victime destinée au sacrifice ${ }^{1}$.

Les inscriptions des monuments assyriens et babyloniens mentionnent aussi et fréquemment les libations et les aspersions, qui.précédaient ou accompagnaient le sacrifice. Quand on élevait un temple, on en arrosait les fondements avec diverses substances consacrées; on en asper'geait également les murs, le seuil et même les serrures et les verrous". "Avec de l'huile parfumée, du miel, de la crème, du vin de sésame, du vin de montagne, j'en arrosai les fondements ", dit l'inscription de la pierre noire ${ }^{3}$. Nabopolassar nous apprend qu'il mit de l'huile excellente et des aromates, etc., sous les fondements des murs du temple de Mardouk ${ }^{4}$. Naboupaliddin, dans son inscription, rapporte aussi qu'il aspergea le seuil du temple de Shamash avec du miel, du vin et de l'hydromel ${ }^{5}$, et Nabonide dit qu'il arrosa les murs du même temple avec du vin de palmier, du vin, de l'huile et du miel. Quand il eut édifié en l'honneur de Shamash le temple Ibarra, ce prince en aspergea aussi soigneusement les seuils, les serrures, les verrous et les portes avec de l'huile, et “ à

Place, Ninive el l'Assyrie, t. III, pl. 57, 1. - Rawlinson, t. I, p. 515 .

1. Musée britannique. Rawlinson, op. laud., t. II, p. 35.

2. Jeremias, Dic Cullustafel von Sippar, p. 273.

3. Beiträge zur Assyriologie, t. III, 2, p. 223.

4. J. N. Strassmaier, Inscriften v. Nabopalassar. (Zeitschr. f. Assyriologic, t. IV, p. 110.) - H. Winckler, Ibid. (Keilinschrifliche Billiothel, t. III, 2, p. 5).

5. Peiser, Inschrift von Nabü-abal-iddin. (Keilinschriftliche Bibliothek, t. III, 1, p. 179.) 
l'entrée de sa sublime divinité, pour la dignité du temple, il le remplit de bomne odeur ${ }^{1}$. "

Ces libations, les onctions d'huile et les fumigations qui les accompagnaient parfois, étaient un symbole ou un moyen de purification. Après avoir répandu l'eau lustrale sur celui qu'on voulait purifier et offert en son nom une libation de vin pur, on lui oignait le corps sept fois ${ }^{2}$. On oignait aussi les statues et les pierres sacrées, ainsi que les inscriptions des rois. Tiglathphalassar rappelle qu'il fit nettoyer avec de l'huile l'inscription de Shamsi-Ramman, son prédécesseur, après quoi il sacrifia ${ }^{3}$. Nabonide, ayant retrouvé l'inscription de Karam-Sin, fils de Sargon, la fait restaurer et oindre d'huile, puis il offre un sacrifice, et il adjure quiconque règnera après lui de respecter aussi son épitaphe, de l'oindre d'huile et d'y joindre son propre nom ${ }^{4}$. Shamashshoumoukin recommande également, dans une inscription, à celui de ses successeurs qui trouvera sa statue de l'oindre d'huile, lui promettant en retour la protection de Nébo ${ }^{5}$.

A còté des libations et des offrandes prenaient place les fumigations; comme en Egypte, elles consistaient en aromates qu'on brûlait devant ou sur l'autel. Dans la scène du sacrifice représenté sur un obélisque de Koyoundjik, dont j'ai parlé plus haut, le roi semble jeter de l'encens sur la flamme allumée devant la chàsse du

1. Peiser, Inscrift von Nabonid. (Keilinschr. Bibliothek, t. III, 2, p. 101 et 116.)

2. Sayce, Leclures, p. 62.

3. H. Winckler, Inschriften Tiglath-Pileser's II. (Keilinschr. Bibliothel, t. I, p. 45.)

4. Keilinschriftliche Bibliothet, t. III, 2, p. 106-107.

5. F. Lehmann, Shamashshumukin, König von Babylonien, p. 11,13 et 77 . 
dieu. Les aromates occupaient une grande place dans les tributs que les monarques assyriens réclamaient des peuples vaincus; ils en offraient aux dieux ${ }^{1}$ la plus grande partie et on en consommait d'énormes quantités dans le culte; Hérodote rapporte qu'à la fète du premier jour de l'année on brûlait mille talents d'encens dans le temple de $\mathrm{Bel}^{2}$.

Les libations et les offrandes dont je viens de parler n'étaient pas particulières aux Babyloniens et aux Assyriens; on les retrouve chez toutes les nations sémi. tiques. La Genèse nous montre Caïn offrant déjà au Seigneur les prémices des fruits de la terre et Jacob oignant d'huile la pierre sur laquelle il avait reposé sa tète, quand il vit en songe l'Eternel ${ }^{3}$. A son retour de Damas, le roi Akhaz offrit un sacrifice et fit des libations sur l'autel qu'Ouriyah avait construit par ses ordres". Sirach nous montre le grand prêtre Simon faisant des libations de vin sur l'autel en présence du peuple ${ }^{5}$. Les libations et les offrandes des produits du sol étaient une partie importante du culte juif; elles constituaient les sacrifices non sanglants - minha - et accompagnaient souvent anssi les sacrifices sanglants - zébah ${ }^{6}$.

Les Juifs offraient à Jahveh les prémices de tous les

1. Inscriptions d'Asshournazirpal et de Sargon. (Keilinschr. Bibliolhek, t. I, p, 67 et t. II, p. 73.)

2. Hislorice, lib. I, cap. 183.

3. Genesis, cap. IV, vers. 3 ; cap. Xxxv, vers. 14.

4. 2 Regna, cap. xvi, vers. 13. 1I. Sayce, The Life and Times of Iscaich, London, 1889, in-16, p. 47, suppose que cet autel était fait à l'imitation de celui du dieu solaire de Syrie.

5. Sapientia Sirach, cap. L, vers. 16.

6. W. Robertson Smith, Lechures on the religion of the Semiles. London, 1889, in-8, p. 218-227. 
fruits de la terre': aromates, huiles, vin, farine, pains, etc. L'huile servait à la fois à alimenter les lampes sacrées et à la préparation des parfums destinés au culte ${ }^{2}$. On l'employait aussi pour faire les pains ou arroser les gâteaux sans levain offerts en actions de grices ${ }^{3}$. Il y avait dans le tabernacle un autel particulier, sur lequel on mettait sur deux rangs douze de ces pains azymes, dits pains de proposition; chacun d'eux était de deux dixièmes d'éfah et fait de la farine la plus fine; à chaque jour de sabbath ils étaient renouvelés * Matin et soir le grand prètre faisait une oblation d'un dixième d'éfah de fleur de farine pétric avec un quart de hin d'huile vierge, et un quart de hin de vin comme libation ${ }^{5}$. Une offrande fréquente consistait en farine fine, sur laquolle on versait de l'huile, en y joignant de l'encens; on la portait aux prètres, qui en brîlaient la part réservée au sacrifice. Au lieu de simple farine, on offrait aussi des gàteaux ou des galettes plates sans levain, pétris ou arrosés arec de l'huile. On offrait encore " comme prémices" des épis grillés au feu ou des grains broyés; on répandait de l'huile dessus arec de l'encens, puis on les remeitait aux prètres pour ètre brûlés ${ }^{6}$.

C'était surtout dans les trois grandes fètes juives, la Pâque, la fête des Semaines" ou la Pentecôte et la

1. Exodus, cap. xxil, vers. 19.

2. Exodus, cap. xxv, vers. 6, cap. xxvir, vers. 20. - Levilicus, cap. xxIr, vers. 2.

3. Exodus, cap. xxix, vers. 2. - Levilicus, cap. VII, vers. 12.

4. Exodus, cap. xxv, vers. 30. - Levilicus, p. xxiv, vers. 5-6.

5. Exodus, cap. Xxix, vers. 40.

6. Levilicus, cap. II, vers. 1-2 et 14-16. 
fète des Tabernacles ", que les plantes et leurs produits jouaient un rôle prédominant. Le lendemain de la Pàque, "au mois des nouveaux blés ", on apportait au prêtre comme prémices une gerbe qu'il agitait devant l'Eternel. Ce même jour on immolait en holocauste un agneau d'un an; puis on offrait deux dixièmes d'éfah de fleur de farine pétrie avec de l'huile, qu'on brûlait " comme un sacrifice d'une odeur agréable à l'Éternel "; on faisait de plus une libation d'un quart de hin de vin, et pendant sept jours le peuple ne mangeait que du pain sans levain ${ }^{2}$,

Au bout de sept semaines, à la Pentecòte, on faisait à l'Éternel une nouvelle offrande publique; elle consistait en deux pains, faits de deux dixièmes d'éfah de farine fine, que l'on portait au prêtre, et l'on y joignait la libation ordinaire ${ }^{3}$. La fête des Tabernaclés, qui commençait le quinzième jour du septième mois, alors que tous les fruits de la terre étaient récoltés, était, comme les deux précédentes, une fête de l'agriculture et de la vie champêtre. Pendant huit jours le peuple habitait sous des tentes faites de branches d'arbres". Le premier jour, portant dans leurs mains le fruit d'un bel arbre - hadar —, des frondes de palmier, des rameaux au feuillage touffu et des saules du ruisseau, tous se réjouissaient devant l'Éternel ${ }^{5}$.

1. J. Wellhausen, Prolegomena zur Geschichte Israels. Berlin, 1886, in-8, p. 84. - W. Smith, A dictionary of the Bible. London, 1863, in-8, art. Feslivals.

2. Levilicus, cap. XxiII, vers. 6 et $10 \cdot 13$.

3. Levilicus, cap. xxiII, vers. 15-17.

4. Néhémie, cap. vil, vers. 15, ordonne de faire ces tentes avec des rameaux d'olivier, de cyprès, de myrte, des frondes de palmier et des branches au feuillage touffu.

5. Levilicus, cap. xxiI, vers. 40-42. - Deuteron., cap. xiri, 
Les offrandes étaient le plus souvent accompagnées de fumigations et de libations ou d'onctions; mais celles-ci étaient aussi, depuis un temps immémorial, employées seules. Jacob, nous l'avons vu, versa, à son réveil, de l'huile sur la pierre qui lui avait servi de cheret pendant la nuit ou l'Éternel lui apparut. Moïse ordonne d'oindre avec l'huile sainte l'arche d'alliance et tout ce qu'elle renfermait: tabernacle, autels, vases sacrés, etc.; c'estavec elle aussi qu'il consacra Aaron et ses fils ${ }^{1}$. Cette huile servit également à Samuel pour oindre Saül et David ${ }^{2}$, et à Sadoc pour sacrer Salomon ${ }^{3}$. Chaque fois qu'on portait aux prêtres des gâteaux sans levain, ils les brûlaient avec l'encens qu'on y joignait. Un autel particulier était réservé dans le tabernacle pour les parfums; matin et soir le grand-prètre brûlait des aromates d'une odeur agréable, au moment où il préparait les lampes sacrées. Après le sacrifice expiatoire, il jetait aussi deux poignées d'encens odoriférant sur de la braise placée derrière le voile du sanctuaire ${ }^{5}$.

Comme dans le culte, les plantes avaient, chez les Sémites, leur place marquée dans les cérémonies funèbres. Chez les Assyriens et les Chaldéens, des pleureuses à gage lavaient et oignaient de parfums le corps

vers. 13-15. - Josèphe, Antiq. jud., lib. III, cap. 10, 4, parle d'un faisceau formé de branches de myrte et de saule auquel on joignait un spathe de dattier et un fruit de perséa. D'après le Talmud, ce fruit aurait été un citron, ce qui ne peut se rapporter qu'à une époque récente.

1. Exodus, cap. xxx, vers. 26-30.

2. 1 Samuel, cap. x, vers. 1 et cap. xvi, vers. 13.

3. 1 Regna, cap. I, vers. 39.

4. Exodus, cap. xxx, vers. 1 et 7.

5. Levilicus, cap. xvi, vers. 13. Cf. Esaias, cap. XlvII, vers. $23 \cdot 24$. 
du défunt; elles le revêtaient ensuite d'une robe d'apparat; enfin elles l'étendaient sur un lit et dressaient à son chevet un petit autel, où étaient placées les offrandes ordinaires d'eau, d'encens et de gâteaux. On croyait que l'esprit du mort - l'élimmou — avait besoin de subsistances dans sa nouvelle demeure; le priver des offrandes et des libations qui lui étaient dues, c'était le condamner à une vie errante et malheureuse ${ }^{1}$; aussi était-il reconnaissant à ceux qui les lui donnaient et il s'en faisait le protecteur?. Dans le tombeau ou le défunt était déposé, des jarres et des plats d'argile et parfois de métal, rangés autour de lui, lui fournissaient sa nourriture et ses boissons journalières, le vin qu'il préférait, des dattes et divers autres mets ${ }^{3}$. Des flacons à parfums et des objets de toilette - plus tard on y mit aussi des fleurs - étaient placés près du corps des jeunes filles et des femmes ${ }^{4}$. D'après la croyance chaldéenne et assyrienne les àmes ordinaires étaient reléguées dans " le pays où l'on ne voit rien ", le sombre royaume de Nergal et d'Allat; mais les àmes de ceux qui s'étaient distingués par leur bonté ou par leur valeur étaient accueillies par les dieux dans les " bocages merveilleux d'une ille fortunée, dont les arbres, comme celui d'Éridou, portaient des pierres précieuses en guise de fruits "; lì

1. Inscription d'Ashshourbanipal. Amiaud, Journal asiatique, an. 1881, p. 237.

2. Perrot et Chipiez, op. laud., t. II, p. 357.

3. Sayce, Social life among the Assyrians and Babylonians. London, 1893, in-16, p. 55. - Maspero, IIistoire ancienne, t. I, p. 68 4.

4. J. E. Taylor, Notes on the ruins of Huqeyer et Noles on Abu-Shahrein and Tell-el-Lahm. (Joumal of the Royal Asiatic Society, t. XV, p. 271-274 et 413-415.) 
les héros, tombés sur le champ de bataille, reposaient sur des couches moelleuses et se désaltéraient à des sources éternellement limpides ${ }^{1}$.

Le culte des morts chez les Sémites occidentaux offrait de grandes ressemblances avec ce qu'il était chez les habitants de la Mésopotamie; parfois leurs tombes étaient, comme chez ces derniers, des caveaux artificiels ${ }^{2}$; mais d'ordinaire elles étaient creusées dans le roc et placées souvent à l'ombre d'un arbre, qui devenait par là sacré. C'est ainsi que la nourrice de Rébecca fut enterrée sous le " chêne des pleurs, " à Bêt-É $]^{3}$, et que les restes de Saül et de ses fils furent déposés sous un térébin the à Jabès". Il semble qu'on prît de bonne heure l'habitude d'oindre d'huile les pierres funéraires, comme on le faisait pour celles qui avaient ou rappelaient quelque de chose remarquable". On plaçait aussi des fioles d'huile dans la tombe du défunt; on voit mème l'image du mort, sculptée sur le couvercle du sarcophage, tenir une fiole d'huile à la main $^{6}$. On plaçait encore dans les tombes des amulettes, des vases contenant des cosmétiques; dans une tombe phénicienne de Tharros, en Sardaigne, on a trouvé aussi des amphores, bouchées avec de l'argile, et qui semblaient avoir été remplies de vin ${ }^{7}$.

1. Hommel, Geschichte Babyloniens und Assyriens, p. 400. - Haupt, Sintfutbericht, p. 10. - Cf. Karppe, Le royaume des morts. (Journal asialique, $\mathrm{n}^{\text {le }}$ série, t. IX (1897), p. 107 et suiv.)

2. Perrot et Chipiez, Histoire de l'art, t. III, p. 163 et 173.

3. Genesis, cap. xxxv, vers. 8.

4. 1 Paralipomena, cap. x, vers. 12. Cf. plus haut, p. 465.

5. Pietschmann, Gesclichte der Phönizier, p. 196-198.

6. Pietschmann, op. laud., p. 207.

7. Perrot et Chipiez, op. laud., t. III, p. 234. 
Les corps des défunts, en Mésopotamie, paraissent avoir été enveloppés de bandelettes bituminées, pour en assurer la conservation; la Genèse rapporte " que Joseph fut embaumé ${ }^{1}$; mais il était mort en Egypte; il n'y a rien là par suite qui prouve que l'embaumement. fìt usité en Palestine; les autres patriarches, les juges et les rois sont presque tous représentés comme léposés seulement dans leur ville ou le tombeau de leurs pères" ; toutefois il est dit d'Asa qu'on "le déposa sur un lit couvert d'aromates et de parfums habilement préparés $)^{3}$. On dut prendre en effet l'habitude de placer à côté du mort des substances aromatiques destinées à en conserver les restes. L'Évangile de Marc et de Luc nous montrent les saintes femmes achetant des aromates pour embaumer le corps du Christ"; et, dans l'Évangile de Jean, Nicodème apporte un mélange de myrrhe et d'aloès du poids d'environ cent livres, et, avec Joseph d'Arimathie, il enveloppe de linges le corps du Saureur arec des aromates, "selon la coutume d'ensevelir parmi les Juifs". "

A ces fêtes funèbres il faut en ajouter une d'un caractère particulier, célébrée en l'honneur d'Adonis; ici point de sacrifices ou de libations, mais des graines hàtives, ou des plantes délicates, semées ou déposées dans des vases en terre, des corbeilles, etc. Ces plantations improvisées, appelées "Jardins d'Adonis ", se

1. Genesis, cap. L, vers. 26.

2. 1 Regna, cap. II, vers. 10 ; cap. XI, vers. 43; cap. XIV, vers. 31 ; cap. xv, vers. 24. - 2 Paralipomena, cap. xit, vers. 26 ; cap. xxxil, vers. 33 ; cap. xxxv, vers. 24.

3. 2 Paralipomena, cap. Xvi, vers. 14. Le livre des Rois ne fait pas mention de ces aromales.

4. Cap. xvi, vers 1 et cap. xxiv, vers 1.

5. Cap. xix, ver's. 39- 10 . 
fanaient rite, image de l'arrèt de la régétation au milieu des chaleurs de l'été; on les jetait alors, avec toutes sortes de manifestations de douleur, dans les sources ou dans la mer'.

Les fètes des dieux el les cérémonies funèbres nétant dordinaire que la reproduction de ce qui se passe dans la vie commune, les plantes avec leurs produits prenaient place dans les fêtes profanes, comme dans les solennités religieuses. Après s'ètre construit un palais, Assarhaddon rapporte qu'il invita les grands de son royaume à un banquet; "De rin, dit-il ², de vin de sésame, je réjouis leur cœur, d'huile excellente jabreuvai leur tète. " Cette dernière phrase nous montre qu'on oignait de parfums la tête des convires. Gilgamès, déplorant la mort de son compagnon d'armes Éabani, dit qu'il ne s'oindra plus de parfums odorants ${ }^{3}$. Cet usage était ou devint général chez tous les Sémites. David s'oint d'huile; Ruth et Judith se parfument le corps"; Amos reproche aux grands de Sidon de s'oindre d'huiles parfumées ${ }^{5}$. Alors que Jésus était à Béthanie dans la maison de Simon, une femme vint à lui, ayant un vase d'albâtre plein de parfum, et le répandit sur sa tète ${ }^{6}$.

On brûlait et on répandait aussi des aromates dans la salle du banquet, ainsi que dans les appartements qu'on habitait. "Les parfums réjouissent le cour ",

1. Clermont-Ganneau, La stèle de Byblos. (Éludes d'archéologie orientale. Paris, 1880, in-8, t. I, p. 27.)

2. B. Neissner und P. Rost, Die Bauinschrift Asarhaddons. (Beitrïge zur Assyriologic, t, IIl, 2, p. 201.)

3. Haupt, Die zwölfle Tafel der Babylon. Nimrodepos, p. 57.

4. 2 Samuel, XII, 20. - Rulh, III, 3. - Judilh, x, 3.

5. Amos, cap. vi, vers. 6. Cf. Psalmus xxiI, vers. 5.

6. Malhacus, cap. xxvi, vers. 6-7. - Lucus, cap. xiv, vers. 3. 
remarquent les Proverbes. "J'ai parfumé ma couche de myrrhe, d'aloès et de cinnamome ", dit la femme adultère ${ }^{1}$. On s'oignait aussi le corps de parfums pour l'embaumer, ainsi que l'air ambiant. "Le nard répandu sur moi a exhalé son parfum ", dit la bien-aimée du Roi dans le Cantique des Cantiques. Et " ses mains distillent la myrrhe et répandent les plus doux parfums sur les verroux ${ }^{2}{ }^{2}$, quand elle va lui ouvrir.

En même temps qu'on les parfumait avec des aromates, on ornait aussi et on embellissait les appartements avec des fleurs. Parmi les serviteurs qui, sur un bas-relief de Koyoundjik, portent dans le palais les apprêts d'un festin ${ }^{3}$, on en voit plusieurs chargés de vases remplis de fleurs; on disposait ces vases sur des supports autour de la salle; on ne parait point les avoir mis sur la table du festin; encore moins, les convives portaient-ils des couronnes de fleurs. Il faut arriver à l'époque grecque pour que cet usage s'établisse ${ }^{4}$; mais on voit les rois assyriens, imitation peut-être de ce qui se faisait en Égypte, porter dans certaines circonstances des lotus sacrés à la main ${ }^{3}$.

II.

Au-dessus des Dieux les Chaldéens admettaient l'existence d'innombrables esprits, les uns bienfaisants, les autres malfaisants. Les premiers étaient les protecteurs de toutes les choses créées et en particulier

1. Proverbia, cap. vil, vers. 6-7 et cap. xxvil, vers. 9:

2. Canlicum, cap. I, vers. 12 et cap. v. vers 5.

3. Layard, The monumenls, ser. I, pl. 8, 1.

4. Si le livre de la Sugesse blàme ceux qui se couronnent de roses, c'est qu'il a été écrit au second siècle avant notre ère.

5. Botta, Monument de Ninive, t. Il, pl. 105. 
des hommes; les seconds, " ennomis d'Éa, comme dit un chant magique', ne se plaisaient qu'it porter le trouble dans la création; ils ne quittaient les lieux sanvages et déserts, leur résidence habitnelle, et ne venaient dans les endroits habités que pour tourmenter les hommes. Les maladies les plus terribles étaient leur œurre; chacun d'eux s'attaquait de préférence à quelque partie du corps; Idpa ou Asakhou, la fièvre, agissait plus particulièrement sur la tête, Alou sur' la poitrine, Gigim sur les entrailles, Namtar, personnification de la peste, s'en prenait à la source même de la vie ${ }^{2}$. Pour se soustraire à leur influence funeste, il fallait s'adresser à un esprit plus puissant. De là ces incantations que le malade prononçait après s'être purifié et avoir bu certaines boissons enchantées.

La peste - Namlar - la fièvre qui emporte les hommes, la consomption qui trouble le genre humain, douloureuse à la chair, injurieuse au corps, l'incube mauvais, le mauvais alou, le mauvais mastim, les maladies des yeux, de la bouche, de la langue, tous, qu'ilss soient expulsés, qu'ils soient chassés de mon corps. Qu'ils ne reviennent jamais contre mon corps, qu'ils n'injurient plus mon œil, qu'ils ne pèsent plus sur mes épaules, qu'ils n'entrent jamais dans ma maison! O esprit du ciel, conjure-les! O esprit de la terre, conjure-les!3.

Au lieu d'être envoyée par un esprit mauvais, la maladie pouvait ètre l'œurre d'un magicien, la consé-

1. Lenormant, Études accadiennes, t. III, p. 83. - Sayce, Lectures, p. 458.

2. A. Laurent, La magie et la divination chez les ChaidéoAssyriens. Paris. 1894, in-8, p. 33-34.

3. Lenormant, La magie chez les Chaldeens, p. 15. - Sayce, Lectures, p. 455. Cette incantation se termine par une invocation adressée successivement à tous les dieux. D'autres incantations ne visaient qu'une maladie ou une souffrance particulière. 
quence d'une imprécation malfaisante, prononcée en l'absence du dieu qui veillait sur le patient. Parfois le sorcier appelait, il semble, à son aide, la vertu des plantes:

La magicienne m'a ensorcelé par son sortilège, lit-on dans une incantation du Musée britannique ${ }^{1}$; le jeteur de sorts a tiré et a imposé son fardeau de peine, le faiseur de philtres a percé, s'est avancé et s'est mis en embuscade en cueillant son herbe, que le dieu du Feu, le héros, dissipe leurs enchantements.

L'intervention seule des dieux pouvait lutter contre de tels maléfices et guérir les maux qu'ils causaicnt; c'étaient eux aussi qui venaient au secours du malade. Dans un vieux texte Mardouk va demander à son père la délivrance d'un homme frappé par un sortilège, et il en apprend un charme qui fera cesser le mal qui tourmente le patient ${ }^{2}$. C'était ce dieu qui avait enseigné aux hommes les incantations toutes puissantes qui délivrent du démon et des sortilèges des magiciens. Une de ces incantations consistait à prendre une gousse d'ail, des dattes, un rameau chargé de fleurs, et à les jeter au feu morceau à morceau, en murmurant une oraison appropriée à l'opération ${ }^{3}$.

De même que cet ail pelé et jeté au feu, la flamme ardente le consume, il ne sera point planté au jardin potager,... sa racine ne s'implantera pas en terre, sa tige ne poussera pas et ne verra pas le soleil; il ne servira pas à la nourriture des dieux ou du roi, de même puisse-t-il emporter l'incantation

1. Lenormant, La magie chez les Chaldiens, p. 48.

2. Jensen, Zeilschrift für Keilschriftforschung, t. I, 4, t. II, 1. - Sayce, Leclures, p. 471. - Maspero, IIisloire ancienne, t. I, p. 780 .

3. Sayce, Leclures, p. 472-473. - Maspero, op. laud., t. I, p. 781. 
mauvaise, puisse-t-il dénouer le lien de la maladie du péché, de la faute, de la perversité, du crime! La maladie qui est en mon corps, en ma chair, en mes muscles, ainsi que cet ail, soitelle pelée et qu'en ce jour la flamme ardente la consume; puisse sortir le sortilège, que je voie la lumière!

Le malade mettait de même en pièces et le feu dévorait le régime de dattes, la branche fleurie, etc. Mais ces régétaux n'agissent ici que comme symboles; lincantation, qui accompagne leur destruction, seule est efficace. C'est aussi moins par leurs vertus curatives que comme talisman qu'agissent les aromates dans un remède prescrit contre la peste ${ }^{1}$.

Le docteur dit: Assieds toi et pétris une pâte d'aromates et fais-en l'image de sa ressemblance (du Namtar, démon de la peste). Applique-la sur la chair de son ventre (du malade), tourne la face de cette image vers le coucher du soleil. Alors la force du mal s'échappera en même temps.

Mème quand on attribuait quelque vertu curative aux plantes, le charme magique, qui en accompagnait ou suivait l'emploi, en assurait à lui seul l'efficacité; c'est ce qu'on voit dans cette formule enseignée par Éa à son fils" : "Prends-un vase et mets-y de l'eau... mets dedans du bois de cèdre blanc, introduis-y le charme qui vient d'Éridou, et complète ainsi puissamment la ver'tu des eaux enchantées. "

On comprend que, avec une telle conception de la cause des maladies et des moyens de les guérir, la thérapeutique eût été poussée peu loin dans l'Assyrie et la Chaldée; si Hérodote a eu tort de dire qu'il n'y

1. Lenormant, La magie chezles Chaldeens, p. 18.

2. Cun. inscriplions of W. Asia. ap. Lenormant, Origines de l'hisloire, t. 1, p. 84, note. 
avait pas de médecins à Babylone ${ }^{1}$, l'art de guérir y était bien plutôt une branche de la magie que de la pharmacopée; il consistait surtout, suivant la remarque de Diodore ${ }^{2}$, en purifications, sacrifices ou incantations et était dans la main des prètres; la pratique en revenait à la seconde classe du corps sacerdotal.

Ce n'est pas à dire toutefois que les habitants de la Mésopotamie aient entièrement et toujours dédaigné les remèdes de la pharmacopée ou lés vertus des plantes ${ }^{3}$; le Musée britannique possède des fragments de l'édition faite pour la Bibliothèque de Ninive d'un vieux traité de médecine babylonien, qui semble sorti de l'école de Borsippa ". A côté d'une tentative pour classifier et décrire les différentes maladies, on y trouve l'énumération des divers remèdes destinés à les combattre. Quelques-unes des recettes sont d'une longueur extraordinaire, comine la plupart de celles de la pharmacopée des Anciens, et renferment un mélange des drogues les plus hétérogènes ". D'autres fois aussi le remède est d'une grande simplicité; ainsi pour stimuler l'appétit le vin de dattes est seul prescrit. Ce liquide entrait dans la composition de la plupart des remèdes, soit comme ingrédient particulier, soit comme diluant. Dans les maladies bilieuses, par exemple, on mêlait ensemble du lait de chèvres, du vin de dattes avec du

1. IIislorice, lib. I, cap. 197.

2. Bibliolheca, lib. II, cap. 29.

3. Il est question dans une incantation d'une "plante qui égaie la vie ». Sayce, Magical lexls III. (Leclures, p. 459.)

4. Sayce, An ancient Babylonian work on Vedicine. (Zeilschrift für Keilschriftorschung und verwandle Gebiete, t. II (an. 1889), p. 1-14 et 207-216.) - Id., Social life among the Assyrians and Babylonians, p. 98.

5. Sayce, Social life, p. 98. 
cyprès, de l'orge et nombre d'autres ingrédients inconnus; contre la fièvre on écrasait ensemble la racine de la "plante de la destinée humaine ${ }^{1}$ " - serait-ce la mandragore? - la racine de l'arbre sousou, du sisi siman et la lang'ue d'un chien, et l'on buvait cette mixtion singulière dans de l'eau ou du vin de dattes ${ }^{2}$.

Ce qui est fait pour surprendre, c'est que le choix était parfois laissé au malade entre les divers remèdes qu'il devait employer; ainsi contre une attaque d'hypocondrie, il pouvait, soit prendre des semences de roseau et des dattes écrasées dans du vin de dattes, soit du lait de génisse et des amers mélangés dans ce mème liquide, soit encore boire une décoction d'ail et d'amers dans du vin de dattes ${ }^{3}$. On a lá un exemple de l'emploi fréquent de ce vin si estimé en Babylonie; il servait de dissolvant à bien d'autres remèdes: cyprès et amers, bois de sousou et amers, feuilles de chêne et amers, d'autres ingrédients encore étaient bus dans ce mème vin. On y ajoutait parfois de l'huile; dans un mélange de ce genre on buvait une préparation d'extrait de bois de sousou et d'amers ". Parmi les remèdes de cet antique traité, ceux qui sont indiqués contre le mal de dents ou les aphtes à la bouche ${ }^{5}$ méritent une

1. Shammou balâti. "L'homme qui peut la cueillir, si vieux qu'il soit, peut rajeunir; s'il est mourant, il revient à la vie ", dit, dans une insignifiante étude. M. A. Boissier, qui parait confondre cette herbe avec la plante merveilleuse, indiquée par Shamashnapishtim à Gilgamès. Liste de planles médicinales. (Revue sémitique d'épigraphie et d'histoire ancienne, t. II (an. 1894), p. 145).

2. Zeitschrift für Keilschriftorschung, t. II, p. 8-13.

3. Sayce, Social life, p. 99.

4. Zeitschrift für Keilschriftorschung, t. II, p. 207.

5. "For a sore mouth", traduit M. Sayce. Zeitschrift, p. 215 . 
mention spéciale. On employait également pour les combattre la racine de la " plante de la destinée humaine ", le fruit de la serpentaire jaune ou les racines d'une épine ${ }^{1}$, qui avait crû sans voir le soleil. Placés sur une dent malade, ces ingrédients la guérissaient.

On voit par ce qui précède que les Assyriens et les Chaldéens croyaient aux propriétés curatives des plantes, mais qu'ils n'en avaient aussi qu'une connaissance bien incertaine; ils attribuaient même des vertus surnaturelles à quelques-unes d'entre elles, rares, il est vrai, et inaccessibles aux mortels. Quand Gilgamès eut été frappé de la lèpre par la vengeance d'Ishtar, le héros se rendit dans l'île fortunée où résidait son ancêtre Shamashnapishtim; celui-ci lui donne le brouet magique, qui doit préparer sa guérison; puis, avant de le renvoyer, il lui fait cueillir une plante, " semblable à l'aubépine par sa fleur et dont les aiguillons piquent comme la vipère, mais qui assure une jeunesse éternelle ", plante merveilleuse qu'un serpent lui ravit? Elle n'était pas la seule que les dieux tinssent cachées aux mortels. Telle était encore celle qui facilitait les naissances et qu'on ne rencontrait qu'au ciel d'A nou. La femme d'Étana ne pouvant enfanter, le héros demande à l'aigle Zou de le porter au lieu où elle croissait; bientôt effrayé toutefois des difficultés de l'ascension, il prie le divin oiseau de redescendre; mais épuisé il tomba brisé sur le sol, sans avoir atteint la plante miraculeuse ${ }^{3}$.

1. Sayce, Social life, p. 99. Dans la Zeitschrift le savant assyriologue traduit par "racines de l'arbre d'Elam ".

2. Haupt, Die Bubylonische Nimrodepos, p. 147. - Naspero, Hisloire ancienne, t. I, p. 527.

3. Ed.-J. Harper, Die babylonischen Legenden von Elana, 
Comme les Assyrio-Chaldéens, les Hébreux, il en ètait sans doute de même des autres Sémites occidentaux, attribuaient les maladies aux puissances divines; c'était Jahveh qui les envoyait, c'était Jahveh aussi qui les guérissait. "Je frappe et je guéris ", dit-il dans le Deutéronome". "Si vous écoutez la voix de l'Éternel votre Dieu ", lit-on dans l'Exode ", " et que vous obéissiez à ses commandements, je n'enverrai sur vous aucun des maux dont j'ai affligé les Égyptiens; car je suis l'Éternel et je vous guérirai. " Le livre de la Sagesse, à une époque où la médecine était connue et pratiquée depuis des siècles chez les Juifs, n'attribue encore qu'à Dieu seul les maladies et leur guérison. "Ce n'est ni une herbe, ni un remède appliqué sur leur plaie, qui les a guéris, mais votre parole, ô Seineur, qui guérit toutes choses ${ }^{3}$. "

D'après cette conception, c'était à Dieu qu'il fallait s'adresser dans les maladies, c'était lui qui faisait connaitre les remèdes qui les guérissaient. Asa, tombé malade, est blâmé de n'avoir point cherché le Seigneur dans son infirmité, mais de s'ètre confié davantage en l'art des médecins ${ }^{4}$. Quand le peuple murmure, dans le désert, contre l'amertume des eaux de Marah, Moïse crie vers l'Éternel, et l'Éternel lui montre un

Zu, Adana und Dibbara. (Beiträge zur Assyriologie, t. II, p. 395 et suiv.) - Naspero, t. I, p. 699.

1. Cap. xxxII, vers. 39.

2. Cap. $x y$, vers. 26.

3. Cap. xvi, vers. 12.

4. 2 Paralipomena, cap. xvi, vers. 12. 
bois qui, jeté dans ces eaux, les rendit douces aussitôt ${ }^{1}$. On comprend d'après cela que la médecine et la pharmacopée furent à l'origine chez les Juifs entre les mains des prêtres; c'était à eux que les malades devaient demander leur guérison, surtout s'ils étaient atteints de maladies contagieuses ou réputées impures, comme la lèpre. Le Lévitique ordonnait de mener devant Aaron ou ses fils l'homme dont la peau en était marquée ${ }^{2}$. C'étaient eux qui devaient suivre les progrès de ce mal terrible, en reconnaître et déclarer la souillure. Quand le lépreux était guéri, il devait encore se présenter devant le prêtre, puis offrir deux passereaux, un morceau de bois de cèdre, du fil de couleur écarlate et de l'hysope. Alors le prêtre le purifiait sept fois en l'aspergeant avec le bois de cèdre et l'hysope trempés dans le sang de l'un des passereaux; puis il l'oignait avec le sang d'un agneau immolé pour racheter son offense ${ }^{3}$; car son mal était considéré comme la punition d'une faute; ensuite il faisait une aspersion arec de l'huile et en faisait une onction sur le bout de l'oreille droite de celui qui venait se purifier, sur le pouce de sa main droite et sur le gros orteil de son pied droit, ainsi que sur sa tête, et en arrosait sept fois la maison.

Le traitement de la lèpre, à ses divers degrés, ressemblait, on le voit, à une cérémonie sacrée, dont tous les détails étaient minutieusement réglés. Toutes les maladies cependant n'étaient pas l'objet de soins pareils, et leur traitement n'était pas toujours entre les

1. Exodus, cap. xv, vers. 23-25.

2. Cap. xul, vers. 2.

3. Levilicus, cap. XIv, vers. $10,15,17,26,28,29,49$ et 51 . 
mains des prètres; l'Exode fait déjà mention de médecins ${ }^{1}$; mais nous ignorons quels remèdes, surtout quels remèdes végétaux, ils employaient. Salomon qui "parla, dit l'Écriture ${ }^{2}$, de toutes les plantes depuis le cèdre jusqu'a l'hysope ", dut connaitre quelquesuns des simples en usage dans la pharmacopée contemporaine; une tradition veut qu'il les ait recueillis et consignés dans le Sepher Rephuot, livre souvent cité dans le Talmud ${ }^{3}$. Une autre tradition faisait, au contraire, un mérite au pieux Ézéchias d'avoir caché les livres qui renfermaient des recettes pour la guérison de toutes les maladies, sans doute parce qu'il voyait dans leur composition un acte de méfiance envers l'intervention divine ${ }^{4}$.

Ce dédain pour la médecine ne derait pas durer; elle participa à l'essor que les sciences prirent en Judée après le retour de la captivité de Babylone; désormais elle fut universellement pratiquée et honorée; sans doute la guérison est toujours considérée comme venant du Très-Haut; c'est lui que le malade doit d'abord invoquer; mais comme "Dieu fait produire à la terre des médicaments ", le sage, dit l'Ecclésiastique $^{3}$, ne doit pas les rejeter; la vertu des plantes est faite pour être connue des hommes; Dieu lui-même leur en a donné la science; c'est avec elles que le médecin guérit et enlève le mal, que le pharmacien prépare ses potions salutaires.

On peut juger d'après cela des progrès que la méde-

1. Cap. xxi, vers. 19.

2. 2 Regna, cap. iv, vers. 32 .

3. Berendes, Die Pharmacie bei den allen Cullurvölliern, p. 88.

4. Talmud Mischnah II, ap. Berendes, ibid., p. 89.

5. Cap. xxxviI, vers. 1-7 et 9. 
cine et la pharmacopée avaient faits en Judée dans les derniers siècles qui précédèrent notre ère. L'établissement d'un collège de médecins à Jérusalem ${ }^{1}$ dut encore en favoriser le développement. L'art de guérir ne ressemble plus à ce qu'il était à l'époque des Juges et des Rois; la thérapeutique chaldéenne et grecque l'avaient transformé. Quant aux remèdes de l'ancienne pharmacopée juive, ils étaient peu nombreux et fort simples; la płupart étaient émpruntés au règne végétal et tirés des substances alimentaires elles-mèmes: vin, bière, vinaigre, huile, figues, dattes, farine de froment, graine de lin, ail, ainsi sans doute que les divers condiments.

Le vin est souvent mentionné comme tonifiant dans l'Ancien Testament; " il réjouit les dieux et les hommes ", dit le livre des Juges ${ }^{2}$; non seulement bu avec sobriété, il est " la santé de l'àme et du corps " ", mais il était recommandé comme stomachique : "Usez d'un peu de vin à cause de votre estomac et de vos fréquentes infirmités ", écrit Paul à Timothée ${ }^{+}$. Le rin de dattes fut probablement aussi employé de bonne heure dans la pharmacopée juive; le Talmud, du moins, en prescrit l'usage conme laxatif. On ne peut guère douter que la bière n'ait été en usage dans la thérapeutique des Sémites occidentaux, comme elle l'était dans celle des Égyptiens; le vinaigre y dut figurer également. Mais l'huile surtout y occupait une place con-

1. Berendes, op.laud., t. I, p. 90.

2. Cap. Ix, vers. 13.

3. Ecclesiasticus, cap. xxxi, vers. 37. "Il est à lhomme comme la vie. " "C'est la joie du cœur et le contentement de l'esprit. "Ibid., vers. 32 et 36 .

4. Epistola, cap. v, vers. 23. 
sidérable; on l'employait contre l'esquinancie, les douleur's intestinales et même la constipation. On s'en servait aussi mèlée avec du vin pour laver les plaies, comme on le voit par la parabole du bon Samaritain'. L'huile la plus usitée était celle d'olive; mais on se servit aussi dans les derniers temps de celle de sésame et de ricin. On employait encore en Judée une huile extraite des graines de la coloquinte?

Les fruits: dattes, grenades, figues, raisins, etc., n'étaient pas moins usités dans la pharmacopée que dans l'alimentation des Sémites occidentanx ; mais nous sarons peu de chose de leur emploi; il fut sans doute chez eux à peu près le même que chez les Égyptiens. Les figues, en particulier, passaient pour émollientes et résolutives; Isaïe guérit les ulcères d’Ézéchias en faisant appliquer des figues fraiches sur ses plaies ${ }^{3}$.

Conme les fruits, les feuilles étaient aussi employées dans la médecine sémitique, mais nous ignorons sous quelle forme. "Leurs fruits serviront pour nourrir les peuples et leurs feuilles les guériront ", dit Ézéchiel ${ }^{4}$, en parlant des arbres de la Jérusalem nouvelle. Les résines et les gommes aromatiques prenaient également place dans la pharmacopée des Chaldéo-Assyriens et des Juifs; ; la myrrhe était regardée comme tonifiante; on l'employait aussi, probablement dissoute dans l'huile, comme émolliente. Le baume de même, si l'on en croit Jérémie", devait être un spécifique précieux. "N'y

1. Lucas, cap. $\mathrm{x}$, vers. 3't.

2. Berendes, op. laud., t. I, p. 95.

3. 2 Regna, cap. xx, vers. 7. - Esaias, cap. xxxviI, vers. 21.

4. Cap. xlviI, vers. 12.

5. Cap. vil, vers. 24 et cap. Xlvi, vers. 11. 
a-t-il plus de baume en Giléad? N'y a-t-il plus là de médecin? " dit-il en faisant allusion à la plaie mortelle dont avaitété frappée la fille d'Israël. Et ailleurs s'adressant à la fille d'Égypte, blessée elle aussi : "Monte à Giléad, s'écrie-t-il, et prends-y du baume. »

A côté de ces remèdes d'origine végétale, il faut placer la mandragore, solanée à la racine fusiforme et bifurquée, aux feuilles radicales d'un vert sombre, aux fleurs purpurines et dont les fruits rouges, semblables à une petite pomme, exhalent une odeur agréable. Cette plante possède des propriétés stupéfiantes et narcotiques ${ }^{1}$; mais les Hébreux paraissent avoir surtout estimé ses prétendues vertus aphrodisiaques. La Genèse raconte ${ }^{2}$ que Ruben ayant trouvé des mandragores - doudaim - dans les champs, au temps de la moisson, les apporta à Léa, sa mère; mais Rachel, qui était stérile, les demanda à celle-ci. Dans le Cantique des Cantiques ${ }^{3}$ la bien-aimée du Roi lui réserve, avec d'autres fruits, des mandragores parfumées, sans doute afin de se l'attacher darantage.

L'emploi des parfums était trop grand et trop général chez les nations sémitiques pour que l'art de la droguerie n'y eut pas été pratiqué de bonne heure,

1. D’après H. Brugsch, Die Alraune als allägyptische Zauberpflanze. (Zeitschrift für ägyptische Sprache, t. XXIX, p. 3133), cétait une mandragore que la plante dont Ra donna à boire une décoction à la déesse chargée de faire périr le genre humain; enivrée par ce breuvage, celle-ci ne reconnut plus les hommes, qui échappèrent ainsi à la destruction. Cf. Pline, xxv, 94.

2. Cap. xxx, vers. 14.

3. Cap. VII, vers. 13. 
mais nous ne savons à peu près rien des progrès qu'il y put faire. Ce que les textes cunéiformes et hébraïques nous apprennent toutefois, c'est que les aromates les plus variés étaient en usage chez les Sémites de la Mésopotamie, comme de la Syrie: résine de cèdre, galbanum, ladanum ', roseau aromatique, lleurs parfumées du henné, safran, baume, myrrhe et stacte ${ }^{2}$, encens, cinnamome et cassie, bois d'aloès et nard, aspalathe. Les Assyriens receraient la résine de cèdre des furèts de l'Amanus et du Liban ${ }^{3}$; le galbanum et le ladanum se trouraient en Syrie; le henné se rencontrait dans la Judée méridionale, ainsi que le roseau aromatique"; le safran ${ }^{5}$ était cultivé dans toute l'Asie antérieure; le baume ${ }^{6}$, acclimaté dans les jardins de Jéricho, était importé de lá en Phénicie. L'encens ou oliban et la myrrhe, arec le stacte, venaient d'Arabie ${ }^{\top}$.

Les " parfums exquis ", que les Sabéens fournissaient à Tyr, d'après le témoignage d'Ézéchiel ${ }^{\text {s }}$, étaient probablement de la myrrhe et de l'encens; eux-mêmes recevaient, pour la plus grande partie, ces aromates du pays des Somalis: précieux produits que leurs caravanes portaient ensuite dans toute la Syrie, ainsi

1. Le galbanum, hébr. khelbenah; le ladanum, hébr. lot.

2. Hébr. nalaph, liquide qui exsude spontanément de l'arbre à myrrhe.

3. V. Scheil, The monolith inscription of Shalmasar II. (Records of the Past, t. IV, p. 63.)

4. Cf. plus haut, p. 253-355. Le nom hébreu du henné est kopher, celuidu roseau aromatique kaneh.

5. Hébr. karkom.

6. Hébr. tsorî. - Ezechiel, cap. xxvir, vers. 17.

7. L'encens, hébr. lebônâh, la myrrhe, hébr. mor. Ed. Schaer. Die ällesten Ileilmittel aus dem Orient, Schaffhausen, 1877, in-8, p. 16.

8. Cap. xxvil, vers. 22. 
que sur les marchés de l'Assyrie et de la Chaldée. Quant all cassie, hébr. kiddâh - Cinnamomum cassia Bl. - au cinnamome, hébr. kinnamon - Cinnamomum zeylanicum Br. - ai nard, hébr. nêrd - Nardostachys Jatamansi DC. - et au bois d'aloès, hébr, uhalim - Aloexylon agallochum Lour., - ils étaient importés dans l'Asie antérieure, ainsi que l'amomum ou cardamome de Molabar - Elettaria cardamomum Mat. et peut-être aussi l'énigmatique aspalathe ${ }^{1}$, par les navigateurs qui faisaient le commerce entre l'Inde et les ports du golfe Persique ${ }^{2}$. De lá ils étaient transportés en Assyrie et en Syrie, d'où ils se répandaient, arec les autres parfums, jusqu'en Grèce et même plus tard en Italie. C'est ainsi que les poètes de ces contrées ont pu parler du nard et de l'amomum d'Assyrie ${ }^{3}$, ainsi que de l'encens et de la myrrhe de Syrie ${ }^{4}$.

Ces aromates, à cause même de leur rareté, aussi bien que de l'usage qu'on en faisait étaient singulièrement recherchés; aussi étaient-ils considérés comme le présent le plus agréable et le plus précieux qu'on pût faire aux hommes et aux dieux. Pour se concilier la faveur de leur frère Joseph, qu'ils n'araient point reconnu, les fils de Jacob lui portent du baume, de la gomme et du ladanum ${ }^{5}$; les Mages, en signe d'hom-

1. Royle incline à voir dans cette plante un Myrica de l'Hindoustan, le M. sapida W. Cf. W. Smith, Dictionary, s. v. J'ai, p. 319, identifié l'aspalathe avec le Convolvulus scoparius I; mais cette plante, d'après De Candolle, n'existant qu'à Ténériffe n'a pu être connue des Anciens.

2. Rawlinson, op. laud., t. I, p. 557.

3. "Assyriàque nardo ». IIor., 2 Od., XI, v. 16. - "Assyrium vulgo nascetur amomum ". Virg., Ecl. IV, v. 25.

4. Eurip., Bacch., v. 14t. - Theocr.: Idyll. XV, v. 114.

5. Genesis, cap. xuml, vers. 11. 
mage suprème, offrent à l'enfant Jésus de l'encens et de la myrrhe'.

Si nous comnaissons les noms et l'origine de la plupart des aromates dont faisaient usage les Sémites occidentaux, nous ignorons presque complètement les préparations dans lesquelles ils entraient. Les parfums d'Assyrie étaient renommés ${ }^{2}$; mais c'est tout ce qu'on en sait. Nous sommes plus heureux en ce qui concerne ceux des Hébreux; si nous ne pouvons dire de quoi se composait au juste l'huile de myrrhe, dont il est sourent question dans l'Ancien Testament, l'Exode nous fait connaitre ${ }^{3}$ les divers ingrédients qui servaient à fabriquer le parfum employé dans les onctions saintes, ainsi que celui qu'on brûlait dans le sanctuaire. Le premier se composait de myrrhe, de cinnamome, de roseau aromatique et de cassie, dissous dans un hin d'huile d'olive; le second était fait avec du stacte, du galbanum et de l'encens pur, mêlés par portions égales, broyés, ensemble, puis réduits en poudre ${ }^{4}$.

1. Mrllhaus, cap. II, vers. 11.

2. "Non soror, Assyrios cineri quae dedat odores." (I Tib., Eleg. IlI, vers. 7.)

3. Cap. xxx, vers. 23-25 et 3́-36.

4. D'après Josèphe, De bello judaico, lib. V, cap. 5, 5, ce parfum aurait renfermé treize aromates; c'étaient, si l'on en croit Maïmonide, outre les précédents, la myrrhe, le cassie, le nard, le safran, le costus et l'herbe macleh âshân, connue des seuls initiés, avec quelques substances minérales. Cf. W. Smith, A diclionary of the Bible, s. v. Incense. 



\section{TABLE}

PRÉFACE Pages,

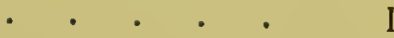

Additions et corrections. . . . . . . . . . . xix

LIVRE PREMIER.

LES PLANTES CIIEZ LES ÉGYPTIENS.

Chapitre Ier. - La flore pharaonique. . . . . . . 1

Chapitre II. - L'agriculture dans l'Égypte ancienne. . 21

$1^{\circ}$ Céréales. . . . . . . . . . . . 26

$2^{\circ}$ Plantes fourragères. . . . . . . . . . . . 36

$3^{\circ}$ Plantes industrielles. . . . . . . . . . . 41

Chapitre III. - L'horticulture dans l'Égypte ancienne. 54

$1^{\circ}$ La culture des plantes potagères. . . . . . 54

$2^{\circ}$ Le jardin pharaonique.. . . . . . . . . 77

Chapitre IV. - Les arbres fruitiers et les arbres d'ornement. Les plantes d'agrément. . . 104

10 Les arbies fruitiers. . . . . . . . . . 104

$2^{\circ}$ Les arbres d'ornement. . . . . . . . . . . . . . 141

$3^{\circ}$ Les fleurs d'agrément. . . . . . . . . . $\quad 157$

Cinspitre V. - Les plantes dans l'alimentation et dans l'industrie des Egyptiens. . . . . 170

10 Les plantes dans l'alimentation. . . . . . 170

$2^{\circ}$ Les plantes dans l"industrie. . . . . . . . . 192

Chapitre VI. - Les plantes danș l'art et dans la poésie des Egyptiens. . . . . . . . 218

$1^{\circ}$ Les plantes dans l'art. . . . . . . . . . 218

$2^{\circ}$ Les plantes dans la poésie. . . . . . . . . 245

Chapitre Vil. - Les plantes dans les légendes divines et dans les cérémonies profanes et religieuses des Egyptiens. . . . . 252

10 Les plantes dans les mythes. . . . . . . 252

$2^{\circ}$ Les plantes dans le culte. . . . . . . . . . $\quad 272$

$3^{\circ}$ Les plantes dans les fêtes profanes. . . . 301

Cinspitre VIII. - Les plantes dans la pharmacopée et la droguerie égyptiennes. Les aromates et leurs usages. . . . . . . . 304

1 L Les plantes dans la pharmacopée. . . . . . 30t

$2^{\circ}$ Les plantes dans la droguerie. . . . . . . . 317

$3^{\circ}$ Les plantes dans les funérailles.. . . . . . . 


\section{LIVRE SECOND.}

LES PLANTES GHEZ LES SÉMITES.

Chapitre Ier. - La flore de l'Asie antérieure. . . . . 327

10 La flore de l'Asie mineure. . . . . . . . 329

$2^{\circ}$ La flore de la Syrie.. . . . . . . . . . 334

30 La flore de la Mésopotamie. . . . . . . . . 339

40 La flore de l'Arabie. . . . . . . . . . . 342

$5^{\circ}$ Les plantes alimentaires et industrielles. Les arbres fruitiers et les aromates de l'Asie antérieure.. . . . . . . 347

60 Les peuples de l'Asie antérieure. . . . . . 356

Chapitre II. - L'agriculture et l'horticulture dans l'Asie antérieure. Les plantes dans l'alimentation et dans l'industrie des Sémites. 368

10 L'agriculture dans la Chaldée. . . . . . . 368

2o L'agriculture dans l'Assyrie. . . . . . . . 376

3 L'horticulture dans l'Assyrie et la Chaldée. . . 381

4o L'agriculture dans la Judée et la Syrie. . . . 386

$5^{\circ}$ L'horticulture dans la Judée et la Syrie. . . . 397

6. Les plantes dans l'alimentation des Sémites. . 400

7o Les plantes dans l'industrie des Sémites. . . 411

Chapitre III. - Les plantes dans l'art et dans la poésie des Sémites. . . . . . . . . . 422

$1^{\circ}$ Les plantes dans l'art des Chaldéens et des Assyriens.

20 Les plantes dans l'art des Hébreus, des Phéniciens et des Hittites. . . . . . . . . 434

30 Les plantes dans la poésie des Sémites. . . . 445

Chapitre IV. - Les plantes dans les légendes divines, dans les cérémonies profanes et religieuses, dans la pharmacopée et daus la droguerie des Sémites. . . . .

10 Les plantes dans les mythes des Chaldéo-As-

20 Les plantes dans les mythes des Sémites occiden-

3o Les plantes dans le culte des Chaldéo-Assyriens et des Hébreux. . . . . . . . . . . 470

4o Les plantes dans la pharmacopée des Sémites. . 486 $5^{\circ}$ Les plantes dans la droguerie des Sémites. . . 498 


\section{LES PLANTES}

\section{DANS L'ANTIQUITE ET AU MOYEN AGE}

HISTOIRE, USAGES ET SYMBOLISME

PREMIERE PARTIE

LES PLANTES DANS I'ORIENT GLASSIQUK

I

Égypte, Chaldẻe, Assyrie, Judée, Phénicie

PAB

Gharles JORET

Professeur à l'Université d'Aix

Correspondant de l'Institut

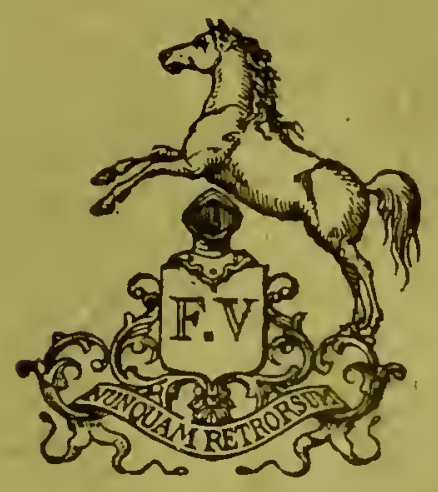

\section{PAR IS}

LIBRAIRIE ÉMIL BOUILLON, ËDITEUR

67, RUE DE RICHELIEU, AU PREMIER

1897

TOUS DROLTS RÉSEKYÉS 


\section{RECHERCHES SUR LEUMPLOI DU GÉNITIF ACGUSATIF}

\section{EN VIEUX SLAVE}

Par A. MEILLET, Directeur d'études, adjoint à l'École des Haules Etudes

Un volume grand in- $\$$. Prix.

$6 \mathrm{fr}$.

DE INDO-EUROPAEA RADICE “ MEN-MIENTE AGTTARE 》 Scripsit A. MEILLET

Brochure in-8. Prix. . . . . . . . . . $3 \mathrm{fr}$.

ÉTUDE sUR LE GREG.DU NOUVEAU TESTAMENT Comparé avec celui des Septante SUJET, GOMPLEMENT ET ATTRIBUT

Par.l'Abbé J. VITE.IU, docteur ès lettres

Un volume grand in-8. Prix. . . . . . . . $12 \mathrm{fr}$.

\section{L'ANNEAU DE LA.MORTE}

HISTOIRE D'UNE LÉGENDE

Par G. PARIS, membre de l'Institut

Brochure in-t. Irix.. . . . . . . . . . . . $2 \mathrm{fr}$.

\section{LA L A N G U E M A D É}

Par J.-B. RAMBAUD, capitaine d'artillerie de marine Brochure grand in-8. Prix. . . . . . . . . . $5 \mathrm{fr}$.

\section{PENSEES DE BLAISE PASGAL} DISPOSÉES SUIVANT L'ORDRE DU CAHIER AUTOGRAPIIE

Texte critique établi d'après le manuscrit original et les deux copies de la

Bibliothèque nationale, avec les variantes des principales éditions

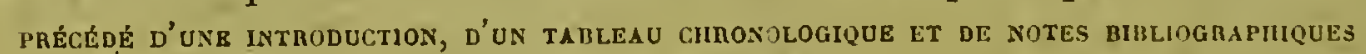
Par G. MICHAUT

Un fort volume grand in-t. Prix.. . . . . $20 \mathrm{fr}$.

\section{ABREGÉ DE LA VIE DE JÉSTS}

Par BL. PASGAL

Texte critique par G. MICHAUT

brochure in-8. Prix.

\section{DELX MINIĖRESS D'ÉCBIRE L'IIISTOIRE}

CRITIMUF, DE BOSSUET, D'AUGUSTIN THERRY ET DF FUSTEL DE COULA.TES

Par Il. d'ARBOIS DE JUBAINVILLE, membre de l'Institut

Un volume in-18 jésus. Prix. . . . . . . . . $4 \mathrm{f}$ 


\section{PERRE DE NOLHAC ET SES TRMAIX}

Essai de contribution aux publications de la Société d'études italiennes Par prerre ne BOClinabo

In rolume grand in-s. Prix. $7 \mathrm{rl} \cdot 50$

\section{G O J O U - B R E I Z}

LÉGENDES, POÉMES ET CONTES BRETONS. PREMLIEE SÉUIE : PIOUGASAOU Par IlExH de KERBEUZliC.

Un rolume in-8. Prix. . . . . . . . . . \& $4 \mathrm{f}$.

RÉPERTOIRE MÉTHODIQUE DU MOYEN AGE FRANGALS HISTOLRE - LTTTERATURE - BEAUX-ARTS

Deuxième année 189. Grand in-8. Prix. . . . . . . 4 [r.

\section{AL-FAKHRI. HISTOIRE DU KIIALIFAT \& DU VIZIRAT'} DEPUIS LEURS ORIGINES JUSQU'A LA CHUTE DU KHALIFAT ABBASIDE DE BAGDADH

(11-6356 de l'Hégire $=632-1258$ de notre ère)

AVEC DES PROLÉGOMEXES SUR LES PRIXCIPES DU GOUVERNENENT Par IBX AT TIĶTAKA

Touvelle édition du texte arabe par H. DERENBOURG Un fort volume grand in-8. Prix. . . . . . . . $25 \mathrm{fr}$.

GLOSSAIRE MOYEN BRETON

Par E. EnNAULT, professeur à la Faculté des lettres de Poiliers Deuxième édition corrigée et augmentée

Deux rolumes grand in-8. Prix.. . . . . . . $30 \mathrm{fr}$.

\section{LES ENFANGES VIVIEN}

\section{CHANSON DE GESTE}

Publiée poui la première fois d'après les manuscrits de Paris, Boulogne-sur-Mer, Londres et Milan

Par C. Wahluxd et II. DE Frhirzex, professeurs à l'Universilé d'Upsal Précédée d'une thèse de doctorat servant d'introduction

par A. Noknfist

Un volume grand in-1. Prix. . . . . . . . $2 j$ fr.

\section{LE PRÉSENT \& SES DÉRIVÉS DANS LA CONJUGAISON LATINE}

$$
\text { D'APRESS LES DONNEES }
$$

DE LA GRAMMARE COMPARE DES LANGULS INDO-LULUREFNES

Par L. Job, professeur au Lycée de Nancy

Un fort volume in-s. Prix. . . . . . . . . $10 \mathrm{fr}$. 


\section{DE GRAMMATICIS VOCABULIS APUD LATINOS}

Paı. L. JOß, professeur au Isycée rle Nancy

Un rolume in-8. Prix. . . . . . . . . . . . 3 fr. 50

R O M A N I A

RECUEIL TRIMESTRIEL CONSACRÉ. a L'ÉTUde des laxgues ET DES LITTÉRATURES ROMANES

Publié par MI. P. MEYER et G. PARIS, membres de l'Institut

Paris : $20 \mathrm{fr}$. - Départements èt Union poslale : $22 \mathrm{fr}$.

\section{REVUE CELTIQUE}

Fondée par H. GAJDOZ

Publiée sous la direction de M. d'Arions df Jubainville, membre de l'Institut, avec le concours de M. J. Loth, doyen de la Faculti des I.ettres de Rennes, et E. Ervault, professeur à la Faculté des Lettres de Poitiers.

Paris: $20 \mathrm{fr}$. - Départements et Union postale: $22 \mathrm{fr}$.

REVUE DE PHILOLOGIE FRANGAISE REGUEIL TRMESTRIEL GONSAGRÉ A L'ÉTUDE DES LANGUES, DIALEGTES ET PATOIS DE LA FRANGE

Publié par L. CLÉDAT, Doyen de la Faculté des Lettres de Lyon

Paris : 1ŏ fr. - Départements et Union postale : $16 \mathrm{fr}$.

\section{LE MOYEN AGE}

REVUE D'HISTOIRE ET DE PHLLLOGIE PARAISSANT TOUS LES DEUX MOIS

Direction : MN. A. MARIGNAN, M. PROU el M. WILMOTTE

Paris : $15 \mathrm{fr}$. - Départements et Union postale : $17 \mathrm{fr}$.

\section{REVUE DES BIBLIOTHĚQUES}

Recueil mensuel dirigé par MM. E. CHATELAIN et L. DOREZ

Paris : 15 fr. - Départements et Union postale : $17 \mathrm{fr}$.

\section{RECUEIL DE TRAVAUX RELATIFS A LA PHILOLOGIE}

ET A L'ARGHÉologie b́gYptienNes et ASSYRIENNES

Pour servir de bulletin à la Mission francaise du Caire, sons la direction de G. MASPERO

Prix d'abonnement au volume complet: Paris, $30 \mathrm{fr}$. -

Départements et Union postale : 32 fr. 





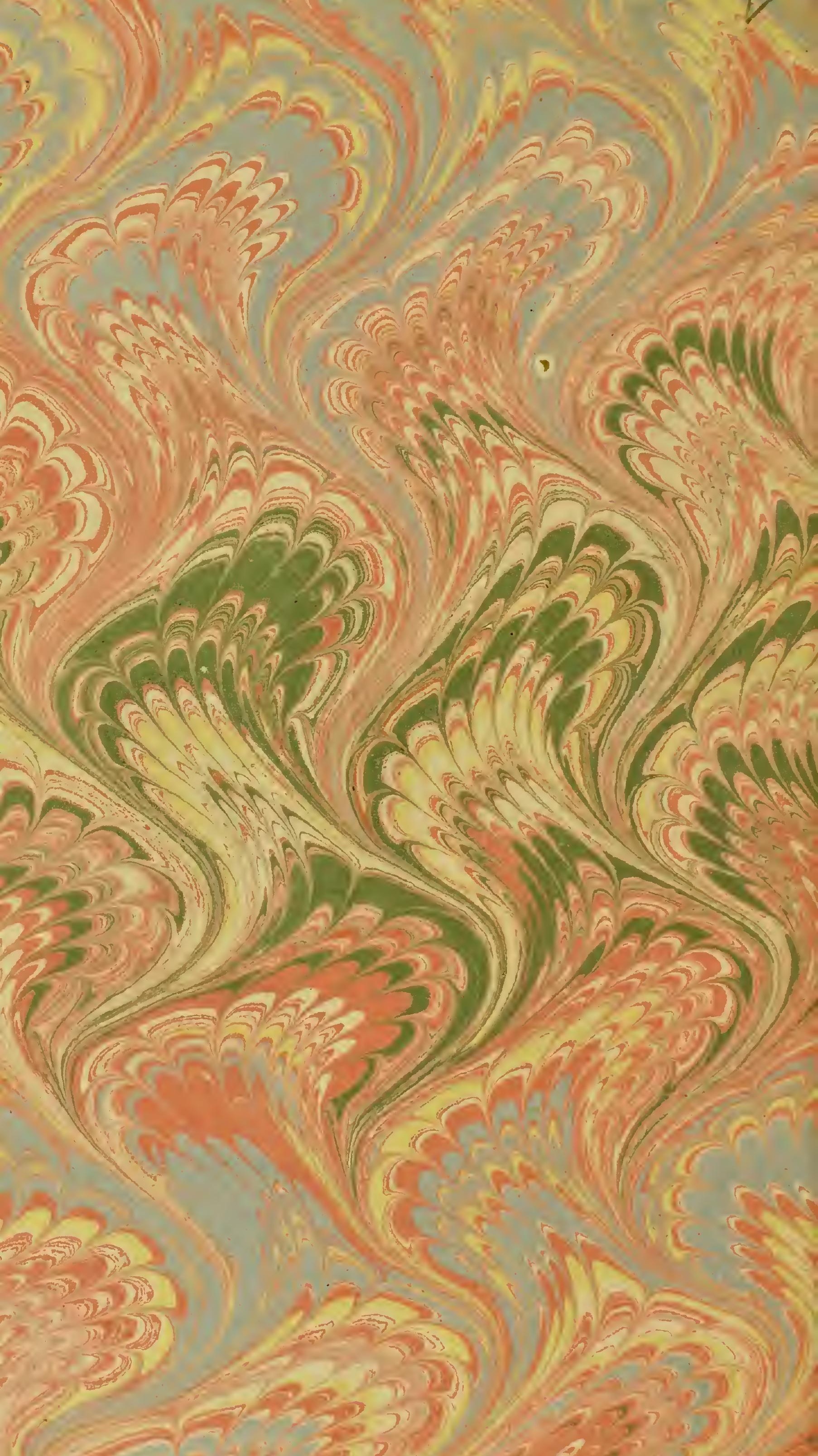




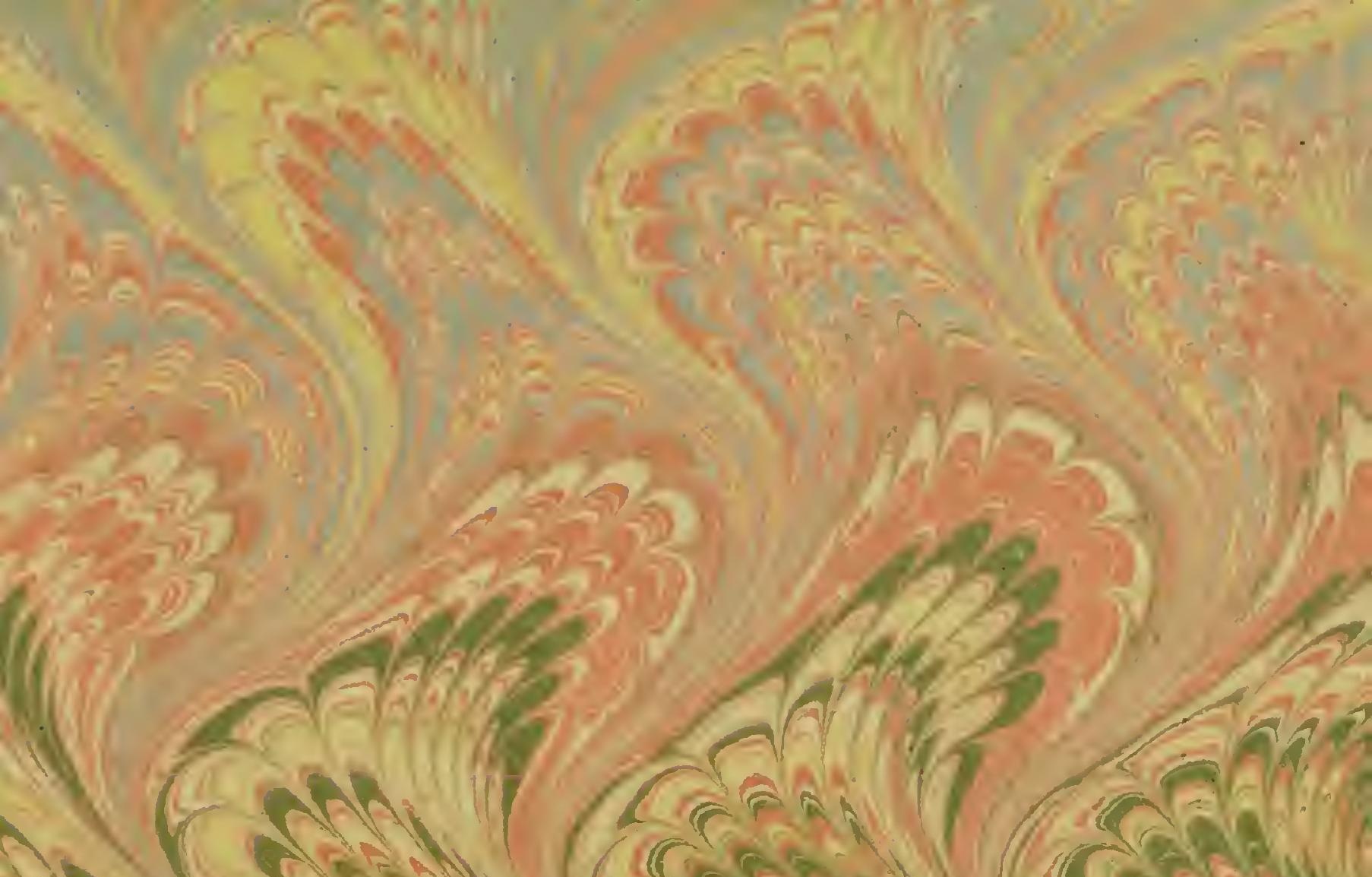


